\title{
Preventing the transmission of mitochondrial diseases
}

Citation for published version (APA):

Sallevelt, S. C. E. H. (2017). Preventing the transmission of mitochondrial diseases. [Doctoral Thesis, Maastricht University]. Datawyse / Universitaire Pers Maastricht. https://doi.org/10.26481/dis.20170203ss

Document status and date:

Published: 01/01/2017

DOI:

10.26481/dis.20170203ss

Document Version:

Publisher's PDF, also known as Version of record

\section{Please check the document version of this publication:}

- A submitted manuscript is the version of the article upon submission and before peer-review. There can be important differences between the submitted version and the official published version of record.

People interested in the research are advised to contact the author for the final version of the publication, or visit the DOI to the publisher's website.

- The final author version and the galley proof are versions of the publication after peer review.

- The final published version features the final layout of the paper including the volume, issue and page numbers.

Link to publication

\footnotetext{
General rights rights.

- You may freely distribute the URL identifying the publication in the public portal. please follow below link for the End User Agreement:

www.umlib.nl/taverne-license

Take down policy

If you believe that this document breaches copyright please contact us at:

repository@maastrichtuniversity.nl

providing details and we will investigate your claim.
}

Copyright and moral rights for the publications made accessible in the public portal are retained by the authors and/or other copyright owners and it is a condition of accessing publications that users recognise and abide by the legal requirements associated with these

- Users may download and print one copy of any publication from the public portal for the purpose of private study or research.

- You may not further distribute the material or use it for any profit-making activity or commercial gain

If the publication is distributed under the terms of Article $25 \mathrm{fa}$ of the Dutch Copyright Act, indicated by the "Taverne" license above, 
Preventing

the thansmission

of mitochondrial diseases

- $\geqslant 1$

$\therefore \therefore(6)$

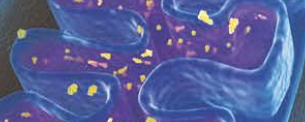

$402: 2$

He: $:$

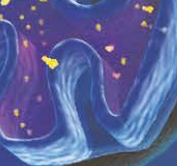

$(i, 2)$

$3, \ldots)$
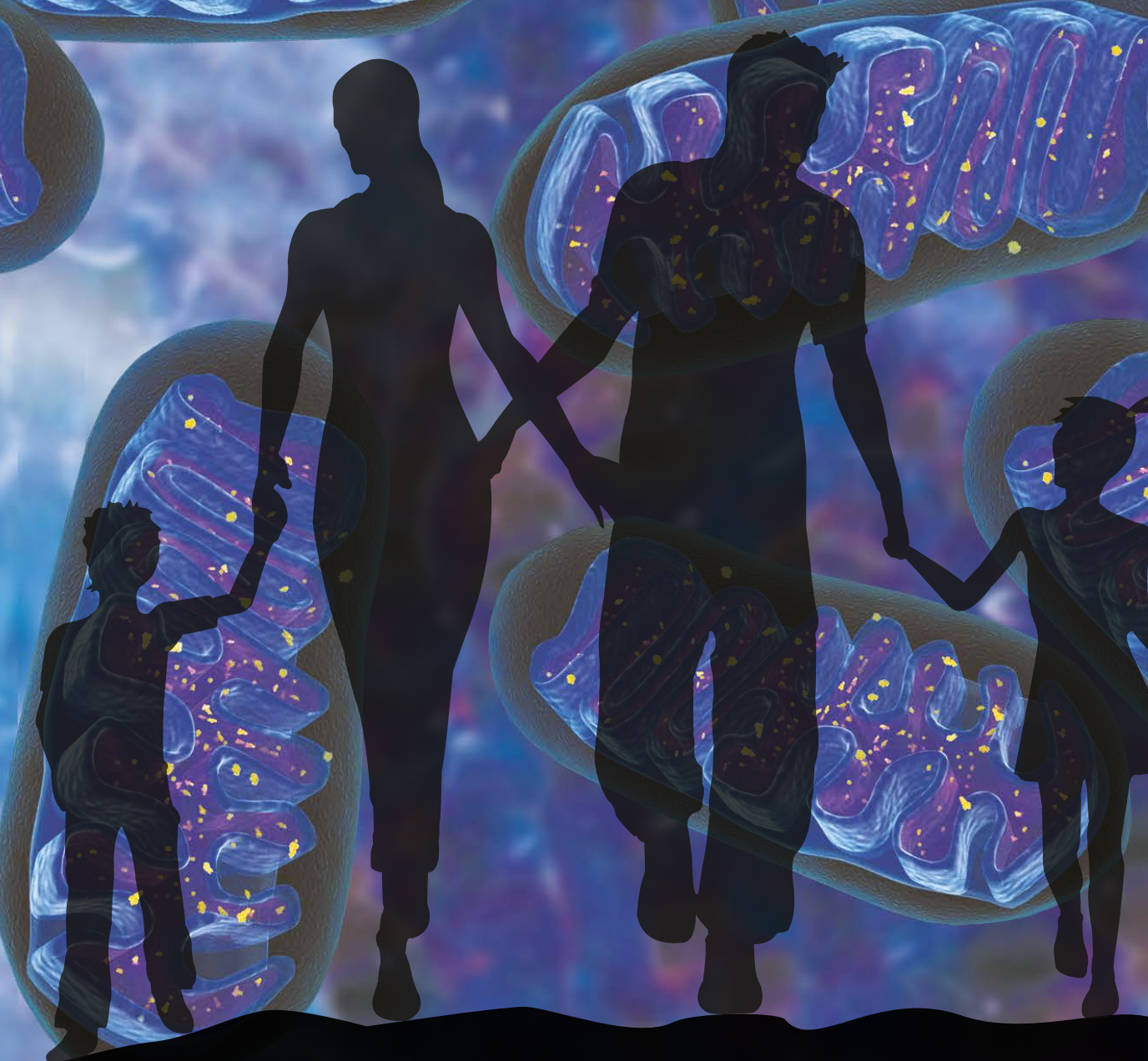

Suzanne Sallevelt 
(C) Suzanne Sallevelt, Maastricht 2017

No parts of this thesis may be reproduced or transmitted in any form or by any means, without prior permission in writing by the author, or when appropriate, by the publishers of the publications.

Lay-out: Tiny Wouters

Cover: Jennie Burgers

Production: Datawyse | Universitaire Pers Maastricht

ISBN: 9789461596581

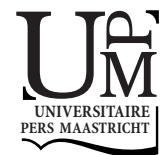




\title{
Preventing the transmission of mitochondrial diseases
}

\author{
PROEFSCHRIFT
}

ter verkrijging van de graad van doctor aan de Universiteit Maastricht, op gezag van de Rector Magnificus, Prof. dr. Rianne M. Letschert, volgens het besluit van het College van Decanen, in het openbaar te verdedigen op vrijdag 3 februari 2017 om 14.00 uur door

\section{Suzanne Cornelia Elisabeth Hélène Sallevelt} Geboren op 23 maart 1985 te Boxmeer 
Promotores:

Prof. dr. H.J.M. Smeets

Prof. dr. C.E.M. de Die-Smulders

\section{Co-promotor:}

Dr. I.F.M. de Coo (Erasmus MC Rotterdam)

\section{Beoordelingscommissie:}

Prof. dr. C.G. Faber (voorzitter)

Prof. dr. J.L.H. Evers

Dr. P. Lakeman (AMC Amsterdam)

Prof. dr. J.A.M. Smeitink (Radboud UMC Nijmegen) 


\section{Contents}

$\begin{array}{ll}\text { Abbreviations } & 7\end{array}$

$\begin{array}{lll}\text { Part I Introduction } & 9\end{array}$

Chapter $1 \quad$ Introduction - Aims and outline of the thesis $\quad 11$

Chapter 2 General overview - Reproductive options for mitochondrial 19 diseases

(C) Springer International Publishing Switzerland.

Screening the Single Euploid Embryo. 2015;Chapter 17:231-246

Part II Primary mutations of the mitochondrial DNA

39

Chapter 3 Preimplantation genetic diagnosis in mitochondrial DNA

disorders: challenge and success

J Med Genet. 2013;50:125-132

Chapter 4 Preimplantation genetic diagnosis for mitochondrial DNA

mutations: analysis of one blastomere suffices

Submitted

Chapter 5 Preimplantation genetic diagnosis for the m.14487T>C mitochondrial DNA mutation resulted in the birth of a healthy boy Accepted for publication in Hum Reprod.

Chapter 6 Mitochondrial DNA inheritance is determined by genetic drift and negative and positive selection

Submitted

Chapter 7 De novo mtDNA point mutations are common and have a low recurrence risk

J Med Genet. 2016 Jul 22 (Epub ahead of print)

Chapter 8 The distribution of mtDNA mutations in human patients is defined by random de novo occurrence followed by pre- and postnatal selection

Submitted 
Part III Primary mutations of the nuclear DNA

Chapter 9 Rapid resolution of blended or composite multigenic disease in infants by whole exome sequencing Accepted for publication in J Pediatr.

Chapter 10 A comprehensive strategy for exome-based preconception carrier screening Genet Med. 2016 Oct 27 (Epub ahead of print)

Part IV

Discussion and summary 237

Chapter 11 General discussion and future perspectives

Summary 275

Part V

Addendum

281

Nederlandse samenvatting 283

Valorisation 289

Dankwoord 297

Curriculum vitae 305

List of publications 309 


\section{Abbreviations}

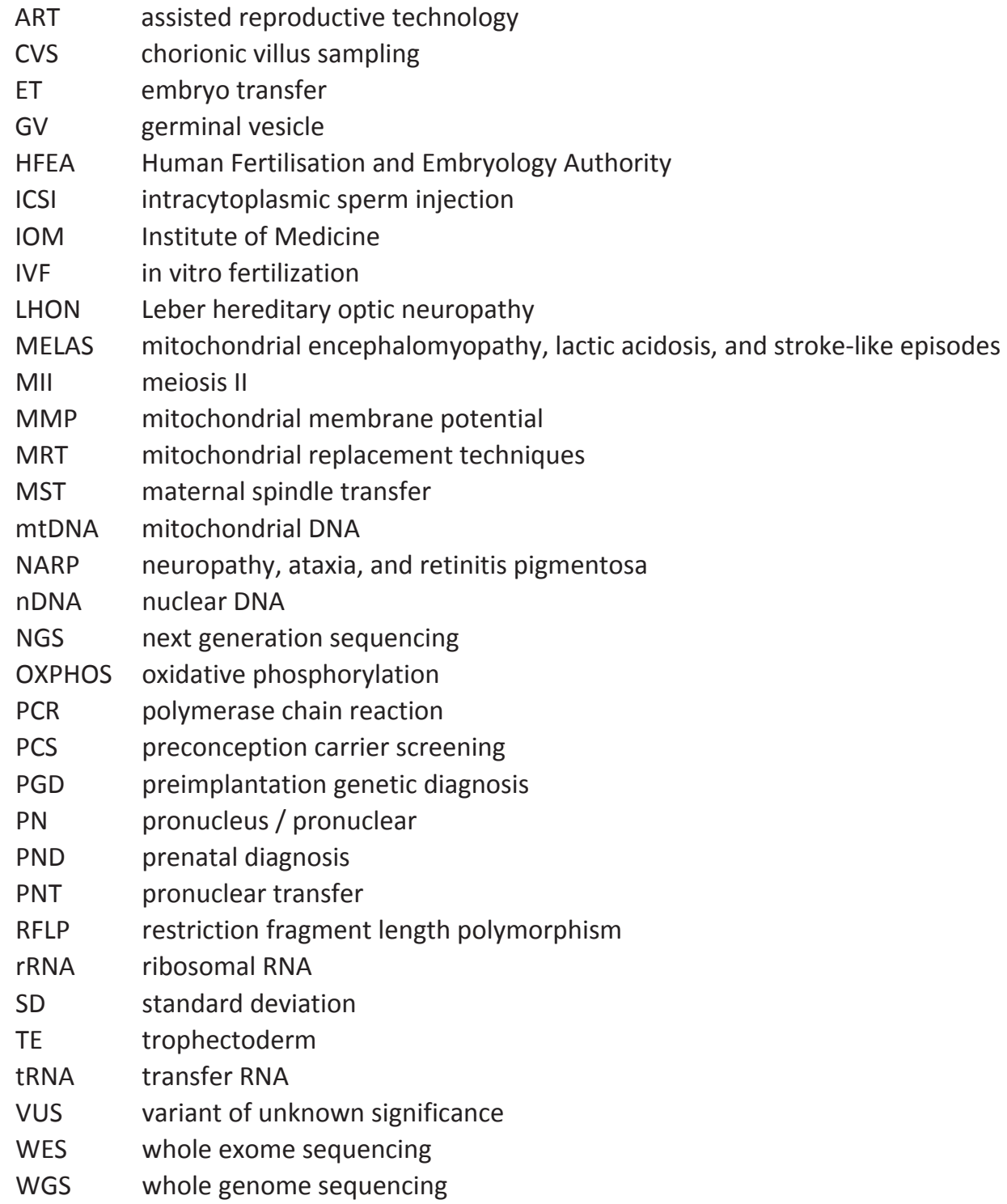





\section{PART |}

Introduction 



\section{CHAPTER 1}

Introduction - Aims and outline of the thesis 

Since the patients are the mean reason we are doing it all for, it seems suitable to start with some illustrative citations of families affected by mitochondrial diseases: "Our son died... He was 2 years and 4 months old. He had very little quality of life and was very ill and suffered greatly, as did the entire family." Another mother writes: "Losing a child is the most devastating thing a parent can ever go through. You never get over it." (Cited from a report of the Nuffield Council on Bioethics $\left.(U K)^{1}\right)$.

Mitochondrial diseases represent the most common inborn errors of metabolism ${ }^{2}$ and are associated with serious morbidity and mortality. ${ }^{3}$ This, combined with the current lack of treatment, regularly results in requests for reproductive counseling and options to prevent the birth of an affected child. The challenge lies in the complex genetics of these disorders, where much has still to be learnt. The primary genetic causes of mitochondrial diseases can be mutations in the mitochondrial DNA (mtDNA) or in the nuclear DNA (nDNA). Recurrence risks for mitochondrial diseases are often difficult to establish due to either the biology -unpredictable segregation patterns of mtDNA mutations- or the lack of a known genetic cause of the disease -particularly an issue for nuclear gene defects-.

Maastricht University and Maastricht University Medical Center harbor an expertise center for mitochondrial disorders, for diagnostic genetic services and for fundamental genetic research, in close collaboration with the Erasmus MC in Rotterdam. Furthermore, Maastricht is a unique center in the Netherlands regarding experience and license for preimplantation genetic diagnosis (PGD). The combination of both provided a unique opportunity for further studies on the transmission of mitochondrial disorders.

The central aim of this thesis is to develop new and optimize existing strategies to prevent the transmission of mitochondrial diseases, either caused by mtDNA mutations or nuclear gene defects, and to embed these in reproductive counseling for mitochondrial disorders. This central aim was splitted in the following concrete objectives:

- Determine the reliability and success of prenatal diagnosis (PND) and preimplantation diagnosis (PGD) for inherited or de novo mtDNA mutations and establish an optimal diagnostic protocol

- Characterize the different mechanisms affecting the germline transmission of mtDNA mutations and the effect this might have for recurrence risks

- Determine the frequency and distribution of de novo mtDNA mutations among mtDNA patients

- Determine the value of whole exome sequencing (WES) for establishing a genetic diagnosis in mitochondrial disease patients 
- Develop a protocol for preconception carrier screening based on whole exome sequencing

Analogous to the two subgroups of primary genetic causes of mitochondrial diseases, each with its own recurrence risks and reproductive counseling challenges, the thesis is divided accordingly.

\section{Primary mutations of the mitochondrial DNA}

Mitochondrial disorders can be caused by primary defects in the mitochondrial DNA (mtDNA), a small circular piece of DNA which is exclusively present in the mitochondria. Each mitochondrion contains many mtDNA copies and each cell contains multiple mitochondria. The mtDNA is only transmitted from a female to her children and is subject to a so-called genetic bottleneck, referring to a drastic decrease in mtDNA copy number during oogenesis. As a result, only a small number of mtDNAs is actually transmitted to the offspring, and subsequently expands to a new mtDNA pool. Consequently, when the mother has a mixture of mutated and wild-type mtDNA -a situation called heteroplasmy- mutation levels in the child can vary widely. The recurrence risk is potentially high but often difficult to specify. Not only predicting the mutation load to be transmitted to offspring, also predicting its phenotypic consequences complicate reproductive counseling of families with mtDNA diseases. The potential differences in mutation load between different tissues/cells of an individual and the -often- lack of a straightforward correlation between heteroplasmy levels and (severity of) symptoms contribute to the complexity of the matter. Particularly the latter is a major limitation in PND for these diseases, making the prediction of disease manifestations in the child difficult. PGD is an attractive alternative, circumventing decision-making regarding a pregnancy termination. As an introduction the available reproductive options for mitochondrial diseases are reviewed in Chapter 2. We investigated the diagnostic applicability of PGD for mtDNA mutations by determining if carriers produce sufficient oocytes with a mtDNA mutation load below the threshold of expression and by studying the predictive value of the test by analyzing interblastomere differences in mutation load (Chapter 3). As our dataset increased we investigated if we could switch from a 2-blastomere to a single blastomere diagnostic protocol, as this would increase the chances of becoming pregnant considerably (Chapter $\mathbf{4}$ ). In Chapter 5 we describe a successful PGD case for a 41-year old carrier of the m.14487T>C mtDNA mutation, illustrating the increasing use of PGD for mtDNA mutations.

Although the general concept of the genetic bottleneck is well-known, the exact underlying mechanisms are poorly understood. Differences in transmission patterns are observed between different mtDNA mutations. Understanding the underlying 
mechanisms are a key to determine the recurrence risk more accurately. We investigated the role of genetic drift and selection in the mtDNA bottleneck on PGD data (mutation loads in oocytes, zygotes and embryos) and data of patient cohorts of three mtDNA mutations and combined these with known data on biological metabolic/mitochondrial processes during oogenesis (Chapter 6).

In cases where an mtDNA point mutation is not inherited from the mother, but arose 'de novo' in the (affected) child, the situation is entirely different regarding the recurrence risk and preferred reproductive options. If the apparently de novo occurrence has been properly established by investigating multiple maternal tissues, the recurrence risk is expected to be low. This had however not been systematically studied so far. In our experience health care professionals are often unaware of it, discussing high recurrence risks and constraints of reproductive options with the parents based on invalid arguments, i.e. high mutation load in the child. We studied the frequency and recurrence risk of apparently de novo mtDNA point mutations, illustrated by some cases (Chapter 7), and provide recommendations for reproductive counseling. Also, the distribution of mutations identified in patients, comparing de novo and familiar cases, was analyzed and explained by different selection processes (Chapter 8).

\section{Primary mutations of the nuclear DNA}

As nuclear genes encode the overwhelming majority of mitochondrial proteins, mitochondrial diseases can also be caused by primary nuclear gene defects. In fact, the majority ( $85 \%)$ of mitochondrial patients have nuclear gene mutations as cause of their disease. These mutations follow the 'simple' Mendelian inheritance patterns and as such are less complex regarding recurrence risks and reproductive options. The main challenge in this subgroup is identifying the genetic cause, which is a prerequisite for establishing recurrence risk and offering reproductive options. With conventional diagnostic techniques the genetic defect could only be identified in a minority of mitochondrial patients without a primary mtDNA defect. Next-generation sequencing (NGS) techniques including unbiased approaches (whole exome sequencing, WES) are promising in increasing these numbers. We illustrate the usefulness of WES in establishing a comprehensive genetic diagnosis in two patients with heterogeneous clinical features (Chapter 9). One of these patients has consanguineous parents. For such consanguineous couples, an increased risk for more than one genetic disorder in their offspring may be present, as is illustrated not only by the reported family but also by multiple unpublished cases from our clinic. Up to now the possibilities to specify such potential risk were limited and often only empirical genetic risks could be provided, without being able to offer concrete reproductive options. With NGS 
techniques getting continuously cheaper and WES becoming routine in clinical genetic practice, the concept of genome-wide preconception carrier screening (PCS), not only for couples who have an a priori increased risk due to for example consanguinity, but for the general population as well, has emerged at the horizon. We investigated the applicability of WES for PCS and propose a strategy for clinical practice (Chapter 10).

In the general discussion (Chapter 11) we further incorporated our work into clinical practice by providing guidelines for reproductive testing strategies of mitochondrial diseases. Furthermore, developments in the field of preventing the transmission of mitochondrial diseases, particularly mitochondrial replacement therapies (MRT), discussed in relation to our findings and the current options available. WES and PCS are more broadly discussed, in the context of counseling and reproductive options, including WES for multigenic disease, incidental findings, the potential benefits of the identified recessive mutations for carrier-screening in the population of origin, and PCS for mtDNA mutations. 


\section{References}

1. Ethics of mitochondrial donation. Lancet (London, England). 2012;379(9834):2314.

2. Thorburn DR. Mitochondrial disorders: prevalence, myths and advances. J Inherit Metab Dis. 2004;27(3): 349-62.

3. Chinnery PF. Mitochondrial Disorders Overview. In: Pagon RA, Adam MP, Ardinger HH, et al., eds. GeneReviews(R). Seattle (WA): University of Washington, Seattle University of Washington, Seattle. All rights reserved.; 1993. Updated 2014 



\section{CHAPTER 2}

\section{General overview - Reproductive options for mitochondrial diseases}

(Published as: Selecting the right embryo in mitochondrial disorders) Suzanne C.E.H. Sallevelt, Joseph C.F.M. Dreesen, Irenaeus F.M. de Coo, Christine E.M. de Die-Smulders, Hubert J.M. Smeets

(c) Springer International Publishing Switzerland. Screening the Single Euploid EmbryoMolecular Genetics in Reproductive Medicine 2015; Chapter 17:231-246 


\section{Abstract}

Mitochondrial disorders are among the most common inborn errors of metabolism. Based on genetic etiology, they can be divided into several subgroups, which require different approaches for reproductive counseling, addressing differences in recurrence risks and reproductive options. The majority are caused by mutations in nuclear genes, which are currently being rapidly resolved by whole-exome sequencing (WES) and which segregate in a Mendelian way. Prenatal diagnosis (PND) or preimplantation genetic diagnosis (PGD) is available for these families to prevent the birth of another severely affected child. In at least $15 \%$ of cases, mitochondrial diseases are caused by mitochondrial DNA (mtDNA) mutations. Such mtDNA mutations can (1) be the result of a nuclear gene defect (multiple mtDNA deletions), (2) occur de novo (point mutations or large single mtDNA rearrangements), or (3) be maternally inherited (generally point mutations). For the maternally inherited heteroplasmic mtDNA mutations, the mitochondrial bottleneck is an important phenomenon defining the mtDNA mutation load in the offspring, with an often high (or unpredictable) recurrence risk and consequently complex counseling. PND to enable testing for mtDNA mutations is technically possible, but for many carriers of mtDNA point mutations, this approach is not applicable given the limitations in predicting phenotype. A total of 44 cases of PND performed in 35 mtDNA mutation carriers (m.3243A $>\mathrm{G}, \mathrm{m} .9176 \mathrm{~T}>\mathrm{C}$, m.8993T>G/C, m.8344A>G, m.13513G >A, m.11777C >A, m.10191T>C, m.10158T>C, m.3688G>A) have been described in literature. One additional unpublished case is presented here (m.3303C $>T$ ). For mtDNA point mutations which are most likely de novo in the affected child, the recurrence risk has been shown to be very low, and PND can be offered for reassurance. We have performed PND in four such cases, and six additional prenatal diagnoses were reported in literature. PND is also the most suitable option for female carriers with a low mutation load, demonstrating extreme skew such as mutations at nucleotide 8993. A fairly new option for preventing the transmission of mtDNA diseases is preimplantation genetic diagnosis (PGD). Embryos with mutant load below a mutation-specific or, if not possible, general expression threshold are transferred. A systematic review showed $\geq 95 \%$ probability of being unaffected at (muscle) mutant level of $18 \%$ or less, irrespective of the mutation. A total of 14 PGD cycles in six female carriers of heteroplasmic mtDNA mutations (m.3243A>G, m.8993T $>\mathrm{G}, \mathrm{m} .8344 \mathrm{~A}>\mathrm{G}$ ) have been completed at our center to date. All carriers produced oocytes below the threshold, and blastomere mutation load was representative for the whole embryo when two blastomeres were analyzed. A total of 12 PGD cycles in nine mtDNA mutation carriers (m.3243A>G, m.8993T>G) have been reported elsewhere. A total of six children were born after PGD and one pregnancy is ongoing. So, PGD is currently the best reproductive option for most maternally transmitted heteroplasmic mtDNA point mutations. Oocyte donation is a safe option to prevent the transmission of mtDNA disease to a future child for couples who reject PGD. Nuclear genome transfer techniques are currently investigated in research settings and might offer additional reproductive options in specific cases of mtDNA disease in the future. 


\section{Introduction}

Mitochondrial diseases are perhaps the most common of all inborn errors of metabolism ${ }^{1}$. They are highly variable in phenotype, ranging from severe and lethal infant/childhood manifestations to relatively mild symptoms with onset at adult age. Generally no treatment is available. The (recurrence) risk is dependent on the nature of the underlying primary genetic defect, and so are the available reproductive options. The primary genetic defect can be located either in nuclear DNA or mitochondrial DNA, and this feature will have direct consequences for the recurrence risk. In case of a nuclear gene defect, the disease segregates in autosomal dominant or recessive fashion with recurrence risks of $50 \%$ or $25 \%$, respectively. De novo nuclear mutations with a lower recurrence risk are rare. For mtDNA defects, the recurrence risk in the family is much more difficult to predict. Age- or drug-induced mtDNA defects (i.e., multiple deletions and mtDNA depletion) occur somatically with no transmission risk at all. However, multiple mtDNA deletions and mtDNA depletion can also be secondary to a primary defect in nuclear genes involved in mtDNA maintenance. In such a case, the recurrence risk is comparable to other nuclear gene mutations. At least $15 \%$ of mitochondrial diseases result from primary mitochondrial DNA (mtDNA) mutations ${ }^{1,2}$ including point mutations and large rearrangements. The recurrence risk of these mtDNA defects can vary between high/unpredictable and very low (in fact even zero for actual de novo mutations) depending on the nature of the underlying defect.

Two characteristics of mtDNA mutations which are key to understanding the complexity of mtDNA disease transmission include mitochondrial heteroplasmy and the genetic bottleneck. The majority of pathogenic mtDNA mutations resulting in severe disease are heteroplasmic, which means a mixture of mutant and wild-type mitochondria within a cell/tissue/individual. Heteroplasmy levels can vary between and within tissues of a carrier. Heteroplasmic mtDNA mutations are characterized by a threshold effect, meaning that there are no symptoms unless the mutant load (proportion of mutant mtDNA) exceeds a certain level. This threshold varies both within tissues and between different mutations and can depend on environmental factors, like the physical condition of the carrier.

Transmission of mtDNA occurs only from females to their offspring and is subject to a so-called genetic bottleneck. During oogenesis, the number of mtDNA molecules to be transmitted is reduced, and the resulting few mtDNAs become the founders for the offspring. In case the transmitting woman carries a heteroplasmic mtDNA mutation, this results in considerable variation in mtDNA mutant load among her individual oocytes ${ }^{3}$ and subsequently among offspring. The exact mechanism of the mitochondrial bottleneck is incompletely known, and some controversy exists concerning the content and "size" of the segregational unit. ${ }^{4-9}$ This size has been hypothesized to depend on 
the type of mtDNA mutation ${ }^{10-13}$ and to be individual dependent for certain mutations, ${ }^{10}$ possibly due to individual differences in initial mitochondrial copy number or genetic background. An important question is whether the bottleneck really is mutation-specific or that only the degree of skewing is mutation-specific, resulting in apparent differences in bottleneck size. Irrespective of the mechanism, the smaller the lowest amount of remaining mtDNA (segregational unit), the more rapid a (complete) shift of the mtDNA genotype can occur.

\section{Primary nuclear defects}

\section{Counseling and recurrence risk}

About $85 \%$ of mitochondrial diseases is caused by mutations in nuclear genes, which are currently being rapidly resolved by whole-exome sequencing (WES) and which segregate in a Mendelian way with recurrence risks of $25 \%$ or $50 \%$. In general, they do not affect the mtDNA, although part of the mtDNA defects, like multiple mtDNA deletions or mtDNA depletion, can be due to defects in nuclear genes involved in mtDNA maintenance. Comparable mtDNA defects can also occur somatically due to a nongenetic cause like aging (e.g., multiple mtDNA deletions) or mitotoxic drugs (e.g., nucleoside reverse transcriptase inhibitors can induce mtDNA depletion) with no recurrence risk at all; it is essential to define the cause of these mtDNA defects for proper estimation of the recurrence risk. De novo nuclear mutations with a low recurrence risk are rare.

\section{Reproductive testing options}

Nuclear gene defects resulting in mitochondrial disease are less complex with regard to reproductive options than primary mtDNA defects. Prenatal diagnosis (PND) and preimplantation genetic diagnosis (PGD) can reliably be offered, provided that the mutation is identified. The latter is the main problem in this category of mitochondrial diseases. Up to now, only in a minority of mitochondrial patients where mtDNA defects have been excluded, the genetic defect has been identified. New sequencing techniques (next-generation sequencing) and unbiased approaches (whole-exome sequencing) are promising in increasing this number, resulting in reproductive options for more at risk couples. In cases where the genetic defect is not known but the mtDNA has been excluded and an enzyme deficiency is detectable in fibroblasts, PND based on biochemical analysis might be an option (e.g., ${ }^{14-17}$ ). However, there are some limitations and pitfalls including sensitivity issues, the absence of the enzymatic defect in fibroblasts in $50 \%$ of patients, and limited knowledge on complex assembly and activity during embryonic development. ${ }^{18}$ 
In patient populations where consanguinity is more common, one should be aware that an increased risk of more than one genetic condition may be present. Thus, when offering reproductive options for a mitochondrial defect, there is still a realistic risk that the resulting child is affected by one or more other genetic abnormalities. Currently, consanguineous couples are empirically counseled regarding genetic risks if no genetic diseases have occurred in their families. In specific ethnic groups, carrier screening is offered for genetic diseases that are frequent in those populations. With the upcoming DNA sequencing techniques, preconception screening will become available on a broader basis.

\section{Familial primary mtDNA mutations}

\section{Counseling and recurrence risk}

The most common heteroplasmic mtDNA point mutation is the $\mathrm{m} .3243 \mathrm{~A}>\mathrm{G}$ mutation in the mitochondrial MT-TL1 gene, causing MELAS syndrome (mitochondrial encephalopathy, lactic acidosis, and stroke-like episodes), among others. This disorder is characterized by highly variable age at onset, symptom severity, and organ involvement. Correlation between the level of mutant mtDNA in blood and clinical features is poor due to the decrease in mutation load in blood cells with time. ${ }^{19-21}$ However, mutation levels in muscle ${ }^{22}$ and urine ${ }^{23-26}$ seem to be of higher prognostic value. Mutant load in oocytes and embryos of m.3243A $>\mathrm{G}$ carriers show large variation $^{10,27}$ approximating a Gaussian distribution, ${ }^{27}$ indicating that the level of mutant mtDNA in oocytes and embryos for this mutation is largely determined by random genetic drift. ${ }^{10,13,27}$ Existing data also point out that although in general a higher mutant load in the mother provides a higher risk of affected offspring, the recurrence risk for an individual $m .3243 A>G$ carrier remains very difficult to predict. ${ }^{27,28}$

\section{mtDNA point mutations demonstrating skewing}

Specific mtDNA mutations such as the nt8993 mutations do not show random transmission as with the m.3243A>G mutation, but rather demonstrate skewing. Due to the skewing to the extremes, there is an overrepresentation of oocytes and subsequent embryos with $0 \%$ and $100 \%$ mutation load. ${ }^{12,27,29-31}$ Accordingly, it is possible for a mother with a high mutant load to have a child with a low mutant load and vice versa. ${ }^{29}$ Compared to other mtDNA mutations however, for these mutations the individual recurrence risk can generally be better predicted and is low (the majority of oocytes not showing the mutation) when the mother's mutant load is low. The proportion of children with a high mutant load increases as the mother's mutant load increases. Other characteristics of the nt 8993 mutations are the rather good correlation between 
mutation load and phenotype ${ }^{29}$ and a quite uniform distribution of the mutation in all tissues. $^{32}$

\section{Reproductive testing options}

\section{Prenatal diagnosis}

In general, PND for mtDNA mutations has several limitations. A key problem is the often unreliable correlation between mutant load and disease severity, making it difficult to predict the clinical disease burden for the child and the likelihood of a couple having severely affected offspring. ${ }^{33}$ Secondly, mutation load in chorionic villi or amniocytes may not be representative for the mutant load in various fetal tissues. Limited available data suggest that the mutant load of extra-embryonic tissues such as chorionic villi can be considered representative for the mutant load in the fetus. ${ }^{29,33-35}$ However, these data predominantly concern the mutations at nucleotide 8993, which are skewing mutations (as discussed above). Other reports, on mtDNA polymorphic variants $^{36}$ and on the $\mathrm{m} .3243 \mathrm{~A}>\mathrm{G}$ mutation ${ }^{10,37}$ indicate that mtDNA mutations may segregate in placenta, questioning the reliability of (a single) CVS sample analysis for mitochondrial disorders carried out in a PND framework. This is further supported by intra-placental mutant load variations up to $55 \%$ which were reported by Monnot et al. [ESHG 2013, Paris]. Finally, the segregation of mtDNA mutations throughout embryofetal development and the distribution of mutation load between different fetal tissues are not fully clarified, although based on existing data from both skewing and non-skewing mutations, these issues do not seem to be a restriction. ${ }^{10,29-31,34,35,38-47}$ Indeed, the data show that the m.3243A>G mutation too segregates quite stable throughout the prenatal period, and this is remarkably different from postnatal segregation.

Advantages of PND include its relatively low cost and lower physical burden compared to IVF procedures and the fact that no oocyte donor needs to be available. Disadvantages of PND are the risk of a miscarriage as a result of the invasive nature of the procedure and the decision the couple has to make with regard to terminating the pregnancy if results are unfavorable. The latter is obviously even more difficult when no firm predictions can be offered concerning the clinical outcome of the fetus. It has been reported that for most mtDNA point mutations, a fetus with mutation load below $\sim 30 \%$ or above approximately $90 \%$ could be cautiously predicted to have a low or high probability, respectively, of being (severely) affected. ${ }^{33}$ However, such guidelines were not based on a systematic analysis and may not be applicable for all mtDNA mutations. A systematic meta-analysis showed $95 \%$ or higher chance of being unaffected at (muscle) mutant level of $\leq 18 \%$, irrespective of the mutation [47]. If possible, mutationspecific thresholds should be established as has been done with the m.3243A>G mutation (15\%) and the skewing m8993T>G mutations (30\%) (See below). A large range 
of mutation loads will fall within a "gray zone" with difficult or impossible interpretation, which is also the case for the m.3243A>G mutation. In 13 proven m.3243A>G carriers a total of 19 (of which 2 occurred in the same twin pregnancy) prenatal diagnoses have been reported. ${ }^{10,45,48,49}$ Another four prenatal diagnoses were performed in three women without any detectable m.3243A>G mutation in leukocytes in two, and leukocytes and urine in one of them, but with considerable risk of being carriers. ${ }^{45,49}$ The m.3243A>G mutation was not detected in chorionic villi or amniocytes when the mutation was absent in maternal leukocytes. ${ }^{45,49}$ This was the case in four females (five prenatal diagnoses), one of them having $3 \%$ mutant load in urinary tract cells. PND might indeed be an option for carriers with very low mutation load of the m.3243A>G mutation, although leukocytes seem not to be the best source to determine this - urine or muscle seem more appropriate. ${ }^{19-26}$ Moreover, analysis of both chorionic villi and amniocytes in a carrier with $21 \%$ mutant load in leukocytes failed to show the mutation in two fetuses. ${ }^{45}$ One of these fetuses was part of a twin pregnancy where mutation loads of $60 \%$ and $63 \%$ were detected in chorionic villi and amniocytes, respectively of the other fetus. The pregnancy was (selectively) reduced for the fetus with the mtDNA mutation. Another example of an m.3243A>G carrier (mutant loads of $1 \%$ in blood and $18 \%$ in urine) without detectable mutation in chorionic villi was reported by Nesbitt et al.. ${ }^{49}$ In two pregnancies of another carrier (with $80 \%$ mutant load in urinary tract cells), mutation loads between $23 \%$ and $35 \%$ were detected; both pregnancies were continued. ${ }^{45}$ The PND cases reported by Monnot et al. and Nesbitt et al. included four pregnancy terminations with mutant loads ranging from $59 \%$ to $77 \%$, whereas, for example, a pregnancy with $79 \%$ mutant load was continued. ${ }^{10,49}$ Chou et al. ${ }^{48}$ were confronted with a carrier when she was already 8 weeks pregnant. This case illustrated the limited value of PND for this particular mutation: both of her children harbored similar (high) levels of mutant mtDNA, the first child was severely affected and died at age $3 \frac{1}{2}$, whereas the second child was healthy at age 4 .

For the (skewing) nt 8993 mutations, PND is more feasible for carriers of a low mutation load, due to the high likelihood of unaffected offspring and a better correlation between mutant load and clinical phenotype. Seventeen cases of PND undertaken in 14 carriers with variable mutation loads of these mutations have been reported. ${ }^{30,35,38,41,42,49,50}$ Prenatal diagnosis of another mtDNA mutation, m.9176T>C, in the ATPase6 gene has been reported in a family after a thorough work-up and counseling $^{51}$ Limited data remain available about this mutation, including the genotype/phenotype correlation. The fetus appeared to have a mutation load of $87 \%$ (CVS)-88\% (amniocentesis), just below the assumed threshold of expression (90\%). The couple decided to continue the pregnancy. A healthy child was born, not showing any abnormalities at the age of 13 . Seven cases of PND in 7 carriers of other mtDNA mutations, namely m.8344A>G, m.13513G $>A$, m.11777C $>A \quad(n=2), m .10191 T>C$, 
m.10158T>C and m.3688G $>A$, respectively, were reported. ${ }^{49}$ In four of them the pregnancy was continued (two without mutation in chorionic villi, one with $3 \%$ mutant load, one with $54 \%$ mutant load), data on pregnancy continuation or termination were not available in the remaining three.

Recently, we performed PND for an unaffected carrier of the m.3303C $>$ T mtDNA mutation (unpublished data). The female's previous child, who had a nearly homoplasmic mtDNA mutation present in the blood and muscle, died at 5 months of age. Based on limited data from the literature and own experience, the expression threshold for this mutation was considered to be very high (90-95\%), and it was assumed that fetal mutant load between 0 and $50 \%$ would likely predict a subsequent child to be unaffected. In amniocytes, a mutant load of $\sim 38 \%$ was detected. The couple decided to continue the pregnancy, which is ongoing and thus far uneventful.

Altogether, PND is not a favorable choice for female carriers of mtDNA mutations with a high or unpredictable recurrence risk and a poor correlation between mutation load and phenotype; this is mainly because of difficulties in predicting the fetal phenotype when a certain mutation load is detected in chorionic villi or amniocytes. Still, when a carrier is already pregnant, PND can be offered with the understanding that a considerable chance exists that no interpretable result can be obtained.

\section{Preimplantation genetic diagnosis}

Another and fairly new option to prevent transmission of mtDNA mutations is preimplantation genetic diagnosis (PGD). ${ }^{10,27,31,52-55}$ In PGD, embryos obtained after in vitro fertilization (IVF) are analyzed at the blastomere stage (day 3), and only those with amounts of mutant mtDNA below the predicted threshold of expression are transferred in the uterus. Our threshold for the m.3243A>G mutation (MELAS) is $15 \%{ }^{22,56}$ and $30 \%$ for the skewing mutation $\mathrm{m} .8993 \mathrm{~T}>\mathrm{G}$ (Leigh). ${ }^{29}$ These guidelines are based on correlations between muscle mutation load and clinical manifestations, assuming that muscle mutation load correlates with the embryonic mutation load. This determination also embraces an arbitrary safety margin to correct for potential errors in determining heteroplasmy levels and for the limited number of data available.

Such thresholds, the preference of individual patients, and input from the clinician all appear to influence the decision on which embryos to be transferred in the setting of mtDNA disease screening. In the Netherlands, the threshold is determined before a cycle will be started, and the couple agrees that the single best embryo below this threshold will be transferred. In other countries like France, the couple has a more decisive role in choosing the embryo for transfer, even if the embryo manifests a mutation load above the threshold of expression. ${ }^{10,54,55}$ For most mtDNA mutations, insufficient data are available to establish a mutation-specific threshold level. A 
systematic meta-analysis showed $95 \%$ or higher chance of being unaffected at (muscle) mutant level of $18 \%$ or less, irrespective of the mutation. ${ }^{56}$ This offers a solution for the difficulties in establishing a transfer threshold for mtDNA mutations and implicates that PGD can be offered for any heteroplasmic mutation. Obviously, this meta-analysis is a guideline, and careful counseling is necessary, stressing the limitations of applying these findings on individual cases when data are scarce.

Prerequisites for PGD in mtDNA mutation carriers are the availability of oocytes with mutation load below the threshold for transfer and a comparable mutation load in all blastomeres of an embryo. We performed 14 PGD cycles in six mtDNA mutation carriers so far: four m.3243A $>\mathrm{G}$ carriers (a total of 9 cycles), one m.8993T $>\mathrm{G}$ carrier (4 IVF cycles), and one m.8344A>G carrier ( 1 cycle) ( ${ }^{27}$ and unpublished data). The m.8993T>G carrier achieved two pregnancies, one resulting in a healthy son and the other pregnancy is ongoing. The PGD results from this series are summarized in Figure 2.1. Here, all carriers did indeed produce oocytes with a mutation load below the threshold, and the blastomere mutation load was generally representative for the whole embryo (although single outliers occasionally occur).

Few additional reports of PGD performed for mtDNA disorders in other centers exist; a total of 12 cycles have been performed in nine mtDNA mutation carriers which resulted in the birth of five children. ${ }^{10,31,52,54,55}$ In general, equal mutation loads we observed for m.8993T>G among single blastomeres were concordant with previous reports. ${ }^{31,52,57}$ of note, interblastomere differences of $11 \%$ have been noted ${ }^{57}$ and fully descriptive data were not provided ${ }^{52}$; in our series blastomeres/embryos with no mutation were overrepresented (25/28 embryos), making it difficult to draw a general conclusion. Interblastomere differences for the m.3243A>G mutation generally larger and occurred more often than previously reported for this mutation, ${ }^{10,54}$ although Monnot et al. did not perform single blastomere analysis in all embryos. ${ }^{10}$ Vanderwoestyne et al. also reported large interblastomere variation of $24 \%$ in an $m .3243 \mathrm{~A}>\mathrm{G}$ embryo. ${ }^{53} \mathrm{As}$ interblastomere variation seems to occur more frequently in certain individuals, this itself might be a phenomenon subject to genetic factors ${ }^{27}$ although insufficient data exist for such individual risk stratifications. All data taken together, nicely plotted in a figure by Steffann et al., ${ }^{55}$ a generally homogeneous distribution of wild-type and mutant mtDNAs can be seen in individual human blastomeres regardless of the mutation, differing remarkably from data on artificially generated heteroplasmic macaque embryos. ${ }^{55,58}$ Based on the human data which shows that single blastomeres can diverge, it is advisable to analyze two blastomeres instead of just one. The adverse risk of removing two cells from the embryo at biopsy is a negative influence on live birth delivery, ${ }^{59}$ illustrating the difficult balance between a safe and correct diagnosis on the one hand and optimizing the chance of pregnancy on the other. 

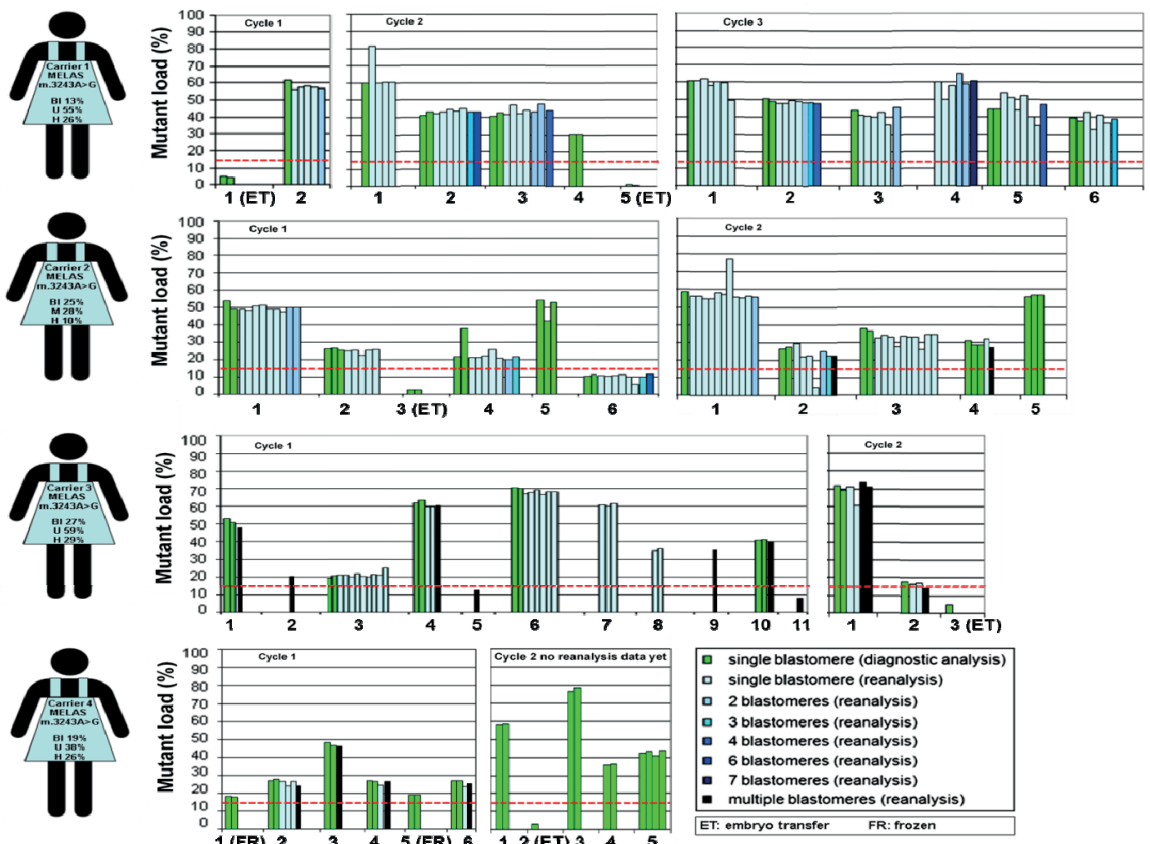

D single blastomere (diagnostic analysis)

- single blastomere (reanalysis)

- 2 blastomeres (reanalysis)

- 3 blastomeres (reanalysis)

- 4 blastomeres (reanalysis)

- 4blastomeres (reanalysis)

- 7 blastomeres (reanalysis)

- multiple blastomeres (reanalysis)

1 (FR) $2 \quad 3 \quad 4 \quad 5$ (FR) $6 \quad 12$ (ET) $3 \quad 4 \quad 5$

ET: embryo transfer $\quad$ FR: frozen
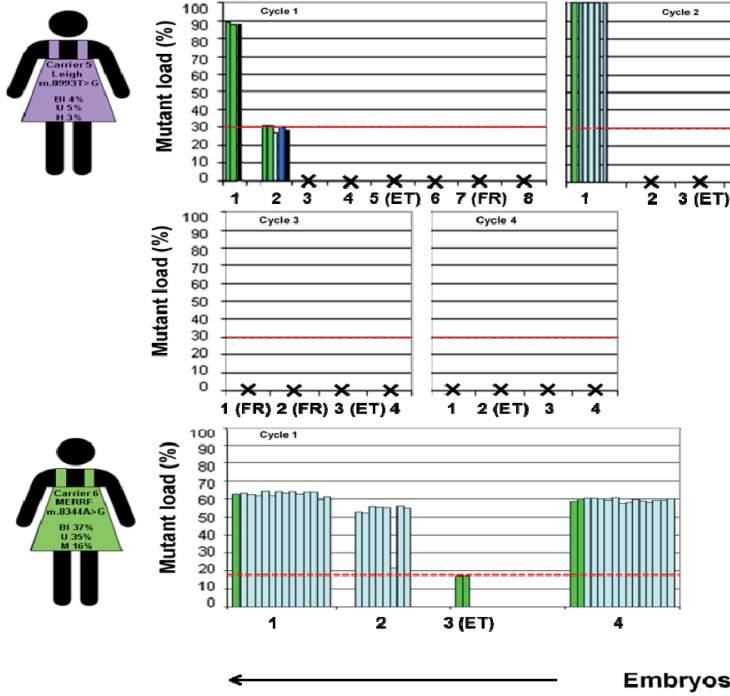

Figure 2.1 PDG cycles of the respective carrier females, performed in our center. Each cluster of bars represents an embryo with its tested blastomeres. The red dotted line represents the threshold level for transfer. For the Leigh carrier, the embryos in which the mutation was not detected are depicted as $\mathrm{X}$. For these embryos, the numbers of analyzed blastomeres are not visible in the figure. ET, embryo transfer; FR, frozen; BI, blood; $U$, urine; $M$, muscle; $H$, hair. 
Throphectoderm biopsy performed at the blastocyst stage provides a larger number of cells for analysis and appears to obviate the negative impact on live birth delivery. This approach would also enable the selection of an embryo based on both mutation load and genetic sex. Male offspring with an mtDNA mutation will not encounter the risk of transmitting the mutation to their offspring. So far, only one blastocyst PGD for an mtDNA mutation (m.3243A>G) has been performed in humans and results were promising with regard to the applicability of blastocyst trophectoderm biopsy and PGD for mtDNA mutation carriers ${ }^{54}$ (which had been supported by mouse data ${ }^{60}$ ). However, recently added follow-up data of the boy born after blastocyst PGD reported clinical symptoms and m.3243A>G mutant loads of $47 \%$ and $46 \%$ in blood and $52 \%$ and $42 \%$ in urine, respectively, at ages of 6 weeks and 18 months ${ }^{61}$. The blastocyst mutation load had been only $12 \% .{ }^{54}$ This contradicts the original report where no abnormal phenotype was reported and follow-up mutation load was $15 \%$ in buccal mucosa at age 1 month; at ages of 5 and 12 months, the mutation load was measured by a commercial lab and found to be $<10 \%$ in blood and undetectable in buccal mucosa and urine ${ }^{54}$. While technical differences do exist between methods used to determine the mutation load, this cannot explain such a large difference. It is unclear what has happened, and the authors of both papers should work collaboratively to clarify this.

Data on the in total 5 children born so far after blastomere PGD at the 8-cell stage are much more reassuring. ${ }^{10,27,31,52,55}$ Besides the balance between optimal diagnostic reliability versus subsequent reproductive outcome (concerning the number of cells to remove from the embryo for analysis), the number of embryos available for analysis also brings some conflicting considerations. From the perspective of geneticists, the more embryos available for study the better, since a larger sample improves the chances of having at least one embryo suitable for transfer (and thus improves the chances of delivering a healthy baby). However, there is a limit to the hormonal (over)stimulation that can be applied during IVF and some clinically affected carriers will be found a priori to be poor candidates for PGD/IVF treatment (based on inacceptable health risks). For mtDNA mutation carriers approved to undergo IVF, it is important to realize that PGD for these indications represents a substantial risk reduction of affected offspring but not an absolute risk exclusion. This should be carefully discussed during patients counseling and the informed consent process. A $0 \%$ mutation load only occurs seldomly (except for skewing mtDNA mutations). Furthermore, current data are suggestive, but not definitive, to guarantee that mutation load in the embryo stage will remain constant throughout life without passing the threshold level for symptoms at some later point. Nevertheless, we feel that for heteroplasmic mtDNA mutation carriers who want to have unaffected offspring who are biologically their own (and therefore not use donor oocytes), PGD represents the best therapeutic option at present. However, it should be acknowledged that PGD is not permitted in all jurisdictions. 
PND versus PGD: specific considerations with respect to skewing (8993) mtDNA point mutations

Although our considerations might be applicable to skewing mutations in general, only for the 8993 mutations do sufficient data currently exist. The characteristics of the nt8993 mutations make PND a feasible option for female carriers, particularly when mutation load is low. PGD is still an alternative in this group of mutation carriers with medium to high mutant load. The chance of producing embryos without the mutation is generally higher than for non-skewing mutations. In cases of high maternal mutation load, the majority of embryos is expected to have high mutation load although PGD will enable selection of those embryos with no or low mutation load. In contrast, PND would lead to the detection of multiple severely affected fetuses and recurrent pregnancy terminations.

If the maternal mutation load is low, the majority of embryos would be expected to be without the mutation. ${ }^{27}$ Due to the linear relationship between the mother's and her offspring's mutation load, ${ }^{29}$ for carriers with intermediate mutation load, the situation will be somewhere in the middle. In the choice between the two reproductive options, also the chances of achieving a pregnancy and the burden of PGD treatment will need to be carefully considered.

\section{Oocyte donation}

Perhaps the safest and most reliable method to prevent transmission of mtDNA disease is the use of donor oocytes accompanied by IVF using the partner's sperm. However, the supply of suitable donors may be limited in some locations, and oocyte donation is not lawfully allowed in every country. Maternal relatives such as sisters will generally not be suitable as oocyte donors, as they are at risk of carrying the mutation in their oocytes as well. The latter cannot be excluded based on the absence of the mutation in blood or other tissues. An important personal reason for couples to reject oocyte donation is the fact that the resulting child would not be genetically related to the mother.

\section{Nuclear transfer}

Nuclear transfer (maternal spindle transfer and pronuclear transfer) entails the transfer of the nuclear genome from an oocyte or zygote with mutated mtDNA in the cytoplasm (donor) to an enucleated acceptor oocyte or zygote of a healthy donor (acceptor) with presumably normal, mutation-free mtDNA. This technique is currently under investigation only in a research setting. ${ }^{62-67}$ Although promising, the safety and efficacy of nuclear transfer which has been noted in primate models has yet to be shown compatible with humans, so this approach requires further study; important ethical issues also require resolution. Whether this technique will be able to completely 
exclude the risk of transmitting an mtDNA mutation or attain merely a reduction of this risk to offspring is still unclear, since nuclear transfer cannot avoid the co-transfer of small amounts ( $<1 \%$ in spindle transfer) of mtDNA from the affected to the donor oocyte/zygote. Nuclear transfer techniques would offer a reproductive option for homoplasmic mtDNA mutation carriers and for heteroplasmic carriers with high mutation load, who might produce no or few oocytes/embryos with mutation load below the threshold.

\section{De novo mtDNA point mutations}

\section{Counseling and recurrence risk}

Besides being maternally inherited, mtDNA point mutation can also occur de novo in the affected individual, and this distinction makes a big difference for recurrence risk. If a de novo mutation is discovered in a child, this mutation is not expected to be present in his/her siblings. Due to the potential intra- and inter-tissue variability of mtDNA mutations, it can never be completely known for sure that the mother of the affected child does not carry any given mutation (i.e., a mutation load beneath the detection level, or the presence of an mtDNA mutation in any non-tested tissue, particularly the oocytes, would be impossible to exclude). However, proper analysis of multiple maternal tissues largely diminishes the residual risk of the mother having the mutation. Accordingly, for such de novo mtDNA point mutations the recurrence risk is low and the mutation is not expected to appear in a subsequent pregnancy. De novo mtDNA mutations are not rare events $\left({ }^{1}\right.$, Chapter 7$)$ yet many such couples may be counseled incorrectly and given a high recurrence risk (erroneously), based on the high mutation load in the child instead of absence of the mutation in the mother.

\section{Reproductive testing options}

Given the low recurrence risk of apparently de novo mtDNA mutations, PND is feasible as reassurance (Sallevelt et al in preparation). In four apparently de novo mtDNA disease cases based on the absence of the mutation in multiple maternal tissues, we have performed PND in a subsequent pregnancy. The mutation was not detected. In 9 of $>100$ reported cases describing apparently de novo mtDNA mutations, PND was performed in one or more subsequent pregnancies with normal findings in the majority, ${ }^{35,68-71}$ but recurrence in one family. ${ }^{49}$ The latter might be the result of gonadal mosaicism, or of failed detection of very low mutation load in the mother's lymphocytes and/or urinary epithelial cells due to the used sequencing method. This is currently being investigated further. PGD is, considering the burden of the treatment, not a favorable alternative in case of such a low recurrence risk. 


\section{mtDNA rearrangements}

\section{Counseling and recurrence risk}

Large, single mtDNA deletions are generally reported to occur sporadically, therefore having a low recurrence risk. ${ }^{72-74}$ Indeed, the available data indicate that a clinically unaffected mother of an affected child has a negligible risk of another affected child. ${ }^{73}$ Even for clinically affected mothers with an mtDNA deletion themselves, the risk of having clinically affected offspring is estimated to be low $(\sim 1: 24) .{ }^{73}$ mtDNA duplications are, like mtDNA point mutations, either maternally inherited or de novo and the same counseling aspects apply.

\section{Reproductive testing options}

PND seems the reproductive option of choice for de novo mtDNA rearrangements. Given the low recurrence risk even for women who carry an mtDNA deletion themselves, PND is the most feasible option in these cases, too. mtDNA duplications are, like mtDNA point mutations, either maternally inherited or de novo. For maternally inherited mtDNA duplications the same considerations regarding reproductive options apply as for maternally inherited mtDNA point mutations.

\section{Conclusion}

Mitochondrial diseases are common metabolic disorders with potentially high morbidity and mortality. Generally, no treatment is available. Couples with a child affected by a mitochondrial disorder or a positive family history and a high risk of affected offspring may request prevention of transmission to a (subsequent) child. Recurrence risks and the applicable reproductive testing options highly depend on the genetic etiology of the mitochondrial disease. For mitochondrial diseases due to nuclear gene defects, Mendelian segregation results in recurrence risks of $25 \%$ or $50 \%$. Both PND and PGD are applicable, once the causative mutation has been identified. Recurrence risks particularly for mtDNA mutations should be determined on an individual basis, for example, taking into account the nature of the mutation and the mutation load in the mother. The risk for female carriers of mtDNA point mutations (such as the m.3243A>G mutation) of having affected offspring is often difficult to establish, but it can be high. In those cases, PND is problematic mainly due to difficulties in predicting the phenotype with a given mutation load. PGD is currently the best reproductive testing option, although it should be regarded as a risk reduction strategy, rather than a method to exclude risk fully. Conversely, PGD is not the reproductive option of choice for apparently de novo mtDNA point mutations which have a very low recurrence risk, making PND feasible for reassurance. The same is true 
for (large) mtDNA deletions which occur almost exclusively de novo. PND is also applicable for skewing mtDNA mutations, particularly when the mother has a low mutation load. The development of nuclear transfer technology would complete the portfolio of reproductive choices to prevent the transmission of mtDNA disease.

\section{Acknowledgements}

"Stichting Metakids"; referring neurologists and clinical geneticists; PND and PGD teams Maastricht University Medical Centre'; Department of Obstetrics \& Gynecology Maastricht University Medical Centre ${ }^{+}$ 


\section{References}

1. Thorburn DR. Mitochondrial disorders: prevalence, myths and advances. J Inherit Metab Dis. 2004;27(3):349-362.

2. Rotig A, Munnich A. Genetic features of mitochondrial respiratory chain disorders. J Am Soc Nephrol. 2003;14(12):2995-3007.

3. Howell N, Halvorson S, Kubacka I, McCullough DA, Bindoff LA, Turnbull DM. Mitochondrial gene segregation in mammals: is the bottleneck always narrow? Hum Genet. 1992;90(1-2):117-120.

4. Lightowlers RN, Chinnery PF, Turnbull DM, Howell N. Mammalian mitochondrial genetics: heredity, heteroplasmy and disease. Trends Genet. 1997;13(11):450-455.

5. Cao L, Shitara H, Horii T, et al. The mitochondrial bottleneck occurs without reduction of mtDNA content in female mouse germ cells. Nat Genet. 2007;39(3):386-390.

6. Cree LM, Samuels DC, de Sousa Lopes SC, et al. A reduction of mitochondrial DNA molecules during embryogenesis explains the rapid segregation of genotypes. Nat Genet. 2008;40(2):249-254.

7. Khrapko K. Two ways to make an mtDNA bottleneck. Nat Genet. 2008;40(2):134-135.

8. Cao L, Shitara H, Sugimoto M, Hayashi J, Abe K, Yonekawa H. New evidence confirms that the mitochondrial bottleneck is generated without reduction of mitochondrial DNA content in early primordial germ cells of mice. PLoS Genet. 2009;5(12):e1000756.

9. Samuels DC, Wonnapinij P, Cree LM, Chinnery PF. Reassessing evidence for a postnatal mitochondrial genetic bottleneck. Nat Genet. 2010;42(6):471-472; author reply 472-473.

10. Monnot S, Gigarel N, Samuels DC, et al. Segregation of mtDNA throughout human embryofetal development: m.3243A>G as a model system. Hum Mutat. 2011;32(1):116-125.

11. Degoul F, Francois D, Diry M, et al. A near homoplasmic T8993G mtDNA mutation in a patient with atypic Leigh syndrome not present in the mother's tissues. J Inherit Metab Dis. 1997;20(1):49-53.

12. Blok RB, Gook DA, Thorburn DR, Dahl HH. Skewed segregation of the mtDNA nt 8993 (T-->G) mutation in human oocytes. Am J Hum Genet. 1997;60(6):1495-1501.

13. Brown DT, Samuels DC, Michael EM, Turnbull DM, Chinnery PF. Random genetic drift determines the level of mutant mtDNA in human primary oocytes. Am J Hum Genet. 2001;68(2):533-536.

14. Wanders RJ, Wijburg FA, Ruiter J, et al. Prenatal diagnosis of systemic disorders of the respiratory chain in cultured amniocytes and chorionic villus fibroblasts by studying the formation of lactate and pyruvate from glucose. J Inherit Metab Dis. 1992;15(1):84-91.

15. Ruitenbeek W, Wendel U, Hamel BC, Trijbels JM. Genetic counselling and prenatal diagnosis in disorders of the mitochondrial energy metabolism. J Inherit Metab Dis. 1996;19(4):581-587.

16. Faivre L, Cormier-Daire V, Chretien D, et al. Determination of enzyme activities for prenatal diagnosis of respiratory chain deficiency. Prenat Diagn. 2000;20(9):732-737.

17. Niers L, van den Heuvel L, Trijbels F, Sengers R, Smeitink J. Prerequisites and strategies for prenatal diagnosis of respiratory chain deficiency in chorionic villi. J Inherit Metab Dis. 2003;26(7):647-658.

18. Jacobs LJ, de Wert G, Geraedts JP, de Coo IF, Smeets HJ. The transmission of OXPHOS disease and methods to prevent this. Hum Reprod Update. 2006;12(2):119-136.

19. t Hart LM, Jansen JJ, Lemkes HH, de Knijff P, Maassen JA. Heteroplasmy levels of a mitochondrial gene mutation associated with diabetes mellitus decrease in leucocyte DNA upon aging. Hum Mutat. 1996;7(3):193-197.

20. Howell N, Ghosh SS, Fahy E, Bindoff LA. Longitudinal analysis of the segregation of mtDNA mutations in heteroplasmic individuals. J Neurol Sci. 2000;172(1):1-6.

21. Rahman S, Poulton J, Marchington D, Suomalainen A. Decrease of 3243 A-->G mtDNA mutation from blood in MELAS syndrome: a longitudinal study. Am J Hum Genet. 2001;68(1): 238-240.

22. Chinnery PF, Howell N, Lightowlers RN, Turnbull DM. Molecular pathology of MELAS and MERRF. The relationship between mutation load and clinical phenotypes. Brain. 1997;120 ( Pt 10):1713-1721.

23. Ma $Y$, Fang $F$, Yang $Y$, et al. The study of mitochondrial A3243G mutation in different samples. Mitochondrion. 2009;9(2):139-143.

24. McDonnell MT, Schaefer AM, Blakely EL, et al. Noninvasive diagnosis of the 3243A > G mitochondrial DNA mutation using urinary epithelial cells. Eur J Hum Genet. 2004;12(9):778-781. 
25. Frederiksen AL, Andersen PH, Kyvik KO, Jeppesen TD, Vissing J, Schwartz M. Tissue specific distribution of the 3243A-> G mtDNA mutation. J Med Genet. 2006;43(8):671-677.

26. Whittaker RG, Blackwood JK, Alston CL, et al. Urine heteroplasmy is the best predictor of clinical outcome in the m.3243A>G mtDNA mutation. Neurology. 2009;72(6):568-569.

27. Sallevelt SC, Dreesen JC, Drusedau M, et al. Preimplantation genetic diagnosis in mitochondrial DNA disorders: challenge and success. J Med Genet. 2013;50(2):125-132.

28. Chinnery PF, Howell N, Lightowlers RN, Turnbull DM. MELAS and MERRF. The relationship between maternal mutation load and the frequency of clinically affected offspring. Brain. 1998;121 (Pt 10):18891894.

29. White SL, Collins VR, Wolfe R, et al. Genetic counseling and prenatal diagnosis for the mitochondrial DNA mutations at nucleotide 8993. Am J Hum Genet. 1999;65(2):474-482.

30. White SL, Shanske S, Biros I, et al. Two cases of prenatal analysis for the pathogenic $T$ to $G$ substitution at nucleotide 8993 in mitochondrial DNA. Prenat Diagn. 1999;19(12):1165-1168.

31. Steffann J, Frydman N, Gigarel N, et al. Analysis of mtDNA variant segregation during early human embryonic development: a tool for successful NARP preimplantation diagnosis. J Med Genet. 2006;43(3):244-247.

32. White SL, Shanske S, McGill JJ, et al. Mitochondrial DNA mutations at nucleotide 8993 show a lack of tissue- or age-related variation. J Inherit Metab Dis. 1999;22(8):899-914.

33. Thorburn DR, Dahl HH. Mitochondrial disorders: genetics, counseling, prenatal diagnosis and reproductive options. Am J Med Genet. 2001;106(1):102-114.

34. Dahl HH, Thorburn DR, White SL. Towards reliable prenatal diagnosis of mtDNA point mutations: studies of nt8993 mutations in oocytes, fetal tissues, children and adults. Hum Reprod. 2000;15 Suppl 2:246-255.

35. Steffann J, Gigarel N, Corcos J, et al. Stability of the m.8993T->G mtDNA mutation load during human embryofetal development has implications for the feasibility of prenatal diagnosis in NARP syndrome. $J$ Med Genet. 2007;44(10):664-669.

36. Marchington DR, Scott-Brown M, Barlow DH, Poulton J. Mosaicism for mitochondrial DNA polymorphic variants in placenta has implications for the feasibility of prenatal diagnosis in mtDNA diseases. Eur $\mathrm{J}$ Hum Genet. 2006;14(7):816-823.

37. Marchington D, Malik S, Banerjee A, et al. Information for genetic management of mtDNA disease: sampling pathogenic mtDNA mutants in the human germline and in placenta. J Med Genet. 2010;47(4):257-261.

38. Harding $A E$, Holt IJ, Sweeney MG, Brockington M, Davis MB. Prenatal diagnosis of mitochondrial DNA8993 T----G disease. Am J Hum Genet. 1992;50(3):629-633.

39. Suomalainen A, Majander A, Pihko H, Peltonen L, Syvanen AC. Quantification of tRNA3243(Leu) point mutation of mitochondrial DNA in MELAS patients and its effects on mitochondrial transcription. Hum Mol Genet. 1993;2(5):525-534.

40. Matthews PM, Hopkin J, Brown RM, Stephenson JB, Hilton-Jones D, Brown GK. Comparison of the relative levels of the 3243 (A-->G) mtDNA mutation in heteroplasmic adult and fetal tissues. J Med Genet. 1994;31(1):41-44.

41. Leshinsky-Silver E, Perach M, Basilevsky E, et al. Prenatal exclusion of Leigh syndrome due to T8993C mutation in the mitochondrial DNA. Prenat Diagn. 2003;23(1):31-33.

42. Ferlin T, Landrieu P, Rambaud C, et al. Segregation of the G8993 mutant mitochondrial DNA through generations and embryonic tissues in a family at risk of Leigh syndrome. J Pediatr. 1997;131(3):447-449.

43. Poulton J, Marchington DR. Progress in genetic counselling and prenatal diagnosis of maternally inherited mtDNA diseases. Neuromuscul Disord. 2000;10(7):484-487.

44. Cardaioli E, Fabrizi GM, Grieco GS, Dotti MT, Federico A. Heteroplasmy of the A3243G transition of mitochondrial tRNA(Leu(UUR)) in a MELAS case and in a 25-week-old miscarried fetus. J Neurol. 2000;247(11):885-887.

45. Bouchet C, Steffann J, Corcos J, et al. Prenatal diagnosis of myopathy, encephalopathy, lactic acidosis, and stroke-like syndrome: contribution to understanding mitochondrial DNA segregation during human embryofetal development. J Med Genet. 2006;43(10):788-792.

46. Jenuth JP, Peterson AC, Fu K, Shoubridge EA. Random genetic drift in the female germline explains the rapid segregation of mammalian mitochondrial DNA. Nat Genet. 1996;14(2):146-151. 
47. Meirelles FV, Smith LC. Mitochondrial genotype segregation in a mouse heteroplasmic lineage produced by embryonic karyoplast transplantation. Genetics. 1997;145(2):445-451.

48. Chou YJ, Ou CY, Hsu TY, et al. Prenatal diagnosis of a fetus harboring an intermediate load of the A3243G mtDNA mutation in a maternal carrier diagnosed with MELAS syndrome. Prenat Diagn. 2004;24(5):367-370.

49. Nesbitt V, Alston CL, Blakely EL, et al. A national perspective on prenatal testing for mitochondrial disease. Eur J Hum Genet. 2014;22(11):1255-1259.

50. Bartley J, Senadheera, D., Park, P., Brar, H., Abad, D., Wong, L-J. Prenatal diagnosis of T8993G mitochondrial DNA point mutation in amniocytes by heteroplasmy detection. Am J Hum Genet. 1996;59:A317.

51. Jacobs LJ, de Coo IF, Nijland JG, et al. Transmission and prenatal diagnosis of the T9176C mitochondrial DNA mutation. Mol Hum Reprod. 2005;11(3):223-228.

52. Thorburn DR, Wilton L, Stock-Myer S. Healthy baby girl born following pre-implantation genetic diagnosis for mitochondrial DNA m.8993T>G mutation. Mol Genet Metab. 2009;98: 5-6.

53. Vandewoestyne M, Heindryckx B, De Gheselle S, et al. Poor correlation between polar bodies and blastomere mutation load in a patient with m.3243A>G tRNALeu(UUR) point mutation. Mitochondrion. 2012;12(4):477-479.

54. Treff NR, Campos J, Tao X, Levy B, Ferry KM, Scott RT, Jr. Blastocyst preimplantation genetic diagnosis (PGD) of a mitochondrial DNA disorder. Fertil Steril. 2012;98(5):1236-1240.

55. Steffann J, Gigarel N, Samuels DC, et al. Data from artificial models of mitochondrial DNA disorders are not always applicable to humans. Cell Rep. 2014;7(4):933-934.

56. Hellebrekers DM, Wolfe R, Hendrickx AT, et al. PGD and heteroplasmic mitochondrial DNA point mutations: a systematic review estimating the chance of healthy offspring. Hum Reprod Update. 2012;18(4):341-349.

57. Tajima H, Sueoka K, Moon SY, et al. The development of novel quantification assay for mitochondrial DNA heteroplasmy aimed at preimplantation genetic diagnosis of Leigh encephalopathy. J Assist Reprod Genet. 2007;24(6):227-232.

58. Lee HS, Ma H, Juanes RC, et al. Rapid mitochondrial DNA segregation in primate preimplantation embryos precedes somatic and germline bottleneck. Cell Rep. 2012;1(5):506-515.

59. De Vos A, Staessen C, De Rycke M, et al. Impact of cleavage-stage embryo biopsy in view of PGD on human blastocyst implantation: a prospective cohort of single embryo transfers. Hum Reprod. 2009;24(12):2988-2996.

60. Neupane J, Vandewoestyne M, Heindryckx B, et al. A systematic analysis of the suitability of preimplantation genetic diagnosis for mitochondrial diseases in a heteroplasmic mitochondrial mouse model. Hum Reprod. 2014;29(4):852-859.

61. Mitalipov S, Amato P, Parry S, Falk MJ. Limitations of preimplantation genetic diagnosis for mitochondrial DNA diseases. Cell Rep. 2014;7(4):935-937.

62. Tachibana M, Sparman M, Sritanaudomchai $H$, et al. Mitochondrial gene replacement in primate offspring and embryonic stem cells. Nature. 2009;461(7262):367-372.

63. Craven L, Tuppen HA, Greggains GD, et al. Pronuclear transfer in human embryos to prevent transmission of mitochondrial DNA disease. Nature. 2010;465(7294):82-85.

64. Craven L, Elson JL, Irving L, et al. Mitochondrial DNA disease: new options for prevention. Hum Mol Genet. 2011;20(R2):R168-174.

65. Tachibana $M$, Amato $P$, Sparman $M$, et al. Towards germline gene therapy of inherited mitochondrial diseases. Nature. 2013;493(7434):627-631.

66. Paull D, Emmanuele V, Weiss KA, et al. Nuclear genome transfer in human oocytes eliminates mitochondrial DNA variants. Nature. 2013;493(7434):632-637.

67. Smeets HJ. Preventing the transmission of mitochondrial DNA disorders: selecting the good guys or kicking out the bad guys. Reprod Biomed Online. 2013;27(6):599-610.

68. Lebon $\mathrm{S}, \mathrm{Chol} \mathrm{M}$, Benit $\mathrm{P}$, et al. Recurrent de novo mitochondrial DNA mutations in respiratory chain deficiency. J Med Genet. 2003;40(12):896-899.

69. Shanske S, Coku J, Lu J, et al. The G13513A mutation in the ND5 gene of mitochondrial DNA as a common cause of MELAS or Leigh syndrome: evidence from 12 cases. Arch Neurol. 2008;65(3):368-372. 
70. Gotz A, Isohanni P, Liljestrom B, et al. Fatal neonatal lactic acidosis caused by a novel de novo mitochondrial G7453A tRNA-Serine ((UCN)) mutation. Pediatr Res. 2012;72(1):90-94.

71. Shanske $S$, Naini $A, C h m a i t ~ R H$, et al. Mutation in an mtDNA protein-coding gene: prenatal diagnosis aided by fetal muscle biopsy. J Child Neurol. 2013;28(2):264-268.

72. Zeviani M, Antozzi C. Mitochondrial disorders. Mol Hum Reprod. 1997;3(2):133-148.

73. Chinnery PF, DiMauro S, Shanske S, et al. Risk of developing a mitochondrial DNA deletion disorder. Lancet. 2004;364(9434):592-596.

74. Chinnery PF, Hudson G. Mitochondrial genetics. Br Med Bull. 2013;106:135-159. 



\section{PART II}

Primary mutations of the mitochondrial DNA 


\section{CHAPTER 3}

\section{Preimplantation genetic diagnosis in mitochondrial}

DNA disorders: challenge and success

Suzanne C.E.H. Sallevelt, Joseph C.F.M. Dreesen, Marion Drüsedau, Sabine Spierts, Edith Coonen, Florence H.J. van Tienen, Ronald J.T. van Golde, Irenaeus F.M. de Coo, Joep P.M. Geraedts, Christine E.M. de Die-Smulders, ${ }^{\#}$ Hubert J.M. Smeets ${ }^{\#}$

\# these authors share senior authorship

J Med Genet. 2013;50:125-132 


\section{Abstract}

\section{Background}

Mitochondrial or oxidative phosphorylation diseases are relatively frequent, multisystem disorders; in about $15 \%$ of cases they are caused by maternally inherited mitochondrial DNA (mtDNA) mutations. Because of the possible severity of the phenotype, the lack of effective treatment, and the high recurrence risk for offspring of carrier females, couples wish to prevent the transmission of these mtDNA disorders to their offspring. Prenatal diagnosis is problematic for several reasons, and concern the often poor correlation between mutation percentages and disease severity and the uncertainties about the representativeness of a fetal sample. A new option for preventing transmission of mtDNA disorders is preimplantation genetic diagnosis (PGD), which circumvents these problems by transferring an embryo below the threshold of clinical expression.

\section{Methods}

We present the data on nine PGD cycles in four female carriers of mitochondrial diseases: three mitochondrial encephalopathy, lactic acidosis and stroke-like episodes (MELAS) (m.3243A>G), and one Leigh (m.8993T>G). Our threshold for transfer after PGD is $15 \%$ for the $\mathrm{m} .3243 \mathrm{~A}>\mathrm{G}$ mutation and $30 \%$ for the $\mathrm{m} .8993 \mathrm{~T}>\mathrm{G}$ mutation.

\section{Results}

All four female carriers produced embryos eligible for transfer. The m.8993T>G mutation in oocytes/embryos showed more skewing than the m.3243A>G. In about $80 \%$ of the embryos the mutation load in the individual blastomeres was fairly constant (interblastomere differences $<10 \%$ ). However, in around $11 \%$ (in embryos with the m.3243A>G mutation only), the mutation load differed substantially $(>15 \%)$ between blastomeres of a single embryo, mostly as a result of one outlier. The m.8993T>G carrier became pregnant and gave birth to a healthy son.

\section{Conclusions}

PGD provides carriers of mtDNA mutations the opportunity to conceive healthy offspring. 


\section{Introduction}

Mitochondrial or oxidative phosphorylation diseases are multisystem disorders, and in about $15 \%$ of cases they are caused by maternally inherited mitochondrial DNA (mtDNA) mutations. ${ }^{1}$ Carrier frequency for pathogenic mtDNA mutations in the normal population is high; one in $400^{2}$ to $>1$ in $200^{3}$. Mitochondrial diseases can manifest with symptoms in many different organs, varying profoundly in severity and age of onset. Fatally affected newborns represent the severe end of the spectrum. The majority of the severe pathogenic mtDNA mutations are heteroplasmic and characterized by a threshold effect, meaning that there are no symptoms unless the mutant load (proportion of mutant mtDNA) exceeds a certain level. This threshold varies both within tissues and between different mutations. In some mtDNA disorders, onset and severity of symptoms are clearly related to mutation load. ${ }^{4,5}$ Often, however, the phenotype and mutation load correlate poorly. ${ }^{6-8}$ MtDNA mutations are transmitted only from females to their offspring. The percentage heteroplasmy inherited by the fetus is affected by the 'mitochondrial bottleneck'. During oogenesis the number of mtDNA molecules to be transmitted is reduced, and the resulting few mtDNAs become the founders for the offspring, resulting in considerable variation in mutant mtDNA among individual oocytes ${ }^{9}$ and subsequently among offspring. The "size" of this bottleneck seems to depend on the type of mtDNA mutation ${ }^{10-12}$ and may even be individualdependent for certain mutations. ${ }^{12}$

Mitochondrial encephalopathy, lactic acidosis and stroke-like episodes (MELAS), mainly caused by the m.3243A>G mutation in the mitochondrial MT-TL1 gene, is a serious, common heteroplasmic mtDNA disorder. ${ }^{13}$ Age at onset, severity of symptoms, as well as organs involved are highly variable. Correlation between the level of mutant mtDNA in blood and clinical features is poor; however, mutation levels in muscle ${ }^{6}$ and urine ${ }^{14-17}$ seem to be of higher prognostic value. The level of mutant mtDNA in blood falls with time and, although rare, may become undetectable in women of fertile age. ${ }^{13}$ The frequency of affected offspring seems to be related to the level of mutant mtDNA in the mother's blood. ${ }^{18}$. Although segregation of the m.3243A>G mutation is thought to be largely determined by random genetic drift, ${ }^{11,12}$ the mutation percentage of the m.3243A>G mutation is reported to be higher in offspring than expected by random transmission only, ${ }^{19,20}$ which might be due to biases ${ }^{19,20}$ or sampling errors. ${ }^{21}$ On the other hand, a selection event against high mutant load might occur during meiosis and mitosis. $^{22}$

A relatively common mtDNA mutation in children is the $\mathrm{m} .8993 \mathrm{~T}>\mathrm{G}$ mutation, leading to Leigh syndrome or NARP (neuropathy, ataxia, and retinitis pigmentosa), depending on mutant load, ${ }^{23}$ which correlates rather well with the phenotype. ${ }^{4}$ Also the risk of affected offspring can quite accurately be predicted by the mother's mutation load. ${ }^{4}$ 
The transmission of the m.8993T>G mutation is generally skewed with an overrepresentation of oocytes with $0 \%$ and $100 \%$ mutation load. ${ }^{4,10,23,24}$ As for the m.3243A>G mutation, the possibility of preferential mutant genome transmission has also been proposed for the $\mathrm{m} .8993 \mathrm{~T}>\mathrm{G} .{ }^{19,20}$

Because of the high frequency of mitochondrial disorders, the possible severity of the phenotype, the lack of effective treatment, and the high recurrence risk for offspring of carrier females, couples may aim to prevent transmission. A key problem in prenatal diagnostics (PND) for mitochondrial disorders is the unreliable correlation between the mutant load and the disease severity, making it difficult to predict the clinical severity of the disease in the child and the likelihood of a couple having affected offspring. ${ }^{13}$ Furthermore, data on the representativeness of chorionic villi or amniocytes for the mutant load in the different fetal tissues are contradicting. ${ }^{4,7,12,25-28}$ Mutant load distribution between different fetal tissues and segregation throughout the embryofetal development does not seem to be a restriction. ${ }^{4,12,23-26,29-38}$

A fairly new option for preventing transmission of mtDNA diseases is preimplantation genetic diagnosis (PGD). ${ }^{12,24,39-41}$ In PGD, embryos obtained after vitro fertilization (IVF) are analysed, and only those with amounts of mutant mtDNA below the predicted threshold of (severe) expression are transferred to the uterus. Uncertainties concerning this threshold make it difficult to develop PGD for all mtDNA mutations, although a recent meta-analysis provided some guidelines. ${ }^{41}$ Our threshold for the m.3243A>G mutation (MELAS) is 15\% and for the m.8993T>G (Leigh) 30\%, based on data of muscle mutation load and clinical manifestations, ${ }^{4,6}$ assuming that the muscle mutation load correlates with the embryonic mutation load, and with a safety margin to correct for potential errors in determining heteroplasmy levels.

We present data on nine PGD cycles in four female carriers of mitochondrial disease; three women with the MELAS m.3243A>G mutation and one with the Leigh m.8993T>G mutation. Worldwide, very few centres perform PGD for mitochondrial diseases, ${ }^{12,24,40}$ and our data will help in understanding the dynamics of mtDNA mutations, evaluating the suitability of PGD for mtDNA disorders, and optimising guidelines regarding the PGD procedures.

\section{Methods}

The study was approved by the local Ethical Committee. 


\section{Patients}

Three couples with the m.3243A>G mutation and one with the m.8993T>G mutation were counseled for PGD. Couples received verbal and written information on IVF and intracytoplasmic sperm injection (ICSI), single cell procedures, success rates of the IVF/PGD treatment, risks of misdiagnosis, and the worldwide limited experience of PGD for mtDNA disorders. Prenatal diagnosis to confirm the PGD diagnosis was offered. Informed consent was given by both partners before treatment, including consent for re-analysis of non-transferred and non-frozen embryos. The threshold level for transfer, being $15 \%$ for the $\mathrm{m} .3243 \mathrm{~A}>\mathrm{G}$ and $30 \%$ for the $\mathrm{m} .8993 \mathrm{~T}>\mathrm{G}$ mutation, was discussed with the couples. The couples could not opt for transfer of embryos above that threshold, for example, in case none of the embryos would have mutation loads below the threshold. The Leigh couple requested transferal of only embryos with $0 \%$ mutation load, but after counseling a threshold level for transfer of $5 \%$ was agreed upon as no severe symptoms will occur at that level. The female carriers underwent a clinical examination to determine contraindications for the IVF procedure. Mutation loads in blood, hair, urine and/or muscle of the carrier females were established.

\section{IVF/ICSI/PGD procedure}

Oocytes were retrieved under ultrasound guidance, following controlled ovarian stimulation. ${ }^{42}$ After $5 \mathrm{~h}$ of maturation, MII oocytes were fertilised with $\mathrm{ICSI}^{43}$ followed by embryo culturing. ${ }^{44}$ At day 3 post-fertilization, blastomeres were biopsied, one blastomere from 4-6-cell stage embryos and two blastomeres from 7-cell stage and beyond. Biopsies were performed in $\mathrm{Ca} 2+$ and $\mathrm{Mg} 2+$ free medium with a micromanipulator (Narishige, ONO-121, Paes Nederland, Zoeterwoude, The Netherlands) mounted on an inverted microscope (Olympus, IX-70, Paes Nederland). Biopsied blastomeres were washed three times in phosphate buffered saline solution with $1 \%$ polyvinylpyrrolidone molecular weight 360000 (Sigma-Aldrich Chemie, Zwijndrecht, the Netherlands) and $0.1 \mathrm{mg} / \mathrm{ml}$ phenol red (Sigma), and transferred to a $0.2 \mathrm{ml} \mathrm{PCR}$ reaction tube. Blank samples were taken from the last washing droplet. After PGD analysis, one (preferably) or two embryos with a mutation load below the threshold were selected for embryo transfer at day 4 after fertilization. Selection was, besides mutation load, based on embryo score criteria. ${ }^{44}$

\section{PCR and single cell quantification assay}

\section{PCR procedure m.3243A>G and 8993T>G mutations}

The alkaline lysis buffer was decontaminated from exogen DNA by $1 \mathrm{~h}$ ultraviolet C (UVC) irradiation using a UV-C lamp type TUV 30W/G30T8 long life (Philips). The PCR mix without the DNA-polymerase was decontaminated by Mnll restriction enzyme $(5 \mathrm{U} / \mu \mathrm{l}$; Biolabs) incubation for $2 \mathrm{~h}$ at $37^{\circ} \mathrm{C}$ followed by 30 min inactivation at $65^{\circ} \mathrm{C}$. Blank 
samples were included in every PCR series to monitor DNA contamination. Cells were lysed by adding alkaline lysis buffer $(50 \mathrm{mM}$ dithiothreitol (DTT) (Pharmacia Biotech)/200 mM NaOH) and $10 \mathrm{~min}$ incubation at $65^{\circ} \mathrm{C}$. After cell lysis, PCR was performed on the GeneAmp PCR System 9700 (Perkin-Elmer Applied Biosystems) in a total volume of $50 \mu \mathrm{l}$, containing $1 \mathrm{X}$ PCR buffer (Invitrogen) and $1 \mathrm{U}$ of Taq DNA polymerase (Invitrogen), $2 \mathrm{mM} \mathrm{MgCl} 2$ (Invitrogen), and $0.1 \mathrm{mM}$ dNTP (Pharmacia). To neutralize the alkaline lysis buffer Tricine (20 mM pH 4.95 (Sigma)) was used. Unlabeled forward and reverse primer sets were used in the first round and varied from 3-15 pmol primer per sample (Table 3.1).

Table 3.1 Primers used for PCR amplification of the m.3243A>G and m.8993T>G mutations and the m.8993T>G digestion control amplicon

\begin{tabular}{llc}
\hline Primer name & Primer sequence & [C ] pmol/ $\boldsymbol{\mu l}$ primer used \\
\hline NARP_294F & CACACCTACACCCCTTATCCC & 0.2 \\
NARP_229R & TCATTATGTGTTGTCGTGCAG & 0.2 \\
NARP229R-VIC & TCATTATGTGTTGTCGTGCAG & 0.3 \\
HpalINARP (6-FAM) & CCTCTACCTGCACGACAACAC & 0.4 \\
HpalINARP-R & GAGGAGCGTTATGGAGTGGA & 0.4 \\
MELAS_200R & tttcgttcggtaagcattag & 0.3 \\
MELAS_82F & caacttagtattatacccacac & 0.06 \\
MELAS_82F-6FAM & caacttagtattatacccacac & 0.3 \\
\hline
\end{tabular}

C, concentration; MELAS, mitochondrial encephalopathy, lactic acidosis and stroke-like episodes; NARP, neuropathy, ataxia, and retinitis pigmentosa.

First round PCR started with 5 min denaturation at $94^{\circ} \mathrm{C}$ followed by 38 cycles of 1 min denaturation at $92^{\circ} \mathrm{C}, 45 \mathrm{~s}$ annealing at $53^{\circ} \mathrm{C}$, and $45 \mathrm{~s}$ elongation at $72^{\circ} \mathrm{C}$, followed by a final elongation step of $7 \mathrm{~min}$ at $72^{\circ} \mathrm{C} .15 \mathrm{ml}$ of first round amplification product was adjusted to $50 \mathrm{ml}$ with second round PCR mix, containing 1 X PCR buffer (Invitrogen) 1 $\mathrm{U}$ of DNA polymerase, $2 \mathrm{mM} \mathrm{MgCl} 2$ (Invitrogen) and fluorescently labeled primer (table 3.1). One final amplification cycle was performed of $5 \mathrm{~min}$ denaturation at $94^{\circ} \mathrm{C}, 1 \mathrm{~min}$ annealing at $53^{\circ} \mathrm{C}$, and 7 min elongation at $72^{\circ} \mathrm{C}$. The labeled second round PCR product $(15 \mathrm{ml})$ was digested in a total volume of $50 \mathrm{ml}$ containing $10 \mathrm{U}$ with Haelll $(10 \mathrm{U} / \mathrm{ml}$; Biolabs) for the m.3243A $>\mathrm{G}$ mutation and Hpall (50 U/ml; Biolabs) for the $\mathrm{m} .8993 \mathrm{~T}>\mathrm{G}$ mutation. The m.3243A>G-amplicon contains an additional Haell restriction site site as an internal control for restriction enzyme digestion completion. The digestion sample of the m.8993T>G is spiked with a labeled amplicon generated by the HpalINARP (6-FAM) and HpalINARP-R primers containing an Hpall site as control. After digestion samples were purified using a QIAquick PCR purification kit (Qiagen). Samples were analyzed by capillary electrophoresis on an ABI Prism 3730 Genetic Analyzer followed by GeneScan analysis. 


\section{Single cell (semi)quantification and statistical analysis}

To calculate the mutation load the area of the mutation peak was divided by the sum of the peak area of the wild type and mutation peak. ${ }^{12,45}$ Five reference samples with a mutation load around the threshold value were included in every series for validation. The reference amplicons were diluted to a single blastomere equivalent amount, approximately 12 500-75 000 mtDNA copies. ${ }^{46}$ A total of 50 of these reference samples were amplified in 10 separate PCR runs during protocol setup and the data were used to construct a Shewhart chart ${ }^{47}$ (Supplementary Figures 3.1A,B), which showed the mean, the mean $\pm 1 S D$, the mean $\pm 2 S D$ and the mean \pm 3 SD. Four of five control observations must fall within the mean \pm 2 SD to approve the PGD PCR analysis. Data were rejected if more than one control sample exceeded the mean \pm 3 SD limit. Due to the linearity of amplification for the different percentages of mutation loads ${ }^{12,45}$ (Supplementary Figures 3.2A,B) only control samples around the threshold mutation load were used for constructing a Shewhart chart.

\section{Analysis of oocytes and remaining embryos}

Surplus embryos not suitable for transfer or cryopreservation were collected on day 4. The zonae pellucidae were removed by incubation in $1 \mathrm{U} / \mathrm{ml}$ pronase (Sigma, Zwijndrecht, The Netherlands). In embryos that could not be dissected in separate blastomeres due to strong intercellular interactions once in the morula stage, it was generally possible to identify the number of blastomeres clustered and the mean mutation load was calculated. Remaining fertilized oocytes, zygotes, embryos or single blastomeres were washed three times in washing buffer, transferred to a $0.2 \mathrm{ml} \mathrm{PCR}$ reaction tube, and stored at $-20^{\circ} \mathrm{C}$ until PCR.

\section{Results}

\section{Patients and PGD cycles}

The four women were aged 36, 30, 28 and 30 years at the start of the first cycle. Pedigrees and mutation loads are given in Figure 3.1 below and Supplementary Table 3.1. The four women did not show any symptoms or abnormalities upon clinical examination. The only probable exception is hearing loss of high frequencies in the first m.3243A $>$ G carrier. The first $m .3243 A>G$ couple had been infertile for many years. The female carrier had a severely affected mother. The second and third m.3243A>G carriers had severely affected brothers, who died at young adult age. The Leigh/NARP couple lost their first child affected by Leigh syndrome in the first year of his life. Nine PGD cycles were performed in total: three, two, and two cycles for each m.3243A>G carrier, respectively, and two for the $\mathrm{m} .8993 \mathrm{~T}>\mathrm{G}$ carrier. The nine cycles resulted in seven transferred embryos (six transfer procedures) (Figure 3.2); two embryos in the 
first m.3243A $>$ G carrier, one in each of the other m.3243A $>$ G carriers, and three in the m.8993T>G carrier, eventually leading to one pregnancy of the latter. PND was refused. Pregnancy follow-up was performed by a gynecologist. A healthy son was born. Cord blood analysis confirmed the initial PGD outcome; no m.8993T>G mutation was demonstrated.

\section{Mutation analysis in embryos, oocytes and zygotes}

A total of 58 embryos, nine oocytes, and 20 zygotes (of which nine were degenerated) could be analyzed (Figure 3.2) (Supplementary Table 3.1). The m.3243A $>$ G mutation was detected in four of four oocytes and in 16 of 18 zygotes (supplementary table 3.1) and in all 38 embryos (100\%, Figure 3.2) from the three carriers. Oocyte mutant load was $37-67 \%$ in the first MELAS carrier (three oocytes analyzed) and $65 \%$ in the second carrier (one oocyte analyzed). Zygote ranges were 0-66\% (11 zygotes analyzed), $15-42 \%$ (four zygotes analyzed), and 0-66\% (three zygotes analyzed), and the average mutation load in the 38 remaining m.3243A>G embryos ranged from 1-65\%, 2-58\%, and $5-70 \%$ for the three women, respectively (Supplementary Table 3.1). The m.8993T>G mutation was only present in one of five oocytes (mutation load 16\%, Supplementary Table 3.1) and in three of 20 embryos (average mutation load $30-100 \%$, Figure 3.2). The histograms concerning the difference in average mutation load between offspring (preimplantation embryos) and the carrier females are shown in Figure 3.3.

\section{Interblastomere differences of mutation load within embryos}

Interblastomere mutation load differed $<10 \%$ in most embryos ( 43 out of $53 ; 81 \%$ ), but in six of 53 tested embryos (11\%) it differed more than 15\%. These were embryos 2.1 (i.e., cycle 2, embryo 1), 3.4, and 3.5 of the first m.3243A>G carrier and embryos 1.4, 2.1 , and 2.2 of the second (figure 3.2), displaying a range of $60-82 \%, 50-65 \%, 35-54 \%$, $21-38 \%, 54-77 \%$, and $5-30 \%$, respectively. So, approximately $18 \%$ of the $\mathrm{m} .3243 \mathrm{~A}>\mathrm{G}$ embryos showed an interblastomere variation of $>15 \%$. In four additional embryos with the m.3243A $>\mathrm{G}$ mutation, we found interblastomere mutation load differences of $>10 \%$. In almost all cases, there was only one outlier responsible for the large range. The only exception was embryo 3.5 of carrier female 1 (Figure 3.2), in which the large range was due to differences between several blastomeres rather than one outlier. 
Pedigree 1 ( $m .3243 A>G$ )

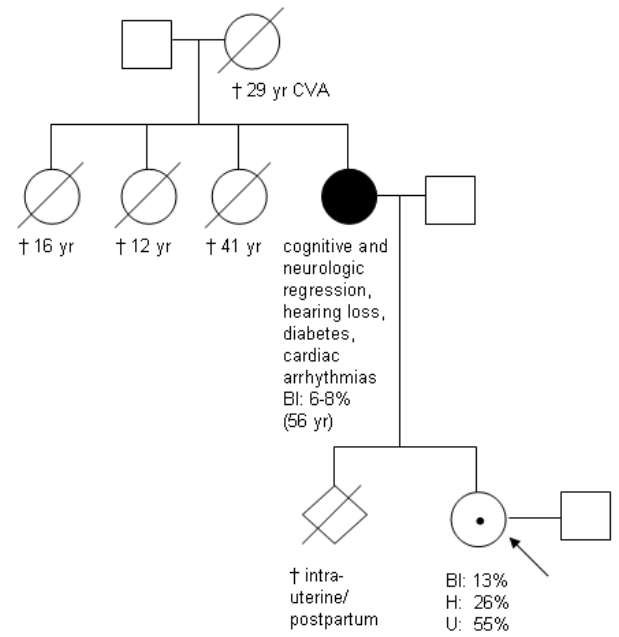

Pedigree $2(m .3243 A>G)$

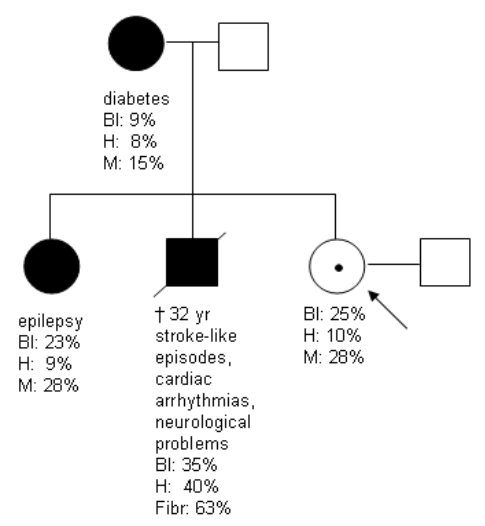

Pedigree $3(\mathrm{~m} .3243 A>G)$

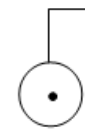

BI: $8 \%$

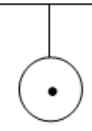

BI: $5-10 \%$
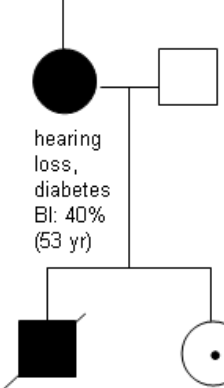

† $31 \mathrm{yr}$

acute

collaps,

death within

3 days, pre-

existing

hearing loss

Bl: $40 \%$
Pedigree $4(\mathrm{m.8993} T>G)$

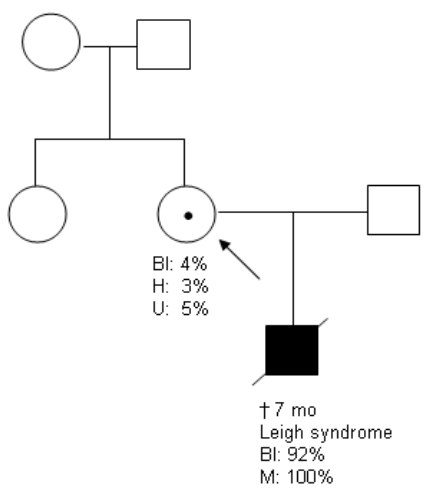

Figure 3.1 Pedigrees with family members showing clinical features and mutant loads. Bl, blood; $\mathrm{H}$, hair follicles; M, muscle; U, urine; Fibr, fibroblasts. 
Carrier 1 m.3243A>G
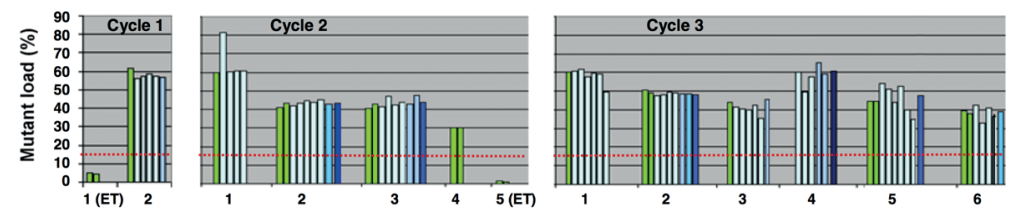

Carrier 2 m.3243A $>$ G
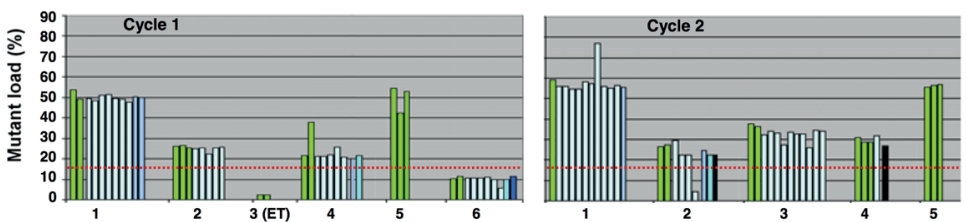

Carrier 3 m.3243A>G

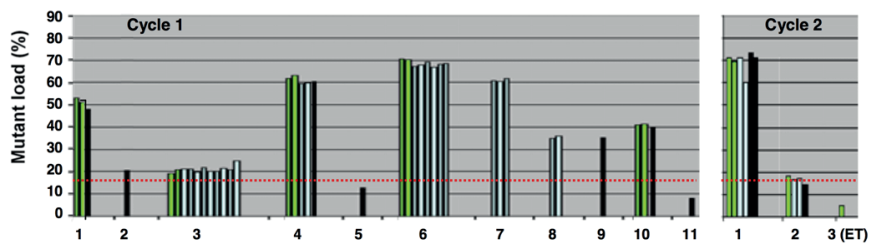

\section{Carrier 4} m.8993T >G

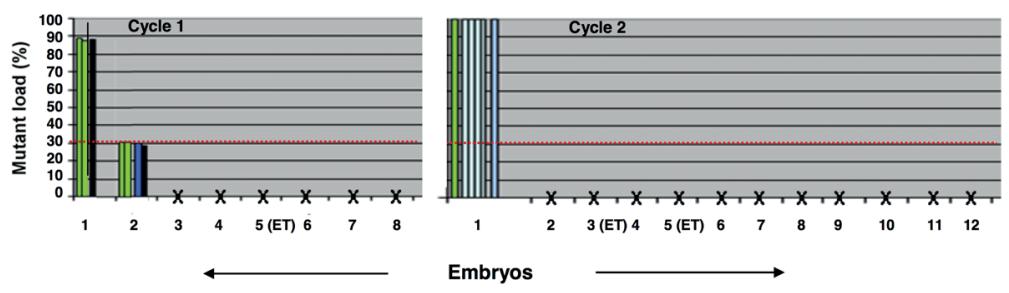

\begin{tabular}{|l|}
\hline$\square$ single blastomere (diagnostic analysis) \\
$\square$ single blastomere (reanalysis) \\
$\square 2$ blastomeres (reanalysis) \\
$\square 3$ blastomeres (reanalysis) \\
$\square 4$ blastomeres (reanalysis) \\
$\square 6$ blastomeres (reanalysis) \\
$\square 7$ blastomeres (reanalysis) \\
$\square$ multiple blastomeres (reanalysis)
\end{tabular}

Figure 3.2 Preimplantation genetic diagnosis cycles of the respective carrier females. Each cluster of bars represents an embryo with its tested blastomeres. The red dotted line represents the threshold level for transfer. For the Leigh carrier, the embryos in which the mutation was not detected are depicted as $\mathrm{X}$. For these embryos the numbers of analyzed blastomeres are not visible in the figure, but are available in online supplementary data. ET, embryo transfer. 


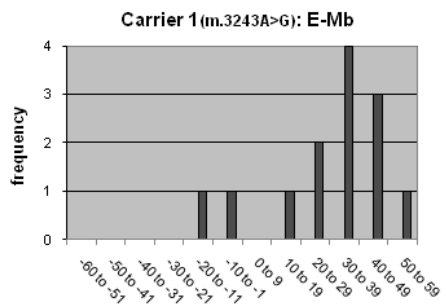

E-Mb

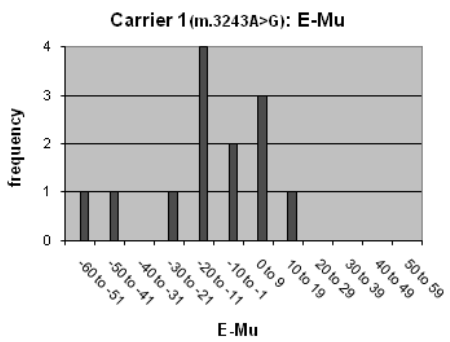

Carrier 3 (m.3243A>G): E-Mb

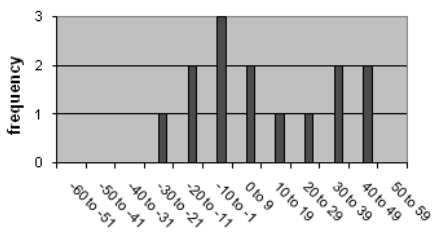

E-Mb

Carrier $3(\mathrm{~m} .3243 \mathrm{~A}>\mathrm{G})$ : E-Mu

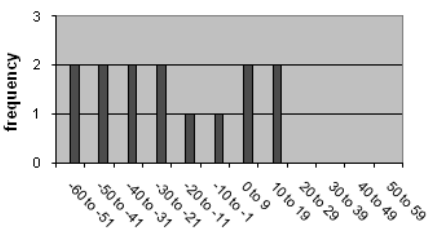

E-Mu
Carrier 2 (m.3243A>G): E-Mb

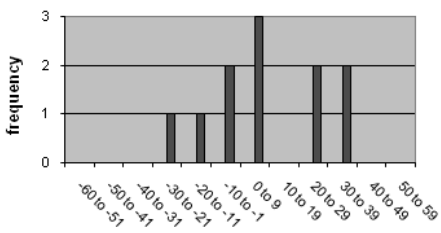

E-Mb

Carrier 2 (m.3243A>G): E-Mm

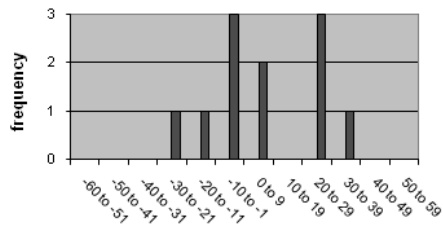

E-Mm

Carrier $4(m .8993 \mathrm{~T}>\mathrm{G})$ : E-Mb

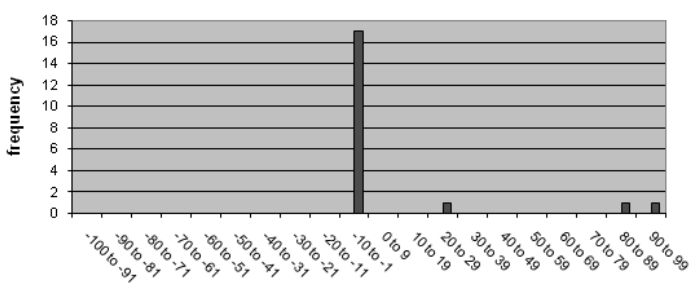

E-Mb

Carrier 4 (m.8993T >G): E-Mu

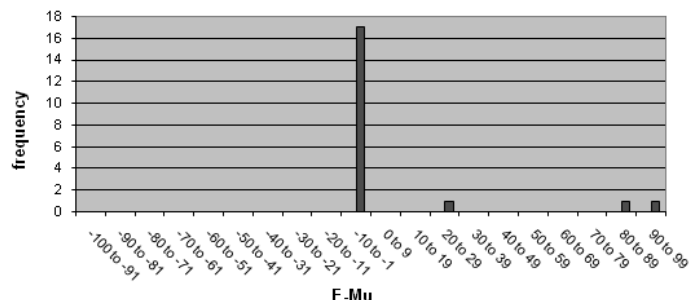

E-Mu

Figure 3.3 Histograms of the difference in average mutant load between the preimplantation embryos (E) and the corresponding carrier females (M). Mb, mutation load in blood; Mm, mutation load in muscle; Mu, mutation load in urine. 


\section{Discussion}

\section{Transmission rates 3243A>G and $8993 \mathrm{~T}>\mathrm{G}$ mutation}

The m.3243A>G mutation was detected in the great majority of oocytes, zygotes and embryos examined, which corroborates earlier reports in which the m.3243A>G was detected in $90 \%$ (74/82) of the primary oocytes of an adult carrier ${ }^{11}$ and in $84 \%(32 / 38)$ of day 3 stage embryos. ${ }^{12}$ In contrast, the m.8993T>G mutation was detected in only a minority of oocytes and embryos examined. Our results differ from Blok et al. ${ }^{10}$ who analysed seven oocytes from a $8993 \mathrm{~T}>\mathrm{G}$ mutation carrier with $50 \%$ mutation load in blood and found only one oocyte lacking the mutation. This is likely due to the difference in maternal mutation load, which is only $4 \%$ in blood in our patient, supporting the observation that the individual recurrence risk, and thus the transmission rate for this mutation, can be quite accurately predicted. ${ }^{4}$

\section{Division of mutation load among oocytes and embryos: segregation patterns}

The large variation in mutant load among oocytes and embryos of the m.3243A>G carriers that we observed is in line with the percentages of $0-77 \%$ found by Monnot et al. ${ }^{12}$. These data, approximating a Gaussian curve (data not shown, derivable from Figure 3.3) support the proposal that the level of mutant mtDNA in human primary oocytes and embryos is largely determined by random genetic drift. ${ }^{11,12}$ Embryonic mutant load that appeared to be higher compared to the mother's mutation load in blood (first and third m.3243A $>$ G carrier, Figure 3.3), appeared on the contrary lower when compared with the mother's mutation load in urine (Figure 3.3). Our data suggest that the reported preferential transmission of mutant genomes may, at least for the m.3243A>G mutation, reflect a positive bias due to differences in mutation load between blood and urine/muscle of carrier females. Based on the longitudinal decrease in $\mathrm{m} .3243 \mathrm{~A}>\mathrm{G}$ mutant load in blood, ${ }^{48-50}$ the difference in mutant load between offspring and their corresponding mothers could be overestimated. ${ }^{19,20}$ However, in only a few of the published pedigrees muscle data were available, and no urine data at all. Furthermore, our results support the hypothesis that a selection event against very high m.3243A>G mutant load in oocytes and subsequent embryos might occur during meiosis. $^{12,22}$

A predominantly skewed segregation of the $\mathrm{m} .8993$ mutations during the preimplantation/prenatal period has been reported. ${ }^{10,24}$ The majority of our embryos indeed had no mutation, which was expected from the low maternal mutation load. However, two embryos had an intermediate mutation load ( $88 \%$ and $30 \%$ ), which has been reported previously. ${ }^{40}$ These findings seem to contradict the hypothesis of highly skewed segregation, but are in accordance with intermediate values of the m.8993T>G mutant load observed after birth. Individual factors influencing a given mutation's 
bottleneck behaviour might contribute to this incomplete skewing, as has been hypothesized before for the m.3243A>G mutation. ${ }^{22}$

\section{Relation to mutation load in the mother: recurrence risk}

The frequency of affected offspring is related to the level of mutant mtDNA in the mother's blood for the m.3243A $>\mathrm{G}$ mutation. ${ }^{18}$ The risk of clinically affected offspring is in our data higher for the first m.3243A>G carrier, with $13 \%$ in blood, than for the second, with $25 \%$. This was unexpected based on risk estimates in previous reports. ${ }^{12,18}$ These data emphasize that, although in general a higher mutant load in the mother provides a higher risk of affected offspring, ${ }^{18}$ the recurrence risk for an individual m.3243A $>$ G carrier remains very difficult to predict. Furthermore, our data underline the conclusion that mothers carrying the m.3243A>G mutation have a high frequency of affected offspring whatever the level of mutant mtDNA in their blood. ${ }^{18}$ Possibly a better prediction can be made based on the mother's mutation load in muscle/urine, but more data are necessary to determine this more accurately.

For the m.8993T>G/C mutations the recurrence risk, defined as the probability of a severe outcome, is related to mutation load of the carrier mother ${ }^{4}$ and would for our carrier be approximately $13 \%$, but with a wide range. This is grossly reflected by our data; the mutation was present in $15 \%(3 / 20)$ of m.8993T>G embryos, in two of them above the threshold of clinical expression.

\section{Interblastomere differences in mutation load}

Mutation load was comparable between blastomeres in the majority of the embryos. In the m.3243A>G subgroup, $18 \%$ of affected embryos showed an interblastomere variation of $>15 \%$. One outlier was responsible for the large range in almost all cases. Blastomeres of mouse embryos have been reported to contain very similar levels of variant mtDNA. ${ }^{37,51,52}$ Steffann et al. $^{24}$ found a small $(\leq 8 \%)$ intercellular variation of mitochondrial heteroplasmy among 12 of 15 human embryos tested for an mtDNA polymorphism. In the remaining three embryos, all originating from the same individual, the intercellular variation was $16-19 \%$. This, combined with our data in which large intercellular variation occurred in only two of three m.3243A>G carriers, suggests that genetic factors could be involved in mitochondrial segregation during embryogenesis. ${ }^{24}$ Monnot et al. ${ }^{12}$ found an equal distribution of mutant DNA among various blastomeres of a carrier embryo until day 5 , with only occasional variability up to $15 \%$ in mutant load between single cell measurements and the whole embryo; this was assumed to result from technical artifacts, or, less likely, physiological distortion in m.3243A>G segregation from day 3 to day 5 . However, in one embryo we found a large variation at initial biopsy instead of at re-analysis, excluding segregation from day 3 to day 5 as an explanation. In our series, interblastomere differences were not only larger, 
they also occurred more often than previously reported, ${ }^{12}$ although Monnot et al. did not perform single blastomere analysis in all embryos. Interestingly, a large interblastomere variation of $24 \%$ in an $\mathrm{m} .3243 \mathrm{~A}>\mathrm{G}$ embryo has been reported by another group. ${ }^{53}$

Differences in mutation load between blastomeres would not be a problem if all values remained above or below the threshold level. However, in one embryo, we found one outlier which would make the embryo eligible for transfer, whereas in fact it was not. Applying a threshold of $30 \%{ }^{12}$ would have caused the same situation in two other embryos (1.4 and 2.3, Supplementary Table 3.1). A false-negative classification can be minimised by analysing two blastomeres, instead of one as proposed before, ${ }^{12}$ although the removal of two cells has been reported to influence the rate of live birth delivery. ${ }^{54}$ This illustrates the difficult balance between a safe and correct diagnosis on the one hand, and optimising the chance of pregnancy on the other.

\section{Conclusion}

Our data from nine PGD cycles in four female carriers of mitochondrial diseases-three MELAS (m.3243A>G) and one Leigh case (m.8993T>G)-show that PGD for mtDNA disorders is useful as all carriers produced oocytes below the threshold. Blastomere mutation load appears to be representative for the whole embryo in the majority of cases. More data are necessary to understand further the segregation of mtDNA mutations throughout human embryo-fetal development, and to optimize guidelines regarding the PGD procedures. Counseling of couples who are at high risk of having offspring with an mtDNA disorder is still a challenge. However, PGD provides those couples with the opportunity to conceive healthy offspring. 


\section{References}

1 A Rotig,A Munnich. Genetic features of mitochondrial respiratory chain disorders. J Am Soc Nephrol. 2003;14(12):2995-3007.

2 N Manwaring, MM Jones, JJ Wang, E Rochtchina, C Howard, P Mitchell,CM Sue. Population prevalence of the MELAS A3243G mutation. Mitochondrion. 2007;7(3):230-3.

3 HR Elliott, DC Samuels, JA Eden, CL Relton,PF Chinnery. Pathogenic mitochondrial DNA mutations are common in the general population. Am J Hum Genet. 2008;83(2):254-60.

4 SL White, VR Collins, R Wolfe, MA Cleary, S Shanske, S DiMauro, HH Dahl,DR Thorburn. Genetic counseling and prenatal diagnosis for the mitochondrial DNA mutations at nucleotide 8993. Am J Hum Genet. 1999;65(2):474-82.

5 GC Black, K Morten, A Laborde,J Poulton. Leber's hereditary optic neuropathy: heteroplasmy is likely to be significant in the expression of LHON in families with the 3460 ND1 mutation. Br J Ophthalmol. 1996;80(10):915-7.

6 PF Chinnery, N Howell, RN Lightowlers,DM Turnbull. Molecular pathology of MELAS and MERRF. The relationship between mutation load and clinical phenotypes. Brain. 1997;120 ( Pt 10):1713-21.

7 DR Thorburn,HH Dahl. Mitochondrial disorders: genetics, counseling, prenatal diagnosis and reproductive options. Am J Med Genet. 2001;106(1):102-14.

8 J Poulton,AL Bredenoord. 174th ENMC international workshop: Applying pre-implantation genetic diagnosis to mtDNA diseases: implications of scientific advances 19-21 March 2010, Naarden, The Netherlands. Neuromuscul Disord. 2010;20(8):559-63.

9 N Howell, S Halvorson, I Kubacka, DA McCullough, LA Bindoff,DM Turnbull. Mitochondrial gene segregation in mammals: is the bottleneck always narrow? Hum Genet. 1992;90(1-2):117-20.

10 RB Blok, DA Gook, DR Thorburn,HH Dahl. Skewed segregation of the mtDNA nt 8993 (T-->G) mutation in human oocytes. Am J Hum Genet. 1997;60(6):1495-501.

11 DT Brown, DC Samuels, EM Michael, DM Turnbull,PF Chinnery. Random genetic drift determines the level of mutant mtDNA in human primary oocytes. Am J Hum Genet. 2001;68(2):533-6.

12 S Monnot, N Gigarel, DC Samuels, P Burlet, L Hesters, N Frydman, R Frydman, V Kerbrat, B Funalot, J Martinovic, A Benachi, J Feingold, A Munnich, JP Bonnefont,J Steffann. Segregation of mtDNA throughout human embryofetal development: m.3243A>G as a model system. Hum Mutat. 2011;32(1): 116-25.

13 J Poulton,DM Turnbull. 74th ENMC international workshop: mitochondrial diseases 19-20 november 1999, Naarden, the netherlands. Neuromuscul Disord. 2000;10(6):460-2.

14 Y Ma, F Fang, Y Yang, L Zou, Y Zhang, S Wang, Y Xu, P Pei,Y Qi. The study of mitochondrial A3243G mutation in different samples. Mitochondrion. 2009;9(2):139-43.

15 MT McDonnell, AM Schaefer, EL Blakely, R McFarland, PF Chinnery, DM Turnbull,RW Taylor. Noninvasive diagnosis of the $3243 \mathrm{~A}>\mathrm{G}$ mitochondrial DNA mutation using urinary epithelial cells. Eur J Hum Genet. 2004;12(9):778-81.

16 AL Frederiksen, PH Andersen, KO Kyvik, TD Jeppesen, J Vissing,M Schwartz. Tissue specific distribution of the 3243A-> G mtDNA mutation. J Med Genet. 2006;43(8):671-7.

17 RG Whittaker, JK Blackwood, CL Alston, EL Blakely, JL Elson, R McFarland, PF Chinnery, DM Turnbull,RW Taylor. Urine heteroplasmy is the best predictor of clinical outcome in the m.3243A>G mtDNA mutation. Neurology. 2009;72(6):568-9.

18 PF Chinnery, N Howell, RN Lightowlers,DM Turnbull. MELAS and MERRF. The relationship between maternal mutation load and the frequency of clinically affected offspring. Brain. 1998;121 (Pt 10):188994.

19 PF Chinnery, DR Thorburn, DC Samuels, SL White, HM Dahl, DM Turnbull, RN Lightowlers, N Howell. The inheritance of mitochondrial DNA heteroplasmy: random drift, selection or both? Trends Genet. 2000;16(11):500-5.

20 LJ Wong, H Wong,A Liu. Intergenerational transmission of pathogenic heteroplasmic mitochondrial DNA. Genet Med. 2002;4(2):78-83. 
21 P Wonnapinij, PF Chinnery,DC Samuels. Previous estimates of mitochondrial DNA mutation level variance did not account for sampling error: comparing the mtDNA genetic bottleneck in mice and humans. Am J Hum Genet. 2010;86(4):540-50.

22 S Monnot, N Gigarel, DC Samuels, P Burlet, L Hesters, N Frydman, R Frydman, V Kerbrat, B Funalot, A Benachi, J Feingold, A Munnich, J Bonnefont,J Steffann. Impact of mtDNA mutations on mtDNA segregation throughout human oogenesis. ESHG conference 2011.

23 SL White, S Shanske, I Biros, L Warwick, HM Dahl, DR Thorburn,S Di Mauro. Two cases of prenatal analysis for the pathogenic $T$ to $G$ substitution at nucleotide 8993 in mitochondrial DNA. Prenat Diagn. 1999;19(12):1165-8.

24 J Steffann, N Frydman, N Gigarel, P Burlet, PF Ray, R Fanchin, E Feyereisen, V Kerbrat, G Tachdjian, JP Bonnefont, R Frydman,A Munnich. Analysis of mtDNA variant segregation during early human embryonic development: a tool for successful NARP preimplantation diagnosis. J Med Genet. 2006;43(3):244-7.

25 HH Dahl, DR Thorburn,SL White. Towards reliable prenatal diagnosis of mtDNA point mutations: studies of nt8993 mutations in oocytes, fetal tissues, children and adults. Hum Reprod. 2000;15 Suppl 2:24655 .

26 J Steffann, N Gigarel, J Corcos, M Bonniere, F Encha-Razavi, M Sinico, S Prevot, Y Dumez, A Yamgnane, R Frydman, A Munnich,JP Bonnefont. Stability of the m.8993T->G mtDNA mutation load during human embryofetal development has implications for the feasibility of prenatal diagnosis in NARP syndrome. $J$ Med Genet. 2007;44(10):664-9.

27 DR Marchington, M Scott-Brown, DH Barlow,J Poulton. Mosaicism for mitochondrial DNA polymorphic variants in placenta has implications for the feasibility of prenatal diagnosis in mtDNA diseases. Eur $J$ Hum Genet. 2006;14(7):816-23.

28 D Marchington, S Malik, A Banerjee, K Turner, D Samuels, V Macaulay, P Oakeshott, C Fratter, S Kennedy,J Poulton. Information for genetic management of mtDNA disease: sampling pathogenic mtDNA mutants in the human germline and in placenta. J Med Genet. 2010;47(4):257-61.

29 AE Harding, IJ Holt, MG Sweeney, M Brockington,MB Davis. Prenatal diagnosis of mitochondrial DNA8993 T----G disease. Am J Hum Genet. 1992;50(3):629-33.

30 A Suomalainen, A Majander, H Pihko, L Peltonen,AC Syvanen. Quantification of tRNA3243(Leu) point mutation of mitochondrial DNA in MELAS patients and its effects on mitochondrial transcription. Hum Mol Genet. 1993;2(5):525-34.

31 PM Matthews, J Hopkin, RM Brown, JB Stephenson, D Hilton-Jones,GK Brown. Comparison of the relative levels of the 3243 (A-->G) mtDNA mutation in heteroplasmic adult and fetal tissues. J Med Genet. 1994;31(1):41-4.

32 E Leshinsky-Silver, M Perach, E Basilevsky, E Hershkovitz, M Yanoov-Sharav, T Lerman-Sagie,D Lev. Prenatal exclusion of Leigh syndrome due to T8993C mutation in the mitochondrial DNA. Prenat Diagn. 2003;23(1):31-3.

33 T Ferlin, P Landrieu, C Rambaud, H Fernandez, R Dumoulin, P Rustin,B Mousson. Segregation of the G8993 mutant mitochondrial DNA through generations and embryonic tissues in a family at risk of Leigh syndrome. J Pediatr. 1997;131(3):447-9.

$34 \mathrm{~J}$ Poulton,DR Marchington. Progress in genetic counselling and prenatal diagnosis of maternally inherited mtDNA diseases. Neuromuscul Disord. 2000;10(7):484-7.

35 E Cardaioli, GM Fabrizi, GS Grieco, MT Dotti,A Federico. Heteroplasmy of the A3243G transition of mitochondrial tRNA(Leu(UUR)) in a MELAS case and in a 25-week-old miscarried fetus. J Neurol. 2000; 247(11):885-7.

36 C Bouchet, J Steffann, J Corcos, S Monnot, V Paquis, A Rotig, S Lebon, P Levy, G Royer, I Giurgea, N Gigarel, A Benachi, Y Dumez, A Munnich,JP Bonnefont. Prenatal diagnosis of myopathy, encephalopathy, lactic acidosis, and stroke-like syndrome: contribution to understanding mitochondrial DNA segregation during human embryofetal development. J Med Genet. 2006;43(10):788-92.

37 JP Jenuth, AC Peterson, K Fu,EA Shoubridge. Random genetic drift in the female germline explains the rapid segregation of mammalian mitochondrial DNA. Nat Genet. 1996;14(2):146-51.

38 FV Meirelles,LC Smith. Mitochondrial genotype segregation in a mouse heteroplasmic lineage produced by embryonic karyoplast transplantation. Genetics. 1997;145(2):445-51. 
39 J Poulton, S Kennedy, P Oakeshott,D Wells. Preventing transmission of maternally inherited mitochondrial DNA diseases. BMJ. 2009;338:b94.

40 DR Thorburn, L Wilton,S Stock-Myer. Healthy baby girl born following pre-implantation genetic diagnosis for mitochondrial DNA m.8993T>G mutation. Mol Genet Metab. 2009;98: 5-6.

41 DM Hellebrekers, R Wolfe, AT Hendrickx, IF de Coo, CE de Die, JP Geraedts, PF Chinnery,HJ Smeets. GD and heteroplasmic mitochondrial DNA point mutations: a systematic review estimating the chance of healthy offspring. Hum Reprod Update. 2012.

42 JA Land, MI Yarmolinskaya, JC Dumoulin,JL Evers. High-dose human menopausal gonadotropin stimulation in poor responders does not improve in vitro fertilization outcome. Fertil Steril. 1996;65(5):961-5.

43 AC Van Steirteghem, Z Nagy, H Joris, J Liu, C Staessen, J Smitz, A Wisanto,P Devroey. High fertilization and implantation rates after intracytoplasmic sperm injection. Hum Reprod. 1993;8(7):1061-6.

44 JC Dumoulin, E Coonen, M Bras, LC van Wissen, R Ignoul-Vanvuchelen, JM Bergers-Jansen, JG Derhaag, JP Geraedts,JL Evers. Comparison of in-vitro development of embryos originating from either conventional in-vitro fertilization or intracytoplasmic sperm injection. Hum Reprod. 2000;15(2):402-9.

45 N Gigarel, PF Ray, P Burlet, N Frydman, G Royer, S Lebon, JP Bonnefont, R Frydman, A Munnich,J Steffann. Single cell quantification of the 8993 T $>$ G NARP mitochondrial DNA mutation by fluorescent PCR. Mol Genet Metab. 2005;84(3):289-92.

46 P Reynier, P May-Panloup, MF Chretien, CJ Morgan, M Jean, F Savagner, P Barriere, Y Malthiery. Mitochondrial DNA content affects the fertilizability of human oocytes. Mol Hum Reprod. 2001;7(5):425-9.

47 JO Westgard, PL Barry, MR Hunt,T Groth. A multi-rule Shewhart chart for quality control in clinical chemistry. Clin Chem. 1981;27(3):493-501.

48 LM t Hart, JJ Jansen, HH Lemkes, P de Knijff,JA Maassen. Heteroplasmy levels of a mitochondrial gene mutation associated with diabetes mellitus decrease in leucocyte DNA upon aging. Hum Mutat. 1996;7(3):193-7.

49 N Howell, SS Ghosh, E Fahy, LA Bindoff. Longitudinal analysis of the segregation of mtDNA mutations in heteroplasmic individuals. J Neurol Sci. 2000;172(1):1-6.

50 S Rahman, J Poulton, D Marchington,A Suomalainen. Decrease of 3243 A-->G mtDNA mutation from blood in MELAS syndrome: a longitudinal study. Am J Hum Genet. 2001;68(1):238-40.

51 NL Dean, BJ Battersby, A Ao, RG Gosden, SL Tan, EA Shoubridge,MJ Molnar. Prospect of preimplantation genetic diagnosis for heritable mitochondrial DNA diseases. Mol Hum Reprod. 2003;9(10):631-8.

52 MJ Molnar,EA Shoubridge. Preimplantation genetic diagnosis for mitochondrial (mt) disorders. Neuromuscul Disord. 1999;9:521.

53 M Vandewoestyne, B Heindryckx, S De Gheselle, T Lepez, J Neupane, J Gerris, R Van Coster, P De Sutter,D Deforce. Poor correlation between polar bodies and blastomere mutation load in a patient with m.3243A>G tRNALeu(UUR) point mutation. Mitochondrion. 2012;12(4):477-9.

54 A De Vos, C Staessen, M De Rycke, W Verpoest, P Haentjens, P Devroey, I Liebaers,H Van de Velde. Impact of cleavage-stage embryo biopsy in view of PGD on human blastocyst implantation: a prospective cohort of single embryo transfers. Hum Reprod. 2009;24(12):2988-96. 


\section{Supplemental materials}

A

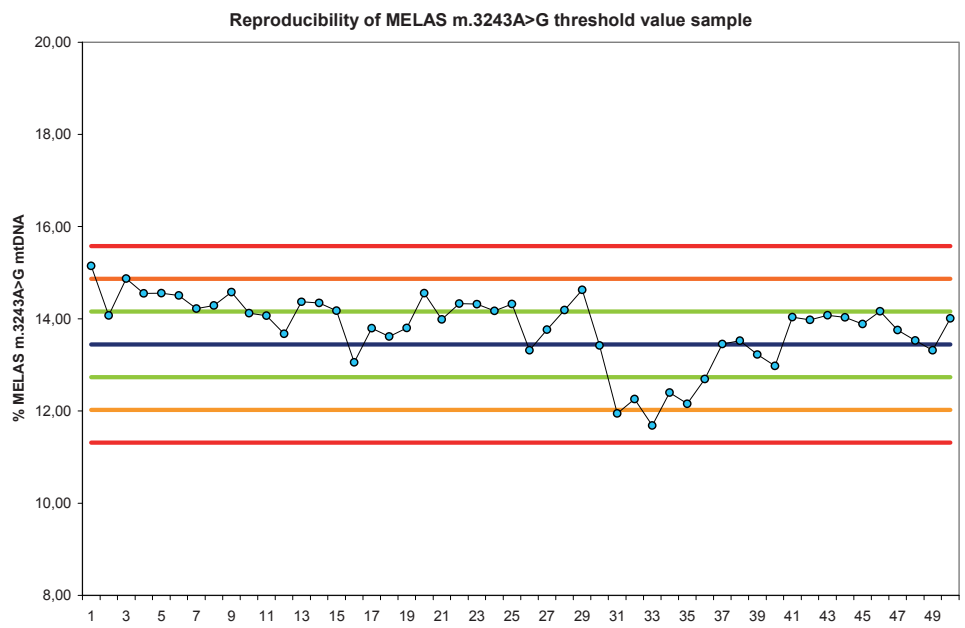

B

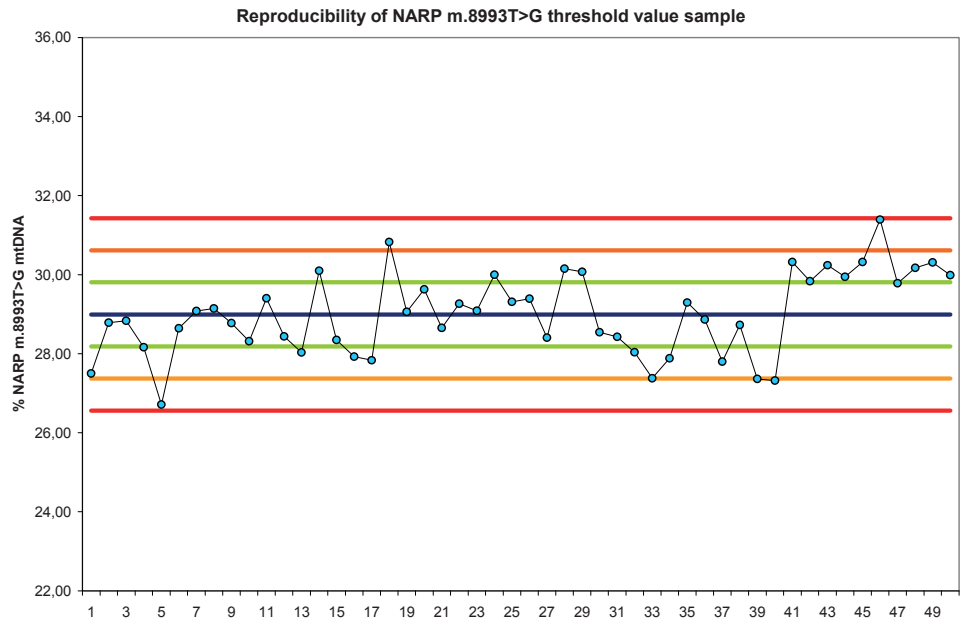

Figures S3.1 Shewhart charts of $\mathrm{m} .3243 \mathrm{~A}>\mathrm{G}(\mathrm{A})$ and $\mathrm{m} .8993 \mathrm{~T}>\mathrm{G}$ (B). To demonstrate reproducibility and verify a valid PCR amplification, five reference samples with a mutation load around the threshold value ( $13 \%$ for the $\mathrm{m} .3243 \mathrm{~A}>\mathrm{G}$ and $29 \%$ for the $\mathrm{m} .8993 \mathrm{~T}>\mathrm{G}$ ) were included in every PCR series for validation. The reference amplicons were diluted to a single blastomere equivalent amount, approximately 12.500-75.000 mtDNA copies. A total of 50 of these reference samples were amplified in 10 separate PCR runs during protocol setup and the data were used to construct a Shewhart chart which showed the mean, the mean $\pm 1 S D$, the mean $\pm 2 S D$ and the mean $\pm 3 S D$. Four of five control observations must fall within the mean $\pm 2 S D$ to approve the PGD PCR analysis. Data were rejected if more then 1 control sample exceeded the mean $\pm 3 S D$ limit. 
A

Expected vs Observed MELAS m.3243A>G mutation load

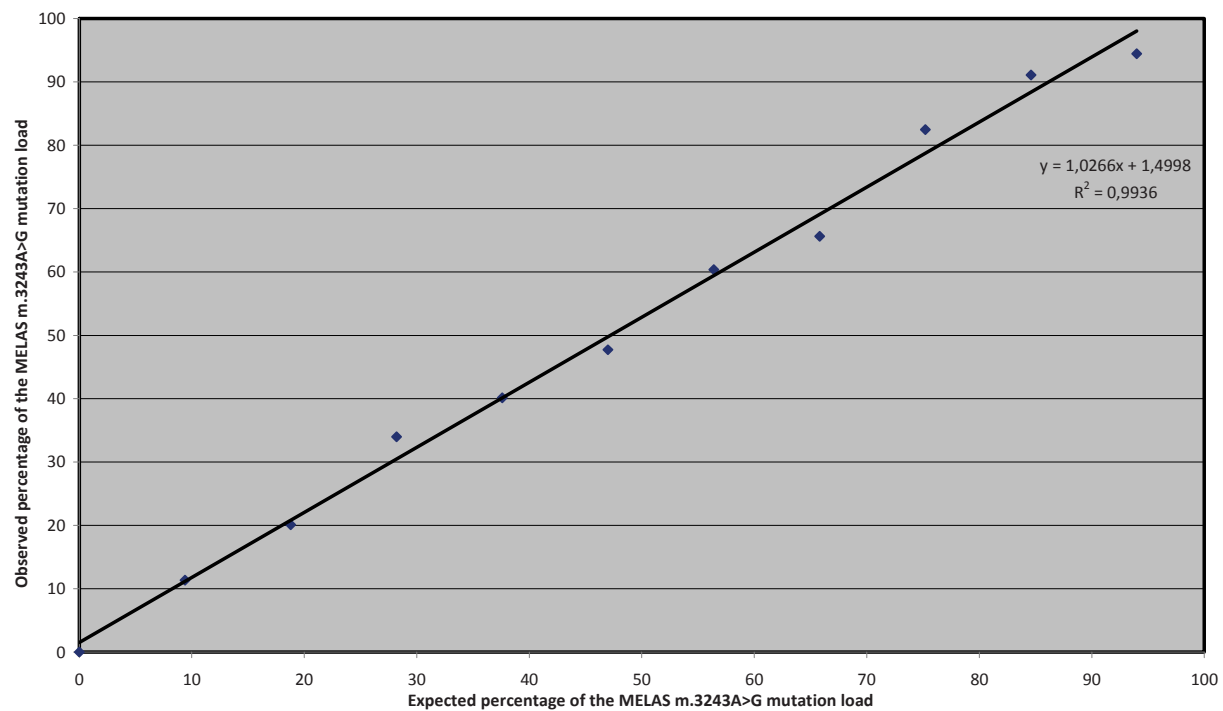

B

Expected vs Observed NARP $m .8993 \mathrm{~T}>\mathrm{G}$ mutation load

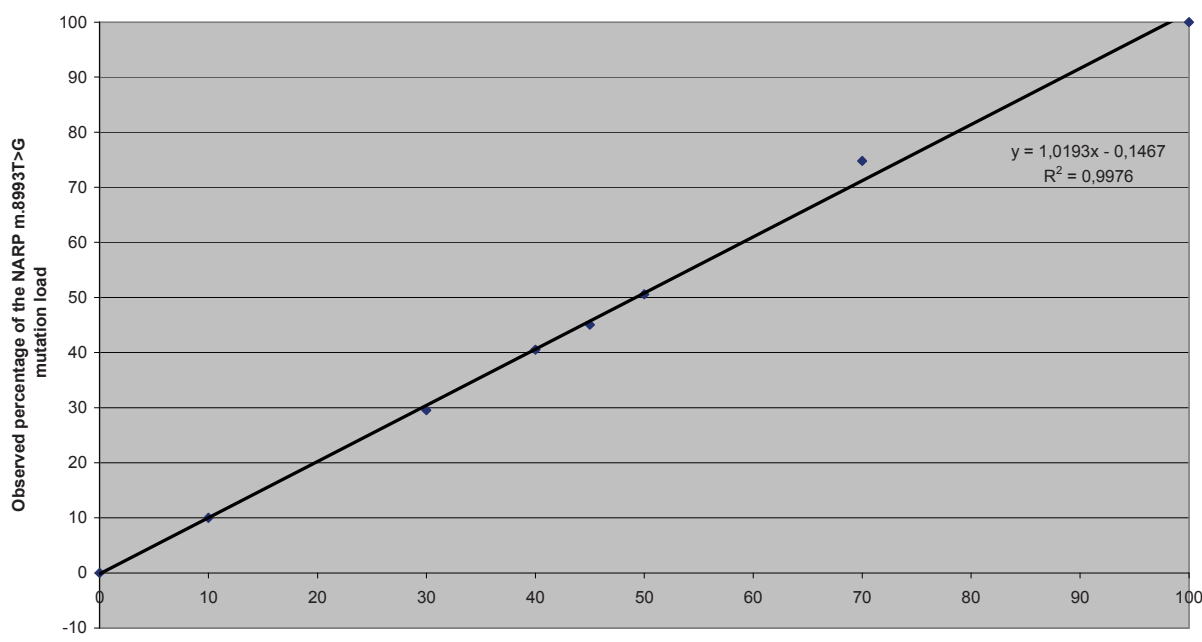

Expected percentage of the NARP m.8993T>G mutation load

Figures S3.2 Serial dilutions of the m.3243A>G amplicon (A) and the m.8993T>G amplicon (B) to demonstrate the linearity of the amplification. The obtained amplicons are diluted to a single blastomere equivalent amount, approximately 12.500-75.000 mtDNA copies with different mutation load percentages. 


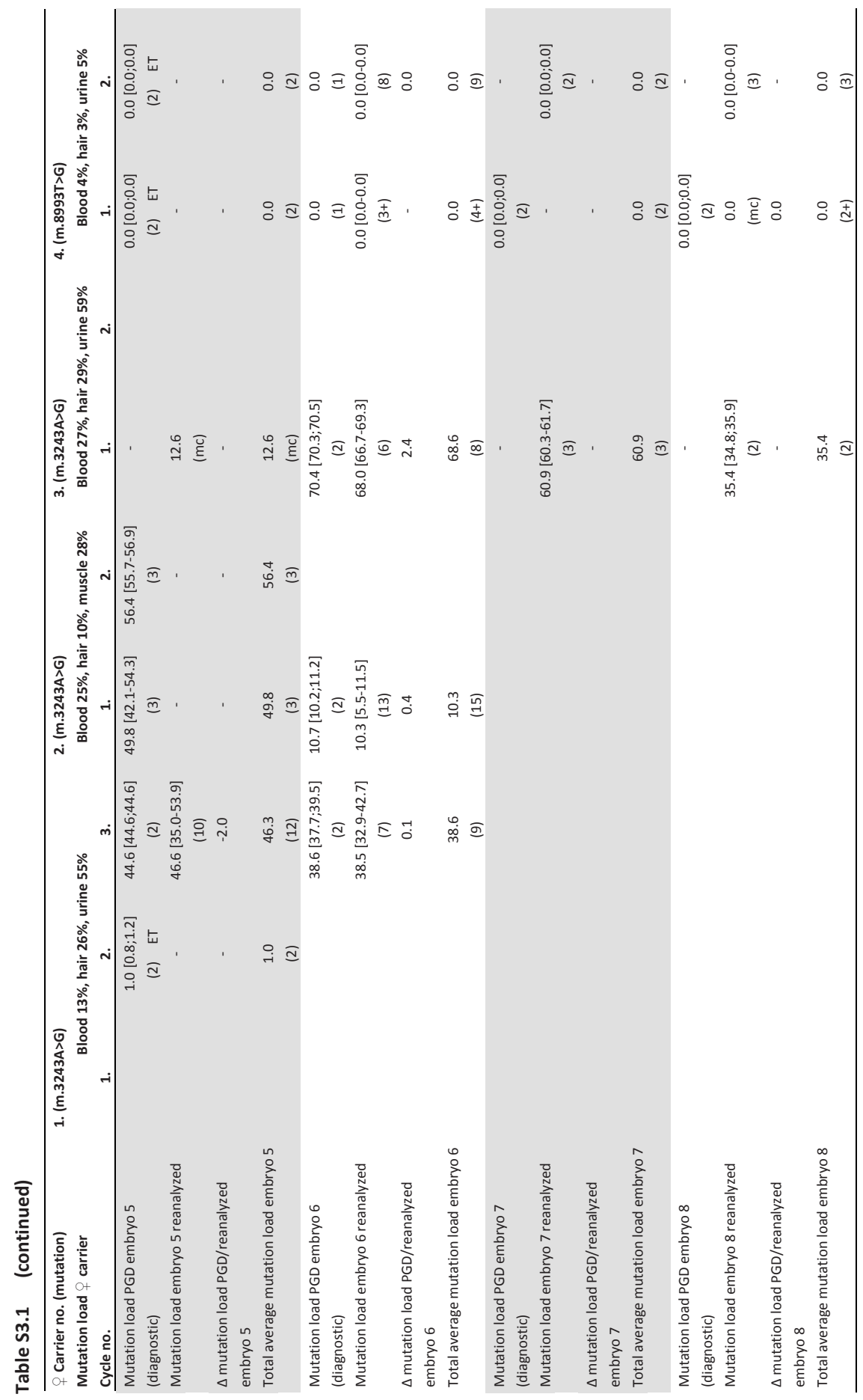




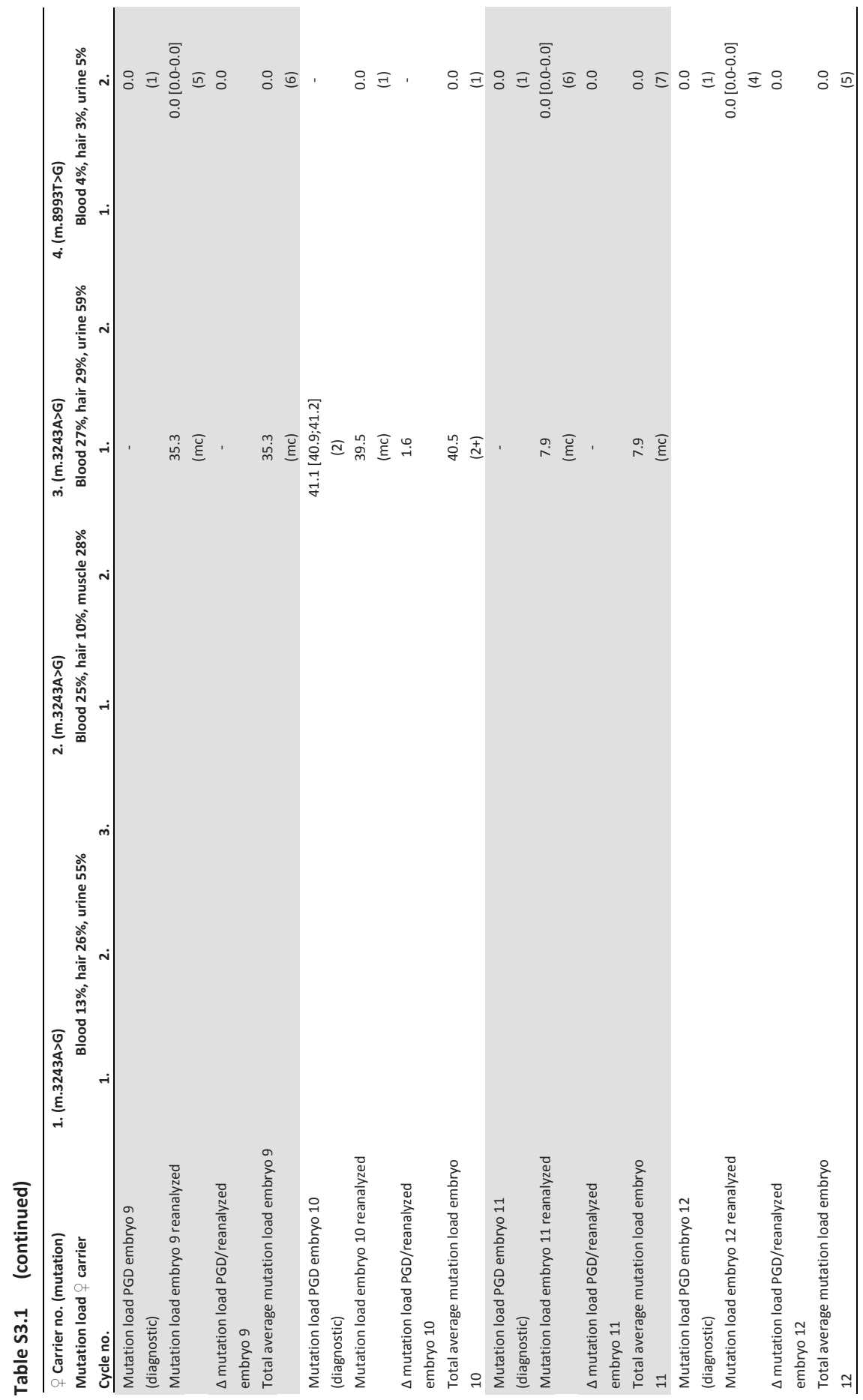


Preimplantation genetic diagnosis in mitochondrial DNA disorders: challenge and success

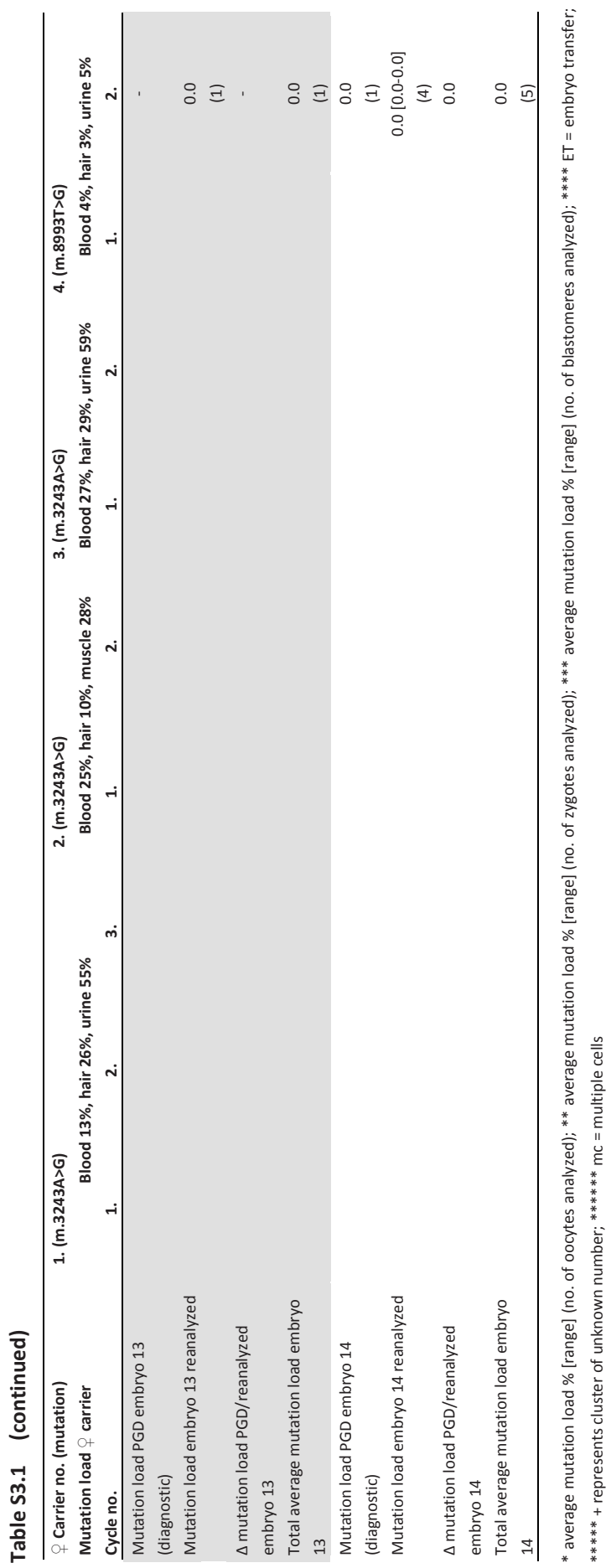





\section{CHAPTER 4}

\section{Preimplantation genetic diagnosis for mitochondrial}

DNA mutations: analysis of one blastomere suffices

Suzanne C.E.H. Sallevelt, Joseph C.F.M. Dreesen, Edith Coonen, Aimee D.C. Paulussen, Debby M.E.I. Hellebrekers, Christine E.M. de Die-Smulders, Hubert J.M. Smeets,

Patrick Lindsey 


\section{Abstract}

\section{Background}

Preimplantation genetic diagnosis (PGD) is a reproductive strategy for mitochondrial DNA (mtDNA) mutation carriers, strongly reducing their risk of affected offspring. Embryos either without the mutation or with mutation load below the phenotypic threshold are transferred to the uterus. Because of incidental heteroplasmy deviations in single blastomeres and the relatively limited data available, we so far preferred relying on two blastomeres rather than one. Considering the negative effect of a two-blastomere compared to a single-blastomere biopsy protocol on live birth delivery rate, we re-evaluated the error rate in our current dataset.

\section{Methods}

For the m.3243A>G mutation sufficient embryos/blastomeres were available for a powerful analysis. The diagnostic error rate, defined as a potential false-negative result, was determined in 294 single blastomeres analyzed in 73 embryos of 9 female m.3243A>G mutation carriers.

\section{Results}

Only one out of 294 single blastomeres (0.34\%) would have resulted in a false-negative diagnosis. False-positive diagnoses were not detected.

\section{Conclusion}

Our findings support a single-blastomere biopsy PGD protocol for the m.3243A $>\mathrm{G}$ mutation as the diagnostic error rate is very low. As in the early preimplantation embryo no mtDNA replication seems to occur and the mtDNA is divided randomly among the daughter cells, we conclude this result to be independent of the specific mutation and therefore applicable to all mtDNA mutations. 


\section{Introduction}

Mitochondrial diseases belong to the most common inborn errors of metabolism. ${ }^{1}$ In at least $15 \%$ of cases, these multisystem disorders are caused by a primary mutation of the mitochondrial DNA (mtDNA), ${ }^{1,2}$ which is exclusively transmitted through the female germline. Female carriers have shown to bear offspring with a wide variety in mutation load, due to the combination of heteroplasmy, meaning a subset of the mtDNAs is mutated, the remainder is wild-type, and a genetic bottleneck upon transmission. ${ }^{3-7}$ Given the potential clinical severity of mitochondrial disorders and the general lack of treatment, female carriers may wish to prevent transmission to their (subsequent) offspring. For biologically own children the two options currently available in clinical practice are prenatal diagnosis $(P N D)^{8-18}$ and preimplantation genetic diagnosis (PGD). ${ }^{17,19-25}$ Mitochondrial replacement techniques are being developed as potential third option ${ }^{26-29}$ and are expected to be applied to the first human carriers in a licensed and tightly regulated clinical investigation setting in the near future (http://www.hfea.gov.uk/9942.html, accessed on 18-05-2016). PND for mtDNA mutations has some drawbacks, ${ }^{30}$ the most important one being the potential difficulties in predicting the future child's phenotype based on the mutation load. Therefore, in the majority of cases PGD is the preferred choice for female carriers. Transmission of the mutation can be prevented by transfer of mutation-free embryos. When these are not available, embryos with mutation loads below a threshold of phenotypic expression are transferred. For some mutations a specific threshold can be established based on available data ${ }^{23}$ whereas for scarcely-reported mutations a generic threshold, calculated by pooling different mtDNA mutations, can be applied. ${ }^{31}$ As such, PGD largely reduces the risk of affected offspring rather than fully excluding it. Prerequisites for PGD to be successful in mtDNA carriers are 1) the presence of embryos with mutation load below the threshold of expression and 2 ) representativeness of the analyzed blastomere(s) for the entire embryo. The first has not been an issue so far, but regarding the second point, until now the analysis of two blastomeres has been performed based on incidental heteroplasmy deviations in single blastomeres. ${ }^{23}$ Although blastomere mutation load appears representative for the whole embryo in the majority of cases, ${ }^{23,24}$ available data were rather limited. The biopsy of two blastomeres has adverse effects on live birth delivery rate compared to the removal of one cell ${ }^{32}$ and for this reason all nuclear PGD-PCR protocols in our center have already switched to one-blastomere biopsy for some years now. ${ }^{33}$ In the present study, we re-evaluate our two-blastomere biopsy protocol for mtDNA mutations in the context of diagnostic reliability on the current dataset. 


\section{Methods}

The error rate was determined in 294 single blastomeres analyzed in 73 embryos of 9 female carriers of the m.3243A $>\mathrm{G}$ mutation. Single blastomere mutation loads from the same embryo were compared. Error rate was defined as a false-negative result, i.e. a single blastomere with mutation load below the threshold whereas the other one or more blastomeres have heteroplasmy levels above the threshold. The opposite situation, when an embryo is discarded unjust (false-positive result), is not clinically relevant because, although a waste, this would not result in the birth of an affected child after a PGD treatment. The proportion of single blastomeres potentially resulting in a false-negative misdiagnosis was calculated from the total number of single blastomeres. To perform the analysis, a logistic linear regression model was constructed with a random intercept using a Gaussian mixing distribution in order to account for the variability between patients or the embryos.

Only embryos of which at least two single blastomeres have been analyzed were included. Diagnostic biopsy data (after which embryos with mutation load below the threshold were transferred, meaning that for those embryos only two single blastomere results were available) as well as reanalysis data of embryos unsuitable for transfer were used. Informed consent for reanalysis was obtained from the female carriers. mtDNA mutations other than m.3243A $>$ G were not included in the current analysis because of insufficient sample sizes. PGD procedures and mutation detection/quantification have been carried out as previously described. ${ }^{23}$

\section{Results}

For single-blastomere biopsy, an error rate of $0.34 \%(1 / 294)$, with a confidence interval of $0.05-2.38 \%$, was calculated based on the presence of one blastomere in our series that would have potentially led to a false-negative diagnosis (Figure 4.1). This particular blastomere harbored $\sim 5 \%$ mutation load whereas heteroplasmies of the other blastomeres were between approximately 22 and 30\% (Figure 4.1). The error rate was zero based on the biopsy of two blastomeres since all potential second blastomeres of this embryo had mutation loads above the threshold and thus would have resulted in a correct diagnosis. The interblastomere heteroplasmy-variability between carriers and embryos were both non-significant (with respectively p-values of 0.954 and 0.642 ). Although not our primary concern, we did not encounter a false-positive diagnosis in our current dataset. 

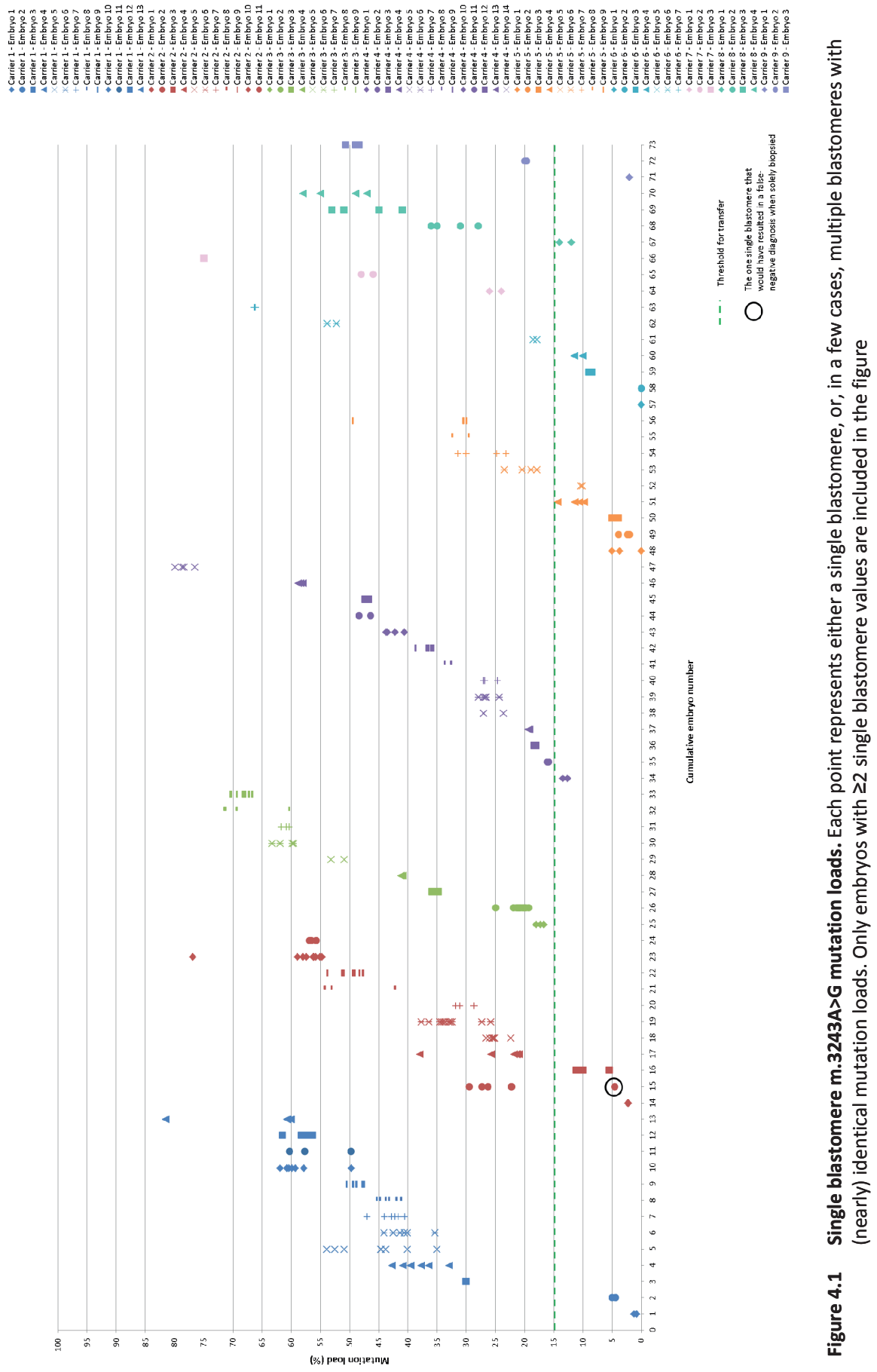


\section{Discussion}

Our results in m.3243A>G embryos indicated a false negative error rate of $0.34 \%$ for single blastomeres. This error rate is defined as the risk that a biopsied single blastomere does not represent the whole embryo's mutation load and would incorrectly label the embryo as eligible for transfer. Notably, false-positive results were absent in our dataset. Our results are generalizable to the population of interest: there was no significant interblastomere heteroplasmy-variability between 1) the carriers, who are assumed to reflect the heterogeneity of the population, or 2) between the embryos, which indicates that it does not matter which embryo from a given carrier is analyzed. The confidence interval is currently relatively wide $(0.05-2.38 \%)$ but is expected to further narrow down below $1 \%$ with additional data numbers. The low false-negative diagnostic error rate justifies single-blastomere analysis for PGD in m.3243A $>\mathrm{G}$ mutation carriers to improve live birth delivery rate.

The fertilized oocyte contains between 50,000 and 550,000 copies of mtDNA. ${ }^{34}$ Upon cleavage, these copies are divided among daughter cells. In viable embryos this occurs in a symmetrical way. ${ }^{35}$ Meanwhile, the total mtDNA amount seems to remain stable during the first three days of preimplantation development (reviewed in reference 34). Therefore, the partitioning of mtDNA among blastomeres in preimplantation embryos is presumed to be a random process. The very high copy number of the fertilized oocyte explains why the Gaussian distribution curves (derivable from Figure 4.1) within cleavage-stage embryos are tight with few deviating values. At the blastomere stage the mtDNA copies are divided among only 6-8 cells, each still containing high amounts. Therefore, if an mtDNA mutation is present, the relative heteroplasmy levels are expected to remain largely the same in each blastomere. This is indeed supported by our data as well as from others. ${ }^{24}$ Should certain processes, such as an active copy number reduction (other than by cell divisions), or significant mtDNA replication, occur in the cleavage-stage embryo, this could result in more pronounced interblastomere variability. Both have been reported in other species ${ }^{36,37}$ but not in humans. mtDNA replication in early human $m .3243 \mathrm{~A}>\mathrm{G}$ preimplantation embryos has been suggested in one study, but this replication could also have occurred in earlier stages (before fertilization). ${ }^{38}$ Moreover, if the observed mtDNA replication indeed took place in the embryo, it may have occurred later, in the trophectoderm at the blastocyst stage. ${ }^{38}$ This timing corroborates other non-human ${ }^{39-41}$ and human ${ }^{29,34}$ data. Thus, the available data indicate that no active events influence the mtDNA segregation during the first three days of preimplantation development in human embryos, in line with the 'quiet embryo' hypothesis, a characteristic of viable embryos. 
Random partitioning among blastomeres does mean, however, that incidental deviations in mutant load can occur by chance. This is indeed illustrated by our data on the m.3243A $>\mathrm{G}$ (reference 23 and current report), m.14487T>C (Sallevelt et al. in preparation) and $\mathrm{m} .8344 \mathrm{~A}>\mathrm{G}^{30}$ mutations. Looking at the $\mathrm{m} .8993 \mathrm{~T}>\mathrm{G}$ and $\mathrm{m} .9176 \mathrm{~T}>\mathrm{C}$ mutations, in our experience blastomere mutation load was very constant in three embryos and 18 embryos, respectively, both of a single carrier (reference 23 and unpublished data) with no occasional variability, but numbers are limited. Notably, the majority of these embryos harbored very high mutant loads ( $90 \%$ and above) and thus rather homogeneous mtDNA populations, for which a more even distribution among daughter cells is more likely upon random distribution.

As interblastomere variability seems determined by a passive and random process of mtDNA partitioning, this is not mutation-dependent, making our conclusions on the m.3243A $>$ G mutation generalizable to all mtDNA mutations.

The biopsy of a single blastomere instead of two has positive implications for embryo development and implantation capacity; an overall live birth rate of $37.4 \%$ for 1 blastomere biopsy versus $22.4 \%$ for two-blastomere biopsy was shown. ${ }^{32}$ Another possibility is to analyze two different conditions in 2 single blastomeres during one PGD treatment. This could be an mtDNA mutation with a nuclear gene defect, which are real examples from our practice (POLG, BRCA2), or an mtDNA mutation with sex-selection on male fetuses to definitely stop the transmission to future generations, which has been requested as well. Although technically possible, Dutch legislation currently does not allow selection of embryos based on sex gender in this situation.

Trophectoderm (TE) biopsy in the blastocyst stage at day $5-6$ postfertilisation ${ }^{42}$ is expected to combine the possibility of multiple analyses at one hand and more favorable embryo viability with better implantation potential on the other. ${ }^{43}$ So far, limited $^{22,25,44}$ and somewhat conflicting ${ }^{22,44}$ data are available on TE biopsy for mtDNA mutations and its outcome. The timing of TE biopsy is closer to the 'narrowest' point of the bottleneck, where mtDNA copy number per cell is lowest and consequently the inter-cell variation highest. Furthermore, evidence from animal studies shows a different mtDNA replication pattern in trophectoderm compared to the inner cell mass, from which the embryo will develop. ${ }^{41}$ Therefore, considering the biological processes, TE biopsy seems less reliable for mtDNA mutations.

In conclusion, PGD for mtDNA mutations is preferably performed on blastomeres in the cleavage-stage preimplantation embryo. Interblastomere heteroplasmy levels are fairly constant in most preimplantation embryos. Here, we specifically show for the m.3243A $>$ G mutation that the biopsy of one blastomere is sufficient for a reliable PGD diagnosis, and we discussed that these results are generalizable for other mtDNA mutations. 


\section{References}

1. Thorburn DR. Mitochondrial disorders: prevalence, myths and advances. J Inherit Metab Dis. 2004;27(3):349-362.

2. Rotig A, Munnich A. Genetic features of mitochondrial respiratory chain disorders. J Am Soc Nephrol. 2003;14(12):2995-3007.

3. Cree LM, Samuels DC, de Sousa Lopes SC, et al. A reduction of mitochondrial DNA molecules during embryogenesis explains the rapid segregation of genotypes. Nat Genet. 2008;40(2):249-254.

4. Khrapko K. Two ways to make an mtDNA bottleneck. Nat Genet. 2008;40(2):134-135.

5. Wai T, Teoli D, Shoubridge EA. The mitochondrial DNA genetic bottleneck results from replication of a subpopulation of genomes. Nat Genet. 2008;40(12):1484-1488.

6. Cao L, Shitara H, Sugimoto M, Hayashi J, Abe K, Yonekawa H. New evidence confirms that the mitochondrial bottleneck is generated without reduction of mitochondrial DNA content in early primordial germ cells of mice. PLoS Genet. 2009;5(12):e1000756.

7. Samuels DC, Wonnapinij P, Cree LM, Chinnery PF. Reassessing evidence for a postnatal mitochondrial genetic bottleneck. Nat Genet. 2010;42(6):471-472; author reply 472-473.

8. Harding AE, Holt IJ, Sweeney MG, Brockington M, Davis MB. Prenatal diagnosis of mitochondrial DNA8993 T----G disease. Am J Hum Genet. 1992;50(3):629-633.

9. Bartley J, Senadheera D, Park P, Brar H, Abad D, Wong L. Prenatal diagnosis of T8993G mitochondrial DNA point mutation in amniocytes by heteroplasmy detection. Am J Hum Genet. 1996;59:A316.

10. Ferlin T, Landrieu P, Rambaud C, et al. Segregation of the G8993 mutant mitochondrial DNA through generations and embryonic tissues in a family at risk of Leigh syndrome. J Pediatr. 1997;131(3):447-449.

11. White SL, Shanske S, Biros I, et al. Two cases of prenatal analysis for the pathogenic T to G substitution at nucleotide 8993 in mitochondrial DNA. Prenat Diagn. 1999;19(12):1165-1168.

12. Leshinsky-Silver E, Perach M, Basilevsky E, et al. Prenatal exclusion of Leigh syndrome due to T8993C mutation in the mitochondrial DNA. Prenat Diagn. 2003;23(1):31-33.

13. Chou YJ, Ou CY, Hsu TY, et al. Prenatal diagnosis of a fetus harboring an intermediate load of the A3243G mtDNA mutation in a maternal carrier diagnosed with MELAS syndrome. Prenat Diagn. 2004;24(5):367-370.

14. Jacobs LJ, de Coo IF, Nijland JG, et al. Transmission and prenatal diagnosis of the T9176C mitochondrial DNA mutation. Mol Hum Reprod. 2005;11(3):223-228.

15. Bouchet C, Steffann J, Corcos J, et al. Prenatal diagnosis of myopathy, encephalopathy, lactic acidosis, and stroke-like syndrome: contribution to understanding mitochondrial DNA segregation during human embryofetal development. J Med Genet. 2006;43(10):788-792.

16. Steffann J, Gigarel N, Corcos J, et al. Stability of the m.8993T->G mtDNA mutation load during human embryofetal development has implications for the feasibility of prenatal diagnosis in NARP syndrome. $J$ Med Genet. 2007;44(10):664-669.

17. Monnot S, Gigarel N, Samuels DC, et al. Segregation of mtDNA throughout human embryofetal development: m.3243A>G as a model system. Hum Mutat. 2011;32(1):116-125.

18. Nesbitt V, Alston $\mathrm{CL}$, Blakely EL, et al. A national perspective on prenatal testing for mitochondrial disease. Eur J Hum Genet. 2014;22(11):1255-1259.

19. Steffann J, Frydman N, Gigarel N, et al. Analysis of mtDNA variant segregation during early human embryonic development: a tool for successful NARP preimplantation diagnosis. J Med Genet. 2006;43(3):244-247.

20. Thorburn DR, Wilton L, Stock-Myer S. Healthy baby girl born following pre-implantation genetic diagnosis for mitochondrial DNA m.8993T>G mutation. Mol Genet Metab. 2009;98: 5-6.

21. Vandewoestyne M, Heindryckx B, De Gheselle $S$, et al. Poor correlation between polar bodies and blastomere mutation load in a patient with m.3243A>G tRNALeu(UUR) point mutation. Mitochondrion. 2012;12(4):477-479.

22. Treff NR, Campos J, Tao X, Levy B, Ferry KM, Scott RT, Jr. Blastocyst preimplantation genetic diagnosis (PGD) of a mitochondrial DNA disorder. Fertil Steril. 2012;98(5):1236-1240.

23. Sallevelt SC, Dreesen JC, Drusedau M, et al. Preimplantation genetic diagnosis in mitochondrial DNA disorders: challenge and success. J Med Genet. 2013;50(2):125-132. 
24. Steffann J, Gigarel N, Samuels DC, et al. Data from artificial models of mitochondrial DNA disorders are not always applicable to humans. Cell Rep. 2014;7(4):933-934.

25. Heindryckx B, Neupane J, Vandewoestyne M, et al. Mutation-free baby born from a mitochondrial encephalopathy, lactic acidosis and stroke-like syndrome carrier after blastocyst trophectoderm preimplantation genetic diagnosis. Mitochondrion. 2014;18:12-17.

26. Tachibana M, Sparman M, Sritanaudomchai $H$, et al. Mitochondrial gene replacement in primate offspring and embryonic stem cells. Nature. 2009;461(7262):367-372.

27. Craven L, Elson JL, Irving L, et al. Mitochondrial DNA disease: new options for prevention. Hum Mol Genet. 2011;20(R2):R168-174.

28. Tachibana M, Amato $P$, Sparman $M$, et al. Towards germline gene therapy of inherited mitochondrial diseases. Nature. 2013;493(7434):627-631.

29. Hyslop LA, Blakeley P, Craven L, et al. Towards clinical application of pronuclear transfer to prevent mitochondrial DNA disease. Nature. 2016;534(7607):383-386.

30. Sallevelt SCEH, J.C.F.M. D, de Coo IFM, de Die-Smulders CEM, Smeets HJM. Selecting the right embryo in mitochondrial disorders. In: Sills ES, ed. Screening the Single Euploid Embryo - Molecular Genetics in Reproductive Medicine: Springer International Publishing Switzerland; 2015:213-246.

31. Hellebrekers DM, Wolfe R, Hendrickx AT, et al. PGD and heteroplasmic mitochondrial DNA point mutations: a systematic review estimating the chance of healthy offspring. Hum Reprod Update. 2012;18(4):341-349.

32. De Vos A, Staessen C, De Rycke M, et al. Impact of cleavage-stage embryo biopsy in view of PGD on human blastocyst implantation: a prospective cohort of single embryo transfers. Hum Reprod. 2009;24(12):2988-2996.

33. Dreesen J, Destouni A, Kourlaba G, et al. Evaluation of PCR-based preimplantation genetic diagnosis applied to monogenic diseases: a collaborative ESHRE PGD consortium study. Eur J Hum Genet. 2014;22(8):1012-1018.

34. Fragouli E, Spath K, Alfarawati S, et al. Altered levels of mitochondrial DNA are associated with female age, aneuploidy, and provide an independent measure of embryonic implantation potential. PLoS Genet. 2015;11(6):e1005241.

35. Van Blerkom J, Davis $\mathrm{P}$, Alexander S. Differential mitochondrial distribution in human pronuclear embryos leads to disproportionate inheritance between blastomeres: relationship to microtubular organization, ATP content and competence. Hum Reprod. 2000;15(12):2621-2633.

36. May-Panloup $\mathrm{P}$, Vignon $\mathrm{X}$, Chretien MF, et al. Increase of mitochondrial DNA content and transcripts in early bovine embryogenesis associated with upregulation of mtTFA and NRF1 transcription factors. Reprod Biol Endocrinol. 2005;3:65.

37. Cagnone GL, Tsai TS, Makanji Y, et al. Restoration of normal embryogenesis by mitochondrial supplementation in pig oocytes exhibiting mitochondrial DNA deficiency. Sci Rep. 2016;6: 23229.

38. Monnot S, Samuels DC, Hesters L, et al. Mutation dependance of the mitochondrial DNA copy number in the first stages of human embryogenesis. Hum Mol Genet. 2013;22(9):1867-1872.

39. Spikings EC, Alderson J, St John JC. Regulated mitochondrial DNA replication during oocyte maturation is essential for successful porcine embryonic development. Biol Reprod. 2007;76(2):327-335.

40. Chiaratti MR, Bressan FF, Ferreira CR, et al. Embryo mitochondrial DNA depletion is reversed during early embryogenesis in cattle. Biol Reprod. 2010;82(1):76-85.

41. St John J. The control of mtDNA replication during differentiation and development. Biochim Biophys Acta. 2014;1840(4):1345-1354.

42. Cimadomo D, Capalbo A, Ubaldi FM, et al. The Impact of Biopsy on Human Embryo Developmental Potential during Preimplantation Genetic Diagnosis. BioMed Res Int. 2016;2016:7193075.

43. Scott RT, Jr., Upham KM, Forman EJ, Zhao T, Treff NR. Cleavage-stage biopsy significantly impairs human embryonic implantation potential while blastocyst biopsy does not: a randomized and paired clinical trial. Fertil Steri. 2013;100(3):624-630.

44. Mitalipov S, Amato P, Parry S, Falk MJ. Limitations of preimplantation genetic diagnosis for mitochondrial DNA diseases. Cell Rep. 2014;7(4):935-937. 



\section{CHAPTER 5}

Preimplantation genetic diagnosis for the

m.14487T >C mitochondrial DNA mutation resulted in

the birth of a healthy boy

Suzanne C.E.H. Sallevelt, Joseph C.F.M. Dreesen, Marion Drusedau, Debby M.E.I. Hellebrekers, Aimee D.C. Paulussen, Edith Coonen, Ronald J.T. van Golde, Joep P.M. Geraedts, Luca Gianaroli, Maria C. Magli, Massimo Zeviani, Hubert J.M. Smeets, Christine E.M. de Die-Smulders Accepted for publication in Hum Reprod. 


\section{Abstract}

We report on the first preimplantation genetic diagnosis (PGD) performed for the m.14487T>C mitochondrial DNA (mtDNA) mutation in the MT-ND6 gene, associated with Leigh syndrome. The female carrier gave birth to a healthy baby boy at age 42 . This case adds to the success of PGD for mtDNA mutations. 


\section{Introduction}

Mitochondrial DNA (mtDNA) is subject to exclusive maternal inheritance. Pathogenic mutations in the mitochondrial DNA (mtDNA) are implicated in a variety of (mostly) multisystem disorders with variable organ involvement, age of onset and severity, generally without treatment options.

Leigh syndrome, a progressive neurodegenerative disorder with multisystem involvement which is often lethal in childhood, is an example at the severe end of the mitochondrial disease spectrum. ${ }^{1}$ Leigh syndrome can be caused by mutations in a variety of genes, either originating from the nuclear DNA, or from the mtDNA. ${ }^{1}$ For the former, transmission to offspring and recurrence risks follow Mendelian patterns, mostly of autosomal recessive inheritance. The applicability of reproductive options, i.e. prenatal diagnosis (PND) and preimplantation genetic diagnosis (PGD), is similar to that of other recessive disorders. However, when caused by an mtDNA mutation, Leigh patients' carrier mothers encounter challenges in pursuing unaffected offspring.

Due to the heteroplasmic nature of mtDNA mutations, referring to the coexistence of mutant and normal mtDNA in the carrier female's cells, and its complex transmission behaviour, ${ }^{2}$ carrier mothers can transmit variable and usually unpredictable amounts of mutant mtDNA to each of their offspring. A threshold effect exists, meaning that a certain level of heteroplasmy is required for symptoms to become manifest. This threshold varies between mutations and tissues and cannot be exactly defined for most individual mtDNA mutations due to lack of data. Consequently, prenatal diagnosis (PND) is hampered by the often limited predictive value of the sample's mutation load for the expected phenotype. Preimplantation genetic diagnosis (PGD) can be an advantageous alternative, ${ }^{3}$ achieving pregnancies from embryos that are either mutation-free or harbor heteroplasmy levels safely below the phenotypic threshold, thereby circumventing arbitrary decisions regarding pregnancy termination. As said, for the majority of mtDNA mutations insufficient data is available to determine a specific threshold. However, a systematic meta-analysis provided a generic threshold by showing a $95 \%$ or higher chance of being unaffected at (muscle) mutant level of $18 \%$ or less, irrespective of the mutation, ${ }^{4}$ which can be used as input for counseling. Worldwide only a handful of centers perform PGD for mtDNA mutations. ${ }^{3,5-9}$

Here we report the first PGD for the m.14487T>C mutation in the MT-ND6 gene causative of Leigh syndrome. For this indication, two blastomeres were analyzed, because data on single blastomere representativeness of the m.14487T $>C$ mutation for the entire embryo were lacking, and for other mtDNA mutations single blastomeres are known to incidentally harbor deviating heteroplasmy levels. ${ }^{3}$ The generic threshold level for transfer of $18 \%$ was applied, ${ }^{4}$ as for the m.14487T $>\mathrm{C}$ mutation relatively 
limited data were available. The mutation has been reported with details on phenotype and mutation load in a total of 22 affected individuals and 14 unaffected family members. ${ }^{10-23}$ It appears that for the m.14487T>C mutation rather high heteroplasmy levels are needed for a clinically significant phenotype; 13 out of 15 (87\%) patients with established muscle mutation loads harbored $\geq 80 \%$ heteroplasmy and 10 out of $15 \geq$ $93 \%$, respectively. The lowest mutation load in muscle detected in an affected individual was $50 \%$. These findings suggest that a higher threshold than the generic threshold of $18 \%$ might be applied for the m.14487T $>C$ mutation. Mutation loads up to $>80 \%$ have been reported in some of the unaffected family members. ${ }^{\text {eg, } 16}$ However, in none of the unaffected carriers mutation load was determined in muscle, making it impossible to reliably evaluate the heteroplasmy range in asymptomatic carriers. Blood mutation levels of the $\mathrm{m} .14487 \mathrm{~T}>\mathrm{C}$ are not representative since these were often significantly lower compared to levels in other tissues in patients as well, even in young and/or seriously affected patients ${ }^{18,19,21}$ and have indeed been shown to decrease over time in one child. ${ }^{11}$

\section{Case report}

\section{Case description}

A 39 year old female carrier of the m.14487T>C mutation in the MTND6 gene ${ }^{13}$ was referred from Italy. She and her husband requested PGD to fulfill their desire for a healthy child. Their son, 8 years of age at that time, had Leigh syndrome with m.14487T $>$ C mutation loads of $>95 \%$ in muscle, fibroblasts and lymphocytes, respectively. Growth delay was noted at 6 months of age. Subsequently, stagnation of psychomotor development with hypotonia became apparent, and his brain MRI showed lesions typical of Leigh syndrome. He had feeding difficulties and recurrent aspiration pneumonias, necessitating tube feeding. At age one a cardiac arrest due to respiratory failure resulted in an intensive care admission. He was discharged with a tracheostomy. Years of intensive home caring followed. Eventually the boy died at age 11 of a respiratory infection. His asymptomatic mother carried the mutation at heteroplasmy levels of $\sim 32 \%$ in lymphocytes, $\sim 65 \%$ in urine and $\sim 55 \%$ in muscle. The mutation was also present in the mother's sister and mother, but not in her maternal grandmother and uncle (Figure 5.1).

The couple was informed of the PGD procedure as customary ${ }^{3}$ and in this case it was specifically made aware that PGD for the m.14487T>C had never been carried out. Written consent, which included the transfer threshold of $18 \%$, was obtained. By the time the PCR protocol had been developed and validated, the female was 40 years old. At that time, her basal FSH and 17-beta-estradiol levels were $5.6 \mathrm{U} / \mathrm{L}$ and $0.15 \mathrm{nmol} / \mathrm{L}$, 
respectively, and antral follicle counts were 12 and 8 at cycle day 4, pointing to a good ovarian reserve despite her age. There were no contraindications for the IVF procedure concerning her own health, a cardiologic evaluation revealed no abnormalities.

\begin{tabular}{|ll|}
\hline M: & Leigh syndrome \\
$\mathrm{F}:$ & fibroblasts \\
$\mathrm{L}:$ & lymphocytes \\
$\mathrm{U}:$ & urine \\
$\mathrm{BM}:$ & buccal mucosa \\
$\mathrm{n}:$ & normal (mutation not detected) \\
$\mathrm{PGD}:$ & preimplantation genetic diagnosis \\
(NL): & genetic analysis performed in the Netherlands, \\
& Maastricht University Medical Centre \\
& (the other analyses have been carried out in Italy, \\
& Milano National Institute of Neurology "C.Besta") \\
\hline
\end{tabular}
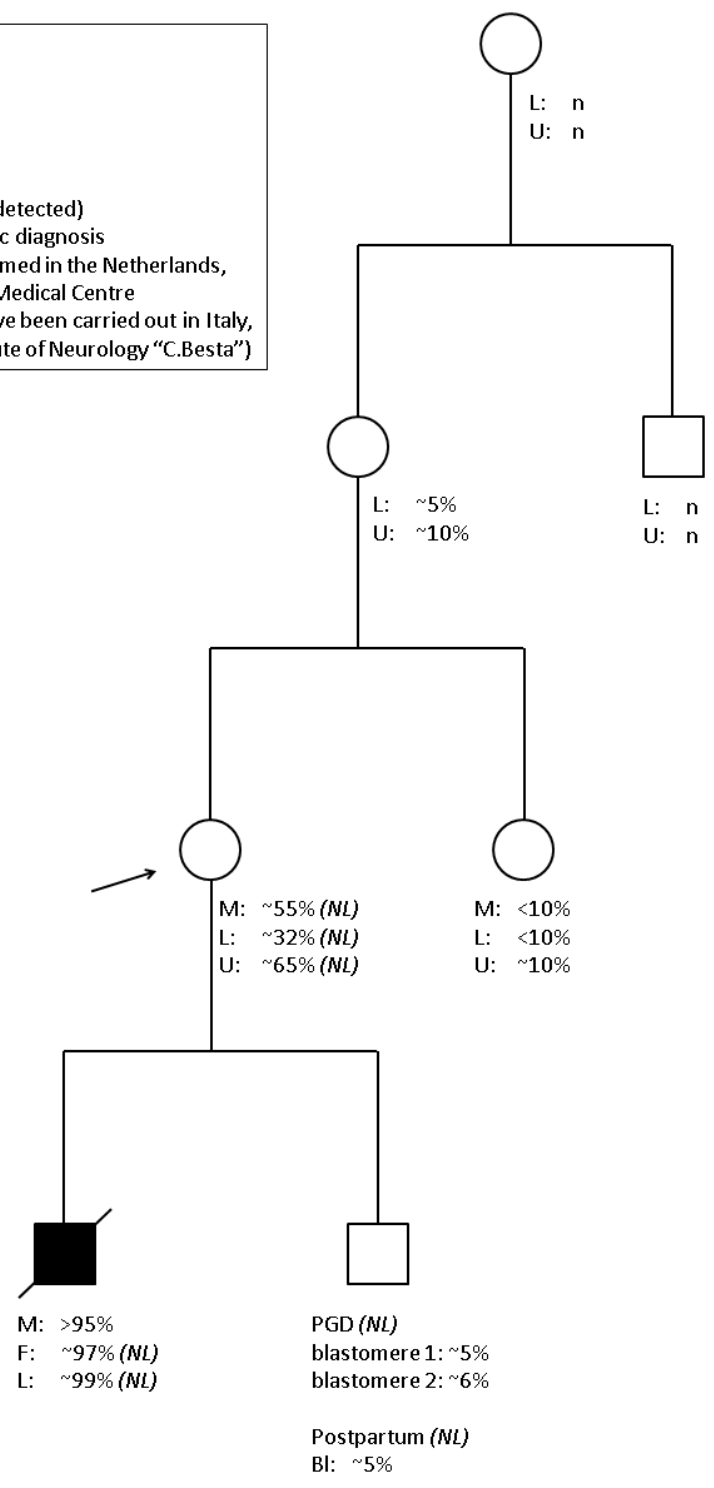

Figure $5.1 \quad$ Pedigree. 


\section{Methods}

m.14487T >C mutation levels in the female, her son and other family members were quantified by fluorescent last-cycle RFLP analysis, using a reverse modified primer and the TspRI enzyme (Department of Molecular Neurogenetics, National Institute of Neurology “C.Besta”, Milano, Italy). ${ }^{13}$

ICSI and PGD procedures (including analysis of oocytes and remaining embryos) were performed as previously described, as were PCR and single cell quantification assay $^{3}$ only with the use of other primers. Mismatch primer ND6_340R ATAGTTTTTTTAATTTATTTAGGGGCA and primer ND6_199F (6-FAM)ATACTCTTTCACCCACAGCAC were used to detect the m.14487T>C mutation by creating a TspRI Restriction site when the mutation was present. The primers SpikeTspRI-F VICAGC TGG TAA AAA GAG GCC TAA C and SpikeTspRI-R GCG CCG AAT AAT AGG TAT AGT $G$ were used to generate an amplicon containing a TspRI site. This VIC labeled amplicon was used to spike the $\mathrm{m} .14487 \mathrm{~T}>\mathrm{C}$ digestion samples to control for complete digestion with the restriction enzyme (TspRI) in order to identify and quantify the m.14487T >C mutation. A calibration curve showing the linearity of the amplification and a Shewhart plot demonstrating the reproducibility are included as Supplementary files (Supplementary Figures S5.1 and S5.2).

Cleavage stage embryos were biopsied at day 3 postfertilization (which is typically the 8-cell stage) and two blastomeres were analyzed. In the individual blastomeres of the non-transferred and non-frozen embryos, as well as in unfertilized oocytes, m.14487T >C heteroplasmy levels were determined in order to obtain further information about interblastomere mutation load variation of this mutation. If embryos could not entirely be dissected in separate blastomeres due to strong intercellular interactions in the morula stage, mean mutation loads of multiple blastomeres were established.

\section{Ethical approval}

The study was approved by the Medical Research Ethics Committee AZM/UM (reference number 03-199).

\section{Patient consent}

Patient consent for publication was obtained.

\section{Results}

Mutation loads identified during the PGD treatment cycles are visualized in Figure 5.2 and annotated in Supplementary Table S5.1. 
The first cycle yielded 12 oocytes, 11 were successfully fertilized and 8 developed into 2-pronuclear (2PN) embryos eligible for 2-blastomere biopsy at day 3 postfertilization. Mutation range varied from $\sim 50 \%$ to $\sim 98 \%$, so unfortunately, all embryos showed $\mathrm{m}$. 14487T $>C$ heteroplasmy levels above the threshold and none could therefore be transferred. Also the unfertilized oocyte showed a high mutation load. Of the 3 remaining fertilized oocytes 2 were degenerated and could not be analyzed, the other one, a single-cell apronuclear (OPN) zygote did not give a result upon testing. In the second cycle 18 oocytes were retrieved and fertilized, resulting in $152 \mathrm{PN}$ embryos and 3 3PN embryos with mutant load ranging from $\sim 6 \%$ to $100 \%$. $132 \mathrm{PN}$ embryos consisted of $\geq 7$ blastomeres enabling 2-blastomere biopsy. Of the 2 embryos consisting of 5 blastomeres, a single cell was biopsied. Two embryos showed heteroplasmy levels below the transfer threshold: mutation loads following 2-blastomere biopsy were $5.4 \%$ and $6.1 \%$ in a 12 -cell embryo (embryo 1 , cycle 2 ) and $12.2 \%$ and $12.3 \%$ in a 13 -cell embryo (embryo 7, cycle 2), respectively. These two embryos were transferred. In three 8-cell embryos and one of the 5-cell embryos the result (in all four above the transfer threshold) was inconclusive because of a positive blank. These are not included in Figure 5.2/Supplementary Table S5.1.

Maximum differences in mutation loads between individual blastomeres were between 0 and 5\% [0.1-3.6\%] in 12 embryos, between 5 and 10\% [5.8-9.1\%] in 5 embryos and $19.4 \%$ (embryo 8 , cycle 1 ) and $30.6 \%$ (embryo 2, cycle 2), in the remaining 2 embryos.

The transfer of the two embryos with mutation loads below $18 \%$ resulted in a viable single pregnancy, from which a healthy son was born at mother's age 42. Prenatal diagnosis to confirm the PGD diagnosis was offered but declined by the couple. In the boy's cord blood the m.14487T>C mutation was shown with 5\% heteroplasmy, which was in accordance with the mutation load of one of the transferred embryos. He is now 7 months old, is healthy and is developing completely normal.

\section{Discussion}

We performed a successful PGD treatment in a female m.14487T >C carrier, resulting in the birth of a healthy boy. This is the first PGD reported for this mutation.

\section{mtDNA segregation and genetic bottleneck}

Our data illustrate the unpredictable segregation of mtDNA mutations from a mother to her offspring. The female carrier is healthy and has moderate mutation loads in her tissues, whereas a significant proportion of her offspring (i.e. the affected child and a subset of the embryos) harbored much higher heteroplasmy levels. The possibility of 
this to occur is inherent to mtDNA inheritance. The number of mtDNA molecules is believed to drastically reduce in the female germline upon transmission, referred to as a 'genetic bottleneck'. ${ }^{\text {eg, } 24}$ As a result, each offspring receives mtDNA that has been formed out of a small (mostly random) sample from the total maternal mtDNA pool. Accordingly, the resulting mutation levels in each offspring may vary widely, both compared to each other and to the mother.

Based on the overrepresentation of embryos with $>90 \%$ mutant load produced by this carrier one may hypothesize the occurrence of selection in favor of the mutation upon transmission, which might at first sight seem corroborated by a number of cases whereby the mother has low mutation load and the child high. ${ }^{11,12,14}$ However, such shifts can occur due to the bottleneck (Otten/Sallevelt et al. submitted) and the reported data mostly represent a single transmission event (one child). Furthermore, there are also reports of more variable segregation patterns, even including the apparent decrease in mutation load upon generations. ${ }^{16,18}$ Thus, current available data are insufficient, both in number and comparability (different tissues tested), to draw conclusions. At the cellular level there is so far no evidence for a selection event for this mutation and its inheritance seems therefore most likely determined by random genetic drift (Otten/Sallevelt et al submitted).

Cycle 1
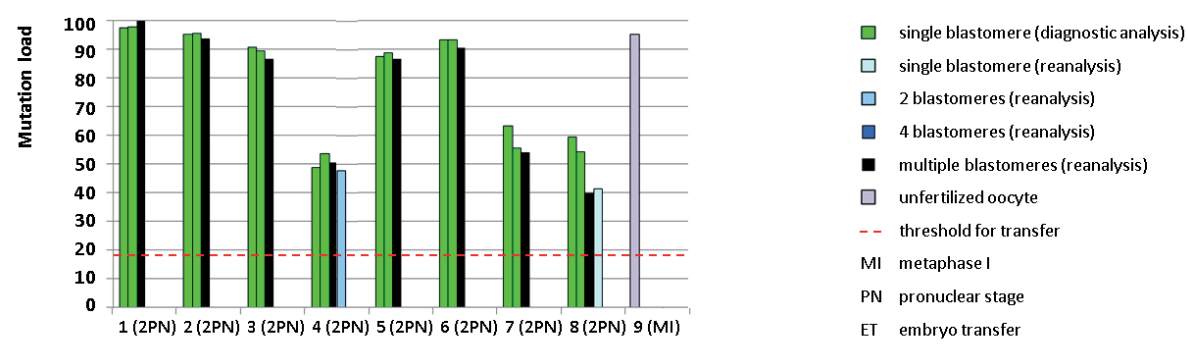

Embryo number (plus one oocyte)

Cycle 2

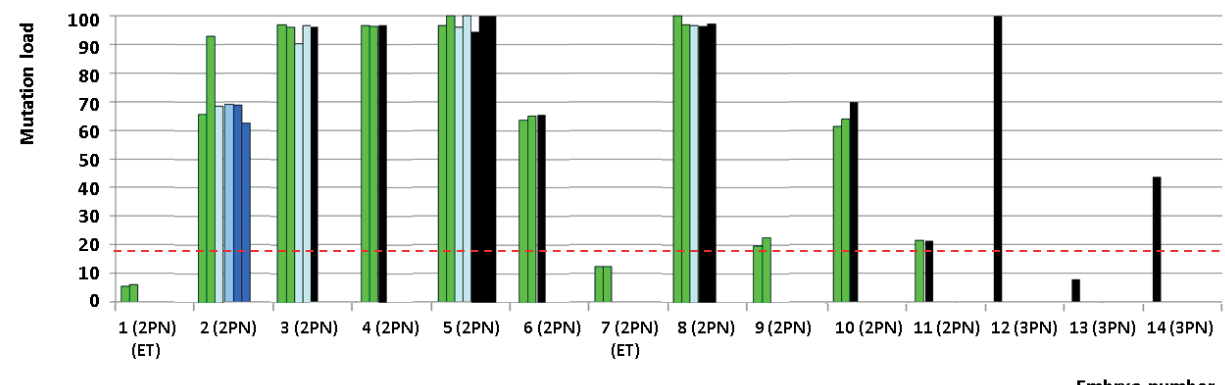

Figure $5.2 \quad$ m.14487T >C PGD treatment cycles. 


\section{Representativeness of blastomere mutation load}

Critical for the reliability of a PGD treatment for mtDNA mutations, is the representativeness of the mutation load in the biopsied blastomere for the entire embryo. Blastomere mutation load is generally fairly constant within the embryo, although single outliers may occur. ${ }^{3,5,25}$ These outliers are probably the result of chance, as the high amount of mtDNA molecules present in the oocyte $\left(50,000-550,000^{26}\right)$ is presumed to be partitioned randomly over the daughter cells (blastomeres) upon cleavage of the early embryo.

We found differences of $<10 \%$ in $\mathrm{m} .14487 \mathrm{~T}>\mathrm{C}$ mutation load between cells of the same embryo in the majority $(90 \% ; 17 / 19)$, whereas in two cleavage stage embryos larger differences of $\sim 20 \%$ and $\sim 30 \%$, respectively, were present. Despite the considerable heteroplasmy variation in these two embryos, neither were at risk of misclassification, because all blastomeres had mutant levels above the transfer threshold. If the one deviating blastomere would have had a mutant level below the threshold whereas the other blastomeres did not, single-blastomere biopsy would have risked the unjust transfer of an embryo. However, in a recent study of another mtDNA mutation $(\mathrm{m} .3243 \mathrm{~A}>\mathrm{G})$ we have shown that this risk is very low $(0.34 \%)$, justifying a single blastomere protocol with advantageous effects on embryo viability, and we conclude that this is applicable to other mtDNA mutations as well (Sallevelt et al submitted). Therefore, henceforth PGD for the m.14487T >C mutation can be carried out on a single blastomere instead of two.

In comparison to trophectoderm (TE) biopsy, blastomere biopsy is more deleterious to the embryo. ${ }^{27}$ However, as long as it has not been shown that TE biopsy gives a true representation of the heteroplasmy percentage in the inner cell mass, blastomere biopsy remains preferred for mtDNA mutations.

In conclusion, the current report illustrates the feasibility of PGD for mtDNA mutation carriers who wish to conceive a biologically-own child, whereby the blastomere mutation load appears representative for postnatal heteroplasmy levels. PGD is clearly preferred compared to PND for carriers such as the female described here, who produce many embryos harboring high mutation loads and a few with low mutation levels which can be conveniently selected this way. This may take more than one PGD treatment or more oocytes per cycle, but likely prevents a large number of abnormal (and/or uninterpretable) PND results. Furthermore, this case confirms earlier reports that the majority of mtDNA mutation carriers is able to benefit from PGD to prevent the transmission of mtDNA disease to their offspring, ${ }^{4,25,28}$ and only a small subset may need the currently still experimental and more controversial nuclear transfer techniques. $^{29-31}$ 


\section{References}

1. Gerards M, Sallevelt SC, Smeets HJ. Leigh syndrome: Resolving the clinical and genetic heterogeneity paves the way for treatment options. Mol Genet Metab. 2016;117(3):300-312.

2. Johnston IG, Burgstaller JP, Havlicek V, et al. Stochastic modelling, Bayesian inference, and new in vivo measurements elucidate the debated mtDNA bottleneck mechanism. elife. 2015;4:e07464.

3. Sallevelt SC, Dreesen JC, Drusedau M, et al. Preimplantation genetic diagnosis in mitochondrial DNA disorders: challenge and success. J Med Genet. 2013;50(2):125-132.

4. Hellebrekers DM, Wolfe R, Hendrickx AT, et al. PGD and heteroplasmic mitochondrial DNA point mutations: a systematic review estimating the chance of healthy offspring. Hum Reprod Update. 2012; 18(4):341-349.

5. Steffann J, Frydman N, Gigarel N, et al. Analysis of mtDNA variant segregation during early human embryonic development: a tool for successful NARP preimplantation diagnosis. J Med Genet. 2006; 43(3):244-247.

6. Thorburn DR, Wilton L, Stock-Myer S. Healthy baby girl born following pre-implantation genetic diagnosis for mitochondrial DNA m.8993T>G mutation. Mol Genet Metab. 2009;98: 5-6.

7. Vandewoestyne M, Heindryckx B, De Gheselle S, et al. Poor correlation between polar bodies and blastomere mutation load in a patient with m.3243A>G tRNALeu(UUR) point mutation. Mitochondrion. 2012;12(4):477-479.

8. Treff NR, Campos J, Tao X, Levy B, Ferry KM, Scott RT, Jr. Blastocyst preimplantation genetic diagnosis (PGD) of a mitochondrial DNA disorder. Fertil Steril. 2012;98(5):1236-1240.

9. Irving L, Craven L, Alston C, et al. Determination of mutation loads in preimplantation embryos to assess the effectiveness of preimplantation genetic diagnosis (PGD) for mitochondrial DNA (mtDNA) inherited disorders. Reprod Biomed Online. 2012;24(supplement 1):S20.

10. Bugiani $M$, Invernizzi $F$, Alberio $S$, et al. Clinical and molecular findings in children with complex I deficiency. Biochim Biophys Acta. 2004;1659(2-3):136-147.

11. Cree LM, Samuels DC, de Sousa Lopes SC, et al. A reduction of mitochondrial DNA molecules during embryogenesis explains the rapid segregation of genotypes. Nat Genet. 2008;40(2):249-254.

12. Lebon $\mathrm{S}$, Chol M, Benit $\mathrm{P}$, et al. Recurrent de novo mitochondrial DNA mutations in respiratory chain deficiency. J Med Genet. 2003;40(12):896-899.

13. Solano A, Roig M, Vives-Bauza $C$, et al. Bilateral striatal necrosis associated with a novel mutation in the mitochondrial ND6 gene. Ann Neurol. 2003;54(4):527-530.

14. Esteitie N, Hinttala R, Wibom R, et al. Secondary metabolic effects in complex I deficiency. Ann Neurol. 2005;58(4):544-552.

15. Wang J, Brautbar A, Chan AK, et al. Two mtDNA mutations 14487T>C (M63V, ND6) and 12297T >C (tRNA Leu) in a Leigh syndrome family. Mol Genet Metab. 2009;96(2):59-65.

16. Dermaut B, Seneca S, Dom L, et al. Progressive myoclonic epilepsy as an adult-onset manifestation of Leigh syndrome due to m.14487T>C. J Neurol Neurosurg Psych. 2010;81(1): 90-93.

17. Ugalde C, Triepels RH, Coenen MJ, et al. Impaired complex I assembly in a Leigh syndrome patient with a novel missense mutation in the ND6 gene. Ann Neurol. 2003;54(5):665-669.

18. Malfatti E, Bugiani M, Invernizzi F, et al. Novel mutations of ND genes in complex I deficiency associated with mitochondrial encephalopathy. Brain. 2007;130(Pt 7):1894-1904.

19. Naess $\mathrm{K}$, Freyer $\mathrm{C}$, Bruhn $\mathrm{H}$, et al. MtDNA mutations are a common cause of severe disease phenotypes in children with Leigh syndrome. Biochim Biophys Acta. 2009;1787(5):484-490.

20. Leshinsky-Silver E, Shuvalov R, Inbar S, Cohen S, Lev D, Lerman-Sagie T. Juvenile Leigh syndrome, optic atrophy, ataxia, dystonia, and epilepsy due to T14487C mutation in the mtDNA-ND6 gene: a mitochondrial syndrome presenting from birth to adolescence. J Child Neurol. 2011;26(4):476-481.

21. Tarnopolsky M, Meaney B, Robinson B, Sheldon K, Boles RG. Severe infantile leigh syndrome associated with a rare mitochondrial ND6 mutation, m.14487T>C. Am J Med Genet. Part A. 2013;161a(8): 2020-2023.

22. Spyropoulos A, Manford M, Horvath R, et al. Near-identical segregation of mtDNA heteroplasmy in blood, muscle, urinary epithelium, and hair follicles in twins with optic atrophy, ptosis, and intractable epilepsy. JAMA Neurol. 2013;70(12):1552-1555. 
23. Eckenweiler M, Catarino CB, Gallenmueller C, et al. Mitochondrial DNA mutation 14487T>C manifesting as Leber's hereditary optic neuropathy. J Neurol. 2015;262(12):2776-2779.

24. Raspall-Chaure M, Solano A, Vazquez E, et al. [A patient with bilateral lesion in the striatum and slowly progressive dystonia secondary to T14487C mutation in the ND6 gene of complex I of the mitochondrial respiratory chain]. Rev Neurol. 2004;39(12):1129-1132.

25. Sallevelt SCEH, J.C.F.M. D, de Coo IFM, de Die-Smulders CEM, Smeets HJM. Selecting the right embryo in mitochondrial disorders. In: Sills ES, ed. Screening the Single Euploid Embryo - Molecular Genetics in Reproductive Medicine: Springer International Publishing Switzerland; 2015:213-246.

26. Fragouli E, Spath K, Alfarawati S, et al. Altered levels of mitochondrial DNA are associated with female age, aneuploidy, and provide an independent measure of embryonic implantation potential. PLoS Genet. 2015;11(6):e1005241.

27. Scott RT, Jr., Upham KM, Forman EJ, Zhao T, Treff NR. Cleavage-stage biopsy significantly impairs human embryonic implantation potential while blastocyst biopsy does not: a randomized and paired clinical trial. Fertil Steril. 2013;100(3):624-630. 


\section{Supplemental material}

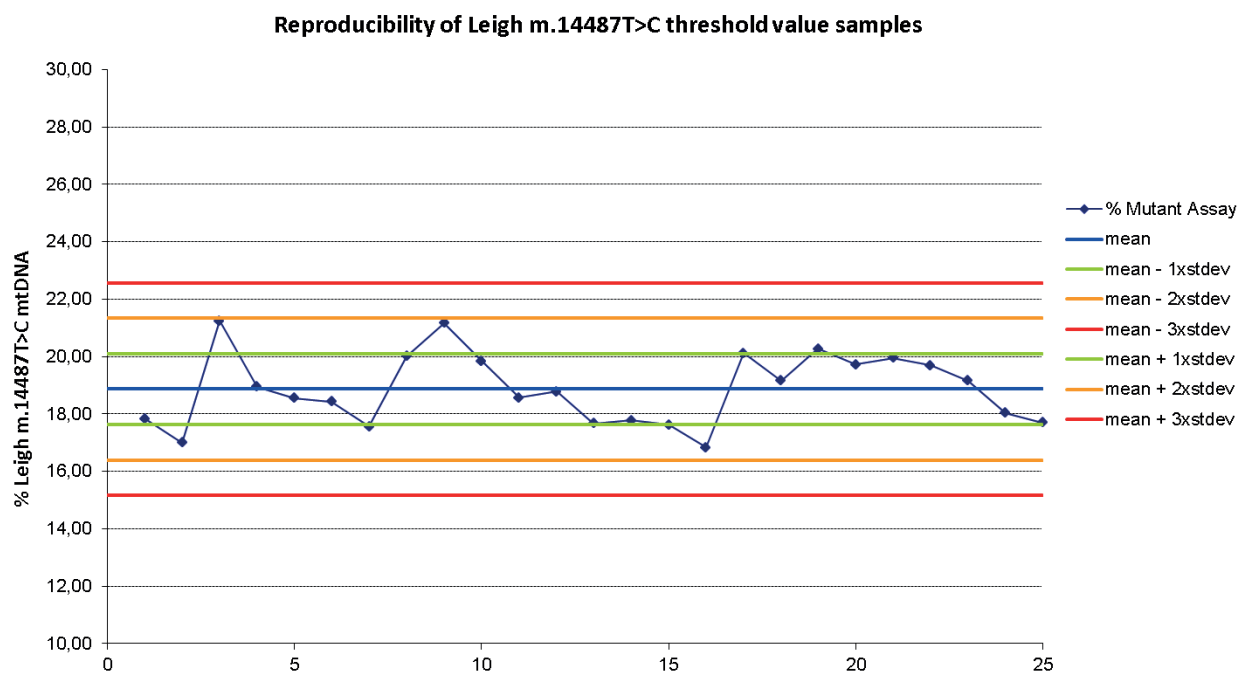

Figure S5.1 Shewhart chart of m.14487T>C. To demonstrate reproducibility and verify a valid PCR amplification, five reference samples with a mutation load around the threshold value ( $18 \%$ for the Leigh m.14487T $>$ C) were included in every PCR series for validation. The reference amplicons were diluted to a single blastomere equivalent amount, approximately 12.500 75.000 mtDNA copies. A total of 25 of these reference samples were amplified in 5 separate PCR runs during protocol setup and the data were used to construct a Shewhart chart which showed the mean, the mean $\pm 1 S D$, the mean $\pm 2 S D$ and the mean $\pm 3 S D$. Four of five control observations must fall within the mean \pm 2 SD to approve the PGD PCR analysis data with a \% mutation load around the threshold value. Data were rejected if more than 1 control sample exceeded the mean $\pm 3 S D$ limit. 
Expected vs Observed Leigh m.14487T $>\mathrm{C}$ mutation load

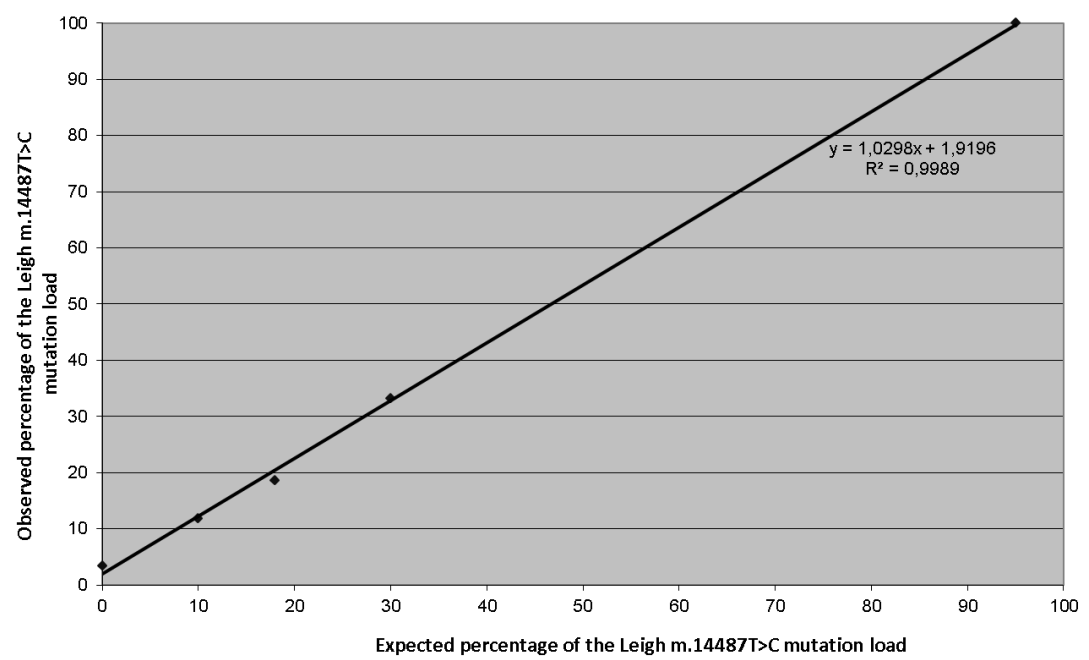

Figure S5.2 Serial dilutions of the Leigh m.14487T>C amplicon to demonstrate the linearity of the amplification. The obtained amplicons are diluted to a single blastomere equivalent amount, approximately 12.500-75.000 mtDNA copies with different mutation load percentages. 


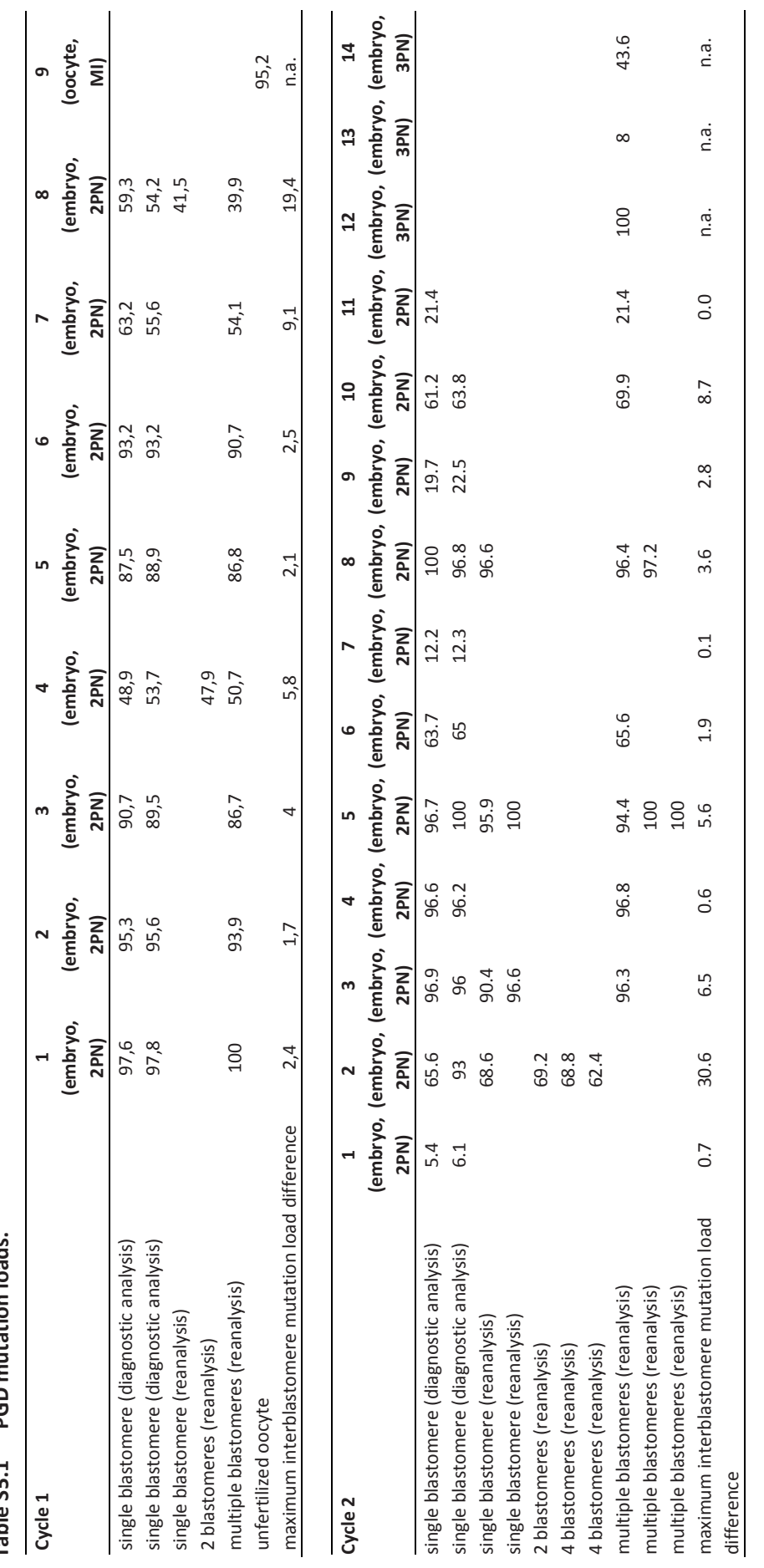




\section{CHAPTER 6}

Mitochondrial DNA inheritance is determined by genetic drift and negative and positive selection

Auke B.C. Otten, ${ }^{\#}$ Suzanne C.E.H. Sallevelt, " Phillippa J. Carling, Joseph C.F.M. Dreesen, Marion Drüsedau, Sabine Spierts, Aimee D.C. Paulussen, Christine E.M. de Die-Smulders, Mary Herbert, Patrick F. Chinnery, David C. Samuels, Hubert J.M. Smeets

\# these authors share first authorship Submitted 


\section{Abstract}

Mitochondrial DNA (mtDNA) inherits maternally through a genetic bottleneck and genetic drift is considered the main driving mechanism. We determined mutation loads of three mtDNA mutations (m.3243A $>\mathrm{G}, \mathrm{m} .8993 \mathrm{~T}>\mathrm{G}$ and $\mathrm{m} .14487 \mathrm{~T}>\mathrm{C}$ ) in preimplantation genetic diagnosis oocytes/zygotes/embryos. Genetic drift occurred, but non-random selection was clearly apparent, based on 1) correlation between m.3243A $>\mathrm{G}$ mutation load and estimated bottleneck size, 2) absence of m.3243A $>$ G mutation loads $>80 \%$, and 3) skewed distribution for the m.8993T $>G$ mutation. Together with data from a meta-analysis this implies that selection mechanisms can have a different effect on different mtDNA mutations. High m.3243A>G mutation loads are selected against, because the resulting complete OXPHOS deficiency prohibits oocyte growth and maturation (progression through meiosis). The OXPHOS deficiency of the m.8993T>G mutation is, even at high mutation loads, less severe. This mutation is positively selected for during oogenesis, because of an increased mitochondrial membrane potential (MMP), a hallmark of healthy mitochondria. The m.14487T >C mutation is transmitted by random genetic drift only. We conclude that non-neutral selection mechanisms occur during mtDNA inheritance, which has implications for current treatment strategies. 


\section{Introduction}

Mitochondrial diseases are a heterogeneous group of progressive multisystemic disorders, which can be caused by mutations in either the nuclear or the mitochondrial DNA (mtDNA). The mtDNA is a small, circular, double stranded, multi-copy genome. Over 150 confirmed pathogenic mutations have been reported in the mtDNA, ${ }^{1}$ affecting about 1 in 5,000 individuals. ${ }^{2}$ In most cases, these pathogenic mtDNA mutations are heteroplasmic, which is a co-existence of wild-type and mutated mtDNA genomes. The mtDNA bottleneck has evolved ${ }^{3}$ to prevent the germline from accumulating, low level heteroplasmic mutations. Based on observations in mice, ${ }^{4,5}$ the mtDNA bottleneck occurs as a result of a restriction in the number of mtDNA molecules during its maternal inheritance, followed by a strong amplification of these founder molecules. In carriers of heteroplasmic mtDNA mutations the bottleneck can have an adverse effect, leading to highly variable mutation loads in their oocytes and an unpredictable recurrence risk. The mechanisms underlying the mtDNA bottleneck are under debate, but to date, although it has been suggested that random genetic drift is predominant mechanism determining the distribution of mtDNA heteroplasmy. ${ }^{6-8}$ When assuming random genetic drift, the segregation pattern of mtDNA mutations can be modeled mathematically by the Kimura distribution. ${ }^{8}$ This model describes the probability distribution of heteroplasmy values in the offspring as a function of the amount of mutation carried over by the mother and the bottleneck size. The segregational unit is either the mtDNA molecule itself or a subset of mtDNA molecules that is preferentially amplified. ${ }^{9,10}$ In mice the physical bottleneck size is estimated to be around 200 mtDNA copies per primordial germ cell (PGC). ${ }^{4,7}$ The exact size of the bottleneck in humans is still under debate, just as the mechanisms underlying the shifts in mutation loads observed. $^{5}$ Some reports describe differences in segregation patterns between different mtDNA mutations, ${ }^{11}$ which, apart from genetic drift, could also be caused by selection events ${ }^{12,13}$ occurring during mtDNA transmission. When selection occurs, a physical and effective bottleneck can be discriminated. The mtDNA molecules that contribute to the mtDNA pool of the next generation determine the effective bottleneck size, which can be lower than the physical bottleneck size when only a subpopulation of mtDNA molecules is preferentially transmitted to the next generation. However, the picture is currently far from clear and the contribution of genetic drift and selection to mtDNA inheritance is still debated.

In the present study we investigated the underlying mechanisms of the mtDNA bottleneck by determining the mutation load of three mtDNA mutations (m.3243A>G, $\mathrm{m} .8993 \mathrm{~T}>\mathrm{G}$ and $\mathrm{m} .14487 \mathrm{~T}>\mathrm{C}$ ) in oocytes, zygotes and embryos from multiple preimplantation genetic diagnosis (PGD) cycles. We observed differences in segregation patterns among these mtDNA mutations, which could not be explained by genetic drift only and our data support the existence of mutation-specific selection factors during 
oogenesis. Besides the mechanisms already proposed in literature, we characterized two selective mechanisms that occurred during oogenesis. As these selective mechanisms alter the effective bottleneck size, these mechanisms give a plausible explanation for the mutation-specific genetic (effective) bottleneck size reported before. ${ }^{14,15}$ Our data was further supported by a meta-analysis of literature data on the segregation of $m .3243 A>G$ and $m .8993 T>G$ mutations. We find that germline selection depends on the degree that mutations affect the cellular OXPHOS-capacity of generating ATP or maintaining a healthy mitochondrial membrane potential (MMP). These findings are important for understanding mtDNA inheritance and will improve the prediction of the recurrence risk for carriers of mtDNA mutations considerably. This has also important implications for preimplantation genetic diagnosis (PGD) and mitochondrial replacement therapy, in which a fraction mutated mtDNA may be carried over during nuclear transplantation. ${ }^{16} \mathrm{~A}$ mechanisms favoring selection, could result in amplification of the mutated genome during embryonic development.

\section{Materials and methods}

The study was performed with informed consent of the participants on rest material of routine clinical procedures, approved by the local ethical committee.

\section{Patients and PGD procedure}

Five female carriers (Table 6.1) of the m.3243A>G mutation, one carrier of the $m .8993 T>G$ mutation and one carrier of the m.14487T $>C$ mutation were counseled for PGD. Verbal and written information on IVF and intracytoplasmic sperm injection (ICSI), single cell procedures, success rates of the procedures, risk of misdiagnosis, and the limited experience on the worldwide application of PGD for mitochondrial disorders was supplied to all carriers and their partners. Before the treatment, informed consent was given by both partners for both the procedure as well as for re-analysis of nontransferred and non-frozen oocytes and embryos. The IVF-ICSI-PGD procedure has been performed as described before. ${ }^{17}$ Briefly, blastomeres were biopsied at 3 days postfertilization and washed three times in phosphate buffered saline solution supplemented with $1 \%$ polyvinylpyrrolidone molecular weight 360000 (Sigma-Aldrich Chemie, Zwijndrecht, the Netherlands) and $0.1 \mathrm{mg} / \mathrm{ml}$ phenol red (Sigma), and transferred to a $0.2 \mathrm{ml} \mathrm{PCR}$ reaction tube, followed by the mutation load analysis. Nontransferrable embryos and embryos not suited for cryopreservation were collected at day 4, as described before. ${ }^{17}$ Remaining fertilized oocytes, zygotes or single blastomeres collected during the procedure, as well as were washed three times in washing buffer, transferred to a $0.2 \mathrm{~mL}$ PCR reaction tube and stored at $-20^{\circ} \mathrm{C}$ until 
mutation load analysis. Furthermore, blood, hair, urine and/or muscle biopsies were taken from all carriers.

Table 6.1 Age and mutation loads in different tissues of carriers of the m.3243A>G, m.8993T>G and m.14487T>C mutation.

\begin{tabular}{|c|c|c|c|c|c|}
\hline m.3243A $>$ G carriers & Carrier \#1 & Carrier \#2 & Carrier \#3 & Carrier \#4 & Carrier \#5 \\
\hline Age first PGD cycle & 36 years & 30 years & 28 years & 32 years & 31 years \\
\hline \multicolumn{6}{|l|}{ Mutation-\% } \\
\hline Blood & $13 \%$ & $25 \%$ & $27 \%$ & $19 \%$ & $16 \%$ \\
\hline Hair & $26 \%$ & $10 \%$ & $29 \%$ & $26 \%$ & $29 \%$ \\
\hline Urine & $55 \%$ & ND & $59 \%$ & $38 \%$ & $28 \%$ \\
\hline Muscle & ND & $28 \%$ & ND & ND & ND \\
\hline Other carriers & $\mathrm{m} .8993 \mathrm{~T}>\mathrm{G}$ & m.14487T $>C$ & & & \\
\hline Age first PGD cycle & 30 years & 40 years & & & \\
\hline \multicolumn{6}{|l|}{ Mutation-\% } \\
\hline Blood & $4 \%$ & $34 \%$ & & & \\
\hline Hair & $3 \%$ & ND & & & \\
\hline Urine & $5 \%$ & $65 \%$ & & & \\
\hline Muscle & ND & $55 \%$ & & & \\
\hline
\end{tabular}

\section{Mutation load analysis}

Primers used for PCR amplification conditions and digestion controls for the m.3243A>G and m.8993T>G mutations were as reported before, ${ }^{17,18}$ while for the m.14487T $>C$ mutation, these were: ND6_199F \& ND6_199F (6-FAM) (ATACTCTTTCACCCACAGCAC), ND6_340R (ATAGTTTTTTTAATTTATTTAGGGCA), SpikeTspRI-F (vic, AGCTGGTAAAAAGAGGCCTAAC) and SpikeTspRI-R (GCGCCGAATAATAGgTATAGTG) and digestion with TspRI. Samples were analyzed by capillary electrophoresis on an ABI Prism 3730 Genetic Analyzer followed by GeneScan analysis. To calculate the mutation load, the area of the mutation peak was divided by the sum of the peak area of the wild-type and mutation peak. The detailed statistical analysis for this procedure has been described before. ${ }^{17}$

For cleavage stage embryos from which multiple blastomeres were available, the average was calculated and reported as the mutation load for this embryo in order to exclude that a single embryo is repeatedly reported. In all cases, the mutation loads in individual blastomeres from a single embryo were not substantially different, consistent with previous findings ${ }^{17}$. Furthermore, mutations loads in blood, hair, urine and/or muscle of varies was established, using the same procedures (Table 6.1).

\section{Estimation of the effective bottleneck size}

We estimated the effective bottleneck size $\left(N_{\text {eff }}\right)$ for a carrier, assuming genetic drift, by using the equation $N_{\text {eff }}=-t / \ln (b)$, where $t$ is the number of mtDNA generations, which has been generally accepted to be 15 during embryogenesis. ${ }^{8}$ As the average 
heteroplasmy level in the dataset $\left(p_{0}\right)$ is taken as an estimate of the amount of mutation carried over from the mother, this is used to determine the bottleneck parameter $(b)$ using equation 6 in reference. ${ }^{8}$

\section{Meta-analysis of published heteroplasmy data (m.3243A>G \& m.8993T>G)}

Blood heteroplasmy data was collected from literature for the m.3243A $>\mathrm{G}(n=111)$ and the m.8993T>G ( $n=118)$ mutation (Supplementary Table S6.1). The blood heteroplasmy levels for the m.3243A>G mutation were age-corrected to account for the loss of the exponential loss of mutated mtDNA in blood leukocytes by using an equation as described before. ${ }^{19}$ Furthermore, patients with mutation load(s) $<15 \%$ were excluded, as this improved the correlation between blood and muscle heteroplasmy (data not shown). For both mutations, the maternal heteroplasmy value was subtracted from the heteroplasmy value in the offspring (O-M). A Gaussian curve was fitted to the frequency histograms. To test whether the samples data from a Gaussian distribution with a mean equal hypothetical value of 0 (no selection bias) we used a one-sample $t$-test. Results were considered significant if the $P$-value was $<0.05$.

\section{Results and discussion}

\section{Individual bottleneck sizes under random genetic drift}

We analyzed the mutation distribution in oocytes, zygotes and blastomeres of five carriers of the m.3243A $>\mathrm{G}$ mutation, one carrier of the m.8993T>G mutation and one carrier of the m.14487T>C mutation, for whom PGD was performed in our clinic (Figure 6.1). Based on these mutation loads, we calculated the effective (or mathematical) bottleneck size (Neff) for every carrier using the Kimura probability distribution 8 (Table 6.2). The Kimura distribution assumes random genetic drift only and gives an estimated bottleneck size value, which might be different from the actual mtDNA copy number value in the PGCs. The calculated Neff values are highly variable, being highest for the m.3243A>G carriers, ranging, on average, from 49-152 mtDNA molecules, whereas Neff for the m.8993T>G and the m.14487T >C carriers are consistently smaller, 10 and 21 respectively. A clear difference (no overlap in confidence interval) exists between the m.8993T $>$ G carrier and the m.3243A $>$ G carrier with the lowest average heteroplasmy (carrier \#5), while there is only a slight overlap in confidence intervals between the m.8993T $>$ G carrier and three of the m.3243A $>$ G carriers. For the m.3243A $>\mathrm{G}$ mutation, the reported values are close to the estimated effective bottleneck size of 108 in a female carrier of the mutation of which 82 single primary oocytes have been analyzed. ${ }^{8}$ 
Table 6.2 Average heteroplasmy levels and estimated bottleneck sizes for carriers with the m.3243A>G, m.8993T > G and m.14487T >C mutation.

\begin{tabular}{lccc}
\hline Carrier & $\mathbf{N}$ & $\begin{array}{c}\boldsymbol{p}_{0}, \begin{array}{c}\text { heteroplasmy in samples } \\
\text { (Average } \pm \text { SEM) }\end{array} \\
\text { m.3242A>G \#1 }\end{array} \quad \begin{array}{c}\text { Effective bottleneck size }\left(\boldsymbol{N}_{\text {eff }}\right) \\
\text { (value [95\% } \mathbf{C l}] \text { ) }\end{array}$ \\
m.3242A>G \#2 & 26 & $0.42 \pm 0.04$ & $83[50-159]$ \\
m.3242A>G \#3 & 16 & $0.34 \pm 0.05$ & $94[50-233]$ \\
m.3242A>G \#4 & 13 & $0.41 \pm 0.07$ & $49[24-117]$ \\
m.3242A>G \#5 & 26 & $0.33 \pm 0.04$ & $92[55-173]$ \\
m.8993T>G & 10 & $0.16 \pm 0.04$ & $152[69-473]$ \\
m.14487T>C & 46 & $0.05 \pm 0.03$ & $10[4-57]$ \\
\hline
\end{tabular}

$\mathrm{n}$, number of unique offspring (oocytes, embryos or zygotes) for every carrier

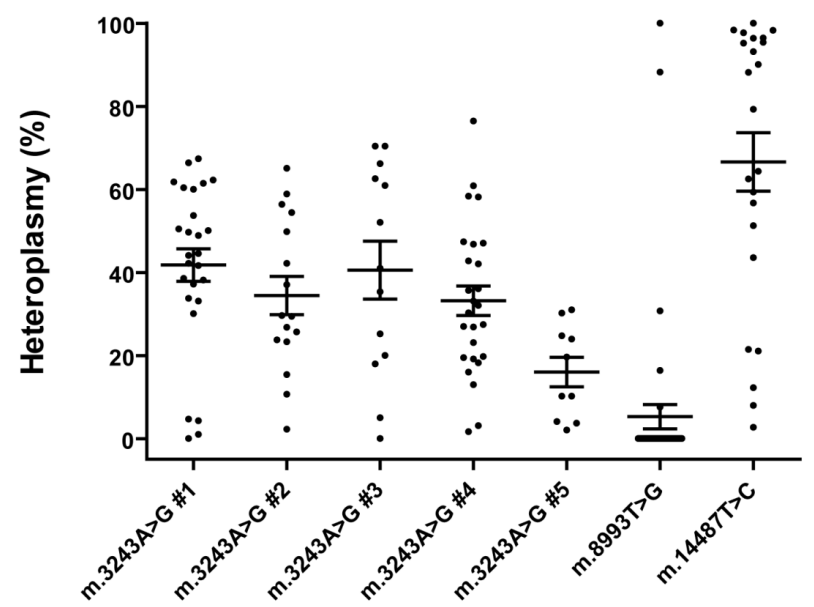

Figure 6.1 Distribution of the percentages of $\mathrm{m} .3243 \mathrm{~A}>\mathrm{G}, \mathrm{m} .8993 \mathrm{~T}>\mathrm{G}$ and $\mathrm{m} .14487 \mathrm{~T}>\mathrm{C}$ mutation in surplus oocytes, blastomeres and zygotes from seven different carriers. When multiple blastomeres of a single cleavage stage embryo were analyzed, the mutation load plotted for this embryo was the average of these blastomeres. Every dot thus represents the (average) mutation load of a single oocyte, embryo or zygote. Horizontal lines indicate average mutation loads with SEM.

\section{Selection occurs during mtDNA inheritance}

The mtDNA segregation pattern can be explained by the Kimura neutral (genetic) drift theory when bottleneck sizes are variable, but the calculated, effective bottleneck sizes may not be equal to the actual physical bottleneck. It has recently been suggested that mtDNA mutations affect their segregation, ${ }^{20}$ implying mechanisms other than random genetic drift to occur. If so, differences in the calculated effective bottleneck size, for which mutation-dependency has been reported, ${ }^{15}$ are not caused by a lower physical bottleneck during germline transmission, but that mtDNA inheritance might be under the influence of mechanisms other than genetic drift. 
Several observations in our data of the carriers indicate non-random mechanisms involved in mtDNA segregation. We observed a strong linear correlation $\left(P\right.$-value $=0.021, R^{2}=-0.93$, Figure 6.2$)$ between the effective bottleneck size and the average $m .3243 A>G$ mutation load in the offspring, which suggest that the effective bottleneck size decreases as the m.3243A>G mutation level in the offspring increases, implying it is not random. Maternal loads of $\sim 50 \%$ are expected, in case of random genetic drift, to yield the whole spectrum of mtDNA mutations in their offspring. Despite sufficient data points for m.3243A>G carrier \#1 and \#3 (mutations loads 50\%, Table 6.1), an absence of high mutation loads (>80\%, Figure 6.1) was noted, whereas we did observe eight samples in the offspring with a mutation load $<20 \%$. Although statistical normality tests are generally too tolerant to demonstrate subtle deviations (due to selection) from a Gaussian distribution, this absence of high mutation loads (>80\%) in offspring of m.3243A $>$ G carriers is consistent with what has been described before, ${ }^{6,18}$ although in one case m.3243A>G mutation between 80 and $90 \%$ have been reported in one oocyte and one cleavage stage embryo. ${ }^{21}$ Furthermore, it has been reported that carriers of the $\mathrm{m} .3243 \mathrm{~A}>\mathrm{G}$ mutation with blood heteroplasmy levels $>50 \%$ tended to have offspring with same or lower heteroplasmy, which was less pronounced for mothers with heteroplasmy $<50 \%{ }^{22}$ Taken together, these data support a strong negative selection above an m.3243A>G mutation load of $80-90 \%$. At lower levels, as demonstrated by a meta-analysis of literature data on differences in blood heteroplasmy levels between mothers and offspring, the role of selection is marginal, as differences in blood heteroplasmy for the m.3243A>G mutation between mothers and offspring (O-M) equals zero (Figure 6.3, $P$-value=0.20).

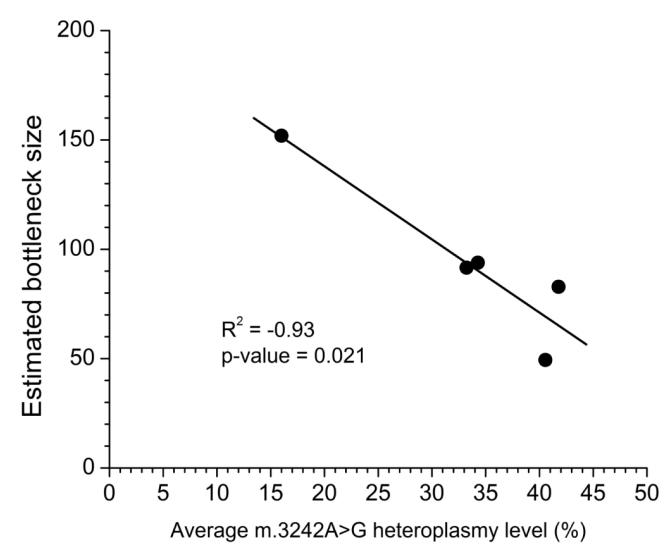

Figure 6.2 Correlation between the average heteroplasmy value in offspring samples (oocytes, zygotes and blastomeres) from the five m.3243A>G carriers and the estimated bottleneck size based on the Kimura distribution. After correlation analysis (Pearson), there was a significant correlation (P-value 0.021 ), indicating that the bottleneck size decreases as the m.3243A>G mutation load increases $\left(R^{2}=-0.93\right)$. 
The distribution of mutation loads in oocytes, zygotes and cleavage embryos of the m.8993T $>$ G carrier was fundamentally different from the m.3243A $>$ G mutation and clearly non-Gaussian, showing a non-random, skewed segregation for this mutation, also indicating selective events are active in during m.8993T>G transmission. Although results for this mutation are limited to one carrier, comparable results have been reported in different studies:

- Seven oocytes from a female m.8993T>G carrier were tested and six oocytes had a mutant load of $>95 \%$, while the other had no signs of the mutation. ${ }^{23}$

- PGD for an asymptomatic carrier of the m.8993T>G mutation resulted in one embryo homoplasmic for the mutation and two embryos that were mutation-free. ${ }^{24}$

- A large collection of pedigrees with a mutation at nucleotide 8993 (which can be either $\mathrm{T}>\mathrm{G}$ or $\mathrm{T}>\mathrm{C}^{25}$ ) showed that the mutation load distribution was skewed to the extremes. The proportion of children with a high mutation load is increased, when the mutant load in the mother is high as well. This is in particular the case for the m.8993T>G mutation. ${ }^{26}$

- Prenatal analysis for the m.8993T>G mutation in six pregnancies indicated three times a mutation load $>95 \%$, two times undetectable mutation loads $(0 \%)$ and one had intermediate (30-80\%) mutation loads. ${ }^{27}$ Intermediate mutation loads (30-40\%) in three embryos after a PGD procedure have been reported only once. ${ }^{28}$ However, the remaining seven embryos analyzed during this PGD procedure had either a very low (one embryo with $2.5 \%$ ) or a very high (six embryos $>95 \%$ ) mutation load, which supports the predominantly skewed segregation of the m.8993T>G mutation. ${ }^{28}$

We obtained additional evidence for a non-random, skewed segregation of the m.8993T>G mutation by performing a meta-analysis (Figure 6.3) of differences in blood heteroplasmy levels between mothers and offspring (O-M). The m.8993T>G mutation had a distribution that was significantly different from zero $(P$-value $<0.0001)$. In these family studies an ascertainment bias could have occurred, because the families were identified through an affected child with a very high mutation load. The impact of such a bias was reduced by ascertainment through the mother, without the proband, which is also the case in offspring analysis as performed in the families we used for metaanalysis, our carriers counseled for PGD, other reported PGD analysis, ${ }^{24,28}$ and prenatal analysis. ${ }^{27}$ Taken together, these data indicate the segregation of the m.8993T>G mutation is under the influence of non-random mechanisms.

For the m.14487T>C mutation limited information is available in literature and only one carrier was assessed in our study. Unlike the distribution in the m.3243A $>\mathrm{G}$ and the m.8993T $>$ G mutation, practically all mutation loads are represented and the distribution appears Gaussian, although due to an overrepresentation of samples with mutation loads $>90 \%$, selection mechanisms cannot be excluded. However, selective events are expected to have a more minor role on inheritance of the m.14487T $>C$ mutation, as all mutation loads were tolerated, but on a single carrier, we cannot draw 
firm conclusions. Obviously, data regarding segregation patterns and bottleneck sizes for mutations in human embryos is limitedly available, but the mtDNA mutation specific mutant load distribution profiles described here are in line with other observations ${ }^{20}$. As these patterns are most likely result from selection events, we investigated the underlying mechanisms.
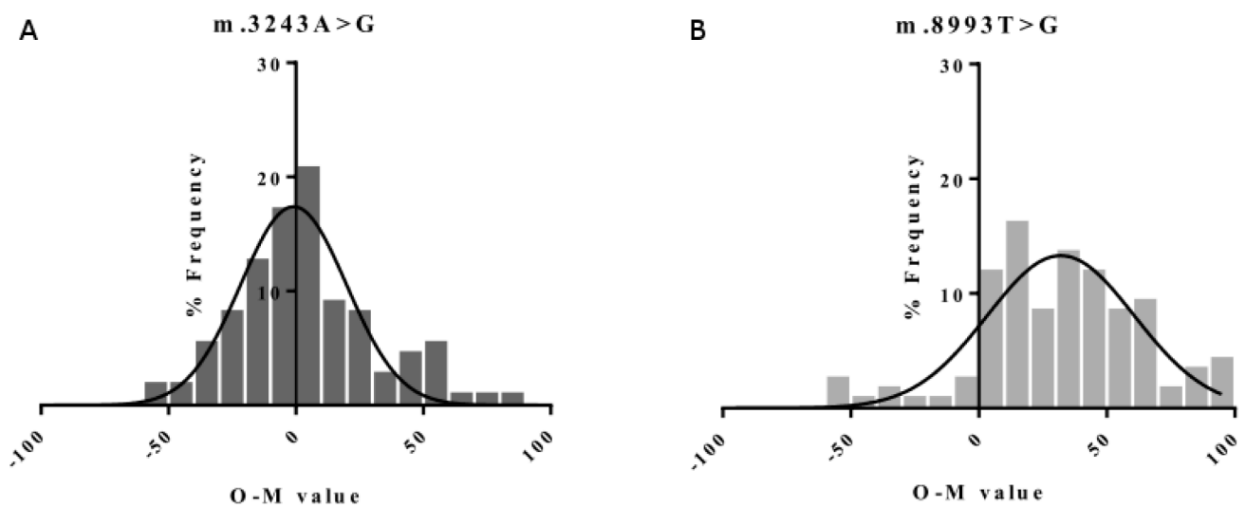

Figure 6.3 Frequency histograms of $\mathrm{O}-\mathrm{M}$ values for $\mathrm{m} .3243 \mathrm{~A}>\mathrm{G}$ and $\mathrm{m} .8993 \mathrm{~T}>\mathrm{G}$ transmissions. Heteroplasmy data from blood for mother to child transmissions were compiled from published studies of the m.3243A $>G \quad(n=111)$ and the m.8993T $>G$ mutation $(n=118)$. The blood heteroplasmy for the m.3243A>G mutation was corrected for age to avoid potential bias. O-M values represent the shift in heteroplasmy between generations ( $O=$ offspring, $M=$ mother) of $A$ ) m.3243A $>G$ and B) m.8993T>G Frequency. Histograms were plotted for both mutations, and a Gaussian curve fitted to each plot.

\section{Selection on ATP production capacity of cellular mitochondria}

Primordial germ cells are highly dependent on glycolysis, ${ }^{29}$ while developing oocytes rely on OXPHOS for ATP production ${ }^{30,31}$ in order to establish a minimal cytoplasmic steady-state ATP level in mature oocytes ${ }^{31,32}$ and (activation of) early embryonic development $^{33}$ (Figure 6.4). The OXPHOS dependency during oogenesis is corroborated by the finding that disturbed mitochondrial metabolism leads to a drop in ATP levels that interferes with oocyte maturation and preimplantation embryogenesis. ${ }^{34}$ The ATP demand can only be satisfied by a highly active mitochondrial OXPHOS, ${ }^{33}$ as the glycolysis pathway is not active. ${ }^{35}$

The m.3243A>G mutation affects the mitochondrial tRNA responsible for leucine-UUR ${ }^{36}$ resulting in defective assembly of complexes I, IV and V in myoblasts homoplasmic for $\mathrm{m} .3243 \mathrm{~A}>\mathrm{G}^{37}$ and, subsequently, in a decrease in the ATP generating capacity. As we did not observe any oocytes, zygotes or blastomeres with $>80 \% \mathrm{~m} .3243 \mathrm{~A}>\mathrm{G}$ mutation load, this is most likely a threshold where the large ATP demands during oogenesis cannot be met anymore despite the high number of mitochondria and 
mtDNA (Figure 6.4), and are selected against by a failure of oocyte maturation. The figure of $80 \%$ is in line with what has been reported for cybrids harboring the m.3243A>G mutation, ${ }^{38,39}$ where oxidative capacity started to decline in cybrids with $60 \%$ mutation load or higher and was completely lost (maximal respiration $<10 \%$ compared to low mutation loads) at $90 \%$ mutation load, due to an almost complete lack of complex I, and severely reduced abundance of complex III and IV. ATP levels rapidly declined in cybrids with $>80 \%$ mutation load with an upregulation of the glycolysis pathway. ${ }^{39}$ This loss in capacity of the mitochondrial energy-generating system has also been observed in muscle biopsies with high m.3243A>G mutation loads. ${ }^{40}$ An adaptive mechanisms has been suggested enabling mutant embryos to escape selection by activating mtDNA replication. ${ }^{20}$ The net result would be a large availability of both mutant and wild-type mtDNA molecules, the latter being able to rescue the energy deficiency induced by the m.3243A>G mutation. This would also explain that very high ( 90\%) m.3243A>G mutations have been reported, ${ }^{21}$ but $100 \%$ mutation loads were never observed.

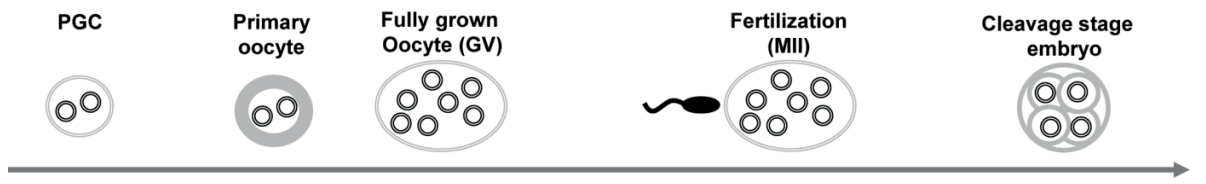

Metabolic dependency Glycolysis OXPHOS

\section{Mitochondrial membrane potential (MMP)}

\section{Total mtDNA content}

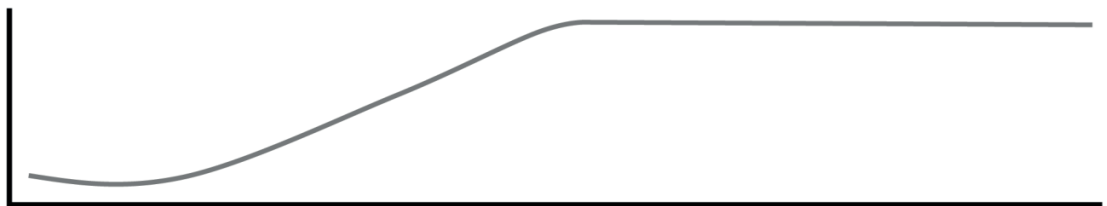

Figure 6.4 Overview of metabolic dependency, mitochondrial membrane potential and mtDNA content during oogenesis and early embryogenesis. The primordial germ cells (PGC) are highly dependent on glycolysis. During oogenesis, the glycolysis becomes blocked and ATP is exclusively produced by mitochondrial OXPHOS, which becomes essential for oocyte maturation and the activation of early embryonic development. Likewise, a high mitochondrial membrane potential (MMP) is essential for oocyte maturation, indicating OXPHOS-dependent ATP production. Furthermore, mtDNA content increases during oogenesis, allowing compensation in OXPHOS defects, as well as selection for highly active mitochondria with high MMP. 
The m.8993T>G mutation affects the ATPase 6 subunit, ${ }^{41}$ and as such impairs the assembly and stability of the terminal complex (complex V) of the OXPHOS machinery, ${ }^{42}$ during which ATP is generated out of a proton gradient. As we observed very high (>95\%) mutation load in oocytes, zygotes and cleavage embryos of carriers, we concluded that negative selection on ATP production capacity did not occur during oogenesis. Indeed, a study in lymphocytes showed that despite high m.8993T>G mutation loads (80-95\%), still $25-35 \%$ capacity in ATP synthesis remained. ${ }^{43,44}$ Likewise, $^{2}$ cybrids homoplasmic for the $\mathrm{m} .8993 \mathrm{~T}>\mathrm{G}$ mutation still displayed $>25 \%$ residual ATP synthesis. ${ }^{45}$ This implies that, even when homoplasmic for the m.8993T>G mutation, oxidative respiration is preserved to a moderate degree, which, in combination with a high abundance of mitochondria in the oocyte (between 19,000 and $25,000^{34}$ ), appears sufficient to produce cytoplasmic ATP for oogenesis to proceed normally.

The m.14487T $>C$ mutation is present in the ND6 subunit of complex I, ${ }^{46}$ thereby affecting its stability and assembly. ${ }^{47}$ The random distribution argues against negative selection and, indeed, biochemical investigations showed that in skeletal muscle, complex I activity was only modestly decreased, ${ }^{48}$ precluding negative selection on ATP production capacity. ${ }^{49}$ Selection on ATP production capacity has also been observed in the germline of a mouse model for mtDNA disease. ${ }^{50}$ A severe ND6 mutation, causing a premature termination of the protein and a subsequent inactivation of OXPHOS, was selectively eliminated during oogenesis within four generations, while a milder mutation in CO1, causing only 50\% decrease in OXPHOS, was retained throughout multiple generations. In conclusion, cellular germline selection against mtDNA mutations depends on the degree that mutations affect the cellular OXPHOS-capacity of generating ATP in combination with the mtDNA copy number.

\section{Selection on membrane potential of mitochondria}

In contrast to the m.3243A $>\mathrm{G}$ and m.14487T $>C$ mutations, oocytes, zygotes and embryos of the m.8993T>G mutation carriers demonstrated skewing to the extremes, which suggests that if the mutation is transmitted above a certain level, it gets rapidly fixed at a high mutation load, indicating positive selection for the m.8993T>G mutation. This can be explained by changes in the mitochondrial membrane potential (MMP). In the metabolically active mitochondria of the oocytes, complex $\mathrm{V}$ is the major regulator of the MMP, which must be maintained to support ATP synthesis. ${ }^{33}$ As a result, an increased MMP is necessary for oocyte maturation and for the competence of an embryo to develop (Figure 6.4). ${ }^{34,51}$ Consequently, MMP has developed as a sensor of normal, healthy OXPHOS activity, which is being selected for during oogenesis at the level of the Balbiani bodies, which contain aggregates of highly active mitochondria with a high MMP. ${ }^{52-54}$ These Balbiani bodies are preferentially transmitted to the PGCs. ${ }^{55}$ It has been reported that the m.8993T>G mutation causes an inefficient coupling between proton transport and ATP production. ${ }^{44}$ In lymphocytes with $>75 \%$ m.8993T>G mutation load or with a blocked complex $\mathrm{V}^{43}$ the MMP was significantly 
increased compared to wild-type mitochondria, because the proton gradient could not be consumed by complex V. Since mitochondrial with high m.8993T>G mutation loads possess a higher MMP, these mitochondria are more likely to be recruited in Balbiani bodies and subsequently transmitted to the offspring, eventually leading to (almost) homoplasmy. This most likely occurs above a certain mutation load, when the selection advantage overrides the genetic drift. Below this threshold, the m.8993T $>\mathrm{G}$ mutation will go to $0 \%$ or rarely generate low-moderate $\mathrm{m} .8993 \mathrm{~T}>\mathrm{G}$ mutation loads ${ }^{28}$ during the 15 germ cell divisions that occur during early embryogenesis. ${ }^{8}$ Together, positive selection events and genetic drift provide for the first time, a plausible explanation for the consistent skewed segregation of the m.8993T>G mutation. By contrast, for the m.8993T >C mutation, which is caused by different substitution of the same nucleotide, a non-skewed, more random segregation pattern has been described, which can be explained by the fact that ATP production is relatively preserved and MMP is not significantly affected by the $\mathrm{T}>\mathrm{G}$ substitution. ${ }^{43}$

In case of the m.3243A>G mutation, the almost complete loss of OXPHOS at high mutation loads causes the MMP to drop, while mitochondria with mainly wild-type mtDNA molecules (with a higher MMP) would therefore have a preference to be recruited in Balbiani bodies, adding up to the absence of high m.3243A $>\mathrm{G}$ mutation loads. Mitochondria with lower mutation loads have a similar MMP compared to those without the mutation and have an equal chance to be recruited in the Balbiani body. This is also the case for the m.14487T >C mutation, which affects the OXPHOS system only modestly and has no effect on the MMP. Although convincing evidence exists for a role of the MMP in selecting mitochondria during oogenesis, definite proof would come from MMP measurements in oocytes harboring mtDNA point mutations.

\section{Conclusion}

Random genetic drift is an important mechanism involved in the segregation of mtDNA. The effects of genetic drift are amplified by the drastic reduction in the mtDNA molecules transmitted to the offspring by the bottleneck mechanism. Based on the mutation distribution in oocytes, zygotes and embryonic cells, a considerable variation in the bottleneck sizes (10 to 152 copies) between carriers and mutations is calculated, causing different rates of genetic drift. However, non-random mutation-specific segregation patterns indicate that other mechanisms are active as well and that genetic drift is supplemented with mutation-specific negative (cellular) and positive (organellar) selection mechanisms, based on optimal OXPHOS capacity and MMP. Future studies are needed to test whether the selective mechanisms we propose here (Table 6.3) are able to explain the segregation of all mtDNA mutations. 
Table 6.3 Relative contribution of genetic drift and selection contribute to the segregation of three mtDNA mutations.

\begin{tabular}{|c|c|c|c|}
\hline Segregational mechanism & $\mathrm{m} .3243 \mathrm{~A}>\mathrm{G}$ & $\mathrm{m} .8993 \mathrm{~T}>\mathrm{G}$ & $\mathrm{m} .14487 \mathrm{~T}>\mathrm{C}$ \\
\hline Genetic drift? & + & + & + \\
\hline Selection on OXPHOS function? & NEGATIVE & POSITIVE & NEUTRAL \\
\hline OXPHOS assembly affected? & $\begin{array}{l}\text { At } 90-100 \% \text { OXPHOS } \\
\text { assembly lost }(\mathrm{Cl})\end{array}$ & Only CV affected & $\begin{array}{l}\text { Stability and assembly } \\
\mathrm{Cl} \text { affected }\end{array}$ \\
\hline ATP generating capacity affected? & $\begin{array}{l}>80 \% \text { mutant load: } \\
\text { respiratory defect, } \\
\text { ATP drops }\end{array}$ & $\begin{array}{l}100 \% \text { mutant load: } \\
\text { residual ATP activity } \\
20-30 \%\end{array}$ & $\begin{array}{l}\text { OXPHOS capacity } \\
\text { preserved }\end{array}$ \\
\hline $\begin{array}{l}\text { Membrane potential ('selection } \\
\text { sensor') affected }\end{array}$ & Severely reduced & $\begin{array}{l}\text { Higher due to } \\
\text { inefficient coupling }\end{array}$ & No effect \\
\hline
\end{tabular}

Cl, complex I. CV, complex V.

Defining the underlying mechanisms of the mtDNA bottleneck are not only important to predict the recurrence risk for mtDNA mutations carriers more accurately, it also has considerable implications for the threshold levels for embryo replacement during PGD, which are currently defined solely on the genetic drift model. Positive selection of a mutation does affect the threshold, below which embryos are being transferred safely, as so far only genetic drift is considered. For mutations under positive selection, the threshold should be reduced. Furthermore, it can have consequences for mitochondrial replacement therapy, a procedure during which the nuclear genome from an mtDNA mutation-harboring zygote of oocytes is transferred to an enucleated acceptor, harboring mutation-free mtDNA. ${ }^{56}$ During this procedure some carry-over of mtDNA can occur, leading to heteroplasmy, which even in the case of neutral variants can be preferentially replicated during ESC propagation. ${ }^{57}$ It is likely that this effect would be amplified in the case of mutations that confer positive selection. It will therefore be particularly important to transfer the lowest possible levels of mtDNA in the event that mitochondrial replacement techniques are used to prevent transmission of such mutations. The combination of genetic drift and (positive) selection leaves a chance that those mtDNA mutations (like the m.8993T>G) can reach higher levels than anticipated by carry-over only. For PGD and mitochondrial replacement therapy this could be taken into account.

\section{Acknowledgements}

We are grateful to GROW-School of Oncology and Developmental Biology for their fellowship to A.B.C.O. and H.J.M.S. 


\section{References}

1. Hellebrekers DM, Wolfe R, Hendrickx AT, et al. PGD and heteroplasmic mitochondrial DNA point mutations: a systematic review estimating the chance of healthy offspring. Hum Reprod Update. 2012;18(4):341-349.

2. Gorman GS, Schaefer AM, Ng Y, et al. Prevalence of nuclear and mitochondrial DNA mutations related to adult mitochondrial disease. Ann Neurol. 2015;77(5):753-759.

3. Otten $A B$, Smeets HJ. Evolutionary defined role of the mitochondrial DNA in fertility, disease and ageing. Hum Reprod Update. 2015.

4. Cree LM, Samuels DC, de Sousa Lopes SC, et al. A reduction of mitochondrial DNA molecules during embryogenesis explains the rapid segregation of genotypes. Nat Genet. 2008;40(2):249-254.

5. Hauswirth WW, Laipis PJ. Mitochondrial DNA polymorphism in a maternal lineage of Holstein cows. Proc Natl Acad Sci U S A. 1982;79(15):4686-4690.

6. Brown DT, Samuels DC, Michael EM, Turnbull DM, Chinnery PF. Random genetic drift determines the level of mutant mtDNA in human primary oocytes. Am J Hum Genet. 2001;68(2):533-536.

7. Jenuth JP, Peterson AC, Fu K, Shoubridge EA. Random genetic drift in the female germline explains the rapid segregation of mammalian mitochondrial DNA. Nat Genet. 1996;14(2):146-151.

8. Wonnapinij P, Chinnery PF, Samuels DC. The distribution of mitochondrial DNA heteroplasmy due to random genetic drift. Am J Hum Genet. 2008;83(5):582-593.

9. Cao L, Shitara $\mathrm{H}$, Horii T, et al. The mitochondrial bottleneck occurs without reduction of mtDNA content in female mouse germ cells. Nat Genet. 2007;39(3):386-390.

10. Wai T, Teoli D, Shoubridge EA. The mitochondrial DNA genetic bottleneck results from replication of a subpopulation of genomes. Nat Genet. 2008;40(12):1484-1488.

11. Howell N, Halvorson S, Kubacka I, McCullough DA, Bindoff LA, Turnbull DM. Mitochondrial gene segregation in mammals: is the bottleneck always narrow? Hum Genet. 1992;90(1-2):117-120.

12. Meiklejohn CD, Montooth KL, Rand DM. Positive and negative selection on the mitochondrial genome. Trends Genet. 2007;23(6):259-263.

13. Stewart JB, Freyer C, Elson JL, et al. Strong purifying selection in transmission of mammalian mitochondrial DNA. PLoS Biol. 2008;6(1):e10.

14. Li M, Rothwell R, Vermaat M, et al. Transmission of human mtDNA heteroplasmy in the Genome of the Netherlands families: support for a variable-size bottleneck. Genome Res. 2016.

15. Wilson IJ, Carling PJ, Alston CL, et al. Mitochondrial DNA sequence characteristics modulate the size of the genetic bottleneck. Hum Mol Genet. 2016;25(5):1031-1041.

16. Mitalipov S, Wolf DP. Clinical and ethical implications of mitochondrial gene transfer. Trends Endocrinol Metab. 2014;25(1):5-7.

17. Sallevelt SC, Dreesen JC, Drusedau M, et al. Preimplantation genetic diagnosis in mitochondrial DNA disorders: challenge and success. J Med Genet. 2013;50(2):125-132.

18. Monnot S, Gigarel N, Samuels DC, et al. Segregation of mtDNA throughout human embryofetal development: m.3243A>G as a model system. Hum Mutat. 2011;32(1):116-125.

19. Rajasimha HK, Chinnery PF, Samuels DC. Selection against pathogenic mtDNA mutations in a stem cell population leads to the loss of the 3243A--> G mutation in blood. Am J Hum Genet. 2008;82(2):333-343.

20. Steffann J, Monnot S, Bonnefont JP. mtDNA mutations variously impact mtDNA maintenance throughout the human embryofetal development. Clin Genet. 2015;88(5):416-424.

21. Treff NR, Campos J, Tao X, Levy B, Ferry KM, Scott RT, Jr. Blastocyst preimplantation genetic diagnosis (PGD) of a mitochondrial DNA disorder. Fertil Steril. 2012;98(5):1236-1240.

22. Uusimaa J, Moilanen JS, Vainionpaa L, et al. Prevalence, segregation, and phenotype of the mitochondrial DNA 3243A>G mutation in children. Ann Neurol. 2007;62(3):278-287.

23. Blok RB, Gook DA, Thorburn DR, Dahl HH. Skewed segregation of the mtDNA nt $8993(T-->G)$ mutation in human oocytes. Am J Hum Genet. 1997;60(6):1495-1501.

24. Steffann J, Frydman N, Gigarel N, et al. Analysis of mtDNA variant segregation during early human embryonic development: a tool for successful NARP preimplantation diagnosis. J Medi Genet. 2006;43(3):244-247. 
25. White SL, Collins VR, Wolfe R, et al. Genetic counseling and prenatal diagnosis for the mitochondrial DNA mutations at nucleotide 8993. Am J Hum Genet. 1999;65(2):474-482.

26. Dahl HH, Thorburn DR, White SL. Towards reliable prenatal diagnosis of mtDNA point mutations: studies of nt8993 mutations in oocytes, fetal tissues, children and adults. Hum Reprod. 2000;15 Suppl 2: 246-255.

27. White SL, Shanske S, Biros I, et al. Two cases of prenatal analysis for the pathogenic T to G substitution at nucleotide 8993 in mitochondrial DNA. Prenat Diagn. 1999;19(12):1165-1168.

28. Thorburn DR, Wilton L, Stock-Myer S. Healthy baby girl born following PGD for the mitochondrial DNA mutation m.8993T>G. Mitochondrion. 2009;10(2):222.

29. Ramalho-Santos J, Varum S, Amaral S, Mota PC, Sousa AP, Amaral A. Mitochondrial functionality in reproduction: from gonads and gametes to embryos and embryonic stem cells. Hum Reprod Update. 2009;15(5):553-572.

30. Dumollard R, Duchen M, Carroll J. The role of mitochondrial function in the oocyte and embryo. Curr Top Dev Biol. 2007;77:21-49.

31. Van Blerkom J, Davis PW, Lee J. ATP content of human oocytes and developmental potential and outcome after in-vitro fertilization and embryo transfer. Hum Reprod. 1995;10(2):415-424.

32. Zeng HT, Yeung WS, Cheung MP, et al. In vitro-matured rat oocytes have low mitochondrial deoxyribonucleic acid and adenosine triphosphate contents and have abnormal mitochondrial redistribution. Fertil Steril. 2009;91(3):900-907.

33. Dumollard R, Marangos P, Fitzharris G, Swann K, Duchen M, Carroll J. Sperm-triggered [Ca2+] oscillations and $\mathrm{Ca} 2+$ homeostasis in the mouse egg have an absolute requirement for mitochondrial ATP production. Development. 2004;131(13):3057-3067.

34. Van Blerkom J. Mitochondrial function in the human oocyte and embryo and their role in developmental competence. Mitochondrion. 2011;11(5):797-813.

35. Shyh-Chang N, Daley GQ, Cantley LC. Stem cell metabolismin tissue development and aging. Development. 2013;140(12):2535-2547.

36. Goto Y, Nonaka I, Horai S. A mutation in the tRNA(Leu)(UUR) gene associated with the MELAS subgroup of mitochondrial encephalomyopathies. Nature. 1990;348(6302):651-653.

37. Sasarman F, Antonicka H, Shoubridge EA. The A3243G tRNALeu(UUR) MELAS mutation causes amino acid misincorporation and a combined respiratory chain assembly defect partially suppressed by overexpression of EFTu and EFG2. Hum Mol Genet. 2008;17(23):3697-3707.

38. Desquiret-Dumas V, Gueguen N, Barth M, et al. Metabolically induced heteroplasmy shifting and Iarginine treatment reduce the energetic defect in a neuronal-like model of MELAS. Biochim Biophys Acta. 2012;1822(6):1019-1029.

39. Picard M, Zhang J, Hancock S, et al. Progressive increase in mtDNA 3243A>G heteroplasmy causes abrupt transcriptional reprogramming. Proc Natl Acad Sci U S A. 2014;111(38):E4033-4042.

40. Janssen AJ, Schuelke M, Smeitink JA, et al. Muscle 3243A-->G mutation load and capacity of the mitochondrial energy-generating system. Ann Neurol. 2008;63(4):473-481.

41. Holt IJ, Harding AE, Petty RK, Morgan-Hughes JA. A new mitochondrial disease associated with mitochondrial DNA heteroplasmy. Am J Hum Genet. 1990;46(3):428-433.

42. Nijtmans LG, Henderson NS, Attardi G, Holt IJ. Impaired ATP synthase assembly associated with a mutation in the human ATP synthase subunit 6 gene. J Biol Chem. 2001;276(9):6755-6762.

43. Baracca A, Sgarbi G, Mattiazzi M, et al. Biochemical phenotypes associated with the mitochondrial ATP6 gene mutations at nt8993. Biochim Biophys Acta. 2007;1767(7):913-919.

44. Sgarbi G, Baracca A, Lenaz G, Valentino LM, Carelli V, Solaini G. Inefficient coupling between proton transport and ATP synthesis may be the pathogenic mechanism for NARP and Leigh syndrome resulting from the T8993G mutation in mtDNA. Biochem J. 2006;395(3):493-500.

45. Manfredi G, Gupta N, Vazquez-Memije ME, et al. Oligomycin induces a decrease in the cellular content of a pathogenic mutation in the human mitochondrial ATPase 6 gene. J Biol Chem. 1999;274(14): 9386-9391.

46. Tarnopolsky M, Meaney B, Robinson B, Sheldon K, Boles RG. Severe infantile leigh syndrome associated with a rare mitochondrial ND6 mutation, m.14487T>C. Am J Med Genet. Part A. 2013;161A(8): 2020-2023. 
47. Ugalde C, Triepels RH, Coenen MJ, et al. Impaired complex I assembly in a Leigh syndrome patient with a novel missense mutation in the ND6 gene. Ann Neurol. 2003;54(5):665-669.

48. Dermaut B, Seneca S, Dom L, et al. Progressive myoclonic epilepsy as an adult-onset manifestation of Leigh syndrome due to m.14487T>C. J Neurol Neurosurg Psych. 2010;81(1):90-93.

49. Gonzalo R, Garcia-Arumi E, Llige D, et al. Free radicals-mediated damage in transmitochondrial cells harboring the T14487C mutation in the ND6 gene of mtDNA. FEBS letters. 2005;579(30):6909-6913.

50. Fan W, Waymire KG, Narula N, et al. A mouse model of mitochondrial disease reveals germline selection against severe mtDNA mutations. Science. 2008;319(5865):958-962.

51. Ge H, Tollner TL, Hu Z, et al. The importance of mitochondrial metabolic activity and mitochondrial DNA replication during oocyte maturation in vitro on oocyte quality and subsequent embryo developmental competence. Mol Reprod Dev. 2012;79(6):392-401.

52. Fosslien E. Mitochondrial medicine--molecular pathology of defective oxidative phosphorylation. Ann Clin :Lab Sci. 2001;31(1):25-67.

53. Zhang YZ, Ouyang YC, Hou Y, Schatten H, Chen DY, Sun QY. Mitochondrial behavior during oogenesis in zebrafish: a confocal microscopy analysis. Development, growth \& differentiation. 2008;50(3):189-201.

54. Zhou RR, Wang B, Wang J, Schatten H, Zhang YZ. Is the mitochondrial cloud the selection machinery for preferentially transmitting wild-type mtDNA between generations? Rewinding Muller's ratchet efficiently. Curr Genet. 2010;56(2):101-107.

55. Milani L. Mitochondrial membrane potential: a trait involved in organelle inheritance? Biol Lett. 2015;11(10).

56. Craven L, Tuppen HA, Greggains GD, et al. Pronuclear transfer in human embryos to prevent transmission of mitochondrial DNA disease. Nature. 2010;465(7294):82-85.

57. Yamada M, Emmanuele V, Sanchez-Quintero MJ, et al. Genetic Drift Can Compromise Mitochondrial Replacement by Nuclear Transfer in Human Oocytes. Cell Stem Cell. 2016. 


\section{Supplemental material}

Table S6.1 Publications contributing heteroplasmy values $\mathrm{m} .3243 \mathrm{~A}>\mathrm{G}$ and $\mathrm{m} .8993 \mathrm{~T}>\mathrm{G}$ for meta-analysis. (Related to Figure 6.3.)

\begin{tabular}{|c|c|c|c|c|c|}
\hline & $\mathrm{m} .3243 \mathrm{~A}>\mathrm{G} \mathrm{mi}$ & utation & & $\mathrm{m} .8993 \mathrm{~T}>\mathrm{G} \mathrm{mu}$ & utation \\
\hline Pedigrees & Transmissions & Reference & Pedigrees & Transmissions & Reference \\
\hline 3 & 8 & (Ciafaloni et al., 1992) $^{1}$ & 1 & 3 & $\left(\right.$ Holt et al., 1990) ${ }^{2}$ \\
\hline 2 & 8 & (Martinuzzi et al., 1992) $^{3}$ & 1 & 1 & (Sakuta et al., 1992) $^{4}$ \\
\hline 1 & 5 & (Huang et al., 1994) $)^{5}$ & 1 & 2 & (Puddu et al., 1993) $^{6}$ \\
\hline 1 & 4 & $\left(\right.$ Liou et al., 1994) ${ }^{7}$ & 1 & 4 & (Shoffner et al., 1992) $^{8}$ \\
\hline 6 & 15 & (Hammans et al., 1995) $^{9}$ & 1 & 5 & (Tatuch et al., 1992) $^{10}$ \\
\hline 3 & 8 & $\left(\right.$ Iwanishi et al., 1995) ${ }^{11}$ & 1 & 2 & $($ Ciafaloni et al., 1993) \\
\hline 1 & 2 & (Rusanen et al., 1995) $^{13}$ & 4 & 6 & (Santorelli et al., 1993) $^{14}$ \\
\hline 1 & 1 & $\left(\right.$ Fabrizi et al., 1996) ${ }^{15}$ & 1 & 5 & $($ Fryer et al., 1994) \\
\hline 1 & 3 & (Huang et al., 1996) $^{17}$ & 1 & 1 & $\left(\right.$ Lodi et al., 1994) ${ }^{18}$ \\
\hline 2 & 8 & $\left(^{(J a n s e n}\right.$ et al., 1997) & 1 & 1 & $($ Pastores et al., 1994) \\
\hline 1 & 2 & $\left(\right.$ Vilarinho et al., 1997) ${ }^{21}$ & 1 & 6 & ${\text { (Degoul et al., } 1995)^{22}}^{22}$ \\
\hline 1 & 1 & (Wilichowski et al., 1998) $^{23}$ & 1 & 3 & $\left(\right.$ Houstek et al., 1995) ${ }^{24}$ \\
\hline 3 & 12 & $(\text { Olsson et al., 1998) })^{25}$ & 1 & 8 & (Makela-Bengs et al., 1995) $^{26}$ \\
\hline 2 & 2 & (Onishi et al., 1998) $^{27}$ & 1 & 2 & $\left(\right.$ Tulinius et al., 1995) ${ }^{28}$ \\
\hline 1 & 2 & (Chinnery et al., 1999) $^{29}$ & 1 & 3 & (Bartley et al., 1996) $^{30}$ \\
\hline 1 & 8 & (Dubeau et al., 2000) $^{31}$ & 1 & 3 & $($ Mak et al., 1996) \\
\hline 1 & 1 & (Hotta et al., 2001) & 1 & 4 & (Ferlin et al., 1997) \\
\hline 1 & 2 & (Ko et al., 2001) & 4 & 9 & $($ Uziel et al., 1997) \\
\hline 1 & 5 & $($ Lien et al., 2001) & 4 & 16 & (White et al., 1999) \\
\hline 1 & 2 & (Morovvati et al., 2002) & 1 & 2 & (Porto et al., 2001) \\
\hline 1 & 3 & (Cervin et al., 2004) ) $^{41}$ & 1 & 2 & (Wong et al., 2002) ${ }^{42}$ \\
\hline 1 & 1 & (Chou et al., 2004) & 1 & 3 & (Enns et al., 2006) $^{44}$ \\
\hline 1 & 1 & (Lu et al., 2002) & 1 & 3 & $\left(\right.$ Steffann et al., 2007) ${ }^{46}$ \\
\hline & & & 2 & 5 & (Mkaouar-Rebai et al., 2009) $^{47}$ \\
\hline
\end{tabular}




\section{Supplemental references}

1. Ciafaloni E, Ricci E, Shanske S, et al. MELAS: clinical features, biochemistry, and molecular genetics. Ann Neurol. 1992;31(4):391-398.

2. Holt IJ, Harding AE, Petty RK, Morgan-Hughes JA. A new mitochondrial disease associated with mitochondrial DNA heteroplasmy. Am J Hum Genet. 1990;46(3):428-433.

3. Martinuzzi A, Bartolomei L, Carrozzo R, et al. Correlation between clinical and molecular features in two MELAS families. J Neurol Sci. 1992;113(2):222-229.

4. Sakuta R, Goto Y, Horai S, et al. Mitochondrial DNA mutation and Leigh's syndrome. Ann Neurol. 1992;32(4):597-598.

5. Huang CC, Chen RS, Chen CM, et al. MELAS syndrome with mitochondrial tRNA(Leu(UUR)) gene mutation in a Chinese family. J Neurol Neurosurg Psychiatry. 1994;57(5):586-589.

6. Puddu $\mathrm{P}$, Barboni $\mathrm{P}$, Mantovani $\mathrm{V}$, et al. Retinitis pigmentosa, ataxia, and mental retardation associated with mitochondrial DNA mutation in an Italian family. Br J Ophthalmol. 1993;77(2):84-88.

7. Liou CW, Huang CC, Chee EC, et al. MELAS syndrome: correlation between clinical features and molecular genetic analysis. Acta Neurol Scand. 1994;90(5):354-359.

8. Shoffner JM, Fernhoff PM, Krawiecki NS, et al. Subacute necrotizing encephalopathy: oxidative phosphorylation defects and the ATPase 6 point mutation. Neurology. 1992;42(11):2168-2174.

9. Hammans SR, Sweeney MG, Hanna MG, Brockington M, Morgan-Hughes JA, Harding AE. The mitochondrial DNA transfer RNALeu(UUR) A-->G(3243) mutation. A clinical and genetic study. Brain. 1995;118 ( Pt 3):721-734.

10. Tatuch Y, Christodoulou J, Feigenbaum A, et al. Heteroplasmic mtDNA mutation (T----G) at 8993 can cause Leigh disease when the percentage of abnormal mtDNA is high. Am J Hum Genet. 1992;50(4): 852-858.

11. Iwanishi M, Obata T, Yamada S, et al. Clinical and laboratory characteristics in the families with diabetes and a mitochondrial tRNA(LEU(UUR)) gene mutation. Diabetes Res Clin Pract. 1995;29(2):75-82.

12. Ciafaloni E, Santorelli FM, Shanske S, et al. Maternally inherited Leigh syndrome. J Pediatr. 1993;122(3):419-422.

13. Rusanen H, Majamaa K, Tolonen U, Remes AM, Myllyla R, Hassinen IE. Demyelinating polyneuropathy in a patient with the tRNA(Leu)(UUR) mutation at base pair 3243 of the mitochondrial DNA. Neurology. 1995;45(6):1188-1192.

14. Santorelli FM, Shanske S, Macaya A, DeVivo DC, DiMauro S. The mutation at nt 8993 of mitochondrial DNA is a common cause of Leigh's syndrome. Ann Neurol. 1993;34(6):827-834.

15. Fabrizi GM, Cardaioli E, Grieco GS, et al. The A to $G$ transition at nt 3243 of the mitochondrial tRNALeu(UUR) may cause an MERRF syndrome. J Neurol Neurosurg Psychiatry. 1996;61(1):47-51.

16. Fryer A, Appleton R, Sweeney MG, Rosenbloom L, Harding AE. Mitochondrial DNA 8993 (NARP) mutation presenting with a heterogeneous phenotype including 'cerebral palsy'. Arch DisChild. 1994;71(5):419-422.

17. Huang CC, Chen RS, Chu NS, Pang CY, Wei YH. Random mitotic segregation of mitochondrial DNA in MELAS syndrome. Acta Neurol Scand. 1996;93(2-3):198-202.

18. Lodi R, Montagna $\mathrm{P}$, lotti S, et al. Brain and muscle energy metabolism studied in vivo by 31P-magnetic resonance spectroscopy in NARP syndrome. J Neurol Neurosurg Psychiatry. 1994;57(12):1492-1496.

19. Jansen JJ, Maassen JA, van der Woude FJ, et al. Mutation in mitochondrial tRNA(Leu(UUR)) gene associated with progressive kidney disease. J Am Soc Nephrol. 1997;8(7):1118-1124.

20. Pastores GM, Santorelli FM, Shanske S, et al. Leigh syndrome and hypertrophic cardiomyopathy in an infant with a mitochondrial DNA point mutation (T8993G). Am J Med Genet. 1994;50(3):265-271.

21. Vilarinho L, Santorelli FM, Rosas MJ, Tavares C, Melo-Pires M, DiMauro S. The mitochondrial A3243G mutation presenting as severe cardiomyopathy. J Med Genet. 1997;34(7):607-609.

22. Degoul F, Diry M, Rodriguez D, et al. Clinical, biochemical, and molecular analysis of a maternally inherited case of Leigh syndrome (MILS) associated with the mtDNA T8993G point mutation. J Inherit Metab Dis. 1995;18(6):682-688. 
23. Wilichowski E, Korenke GC, Ruitenbeek W, et al. Pyruvate dehydrogenase complex deficiency and altered respiratory chain function in a patient with Kearns-Sayre/MELAS overlap syndrome and A3243G mtDNA mutation. J Neurol Sci. 1998;157(2):206-213.

24. Houstek J, Klement P, Hermanska J, et al. Altered properties of mitochondrial ATP-synthase in patients with a T-->G mutation in the ATPase 6 (subunit a) gene at position 8993 of mtDNA. Biochim Biophys Acta. 1995;1271(2-3):349-357.

25. Olsson C, Zethelius B, Lagerstrom-Fermer M, Asplund J, Berne C, Landegren U. Level of heteroplasmy for the mitochondrial mutation A3243G correlates with age at onset of diabetes and deafness. Hum Mutat. 1998;12(1):52-58.

26. Makela-Bengs P, Suomalainen A, Majander A, et al. Correlation between the clinical symptoms and the proportion of mitochondrial DNA carrying the 8993 point mutation in the NARP syndrome. Pediatr Res. 1995;37(5):634-639.

27. Onishi $\mathrm{H}$, Hanihara $\mathrm{T}$, Sugiyama $\mathrm{N}$, et al. Pancreatic exocrine dysfunction associated with mitochondrial tRNA(Leu)(UUR) mutation. J Med Genet. 1998;35(3):255-257.

28. Tulinius $\mathrm{MH}$, Houshmand $\mathrm{M}$, Larsson NG, et al. De novo mutation in the mitochondrial ATP synthase subunit 6 gene (T8993G) with rapid segregation resulting in Leigh syndrome in the offspring. Hum Genet. 1995;96(3):290-294.

29. Chinnery PF, Zwijnenburg PJ, Walker M, et al. Nonrandom tissue distribution of mutant mtDNA. Am J Med Genet. 1999;85(5):498-501.

30. Bartley J, Senadheera D, Park P, Brar H, Wong LJ. Prenatal diagnosis of T8993G mitochondrial DNA point mutation in amniocytes by heteroplasmy detection. Am J Hum Genet. 1996;59:A316.

31. Dubeau F, De Stefano N, Zifkin BG, Arnold DL, Shoubridge EA. Oxidative phosphorylation defect in the brains of carriers of the tRNAleu(UUR) A3243G mutation in a MELAS pedigree. Ann Neurol. 2000;47(2):179-185.

32. Mak SC, Chi CS, Liu CY, Pang CY, Wei YH. Leigh syndrome associated with mitochondrial DNA 8993 T-$>$ G mutation and ragged-red fibers. Pediatr Neurol. 1996;15(1):72-75.

33. Hotta O, Inoue CN, Miyabayashi S, Furuta T, Takeuchi A, Taguma Y. Clinical and pathologic features of focal segmental glomerulosclerosis with mitochondrial tRNALeu(UUR) gene mutation. Kidney Int. 2001;59(4):1236-1243.

34. Ferlin T, Landrieu P, Rambaud C, et al. Segregation of the G8993 mutant mitochondrial DNA through generations and embryonic tissues in a family at risk of Leigh syndrome. J Pediatr. 1997;131(3):447-449.

35. Ko CH, Lam CW, Tse PW, Kong CK, Chan AK, Wong LJ. De novo mutation in the mitochondrial tRNALeu(UUR) gene (A3243G) with rapid segregation resulting in MELAS in the offspring. J Paediatr Child Health. 2001;37(1):87-90.

36. Uziel G, Moroni I, Lamantea E, et al. Mitochondrial disease associated with the T8993G mutation of the mitochondrial ATPase 6 gene: a clinical, biochemical, and molecular study in six families. J Neurol Neurosurg Psychiatry. 1997;63(1):16-22.

37. Lien LM, Lee HC, Wang KL, Chiu JC, Chiu HC, Wei YH. Involvement of nervous system in maternally inherited diabetes and deafness (MIDD) with the A3243G mutation of mitochondrial DNA. Acta Neurol Scand. 2001;103(3):159-165.

38. White SL, Shanske S, McGill JJ, et al. Mitochondrial DNA mutations at nucleotide 8993 show a lack of tissue- or age-related variation. J Inherit Metab Dis. 1999;22(8):899-914.

39. Morovvati S, Nakagawa M, Sato Y, Hamada K, Higuchi I, Osame M. Phenotypes and mitochondrial DNA substitutions in families with A3243G mutation. Acta Neurol Scand. 2002;106(2):104-108.

40. Porto FB, Mack G, Sterboul MJ, et al. Isolated late-onset cone-rod dystrophy revealing a familial neurogenic muscle weakness, ataxia, and retinitis pigmentosa syndrome with the T8993G mitochondrial mutation. Am J Ophthalmol. 2001;132(6):935-937.

41. Cervin C, Liljestrom B, Tuomi T, et al. Cosegregation of MIDD and MODY in a pedigree: functional and clinical consequences. Diabetes. 2004;53(7):1894-1899.

42. Wong LJ, Wong $\mathrm{H}$, Liu A. Intergenerational transmission of pathogenic heteroplasmic mitochondrial DNA. Genet Med. 2002;4(2):78-83.

43. Chou YJ, Ou CY, Hsu TY, et al. Prenatal diagnosis of a fetus harboring an intermediate load of the A3243G mtDNA mutation in a maternal carrier diagnosed with MELAS syndrome. Prenat Diagn. 2004;24(5):367-370. 
44. Enns GM, Bai RK, Beck AE, Wong LJ. Molecular-clinical correlations in a family with variable tissue mitochondrial DNA T8993G mutant load. Mol Genet Metab. 2006;88(4):364-371.

45. Lu CY, Tso DJ, Yang T, Jong YJ, Wei YH. Detection of DNA mutations associated with mitochondrial diseases by Agilent 2100 bioanalyzer. Clin Chim Acta. 2002;318(1-2):97-105.

46. Steffann J, Gigarel N, Corcos J, et al. Stability of the m.8993T->G mtDNA mutation load during human embryofetal development has implications for the feasibility of prenatal diagnosis in NARP syndrome. $J$ Med Genet. 2007;44(10):664-669.

47. Mkaouar-Rebai E, Chaari W, Younes S, Bousoffara R, Sfar MT, Fakhfakh F. Maternally inherited Leigh syndrome: T8993G mutation in a Tunisian family. Pediatr Neurol. 2009;40(6):437-442. 



\section{CHAPTER 7}

De novo mtDNA point mutations are common and

have a low recurrence risk

Suzanne C.E.H. Sallevelt, Christine E.M. de Die-Smulders, Alexandra T.M. Hendrickx, Debby M.E.I. Hellebrekers, Irenaeus F.M. de Coo, Charlotte L. Alston, Charlotte Knowles, Robert W. Taylor, Robert McFarland, Hubert J.M. Smeets

J Med Genet. 2016 Jul 22 (Epub ahead of print) 


\section{Abstract}

\section{Background}

Severe, disease-causing germline mitochondrial (mt)DNA mutations are maternally-inherited, or arise de novo. Strategies to prevent transmission are generally available, but depend on recurrence risks, ranging from high/unpredictable for many familial mtDNA point mutations to very low for sporadic, large-scale single mtDNA deletions. Comprehensive data are lacking for de novo mtDNA point mutations, often leading to misconceptions and incorrect counseling regarding recurrence risk and reproductive options. We aim to study the relevance and recurrence risk of apparently de novo mtDNA point mutations.

\section{Methods}

Systematic study of prenatal diagnosis (PND) and recurrence of mtDNA point mutations in families with de novo cases, including new and published data. "De novo" based on the absence of the mutation in multiple (postmitotic) maternal tissues is preferred, but mutations absent in maternal blood only were also included.

\section{Results}

In our series of 105 index-patients ( 33 children and 72 adults) with (likely) pathogenic mtDNA point mutations, the de novo frequency was $24.6 \%$, the majority being pediatric. PND was performed in subsequent pregnancies of mothers of 4 de novo cases. A fifth mother opted for Preimplantation Genetic Diagnosis (PGD) because of a coexisting Mendelian genetic disorder. The mtDNA mutation was absent in all 4 prenatal samples and all 11 oocytes/embryos tested. A literature survey revealed 137 de novo cases, but PND was only performed for 9 (including 1 unpublished) mothers. In one, recurrence occurred in 2 subsequent pregnancies, presumably due to germline mosaicism.

\section{Conclusions}

De novo mtDNA point mutations are a common cause of mtDNA disease. Recurrence risk is low. This is relevant for genetic counseling, particularly for reproductive options. PND can be offered for reassurance. 


\section{Introduction}

Mitochondrial diseases due to defective oxidative phosphorylation are the most common inborn errors of metabolism, ${ }^{1}$ with between $15 \%$ and $25 \%$ of cases caused by pathogenic mitochondrial (mt)DNA mutations. ${ }^{1,2}$ In the majority of cases these mtDNA mutations are heteroplasmic, a mixture of mutated and wild-type mtDNA molecules in cells and/or tissues of an individual. At a certain level of mtDNA mutant load, the cell expresses dysfunction and symptoms will occur, the so-called threshold effect. This threshold varies within tissues and between different mutations and is difficult to specify for most mtDNA mutations. Nevertheless, mutant loads of below $\sim 18 \%$ are not considered to cause symptoms in $>95 \%$ of the cases. ${ }^{3}$ Severity of clinical involvement broadly increases with a higher heteroplasmy level although clearly this is not the only factor. Individuals exclusively receive their mtDNA from their mother. It is transmitted, at least in part, through a genetic bottleneck induced by a drastic reduction in the number of mtDNA molecules per primordial germ cell during oogenesis, leaving a few mtDNAs to become the founders for the offspring, although the exact nature and timing of events remain topic of debate. ${ }^{4-8}$ The result is, however, indisputable: children of a woman carrying a heteroplasmic mtDNA mutation can display a wide variety of mutation loads.

Disease-causing mtDNA mutations can be point mutations or single, large-scale deletions/rearrangements as a primary cause, or multiple deletions and depletions usually secondary to a nuclear gene defect or environmental factor (e.g., nucleoside RT inhibitors and ageing). The recurrence risk in a subsequent pregnancy depends on the underlying primary genetic defect. The potential of a severe phenotype and the lack of effective treatment often prompts couples who have affected offspring or a positive family history of mtDNA disease to request intervention to prevent transmission.

Women harboring an mtDNA deletion have a low risk ( 1 in 24) of clinically-affected offspring, ${ }^{9}$ whereas females with an mtDNA point mutation potentially have a high risk of recurrence. Often, for female carriers the risk of having an affected child is difficult to predict. However, a substantial proportion of the children with a single, large-scale mtDNA deletion or point mutation have a de novo mutation not inherited from their mother. ${ }^{1}$ The recurrence risk of de novo, single large-scale mtDNA deletions in subsequent offspring is low. ${ }^{9-11}$ For de novo point mutations one would expect the recurrence risk to be similarly low although this has not been systematically investigated to date.

In reproductive counseling of mtDNA mutations, the choice between prenatal diagnosis (PND) and preimplantation genetic diagnosis (PGD) depends mainly on the recurrence risk and on the expected predictive value of the test. The latter can be problematic in 
PND given the potential difficulties of interpreting results for most mtDNA mutations when fetal mutant load falls within a 'grey zone' where correlation between genotype and phenotype is unclear. However, when the likelihood of offspring with either no mutation or a mutant load below the heteroplasmic threshold of disease expression, is high, PND can be applied for reassurance. This is the case for single, large-scale mtDNA deletions $^{9-12}$ and for low level mtDNA mutations demonstrating skewing to the extremes (e.g., m.8993T>G) in the mother. ${ }^{13}$ Also, when maternal mutation load of a non-skewing mtDNA mutation is very low, PND could be considered. For familial mtDNA mutations showing an unpredictable and/or high recurrence risk, Preimplantation Genetic Diagnosis (PGD) is an attractive option ${ }^{3,14}$ although patient preference and choice plays an important role in the decision-making process. Moreover, other factors including maternal age or pre-existent fertility problems may influence an informed decision regarding preferred reproductive options.

In order to study the relevance and recurrence risk of apparently de novo mtDNA point mutations, we evaluated the occurrence of de novo mtDNA point mutations in our own experience and in the literature, and present data on five couples presenting to our own clinical services who have had an affected child and for whom PND or PGD has been performed in a subsequent pregnancy. We offer recommendations for reproductive counseling including strategies to prevent the birth of children affected by mtDNA disease.

\section{Materials and methods}

\section{Patients and patient data}

The frequency of de novo versus recurrent mtDNA disease was studied by cataloguing all (likely) pathogenic mtDNA point mutations identified in our diagnostic laboratory either by specifically screening for known mutations (m.3243A>G, m.8344A>G, $\mathrm{m} .8993 \mathrm{~T}>\mathrm{C} / \mathrm{G}$ ) or sequencing the entire mitochondrial genome. 'Likely' pathogenic refers to novel mutations that are suspected to be pathogenic based on wellestablished prediction tools and accepted criteria, ${ }^{15,16}$ however this was not in all cases proven by functional (e.g., single fiber segregation, transmitochondrial cybrid) studies. In addition, we documented whether maternal relatives of the index patients were tested to determine presence and, if applicable, heteroplasmy levels of the mtDNA mutation. Apparent de novo mtDNA mutations were defined by their absence in one or more accessible maternal tissues (e.g., blood, urinary sediment, buccal epithelia) and, if tested, absence in other matrilineal relatives. Conversely, if the mtDNA mutation was detected in the mother and/or other maternal relatives, it was classified as maternallyinherited/familial. The de novo frequency was calculated by taking the proportion of 
apparently de novo mutations from all mtDNA mutations identified between January 1996 and March 2015. Cases where it was not clear whether the reported mtDNA mutation was (apparently) de novo or maternally-inherited/familial because additional familial testing was not possible, were excluded from the analysis.

\section{Literature search}

PubMed was systematically searched as of December 2015 for cases of de novo mtDNA mutations. Search terms were "mtDNA de novo mutation". PubMed's automated query translation, incorporating $\mathrm{MeSH}$ terms and enhancements from the Unified Medical Language System (UMLS ${ }^{\circledR}$ ), was as follows: "dna, mitochondrial"[MeSH Terms] OR ("dna"[All Fields] AND "mitochondrial"[All Fields]) OR "mitochondrial dna"[All Fields] OR "mtdna"[All Fields]) AND "de novo"[All Fields] AND ("mutation"[MeSH Terms] OR "mutation"[All Fields]. Also 'related citations' of de novo reports in PubMed were screened.

\section{Prenatal diagnosis and preimplantation genetic diagnosis}

Chorionic villus sampling (CVS) samples were obtained at $10+4$ weeks and $11+2$ weeks gestation, respectively, amniotic fluid sampling at $16+2$ weeks and $16+6$ weeks, respectively. DNA extraction from prenatal and postnatal tissues, quantitative analyses of the mtDNA mutations as well as the PGD procedure were performed as previously described. ${ }^{14,16,17}$ Primers used for the Maastricht cases are for m.8993T>C/G: CACACCTACACCCCTTATCCC (forward) and TCATTATGTGTTGTCGTGCAG (reverse); for m.5556G>A: CACCATCATAGCCACCATCA (forward) and GGCTGAGTGAAGCATTGGAC (reverse); for m.8969G>A: GCTTCATTCATTGCCCCCAC (forward) and AGGGCTATTGGTTGAATGAGTAAG (reverse); and for m.3243A>G: CAACTTAGTATTATACCCACAC (forward) and TTTCGTTCGGTAAGCATTAG (reverse). Mutation-specific restriction enzymes are: Hpall (Roche) for m.8993T>C/G, Ddel (Roche) for m.5556G>A, Alul (Roche) for m.8969G $>$ A and Haell (Roche) for m.3243A>G. For the Newcastle cases (ref. ${ }^{18}$ and unpublished data), pyrosequencing was used to quantitatively assess mtDNA mutation heteroplasmy levels as described previously $^{19}$ (primer sequences available on request). For one patient, further assessment of mtDNA heteroplasmy was undertaken using NGS-based mtDNA sequencing; briefly, patient DNA samples were amplified by long-range PCR, sheared using the Ion Xpress ${ }^{\mathrm{TM}}$ Plus Fragment Library Kit and sequenced on an Ion Torrent PGM platform according to manufacturer's protocols (Life Technologies, Foster City, California, USA). 


\section{Results}

\section{De novo mtDNA point mutations}

In our diagnostic laboratory, 105 index cases were identified based on laboratory diagnoses with a (likely) pathogenic mtDNA point mutation (Table 7.1 and Supplementary Table S7.1). The majority (72/105) being adults, our cohort seems a fair representation of the patient population. A subset of 17 patients were found to harbor an apparently de novo mtDNA mutation (Table 7.1), of which 12 were between 0 and 3 years of age at investigation, whereas 5 were $>18$ years old. Of note, in 3 of 17 cases only maternal blood was analyzed, and with a semi-quantitative method. In two other mothers, besides blood only hair, and hair plus fibroblasts, respectively, were tested. These cases have a lower probability of being truly de novo than the remaining 12 where besides blood also maternal urine and/or muscle has been investigated. Maternal inheritance was firmly established for 52 patients, 35 of whom were adults (Supplementary Table S7.1). Notably, for one of these cases (patient 44) we were able to demonstrate de novo occurrence of the pathogenic mtDNA mutation in the mother of the patient, which might also be the case for two other families (families 14 and 20). For the outstanding 36 patients, it remains unknown whether the mtDNA mutation arose de novo in the index patient or not (supplementary table 7.1). Accordingly, 17/69 $(24.6 \%)$ of the (likely) pathogenic mtDNA point mutations occurred de novo in the index patient in our series. Additionally, we identified a further 137 de novo cases in the literature (Supplementary Table S7.2; those published from our own center and therefore shown in Table 7.1 or Supplementary Table 7.1 are not included). These are listed according to whether the mother and/or siblings were tested as well, and if so, whether this was carried out in one or more tissues to assess mtDNA heteroplasmy levels. Reports with apparently de novo mtDNA mutations where no (close/relevant) relatives were tested (e.g., ${ }^{20-24}$ ), are not included in the table.

\section{PND/PGD in subsequent pregnancies}

The parents of four of the five children described below with apparently de novo mtDNA disease (Figure 7.1, Tables 7.1 and 7.2) were counseled in the outpatient department of Clinical Genetics in Maastricht. For case 3, the parents were counseled at another center while mtDNA analyses were performed in Maastricht. 


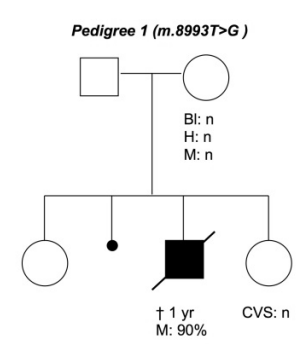

Pedigree 3 ( $m .8969 G>A)$
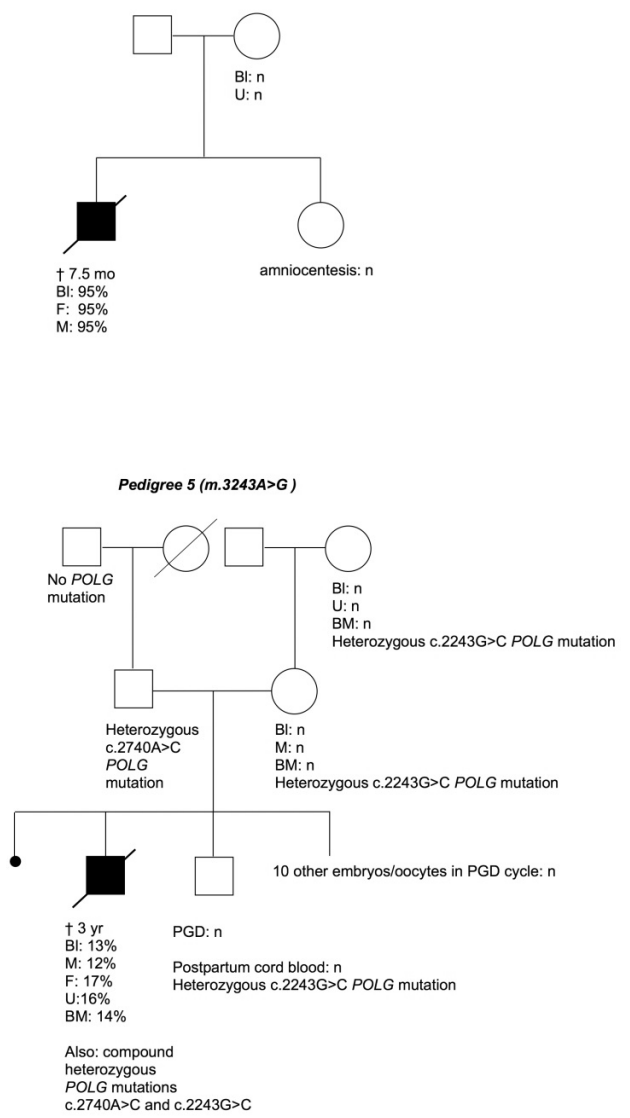

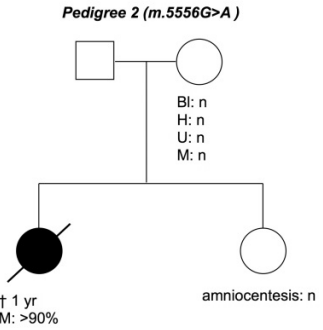

Pedigree 4 (m.8993T>G)

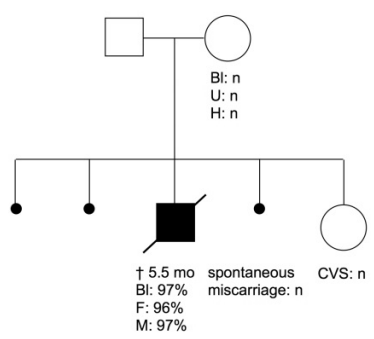

Figure 7.1 Pedigrees of the five case descriptions in this report.

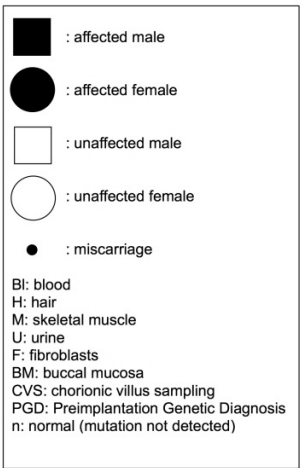




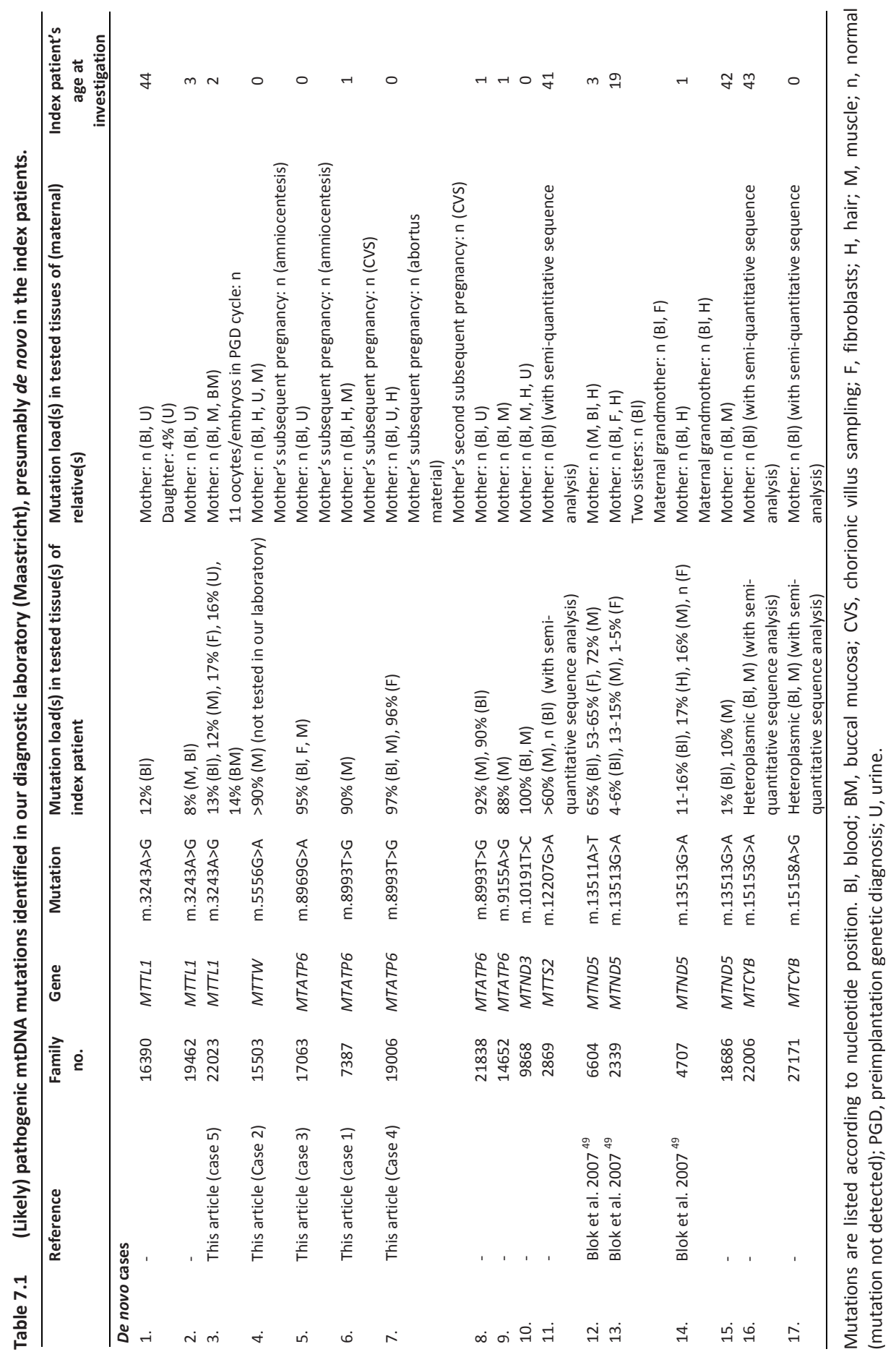


De novo mtDNA point mutations are common and have a low recurrence risk

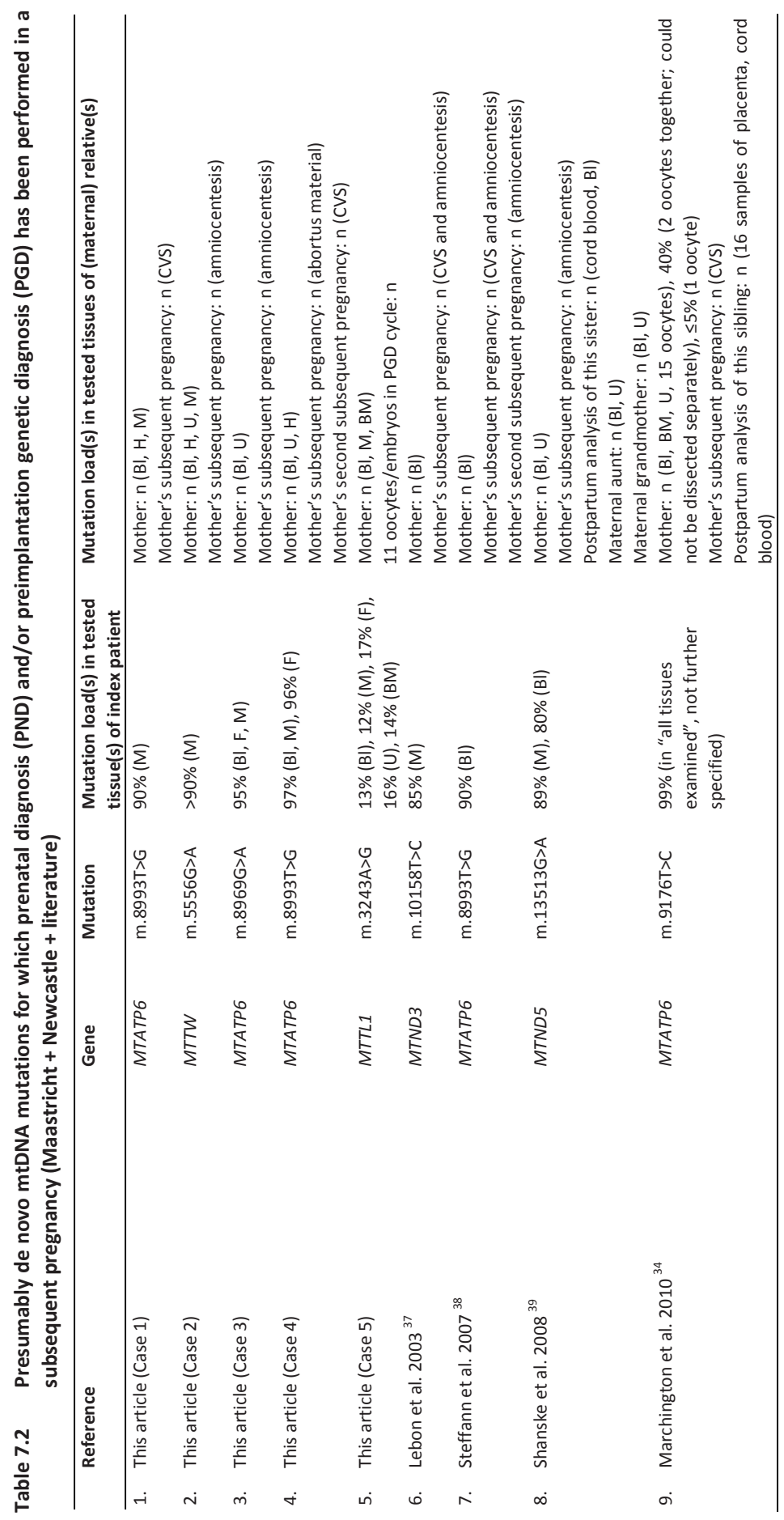




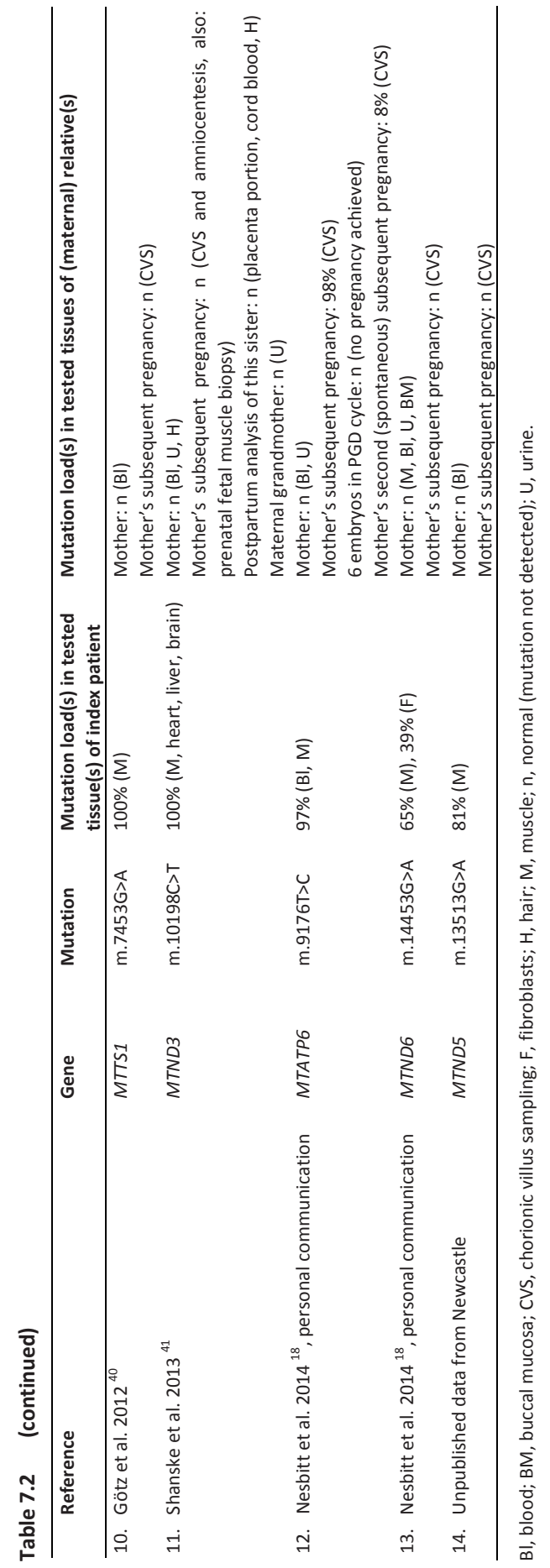




\section{Case 1}

Couple 1 was referred for PGD because their son had Leigh syndrome due to the m.8993T>G mutation in the MTATP6 gene, with 90\% mutant load in skeletal muscle. He died at the age of 1 . Family history was otherwise negative. His healthy older sister, 6 years of age, was not tested for the mutation. In the mother's blood, hair and muscle the $\mathrm{m} .8993 \mathrm{~T}>\mathrm{G}$ mutation was absent (detection level $<1 \%$ ). The mutation seeming to have arisen de novo in their son, a low recurrence risk was estimated and PND was offered for reassurance. The couple were surprised because maternal inheritance of the $\mathrm{m} .8993 \mathrm{~T}>\mathrm{G}$ mutation was assumed, based on the high mutation percentage and severe disease in the affected child, and they were counseled as having a high recurrence risk. Furthermore, they were informed that PND was not an option because of limitations in predicting the phenotype of the offspring and PGD was recommended. The couple opted for PND. The mutation was not detected in chorionic villus DNA and a healthy daughter was born. The child has not been genetically tested postpartum, but is still doing well at the age of 11.

\section{Case 2}

Couple 2 was referred for PGD. Their daughter had a mitochondrial disorder due to oxidative phosphorylation defects (strongly diminished complex I, III and IV activities). She was shown to carry an m.5556G>A mutation in the MTTW gene, with $>90 \%$ heteroplasmy level in skeletal muscle. This mutation had not been described before. Pathogenicity was based on the disruption of the tertiary structure of mt-tRNA ${ }^{\text {Trp }}$ by the mutation, and the compatibility of the mutation with the combined deficiency of complexes I, III and IV, which was also demonstrated in transmitochondrial cybrids. ${ }^{25}$ She died when she was 1.5 years old. Family history was negative. The mother's blood, hair, urine and muscle did not show the m.5556G>A mutation (detection level $<1 \%$ ), and accordingly, the mutation was considered de novo in their daughter, further supporting a pathogenic role. The recurrence risk was regarded as low and PND was offered. PGD seemed a less suitable option because of the presumed low recurrence risk and the maternal age (38 years). As in case 1 , the couple was surprised since at the referring center PND was not considered an option because of uncertainties about the representativeness of the prenatal sample for the fetus, about the stability of the mutant load over time, and about the clinical phenotype to be expected. Amniocentesis revealed no mutation. A healthy daughter was born, who is now 4 years old. No mutation analysis was performed postpartum.

\section{Case 3}

The son of couple 3 died at age 7.5 months carrying the m.8969G $>$ A mutation in the MTATP6 gene with $95 \%$ heteroplasmy in blood, fibroblasts and skeletal muscle. The m.8969G >A mutation was not detected in the mother's blood and urine (detection 
level $<1 \%$ ). The couple opted for PND in a subsequent pregnancy and amniocentesis showed no mutation. A healthy girl was born, now almost 4 years old. She has not been tested for the mutation postpartum.

\section{Case 4}

Couple 4 was referred to discuss their reproductive options because their son had Leigh syndrome caused by an almost homoplasmic m.8993T>G mutation in the MTATP6 gene, demonstrated in his blood, fibroblasts and skeletal muscle. He died when he was 5.5 months of age. In the mother's blood, urine and hair the m.8993T>G mutation was not present (detection level $<1 \%$ ). The mutation therefore appeared de novo in their son, resulting in a low recurrence risk. The boy also had neurofibromatosis type 1 (NF1) caused by a de novo mutation c.2155dupA in the NF1 gene. Since recurrence risks of both the m.8993T>G and the NF1 mutation were low, PND was offered. The subsequent pregnancy ended in a spontaneous miscarriage. The m.8993T>G mutation was not detected in the abortus. Subsequently a further spontaneous pregnancy was achieved. CVS was performed and the m.8993T $>\mathrm{G}$ was not detected in chorionic villi. A healthy daughter was born, who is presently 2 years old. No mutation analysis was performed postpartum.

\section{Case 5}

Couple 5 was referred to us to discuss the possibility of PGD because their son harbored the m.3243A>G MTTL1 mutation. Mutation loads were 13\%, 12\%, 17\%, 16\% and $14 \%$ in his blood, skeletal muscle, fibroblasts, urine and buccal mucosa cells, respectively. The mutation was absent in the mother's blood, muscle and buccal mucosa (detection level $<1 \%)$; the mutation was also absent in the maternal grandmother's blood, urine and buccal mucosa, consistent with a de novo mutation in the index patient and a low recurrence risk of m.3243A>G-related disease. It was doubtful, however, whether the boy's severe, infantile-onset, clinical presentation with hypotonia, feeding problems, psychomotor retardation and intractable epilepsy could be explained by the relatively low mutation load of the m.3243A>G mutation, and just prior to his death at age 3, compound heterozygosity for the POLG mutations c.2740A>C, p.(Thr914Pro) and c.2243G >C, p.(Trp748Ser) was diagnosed, which was consistent with his clinical features of Alpers' syndrome. Both parents were carriers (father c.2740A $>C$, p.(Thr914Pro) and mother c.2243G >C,p.(Trp748Ser)), resulting in a recurrence risk of $25 \%$ for the recessive POLG mutations. The couple preferred PGD to PND. Despite the presumed low recurrence risk for the m.3243A>G mutation, they also requested analysis of the embryos for the m.3243A>G mutation. It was agreed that of the embryos one blastomere was tested for POLG and one blastomere for m.3243A $>G$. Since testing for the m.3243A>G mutation was performed as reassurance, analysis of one blastomere seemed reasonable, although two blastomeres are usually analyzed. ${ }^{14}$ 
In none of the 11 embryos and oocytes the m.3243A>G mutation was detected. One embryo, heterozygous for one of the POLG mutations, was transferred and resulted in a successful pregnancy. The couple did not opt for PND to confirm the PGD result. A healthy son was born. Postpartum DNA-analysis was performed in cord blood and confirmed the blastomere genotype and absence of the m.3243A>G mutation.

In the literature, together with one unpublished case from Newcastle, we identified a further 11 prenatal diagnoses, performed in 9 pregnant mothers of a previously identified case of de novo mtDNA disease. These are listed in Table 7.2, together with our own five cases. All but one case (case 12) showed normal prenatal results. One of the mothers (also case 12) additionally underwent one PGD treatment.

\section{Discussion}

Irrespective of the mechanism leading to mtDNA disease in a child, parents may desire to prevent disease in a subsequent child. The reproductive options available to such couples largely depend on the genetic aetiology. Based on data provided from our own clinical experience and from cases published in the literature, we conclude that mtDNA mutations arise de novo in a significant number of cases and that the recurrence risk for apparently de novo mtDNA mutations is low.

A common approach to determine whether an mtDNA mutation occurred de novo is by testing multiple tissues from the mother for the heteroplasmic mtDNA variant. It is, however, remarkable that often only the mother's blood is analysed even when the mutation was not tested or detected in the affected child's blood (Supplementary Table S7.2). It is well-known, at least for certain mtDNA mutations, ${ }^{26-28}$ that mutant load in blood, a rapidly-dividing tissue, can decrease over time due to negative selection. This clearly has implications for the reliability of maternal blood in evaluating de novo occurrence of an mtDNA mutation. Therefore, preferably muscle (a post-mitotic tissue) should be included in the maternal analysis, which has however the drawback of an invasive procedure. Notably, needle muscle biopsy sampling nowadays offers a less invasive, more rapid alternative to conventional open muscle biopsies, yielding a sufficient muscle amount for DNA analysis. Urine epithelium has been shown to be a reliable non-invasive alternative for the $\mathrm{m} .3243 \mathrm{~A}>\mathrm{G}$ mutation. ${ }^{29}$ This may also be true for other pathogenic mtDNA point mutations ${ }^{30}$ although urine mutant load has not been compared with muscle levels for these. Evaluation of tissue distribution is also critical in light of potential selection events in the germline. Negative selection has been suggested for pathogenic tRNA mutations with low blood mutant levels (pointing to detrimental effects in replicating cells), which are less likely to be transmitted and as a consequence occur more often in isolated cases. ${ }^{31}$ Similarly, negative germline 
selection has been proposed for deleterious heteroplasmic mtDNA mutations and for de novo mutations in a recent study of healthy humans. ${ }^{32}$ These findings implicate that mutations that are absent or (very) low-level in the index patient's blood may be more likely to have indeed occurred de novo. This cannot, however, be assumed for individual mutations without careful analysis of the mother. Furthermore, conversely, potential positive selection events in the germline whereby high mutation loads in offspring could result from low maternal levels cannot be excluded and again stress the importance of thorough maternal investigation. In addition to analysis of maternal tissues, testing apparently healthy siblings of the affected child can contribute to the likelihood that a mutation occurred de novo. The method used to assess mtDNA heteroplasmy is of critical importance. Fluorescent last-cycle RFLP analysis has a detection level of $<1 \%$, whereas Sanger sequencing has a sensitivity of between $5 \%$ and $30 \%$ to detect different heteroplasmic mutations (unpublished laboratory findings). Even using last-fluorescent RFLP analysis, the absence of a mutation in the mother is obviously not definitive, as a mutation load below the detection threshold for the assay in the mother cannot be excluded, neither can the presence of the mutation in her untested tissues, particularly oocytes.

\section{Proportion of mtDNA point mutations arising de novo in patients with mtDNA disease}

Based on the absence of the mtDNA mutation in (mostly multiple) maternal tissue(s), $24.6 \%$ of the putative pathogenic mtDNA point mutations in our cohort were de novo, a significant subset of cases which is in agreement with available data from other centres (e.g., ${ }^{1}$ Supplementary Table S7.2). Since paediatric patients seem over-represented in the de novo subgroup, whereas the majority of the entire cohort are adult patients, the proportion of de novo mutations is likely higher in the paediatric patient population and lower in adults.

De novo mtDNA point mutations manifesting below the threshold required for phenotypic expression are clearly not included in this number. It is, however, important to realise that de novo mutations in asymptomatic individuals could potentially segregate to high levels and thus cause mtDNA diseases in subsequent generations. This mechanism is also illustrated by our cases (grey-coloured cases in Supplementary Tables S7.1 and S7.2) where a mutation did not occur de novo in the index patient, but in the healthy mother (or another maternal relative). Such carriers with low mtDNA mutation loads themselves will only be identified if they have a clinically affected child.

\section{Recurrence risk of de novo mtDNA mutation or disease}

The recurrence risk of a de novo mtDNA mutation depends on the moment at which the mutation arose. A germline de novo mutation event, which reaches clinical significance, most likely occurs at the lowest point of the bottleneck during oogenesis, 
when the mtDNA copy number is lowest. The same de novo event is not expected to happen twice and the recurrence risk is therefore negligible. Mutations may also be pre-existent in (some) maternal oocytes, representing gonadal mosaicism and resulting in a potential recurrence risk. Oocyte sampling has been used to further estimate the recurrence risks of mtDNA mutations, ${ }^{33,34}$ although the invasive nature of this procedure may pose ethical questions if no assisted fertility treatment is intended. Also, it is still not guaranteed that the analyzed oocytes are representative of the entire oocyte pool. Finally, a de novo mutation may represent a de novo somatic mutation (being by definition not present in the mother) rather than a de novo germ line mutation. In the latter scenario, there is no risk of recurrence. Somatic mutations may be present in only one tissue (eg, skeletal muscle); however, also when detected in several tissues, even of different embryonic origin (such as muscle and urinary epithelial cells, ${ }^{35}$ or muscle and hair roots, ${ }^{36}$ respectively), the mtDNA mutation can be somatic, having occurred very early in embryological development. Taken together, it is nigh impossible to distinguish whether an mtDNA mutation occurred de novo somatically or in the germline (and if so: at what point in the germline) in single disease cases. In practice, all three scenarios should be considered in cases of apparently de novo mtDNA mutations and as such are taken together in empirically established recurrence risks as discussed below.

Our PND/PGD data of subsequent pregnancies obtained from five mothers of patients with de novo mtDNA disease indicate a very low recurrence risk for de novo mtDNA mutations. These results are supported by (un)published data in which PND in subsequent pregnancies following affected children with an apparently de novo mtDNA mutation were reported in nine mothers (Table 7.2, ${ }^{18,34,37-41}$ ). In eight of these, the mtDNA mutation was not detected in the prenatal sample(s). The absence of the mtDNA mutation in 97 siblings of individuals with a presumed de novo mtDNA mutation further adds to the low recurrence risk of these mutations (Tables 7.1 and Supplementary Table S7.2). However, in most asymptomatic siblings of an index patient (a low) mtDNA mutation load could not be excluded as they were not tested for the mutation on ethical grounds. Recurrence risk is increased in the case of gonadal mosaicism, which was shown for one of the de novo cases in the literature (case 9, Table 7.2 /case 58, supplementary table $7.2^{34}$ ). The single case where the mutation was present in subsequent pregnancies of the mother (case 12, Table 7.2/case 59,

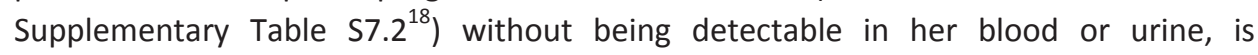
presumably also an example of gonadal mosaicism. The maternal mutation analysis was repeated using deep NGS analysis, but even with this the mtDNA mutation could not be detected in the mother. It is the only case known so far where three offspring have the same mtDNA mutation that is not detectable in the mother. The pattern of distribution of mutation load in this mother's offspring with very high levels in some (her affected child and one of the prenatal samples), but no mutation in the majority (six embryos), is 
quite similar to an m.8993T>G carrier with low mutant load we previously described (case 44, Supplementary Table S7.1 ${ }^{14}$ ). Interestingly, the two cases of (presumed) maternal gonadal mosaicism in Table 7.2 concern the same mtDNA mutation, m.9176T>C. ${ }^{18,34}$

The total number of prenatal/preimplantation samples described in this paper is 50 , including multiple pregnancies per female (cases 7 and 12, Table 7.2) multiple oocytes and/or embryos per female (cases 5, 9 and 12, Table 7.2) and analyzed abortus material (case 4, Table 7.2). In four of these samples, the mtDNA mutation present in the index patient was detected, indicating a recurrence risk of $8 \%(4 / 50)$. Larger numbers of (normal) prenatal diagnoses are presently not available to include in our analysis. However, we do have results in siblings that can be added. Also, 100 siblings of 57 individuals with an apparently de novo mtDNA mutation based on absence of the mutation in the mother (note: families where the mother of such an individual was not analysed, cases 79 and 80 in Supplementary Table S7.2, were not included in this calculation) were tested, both from our own centre and from the literature. In one of these, the mtDNA mutation might be present at low levels in a clinically-unaffected sibling (case 108, Supplementary Table $57.2^{36}$ ) although recurrence is debatable here since the sibling's mutation load is at the limit of detection. Besides, in the mother only blood was tested, which is also the case in a second recurrence example (case 97, Supplementary Table $57.2^{42}$ ). In a third case, the mutation was present (3\% mutant load) in the sibling's urine whereas it was absent in the mother's blood and urine (case 122, Supplementary Table $S 7.2^{43}$ ). Considering both the prenatal/pre-implantation data $(n=50)$ and the sibling data $(n=100)$, a recurrence risk of approximately $4 \%$ is calculated $(5-7 / 150)$ in this dataset. It is likely that this percentage further decreases when all healthy siblings that were not tested could be included.

Few other reports from family studies potentially describe recurrence, but the data are not unambiguous. These include a case where the similarly affected sibling was not tested for the mtDNA mutation and pathogenicity of the mutation has not been proven (case 93, Supplementary Table S7.2 ${ }^{44}$ ); and two cases where relatives with neurological symptoms were not extensively or not at all tested for the mtDNA mutation, whereby it remained unclear whether these symptoms are related to the familial mtDNA mutation or represent a separate disease (cases 50 and 79, Supplementary Table S7.2 ${ }^{45,46}$ ). Presence of the m.14484T>C mutation in an unaffected sibling, when no mutation was detectable in the mother, was reported in a monozygotic twin (case 136, Supplementary Table $\mathrm{S7.} .2^{47}$ ), consistent with no recurrence.

Even if all the cases discussed (with the exception of the monozygotic twin) would actually represent recurrence despite absence of the mutation in the mother, 8 examples in 154 cases (17 from our own centre, 137 from literature) would still 
support our hypothesis of a low recurrence risk, considering the likely bias that exceptional cases may be more easily reported. Furthermore, it is important to note that in none of the (potential) recurrence cases (including the proven gonadal mosaicism one) postmitotic tissue such as muscle was analyzed in the mother. None of these involved the m.3243A>G mutation.

\section{Reproductive counselling strategy for mtDNA disease (Figure. 7.2)}

When a couple with a child affected by mtDNA disease seeks counselling regarding the likelihood of having subsequent unaffected offspring, an individual risk assessment should be performed, leading to personalised advice. The first step is to ascertain the genetic cause of the disease. As is illustrated by case 5 , finding a pathogenic mtDNA mutation does not always provide an unequivocal answer and the mtDNA heteroplasmy level and clinical phenotype should be consistent with previous reports of affected individuals. If a causative mtDNA mutation is identified, the next step is to evaluate whether the mutation occurred de novo or was maternally-inherited. It is necessary to examine multiple tissues in the mother preferably including a post-mitotic tissue such as muscle if possible (needle-biopsy suffices), and being mindful of the tissues anticipated to harbour the mutation at detectable levels. For the m.3243A>G mutation, urine can be considered a reliable alternative for muscle, but for other mutations this correlation has not been validated. A quantitative method with low detection level $(<1-2 \%)$, such as fluorescent restriction fragment length polymorphism (RFLP), pyrosequencing, or NGS, should be used. Testing of unaffected siblings of the affected child should be considered but may be deemed unacceptable on ethical grounds. As it will be impossible to fully exclude a mutation in the mother, partly due to technical reasons and the availability of tissues or oocytes (note: oocyte sampling might be considered), couples should be aware of a small residual risk. Based on our current calculation this risk may be $\sim 4 \%$, although the actual risk is likely to be lower. If a couple wishes reproductive genetic testing, PND is the preferred option given the high likelihood that the mutation will not be present in the fetus. PND is also a fair option for carriers with low mutation load (eg, <10\%) of familial mutations, particularly for an mtDNA mutation that manifests skewed segregation, and for single, large-scale mtDNA deletions, the latter both for healthy mothers of an affected child and for affected women themselves. 


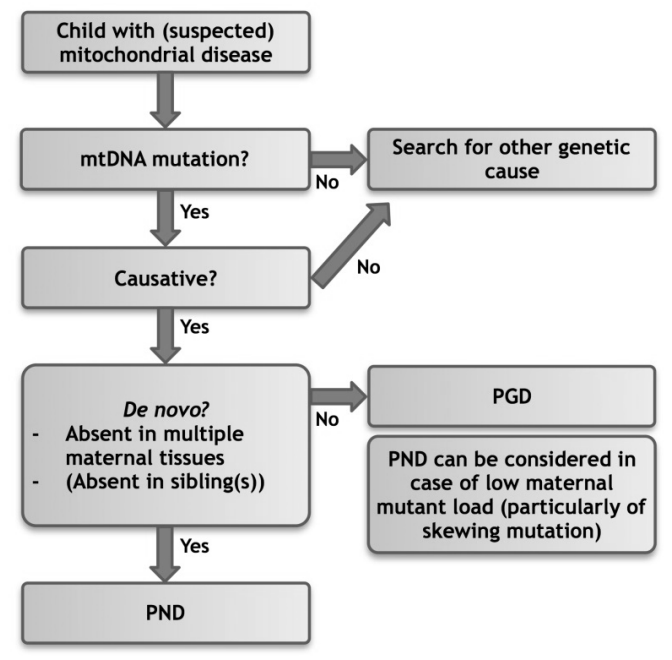

Figure 7.2 Flow chart: simplified reproductive counselling strategy for mitochondrial (mt)DNA disease. Patient's preferences have not been explicitly included in this algorithm; however, they obviously are an important factor as well. Choices are made on a case-by-case basis. PGD, preimplantation genetic diagnosis; PND, prenatal diagnosis

Although amniocentesis seems to be the preferred method for some mtDNA mutations, ${ }^{48}$ one can discuss the relevance of this in situations where no mutation is to be expected at all. CVS, on the other hand, has the advantage that results can be obtained earlier in the pregnancy. In most familial mtDNA mutations, with a high or unpredictable recurrence risk in offspring, and given the potential difficulties of interpreting PND results, PGD is currently the best reproductive option. ${ }^{14}$ Decisions on reproductive options naturally also depend on personal considerations of the individual couple; providing all the available options and information is the key to being able to make a well-informed decision.

\section{Acknowledgements}

The authors thank the families who have been included in this report. 


\section{References}

1. Thorburn DR. Mitochondrial disorders: prevalence, myths and advances. J Inherit Metab Dis. 2004;27(3):349-362.

2. Rotig A, Munnich A. Genetic features of mitochondrial respiratory chain disorders. J Am Soc Nephrol. 2003;14(12):2995-3007.

3. Hellebrekers DM, Wolfe R, Hendrickx AT, et al. PGD and heteroplasmic mitochondrial DNA point mutations: a systematic review estimating the chance of healthy offspring. Hum Reprod Update. 2012;18(4):341-349.

4. Cree LM, Samuels DC, de Sousa Lopes SC, et al. A reduction of mitochondrial DNA molecules during embryogenesis explains the rapid segregation of genotypes. Nat Genet. 2008;40(2):249-254.

5. Khrapko K. Two ways to make an mtDNA bottleneck. Nat Genet. 2008;40(2):134-135.

6. Wai T, Teoli D, Shoubridge EA. The mitochondrial DNA genetic bottleneck results from replication of a subpopulation of genomes. Nat Genet. 2008;40(12):1484-1488.

7. Cao L, Shitara H, Sugimoto M, Hayashi J, Abe K, Yonekawa H. New evidence confirms that the mitochondrial bottleneck is generated without reduction of mitochondrial DNA content in early primordial germ cells of mice. PLoS Genet. 2009;5(12):e1000756.

8. Samuels DC, Wonnapinij P, Cree LM, Chinnery PF. Reassessing evidence for a postnatal mitochondrial genetic bottleneck. Nat Genet. 2010;42(6):471-472; author reply 472-473.

9. Chinnery PF, DiMauro S, Shanske S, et al. Risk of developing a mitochondrial DNA deletion disorder. Lancet. 2004;364(9434):592-596.

10. Zeviani M, Antozzi C. Mitochondrial disorders. Mol Hum Reprod. 1997;3(2):133-148.

11. Chinnery PF, Hudson G. Mitochondrial genetics. Br Med Bull. 2013;106:135-159.

12. DiMauro S, Hirano M. Mitochondrial DNA Deletion Syndromes. In: Pagon RA, Adam MP, Ardinger HH, et al., eds. GeneReviews(R). Seattle WA: University of Washington, Seattle; 1993.

13. White SL, Collins VR, Wolfe R, et al. Genetic counseling and prenatal diagnosis for the mitochondrial DNA mutations at nucleotide 8993. Am J Hum Genet. 1999;65(2):474-482.

14. Sallevelt SC, Dreesen JC, Drusedau M, et al. Preimplantation genetic diagnosis in mitochondrial DNA disorders: challenge and success. J Med Genet. 2013;50(2):125-132.

15. McFarland R, Elson JL, Taylor RW, Howell N, Turnbull DM. Assigning pathogenicity to mitochondrial tRNA mutations: when "definitely maybe" is not good enough. Trends Genet. 2004;20(12):591-596.

16. Richards S, Aziz N, Bale S, et al. Standards and guidelines for the interpretation of sequence variants: a joint consensus recommendation of the American College of Medical Genetics and Genomics and the Association for Molecular Pathology. Genet Med. 2015;17(5):405-424.

17. Jacobs LJ, de Coo IF, Nijland JG, et al. Transmission and prenatal diagnosis of the T9176C mitochondrial DNA mutation. Mol Hum Reprod. 2005;11(3):223-228.

18. Nesbitt $\mathrm{V}$, Alston $\mathrm{CL}$, Blakely $\mathrm{EL}$, et al. A national perspective on prenatal testing for mitochondrial disease. Eur J Hum Genet. 2014;22(11):1255-1259.

19. Blakely EL, Yarham JW, Alston CL, et al. Pathogenic mitochondrial tRNA point mutations: nine novel mutations affirm their importance as a cause of mitochondrial disease. Hum Mutat. 2013;34(9):12601268.

20. Legros $F$, Chatzoglou E, Frachon $P$, et al. Functional characterization of novel mutations in the human cytochrome b gene. Eur J Hum Genet. 2001;9(7):510-518.

21. Bruno C, Santorelli FM, Assereto $S$, et al. Progressive exercise intolerance associated with a new musclerestricted nonsense mutation $(\mathrm{G} 142 \mathrm{X})$ in the mitochondrial cytochrome b gene. Muscle Nerve. 2003;28(4):508-511.

22. Andreu AL, Hanna MG, Reichmann $\mathrm{H}$, et al. Exercise intolerance due to mutations in the cytochrome $b$ gene of mitochondrial DNA. N Engl J Med. 1999;341(14):1037-1044.

23. Andreu AL, Checcarelli N, Iwata S, Shanske S, DiMauro S. A missense mutation in the mitochondrial cytochrome b gene in a revisited case with histiocytoid cardiomyopathy. Pediatr Res. 2000;48(3):311314. 
24. Zhadanov SI, Atamanov VV, Zhadanov NI, Schurr TG. De novo COX2 mutation in a LHON family of Caucasian origin: implication for the role of mtDNA polymorphism in human pathology. J Hum Genet. 2006;51(3):161-170.

25. Smits P, Mattijssen S, Morava E, et al. Functional consequences of mitochondrial tRNA Trp and tRNA Arg mutations causing combined OXPHOS defects. Eur J Hum Genet. 2010;18(3):324-329.

26. $t$ Hart LM, Jansen JJ, Lemkes HH, de Knijff P, Maassen JA. Heteroplasmy levels of a mitochondrial gene mutation associated with diabetes mellitus decrease in leucocyte DNA upon aging. Hum Mutat. 1996;7(3):193-197.

27. Howell N, Ghosh SS, Fahy E, Bindoff LA. Longitudinal analysis of the segregation of mtDNA mutations in heteroplasmic individuals. J Neurol Sci. 2000;172(1):1-6.

28. Rahman S, Poulton J, Marchington D, Suomalainen A. Decrease of 3243 A-->G mtDNA mutation from blood in MELAS syndrome: a longitudinal study. Am J Hum Genet. 2001;68(1):238-240.

29. McDonnell MT, Schaefer AM, Blakely EL, et al. Noninvasive diagnosis of the 3243A > G mitochondrial DNA mutation using urinary epithelial cells. Eur J Hum Genet. 2004;12(9):778-781.

30. O'Callaghan MM, Emperador S, Pineda M, et al. Mutation loads in different tissues from six pathogenic mtDNA point mutations. Mitochondrion. 2015;22:17-22

31. Elson JL, Swalwell H, Blakely EL, McFarland R, Taylor RW, Turnbull DM. Pathogenic mitochondrial tRNA mutations--which mutations are inherited and why? Hum Mutat. 2009;30(11):E984-992.

32. Li M, Rothwell R, Vermaat M, et al. Transmission of human mtDNA heteroplasmy in the Genome of the Netherlands families: support for a variable-size bottleneck. Genome Res. 2016;26(4):417-426.

33. Poulton J, Marchington DR. Segregation of mitochondrial DNA (mtDNA) in human oocytes and in animal models of mtDNA disease: clinical implications. Reproduction (Cambridge, England). 2002;123(6): 751-755.

34. Marchington D, Malik S, Banerjee A, et al. Information for genetic management of mtDNA disease: sampling pathogenic mtDNA mutants in the human germline and in placenta. J Med Genet. 2010;47(4):257-261.

35. Mancuso M, Filosto M, Stevens JC, et al. Mitochondrial myopathy and complex III deficiency in a patient with a new stop-codon mutation (G339X) in the cytochrome b gene. J Neurol Sci. 2003;209(1-2):61-63.

36. Keightley JA, Anitori R, Burton MD, Quan F, Buist NR, Kennaway NG. Mitochondrial encephalomyopathy and complex III deficiency associated with a stop-codon mutation in the cytochrome b gene. Am J Hum Genet. 2000;67(6):1400-1410.

37. Lebon $\mathrm{S}, \mathrm{Chol} \mathrm{M}$, Benit $\mathrm{P}$, et al. Recurrent de novo mitochondrial DNA mutations in respiratory chain deficiency. J Med Genet. 2003;40(12):896-899.

38. Steffann J, Gigarel N, Corcos J, et al. Stability of the m.8993T->G mtDNA mutation load during human embryofetal development has implications for the feasibility of prenatal diagnosis in NARP syndrome. $J$ Med Genet. 2007;44(10):664-669.

39. Shanske S, Coku J, Lu J, et al. The G13513A mutation in the ND5 gene of mitochondrial DNA as a common cause of MELAS or Leigh syndrome: evidence from 12 cases. Arch Neurol. 2008;65(3):368-372.

40. Gotz A, Isohanni P, Liljestrom B, et al. Fatal neonatal lactic acidosis caused by a novel de novo mitochondrial G7453A tRNA-Serine ((UCN)) mutation. Pediatr Res. 2012;72(1):90-94.

41. Shanske $\mathrm{S}$, Naini $\mathrm{A}$, Chmait $\mathrm{RH}$, et al. Mutation in an mtDNA protein-coding gene: prenatal diagnosis aided by fetal muscle biopsy. J Child Neurol. 2013;28(2):264-268.

42. White SL, Shanske S, McGill JJ, et al. Mitochondrial DNA mutations at nucleotide 8993 show a lack of tissue- or age-related variation. J Inherit Metab Dis. 1999;22(8):899-914.

43. Kirby DM, McFarland R, Ohtake A, et al. Mutations of the mitochondrial ND1 gene as a cause of MELAS. J Med Genet. 2004;41(10):784-789.

44. Mkaouar-Rebai E, Kammoun F, Chamkha I, et al. A de novo mutation in the adenosine triphosphatase (ATPase) 8 gene in a patient with mitochondrial disorder. J Child Neurol. 2010;25(6):770-775.

45. Baric I, Fumic K, Petkovic Ramadza D, et al. Mitochondrial myopathy associated with a novel 5522G $>A$ mutation in the mitochondrial tRNA(Trp) gene. Eur J Hum Genet. 2013;21(8):871-875.

46. Anitori R, Manning K, Quan F, et al. Contrasting phenotypes in three patients with novel mutations in mitochondrial tRNA genes. Mol Genet Metab. 2005;84(2):176-188.

47. Biousse V, Brown MD, Newman NJ, et al. De novo 14484 mitochondrial DNA mutation in monozygotic twins discordant for Leber's hereditary optic neuropathy. Neurology. 1997;49(4):1136-1138. 
48. Steffann J, Monnot S, Bonnefont JP. mtDNA mutations variously impact mtDNA maintenance throughout the human embryofetal development. Clin Genet. 2015;88(5):416-424.

49. Blok MJ, Spruijt L, de Coo IF, Schoonderwoerd K, Hendrickx A, Smeets HJ. Mutations in the ND5 subunit of complex I of the mitochondrial DNA are a frequent cause of oxidative phosphorylation disease. J Med Genet. 2007;44(4):e74.

50. van den Bosch BJ, de Coo IF, Hendrickx AT, et al. Increased risk for cardiorespiratory failure associated with the A3302G mutation in the mitochondrial DNA encoded tRNALeu(UUR) gene. Neuromuscul Disord. 2004;14(10):683-688.

51. Spruijt L, Smeets HJ, Hendrickx A, et al. A MELAS-associated ND1 mutation causing leber hereditary optic neuropathy and spastic dystonia. Arch Neurol. 2007;64(6):890-893.

52. Hanna MG, Nelson IP, Morgan-Hughes JA, Wood NW. MELAS: a new disease associated mitochondrial DNA mutation and evidence for further genetic heterogeneity. I Neurol Neurosurg Psychiatry. 1998;65(4):512-517.

53. Darin N, Kollberg G, Moslemi AR, et al. Mitochondrial myopathy with exercise intolerance and retinal dystrophy in a sporadic patient with a G583A mutation in the mt tRNA(phe) gene. Neuromuscul Disord. 2006;16(8):504-506.

54. Shoffner JM, Bialer MG, Pavlakis SG, et al. Mitochondrial encephalomyopathy associated with a single nucleotide pair deletion in the mitochondrial tRNALeu(UUR) gene. Neurology. 1995;45(2):286-292.

55. Ugalde C, Hinttala R, Timal S, et al. Mutated ND2 impairs mitochondrial complex I assembly and leads to Leigh syndrome. Mol Genet Metab. 2007;90(1):10-14.

56. Granadillo JL, Moss T, Lewis RA, et al. Early Onset and Severe Clinical Course Associated with the m.5540G>A Mutation in. Mol Genet Metab Rep. 2014;1:61-65.

57. Moraes CT, Ciacci F, Bonilla E, et al. Two novel pathogenic mitochondrial DNA mutations affecting organelle number and protein synthesis. Is the tRNA(Leu(UUR)) gene an etiologic hot spot? J Clin Invest. 1993;92(6):2906-2915.

58. Valente L, Piga D, Lamantea E, et al. Identification of novel mutations in five patients with mitochondrial encephalomyopathy. Biochim Biophys Acta. 2009;1787(5):491-501.

59. Kollberg G, Jansson M, Perez-Bercoff A, et al. Low frequency of mtDNA point mutations in patients with PEO associated with POLG1 mutations. Eur J Hum Genet. 2005;13(4):463-469.

60. Gattermann N, Retzlaff S, Wang YL, et al. Heteroplasmic point mutations of mitochondrial DNA affecting subunit I of cytochrome c oxidase in two patients with acquired idiopathic sideroblastic anemia. Blood. 1997;90(12):4961-4972.

61. Seneca S, Goemans N, Van Coster R, et al. A mitochondrial tRNA aspartate mutation causing isolated mitochondrial myopathy. Am J Med Genet. Part A. 2005;137(2):170-175.

62. Rahman S, Taanman JW, Cooper JM, et al. A missense mutation of cytochrome oxidase subunit II causes defective assembly and myopathy. Am J Hum Genet. 1999;65(4):1030-1039.

63. McFarland R, Taylor RW, Chinnery PF, Howell N, Turnbull DM. A novel sporadic mutation in cytochrome c oxidase subunit II as a cause of rhabdomyolysis. Neuromuscul Disord. 2004;14(2):162-166.

64. De Meirleir L, Seneca S, Lissens W, Schoentjes E, Desprechins B. Bilateral striatal necrosis with a novel point mutation in the mitochondrial ATPase 6 gene. Pediatr Neurol. 1995;13(3):242-246.

65. Duno M, Wibrand F, Baggesen K, Rosenberg T, Kjaer N, Frederiksen AL. A novel mitochondrial mutation m.8989G $>C$ associated with neuropathy, ataxia, retinitis pigmentosa - the NARP syndrome. Gene. 2013;515(2):372-375

66. Santorelli FM, Shanske S, Macaya A, DeVivo DC, DiMauro S. The mutation at nt 8993 of mitochondrial DNA is a common cause of Leigh's syndrome. Ann Neurol. 1993;34(6):827-834.

67. Pastores GM, Santorelli FM, Shanske S, et al. Leigh syndrome and hypertrophic cardiomyopathy in an infant with a mitochondrial DNA point mutation (T8993G). Am J Med Genet. 1994;50(3):265-271.

68. Uziel G, Moroni I, Lamantea E, et al. Mitochondrial disease associated with the T8993G mutation of the mitochondrial ATPase 6 gene: a clinical, biochemical, and molecular study in six families. J Neurol Neurosurg Psychiatry. 1997;63(1):16-22.

69. Playan A, Solano-Palacios A, Gonzalez de la Rosa JB, et al. [Leigh syndrome resulting from a de novo mitochondrial DNA mutation (T8993G)]. Rev Neurol. 2002;34(12):1124-1126.

70. Keightley JA, Hoffbuhr KC, Burton MD, et al. A microdeletion in cytochrome c oxidase (COX) subunit III associated with COX deficiency and recurrent myoglobinuria. Nat Genet. 1996;12(4):410-416. 
71. McFarland R, Kirby DM, Fowler KJ, et al. De novo mutations in the mitochondrial ND3 gene as a cause of infantile mitochondrial encephalopathy and complex I deficiency. Ann Neurol. 2004;55(1):58-64.

72. Bannwarth S, Procaccio V, Rouzier C, et al. Rapid identification of mitochondrial DNA (mtDNA) mutations in neuromuscular disorders by using surveyor strategy. Mitochondrion. 2008;8(2):136-145.

73. Werner KG, Morel CF, Kirton A, et al. Rolandic mitochondrial encephalomyelopathy and MT-ND3 mutations. Pediatr Neurol. 2009;41(1):27-33.

74. Komaki H, Akanuma J, Iwata H, et al. A novel mtDNA C11777A mutation in Leigh syndrome. Mitochondrion. 2003;2(4):293-304.

75. Zhadanov SI, Grechanina EY, Grechanina YB, et al. Fatal manifestation of a de novo ND5 mutation: Insights into the pathogenetic mechanisms of mtDNA ND5 gene defects. Mitochondrion. 2007;7(4):260266.

76. Kirby DM, Kahler SG, Freckmann ML, Reddihough D, Thorburn DR. Leigh disease caused by the mitochondrial DNA G14459A mutation in unrelated families. Ann Neurol. 2000;48(1):102-104.

77. Valnot I, Kassis J, Chretien D, et al. A mitochondrial cytochrome b mutation but no mutations of nuclearly encoded subunits in ubiquinol cytochrome c reductase (complex III) deficiency. Hum Genet. 1999;104(6):460-466.

78. Wibrand F, Ravn K, Schwartz M, Rosenberg T, Horn N, Vissing J. Multisystem disorder associated with a missense mutation in the mitochondrial cytochrome b gene. Ann Neurol. 2001;50(4):540-543.

79. Lamantea E, Carrara F, Mariotti C, Morandi L, Tiranti V, Zeviani M. A novel nonsense mutation (Q352X) in the mitochondrial cytochrome $b$ gene associated with a combined deficiency of complexes I and III. Neuromuscul Disord. 2002;12(1):49-52.

80. Schaller A, Desetty R, Hahn D, et al. Impairment of mitochondrial tRNAlle processing by a novel mutation associated with chronic progressive external ophthalmoplegia. Mitochondrion. 2011;11(3): 488-496.

81. Blakely EL, Swalwell H, Petty RK, McFarland R, Turnbull DM, Taylor RW. Sporadic myopathy and exercise intolerance associated with the mitochondrial 8328G>A tRNALys mutation. J Neurol. 2007;254(9): 1283-1285.

82. Jeppesen TD, Duno M, Risom L, et al. A novel de novo mutation of the mitochondrial tRNAlys gene mt.8340G>a associated with pure myopathy. Neuromuscul Disord. 2014;24(2):162-166.

83. Biancheri R, Rossi D, Cassandrini D, Rossi A, Bruno C, Santorelli FM. Cavitating leukoencephalopathy in a child carrying the mitochondrial A8344G mutation. AJNR Am J Neuroradiol. 2010;31(9):E78-79.

84. Burrage LC, Tang S, Wang J, et al. Mitochondrial myopathy, lactic acidosis, and sideroblastic anemia (MLASA) plus associated with a novel de novo mutation (m.8969G $>A$ ) in the mitochondrial encoded ATP6 gene. Mol Genet Metab. 2014;113(3):207-212.

85. Seller A, Kennedy CR, Temple IK, Brown GK. Leigh syndrome resulting from de novo mutation at position 8993 of mitochondrial DNA. J Inherit Metab Dis. 1997;20(1):102-103.

86. De Praeter $C$, Vanlander A, Vanhaesebrouck $P$, et al. Extremely high mutation load of the mitochondrial 8993 T>G mutation in a newborn: implications for prognosis and family planning decisions. Eur J Pediatr. 2015;174(2):267-270.

87. Leng Y, Liu Y, Fang X, et al. The mitochondrial DNA $10197 \mathrm{G}>$ A mutation causes MELAS/Leigh overlap syndrome presenting with acute auditory agnosia. Mitochondrial DNA. 2015;26(2):208-212.

88. Nesbitt V, Morrison PJ, Crushell E, et al. The clinical spectrum of the m.10191T>C mutation in complex Ideficient Leigh syndrome. Dev Med Child Neurol. 2012;54(6):500-506.

89. Roos S, Darin N, Kollberg G, et al. A novel mitochondrial tRNA Arg mutation resulting in an anticodon swap in a patient with mitochondrial encephalomyopathy. Eur J Hum Genet. 2013;21(5):571-573.

90. Wong LJ, Yim D, Bai RK, et al. A novel mutation in the mitochondrial tRNA(Ser(AGY)) gene associated with mitochondrial myopathy, encephalopathy, and complex I deficiency. J Med Genet. 2006;43(9):e46.

91. Karadimas CL, Salviati L, Sacconi S, et al. Mitochondrial myopathy and ophthalmoplegia in a sporadic patient with the G12315A mutation in mitochondrial DNA. Neuromuscul Disord. 2002;12(9):865-868.

92. Alston $\mathrm{CL}$, Morak M, Reid C, et al. A novel mitochondrial MTND5 frameshift mutation causing isolated complex I deficiency, renal failure and myopathy. Neuromuscul Disord. 2010;20(2):131-135.

93. Slawek J, Kierdaszuk B, Tonska K, et al. Mitochondrial encephalopathy in a patient with a 13042G>A de novo mutation. J Clin Pathol. 2012;65(12):1147-1149. 
94. Leshinsky-Silver E, Shuvalov R, Inbar S, Cohen S, Lev D, Lerman-Sagie T. Juvenile Leigh syndrome, optic atrophy, ataxia, dystonia, and epilepsy due to T14487C mutation in the mtDNA-ND6 gene: a mitochondrial syndrome presenting from birth to adolescence. J Child Neurol. 2011;26(4):476-481.

95. Lax NZ, Gnanapavan S, Dowson SJ, et al. Early-onset cataracts, spastic paraparesis, and ataxia caused by a novel mitochondrial tRNAGlu (MT-TE) gene mutation causing severe complex I deficiency: a clinical, molecular, and neuropathologic study. J Neuropathol Exp Neurol. 2013;72(2):164-175.

96. Mayr JA, Moslemi AR, Forster H, et al. A novel sporadic mutation G14739A of the mitochondrial tRNA(Glu) in a girl with exercise intolerance. Neuromuscul Disord. 2006;16(12):874-877.

97. De Coo IF, Renier WO, Ruitenbeek W, et al. A 4-base pair deletion in the mitochondrial cytochrome b gene associated with parkinsonism/MELAS overlap syndrome. Ann Neurol. 1999;45(1):130-133.

98. Emmanuele V, Sotiriou E, Rios PG, et al. A novel mutation in the mitochondrial DNA cytochrome b gene (MTCYB) in a patient with mitochondrial encephalomyopathy, lactic acidosis, and strokelike episodes syndrome. J Child Neurol. 2013;28(2):236-242.

99. Fragaki K, Procaccio V, Bannwarth S, et al. A neonatal polyvisceral failure linked to a de novo homoplasmic mutation in the mitochondrially encoded cytochrome b gene. Mitochondrion. 2009;9(5): 346-352.

100. Bataillard M, Chatzoglou E, Rumbach L, et al. Atypical MELAS syndrome associated with a new mitochondrial tRNA glutamine point mutation. Neurology. 2001;56(3):405-407.

101. Dey R, Tengan CH, Morita MP, Kiyomoto BH, Moraes CT. A novel myopathy-associated mitochondrial DNA mutation altering the conserved size of the tRNA(Gln) anticodon loop. Neuromuscul Disord. 2000;10(7):488-492.

102. Vissing J, Salamon MB, Arlien-Soborg P, et al. A new mitochondrial tRNA(Met) gene mutation in a patient with dystrophic muscle and exercise intolerance. Neurology. 1998;50(6):1875-1878.

103. Pulkes T, Siddiqui A, Morgan-Hughes JA, Hanna MG. A novel mutation in the mitochondrial tRNA(TYr) gene associated with exercise intolerance. Neurology. 2000;55(8):1210-1212.

104. Karadimas CL, Greenstein $\mathrm{P}$, Sue CM, et al. Recurrent myoglobinuria due to a nonsense mutation in the COX I gene of mitochondrial DNA. Neurology. 2000;55(5):644-649.

105. Comi GP, Bordoni A, Salani S, et al. Cytochrome c oxidase subunit I microdeletion in a patient with motor neuron disease. Ann Neurol. 1998;43(1):110-116.

106. Bruno C, Martinuzzi A, Tang Y, et al. A stop-codon mutation in the human mtDNA cytochrome c oxidase I gene disrupts the functional structure of complex IV. Am J Hum Genet. 1999;65(3):611-620.

107. Bidooki S, Jackson MJ, Johnson MA, et al. Sporadic mitochondrial myopathy due to a new mutation in the mitochondrial tRNASer(UCN) gene. Neuromuscul Disord. 2004;14(7):417-420.

108. Campos Y, Garcia-Redondo A, Fernandez-Moreno MA, et al. Early-onset multisystem mitochondrial disorder caused by a nonsense mutation in the mitochondrial DNA cytochrome C oxidase II gene. Ann Neurol. 2001;50(3):409-413.

109. Verma A, Piccoli DA, Bonilla E, Berry GT, DiMauro S, Moraes CT. A novel mitochondrial G8313A mutation associated with prominent initial gastrointestinal symptoms and progressive encephaloneuropathy. Pediatr Res. 1997;42(4):448-454.

110. Lopez-Gallardo E, Solano A, Herrero-Martin MD, et al. NARP syndrome in a patient harbouring an insertion in the MT-ATP6 gene that results in a truncated protein. J Med Genet. 2009;46(1):64-67.

111. Moslemi AR, Darin N, Tulinius M, Oldfors A, Holme E. Two new mutations in the MTATP6 gene associated with Leigh syndrome. Neuropediatrics. 2005;36(5):314-318.

112. Ruiter EM, Siers MH, van den Elzen C, et al. The mitochondrial 13513G > A mutation is most frequent in Leigh syndrome combined with reduced complex I activity, optic atrophy and/or Wolff-Parkinson-White. Eur J Hum Genet. 2007;15(2):155-161.

113. Tarnopolsky M, Meaney B, Robinson B, Sheldon K, Boles RG. Severe infantile leigh syndrome associated with a rare mitochondrial ND6 mutation, m.14487T>C. Am J Med Genet. Part A. 2013.

114. Pereira C, Nogueira C, Barbot C, et al. Identification of a new mtDNA mutation (14724G>A) associated with mitochondrial leukoencephalopathy. Biochem Biophys Res Commun. 2007;354(4):937-941.

115. Schuelke M, Krude H, Finckh B, et al. Septo-optic dysplasia associated with a new mitochondrial cytochrome b mutation. Ann Neurol. 2002;51(3):388-392.

116. Andreu AL, Bruno C, Dunne TC, et al. A nonsense mutation (G15059A) in the cytochrome b gene in a patient with exercise intolerance and myoglobinuria. Ann Neurol. 1999;45(1):127-130. 
117. Dumoulin R, Sagnol I, Ferlin T, Bozon D, Stepien G, Mousson B. A novel gly290asp mitochondrial cytochrome b mutation linked to a complex III deficiency in progressive exercise intolerance. Mol Cell Probes. 1996;10(5):389-391.

118. Blakely EL, Mitchell AL, Fisher N, et al. A mitochondrial cytochrome b mutation causing severe respiratory chain enzyme deficiency in humans and yeast. FEBS J. 2005;272(14):3583-3592.

119. Andreu AL, Bruno $C$, Shanske $S$, et al. Missense mutation in the mtDNA cytochrome b gene in a patient with myopathy. Neurology. 1998;51(5):1444-1447.

120. Nishino I, Seki A, Maegaki Y, et al. A novel mutation in the mitochondrial tRNA(Thr) gene associated with a mitochondrial encephalomyopathy. Biochem Biophys Res Commun. 1996;225(1):180-185.

121. Ionasescu VV, Hart M, DiMauro S, Moraes CT. Clinical and morphologic features of a myopathy associated with a point mutation in the mitochondrial tRNA(Pro) gene. Neurology. 1994;44(5):975-977.

122. Mancuso M, Filosto M, Mootha VK, et al. A novel mitochondrial tRNAPhe mutation causes MERRF syndrome. Neurology. 2004;62(11):2119-2121.

123. Coulbault L, Deslandes B, Herlicoviez D, et al. A novel mutation $3090 \mathrm{G}>\mathrm{A}$ of the mitochondrial $16 \mathrm{~S}$ ribosomal RNA associated with myopathy. Biochem Biophys Res Commun. 2007;362(3):601-605.

124. Campos Y, Martin MA, Lorenzo G, Aparicio M, Cabello A, Arenas J. Sporadic MERRF/MELAS overlap syndrome associated with the $3243 \operatorname{tRNA}(\operatorname{Leu}(\mathrm{UUR}))$ mutation of mitochondrial DNA. Muscle Nerve. 1996;19(2):187-190.

125. Yamamoto M. Did de novo MELAS common mitochondrial DNA point mutation (mtDNA 3243, A-->G transition) occur in the mother of a proband of a Japanese MELAS pedigree? J Neurol Sci. 1996;135(1): 81-84.

126. Ko CH, Lam CW, Tse PW, Kong CK, Chan AK, Wong LJ. De novo mutation in the mitochondrial tRNALeu(UUR) gene (A3243G) with rapid segregation resulting in MELAS in the offspring. J Paediatr Child Health. 2001;37(1):87-90.

127. Maassen JA, Biberoglu S, t Hart LM, Bakker E, de Knijff P. A case of a de novo A3243G mutation in mitochondrial DNA in a patient with diabetes and deafness. Arch Physiol Biochem. 2002;110(3):186-188.

128. Pallotti F, Binelli G, Fabbri R, et al. A wide range of 3243A>G/tRNALeu(UUR) (MELAS) mutation loads may segregate in offspring through the female germline bottleneck. PloS One. 2014;9(5):e96663.

129. Blakely EL, de Silva R, King A, et al. LHON/MELAS overlap syndrome associated with a mitochondrial MTND1 gene mutation. Eur J Hum Genet. 2005;13(5):623-627.

130. Taylor RW, Schaefer AM, McFarland R, Maddison P, Turnbull DM. A novel mitochondrial DNA tRNA(Ile) (A4267G) mutation in a sporadic patient with mitochondrial myopathy. Neuromuscul Disord. 2002;12(78):659-664.

131. Houshmand M, Lindberg C, Moslemi AR, Oldfors A, Holme E. A novel heteroplasmic point mutation in the mitochondrial tRNA(Lys) gene in a sporadic case of mitochondrial encephalomyopathy: de novo mutation and no transmission to the offspring. Hum Mutat. 1999;13(3):203-209.

132. Brinckmann A, Ruther K, Williamson K, et al. De novo double mutation in PAX6 and mtDNA tRNA(Lys) associated with atypical aniridia and mitochondrial disease. J Mol Med. 2007;85(2):163-168.

133. Tulinius $\mathrm{MH}$, Houshmand $\mathrm{M}$, Larsson NG, et al. De novo mutation in the mitochondrial ATP synthase subunit 6 gene (T8993G) with rapid segregation resulting in Leigh syndrome in the offspring. Hum Genet. 1995;96(3):290-294.

134. de Coo IF, Smeets HJ, Gabreels FJ, Arts N, van Oost BA. Isolated case of mental retardation and ataxia due to a de novo mitochondrial T8993G mutation. Am J Hum Genet. 1996;58(3):636-638.

135. Degoul F, Francois D, Diry M, et al. A near homoplasmic T8993G mtDNA mutation in a patient with atypic Leigh syndrome not present in the mother's tissues. J Inherit Metab Dis. 1997;20(1):49-53.

136. Santorelli FM, Tanji K, Shanske S, DiMauro S. Heterogeneous clinical presentation of the mtDNA NARP/T8993G mutation. Neurology. 1997;49(1):270-273.

137. Takahashi S, Makita Y, Oki J, et al. De novo mtDNA nt 8993 (T-->G) mutation resulting in Leigh syndrome. Am J Hum Genet. 1998;62(3):717-719.

138. Horvath R, Scharfe C, Hoeltzenbein M, et al. Childhood onset mitochondrial myopathy and lactic acidosis caused by a stop mutation in the mitochondrial cytochrome c oxidase III gene. J Med Genet. 2002;39(11):812-816. 
139. Tiranti V, Corona P, Greco M, et al. A novel frameshift mutation of the mtDNA COIII gene leads to impaired assembly of cytochrome c oxidase in a patient affected by Leigh-like syndrome. Hum Mol Genet. 2000;9(18):2733-2742.

140. Pancrudo J, Shanske S, Coku J, et al. Mitochondrial myopathy associated with a novel mutation in mtDNA. Neuromuscul Disord. 2007;17(8):651-654.

141. Taylor RW, Schaefer AM, McDonnell MT, et al. Catastrophic presentation of mitochondrial disease due to a mutation in the tRNA(His) gene. Neurology. 2004;62(8):1420-1423.

142. Melone MA, Tessa A, Petrini S, et al. Revelation of a new mitochondrial DNA mutation (G12147A) in a MELAS/MERFF phenotype. Arch Neurol. 2004;61(2):269-272.

143. Blakely EL, Trip SA, Swalwell H, et al. A new mitochondrial transfer RNAPro gene mutation associated with myoclonic epilepsy with ragged-red fibers and other neurological features. Arch Neurol. 2009; 66(3):399-402. 


\section{Supplemental materials}

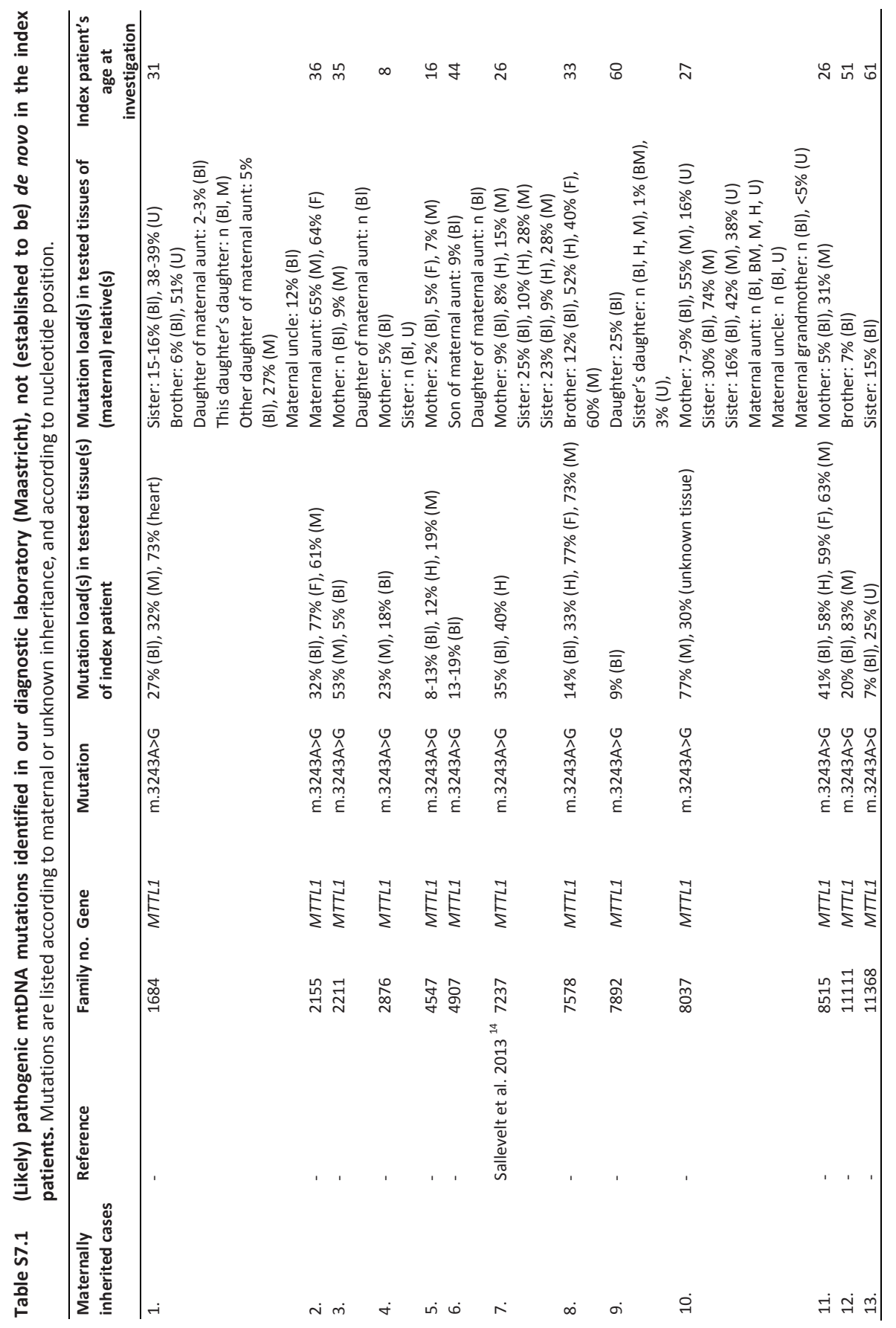




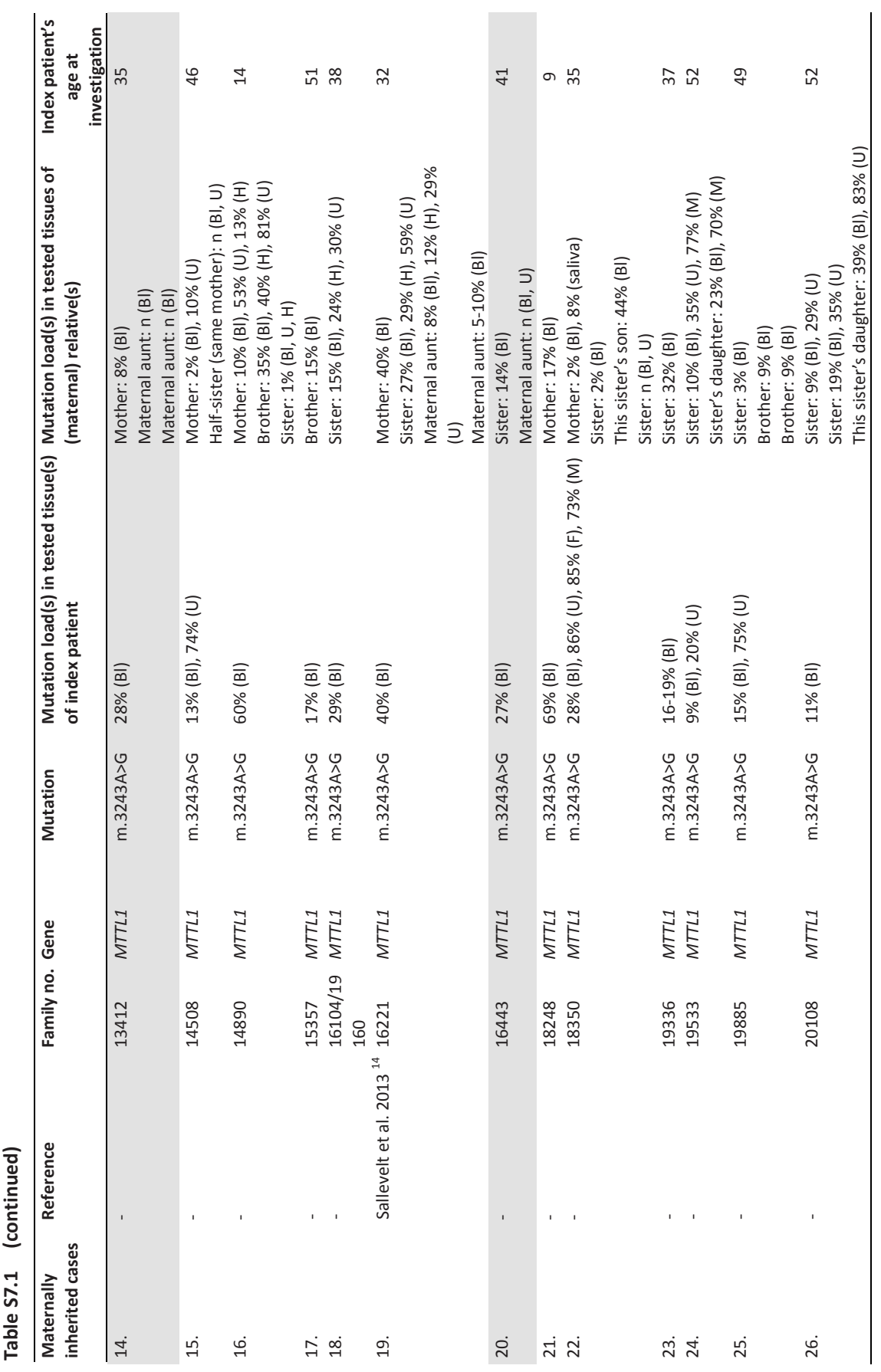




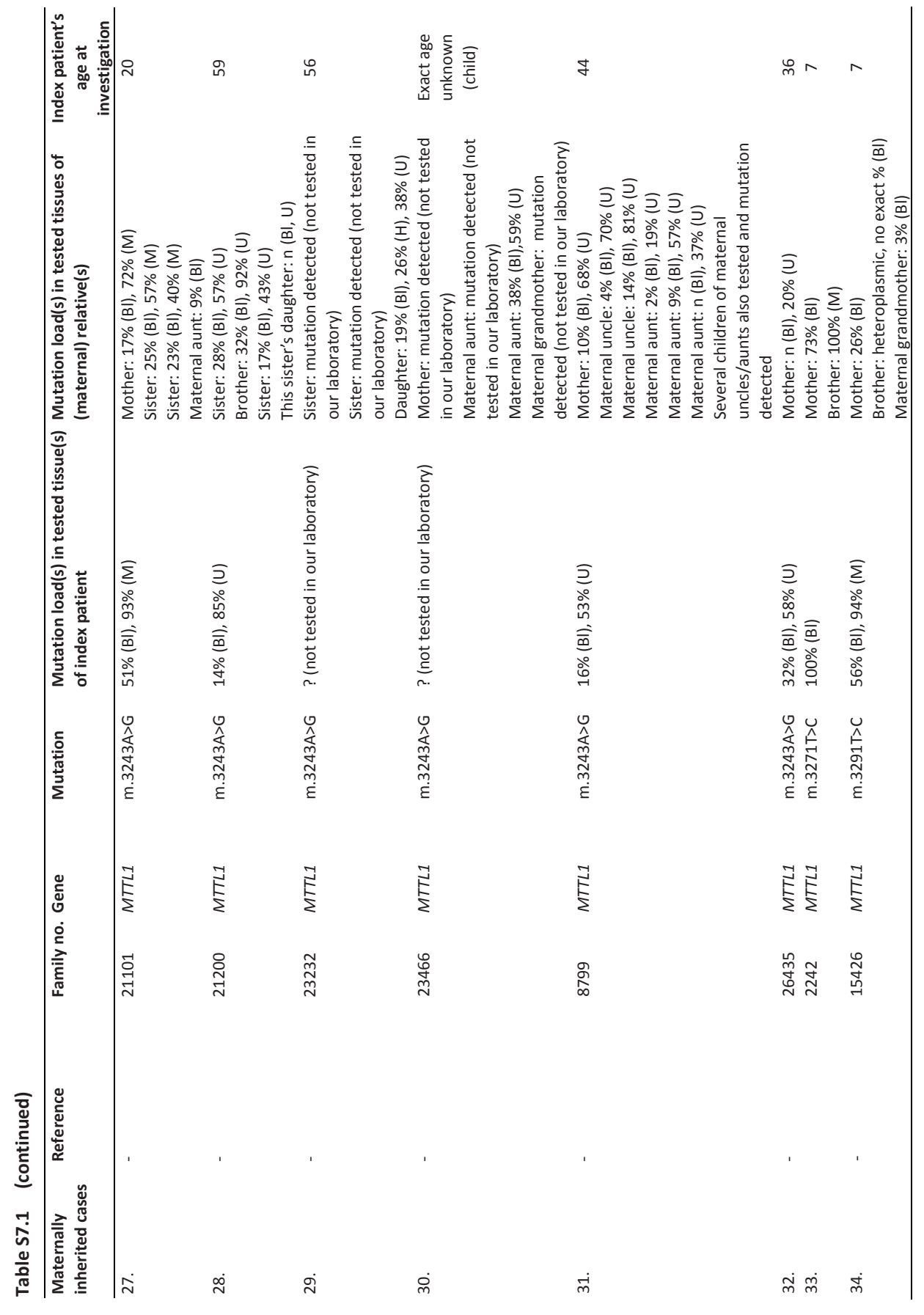




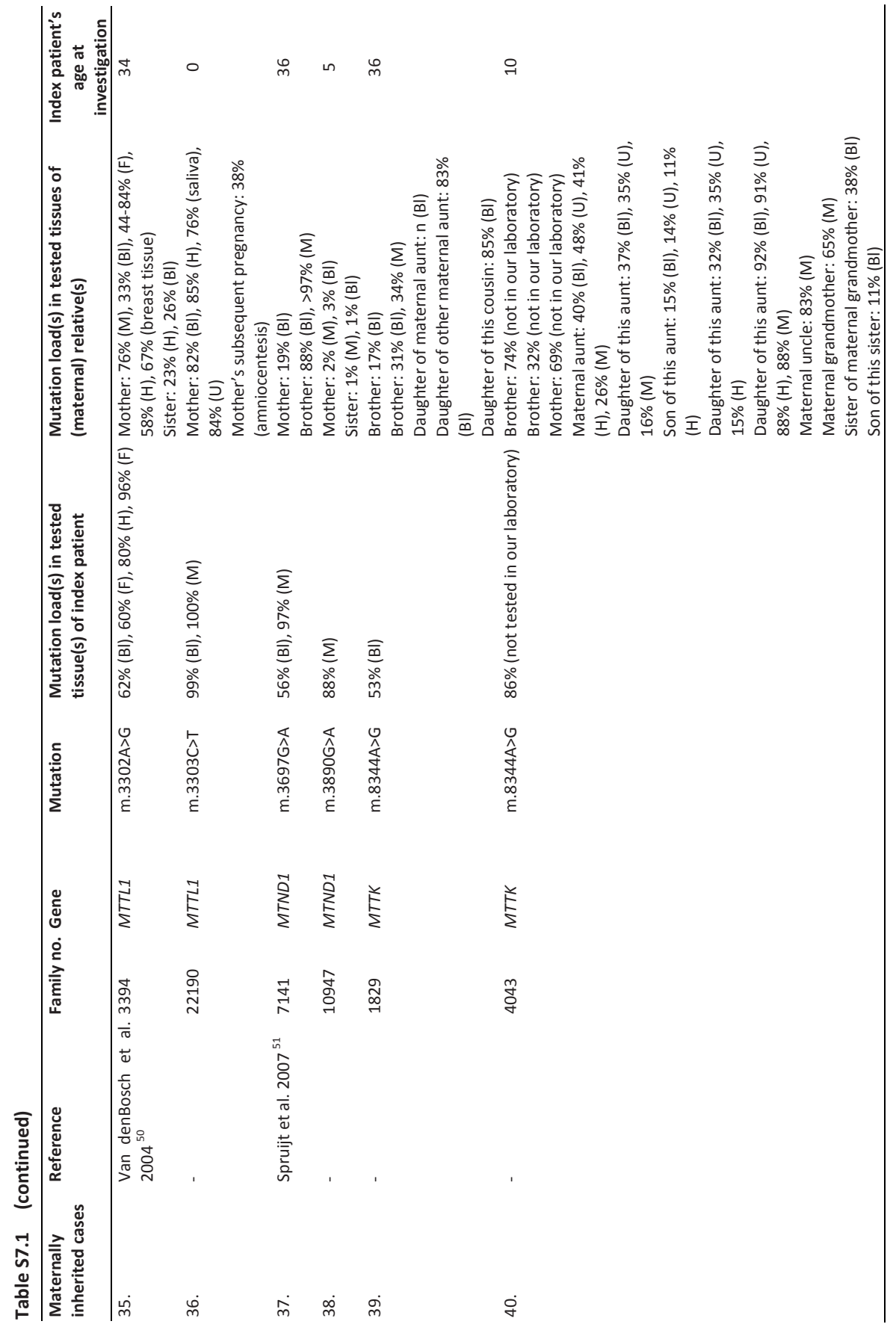




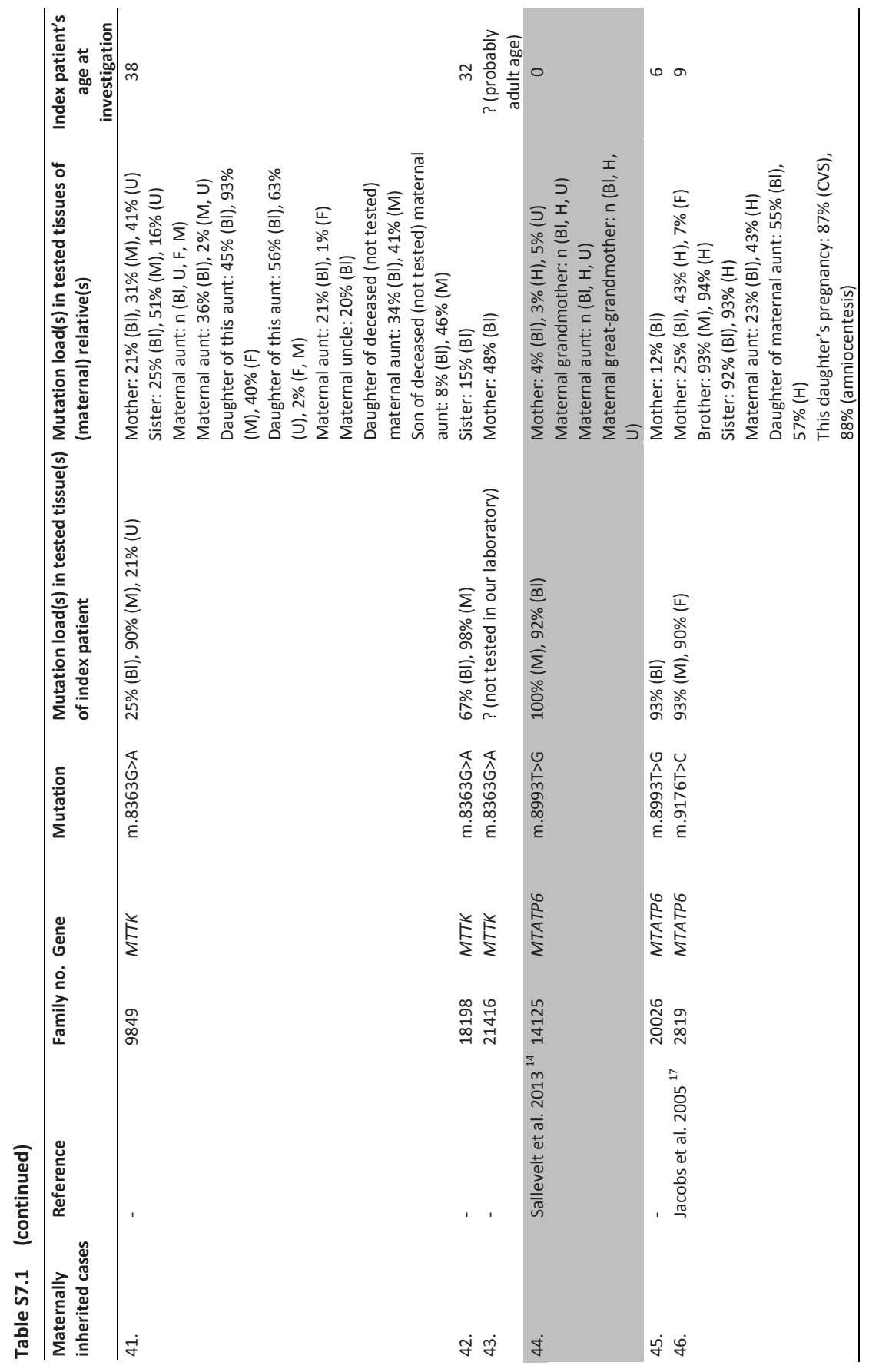




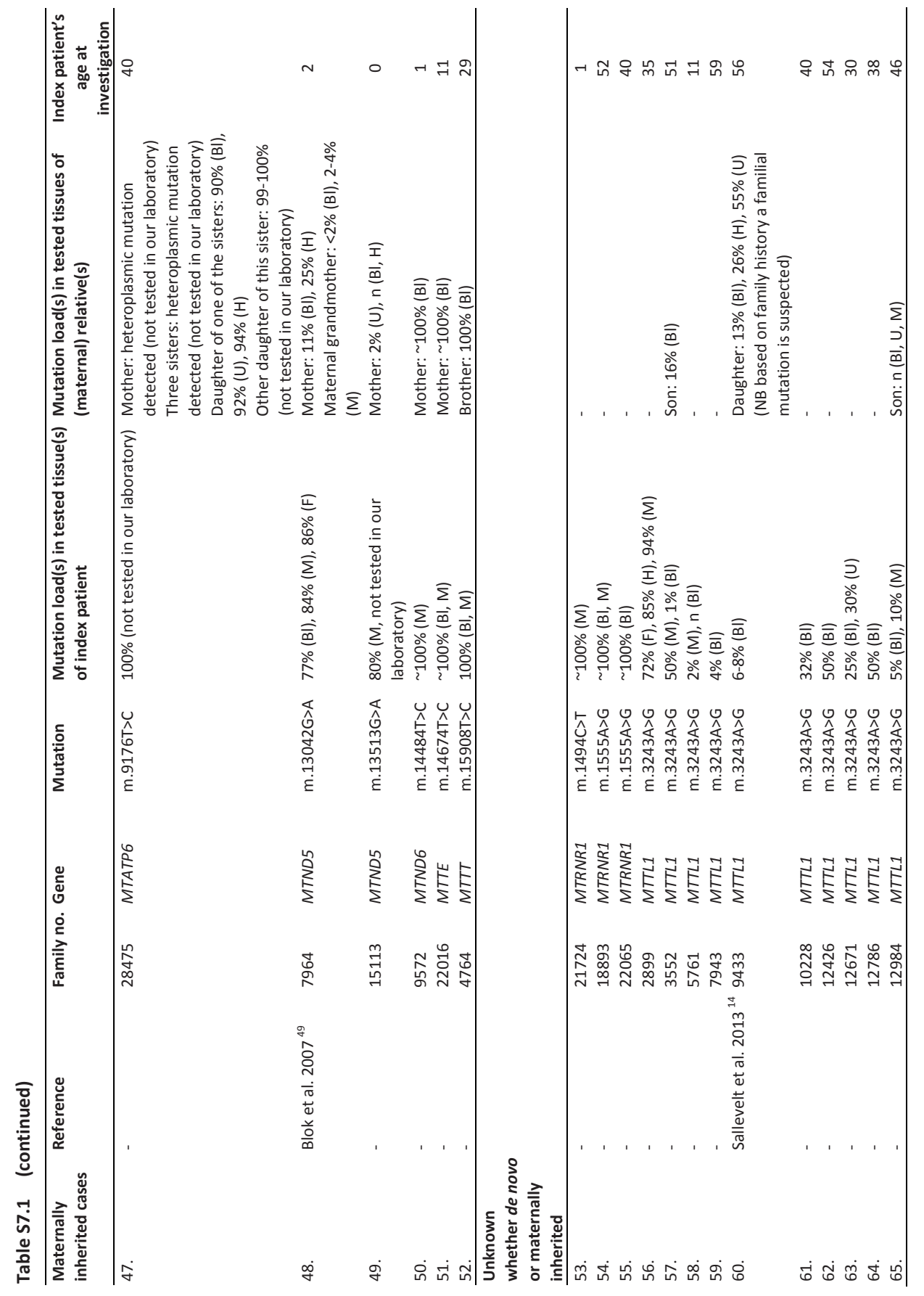




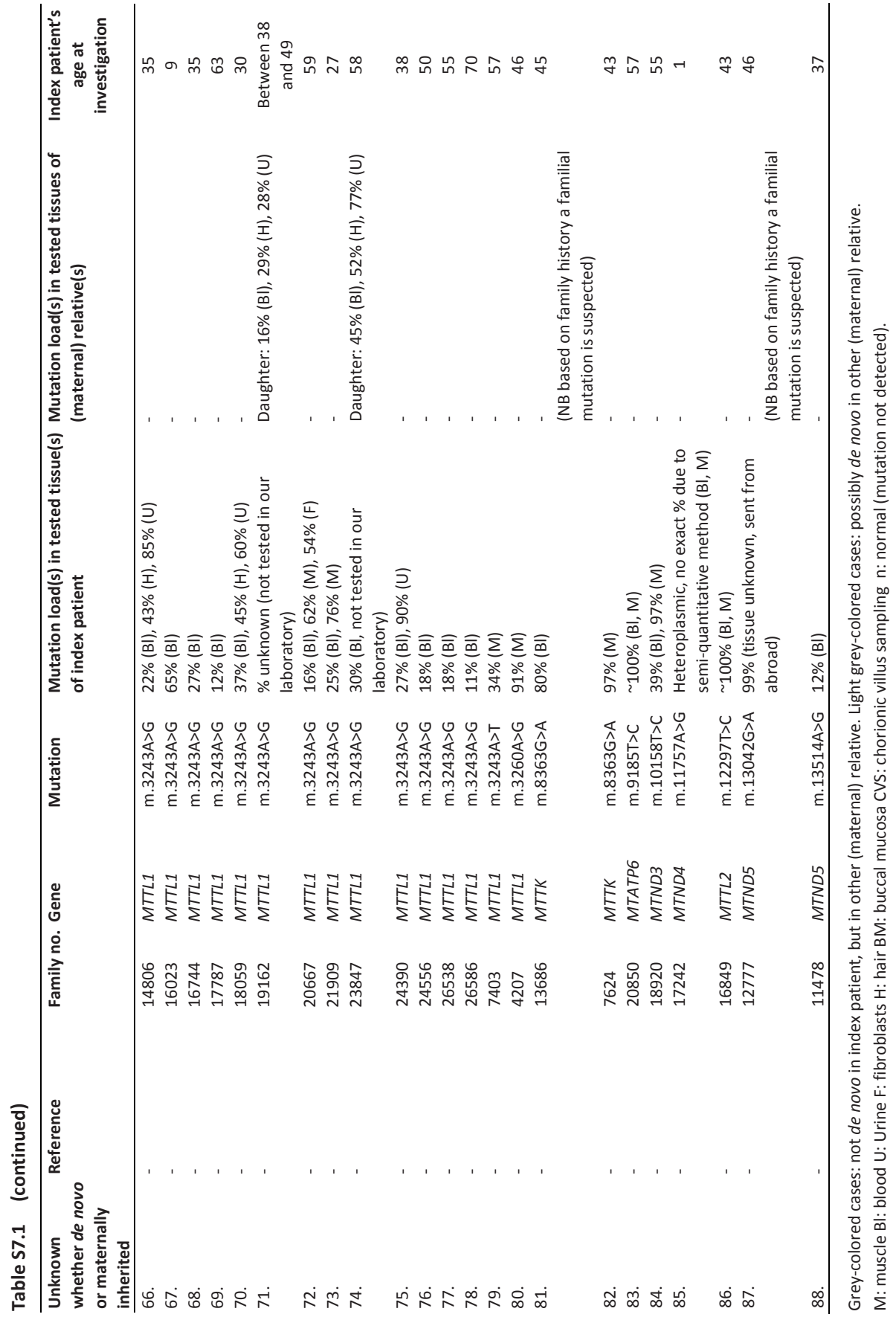




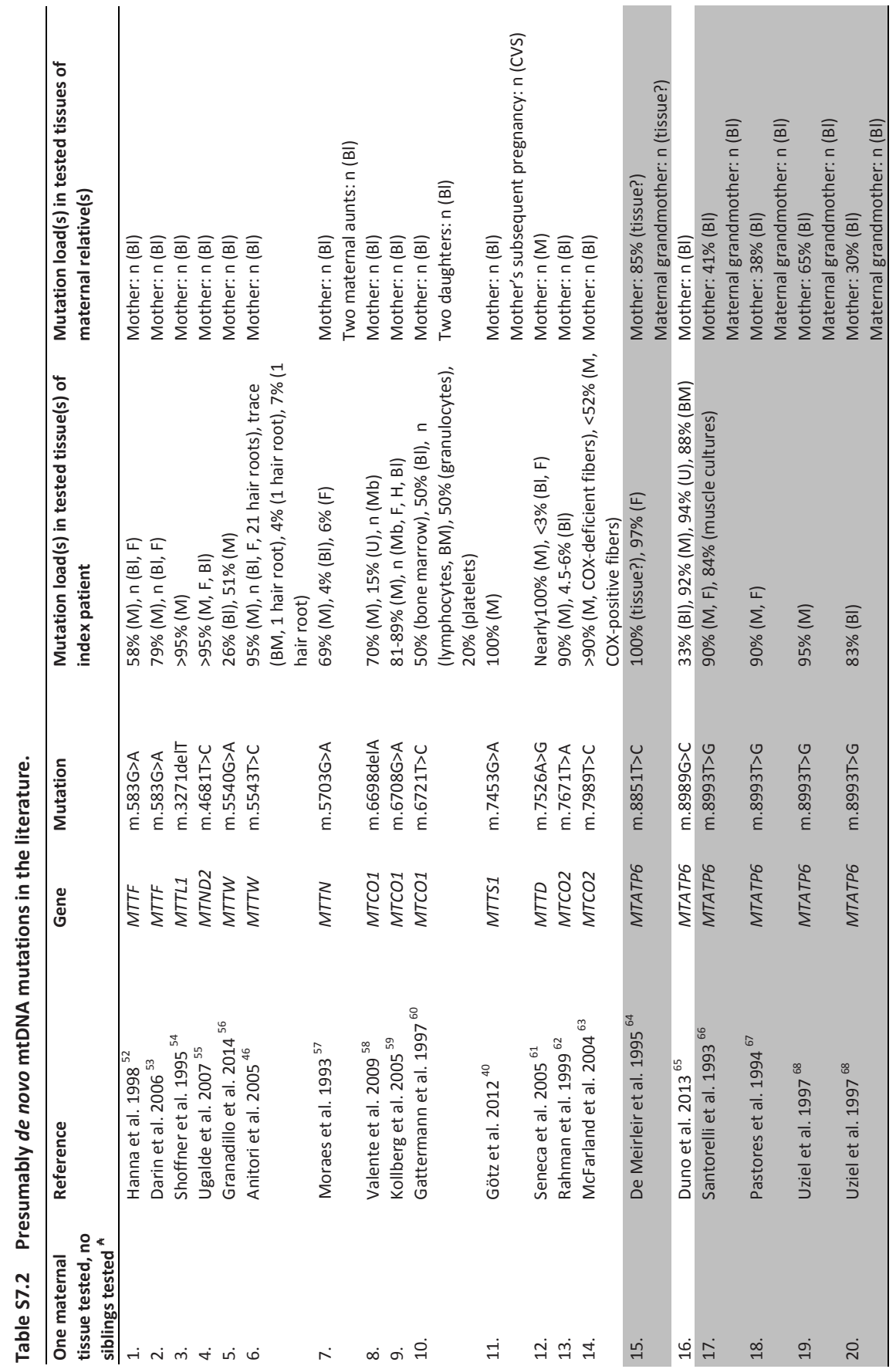




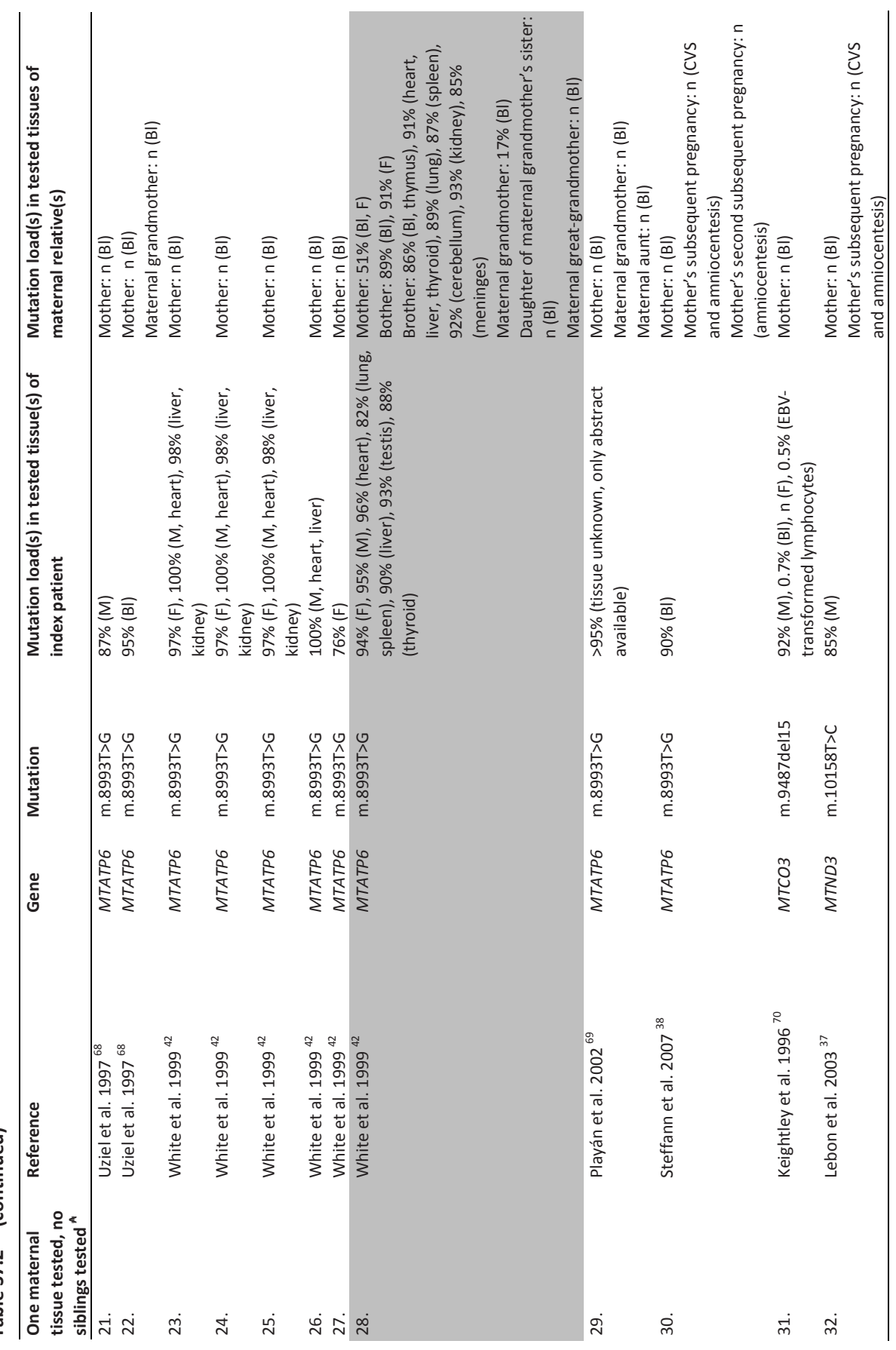




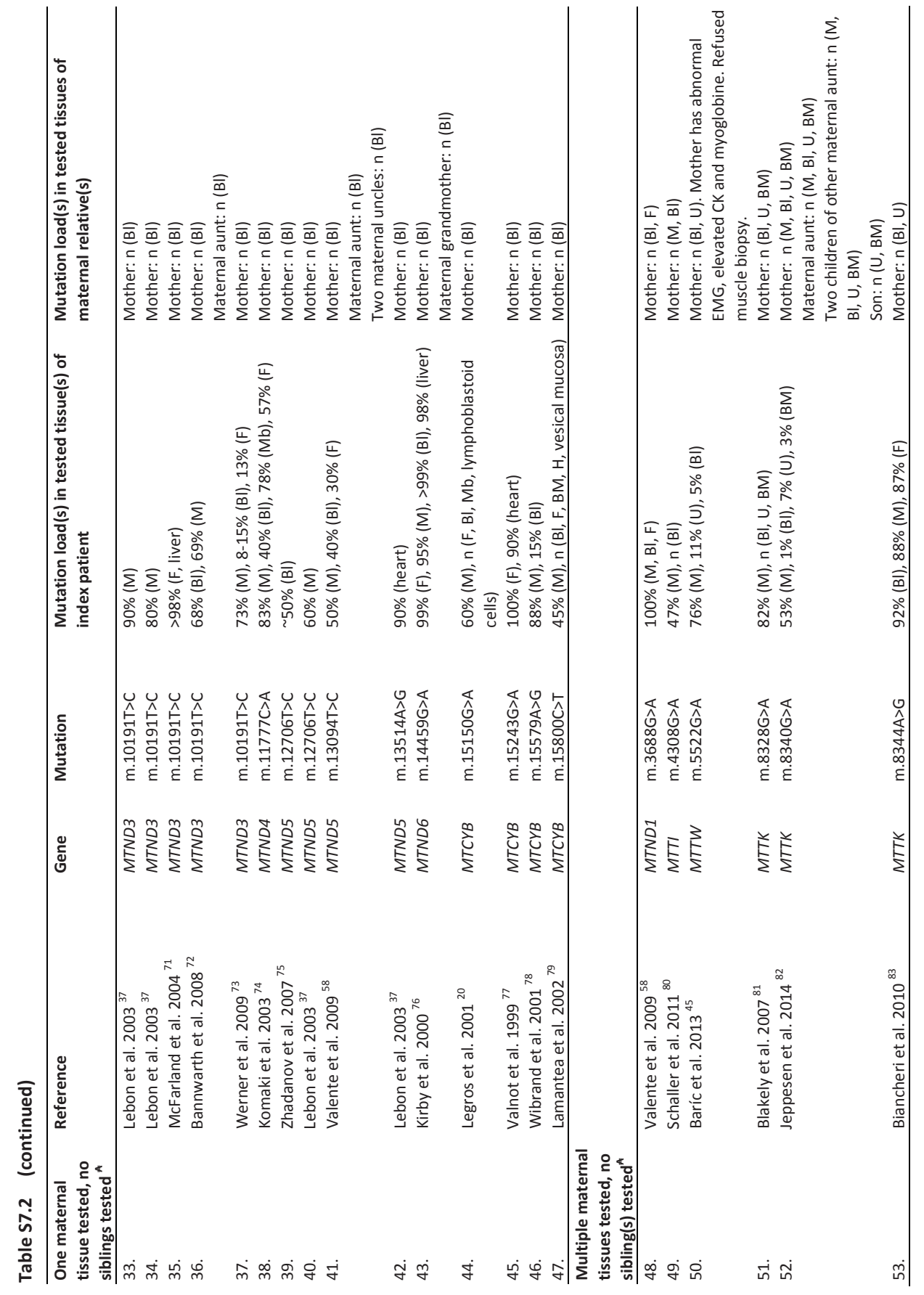




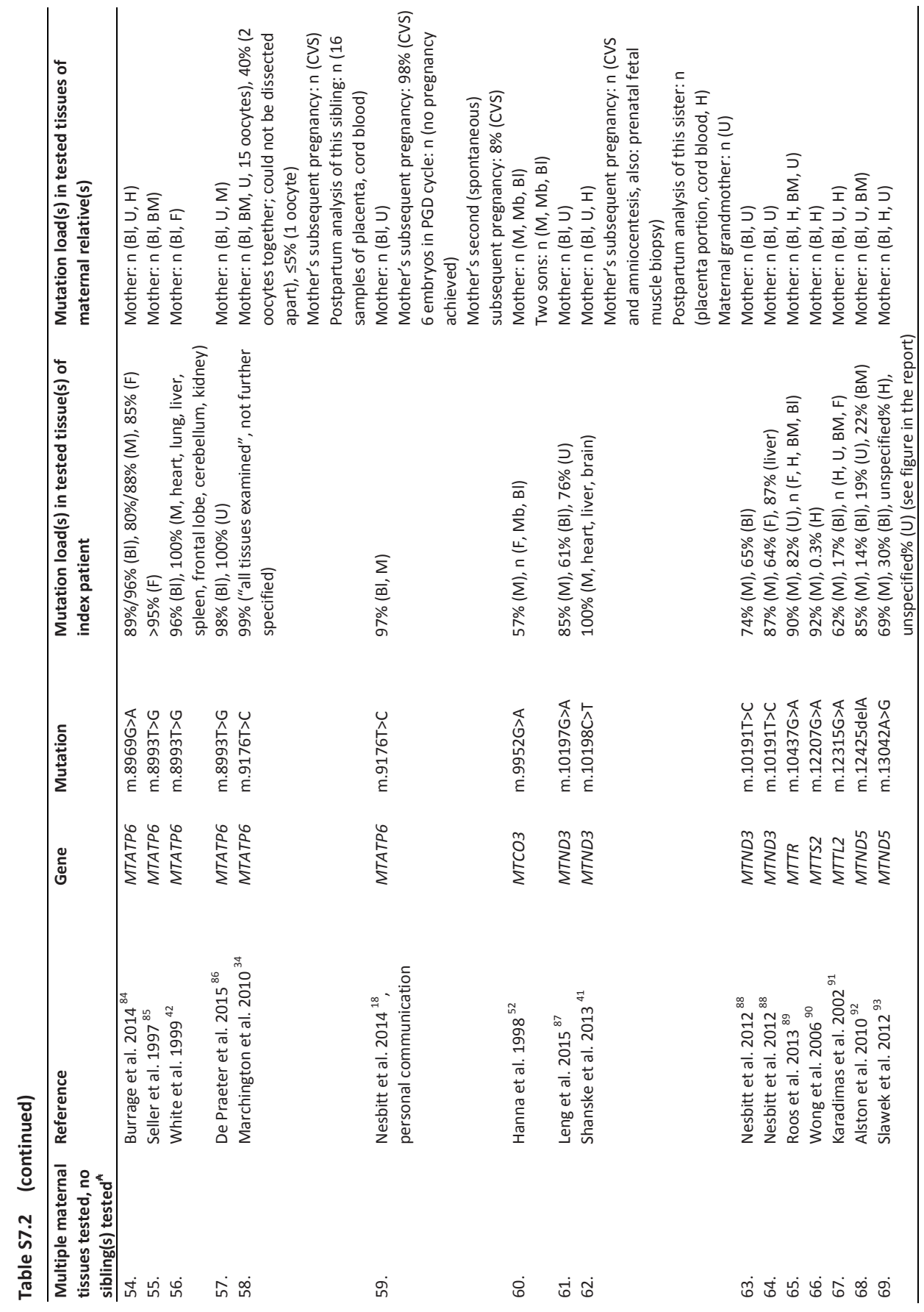




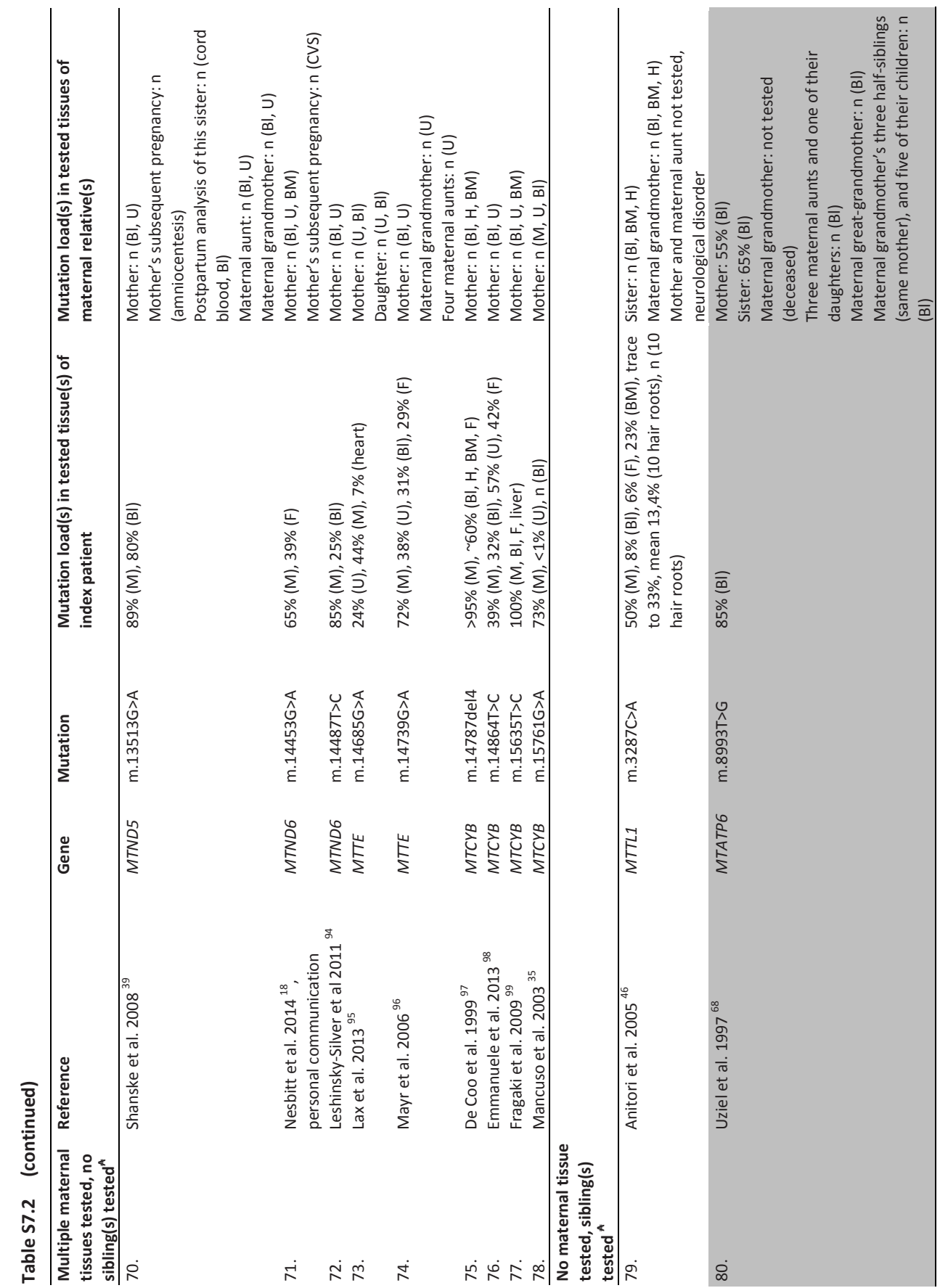




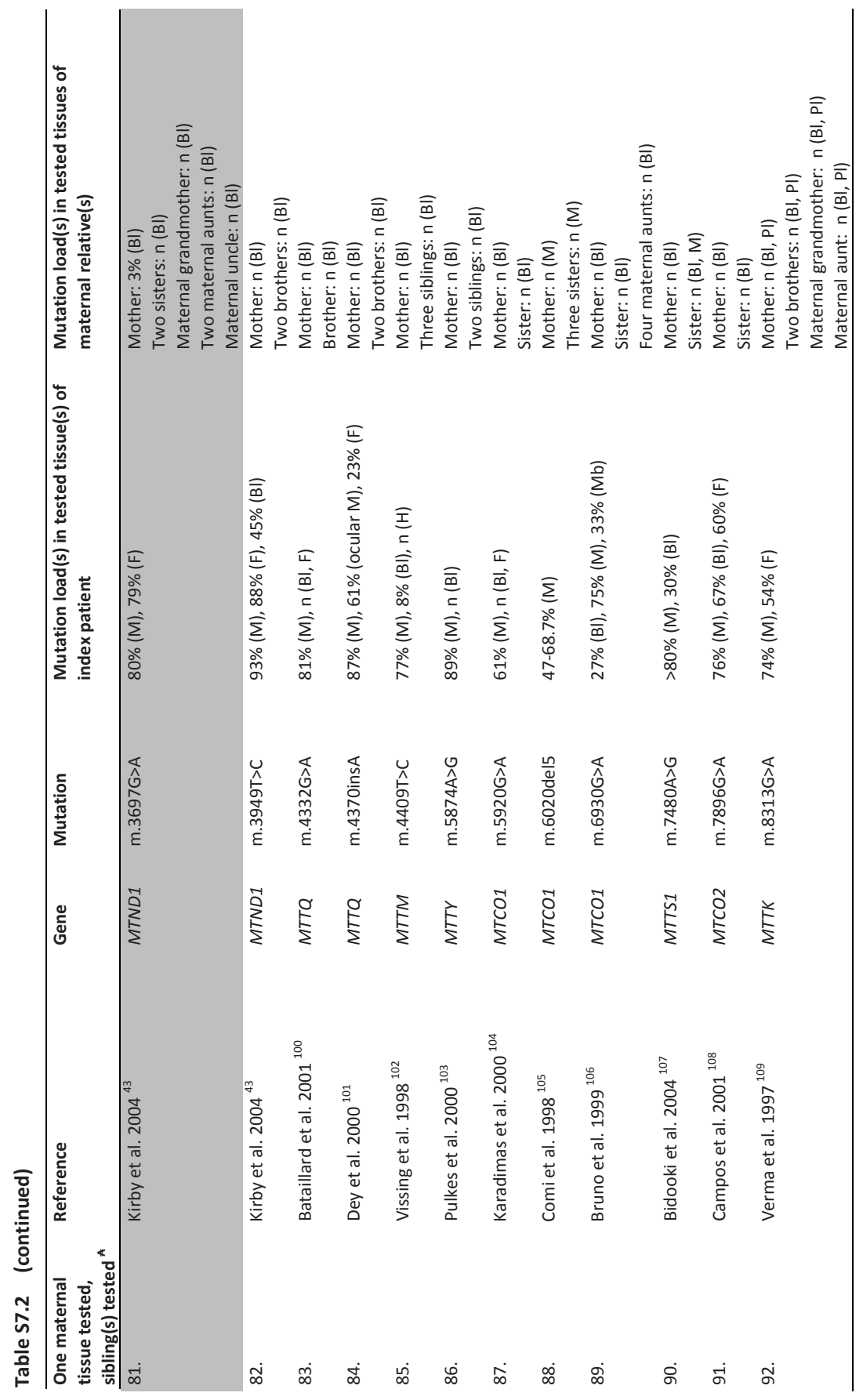




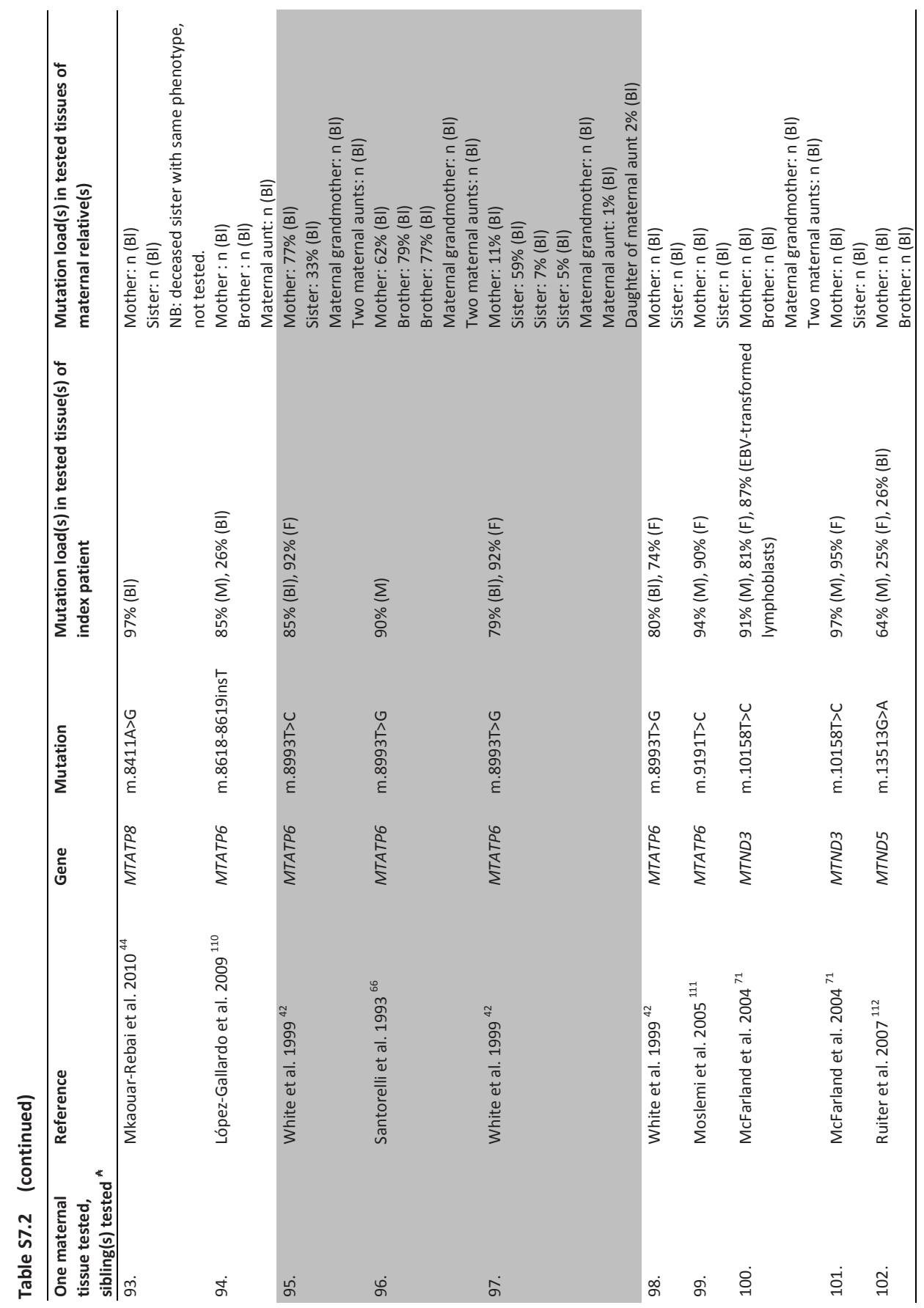




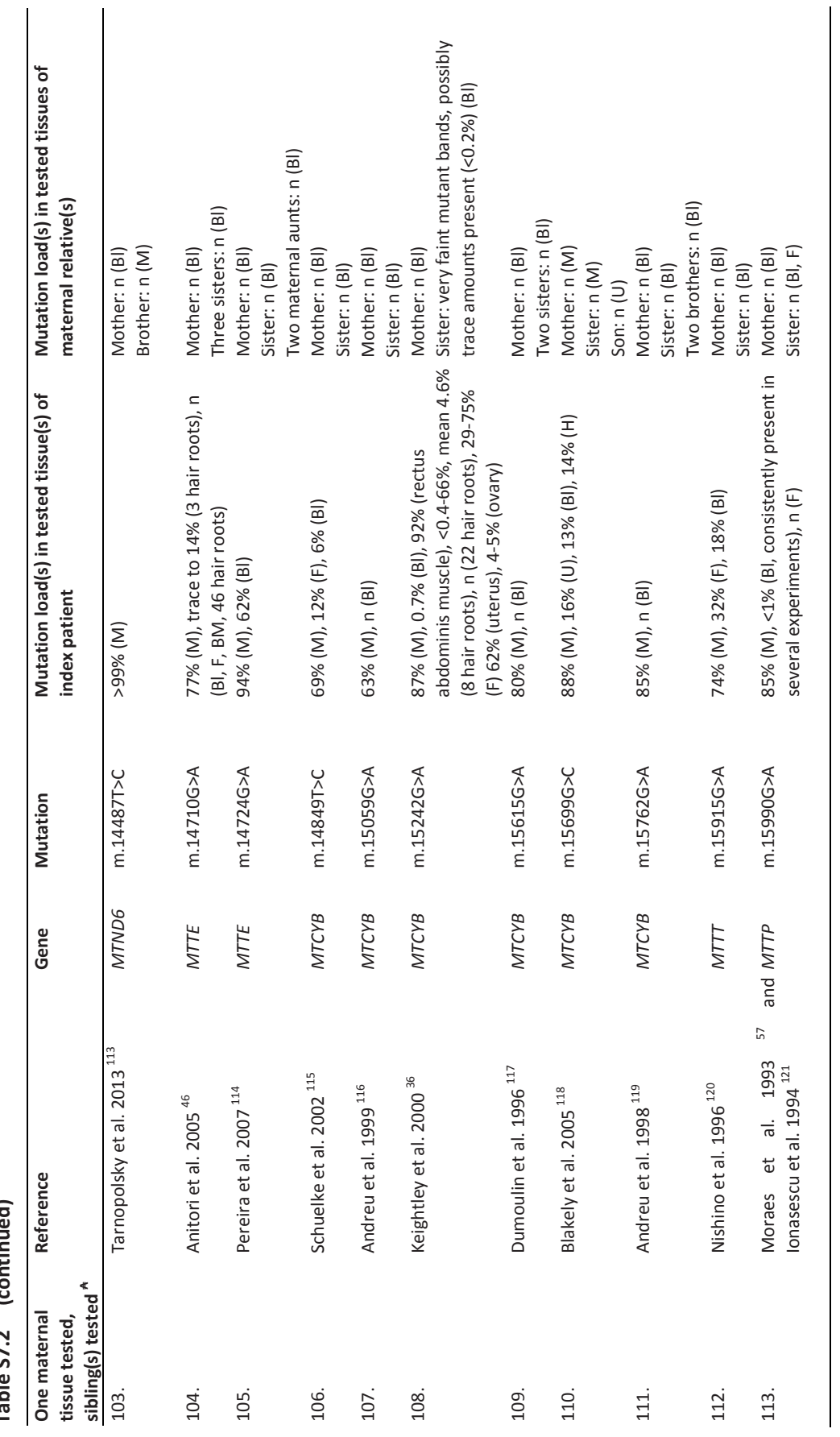




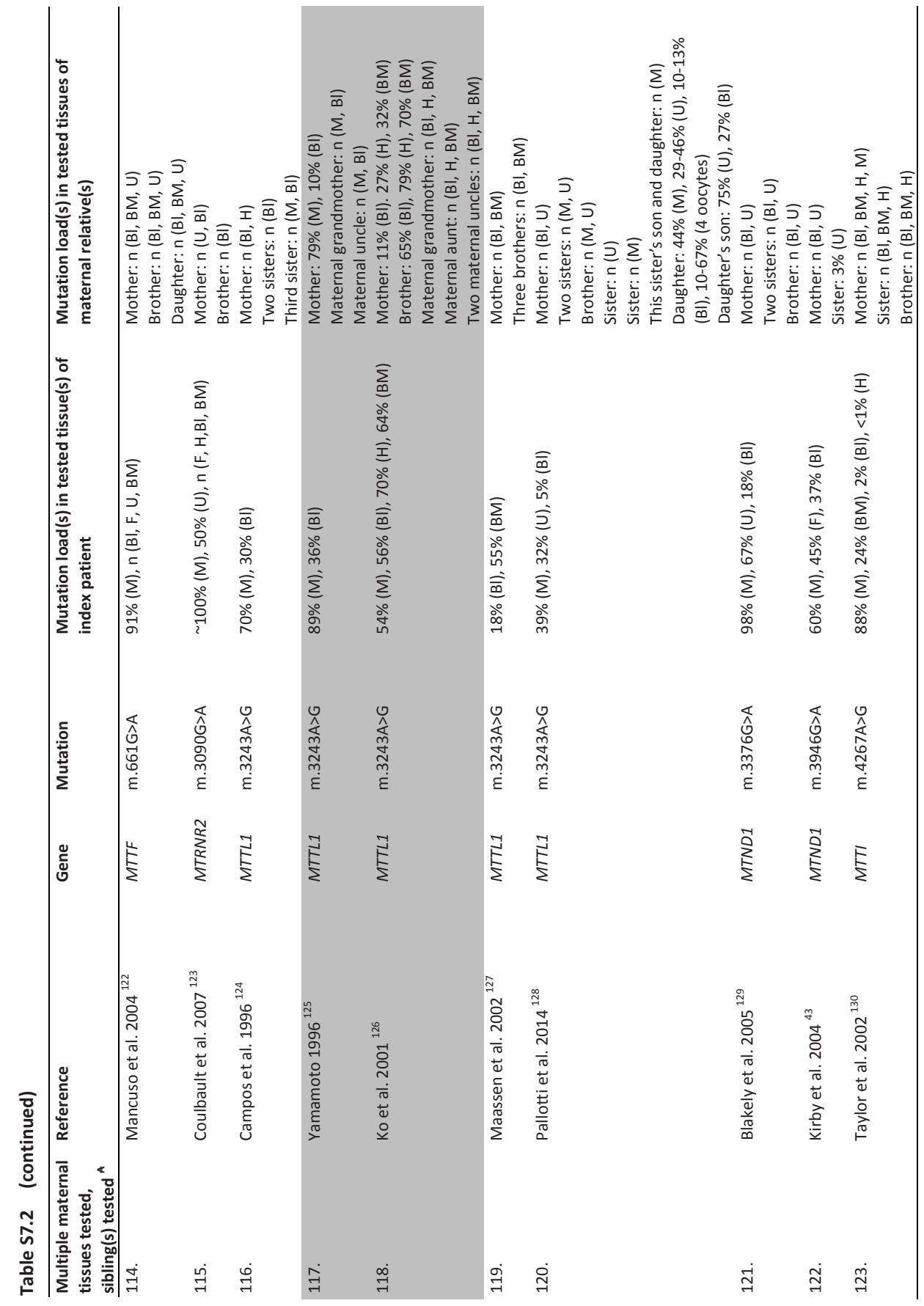




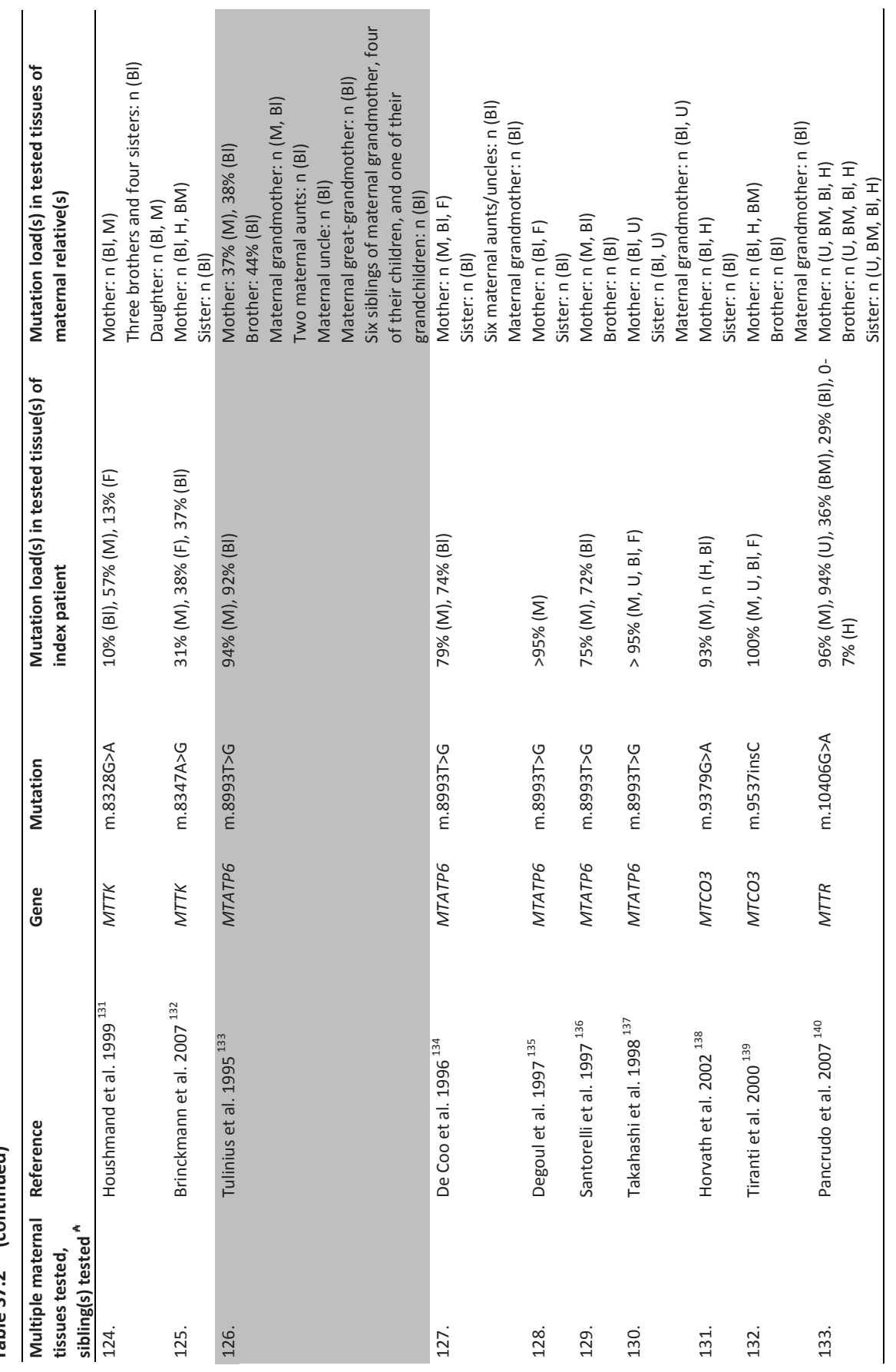




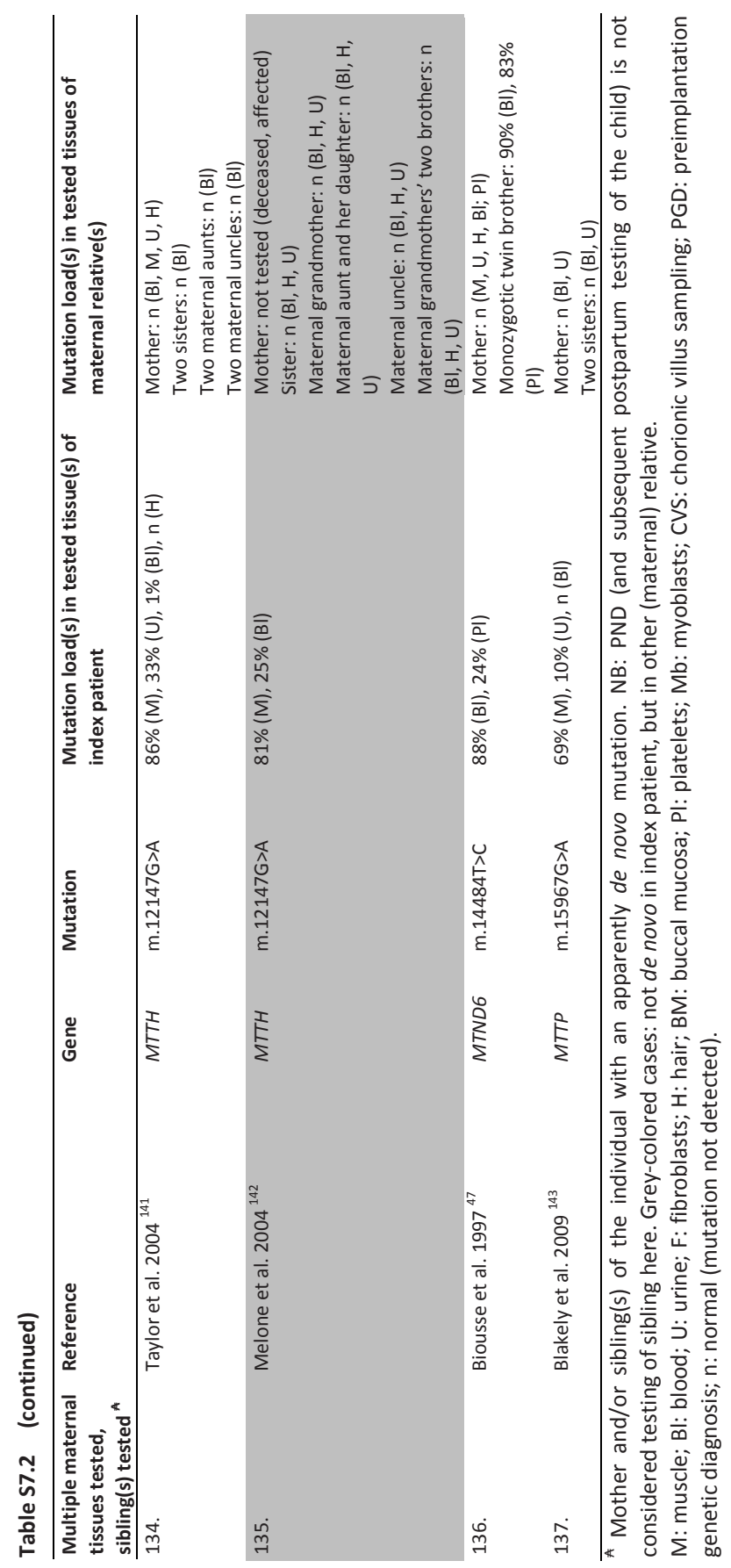



This chapter is embargoed at request

\section{CHAPTER 8}

The distribution of mtDNA mutations in human patients is defined by random de novo occurrence followed by pre-and postnatal selection

Suzanne C.E.H. Sallevelt, Christine E.M. de Die-Smulders, Hubert J.M. Smeets Submitted 


\section{PART III}

Primary mutations of the nuclear DNA 



\section{CHAPTER 9}

\section{Rapid resolution of blended or composite multigenic disease in infants by whole exome sequencing}

Tom E.J. Theunissen*, Suzanne C.E.H. Sallevelt*, Debby M.E.I. Hellebrekers, Bart de Koning, Alexandra T.M. Hendrickx, Bianca J.C. van den Bosch, Rick Kamps, Kees Schoonderwoerd, Radek Szklarczyk, Elvira N.M. Mulder-den Hartog, Irenaeus F.M. de Coo, Hubert J.M. Smeets

* these authors share first authorship Accepted for publication in J Pediatr. 


\section{Abstract}

Establishing a genetic diagnosis in patients, who manifest with a broad variety of clinical symptoms, is a major challenge, often requiring multiple rounds of clinical investigations. Phenotypic complexity might be explained by heterogeneous expression of a single-gene defect or a combination of different single-gene defects. Whereas candidate gene approaches often fall short in explaining the entire clinical picture, whole-exome sequencing provides an unbiased solution for fully resolving composite or blended phenotypes, which can be explained by multiple gene defects. Two patients suffering from a broad variety of clinical symptoms were analyzed by whole-exome sequencing. Consanguineous patient 1 had a neurological disorder involving psychomotor retardation with Leigh-like brain MRI. Three homozygous mutations, located in SERAC1, ACY1 and ANTXR2A, were found to underlie this blended phenotype. Nonconsanguineous patient 2 had arthrogryposis and oculocutaneous albinism, carrying a compound heterozygous HPS1 mutation combined with a dominant de novo mutation in BICD2, each gene defect explaining part of the composite clinical manifestation. Our data illustrates the importance of pursuing exome analysis until a genetic explanation is found for each individual symptom. Whole-exome analysis as a first test is the easiest and fastest way to establish a comprehensive genetic diagnosis in patients with heterogeneous clinical features. 


\section{Introduction}

A variety of disease manifestations in a patient, explainable by combinations of genetic and environmental factors, can hamper a correct and complete genetic diagnosis. Targeted analysis of candidate genes often falls short in explaining the complete clinical picture. Whole-exome sequencing (WES) is unbiased, allowing identification and evaluation of the majority of the patient's genetic variants. In clinical practice, WES has become the prime test for genetically heterogeneous monogenic disorders, with no straightforward genotype-phenotype correlation and unknown genes involved. ${ }^{1}$ WES could also be preferable to decipher complex disease cases, which could be caused by genes with overlapping (blended) or discrete (composite) manifestations. This is often the case in consanguineous patients. Yet, there has been little reported on the use of WES in presumably multi-genic disease, where complex phenotypes are caused by at least two different gene defects. ${ }^{2,3}$

We applied WES on two patients suffering from a variety of clinical symptoms, most likely not due to a single monogenic disorder and found that a combination of single gene defects was responsible for the complex picture of metabolic and phenotypic observations in these patients. Our data show the power of exome sequencing to resolve multiple monogenic phenotypes in isolated cases, which is important for diagnostics and prognosis, but also for accurate treatment and prevention of disease transmission to future children.

\section{Materials and methods}

\section{Informed consent}

Informed consent was obtained from all participants.

\section{Patients}

Patient 1, the second child of healthy consanguineous Moroccan parents (first-degree cousins), was hospitalized with septic-like symptoms, lower consciousness and hypotonia. At the fifth month of life, she suffered from growth delay, failure to thrive, hypotonia of extremities and neck, axial hypertonia, developmental delay and hypermetropia. Metabolic screening revealed hyperammonemia and hypoglycemia. Further biochemical findings revealed signs of liver dysfunction, high plasma lactate levels and mildly increased serine and alanine amino-acid levels in plasma. Plasma acylcarnitine analysis indicated mild increased free carnitine and 3-hydroxybutyrylcarnitine. Significant increased levels of 3-methylglutaconic acid, 3-methylglutaric acid and $\mathrm{N}$-acetyl amino acids were measured in urine. Because of epileptic 
activity, Magnetic Resonance Imaging (MRI) of the brain was performed, showing Leighlike features. During the following months, progressive hearing loss, severe reflux esophagitis and liver cirrhosis developed, also gingival hyperplasia was noted. She died at two years of age. Her sister showed only skin nodules and gingival hyperplasia.

Patient 2, a girl, born to healthy non-consanguineous Dutch parents, expressed symptoms of oculocutaneous albinism and abnormalities of the lower extremities, such as bilateral congenital hip dislocation, hyperextension of the right knee, limited extension of the left knee and bilateral foot deformities with talipes calcaneovalgus on the right and talipes equinovarus on the left. Examination of the upper extremities showed bilateral thumb adduction and radial deviation of the fingers. Echography and $\mathrm{MRI}$ of the vertebral column-myelum only revealed notable ectasia of the lumbar spinal canal. Distal skeletal muscles revealed excessive lipid accumulation and patient fibroblasts showed deficiency of mitochondrial complex IV activity.

\section{Homozygosity mapping}

DNA was prepared and labeled according to the manufacturer's manual (Affymetrix Human Mapping 250K array). Affymetrix Genotyping console 4.0 was used for SNP genotyping and homozygosity regions $(\mathrm{ROH})$ were determined by "Homozygosity mapper". A cutoff of $5 \mathrm{MB}$ was use to define these regions.

\section{Whole exome sequencing and data analysis}

Exonic regions were captured (Agilent SureSelect-v4 with UTRs), according to the manufacturers' protocol. Massive parallel sequencing was performed on an Illumina HiSeq2000 platform, using a 2x100 bp paired end setting. Exome data of patient 1 were filtered for homozygous variants within homozygosity regions. Only variants with allele frequencies lower than $1 \%$ (dbSNP137) and a coverage of $>10$ reads, consisting of nonsynonymous substitutions, INDELs (in-frame and frameshift), nonsense mutations and splice-variants, were evaluated. Non-annotated variants were maintained, unless allele frequencies exceeded $5 \%$ prevalence in our in-house patient database. Pathogenicity of non-synonymous missense mutation was estimated by PolyPhen-2, SIFT, PROVEAN and Mutation Taster. Nonsense, frameshift and splice-site mutations were maintained. WES data of patient 2 was filtered for compound heterozygous, homozygous and dominant de novo mutations. The filtering strategy was comparable to patient 1 . The familial segregation pattern of each mutation was determined by Sanger sequencing. 


\section{Results}

\section{Patient 1: SERAC1, ACY1 and ANTXR2 mutations}

In patient 1, 15 homozygous missense mutations and 4 homozygous INDELs were detected. In silico pathogenicity predictions indicated three variants as potentially disease causing.

A homozygous in-frame insertion was identified in the serine active site-containing protein 1 gene (SERAC1, NC_000006.12, NM_032861.3:c.1349_1350insATC). The insertion in exon 13 results in an additional serine residue (p.Ser450) at a highly conserved site in animals. It is a new and pathogenic predicted mutation (Provean, MutationTaster). Both parents were carriers and her sister was homozygous wild-type (Supplementary Figure S9.1). A second homozygous mutation was identified in the aminoacylase-1 gene (ACY1, NG_012036.1, NM_000666.2:c.811G>A), resulting in a non-polar alanine to polar threonine alteration (p.(Ala271Thr)) in a conserved M20 dimerization domain (Pfam07687). This is again a non-reported mutation (rs765511074), classified as disease causing by Polyphen2 and Mutation taster. Both parents and the sister were heterozygous for the mutation (Supplementary Figure S9.1). The Anthrax Toxin Receptor 2 (ANTXR2) gene contained a homozygous missense mutation (NG_015987.1, NM_001145794.1:c.1142A>G), changing an evolutionary conserved tyrosine to cysteine (p.(Tyr381Cys)) within the cytoplasmic domain. The mutation has been reported as disease causing (HGMD database - CM033747). ${ }^{4}$ The parents were heterozygous and both the index patient and her sister homozygous (Supplementary Figure S9.1).

\section{Patient 2: HPS1 and BICD2 mutations}

WES data was filtered for compound heterozygous, homozygous and dominant de novo mutations. A total of six compound heterozygous and two homozygous mutations passed the inclusion criteria and were further evaluated for pathogenicity and segregation in the family. A mutation in the Hermansky-Pudlak 1 gene (HPS1) and in the Bicaudal D Homolog 2 gene (BICD2), were considered relevant for the disease manifestations.

The index patient was compound heterozygous for two HPS1 mutations (Supplementary Figure S9.2), p.(Arg173*) and p.(GIn397Serfs*2) (NG_009646.1, NM_000195.4:c.[517C>T];[1189delC]). The novel premature stop codon at p.173 (dbSNP rs538274657) would cause a loss of 527 amino acids or, more likely, nonsensemediated decay of the mRNA. The second HPS1 mutation (p.(GIn397Serfs*2)), resulted in a frameshift with a premature stop codon or, most likely, nonsense-mediated mRNA decay. The mutation has been reported as disease causing (HGMD database CD982692). ${ }^{5}$ In addition, a dominant missense mutation (p.(Asp180Ala)) was identified at a conserved amino acid position in the BICD2 gene (NG_033908.1, 
NM_001003800.1:c.539A>C). PROVEAN, SIFT and PolyPhen2 called this novel mutation highly damaging. Sanger sequencing and paternity testing showed that the BICD2 mutation was de novo (Supplementary Figure S9.2).

\section{Discussion}

\section{Gene-phenotype relations}

Clinical symptoms of patient 1 were compared with other patients, having mutations in these three genes (Table 9.1). Similar to the SERAC1 cases $^{6}$, patient 1 showed Leigh syndrome-related disease manifestations, involving characteristic MRI and neurological and muscular impairments combined with respiratory and metabolic findings (Table 9.1). ${ }^{6}$ Although Leigh-like features are genetically heterogeneous, involvement of 3-methylglutaconic aciduria with sensorineural deafness and encephalopathy is typically explained by SERAC1 mutations, underlying MEGDEL syndrome (3-methylglutaconic aciduria, deafness, encephalopathy, and Leigh-like disease). The majority of the MEGDEL reported symptoms were found in patient 1 (Table 9.1).

Table 9.1 Symptoms caused by SERAC1 (NM_032861.3:c.1349_1350insATC), ACY1

(NM_000666.2:c.811G>A) and ANTXR2 (NM_001145794.1:c.1142A>G) mutations.

\begin{tabular}{|c|c|c|c|}
\hline & & Patient 1 (index) & sister \\
\hline \multirow[t]{5}{*}{$\begin{array}{l}\overline{\overline{0}} \\
\frac{0}{\square} \\
\bar{\Xi}\end{array}$} & $\begin{array}{l}\text { Patient description } \\
\text { genetic variants }\end{array}$ & $\begin{array}{c}\text { SERAC1:p.450insS, ACY1:p.(S271T), } \\
\text { ANTRX2:p.(Y381C) }\end{array}$ & ANTRX2:p.(Y381C) \\
\hline & alive (age) & no (died at 2 yrs.) & yes (age 8 yrs.) \\
\hline & first symptoms (age) & birth & 1 year \\
\hline & length and weight & $-3 S D$ & n.d. \\
\hline & birth weight & $2,300 \mathrm{~g}(-2 \mathrm{SD})$ & n.d. \\
\hline \multirow{17}{*}{ 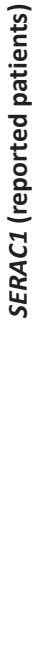 } & Clinical findings & & \\
\hline & sepsis-like features & yes & \\
\hline & feeding problems & yes & \\
\hline & failure to thrive / growth delay & yes & \\
\hline & microcephaly (1 patient) & borderline (-2 SD) & \\
\hline & hypotonia & yes & \\
\hline & psychomotor / developmental delay & yes & \\
\hline & developmental regression & yes & \\
\hline & absent speech & $\begin{array}{l}\text { yes; elementary speech development } \\
\text { disappeared }\end{array}$ & \\
\hline & epilepsy & yes & \\
\hline & encephalopathy & yes & \\
\hline & Leigh-like brain imaging (MRI/MRS) & yes & \\
\hline & dystonia / spasticity & yes & \\
\hline & sensorineural hearing loss & yes & \\
\hline & optic atrophy (bilateral) (1 patient) & $\begin{array}{c}\text { no; however, bilateral hyperopia (+6 } \\
\text { dpt) was diagnosed }\end{array}$ & \\
\hline & hepatopathy & yes & \\
\hline & respiratory dysfunction & yes & \\
\hline
\end{tabular}


Table 9.1 (continued)

\begin{tabular}{|c|c|c|c|}
\hline & Biochemical data & & \\
\hline & $\begin{array}{l}\text { hypoglycemia (neonatal) } \\
\text { hyperammonemia (neonatal) }\end{array}$ & $\begin{array}{l}\text { yes } \\
\text { yes }\end{array}$ & \\
\hline & increased 3-methylglutaconate in urine & yes & \\
\hline & increased 3-methylglurarate in urine & yes & \\
\hline & increased alanine & yes (slightly) & \\
\hline & increased lactate in blood & yes & \\
\hline & accumulation of free intracellular & n.d. & \\
\hline & cholesterol in fibroblasts & & \\
\hline & increased phosphatidylglycerol-34:1 to & n.d. & \\
\hline & phosphatidylglycerol-36:1 ratio in & & \\
\hline & fibroblasts & & \\
\hline & decreased blood cholesterol levels & no & \\
\hline & increased concentrations of specific & n.d. & \\
\hline & cardiolipin species in fibroblasts & & \\
\hline & Mitochondrial respiratory chain activities & & \\
\hline & muscle tissue & n.d. & n.d. \\
\hline & fibroblasts & no abnormalities & \\
\hline & Enzymatic activities & & \\
\hline & 3-methylglutaconyl-CoA hydratase & n.d. & \\
\hline $\bar{n}$ & Clinical findings & & \\
\hline .气 & skin nodules and/or papules & & yes \\
\hline$\stackrel{\pi}{\pi}$ & subcutaneous tumors & & \\
\hline $\bar{g}$ & hyaline deposition in dermis & & \\
\hline$\frac{\pi}{2}$ & gingival hypertrophy & yes & yes \\
\hline$\frac{0}{0}$ & progressive joint contractures & & \\
\hline$\stackrel{\sim}{\approx}$ & bone deformities & & \\
\hline$\underset{x}{x}$ & persistent diarrhea & & \\
\hline$\sum_{\alpha}^{5}$ & sepsis-like features & yes & \\
\hline & failure to thrive & yes & \\
\hline & growth delay & yes & \\
\hline & visceral involvement & & \\
\hline & coarse facial features & & \\
\hline & respiratory distress & yes & \\
\hline $\bar{n}$ & Clinical findings & & \\
\hline . & encephalopathy & yes & \\
\hline 范 & autistic features & & \\
\hline రृ & epilepsy & yes & \\
\hline tั & sensorineural hearing loss & yes & \\
\hline$\frac{0}{2}$ & growth delay & yes & \\
\hline$\stackrel{2}{-1}$ & psychomotor / developmental delay & yes & \\
\hline ঠे & opisthotonic spasms (1 patient) & no; only generalized spasticity involved & \\
\hline & hypotonia & yes & \\
\hline & Biochemical data & & \\
\hline & $\begin{array}{l}\text { increased } \mathrm{N} \text {-acetylated amino acid levels } \\
\text { (urine); indicating amino-acylase } 1 \\
\text { deficiency }\end{array}$ & yes & \\
\hline
\end{tabular}

ANTXR2 gene defects can lead to Juvenile hyaline fibromatosis (JHF) or infantile systemic hyalinosis (ISH) with clinical similarity involving subcutaneous skin nodules, gingival hypertrophy and joint contractures. Hyaline depositions are more widespread 
over different tissues in ISH, causing a more severe phenotype. ${ }^{4}$ Both affected siblings were homozygous for the p.(Tyr381Cys) ANTXR2 mutation, similar to two Moroccan siblings with JHF, ${ }^{4}$ which explains the JHF symptoms in the two sibs.

Mutations in ACY1 have been reported to underlie deficiency of aminoacylase 1. Urinary metabolite screening of patient 1 provided a specific profile of accumulating $\mathrm{N}$-acetylated amino acids, including $\mathrm{N}$-acetylalanine, $\mathrm{N}$-acetylleucine and $\mathrm{N}$-acetylglutamate, characteristic for ACY1 deficiency. Comparable to other ACY1 cases, patient 1 suffered from pronounced neurological symptoms with hypotonia, spasticity and growth delay (Table 9.1). ${ }^{7,8}$ Although several patient reports highlight a role for aminoacylase deficiency in disease manifestation, ${ }^{7}$ the clinical significance of the enzyme deficiency has also been questioned. ${ }^{9,10}$

Symptoms deriving from the three individual gene defects showed overlap in patient 1 (Figure 9.1, a blended phenotype). Since all neurological features could be explained from the SERAC1 defect, it is uncertain whether ACY1 deficiency contributed to disease manifestation or its severity. Based on the available data, a possible role cannot be excluded. If parents would opt for prenatal diagnosis (PND) in a subsequent pregnancy, ACY1 cannot be ignored, having implications for the number of gene defects tested. Discussions on the clinical impact of a genetic defect can cause dilemmas in a PNDcontext, as is the case for JHF, in which symptom manifestation might be relatively mild. Preimplantation genetic diagnosis (PGD) is currently possible for a maximum of two separate gene defects on individual blastomeres. Selection for three or more diseases is only possible after trophectoderm biopsy, or with new single cell analysis methods. Although, an increase in the number of conditions tested reduces the number of embryos suitable for transfer.

Mutations in HPS1 underlie Hermansky-Pudlak syndrome type 1, a rare autosomal recessive disorder, characterized by oculocutaneous albinism. ${ }^{11}$ Oculocutaneous albinism was pronounced in patient 2 , however no indications were found for other reported symptoms (Table 9.2). A second gene defect was found in BICD2, in which several autosomal dominant BICD2 mutations have been reported in patients with Spinal Muscular Atrophy, Lower Extremity-preDominant type2 (SMALED2). SMALED2 is a dominant spinal muscular atrophy with congenital onset and a variety of symptoms, mainly causing weakness of the lower limb muscles and affecting joint function (contractures) of the lower extremities. ${ }^{12}$ For some patients involvement of the upper extremities has been reported as well. Symptoms of patient 2 overlapped with previously reported BICD2 symptoms (Table 9.2). c.539A $>C$ (p.(Asp180Ala)) is located in the same domain as a SMALED2 mutation reported to cause Golgi-apparatus fragmentation. ${ }^{13}$ The $c .539 \mathrm{~A}>\mathrm{C}$ mutation in $B I C D 2$ therefore likely explains the remaining clinical symptoms in patient 2 , having a composite phenotype. 


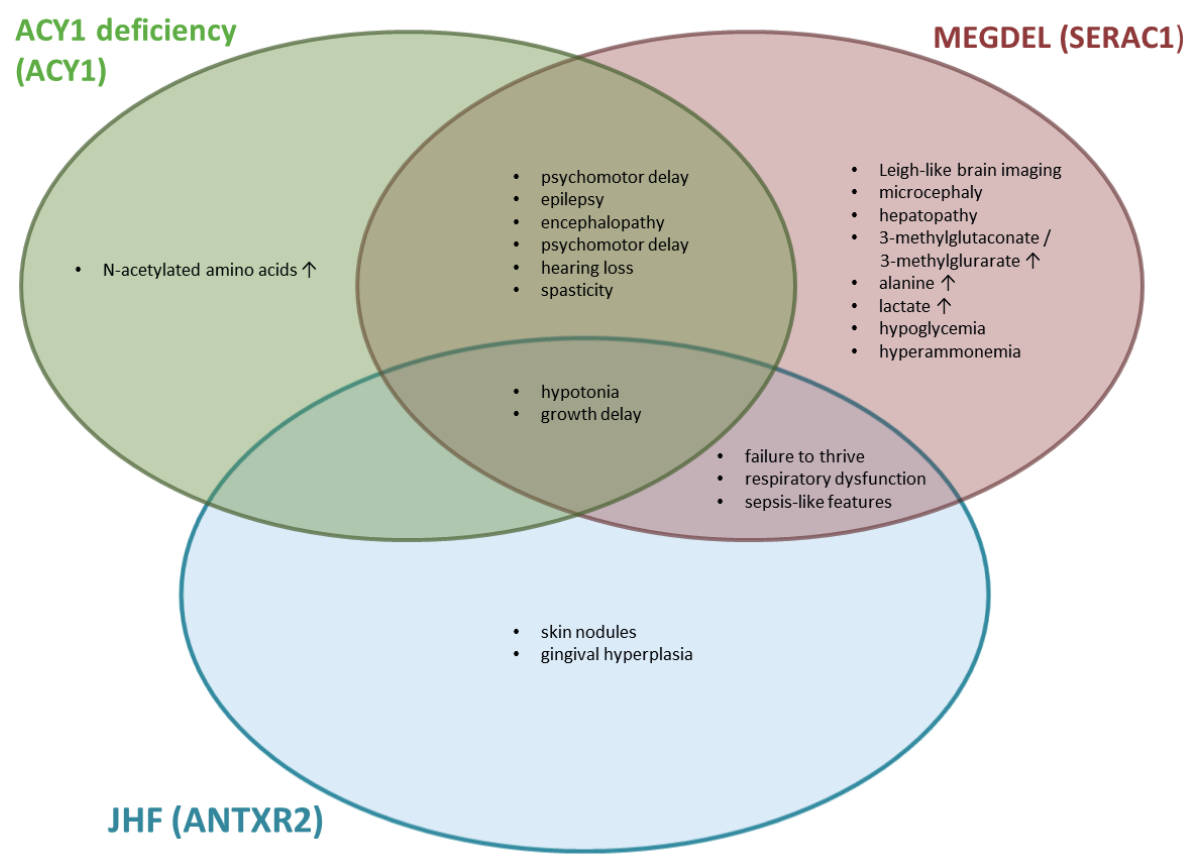

Figure 9.1 Index patient 1, categorization of observed clinical features according to three individual gene defects. Large overlap in neurological features was especially found between ACY1 and SERAC1 symptoms. The index's sister was solely affected by JHF symptoms (ANTXR2)

\section{WES in multi-genic disease}

Our paper shows the power of unbiased WES in the characterization of complex patient phenotypes that can be explained from multiple gene defects, enabling precision medicine and personalized care for individuals with unique combined disease manifestations. Based on the gene defects identified, clinical symptoms can be grouped into overlapping (blended) or discrete (composite) sub-phenotypes, which can guide further clinical investigations. The latter is important, as sometimes the same clinical symptoms can be explained by two independent gene defects and specific follow-up investigation might help to discriminate the individual contribution. This is also important if parents opt for prenatal diagnosis to get an unaffected child, as it should be clear what the consequences of individual gene defects are, either in isolation or in combination with other gene defects. This dilemma is illustrated by the first pedigree, where the contribution of the ACY1 mutation to the phenotype is currently unclear, and where it is shown that inheritance of the ANTXR2 mutation as a single gene defect can still result in good life quality. Whereas in clinical practice a genetic diagnosis is often completed once a gene defect has been identified, our data illustrate the importance of pursuing genetic analysis until each individual symptom can be explained. WES will 
become the first, rapid test for patients with heterogenic or multi-genetic disease, which would otherwise require expensive and time-consuming clinical investigations. This could especially apply to patients from consanguineous marriages, where the parents potentially share carrier-ship of more genetic diseases than outbred marriages.

\section{Acknowledgements}

This work was supported by a grant obtained from Princes Beatrix Spierfonds (W.OR11-). 


\section{References}

1. Rabbani B, Tekin M, Mahdieh N. The promise of whole-exome sequencing in medical genetics. J Hum Genet. 2014;59(1):5-15.

2. Khalifa M, Naffaa L. Exome sequencing reveals a novel WDR45 frameshift mutation and inherited POLR3A heterozygous variants in a female with a complex phenotype and mixed brain MRI findings. Eur J Med Genet. 2015;58(8):381-386.

3. Cullinane AR, Vilboux T, O'Brien K, et al. Homozygosity mapping and whole-exome sequencing to detect SLC45A2 and G6PC3 mutations in a single patient with oculocutaneous albinism and neutropenia. J Invest Dermatol. 2011;131(10):2017-2025.

4. Hanks S, Adams S, Douglas J, et al. Mutations in the gene encoding capillary morphogenesis protein 2 cause juvenile hyaline fibromatosis and infantile systemic hyalinosis. Am J Hum Genet. 2003;73(4): 791-800.

5. Oh J, Ho L, Ala-Mello S, et al. Mutation analysis of patients with Hermansky-Pudlak syndrome: a frameshift hot spot in the HPS gene and apparent locus heterogeneity. Am J Hum Genet. 1998;62(3):593-598.

6. Sarig O, Goldsher D, Nousbeck J, et al. Infantile mitochondrial hepatopathy is a cardinal feature of MEGDEL syndrome (3-methylglutaconic aciduria type IV with sensorineural deafness, encephalopathy and Leigh-like syndrome) caused by novel mutations in SERAC1. Am J Med Genet A. 2013;161A(9): 2204-2215.

7. Alessandri MG, Casarano M, Pezzini I, et al. Isolated mild intellectual disability expands the aminoacylase 1 phenotype spectrum. JIMD Rep. 2014;16:81-87.

8. Sass JO, Olbrich $\mathrm{H}$, Mohr V, et al. Neurological findings in aminoacylase 1 deficiency. Neurology. 2007;68(24):2151-2153.

9. Sass JO, Mohr V, Olbrich $\mathrm{H}$, et al. Mutations in ACY1, the gene encoding aminoacylase 1, cause a novel inborn error of metabolism. Am J Hum Genet. 2006;78(3):401-409.

10. Van Coster RN, Gerlo EA, Giardina TG, et al. Aminoacylase I deficiency: a novel inborn error of metabolism. Biochem Biophys Res Commun. 2005;338(3):1322-1326.

11. Gahl WA, Brantly M, Kaiser-Kupfer MI, et al. Genetic defects and clinical characteristics of patients with a form of oculocutaneous albinism (Hermansky-Pudlak syndrome). N Engl J Med. 1998;338(18):12581264.

12. Rossor AM, Oates EC, Salter HK, et al. Phenotypic and molecular insights into spinal muscular atrophy due to mutations in BICD2. Brain. 2015;138(Pt 2):293-310.

13. Neveling K, Martinez-Carrera LA, Holker I, et al. Mutations in BICD2, which encodes a golgin and important motor adaptor, cause congenital autosomal-dominant spinal muscular atrophy. Am J Hum Genet. 2013;92(6):946-954. 


\section{Supplemental material}

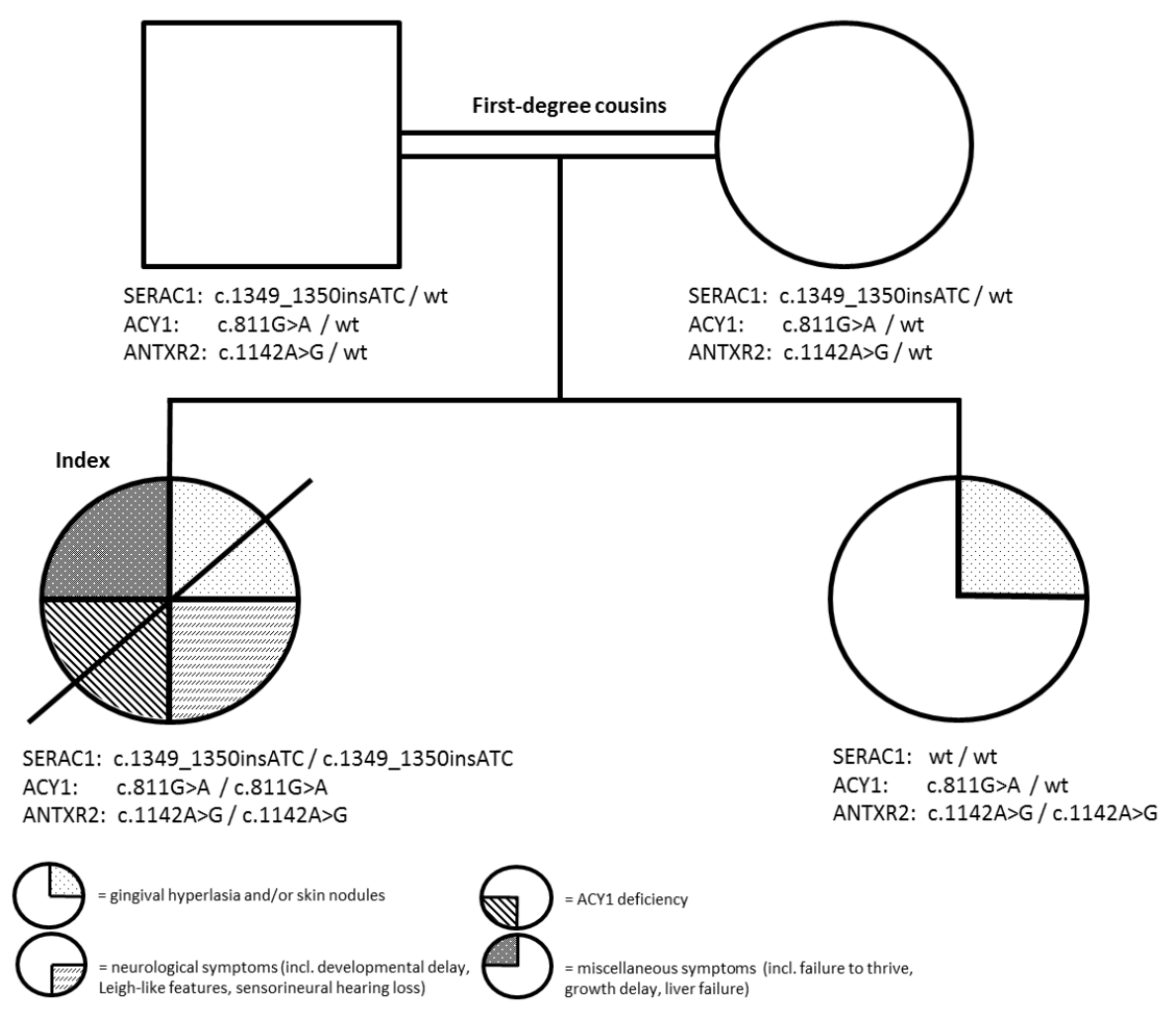

Figure S9.1 Patient case 1, the family segregation pattern of three WES identified mutations (confirmed by Sanger sequencing). Mutations were present in SERAC1 ((NM_032861.3:c.1349_1350insATC), ACY1 (NM_000666.2:c.811G>A) and ANTXR2 (NM_001145794.1:c.1142A>G) 


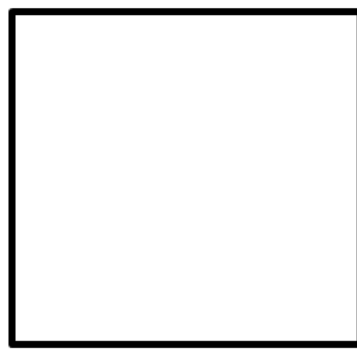

HSP1: c.1189delC/wt

BICD2: wt / wt

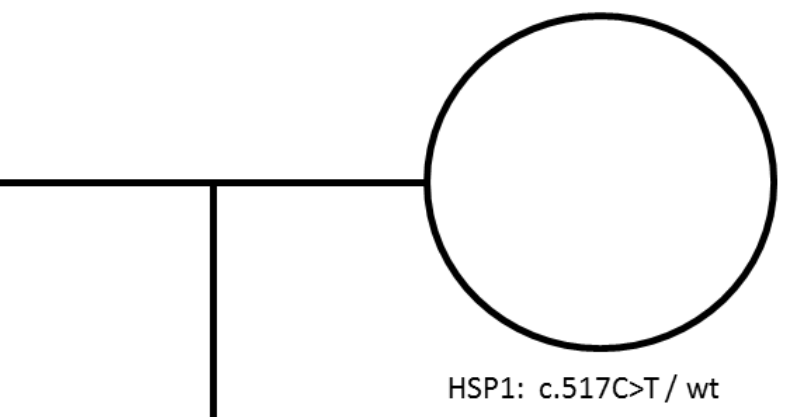

BICD2: wt / wt

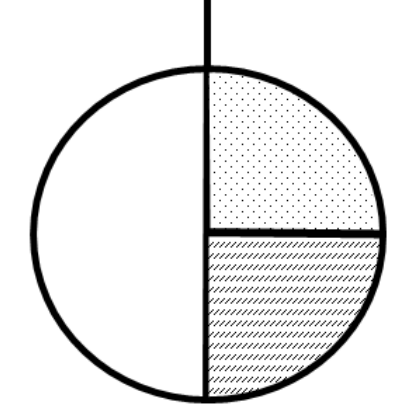

HSP1: c.1189delC/ c.517C >T

BICD2: c.539A>C/wt

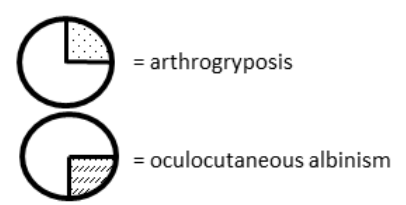

Figure S9.2 Patient case 2, Sanger sequencing confirmed both parents as heterozygous carriers of a HPS1 mutation, indicating a compound heterozygous orientation of the index's HPS1 mutations (NM_000195.4:c.[517C>T];[1189delC]). The dominant BICD2 mutation (NM_001003800.1: c.539A $>C$ ) was introduced de novo, because it was not present in both parents (non-paternity was excluded) 



\section{CHAPTER 10}

\section{A comprehensive strategy for exome-based preconception carrier screening}

Suzanne C.E.H. Sallevelt, Bart de Koning, Radek Szklarczyk, Aimee D.C. Paulussen, Christine E.M. de Die-Smulders, Hubert J.M. Smeets Genet Med. 2016 Oct 27 (Epub ahead of print) 


\section{ABSTRACT}

Purpose

Whole exome sequencing (WES) provides the possibility of genome-wide preconception carrier screening (PCS). Here, we propose a filter strategy to rapidly identify the majority of relevant pathogenic mutations.

\section{Methods}

Our strategy was developed on WES data from 8 consanguineous and 5 fictive nonconsanguineous couples, and subsequently applied to 20 other fictive non-consanguineous couples. Presumably pathogenic variants based on frequency and 1) database annotations (HGMD/ClinVar) or 2) generic characteristics and mutation type (nonsense, frameshift and splicesite mutations), were selected in genes shared by the couple and in the female's X-chromosome. Unclassified variants were not included.

\section{Results}

This yielded on average 29 [19-51] variants in genes shared by the consanguineous couples $(n=8)$ and 15 [6-30] by the non-consanguineous $(n=25)$. For $X$-linked variants the numbers per female were 3 [1-5] and 1 [0-3], respectively. Remaining variants were verified manually. The majority could be quickly discarded, effectively leaving true pathogenic variants. Dependent on the clinical consequences, reproductive choices can be offered to the couple.

\section{Conclusion}

We conclude that WES is eligible for PCS, both for consanguineous and non-consanguineous couples, with the remaining number of variants being manageable in a clinical setting. Addition of gene panels for filtering was not favorable, as it resulted in missing pathogenic variants. It is important to develop and continuously curate databases with pathogenic mutations in order to further increase sensitivity of WES-based PCS. 


\section{Introduction}

Preconception carrier screening (PCS) refers to genetic testing of couples before pregnancy. These couples do not per se have an a priori increased risk of having a child with a recessive genetic disease.

More than 1300 (autosomal and X-linked) recessive disorders have been identified so far, ${ }^{1}$ varying greatly in severity and age of onset. Although individually uncommon in general populations, Mendelian diseases are collectively reported to account for $\sim 20 \%$ of infant mortality and $\sim 10 \%$ of pediatric hospitalizations. ${ }^{2}$ By detecting carrier status, couples having a $25 \%$ risk of affected offspring can be identified. The primary aim of PCS is to provide such couples informed reproductive choices, which include prenatal diagnosis (PND), preimplantation genetic diagnosis (PGD), accepting the genetic risk and prepare themselves of (the possibility of) having a child with a certain disease, sperm/egg donation, adoption, or refraining from having children. In certain communities, particularly with high incidence of specific severe diseases, disease prevention may be viewed as the primary goal and as such the reduction in disease incidence is regarded as success measure. ${ }^{3}$ Another potentially favorable consequence of carrier screening is enabling early perinatal diagnosis and treatment which can profoundly reduce morbidity and mortality. Considering the primary aim of carrier screening being the facilitation of autonomous choices, the preconception period is considered as the optimal timing since only then all aforementioned reproductive options are still applicable. In contrast, when carrier status is identified during pregnancy, only PND or accepting the risk of an affected child without further diagnostic testing remain as options. Additionally, in a prenatal setting, time available to perform analyses and to make, often difficult, decisions is limited.

The prevalence of offspring with major anomalies is increased among consanguineous couples compared to non-consanguineous couples, ${ }^{4}$ mostly attributable to autosomal recessive disorders. Increased genetic risks are also applicable to subpopulations with high carrier frequencies of specific mutations, like founder mutations. ${ }^{5}$ The concept of PCS has been applied already for decades in some of these populations. ${ }^{6}$ Typically one or a few diseases with relatively high incidence in a population, or subpopulation, are tested in a targeted manner. In the Netherlands, this concept is exemplified by carrier screening in a genetically isolated community. ${ }^{5}$ However, with the introduction of next generation sequencing (NGS) techniques, simultaneous testing of much larger gene numbers has become possible and cost-effective. Until now, particularly targeted, gene panel-based, NGS approaches have been described. ${ }^{2,7-9}$ An approach using targeted analysis after untargeted whole exome sequencing (WES) has been explored in one study, filtering for identical mutations in about 500 genes in both partners of four consanguineous couples. ${ }^{10}$ However, systematic assessment of the use of WES for PCS has not been performed so far. In our study we aim to investigate WES as basis for PCS in a broader setting and develop a filter strategy that could be used both for 
consanguineous and non-consanguineous couples.

\section{Methods}

Different filter strategies for PCS were explored in WES data from 8 consanguineous (C) couples and 5 fictive non-consanguineous (NC) couples. The resulting proposed filter strategy was applied to a further 20 non-consanguineous fictive couples.

\section{Subjects}

The 8 consanguineous couples all have a child with suspected mitochondrial, or mitochondrial like, disease. They gave consent for WES, including their own DNA, in a research setting. The genetic cause of the child's disease has been established in four couples (1, 5, 6 and 7) (Table 10.1), whereby the affected child was homozygous for the causative mutation(s) and the parents were identified as heterozygous carriers. Couples 1, 3, 5, 6 and 7 are of Moroccan origin, couples 4 and 8 are from Turkey and couple 2 from Iraq (Table S10.1). For details on the degrees of consanguinity of the 8 couples, see Supplementary Table S10.1.

The non-consanguineous couples, originating from the Dutch population, were anonymized male and female exomes from existing diagnostic data sets, which were randomly mixed. For the current proof of concept study, it was not necessary to use actual couples. The study was approved by the local Medical Ethical Committee.

\section{Analysis}

WES for the consanguineous couples and the non-consanguineous couples was performed as previously described ( ${ }^{11}$ and ${ }^{12}$, respectively). Standard filter steps were location (exonic and canonical splice, the latter defined as $\leq 2$ nucleotides intronic), sequencing depth/reads $(\geq 8)$ and zygosity (homozygous variants removed). Variably applied filter steps were amino acid alteration (synonymous variants removed), annotation in databases (HGMD and/or ClinVar), allele frequency (dbSNP $<5 \%$ versus $<1 \%$ ), mutation type (frameshift, nonsense, splice site, missense), presence of identical variants in both partners of the couple, presence of variants in the same genes in both partners of the couple and presence of variants in genes included in gene panels. In order to establish an adequate cut-off for the allele frequency, disease prevalences were reviewed. Allele frequencies were calculated by taking the square root of the prevalence. ClinVar database on pathogenicity of known variants was downloaded from ftp://ftp.ncbi.nlm.nih.gov/pub/clinvar on 2 March 2016 in a VCF format. These were matched to the patient exome data using SNP identifiers, accounting for multiple SNPs at a given position. For multiple pathogenicity classes assigned to a genetic variant only classes 4 ("likely pathogenic") and 5 ("pathogenic") were taken into consideration for further analysis. 


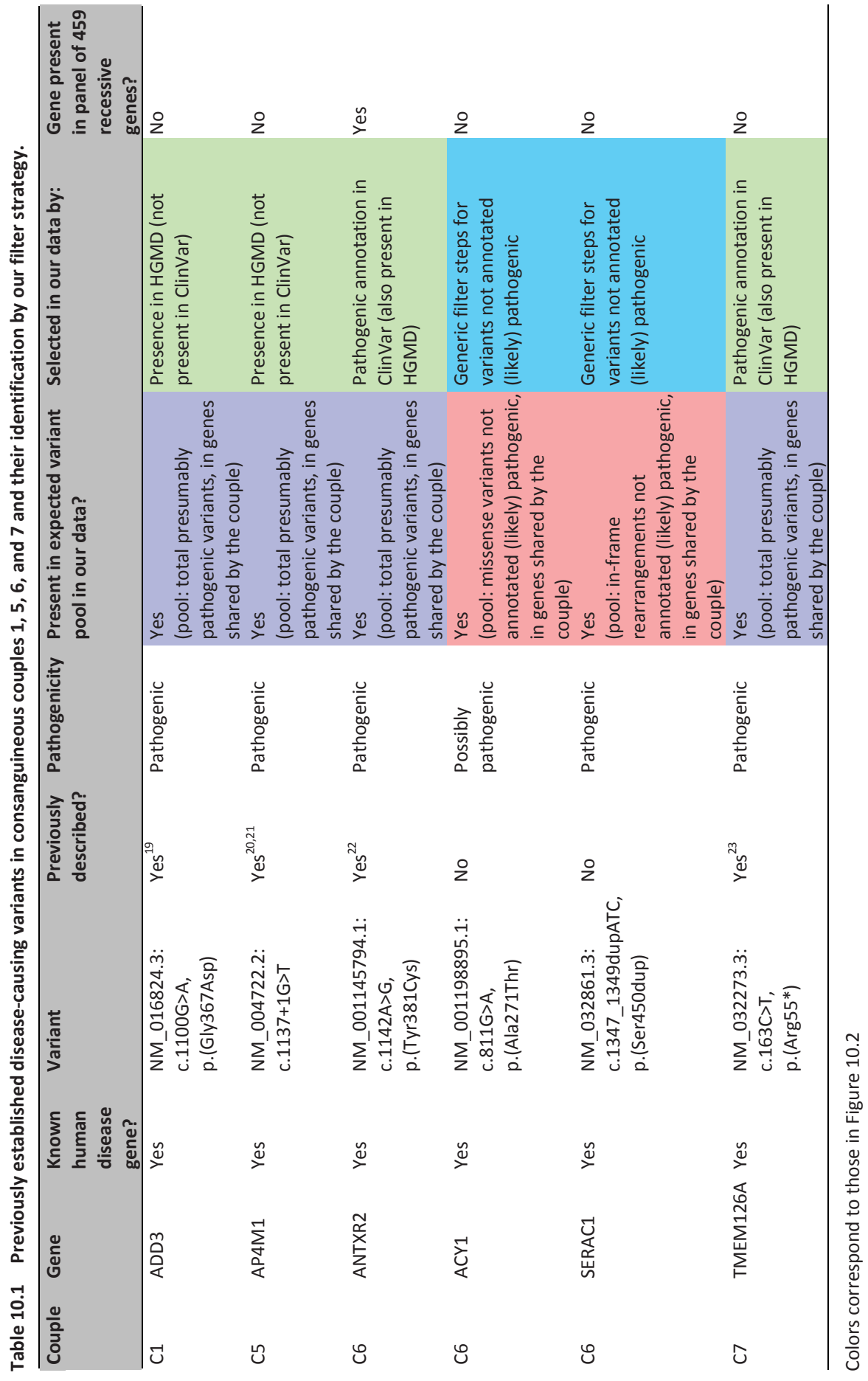




\section{Gene panel}

The gene list described by Bell et al. and Kingsmore, ${ }^{2,7}$ consisting of 508 items, comprising 437 genes supposedly causing 448 severe recessive diseases, was studied. A curated list of 459 genes after the removal of some (particularly non-recessive) genes/diseases and the addition of the genes/diseases for which PGD has been performed for in our center as well as which are included in a PCS pilot in Groningen, the Netherlands, ${ }^{13}$ was used in our analysis.

\section{Results}

A first observation is the larger number of variants per individual in the consanguineous individuals compared to the non-consanguineous. Considering the ethnicity of the consanguineous couples (Table S10.1), this likely reflects population differences: genetic diversity is known to vary between populations, related to human evolutionary origin and migration. ${ }^{14}$

\section{Proposed filter strategy}

Variant filtering in all genes interrogated by WES (Figures 10.1 and 10.2, Tables 10.1 and 10.2, Supplementary Tables 10.2, 10.3, 10.4)

As a first and separate step, variants that are annotated as pathogenic or likely pathogenic in ClinVar and have a $<1 \%$ or unknown dbSNP frequency are selected from the exome data, yielding an average of 8 [5-13] variants per individual $(n=16)$ in the consanguineous couples and $5([0-11], n=50)$ in the non-consanguineous. From the remainder, HGMD-annotated variants with SNP frequency $<1 \% /$ unknown are selected, resulting in 20 [11-33] and 11 [1-21] variants, respectively. The residual data are initially filtered for location, sequencing depth, zygosity, amino acid alteration and SNP frequency. Subsequently, frameshift, nonsense and splice site mutations are selected and added up to the ClinVar- and HGMD-selected variants. This total of presumably pathogenic mutations yielded on average 100 [84-151] variants in the eight consanguineous individuals and 80 [59-98] variants in the 25 non-consanguineous. These were compared between both partners of the couple, thereby selecting the variants in genes where the partner has at least one presumably pathogenic variant as well, and vice versa, resulting in an average of 29 [19-51] variants in the consanguineous couples and 15 [6-30] in the non-consanguineous. X-linked variants were separately selected in the females, yielding 3 [1-5] and 1 [0-3] variants, respectively. All six previously identified disease-causing variants were present in the expected variant pools in our dataset of the consanguineous carriers (Table 10.1). 


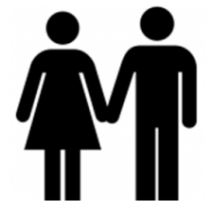

Couple with a child wish

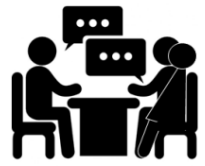

Pre-test counseling

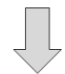

Perform whole exome sequencing in both partners

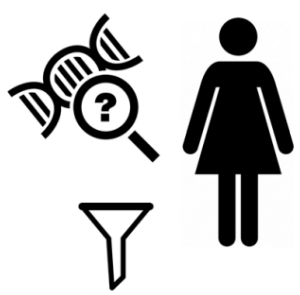

1) Select presumably pathogenic variants from databases:

- Annotation pathogenic or likely pathogenic in ClinVar and

frequency $<1 \%$ or unknown

- Annotation in HGMD and frequency $<1 \%$ or unknown

2) Apply generic filter steps on the remaining variants:

- Exonic and canonical splice (+/-2nt)

Depth/reads $\geq 8$

Homozygous variants removed

Synonymous variants removed

Frequency $<1 \%$ or unknown

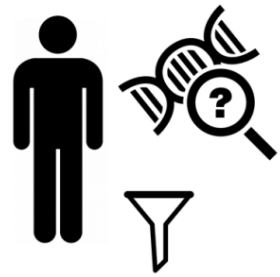

3) Select presumably pathogenic variants based on mutation type:

- Frameshift

- Nonsense

- Splice site

Add up 1 and 3 to get the total number of presumably pathogenic variants

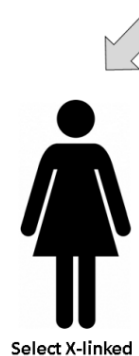

Select X-linked variants in우

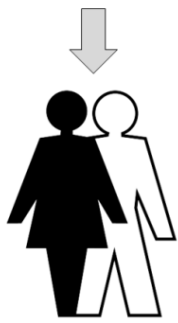

Compare $\sigma^{\prime}$ and 우, select variants in overlapping genes
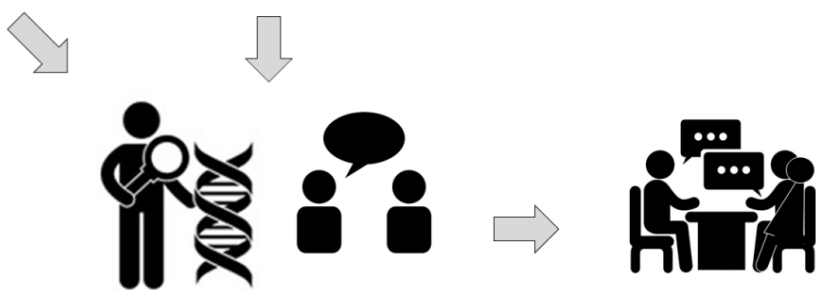

Evaluate variants manually (true variants?

Post-test counseling

true pathogenic variants? known associated disease? and discuss in multidisciplinary PCS team (clinical implications?)

Figure 10.1 Strategy for WES-based PCS. 


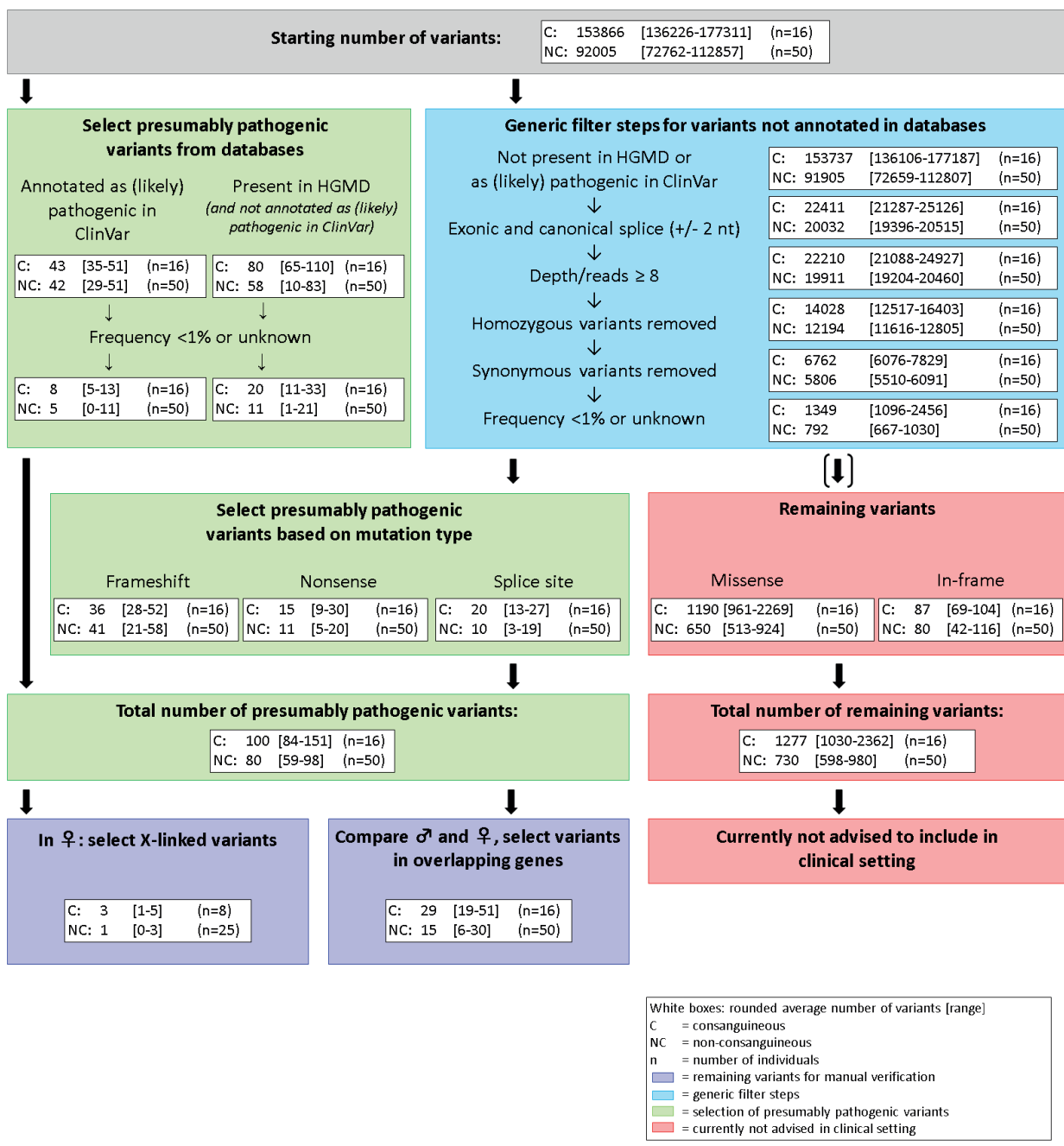

Figure 10.2 Average variant numbers resulting from our filter strategy for WES-based PCS. 


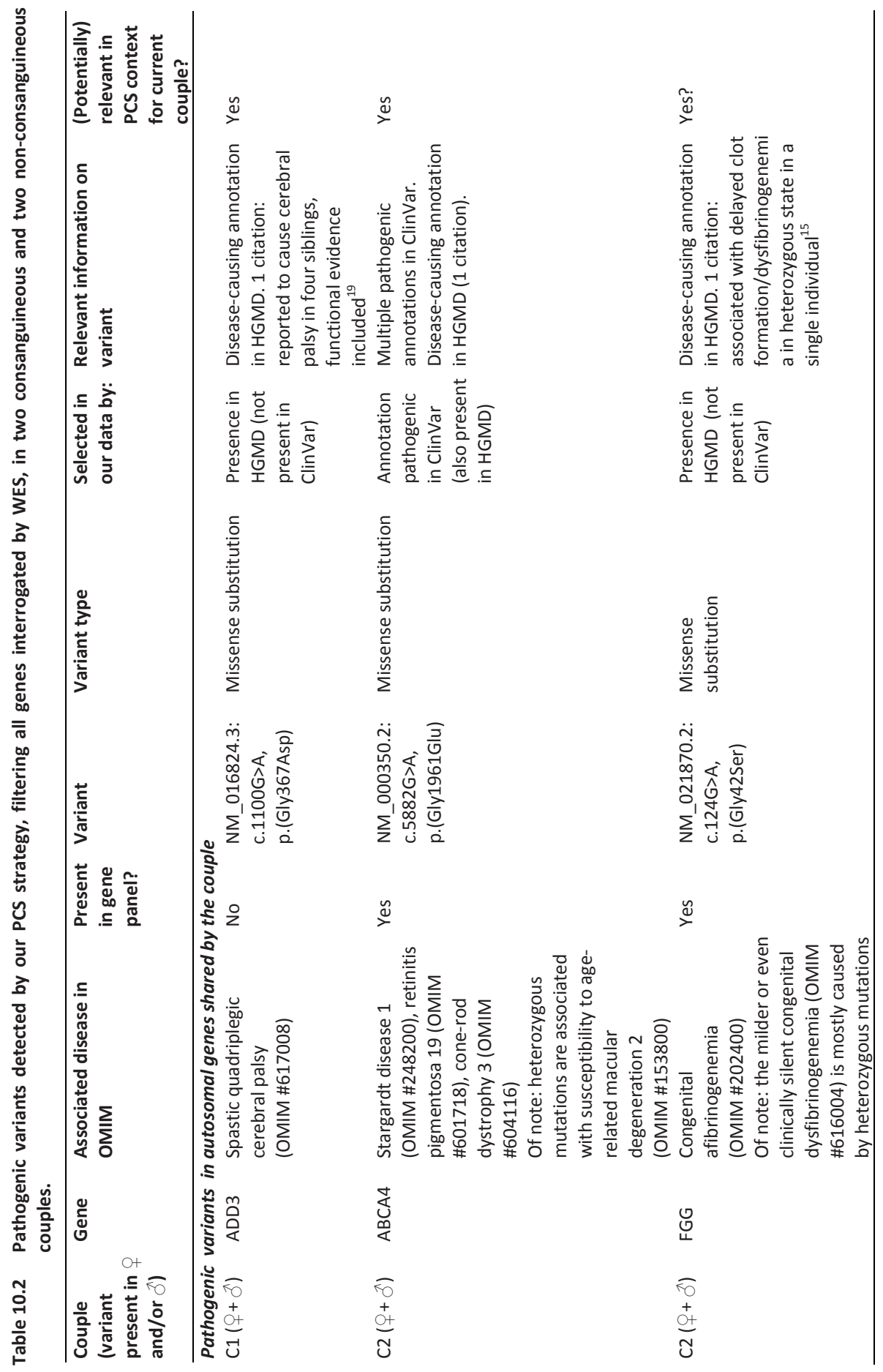




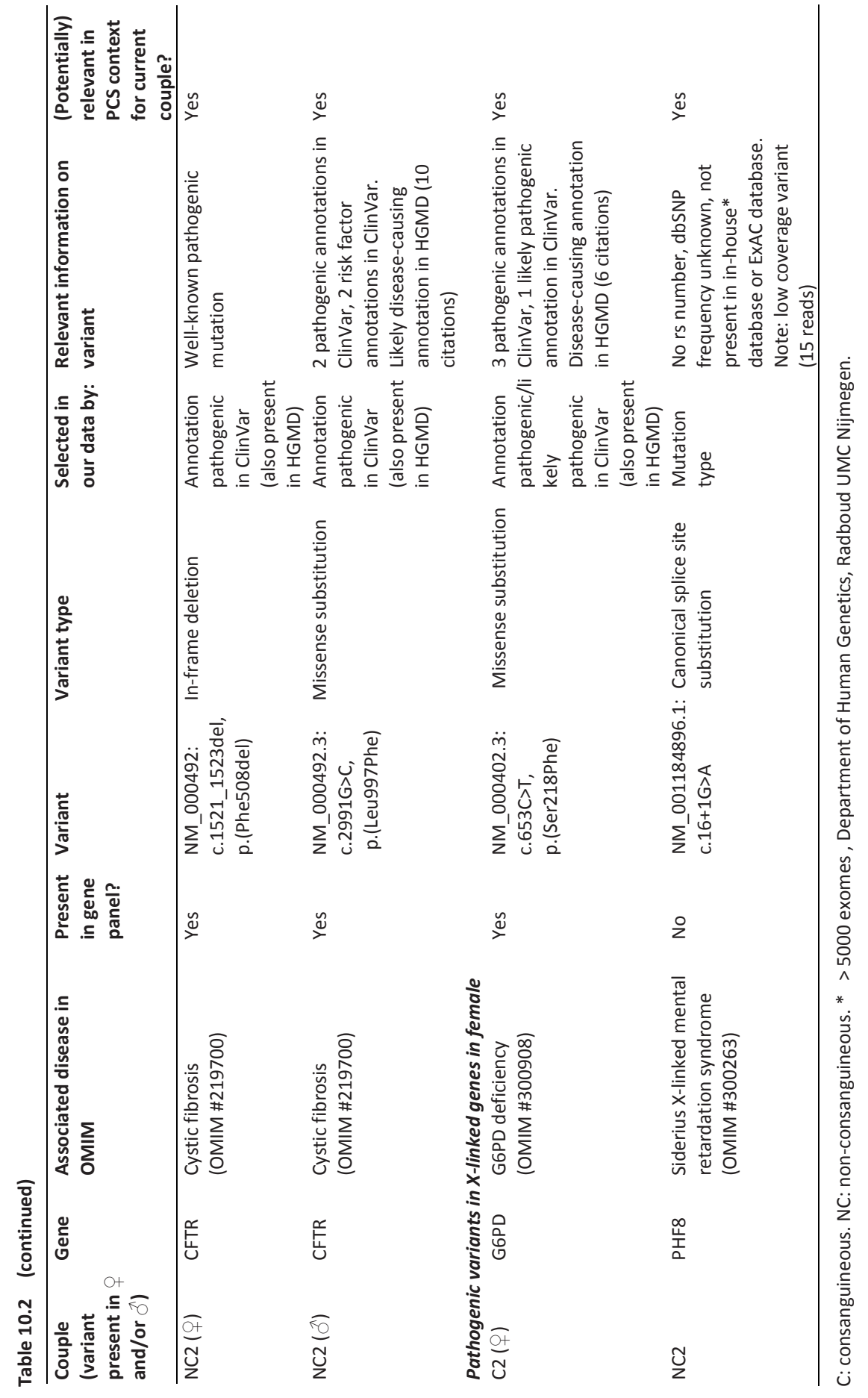


For the first two consanguineous and non-consanguineous couples, the presumably pathogenic variants in shared genes are displayed in Supplementary Table S10.3 as examples. The majority could be discarded based on 1) high in-house and/or ExAC (http://exac.broadinstitute.org/) frequency, in an absolute manner or relative to disease prevalence, and/or 2) on the absence of a monogenic disease association, and/or 3) because they appeared to be a sequencing artifact (Supplementary Table S10.3). The remaining relevant variants are summarized in Table 10.2. If necessary, low coverage variants may be confirmed by Sanger sequencing. C1 was identified as clear carrier couple only for the pathogenic mutation in the $A D D 3$ gene, causative of their child's cerebral palsy. For the ABCA4 gene and the PRPH2 gene, both implicated in ophthalmologic phenotypes, only in one of the partners did a pathogenic mutation remain (Supplementary Table S10.3), therefore the finding is not relevant in a PCS context. C2 was identified as carrier couple of a known pathogenic ABCA4 mutation and of a FGG mutation that has been reported once in association with delayed clot formation/dysfibrinogenemia in heterozygous state. ${ }^{15}$ It is unknown whether homozygosity for this particular mutation would result in a more severe phenotype (congenital afibrinogenemia, OMIM \#616004). Furthermore, in both partners a rare PRRX1 missense substitution was detected (Supplementary Table S10.3). The latter has been reported in one association study of atrial fibrillation, ${ }^{16}$ whereas mutations in this gene are otherwise implicated in agnathia-otocephaly complex (OMIM \#202650), mostly in heterozygous state although one homozygous mutation has been reported. In NC1 no relevant presumably pathogenic variants were found. NC2 was identified as CFTR (cystic fibrosis, OMIM \#219700) carrier couple, the female carrying the classic DF508 mutation and the male the mild/non-classical CFTR mutation L997F (e.g. ${ }^{17,18}$ ).

In X-linked genes a pathogenic G6PD mutation (G6PD deficiency, OMIM \#300908) was identified in consanguineous female 2 and a canonical splice site substitution in the PHF8 gene (siderius $\mathrm{X}$-linked mental retardation syndrome, OMIM\#300263) in nonconsanguineous female 2 (Table 10.2, and Supplementary Table S10.4).

To compare our results to those after adding an extensive gene panel, the same four couples were filtered for 459 autosomal and X-linked recessive genes. This failed to identify the pathogenic $A D D 3$ variant in $\mathrm{C} 1$, the pathogenic $A B C A 4$ variant in $\mathrm{C} 2$ and the PHF8 splice site substitution in NC2. The identification of mutations that pose some uncertainties, for example regarding predicting homozygous phenotype, was not prevented by applying the gene panel (e.g. FGG). Only one (ANTXR2) of the six genes that were disease-causing in the consanguineous families was included in the gene panel. 


\section{Remaining variants (Figure 10.2 and Supplementary Figure 10.1, Supplementary Table} 10.2)

Beyond the annotated variants and presumed pathogenic variants, two variant categories remain. The first consists of missense variants that are not present in HGMD or annotated as at least likely pathogenic in ClinVar, with on average 1190 ([961-2269], $n=16$ ) variants per individual in the consanguineous couples and 650 ([513-924], $n=50$ ) in the non-consanguineous. Secondly, non-annotated in-frame deletions, insertions and complex rearrangements remain: an average of 87 [69-104] in the consanguineous and 80 [42-116] in the non-consanguineous individuals. These were added up to a total of 1277 [1030-2362] and 730 [598-980] remaining variants, respectively. The selection of variants present in the same genes in both partners reduces the numbers to 534 [385$1150]$ and 178 [91-329]. A further reduction could be achieved by applying the gene list: 15 [8-35] and 5 [0-10] variants, respectively.

We used the data from the gene panel to assess the number of carrier couples in the entire cohort (Supplementary Figure S10.1, Supplementary Tables S10.2 and S10.5). By extrapolating this to the expected number of carrier couples, this gives an idea of the proportion of pathogenic variants that is missed by our strategy (see discussion). A total of 5 unique variants in X-linked genes and 21 in the overlapping genes) were found in the 33 couples (Table S10.5). Again, not all variants remain as (likely) pathogenic. In C2 the above-discussed FGG variant was found. Additionally, as anticipated, C6 was identified as carrier couple of the pathogenic ANTXR2 mutation (Table 10.1). In the non-consanguineous couples, no other than the previously mentioned CFTR carrier couple was identified (NC2). In NC3 only one partner carried a pathogenic PKHD1 mutation (AR polycystic kidney disease, OMIM \#263200), whereas the PKHD1 variant in the other partner could be discarded. In the X-linked analysis, only one relevant variant other than the G6PD (C2) variant was identified: a missense substitution in the $A R$ gene (NC21), which has been reported in two boys with severe virilization defects due to partial androgen insensitivity, ${ }^{24,25}$ but also in a boy with normal phenotype. ${ }^{26}$ Whether the mutation has a phenotypic consequence appears to depend on the size of polymorphic repeats. ${ }^{25}$

Altogether, 4 out of the 8 consanguineous couples and 1 out of the 25 fictive nonconsanguineous couples were identified as autosomal recessive carrier couples (Tables 10.1, 10.2, S10.5). X-linked carriership was established in 1 consanguineous and 2 nonconsanguineous females (Tables 10.2, S10.5).

\section{Discussion}

We explored WES as method for PCS and provide a filter strategy to identify rapidly the majority of pathogenic mutations in genes shared by the couple and in the female's 
X-linked genes. A major advantage of our PCS-approach is that WES, being used increasingly as standard cost-effective diagnostic technique with a short turn-aroundtime of a few months, could easily be implemented in the same flow. Whether WESPCS will be cost-effective will require a proper health technology assessment (HTA) study, but it is clear that in genetic testing the prevention of disease occurrence is costwise most rewarding and it is likely, given the high number of unexpected recessive disease cases occurring every year, that this is or will become cost-effective shortly. Furthermore, using WES enables flexible adjustments of the genes included in the PCS test and of the exact filter strategies used. The most important benefit of PCS based on WES from a clinical point of view is the possibility to screen all known disease genes instead of a selected and therefore by definition limited/arbitrary gene panel. Particularly the very rare recessive disease alleles, mainly relevant for consanguineous couples, are not likely to be included in such panels.

One of 25 non-consanguineous couples was identified as a carrier couple. The expected number of non-consanguineous carrier couples can be derived from the cumulative risk of being a carrier couple of any autosomal recessive disease in the population concerned. The latter was calculated/estimated to be $\sim 2 \%$ (based on prevalence numbers of rare diseases provided by Orphanet, ${ }^{27}$ whereby only diseases which can technically be identified by WES were considered and prevalences applicable to our Northern-European population were applied), including disorders ranging from severe to mild. Although our numbers are small, finding 1 non-consanguineous carrier couple out of 25 may be grossly in line with this. The one identified couple being CFTR carrier is also in agreement with the relatively common CFTR carriership in Northern-Europe. ${ }^{28}$ For females a cumulative $X$-linked carrier risk of $\sim 1 \%$ was estimated, ${ }^{27}$ again exclusively based on diseases that can be picked up by WES, and including conditions with limited clinical relevance, whereby a de novo rate of $1 / 3$ for lethal/non-reproducing $X$-linked disorders was taken into account. ${ }^{29}$ Our findings of 3 (presumed) carriers out of 33 exceed this estimation, indicating, although in a limited dataset, that probably not many variants are missed. For X-linked carriership no differences are expected between consanguineous and non-consanguineous couples, which is indeed illustrated by our data.

\section{Selection of pathogenic variants}

We selected presumably pathogenic variants based on published data (databases) and on predictions by the nature of the variant. Although databases are known to be imperfect, we initially hypothesized that by selecting variants associated with human disease, annotated in the Human Genome Mutation Database (HGMD, http://www.hgmd.cf.ac.uk/ac/index.php), ${ }^{30}$ additional filter steps such as frequency would not be necessary. However, the remaining variant numbers were much higher than expected and included high-frequency benign variants or variants associated with 
multifactorial context. The same was true for variants that are annotated as pathogenic or likely pathogenic in ClinVar. Therefore, we added frequency as additional step, largely reducing the numbers, without removing presumably pathogenic variants. In addition, we selected non-annotated (likely) pathogenic variants in our strategy. Synonymous variants as well as homozygous variants in healthy adults were discarded from the variant pool. Further criteria in determining a variant's pathogenicity are location and frequency. An allele frequency cut-off of $<1 \%$ (corresponding with a disease prevalence of $<1 / 10,000$ for autosomal recessive diseases) was considered justifiable based on the afore-mentioned Orphanet disease frequency data. ${ }^{27}$ Most diseases with prevalences above this cut-off either have limited clinical relevance (e.g. congenital isolated thyroxine-binding globulin deficiency), are caused by mutation types that are not detected by sequencing analysis and therefore require a separate test anyway (e.g. SMA, Fragile $X$ syndrome), or are higher-frequent only in specific populations (e.g. thalassemia). The latter is generally well-known for such populations, thereby enabling the addition of population-specific tests. Moreover, an allele frequency of $<1 \%$ in the filter algorithm represents the frequency of a single disease allele, while for most diseases multiple disease alleles exist. Exceptions may be disease alleles which are responsible for the vast majority of disease cases (e.g. p.Phe508del mutation in cystic fibrosis or p.Lys304Glu mutation in MCAD deficiency) or, again population-specific, certain founder mutations. These can be assessed in or added to the data separately.

A frequency cut-off of $<5 \%$ was also considered but resulted in a $50 \%$ increase in remaining variants (data not shown), which is expected to be particularly attributable to non-pathogenic variants. This will significantly increase interpretation load at the end and in our opinion does not outweigh the effort of separate mutation analysis/testing that may be necessary in specific populations. After filtering for generic filter steps, we selected frameshift mutations, nonsense mutations and canonical splice site mutations, which are considered often pathogenic due to predicted effects on the protein. Obviously, as for the annotated variants, all resulting variants will have to be evaluated since for various reasons (annotation errors, database errors) not every one of these is actually pathogenic. Also, if coverage is borderline, validation by Sanger sequencing is advisable. The number of variants resulting from our filter strategy for PCS was small enough to enable such follow-up in a diagnostic setting. With our strategy, only between 0 and $\sim 20$ variants needed manual verification, varying from fast checks (e.g. HGMD/ClinVar, OMIM, Orphanet prevalence list) in most to more extensive evaluation in a few. Of note, in-house variant frequency databases as well as the ExAC database appeared very useful in relatively quickly discarding a first set of variants, even though only variants with low or unknown dbSNP frequency were selected in our algorithm. Benign variants or variants of uncertain significance (VUS) may be present in the final results, the latter posing the greatest clinical challenge. 
However, the majority of variants, even when analyzing the entire exome, could be clearly assigned either benign or pathogenic. A complicating factor in PCS compared to genetic testing in affected individuals is the lack of a phenotype to relate the genetic findings to. This is not a WES-specific issue; it also applies to, for example, targeted sequencing of specific genes. The only way to circumvent this issue is to exclusively test for known pathogenic mutations. However, collecting these mutations is very timeconsuming and, more importantly, once designed a certain mutation-based array, mutations are not easily added. Given the daily basis on which new pathogenic mutations are identified, such method would be outdated in no time.

\section{Pathogenic mutations that may be missed by WES-based PCS}

Pathogenic mutations may be missed either 1 ) because they are not recognized as being pathogenic in the filtering process or 2) because they are not detected by the sequencing technique. 1) The first category mainly consists of amino acid substitutions (missense variants) and, to a lesser extent, in-frame rearrangements not yet known or described to be pathogenic. These variants are often difficult to interpret regarding pathogenicity. As illustrated by our data, their amount in genes shared by the couple can be reduced to manageable numbers by applying gene panels. However, reducing the variant numbers will not solve the interpretation issues. Therefore, we feel this category is not recommended in a diagnostic setting at this point. It is necessary to obtain more experience (in a research setting) with the nature of the resulting variants to further evaluate its clinical practice. More importantly, increasing knowledge of pathogenic mutations, and collection of these mutations in an aforementioned database, will eventually decrease this variant category. Also, newer filtering pipelines being able to identify other presumably pathogenic subcategories (e.g. stop-loss, startloss and/or glycosylation site missense variants) will move variants from the unknown to the presumably pathogenic variant pool. The same will in time be true for intronic variants, microRNA variants, etc. Conservation being an important factor in pathogenicity, one may argue to include this in the filter strategy. However, conservation scores, predicting (potential) variant pathogenicity either isolated (e.g. GERP, ${ }^{31}$ PhyloP $^{32}$ ) or incorporated in more comprehensive software tools (e.g. SIFT, ${ }^{33}$ PolyPhen, ${ }^{34} \mathrm{CADD}^{35}$ ) are merely predictions and may give conflicting results, if at all accurate to distinguish pathogenic from non-pathogenic rare alleles. ${ }^{36}$ This was also illustrated by a PolyPhen-based spot check in our own data whereby 134 homozygous variants in a healthy individual were assigned pathogenic (data not shown). Taken together, conservation and pathogenicity predictions are more suited as part of manual variant verification than as standard filter step. Notably, the issues of discarding unknown pathogenic mutations and encountering interpretation problems are not WES-specific but applicable to other sequencing methods as well. Another reason why disease-causing variants may be discarded in the filter process is because allele frequencies exceed the filter cut-off. If relevant for the population concerned, a 
complementary test and/or panel can be offered. 2) Secondly, there is the category of variants that are not detected by WES. One of the potential causes is insufficient callable coverage. Although WES coverage has improved considerably over the last years ( 95\% capturing of the exome $\left.{ }^{37}\right)$, coverage is not complete. Whole genome sequencing (WGS) techniques will in time replace WES in clinical genetic practice, thereby further improving completeness of the data. ${ }^{37}$ To ensure that the most relevant mutations in the population concerned are covered sufficiently, the presence (and expected frequencies) of these mutations should be evaluated in WES-data available to the laboratory. If needed, a specific complementary mutation panel could be offered. Another, non-WES-specific cause of missing mutations by sequencing analysis is due to technical characteristics of the test. The ability to detect exon deletions and repeat expansions vary between sequencing techniques. If desired to include these in PCS, additional test methods may be necessary. Some relevant examples have been indicated previously.

\section{Gene panels}

Our data show that the number of presumably pathogenic variants resulting from WESbased PCS is small enough not to need a gene panel. In fact, by using an extensive gene panel, three of the four known pathogenic mutations in the consanguineous couples were discarded, whereas all four were identified in our algorithm without gene panel. Also our other data illustrate that several relevant disease-associated genes are not included in the panel (Tables S10.3, S10.4). The occasional encounter of a variant with interpretation uncertainties was not prevented by applying gene panels. Therefore, taken together, our results argue against the use of gene panels, especially when aiming a generic PCS test applicable to a broad population with varying ethnicities. The main rationale behind gene panels is to pre-filter out certain variants or genes that are deemed undesirable to report for preconception carrier status. Although we do not consider this necessary, if preferred a gene panel could be easily incorporated in our pipeline, before manual evaluation. Also, creating a gene panel which is, given certain agreed criteria, complete, is hardly possible. When omitting gene panels, thereby not restricting the analysis to autosomal recessive genes, one might encounter autosomal dominant mutations with potential clinical consequences for the tested individual. In that case PCS transforms into presymptomatic testing, which is not deemed desirable in this context. However, when combining the data of both partners the risk is very small to identify dominant disease genes. Furthermore, if offspring is at risk of inheriting two dominant disease alleles this is of particular relevance in a PCS context. Another effect of leaving gene panels out is that carrier couples of for example relatively mild and/or treatable disease may be identified. We feel this does not differ significantly from issues encountered in current clinical genetics practice. Adequate counseling is the key to come to a responsible consideration of consequences. Moreover, the manual evaluation of remaining variants does not only include pathogenicity, but also their 
clinical implications. With such a 'post hoc approach', the chances of missing highly relevant pathogenic mutations or genes is smallest. Defining the exact criteria of the findings to be reported is a difficult/arbitrary discussion upfront and can be more easily done on the small number of remaining variants, in case-by-case decisions (Tables S10.3-S10.5) from clinicians and laboratory geneticists. This stresses the importance of carrying out WES-PCS in a specialized, academic setting.

In conclusion, our results show that WES is eligible for PCS, both for consanguineous and non-consanguineous couples, with the remaining number of variants being manageable in a clinical setting. Although pathogenic mutations may be missed either due to technical limitations or current lack of knowledge on new mutations, respectively, only the first is WES-specific and, with current WES-performance, is not a major issue. Specific mutations or genes may be tested complementarily. We think that WES is overall able to identify a higher proportion of relevant carrier couples compared to other PCS tests. The omission of gene panels convincingly added value in our experience. Adequate counseling of couples opting for WES-based PCS is critical. PCS may clearly identify a carrier couple or may pose some additional questions, for example concerning disease severity, the difficulty to predict the phenotype and the issue of possibly manifesting carriership of X-linked disease, of which discussion in a multidisciplinary PCS team is crucial. Each couple should also be informed that the test is not $100 \%$ sensitive and therefore residual carrier risks remain. It is important to develop and continuously curate databases with pathogenic mutations in order to reduce manual work in variant analysis and increase sensitivity of PCS.

\section{Acknowledgements}

We would like to acknowledge the contribution of the Department of Human Genetics, Radboud UMC, Nijmegen, The Netherlands (involved colleagues: T. Rinne, H. Scheffer and C. Gillissen, among others), for providing the non-consanguineous exome data. Furthermore, we would like to thank I.F.M. de Coo, Department of Neurology, Erasmus MC-Sophia Children's Hospital Rotterdam, Rotterdam, The Netherlands, for enrolling the consanguineous families in the Rotterdam-Maastricht collaboration for diagnosing mitochondrial disorders, and D.M.E.I. Hellebrekers, Department of Clinical Genetics, Maastricht UMC+ for her diagnostic work in establishing the disease-causing variants in the affected children of the consanguineous couples.

\section{Funding}

This work was supported by Stichting Metakids and Prinses Beatrix Spierfonds. 


\section{References}

1. Henneman L, Borry P, Chokoshvili D, et al. Responsible implementation of expanded carrier screening. Eur J Hum Genet. 2016;24(6):e1-e12.

2. Bell CJ, Dinwiddie DL, Miller NA, et al. Carrier testing for severe childhood recessive diseases by nextgeneration sequencing. Sci Trans/ Med. 2011;3(65):65ra64.

3. De Wert GM, Dondorp WJ, Knoppers BM. Preconception care and genetic risk: ethical issues. J Community Genet. 2012;3(3):221-228.

4. Becker R, Keller T, Wegner RD, et al. Consanguinity and pregnancy outcomes in a multi-ethnic, metropolitan European population. Prenat Diagn. 2015;35(1):81-89.

5. Mathijssen IB, Henneman L, van Eeten-Nijman JM, et al. Targeted carrier screening for four recessive disorders: high detection rate within a founder population. Eur J Med Genet. 2015;58(3):123-128.

6. Bajaj K. GSJ. Carrier screening: past, present, and future. J Clin Med. 2014;3(3):1033-1042.

7. Kingsmore S. Comprehensive carrier screening and molecular diagnostic testing for recessive childhood diseases. PLoS Curr. 2012:e4f9877ab9878ffa9879.

8. Martin J, Asan, Yi Y, et al. Comprehensive carrier genetic test using next-generation deoxyribonucleic acid sequencing in infertile couples wishing to conceive through assisted reproductive technology. Fertil Steril. 2015;104(5):1286-1293.

9. Abuli A, Boada M, Rodriguez-Santiago B, et al. NGS-Based Assay for the Identification of Individuals Carrying Recessive Genetic Mutations in Reproductive Medicine. Hum Mutat. 2016;37(6):516-523.

10. Teeuw M, Waisfisz $Q$, Zwijnenburg PJ, et al. First steps in exploring prospective exome sequencing of consanguineous couples. Eur J Med Genet. 2014;57(11-12):613-616.

11. Nguyen M, Boesten I, Hellebrekers DM, et al. Pathogenic CWF19L1 variants as a novel cause of autosomal recessive cerebellar ataxia and atrophy. Eur J Hum Genet. 2016;24(4):619-622.

12. Gilissen C, Hehir-Kwa JY, Thung DT, et al. Genome sequencing identifies major causes of severe intellectual disability. Nature. 2014;511(7509):344-347.

13. Plantinga $M$, Birnie $E$, Abbott $K M$, et al. Population-based preconception carrier screening: how potential users from the general population view a test for 50 serious diseases. Eur J Hum Genet. 2016.

14. Campbell MC, Tishkoff SA. African genetic diversity: implications for human demographic history, modern human origins, and complex disease mapping. Annu Rev Genomics Hum Genet. 2008;9:403-433.

15. Pietrys D, Balwierz W, Iwaniec T, Vorjohann S, Neerman-Arbez M, Undas A. Two different fibrinogen gene mutations associated with bleeding in the same family (A alphaGly13Glu and gammaGly16Ser) and their impact on fibrin clot properties: fibrinogen Krakow II and Krakow III. Thromb Haemost. 2011;106(3):558-560.

16. Lin $\mathrm{H}$, Sinner MF, Brody JA, et al. Targeted sequencing in candidate genes for atrial fibrillation: the Cohorts for Heart and Aging Research in Genomic Epidemiology (CHARGE) Targeted Sequencing Study. Heart Rhythm. 2014;11(3):452-457.

17. Strom CM, Redman JB, Peng M. The dangers of including nonclassical cystic fibrosis variants in population-based screening panels: p.L997F, further genotype/phenotype correlation data. Genet Med. 2011;13(12):1042-1044.

18. Schippa S, lebba V, Santangelo F, et al. Cystic fibrosis transmembrane conductance regulator (CFTR) allelic variants relate to shifts in faecal microbiota of cystic fibrosis patients. PloS One. 2013;8(4): e61176.

19. Kruer MC, Jepperson T, Dutta S, et al. Mutations in gamma adducin are associated with inherited cerebral palsy. Ann Neurol. 2013;74(6):805-814

20. Verkerk AJ, Schot R, Dumee B, et al. Mutation in the AP4M1 gene provides a model for neuroaxonal injury in cerebral palsy. Am J Hum Genet. 2009;85(1):40-52.

21. Langouet M, Siquier-Pernet K, Sanquer S, et al. Contiguous mutation syndrome in the era of highthroughput sequencing. Mol Genet Genomic Med. 2015;3(3):215-220.

22. Hanks S, Adams S, Douglas J, et al. Mutations in the gene encoding capillary morphogenesis protein 2 cause juvenile hyaline fibromatosis and infantile systemic hyalinosis. Am J Hum Genet. 2003;73(4): 791-800. 
23. Hanein S, Perrault I, Roche O, et al. TMEM126A, encoding a mitochondrial protein, is mutated in autosomal-recessive nonsyndromic optic atrophy. Am J Hum Genet. 2009;84(4):493-498.

24. Hiort O, Sinnecker GH, Holterhus PM, Nitsche EM, Kruse K. The clinical and molecular spectrum of androgen insensitivity syndromes. Am J Med Genet. 1996;63(1):218-222.

25. Werner R, Holterhus PM, Binder $G$, et al. The A645D mutation in the hinge region of the human androgen receptor (AR) gene modulates AR activity, depending on the context of the polymorphic glutamine and glycine repeats. J Clin Endocrinol Metab. 2006;91(9):3515-3520.

26. Nordenskjold A, Soderhall S. An androgen receptor gene mutation (A645D) in a boy with a normal phenotype. Hum Mutat. 1998;11(4):339.

27. Orphanet. Orphanet Report Series - Prevalence of rare diseases: Bibliographic data. http://www.orpha.net/orphacom/cahiers/docs/GB/Prevalence_of_rare_diseases_by_alphabetical_list. pdf and http://www.orpha.net/orphacom/cahiers/docs/GB/Prevalence_of_rare_diseases_by_decreasing_preva lence_or_cases.pdf. 2016.

28. Moskowitz SM, Chmiel JF, Sternen DL, Cheng E, Cutting GR. CFTR-Related Disorders. In: Pagon RA, Adam $\mathrm{MP}$, Ardinger HH, et al., eds. GeneReviews(R). Seattle (WA): University of Washington, Seattle. All rights reserved.; 1993.

29. Nussbaum R.L. MRR, Willard H.F. Thompson \& Thompson Genetics in Medicine. 8th ed: Elsevier/Saunders; 2015.

30. Stenson PD, Mort M, Ball EV, Shaw K, Phillips A, Cooper DN. The Human Gene Mutation Database: building a comprehensive mutation repository for clinical and molecular genetics, diagnostic testing and personalized genomic medicine. Hum Genet. 2014;133(1):1-9.

31. Cooper GM, Stone EA, Asimenos G, Green ED, Batzoglou S, Sidow A. Distribution and intensity of constraint in mammalian genomic sequence. Genome Res. 2005;15(7):901-913.

32. Pollard KS, Hubisz MJ, Rosenbloom KR, Siepel A. Detection of nonneutral substitution rates on mammalian phylogenies. Genome Res. 2010;20(1):110-121

33. Ng PC, Henikoff S. SIFT: Predicting amino acid changes that affect protein function. Nucleic Acids Res. 2003;31(13):3812-3814.

34. Adzhubei IA, Schmidt S, Peshkin L, et al. A method and server for predicting damaging missense mutations. Nat Methods. 2010;7(4):248-249.

35. Kircher M, Witten DM, Jain P, O'Roak BJ, Cooper GM, Shendure J. A general framework for estimating the relative pathogenicity of human genetic variants. Nat Genetics. 2014;46(3):310-315.

36. Li MX, Kwan JS, Bao SY, et al. Predicting mendelian disease-causing non-synonymous single nucleotide variants in exome sequencing studies. PLoS Genetics. 2013;9(1):e1003143.

37. Lelieveld SH, Spielmann M, Mundlos S, Veltman JA, Gilissen C. Comparison of Exome and Genome Sequencing Technologies for the Complete Capture of Protein-Coding Regions. Hum Mutat. 2015;36(8):815-822. 


\section{Supplementary material}

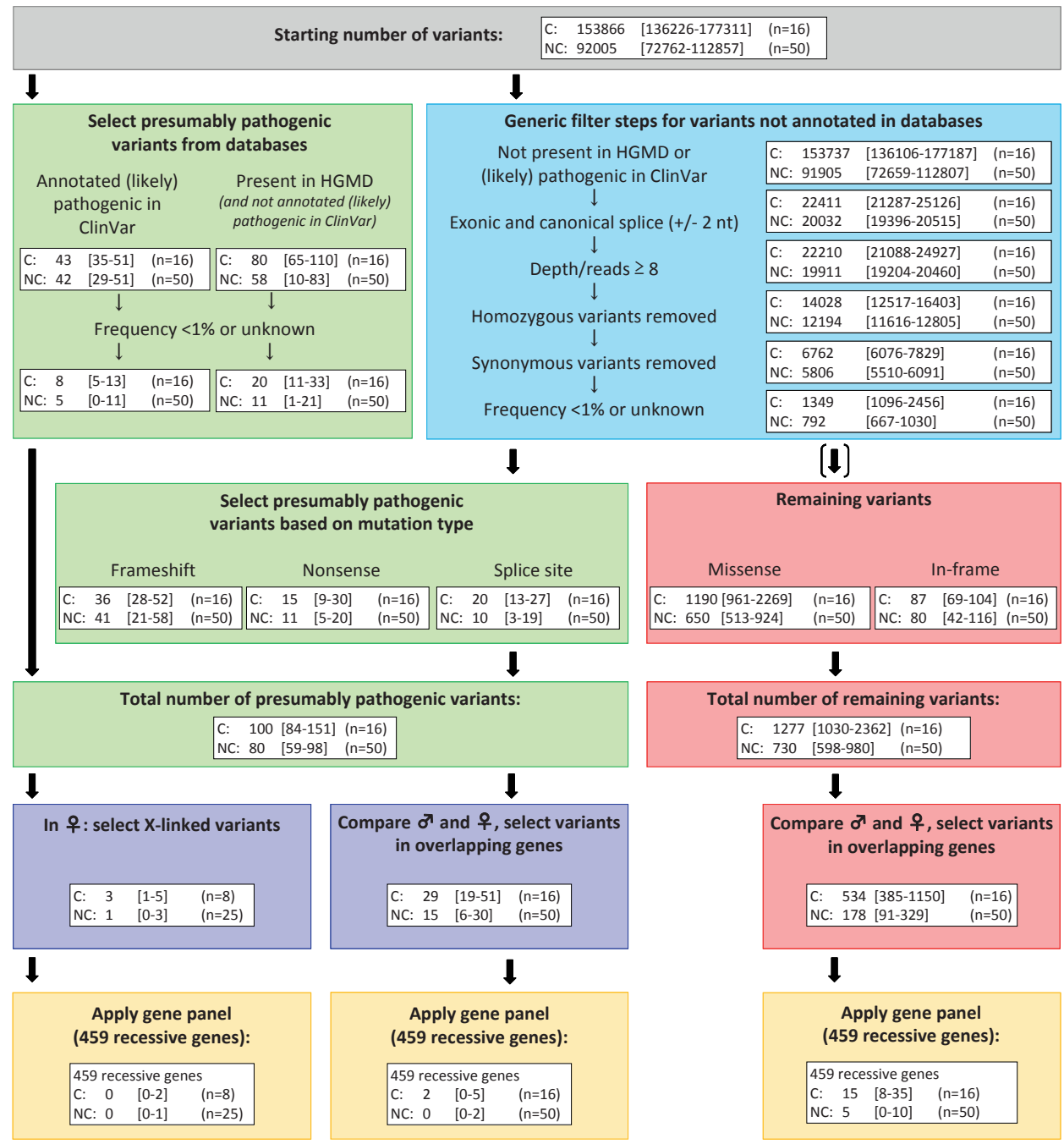

\begin{tabular}{|c|}
\hline $\begin{aligned} & =\text { consanguineous } \\
& =\text { non-consanguineous } \\
& =\text { number of individuals } \\
& =\text { remaining variants for manual verification from our proposed strategy } \\
& =\text { remaining variants for manual verification after adding the gene panel } \\
& =\text { generic filter steps } \\
& =\text { selection of presumably pathogenic variants }\end{aligned}$ \\
\hline
\end{tabular}

Figure S10.1 Filter strategy for WES-based PCS with the addition of a gene panel. 
Table S10.1 Ethnicity and degree of consanguinity of the 8 consanguineous couples.

\begin{tabular}{lcccc}
\hline Couple & Country of origin & Degree of consanguinity & $\begin{array}{c}\text { Remark } \\
\text { Bdentified in affected } \\
\text { child? }\end{array}$ \\
\hline 1 & Morocco & First cousins & $\begin{array}{c}\text { Belong to the same } \\
\text { family as couple } 5\end{array}$ & Yes \\
2 & Iraq & Second cousins & No \\
3 & Morocco & First cousins & No \\
4 & Turkey & First cousins & No \\
5 & Morocco & Second cousins & Belong to the same & Yes \\
6 & Morocco & family as couple 1 & Yes \\
7 & Morocco & First cousins & & Yes \\
8 & Turkey & Second cousins & & No \\
\hline
\end{tabular}


Table S10.2 Variant numbers resulting from our PCS filter strategy (corresponding to Figure 10.2) and from the addition of a gene panel (corresponding to Figure S10.1)

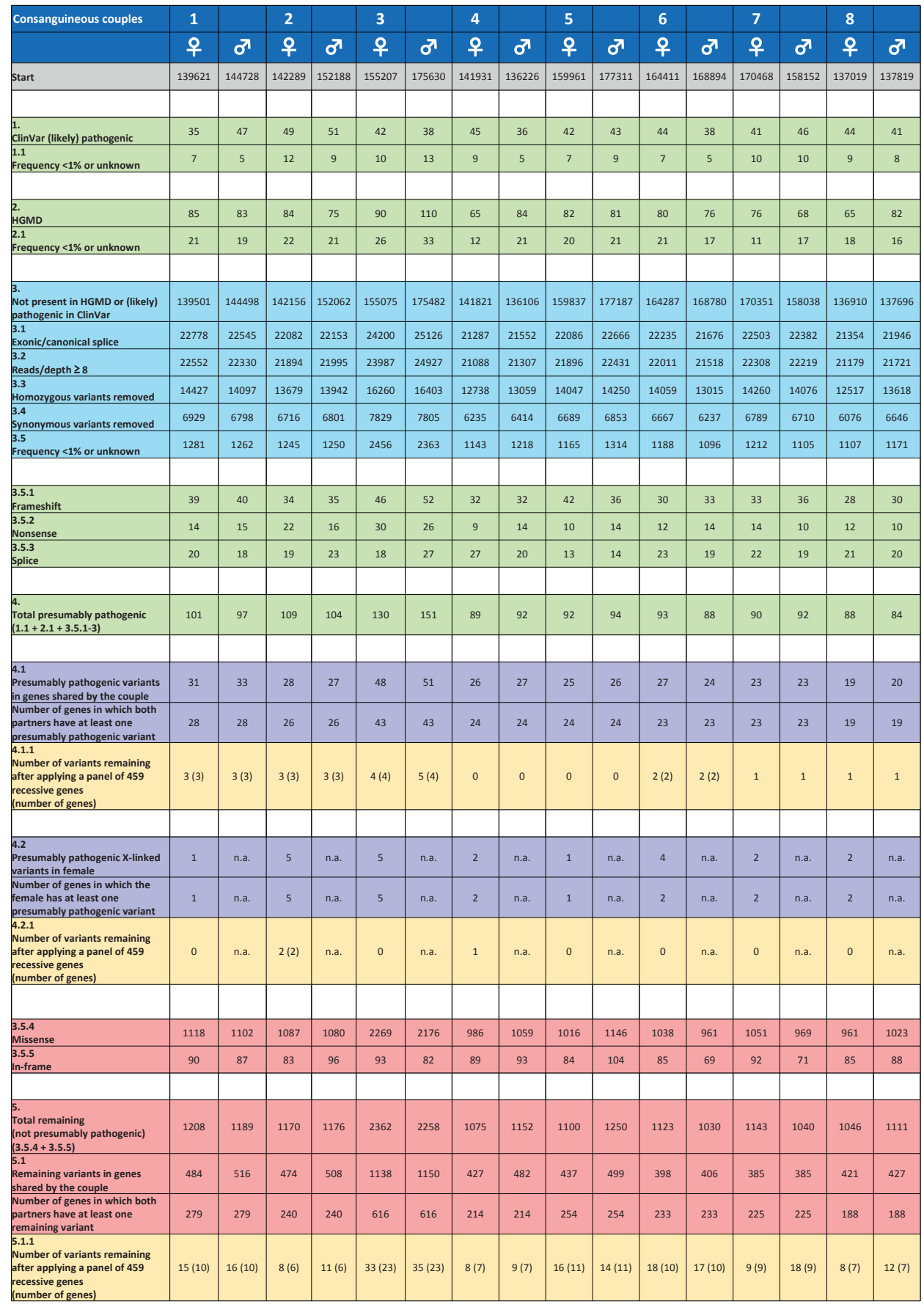


Table S10.2 (continued)

\begin{tabular}{|c|c|c|c|c|c|c|c|c|c|c|}
\hline Non-consanguineous couples & 1 & & 2 & & 3 & & 4 & & 5 & \\
\hline & 우 & $\sigma^{7}$ & 9 & $\bar{\sigma}$ & 9 & $\bar{\sigma}$ & 9 & 7 & 우 & ग \\
\hline Start & 97840 & 103560 & 87332 & 92181 & 73552 & 103978 & 77089 & 72762 & 97846 & 95298 \\
\hline $\begin{array}{l}1 . \\
\text { ClinVar (likely) pathogenic }\end{array}$ & 40 & 41 & 51 & 36 & 38 & 42 & 41 & 39 & 44 & 35 \\
\hline \begin{tabular}{|l|l|}
1.1 \\
Frequency $<1 \%$ or unknown
\end{tabular} & 4 & 5 & 11 & 6 & 7 & 2 & 8 & 5 & 5 & 3 \\
\hline \begin{tabular}{|l|}
2. \\
HGMD \\
\end{tabular} & 78 & 21 & 76 & 63 & 73 & 73 & 58 & 64 & 67 & 59 \\
\hline \begin{tabular}{|l|}
.1 \\
Frequency $<1 \%$ or unknown \\
\end{tabular} & 4 & 5 & 16 & 16 & 7 & 2 & 8 & 5 & 5 & 3 \\
\hline $\begin{array}{l}\text { 3. } \\
\text { Not present in HGMD or (likely) } \\
\text { pathogenic in ClinVar }\end{array}$ & 97722 & 103498 & 87205 & 92082 & 73441 & 103863 & 76990 & 72659 & 97735 & 95204 \\
\hline $\begin{array}{l}3.1 \\
\text { Exonic/canonical splice }\end{array}$ & 20479 & 19756 & 20107 & 20077 & 19527 & 20274 & 19877 & 19396 & 20187 & 19823 \\
\hline $\begin{array}{l}3.2 \\
\text { Reads/depth } \geq 8\end{array}$ & 20396 & 19692 & 19985 & 19971 & 19315 & 20203 & 19706 & 19204 & 20098 & 19710 \\
\hline $\begin{array}{l}3.3 \\
\text { Homozygous variants removed }\end{array}$ & 12805 & 12041 & 12318 & 12078 & 11904 & 12185 & 12389 & 11687 & 12473 & 11956 \\
\hline $\begin{array}{l}3.4 \\
\text { Synonymous variants removed }\end{array}$ & 6086 & 5800 & 5933 & 5828 & 5649 & 5774 & 5956 & 5588 & 5948 & 5649 \\
\hline $\begin{array}{l}3.5 \\
\text { Frequency }<1 \% \text { or unknown } \\
\end{array}$ & 773 & 722 & 751 & 738 & 865 & 732 & 860 & 857 & 751 & 669 \\
\hline \begin{tabular}{|l|}
3.5 .1 \\
Frameshift \\
\end{tabular} & 47 & 39 & 46 & 51 & 38 & 57 & 24 & 21 & 34 & 47 \\
\hline \begin{tabular}{|l|}
3.5 .2 \\
Nonsense \\
\end{tabular} & 12 & 10 & 11 & 10 & 9 & 9 & 12 & 15 & 9 & 13 \\
\hline \begin{tabular}{|l|}
3.5 .3 \\
Splice \\
\end{tabular} & 13 & 17 & 12 & 13 & 10 & 10 & 6 & 7 & 9 & 11 \\
\hline \begin{tabular}{|l}
4. \\
Total presumably pathogenic \\
$(1.1+2.1+3.5 .1-3)$ \\
\end{tabular} & 96 & 79 & 96 & 96 & 82 & 90 & 64 & 65 & 66 & 88 \\
\hline $\begin{array}{l}4.1 \\
\text { Presumably pathogenic variants } \\
\text { in genes shared by the couple }\end{array}$ & 16 & 15 & 25 & 22 & 11 & 11 & 17 & 18 & 9 & 9 \\
\hline $\begin{array}{l}\text { Number of genes in which both } \\
\text { partners have at least one } \\
\text { presumably pathogenic variant }\end{array}$ & 15 & 15 & 21 & 21 & 10 & 10 & 15 & 15 & 9 & 9 \\
\hline $\begin{array}{l}4.1 .1 \\
\text { Number of variants remaining } \\
\text { after applying a panel of } 459 \\
\text { recessive genes } \\
\text { (number of genes) } \\
\end{array}$ & 0 & 0 & $2(2)$ & $2(2)$ & $2(2)$ & $2(2)$ & 0 & 0 & 1 & 1 \\
\hline \begin{tabular}{|l}
.2 \\
Presumably pathogenic X-linked \\
variants in female
\end{tabular} & 1 & n.a. & 3 & n.a. & 2 & n.a. & 0 & n.a. & 0 & n.a. \\
\hline $\begin{array}{l}\text { Number of genes in which the } \\
\text { female has at least one } \\
\text { presumably pathogenic variant }\end{array}$ & 1 & n.a. & 3 & n.a. & 2 & n.a. & 0 & n.a. & 0 & n.a. \\
\hline $\begin{array}{l}4.2 .1 \\
\text { Number of variants remaining } \\
\text { after applying a panel of } 459 \\
\text { recessive genes }\end{array}$ & 0 & n.a. & 0 & n.a. & 1 & n.a. & - & n.a. & - & n.a. \\
\hline \begin{tabular}{|l|}
3.5 .4 \\
Missense \\
\end{tabular} & 605 & 567 & 593 & 562 & 740 & 547 & 762 & 757 & 606 & 513 \\
\hline \begin{tabular}{|l}
3.5 .5 \\
In-frame \\
\end{tabular} & 96 & 89 & 89 & 102 & 68 & 109 & 56 & 57 & 93 & 85 \\
\hline \begin{tabular}{|l}
5 \\
Total remaining \\
(not presumably pathogenic) \\
$(3.5 .4+3.5 .5)$
\end{tabular} & 701 & 656 & 682 & 664 & 808 & 656 & 818 & 814 & 699 & 598 \\
\hline 5.1 & & & & & & & & & & \\
\hline $\begin{array}{l}\text { Remaining variants in genes } \\
\text { shared by the couple }\end{array}$ & 149 & 143 & 155 & 153 & 235 & 132 & 285 & 280 & 160 & 155 \\
\hline $\begin{array}{l}\text { Number of genes in which both } \\
\text { partners have at least one } \\
\text { remaining variant }\end{array}$ & 110 & 110 & 112 & 112 & 92 & 92 & 129 & 129 & 115 & 115 \\
\hline $\begin{array}{l}5.1 .1 \\
\text { Number of variants remaining } \\
\text { after applying a panel of } 459 \\
\text { recessive genes } \\
\text { (number of genes) }\end{array}$ & $5(4)$ & $4(4)$ & $3(3)$ & $5(3)$ & $7(3)$ & $8(3)$ & $10(8)$ & $9(8)$ & $5(3)$ & $3(3)$ \\
\hline
\end{tabular}


Table S10.2 (continued)

\begin{tabular}{|c|c|c|c|c|c|c|c|c|c|c|}
\hline Non-consanguineous couples & 6 & & 7 & & 8 & & 9 & & 10 & \\
\hline & 9 & $\bar{\sigma}$ & 우 & $\overline{0}$ & 우 & क & 우 & d & 우 & ग' \\
\hline Start & 89001 & 107857 & 105209 & 75021 & 109929 & 74152 & 95631 & 104008 & 91638 & 85551 \\
\hline $\begin{array}{l}\text { l. } \\
\text { ClinVar (likely) pathogenic }\end{array}$ & 29 & 45 & 49 & 39 & 46 & 50 & 46 & 45 & 47 & 42 \\
\hline \begin{tabular}{|l|l|}
1.1 \\
Frequency $<1 \%$ or unknown
\end{tabular} & 0 & 4 & 7 & 7 & 7 & 7 & 1 & 1 & 8 & 2 \\
\hline $\begin{array}{ll}\text { HGMD } \\
\end{array}$ & 63 & 75 & 69 & 70 & 13 & 63 & 70 & 15 & 75 & 72 \\
\hline \begin{tabular}{|l|}
2.1 \\
Frequency $<1 \%$ or unknown \\
\end{tabular} & 10 & 17 & 14 & 11 & 3 & 11 & 11 & 5 & 21 & 10 \\
\hline $\begin{array}{l}\text { 3. } \\
\text { Not present in HGMD or (likely) } \\
\text { pathogenic in ClinVar }\end{array}$ & 88909 & 107737 & 105091 & 74912 & 109870 & 74039 & 95515 & 103948 & 91516 & 85437 \\
\hline $\begin{array}{l}3.1 \\
\text { Exonic/canonical splice }\end{array}$ & 20158 & 20196 & 20260 & 19768 & 20345 & 19608 & 20049 & 20043 & 19964 & 20381 \\
\hline $\begin{array}{l}3.2 \\
\text { Reads/depth } \geq 8\end{array}$ & 20020 & 20093 & 20193 & 19561 & 20300 & 19426 & 19929 & 19987 & 19859 & 20165 \\
\hline $\begin{array}{l}3.3 \\
\text { Homozygous variants removed }\end{array}$ & 12507 & 12100 & 12422 & 12060 & 12575 & 11904 & 12301 & 12214 & 11924 & 12274 \\
\hline $\begin{array}{l}3.4 \\
\text { Synonymous variants removed }\end{array}$ & 5914 & 5732 & 5935 & 5704 & 6064 & 5605 & 5851 & 5788 & 5623 & 5803 \\
\hline $\begin{array}{l}3.5 \\
\text { Frequency }<1 \% \text { or unknown }\end{array}$ & 802 & 712 & 720 & 869 & 790 & 845 & 726 & 700 & 680 & 953 \\
\hline \begin{tabular}{|l|} 
Fr.1 \\
Frameshift
\end{tabular} & 58 & 45 & 46 & 29 & 54 & 32 & 49 & 49 & 31 & 52 \\
\hline \begin{tabular}{|l|}
3.5 .2 \\
Nonsense
\end{tabular} & 11 & 12 & 11 & 15 & 5 & 8 & 9 & 11 & 11 & 11 \\
\hline $\begin{array}{l}3.5 .3 \\
\text { Splice } \\
\end{array}$ & 12 & 9 & 14 & 7 & 12 & 7 & 8 & 19 & 14 & 9 \\
\hline $\begin{array}{l}4 . \\
\text { Total presumably pathogenic } \\
(1.1+2.1+3.5 .1-3)\end{array}$ & 91 & 87 & 92 & 69 & 81 & 65 & 78 & 85 & 85 & 84 \\
\hline \begin{tabular}{|l}
4.1 \\
Presumably pathogenic variants \\
in genes shared by the couple
\end{tabular} & 24 & 26 & 13 & 12 & 7 & 8 & 19 & 19 & 16 & 17 \\
\hline $\begin{array}{l}\text { Number of genes in which both } \\
\text { partners have at least one } \\
\text { presumably pathogenic variant }\end{array}$ & 24 & 24 & 11 & 11 & 7 & 7 & 19 & 19 & 15 & 15 \\
\hline $\begin{array}{l}4.1 .1 \\
\text { Number of variants remaining } \\
\text { after applying a panel of } 459 \\
\text { recessive genes }\end{array}$ & 1 & 1 & 0 & 0 & 1 & 1 & 0 & 0 & 0 & 0 \\
\hline $\begin{array}{l}4.2 \\
\text { Presumably pathogenic X-linked } \\
\text { variants in female }\end{array}$ & 3 & n.a. & 0 & n.a. & 1 & n.a. & 3 & n.a. & 1 & n.a. \\
\hline $\begin{array}{l}\text { Number of genes in which the } \\
\text { female has at least one } \\
\text { presumably pathogenic variant }\end{array}$ & 3 & n.a. & 0 & n.a. & 1 & n.a. & 3 & n.a. & 1 & n.a. \\
\hline $\begin{array}{l}4.2 .1 \\
\text { Number of variants remaining } \\
\text { after applying a panel of } 459 \\
\text { recessive genes }\end{array}$ & 0 & n.a. & - & n.a. & 0 & n.a. & 0 & n.a. & 0 & n.a. \\
\hline \begin{tabular}{|l}
3.5 .4 \\
Missense
\end{tabular} & 642 & 564 & 552 & 769 & 622 & 742 & 561 & 539 & 540 & 817 \\
\hline $\begin{array}{l}3.5 .5 \\
\text { In-frame }\end{array}$ & 79 & 82 & 97 & 49 & 97 & 56 & 99 & 82 & 84 & 64 \\
\hline $\begin{array}{l}5 . \\
\text { Total remaining } \\
\text { (not presumably pathogenic) } \\
(3.5 .4+3.5 .5)\end{array}$ & 721 & 646 & 649 & 818 & 719 & 798 & 660 & 621 & 624 & 881 \\
\hline $\begin{array}{l}5.1 \\
\text { Remaining variants in genes } \\
\text { shared by the couple }\end{array}$ & 160 & 160 & 138 & 236 & 124 & 222 & 148 & 151 & 140 & 152 \\
\hline $\begin{array}{l}\text { Number of genes in which both } \\
\text { partners have at least one } \\
\text { remaining variant }\end{array}$ & 113 & 113 & 89 & 89 & 89 & 89 & 115 & 115 & 102 & 102 \\
\hline $\begin{array}{l}\text { 5.1.1 } \\
\text { Number of variants remaining } \\
\text { after applying a panel of } 459 \\
\text { recessive genes } \\
\text { (number of genes) }\end{array}$ & $6(3)$ & $5(3)$ & $6(4)$ & $4(4)$ & $7(6)$ & $7(6)$ & $5(5)$ & $7(5)$ & $1(1)$ & $1(1)$ \\
\hline
\end{tabular}


Table S10.2 (continued)

\begin{tabular}{|c|c|c|c|c|c|c|c|c|c|c|}
\hline \begin{tabular}{|l|} 
(B) \\
Non-consanguineous couples
\end{tabular} & 16 & & 17 & & 18 & & 19 & & 20 & \\
\hline & 우 & ग & 우 & व & 우 & $\overline{0}$ & 우 & $\bar{d}$ & 우 & d \\
\hline Start & 79521 & 92281 & 77497 & 93127 & 107279 & 73572 & 94038 & 78776 & 92910 & 95158 \\
\hline $\begin{array}{l}1 . \\
\text { ClinVar (likely) pathogenic }\end{array}$ & 42 & 46 & 48 & 39 & 46 & 48 & 46 & 32 & 41 & 43 \\
\hline $\begin{array}{l}1.1 \\
\text { Frequency }<1 \% \text { or unknown }\end{array}$ & 6 & 7 & 6 & 8 & 4 & 11 & 7 & 5 & 3 & 7 \\
\hline \begin{tabular}{|l|} 
HGMD \\
HGM
\end{tabular} & 70 & 66 & 69 & 63 & 69 & 68 & 67 & 72 & 70 & 74 \\
\hline $\begin{array}{l}2.1 \\
\text { Frequency }<1 \% \text { or unknown }\end{array}$ & 19 & 11 & 21 & 8 & 15 & 13 & 16 & 18 & 12 & 16 \\
\hline $\begin{array}{l}\text { 3. } \\
\text { Not present in HGMD or (likely) } \\
\text { pathogenic in ClinVar }\end{array}$ & 79409 & 92169 & 77380 & 93025 & 107164 & 73456 & 93925 & 78672 & 92799 & 95041 \\
\hline $\begin{array}{l}3.1 \\
\text { Exonic/canonical splice }\end{array}$ & 20373 & 19764 & 19690 & 20133 & 19997 & 19457 & 19922 & 20163 & 20181 & 20124 \\
\hline $\begin{array}{l}\text { R.2 } \\
\text { Reads/depth } \geq 8\end{array}$ & 20198 & 19651 & 19486 & 20051 & 19920 & 19231 & 19825 & 19969 & 20095 & 20029 \\
\hline $\begin{array}{l}3.3 \\
\text { Homozygous variants removed }\end{array}$ & 12707 & 11770 & 11907 & 12205 & 12341 & 11616 & 12104 & 12156 & 12219 & 12135 \\
\hline $\begin{array}{l}3.4 \\
\text { Synonymous variants removed }\end{array}$ & 6053 & 5633 & 5681 & 5825 & 5861 & 5510 & 5804 & 5726 & 5899 & 5745 \\
\hline $\begin{array}{l}3.5 \\
\text { Frequency }<1 \% \text { or unknown }\end{array}$ & 957 & 673 & 854 & 706 & 694 & 808 & 720 & 1030 & 800 & 956 \\
\hline \begin{tabular}{|l|}
3.5 .1 \\
Frameshift
\end{tabular} & 28 & 46 & 28 & 43 & 39 & 27 & 42 & 32 & 48 & 35 \\
\hline \begin{tabular}{|l|}
3.5 .2 \\
Nonsense \\
\end{tabular} & 20 & 8 & 13 & 11 & 7 & 10 & 12 & 13 & 9 & 10 \\
\hline $\begin{array}{l}3.5 .3 \\
\text { Splice }\end{array}$ & 9 & 6 & 5 & 3 & 12 & 3 & 12 & 5 & 15 & 9 \\
\hline $\begin{array}{l}4 . \\
\text { Total presumably pathogenic } \\
(1.1+2.1+3.5 .1-3)\end{array}$ & 82 & 78 & 73 & 73 & 77 & 64 & 89 & 73 & 87 & 77 \\
\hline \begin{tabular}{|l}
4.1 \\
Presumably pathogenic variants \\
in genes shared by the couple
\end{tabular} & 11 & 9 & 10 & 11 & 7 & 9 & 10 & 9 & 14 & 15 \\
\hline $\begin{array}{l}\text { Number of genes in which both } \\
\text { partners have at least one } \\
\text { presumably pathogenic variant }\end{array}$ & 8 & 8 & 8 & 8 & 7 & 7 & 8 & 8 & 12 & 12 \\
\hline $\begin{array}{l}4.1 .1 \\
\text { Number of variants remaining } \\
\text { after applying a panel of } 459 \\
\text { recessive genes } \\
\text { (number of genes) }\end{array}$ & $0(0)$ & $0(0)$ & $0(0)$ & $0(0)$ & $0(0)$ & $0(0)$ & $1(1)$ & $1(1)$ & $0(0)$ & $0(0)$ \\
\hline $\begin{array}{l}4.2 \\
\text { Presumably pathogenic X-linked } \\
\text { variants in female }\end{array}$ & 1 & n.a. & 1 & n.a. & 3 & n.a. & 3 & n.a. & 0 & n.a. \\
\hline $\begin{array}{l}\text { Number of genes in which the } \\
\text { female has at least one } \\
\text { presumably pathogenic variant }\end{array}$ & 1 & n.a. & 1 & n.a. & 3 & n.a. & 3 & n.a. & 0 & n.a. \\
\hline $\begin{array}{l}4.2 .1 \\
\text { Number of variants remaining } \\
\text { after applying a panel of } 459 \\
\text { recessive genes }\end{array}$ & 1 & n.a. & 0 & n.a. & 0 & n.a. & 1 & n.a. & - & n.a. \\
\hline \begin{tabular}{|l|}
3.5 .4 \\
Missense
\end{tabular} & 849 & 531 & 739 & 561 & 544 & 726 & 574 & 924 & 612 & 813 \\
\hline $\begin{array}{l}3.5 .5 \\
\text { In-frame }\end{array}$ & 51 & 82 & 69 & 88 & 92 & 42 & 80 & 56 & 116 & 89 \\
\hline \begin{tabular}{|l}
5. \\
Total remaining \\
(not presumably pathogenic) \\
$(3.5 .4+3.5 .5)$
\end{tabular} & 900 & 613 & 808 & 649 & 636 & 768 & 654 & 980 & 728 & 902 \\
\hline $\begin{array}{l}5.1 \\
\text { Remaining variants in genes } \\
\text { shared by the couple }\end{array}$ & 249 & 129 & 225 & 108 & 91 & 186 & 127 & 234 & 134 & 248 \\
\hline $\begin{array}{l}\text { Number of genes in which both } \\
\text { partners have at least one } \\
\text { remaining variant }\end{array}$ & 83 & 83 & 74 & 74 & 60 & 60 & 87 & 87 & 85 & 85 \\
\hline $\begin{array}{l}5.1 .1 \\
\text { Number of variants remaining } \\
\text { after applying a panel of } 459 \\
\text { recessive genes } \\
\text { (number of genes) }\end{array}$ & 8 & 4 & 5 & 6 & 3 & 3 & 6 & 10 & 1 & 1 \\
\hline
\end{tabular}


Table S10.2 (continued)

\begin{tabular}{|c|c|c|c|c|c|c|c|c|c|c|}
\hline \begin{tabular}{|l|} 
(B) \\
Non-consanguineous couples
\end{tabular} & 16 & & 17 & & 18 & & 19 & & 20 & \\
\hline & 우 & or & 우 & $\sigma^{-1}$ & 우 & $\sigma^{1}$ & 우 & $\overline{0}$ & 우 & or \\
\hline Start & 79521 & 92281 & 77497 & 93127 & 107279 & 73572 & 94038 & 78776 & 92910 & 95158 \\
\hline $\begin{array}{l}1 . \\
\text { ClinVar (likely) pathogenic }\end{array}$ & 42 & 46 & 48 & 39 & 46 & 48 & 46 & 32 & 41 & 43 \\
\hline \begin{tabular}{|l|}
1.1 \\
Frequency $<1 \%$ or unknown \\
\end{tabular} & 6 & 7 & 6 & 8 & 4 & 11 & 7 & 5 & 3 & 7 \\
\hline \begin{tabular}{|l|}
2. \\
HGMD
\end{tabular} & 70 & 66 & 69 & 63 & 69 & 68 & 67 & 72 & 70 & 74 \\
\hline \begin{tabular}{|l|}
2.1 \\
Frequency $<1 \%$ or unknown
\end{tabular} & 19 & 11 & 21 & 8 & 15 & 13 & 16 & 18 & 12 & 16 \\
\hline $\begin{array}{l}\text { 3. } \\
\text { Not present in HGMD or (likely) } \\
\text { pathogenic in ClinVar }\end{array}$ & 79409 & 92169 & 77380 & 93025 & 107164 & 73456 & 93925 & 78672 & 92799 & 95041 \\
\hline $\begin{array}{l}3.1 \\
\text { Exonic/canonical splice }\end{array}$ & 20373 & 19764 & 19690 & 20133 & 19997 & 19457 & 19922 & 20163 & 20181 & 20124 \\
\hline Reads/depth $\geq 8$ & 20198 & 19651 & 19486 & 20051 & 19920 & 19231 & 19825 & 19969 & 20095 & 20029 \\
\hline $\begin{array}{l}3.3 \\
\text { Homozygous variants removed }\end{array}$ & 12707 & 11770 & 11907 & 12205 & 12341 & 11616 & 12104 & 12156 & 12219 & 12135 \\
\hline $\begin{array}{l}3.4 \\
\text { Synonymous variants removed }\end{array}$ & 6053 & 5633 & 5681 & 5825 & 5861 & 5510 & 5804 & 5726 & 5899 & 5745 \\
\hline $\begin{array}{l}3.5 \\
\text { Erequency }<1 \% \text { or unknown }\end{array}$ & 957 & 673 & 854 & 706 & 694 & 808 & 720 & 1030 & 800 & 956 \\
\hline $\begin{array}{l}3.5 .1 \\
\text { Frameshift }\end{array}$ & 28 & 46 & 28 & 43 & 39 & 27 & 42 & 32 & 48 & 35 \\
\hline \begin{tabular}{|l|}
3.5 .2 \\
Nonsense
\end{tabular} & 20 & 8 & 13 & 11 & 7 & 10 & 12 & 13 & 9 & 10 \\
\hline $\begin{array}{l}3.5 .3 \\
\text { Splice }\end{array}$ & 9 & 6 & 5 & 3 & 12 & 3 & 12 & 5 & 15 & 9 \\
\hline \begin{tabular}{|l}
4. \\
Total presumably pathogenic \\
$(1.1+2.1+3.5 .1-3)$ \\
\end{tabular} & 82 & 78 & 73 & 73 & 77 & 64 & 89 & 73 & 87 & 77 \\
\hline $\begin{array}{l}4.1 \\
\text { Presumably pathogenic variants } \\
\text { in genes shared by the couple }\end{array}$ & 11 & 9 & 10 & 11 & 7 & 9 & 10 & 9 & 14 & 15 \\
\hline $\begin{array}{l}\text { Number of genes in which both } \\
\text { partners have at least one } \\
\text { presumably pathogenic variant }\end{array}$ & 8 & 8 & 8 & 8 & 7 & 7 & 8 & 8 & 12 & 12 \\
\hline $\begin{array}{l}4.1 .1 \\
\text { Number of variants remaining } \\
\text { after applying a panel of } 459 \\
\text { recessive genes } \\
\text { (number of genes) }\end{array}$ & $0(0)$ & $0(0)$ & $0(0)$ & $0(0)$ & $0(0)$ & $0(0)$ & $1(1)$ & $1(1)$ & $0(0)$ & $0(0)$ \\
\hline \begin{tabular}{|l|}
4.2 \\
Presumably pathogenic X-linked \\
variants in female
\end{tabular} & 1 & n.a. & 1 & n.a. & 3 & n.a. & 3 & n.a. & 0 & n.a. \\
\hline $\begin{array}{l}\text { Number of genes in which the } \\
\text { female has at least one } \\
\text { presumably pathogenic variant }\end{array}$ & 1 & n.a. & 1 & n.a. & 3 & n.a. & 3 & n.a. & 0 & n.a. \\
\hline $\begin{array}{l}4.2 .1 \\
\text { Number of variants remaining } \\
\text { after applying a panel of } 459 \\
\text { recessive genes }\end{array}$ & 1 & n.a. & 0 & n.a. & 0 & n.a. & 1 & n.a. & - & n.a. \\
\hline \begin{tabular}{|l}
3.5 .4 \\
Missense
\end{tabular} & 849 & 531 & 739 & 561 & 544 & 726 & 574 & 924 & 612 & 813 \\
\hline $\begin{array}{l}3.5 .5 \\
\text { In-frame } \\
\end{array}$ & 51 & 82 & 69 & 88 & 92 & 42 & 80 & 56 & 116 & 89 \\
\hline $\begin{array}{l}5 . \\
\text { Total remaining } \\
\text { (not presumably pathogenic) } \\
(3.5 .4+3.5 .5)\end{array}$ & 900 & 613 & 808 & 649 & 636 & 768 & 654 & 980 & 728 & 902 \\
\hline $\begin{array}{l}5.1 \\
\text { Remaining variants in genes } \\
\text { shared by the couple }\end{array}$ & 249 & 129 & 225 & 108 & 91 & 186 & 127 & 234 & 134 & 248 \\
\hline $\begin{array}{l}\text { Number of genes in which both } \\
\text { partners have at least one } \\
\text { remaining variant }\end{array}$ & 83 & 83 & 74 & 74 & 60 & 60 & 87 & 87 & 85 & 85 \\
\hline $\begin{array}{l}5.1 .1 \\
\text { Number of variants remaining } \\
\text { after applying a panel of } 459 \\
\text { recessive genes } \\
\text { (number of genes) }\end{array}$ & 8 & 4 & 5 & 6 & 3 & 3 & 6 & 10 & 1 & 1 \\
\hline
\end{tabular}


Table S10.2 (continued)

\begin{tabular}{|c|c|c|c|c|c|c|c|c|c|c|}
\hline Non-consanguineous couples & 21 & & 22 & & 23 & & 24 & & 25 & \\
\hline & 우 & 万' & 우 & 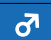 & 우 & $\sigma^{7}$ & 우 & б' & 우 & 万' \\
\hline Start & 96180 & 91240 & 91900 & 84745 & 77456 & 88487 & 106279 & 93852 & 90911 & 111071 \\
\hline $\begin{array}{l}. \\
\text { ClinVar (likely) pathogenic }\end{array}$ & 36 & 44 & 42 & 43 & 35 & 42 & 46 & 39 & 42 & 46 \\
\hline $\begin{array}{l}1.1 \\
\text { Frequency }<1 \% \text { or unknown }\end{array}$ & 4 & 3 & 10 & 5 & 4 & 3 & 7 & 6 & 5 & 9 \\
\hline \begin{tabular}{|l}
2. \\
HGMD
\end{tabular} & 10 & 77 & 77 & 70 & 59 & 15 & 17 & 56 & 77 & 19 \\
\hline \begin{tabular}{|l|}
2.1 \\
Frequency $<1 \%$ or unknown
\end{tabular} & 1 & 13 & 18 & 21 & 16 & 2 & 4 & 12 & 17 & 7 \\
\hline $\begin{array}{l}\text { 3. } \\
\text { Not present in HGMD or (likely) } \\
\text { pathogenic in ClinVar }\end{array}$ & 96134 & 91119 & 91781 & 84632 & 77362 & 88430 & 106216 & 93757 & 90792 & 111006 \\
\hline $\begin{array}{l}3.1 \\
\text { Exonic/canonical splice } \\
\text { E }\end{array}$ & 20008 & 19894 & 19815 & 19900 & 19687 & 20040 & 20326 & 20350 & 19970 & 20515 \\
\hline $\begin{array}{l}3.2 \\
\text { Reads/depth } \geq 8\end{array}$ & 19891 & 19760 & 19718 & 19732 & 19498 & 19885 & 20258 & 20222 & 19839 & 20460 \\
\hline $\begin{array}{l}3.3 \\
\text { Homozygous variants removed }\end{array}$ & 12096 & 11910 & 12063 & 12022 & 11858 & 12081 & 12472 & 12636 & 12149 & 12628 \\
\hline $\begin{array}{l}3.4 \\
\text { Synonymous variants removed }\end{array}$ & 5802 & 5624 & 5749 & 5693 & 5649 & 5839 & 5995 & 5984 & 5745 & 6091 \\
\hline $\begin{array}{l}3.5 \\
\text { Frequency }<1 \% \text { or unknown }\end{array}$ & 688 & 667 & 906 & 861 & 901 & 704 & 778 & 966 & 715 & 781 \\
\hline \begin{tabular}{|l|} 
Frameshift \\
\end{tabular} & 41 & 43 & 34 & 29 & 24 & 50 & 49 & 22 & 48 & 56 \\
\hline \begin{tabular}{|l|}
3.5 .2 \\
Nonsense \\
\end{tabular} & 10 & 10 & 11 & 14 & 12 & 20 & 7 & 10 & 10 & 12 \\
\hline $\begin{array}{l}.5 .3 \\
\text { Splice }\end{array}$ & 16 & 11 & 9 & 7 & 9 & 11 & 16 & 9 & 13 & 14 \\
\hline \begin{tabular}{|l}
4. \\
Total presumably pathogenic \\
$(1.1+2.1+3.5 .1-3)$
\end{tabular} & 72 & 80 & 82 & 76 & 65 & 86 & 83 & 59 & 93 & 98 \\
\hline $\begin{array}{l}4.1 \\
\text { Presumably pathogenic variants } \\
\text { in genes shared by the couple }\end{array}$ & 18 & 18 & 20 & 18 & 10 & 10 & 6 & 6 & 30 & 28 \\
\hline $\begin{array}{l}\text { Number of genes in which both } \\
\text { partners have at least one } \\
\text { presumably pathogenic variant }\end{array}$ & 17 & 17 & 15 & 15 & 7 & 7 & 6 & 6 & 27 & 27 \\
\hline $\begin{array}{l}4.1 .1 \\
\text { Number of variants remaining } \\
\text { after applying a panel of } 459 \\
\text { recessive genes } \\
\text { (number of genes) }\end{array}$ & $0(0)$ & $0(0)$ & $0(0)$ & $0(0)$ & $0(0)$ & $0(0)$ & $0(0)$ & $0(0)$ & $1(1)$ & $1(1)$ \\
\hline $\begin{array}{l}4.2 \\
\text { Presumably pathogenic X-linked } \\
\text { variants in female }\end{array}$ & 1 & n.a. & 1 & n.a. & 0 & n.a. & 0 & n.a. & 0 & n.a. \\
\hline $\begin{array}{l}\text { Number of genes in which the } \\
\text { female has at least one } \\
\text { presumably pathogenic variant }\end{array}$ & 1 & n.a. & 1 & n.a. & 0 & n.a. & 0 & n.a. & 0 & n.a. \\
\hline $\begin{array}{l}4.2 .1 \\
\text { Number of variants remaining } \\
\text { after applying a panel of } 459 \\
\text { recessive genes }\end{array}$ & 1 & n.a. & 0 & n.a. & - & n.a. & - & n.a. & - & n.a. \\
\hline \begin{tabular}{|l|}
3.5 .4 \\
Missense \\
\end{tabular} & 557 & 533 & 787 & 744 & 785 & 545 & 613 & 856 & 554 & 599 \\
\hline $\begin{array}{l}3.5 .5 \\
\text { In-frame }\end{array}$ & 64 & 70 & 65 & 67 & 71 & 78 & 93 & 69 & 90 & 100 \\
\hline \begin{tabular}{|l}
5. \\
Total remaining \\
(not presumably pathogenic) \\
$(3.5 .4+3.5 .5)$
\end{tabular} & 621 & 603 & 852 & 811 & 856 & 623 & 706 & 925 & 644 & 699 \\
\hline $\begin{array}{l}5.1 \\
\text { Remaining variants in genes } \\
\text { shared by the couple }\end{array}$ & 133 & 141 & 329 & 321 & 239 & 127 & 131 & 237 & 155 & 172 \\
\hline $\begin{array}{l}\text { Number of genes in which both } \\
\text { partners have at least one } \\
\text { remaining variant }\end{array}$ & 103 & 103 & 165 & 165 & 88 & 88 & 88 & 88 & 107 & 107 \\
\hline $\begin{array}{l}\text { 5.1.1 } \\
\text { Number of variants remaining } \\
\text { after applying a panel of } 459 \\
\text { recessive genes } \\
\text { (number of genes) }\end{array}$ & $3(3)$ & $3(3)$ & $8(8)$ & $8(8)$ & $8(4)$ & $4(4)$ & $4(4)$ & $6(4)$ & $4(4)$ & $6(4)$ \\
\hline
\end{tabular}




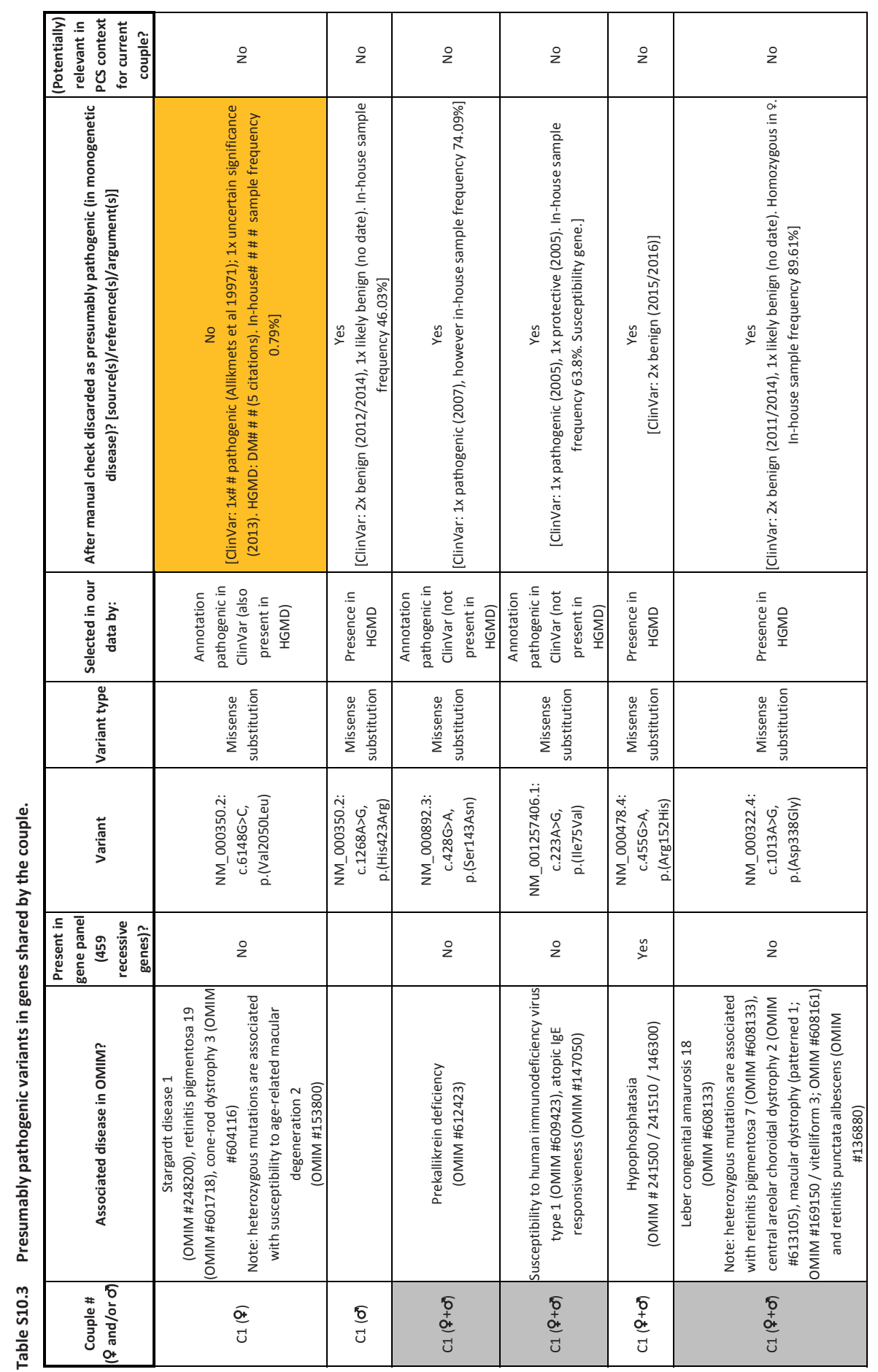


A comprehensive strategy for exome-based preconception carrier screening

\begin{tabular}{|c|c|c|c|c|c|c|c|}
\hline 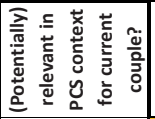 & $\stackrel{0}{z}$ & 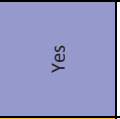 & $\stackrel{\circ}{z}$ & $\stackrel{\circ}{2}$ & $\stackrel{\circ}{z}$ & $\stackrel{0}{z}$ & $\stackrel{2}{2}$ \\
\hline 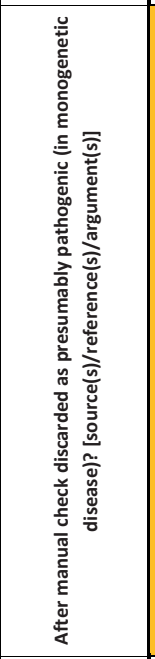 & 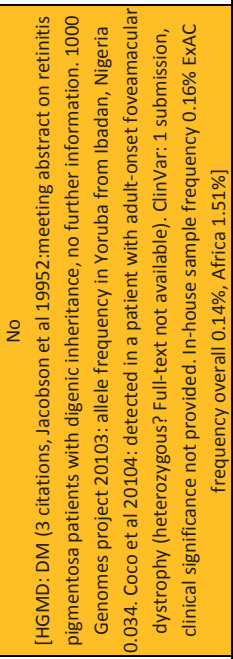 & 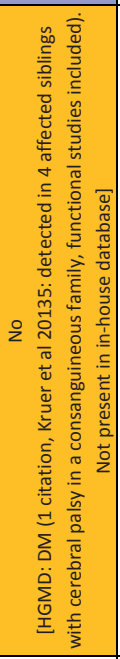 & 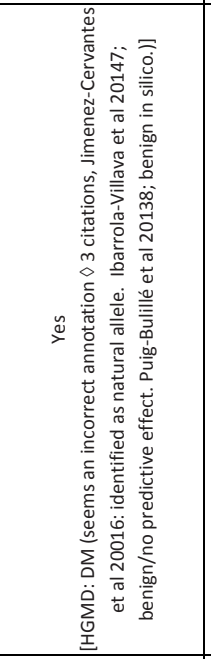 & 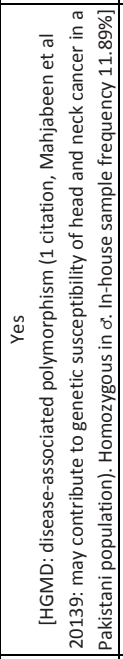 & 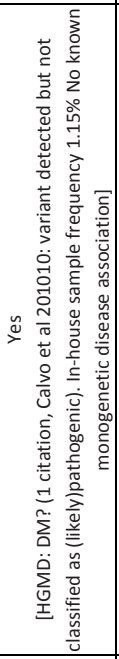 & 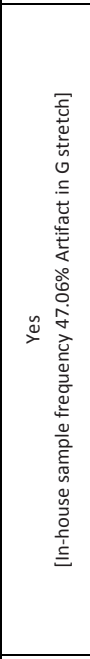 & 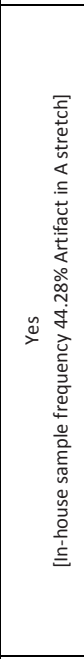 \\
\hline 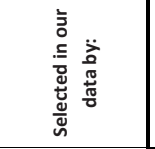 & 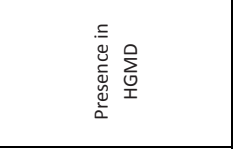 & 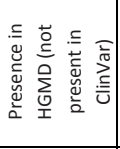 & 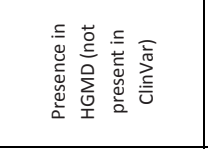 & 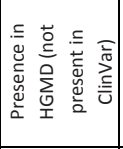 & 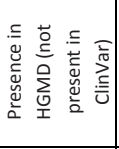 & 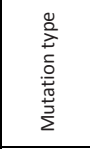 & 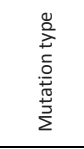 \\
\hline 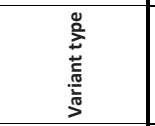 & 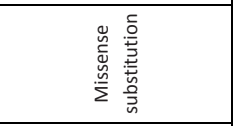 & 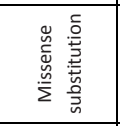 & 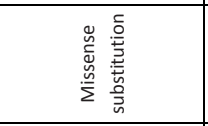 & 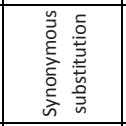 & 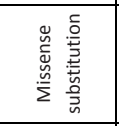 & 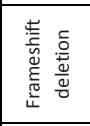 & 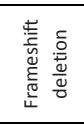 \\
\hline 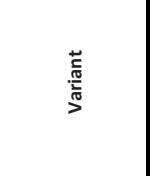 & 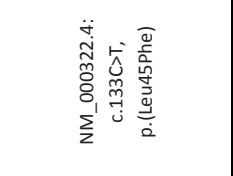 & 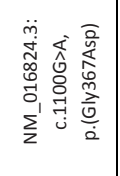 & 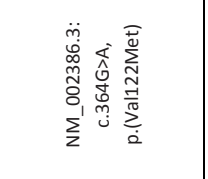 & 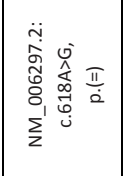 & 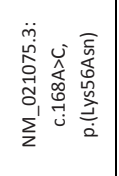 & 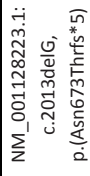 & 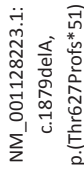 \\
\hline 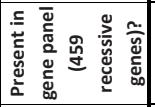 & & $\stackrel{0}{z}$ & $\stackrel{0}{z}$ & $\stackrel{\circ}{2}$ & $\stackrel{0}{z}$ & $\stackrel{0}{2}$ & \\
\hline 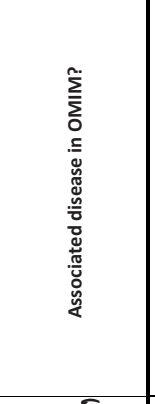 & & 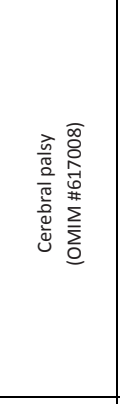 & 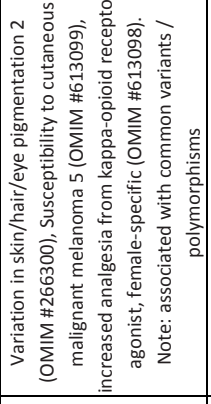 & 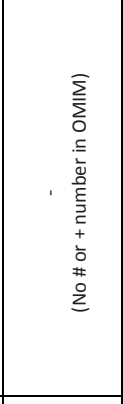 & 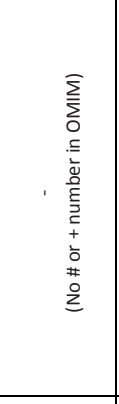 & 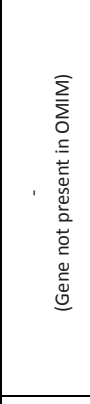 & \\
\hline 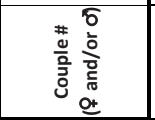 & $\frac{\bar{\sigma}}{\mathrm{y}}$ & 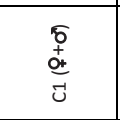 & $\begin{array}{l}5 \\
0 \\
+ \\
\text { ot } \\
\text { U }\end{array}$ & 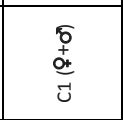 & $\begin{array}{l}\bar{\delta} \\
+ \\
\text { o+ } \\
\text { ป }\end{array}$ & $\begin{array}{l}5 \\
0 \\
+ \\
\text { ot } \\
-1\end{array}$ & 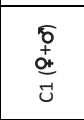 \\
\hline
\end{tabular}




\begin{tabular}{|c|c|c|c|c|c|c|c|c|c|c|}
\hline 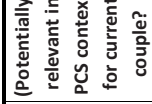 & $\stackrel{\circ}{z}$ & $\stackrel{0}{z}$ & $\stackrel{0}{2}$ & $\stackrel{2}{z}$ & $\stackrel{\circ}{z}$ & $\stackrel{0}{z}$ & $\stackrel{0}{2}$ & $\stackrel{\circ}{z}$ & $\frac{8}{2}$ & $\stackrel{\circ}{2}$ \\
\hline 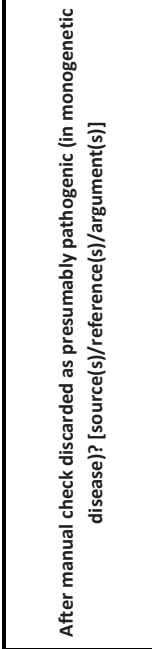 & 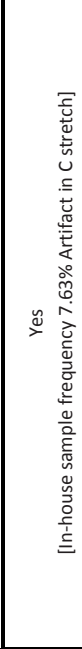 & 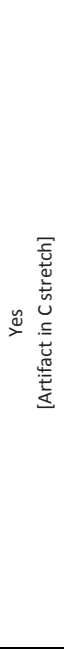 & 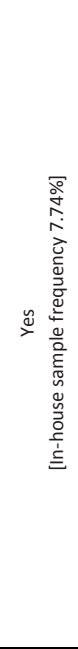 & 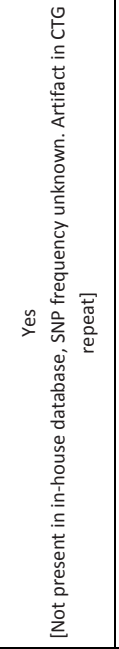 & 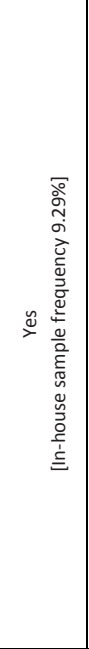 & 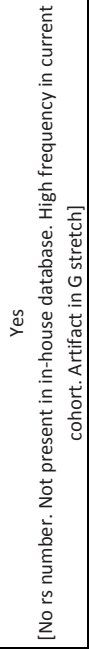 & 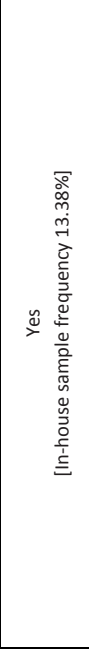 & 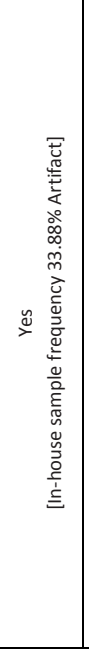 & 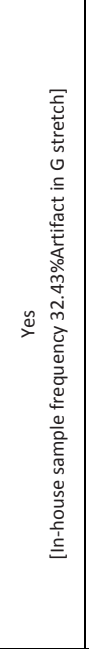 & 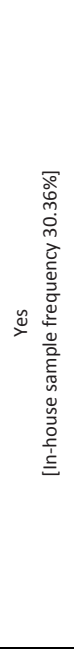 \\
\hline 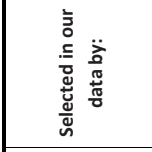 & 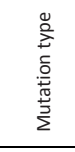 & 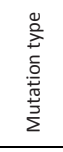 & 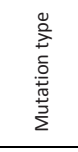 & 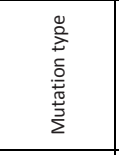 & 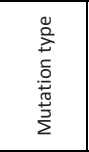 & 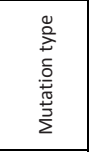 & 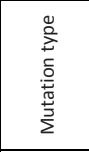 & 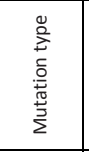 & 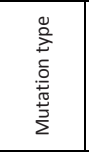 & 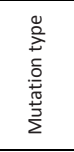 \\
\hline 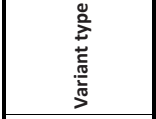 & 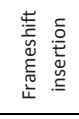 & 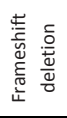 & 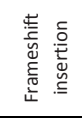 & 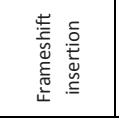 & 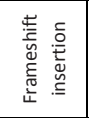 & 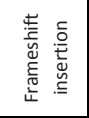 & 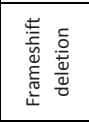 & 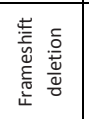 & 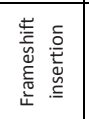 & 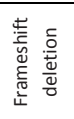 \\
\hline 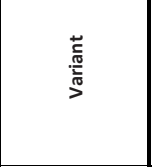 & 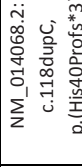 & & 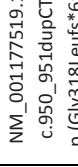 & 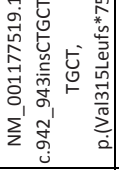 & 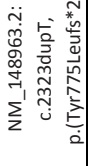 & 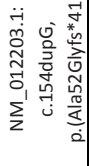 & 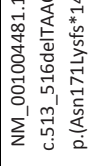 & & 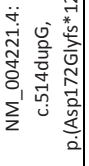 & 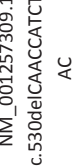 \\
\hline 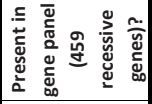 & $\stackrel{8}{z}$ & & $\stackrel{\circ}{z}$ & & $\stackrel{8}{z}$ & 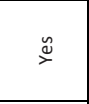 & $\stackrel{\circ}{z}$ & z & $\frac{8}{2}$ & $\stackrel{\circ}{z}$ \\
\hline 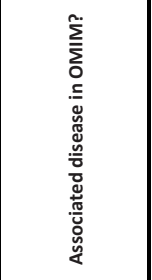 & 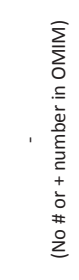 & & 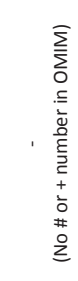 & & 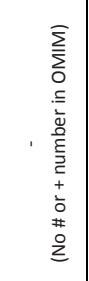 & 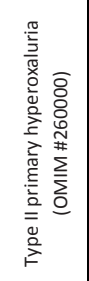 & 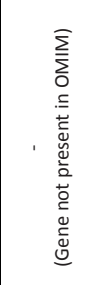 & 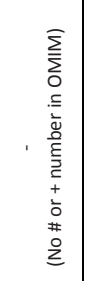 & 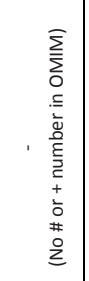 & 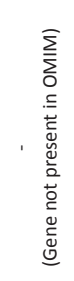 \\
\hline 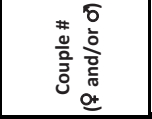 & $\frac{\bar{\partial}}{\vec{U}}$ & $\frac{\delta}{\sigma}$ & 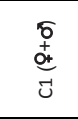 & $\frac{\delta}{\sigma}$ & 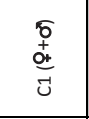 & 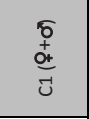 & 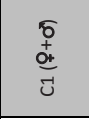 & $\begin{array}{l}\sigma \\
\vdots \\
\text { ot } \\
\end{array}$ & 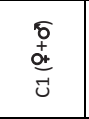 & 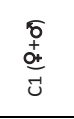 \\
\hline
\end{tabular}


A comprehensive strategy for exome-based preconception carrier screening

\begin{tabular}{|c|c|c|c|c|c|c|c|c|c|c|}
\hline 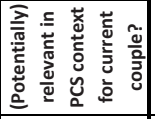 & z & $\frac{0}{2}$ & zo & $\stackrel{0}{2}$ & $\stackrel{\circ}{z}$ & $\frac{0}{2}$ & $\frac{0}{2}$ & $\frac{0}{2}$ & z & $\stackrel{\circ}{z}$ \\
\hline 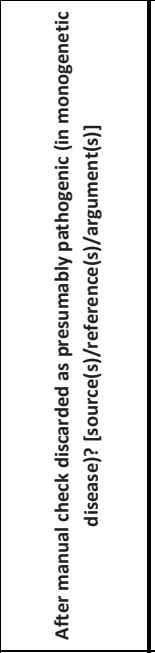 & 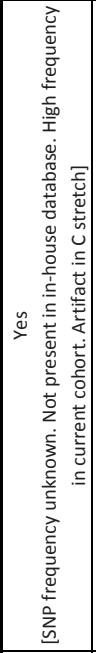 & 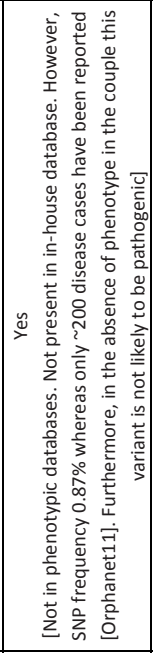 & 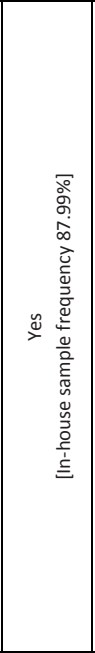 & 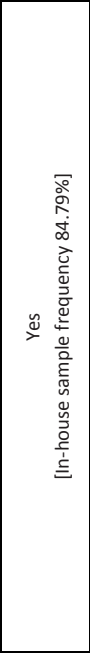 & 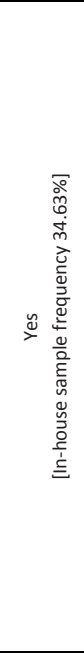 & 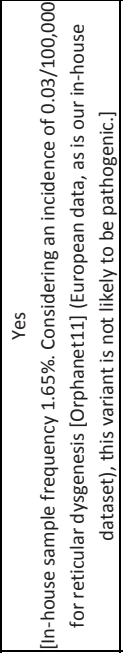 & 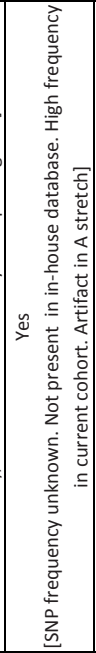 & 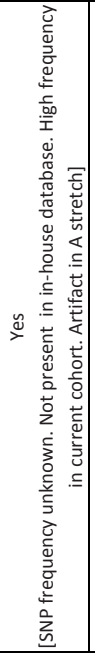 & 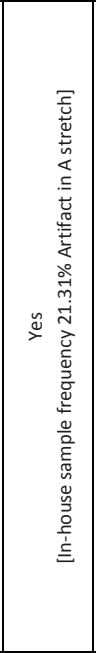 & 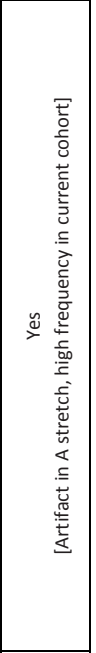 \\
\hline 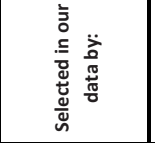 & 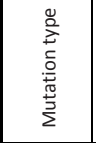 & 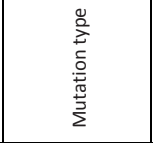 & 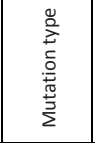 & 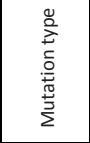 & 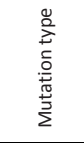 & 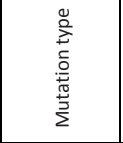 & 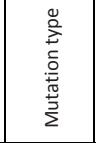 & 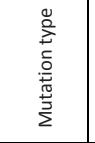 & 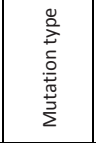 & 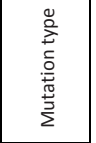 \\
\hline 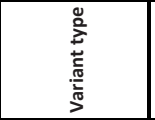 & 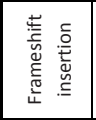 & 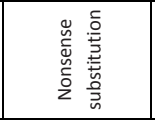 & 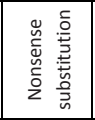 & 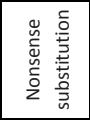 & 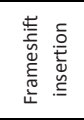 & 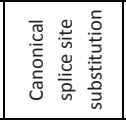 & 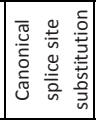 & 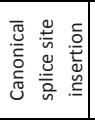 & 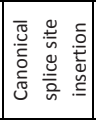 & 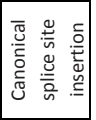 \\
\hline 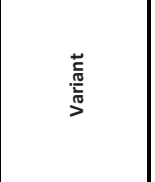 & 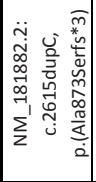 & 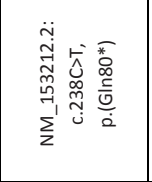 & 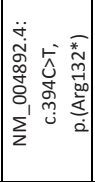 & 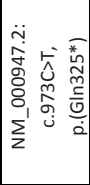 & 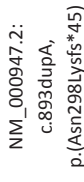 & 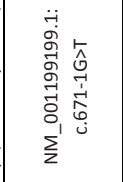 & 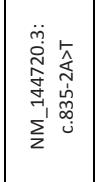 & 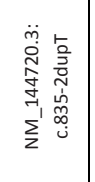 & 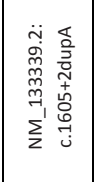 & 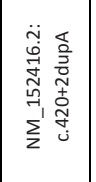 \\
\hline 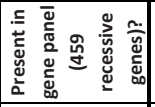 & $\stackrel{\mathscr{u}}{\rightleftharpoons}$ & $z^{\circ}$ & $\stackrel{0}{z}$ & $\stackrel{\circ}{z}$ & & $\stackrel{\circ}{2}$ & $\stackrel{0}{2}$ & & $\stackrel{\circ}{2}$ & $\stackrel{0}{z}$ \\
\hline 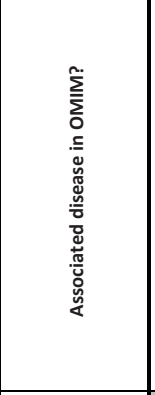 & 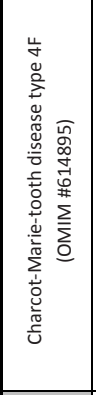 & 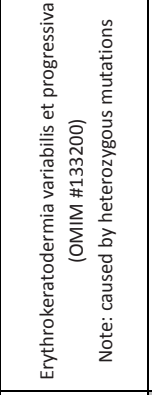 & 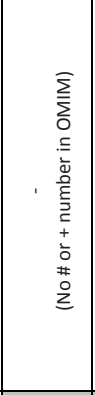 & 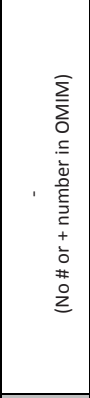 & & 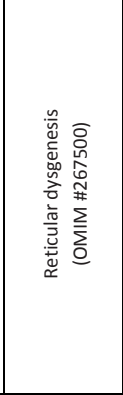 & 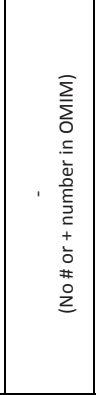 & & 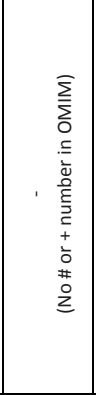 & 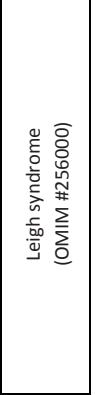 \\
\hline 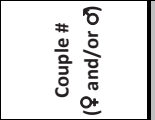 & $\begin{array}{l}\overline{0} \\
+ \\
\text { O+ } \\
\bar{y}\end{array}$ & 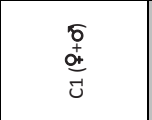 & $\begin{array}{l}\delta \\
0 \\
+ \\
\text { ot } \\
\text { ป }\end{array}$ & $\begin{array}{l}5 \\
0 \\
+ \\
\text { ot } \\
\overline{1}\end{array}$ & $\frac{\delta}{\sigma}$ & $\begin{array}{l}5 \\
0 \\
0+ \\
\\
-1\end{array}$ & $\begin{array}{l}5 \\
0 \\
\text { o+ } \\
\overline{-}\end{array}$ & क्षे & $\begin{array}{l}5 \\
5 \\
\text { o+ } \\
-1\end{array}$ & $\begin{array}{l}5 \\
0 \\
+ \\
\text { o+ } \\
-1\end{array}$ \\
\hline
\end{tabular}




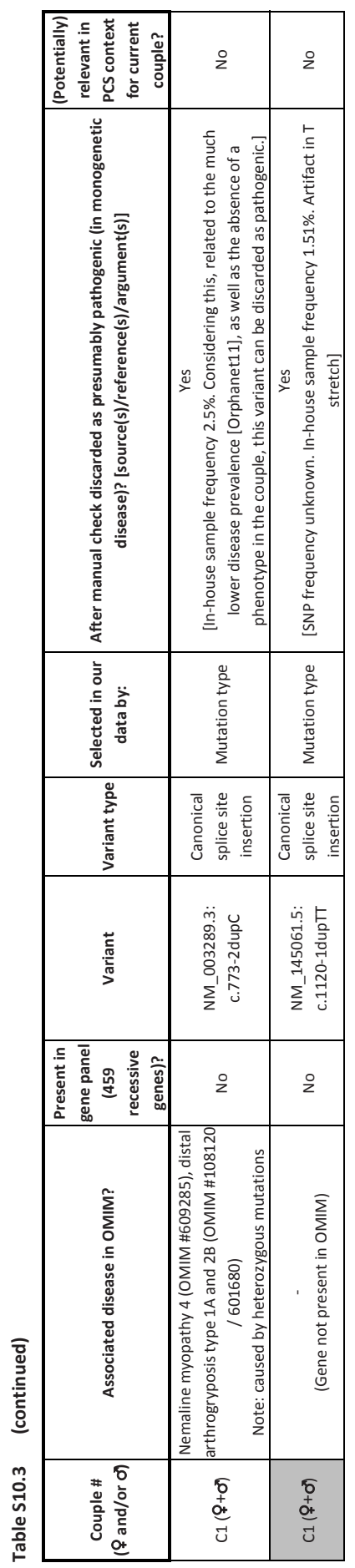


A comprehensive strategy for exome-based preconception carrier screening

\begin{tabular}{|c|c|c|c|c|c|c|}
\hline 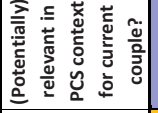 & $\stackrel{\check{\nu}}{\check{\nu}}$ & $\stackrel{\circ}{z}$ & $\stackrel{\circ}{z}$ & $\stackrel{\circ}{z}$ & $\stackrel{\circ}{z}$ & $\stackrel{0}{z}$ \\
\hline 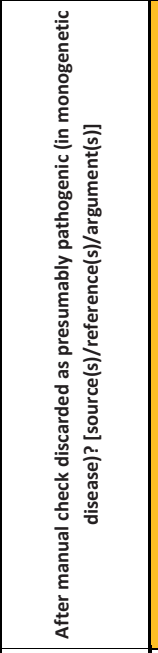 & 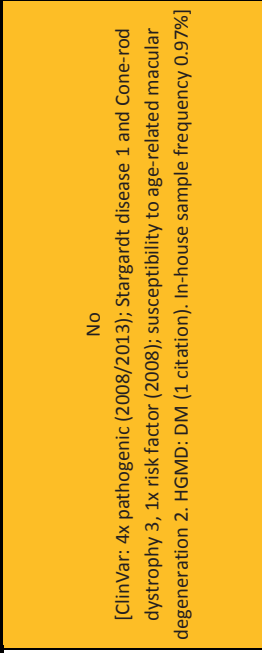 & 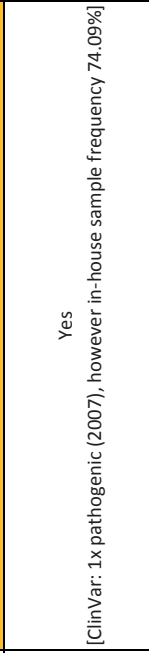 & 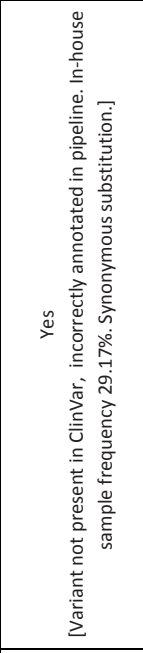 & 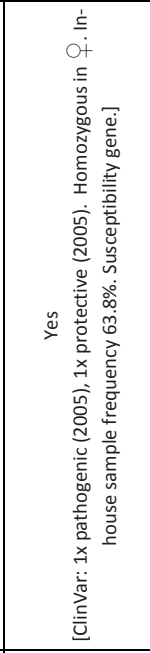 & 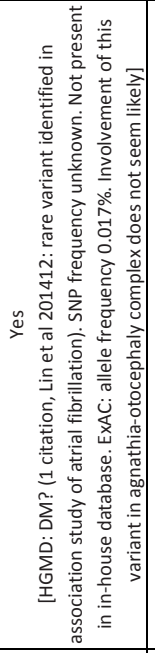 & 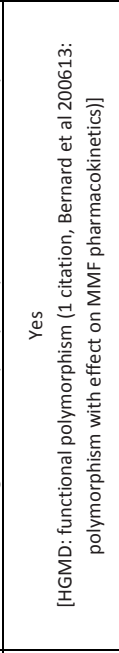 \\
\hline 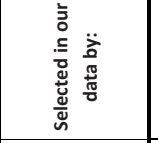 & 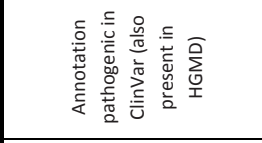 & 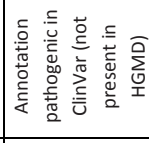 & 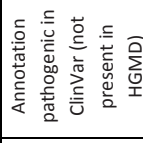 & 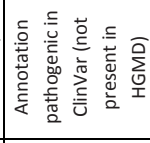 & 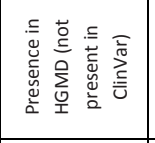 & 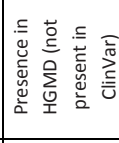 \\
\hline 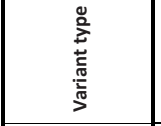 & 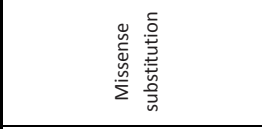 & 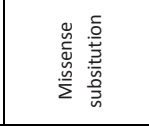 & 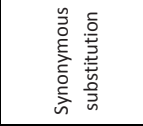 & 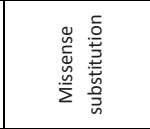 & 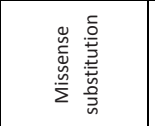 & 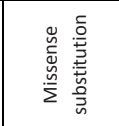 \\
\hline 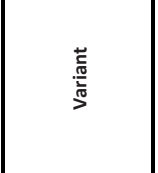 & 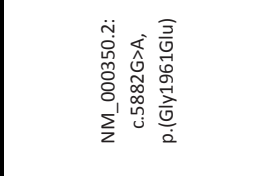 & 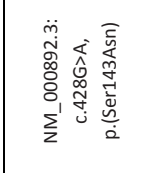 & 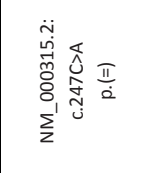 & 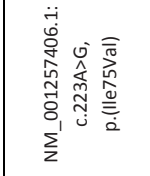 & 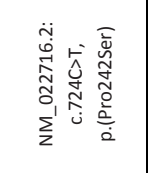 & 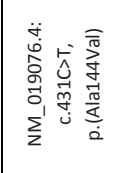 \\
\hline 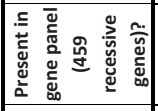 & $\stackrel{\check{\nu}}{\check{\nu}}$ & $\stackrel{\circ}{z}$ & $\stackrel{0}{z}$ & $\stackrel{0}{2}$ & $\frac{0}{2}$ & $\stackrel{\circ}{z}$ \\
\hline 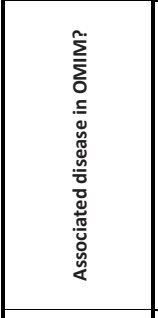 & 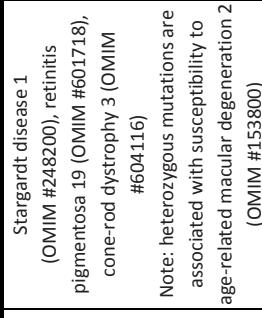 & 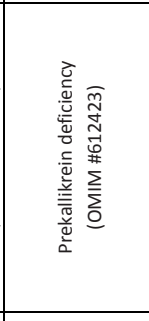 & 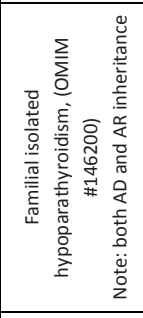 & 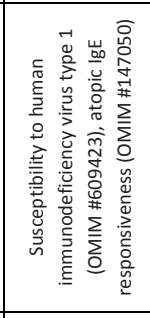 & 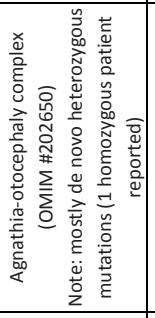 & 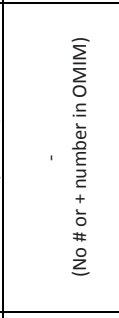 \\
\hline 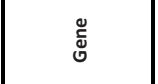 & $\begin{array}{l}\text { 莣 } \\
\text { 要 }\end{array}$ & $\begin{array}{l}\vec{p} \\
\overrightarrow{\underline{y}}\end{array}$ & 吾 & $\stackrel{\text { 寽 }}{=}$ & 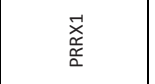 & 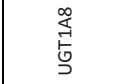 \\
\hline 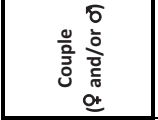 & 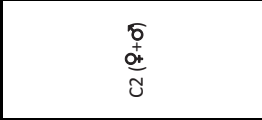 & 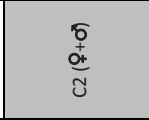 & 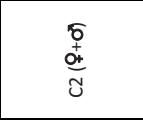 & 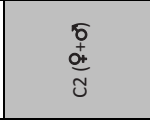 & 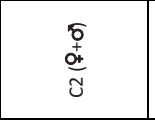 & 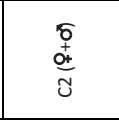 \\
\hline
\end{tabular}




\begin{tabular}{|c|c|c|c|c|c|c|c|c|}
\hline 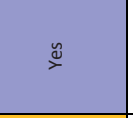 & z & $\stackrel{\circ}{z}$ & $\stackrel{0}{2}$ & $\stackrel{0}{z}$ & $\stackrel{2}{2}$ & $\frac{8}{2}$ & $\stackrel{0}{2}$ & $\stackrel{8}{z}$ \\
\hline 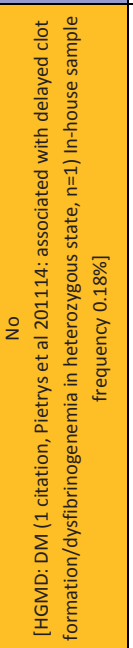 & 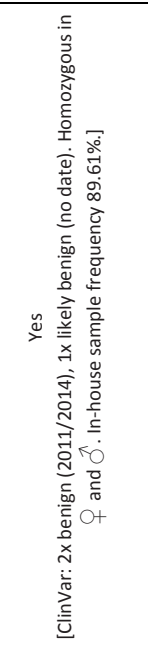 & 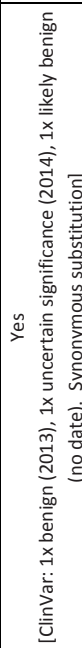 & 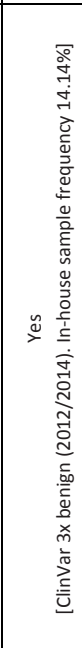 & 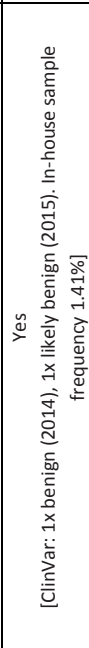 & 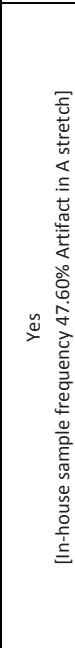 & 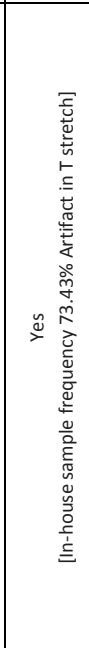 & 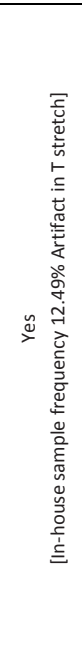 & 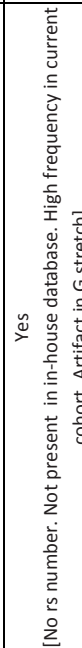 \\
\hline 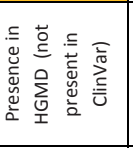 & & 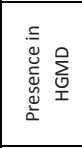 & 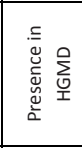 & 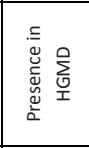 & 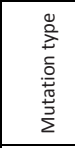 & 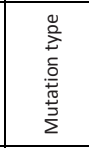 & 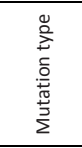 & 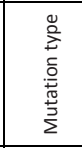 \\
\hline 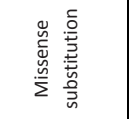 & 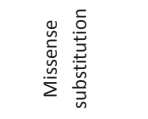 & 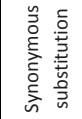 & 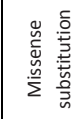 & 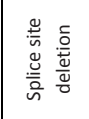 & 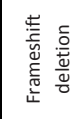 & 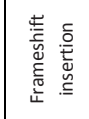 & 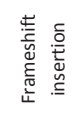 & 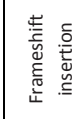 \\
\hline 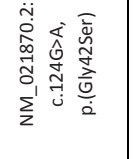 & 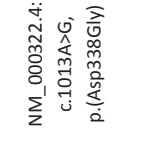 & {$\left[\begin{array}{ll}0 \\
0 \\
1 \\
0 \\
0\end{array}\right.$} & 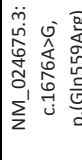 & 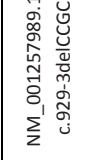 & 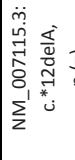 & 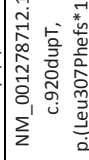 & & 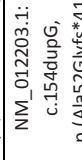 \\
\hline$\stackrel{\check{\nu}}{\rightleftharpoons}$ & 2 & $\stackrel{\square}{\nearrow}$ & $\stackrel{8}{2}$ & 2 & $\stackrel{2}{2}$ & $\stackrel{8}{z}$ & $\stackrel{8}{2}$ & $\stackrel{\mathscr{u}}{x}$ \\
\hline 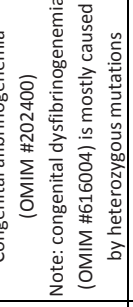 & 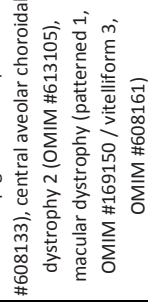 & 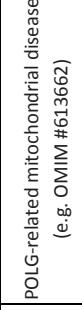 & 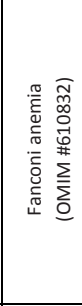 & 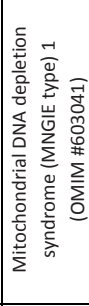 & 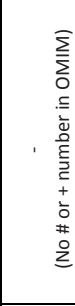 & 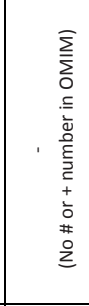 & 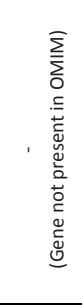 & 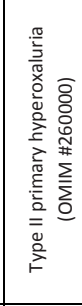 \\
\hline 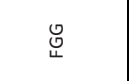 & 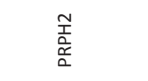 & 홍 & $\frac{\tilde{x}}{\alpha}$ & $\sum_{i=}^{n}$ & 产 & 尊 & $\frac{\stackrel{o}{0}}{\frac{o}{d}}$ & 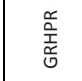 \\
\hline $\begin{array}{l}\frac{0}{0+} \\
\frac{\delta}{\delta}\end{array}$ & 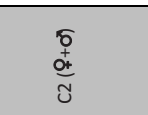 & $\begin{array}{l}\frac{\sigma}{0} \\
\frac{0}{\alpha+} \\
\tilde{\sigma}\end{array}$ & 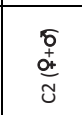 & 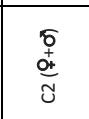 & 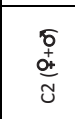 & 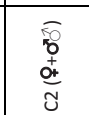 & 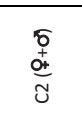 & 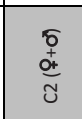 \\
\hline
\end{tabular}


A comprehensive strategy for exome-based preconception carrier screening

\begin{tabular}{|c|c|c|c|c|c|c|c|c|c|c|}
\hline 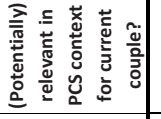 & $z$ & $\stackrel{0}{z}$ & $\stackrel{0}{2}$ & $\stackrel{\circ}{2}$ & $\stackrel{\circ}{2}$ & $\stackrel{\circ}{2}$ & $\stackrel{0}{2}$ & $\stackrel{0}{z}$ & $\frac{0}{2}$ & $\stackrel{0}{2}$ \\
\hline 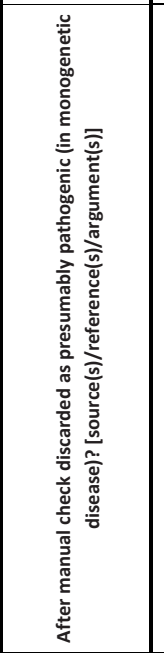 & 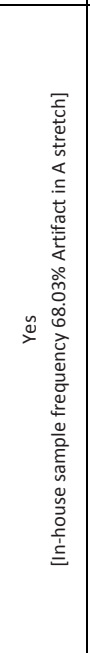 & 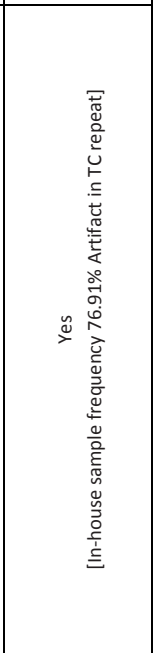 & 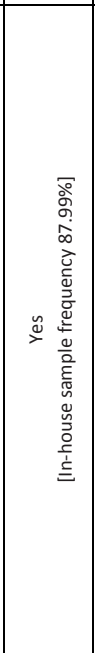 & 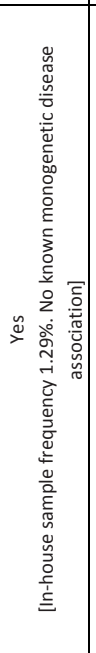 & 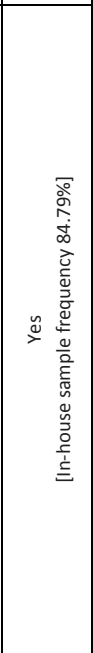 & 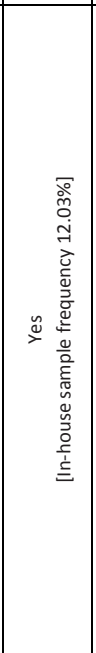 & 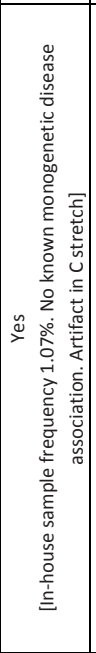 & 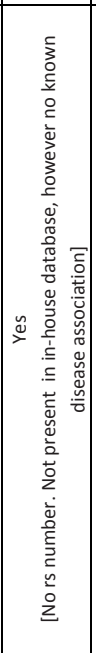 & 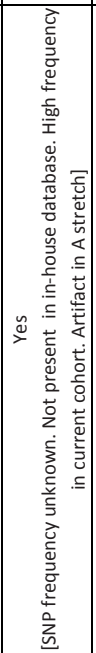 & 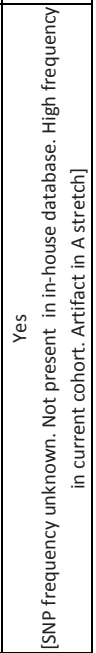 \\
\hline 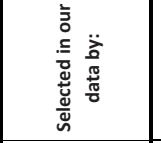 & 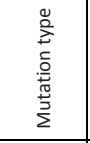 & 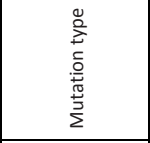 & 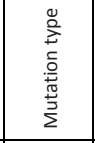 & 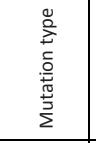 & 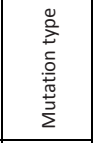 & 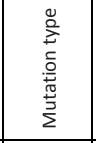 & 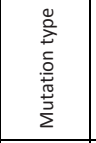 & 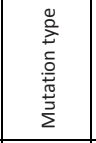 & 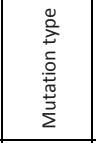 & 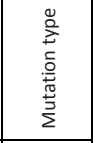 \\
\hline 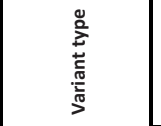 & 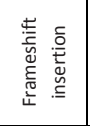 & 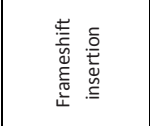 & 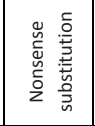 & 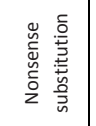 & 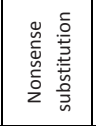 & 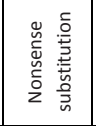 & 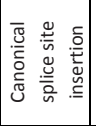 & 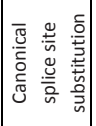 & 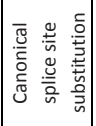 & 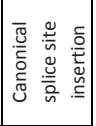 \\
\hline 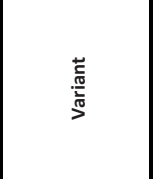 & 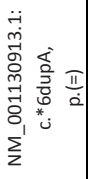 & 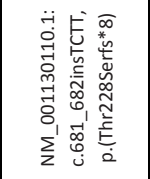 & 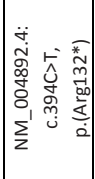 & 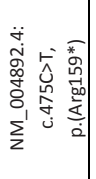 & 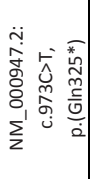 & 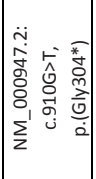 & 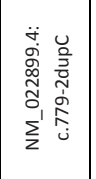 & 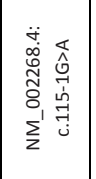 & 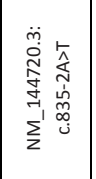 & 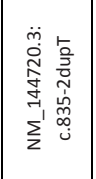 \\
\hline 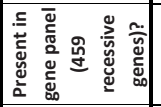 & $\stackrel{0}{2}$ & $\frac{0}{2}$ & $\frac{0}{2}$ & & $\frac{0}{2}$ & & $\stackrel{0}{2}$ & $\frac{0}{2}$ & $\frac{0}{2}$ & \\
\hline 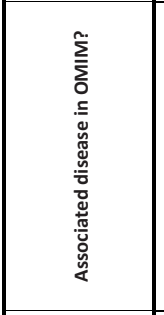 & 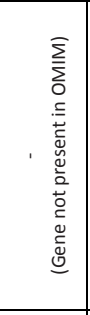 & 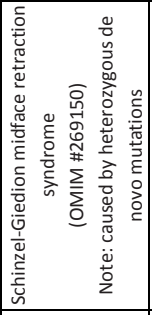 & 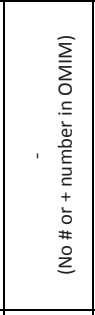 & & 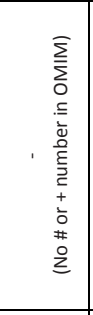 & & 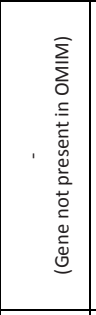 & 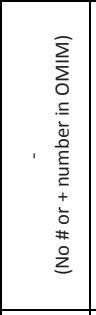 & 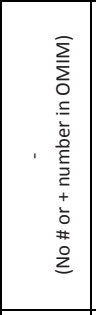 & \\
\hline 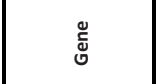 & 吝 & $\begin{array}{l}\overrightarrow{\vec{\omega}} \\
\stackrel{\vec{\omega}}{\mathrm{w}}\end{array}$ & $\begin{array}{l}\underset{\tilde{J}}{\tilde{W}} \\
\text { }\end{array}$ & & $\sum_{\substack{\alpha \\
\alpha}}^{\tilde{N}}$ & & 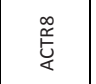 & 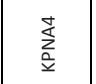 & $\begin{array}{l}\overrightarrow{\underline{0}} \\
\bar{\sum}\end{array}$ & \\
\hline 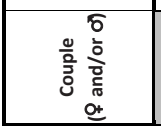 & 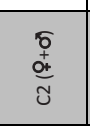 & 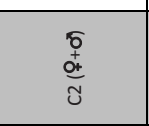 & $\frac{\Phi+}{\tilde{\sigma}}$ & $\frac{\delta}{\sigma}$ & 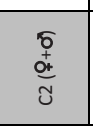 & $\overline{\sigma f}$ & 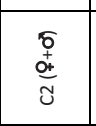 & 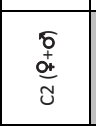 & $\frac{\partial t}{\sigma}$ & $\frac{\delta}{\tilde{c}}$ \\
\hline
\end{tabular}




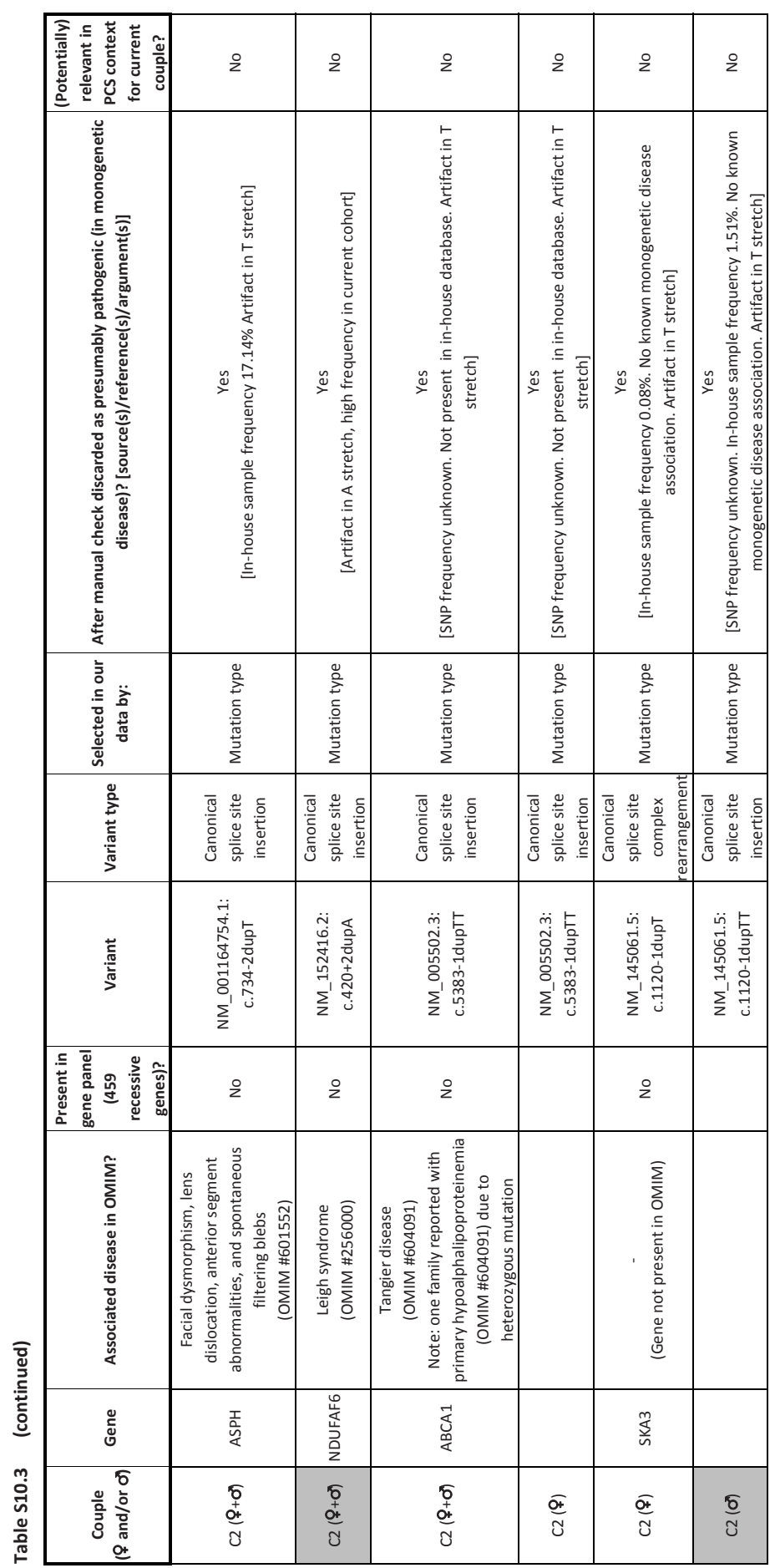


A comprehensive strategy for exome-based preconception carrier screening

\begin{tabular}{|c|c|c|c|c|c|c|c|c|c|c|}
\hline 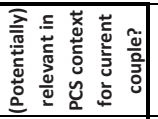 & $\stackrel{0}{z}$ & $\stackrel{0}{z}$ & $\stackrel{0}{2}$ & $\stackrel{0}{z}$ & $\stackrel{0}{z}$ & 울 & 울 & qo & 울 & 울 \\
\hline 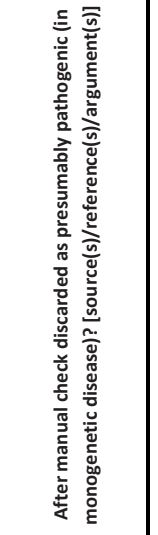 & 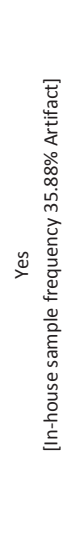 & 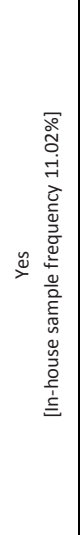 & 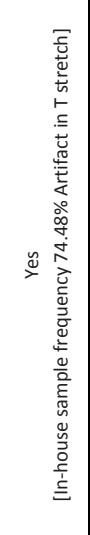 & 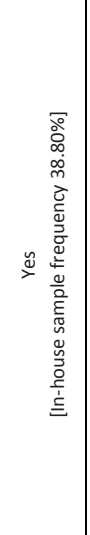 & 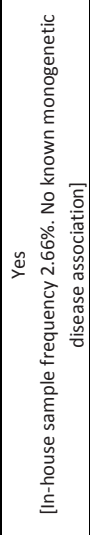 & 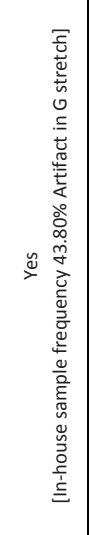 & 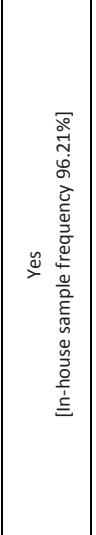 & 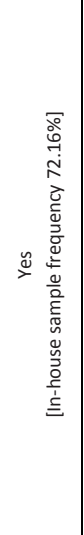 & 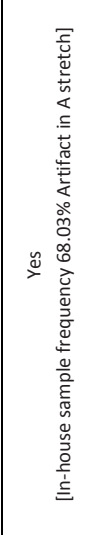 & 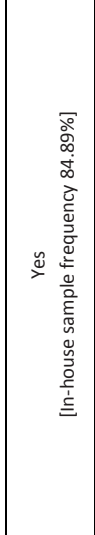 \\
\hline 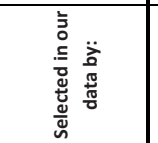 & 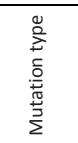 & 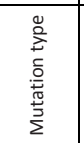 & 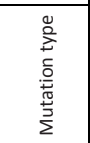 & 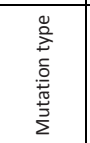 & 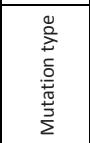 & 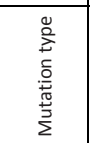 & 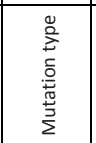 & 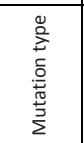 & 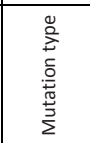 & 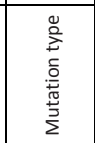 \\
\hline 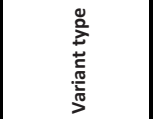 & 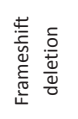 & 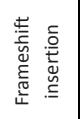 & 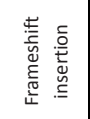 & 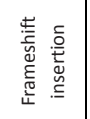 & 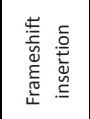 & 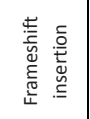 & 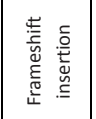 & 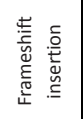 & 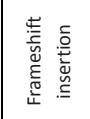 & 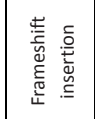 \\
\hline 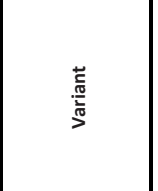 & 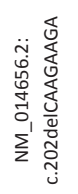 & $\sim$ & 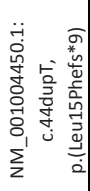 & 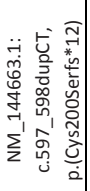 & 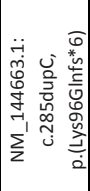 & 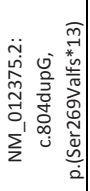 & 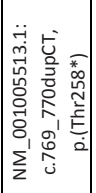 & 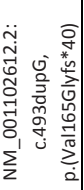 & 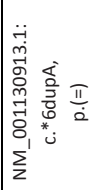 & 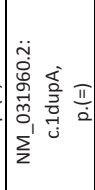 \\
\hline 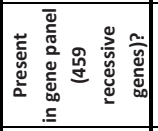 & $\stackrel{\circ}{z}$ & $\stackrel{2}{2}$ & $\stackrel{\circ}{2}$ & $\stackrel{\circ}{2}$ & & z & $\stackrel{\circ}{2}$ & 2 & 운 & 운 \\
\hline 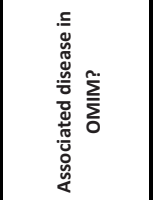 & 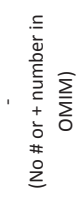 & 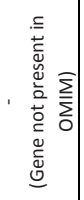 & 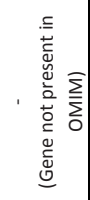 & 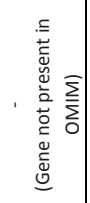 & & 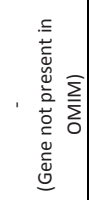 & 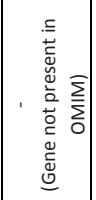 & 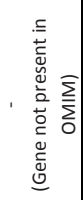 & 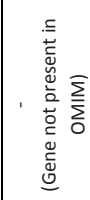 & 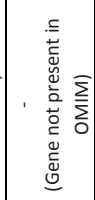 \\
\hline 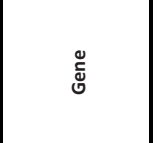 & $\begin{array}{l}\text { ơ } \\
0 \\
\frac{1}{4} \\
\frac{1}{2}\end{array}$ & 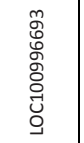 & 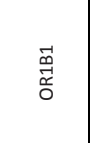 & 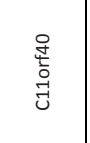 & & 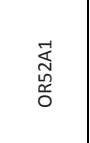 & $\begin{array}{l}\text { 导 } \\
\text { ỡ }\end{array}$ & 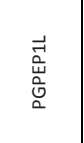 & 胥 & 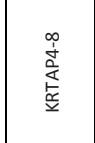 \\
\hline 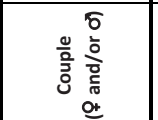 & $\begin{array}{l}\delta \\
+ \\
\stackrel{+}{+} \\
\frac{\tilde{J}}{z}\end{array}$ & $\begin{array}{l}\bar{p} \\
+ \\
\text { ot } \\
\bar{J} \\
z\end{array}$ & $\begin{array}{l}\bar{\delta} \\
+ \\
\text { ot } \\
\bar{J} \\
z\end{array}$ & $\begin{array}{l}\bar{\gamma} \\
+ \\
\text { ot } \\
\bar{J} \\
z\end{array}$ & $\begin{array}{l}\frac{\partial+}{\partial} \\
\bar{z}\end{array}$ & 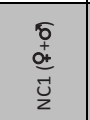 & $\begin{array}{l}\bar{\delta} \\
+ \\
o+ \\
\bar{J} \\
z\end{array}$ & 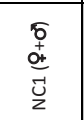 & 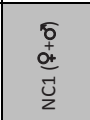 & 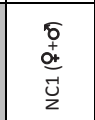 \\
\hline
\end{tabular}




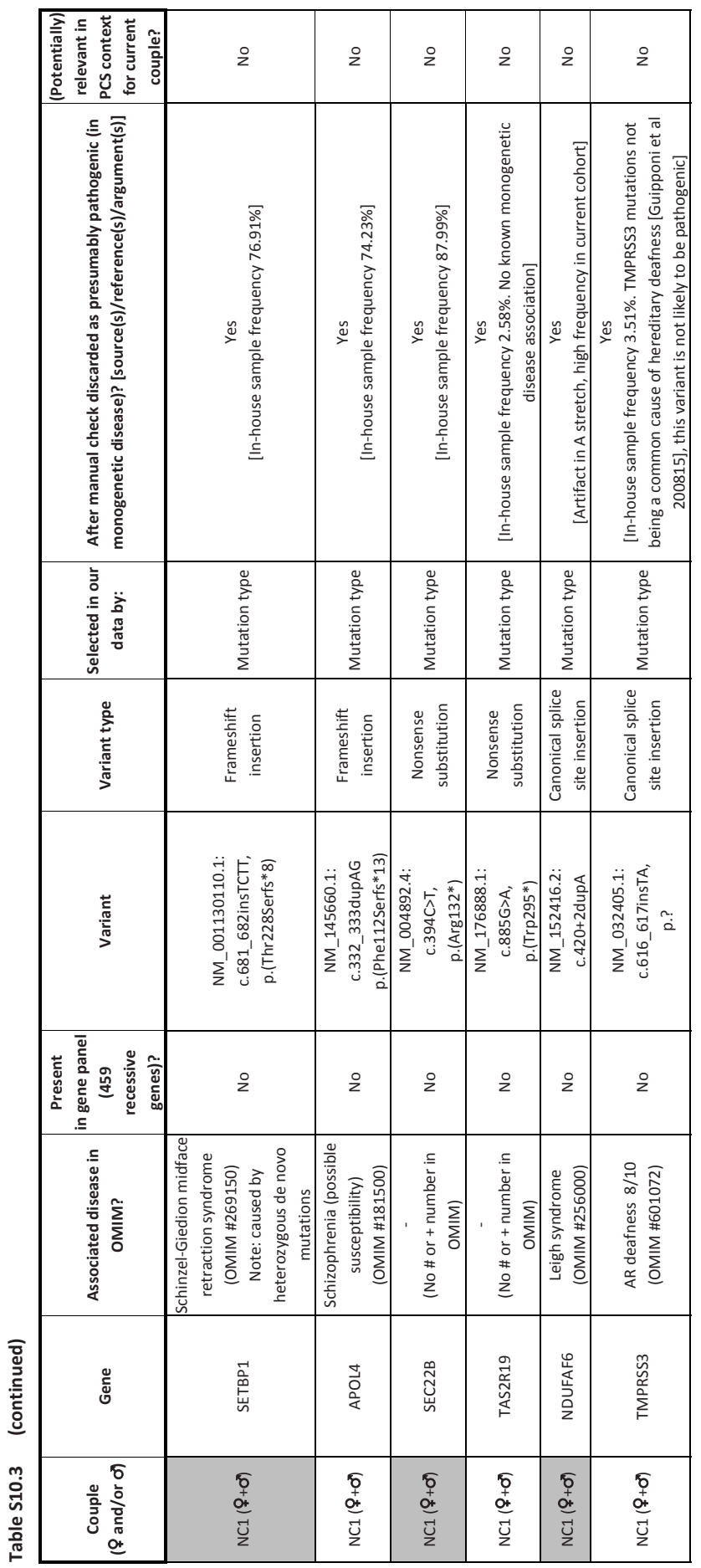




\begin{tabular}{|c|c|c|c|c|c|c|c|}
\hline 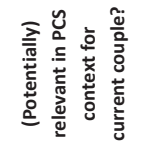 & $\stackrel{\check{\nu}}{\rightleftharpoons}$ & 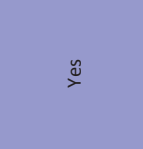 & $\stackrel{0}{2}$ & $\stackrel{\circ}{z}$ & $\stackrel{\circ}{2}$ & $\stackrel{\circ}{2}$ & $\stackrel{\circ}{2}$ \\
\hline 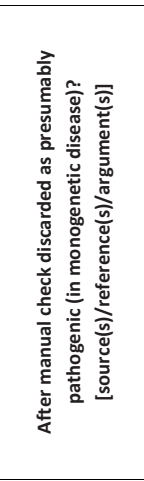 & 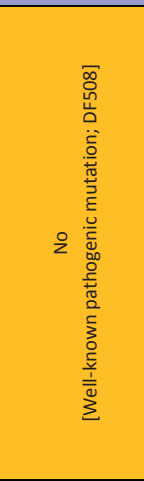 & 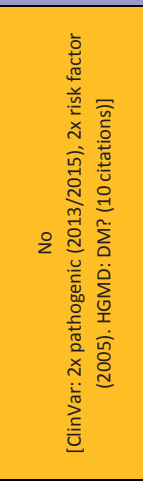 & 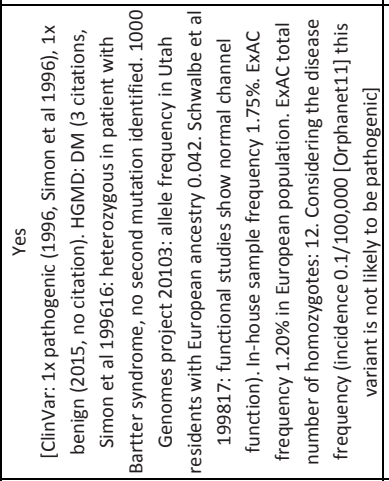 & 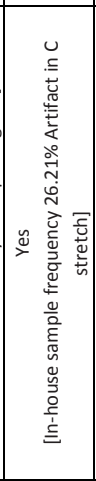 & 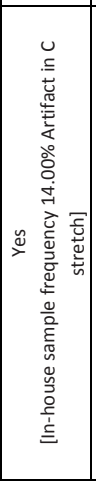 & 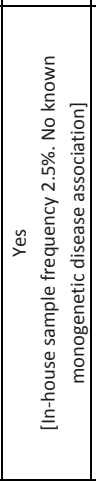 & 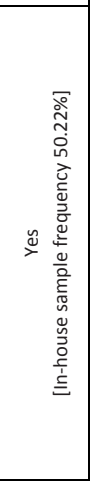 \\
\hline 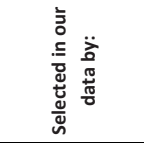 & 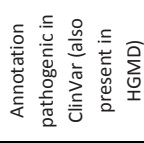 & 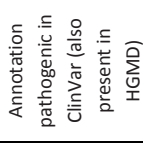 & 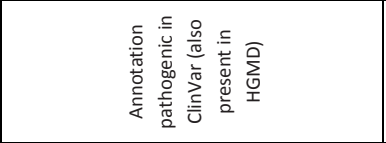 & 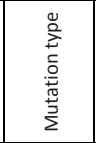 & 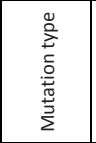 & 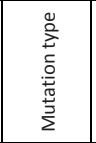 & 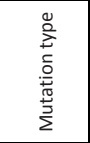 \\
\hline 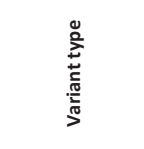 & 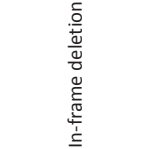 & 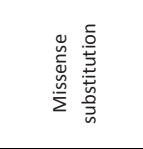 & 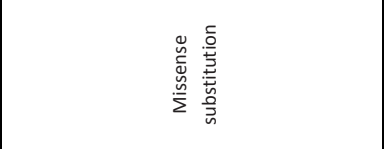 & 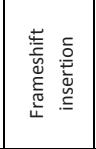 & 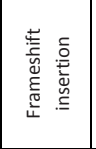 & 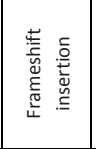 & 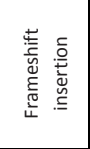 \\
\hline 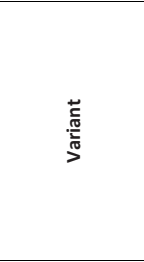 & 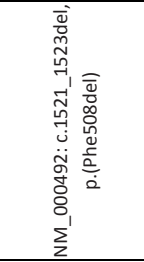 & 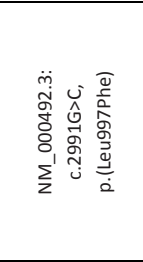 & 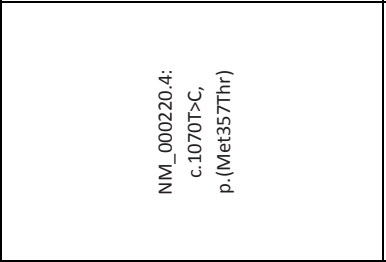 & 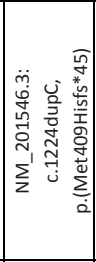 & 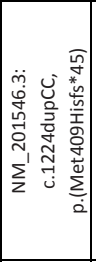 & 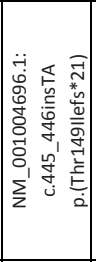 & 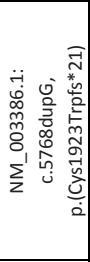 \\
\hline 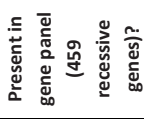 & $\stackrel{\check{\varpi}}{\check{\nu}}$ & & $\stackrel{巳}{\rightleftharpoons}$ & $\stackrel{0}{z}$ & & $\stackrel{\circ}{2}$ & $\stackrel{0}{2}$ \\
\hline 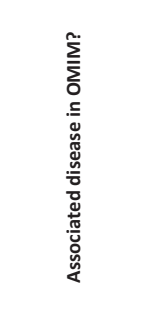 & 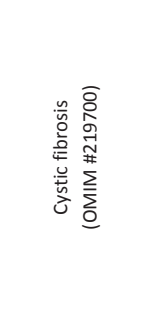 & & 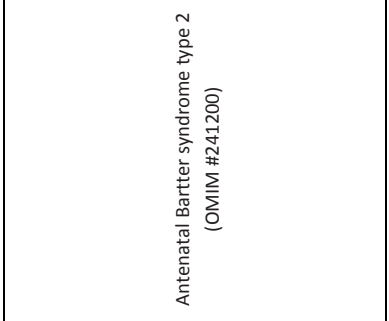 & 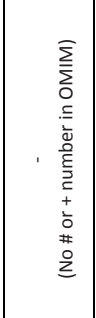 & & 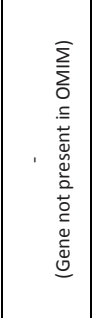 & 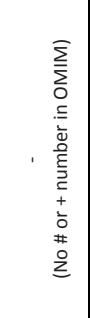 \\
\hline 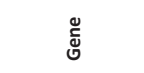 & 誉 & & $\underset{⿱ 亠 䒑}{\vec{z}}$ & $\begin{array}{l}\tilde{u} \\
\tilde{u}\end{array}$ & & $\begin{array}{l}\underset{\sim}{\Delta} \\
\underset{\widetilde{\sigma}}{\Delta}\end{array}$ & 录 \\
\hline 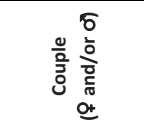 & $\begin{array}{l}\frac{\partial+}{\tilde{N}} \\
\frac{\mathrm{O}}{z}\end{array}$ & $\frac{\delta}{\frac{\delta}{Z}}$ & 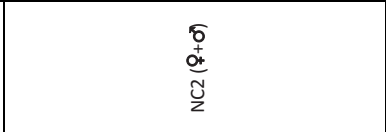 & 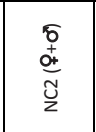 & $\begin{array}{l}\frac{\overline{O_{t}}}{\tilde{N}} \\
\frac{2}{2}\end{array}$ & 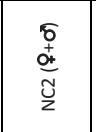 & 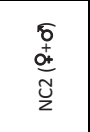 \\
\hline
\end{tabular}




\begin{tabular}{|c|c|c|c|c|c|c|c|c|c|}
\hline 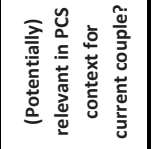 & $z^{\circ}$ & z & $z_{z}^{\circ}$ & $\stackrel{0}{z}$ & $\stackrel{0}{z}$ & $\stackrel{0}{z}$ & $\stackrel{0}{z}$ & $\stackrel{0}{z}$ & $\stackrel{\circ}{z}$ \\
\hline 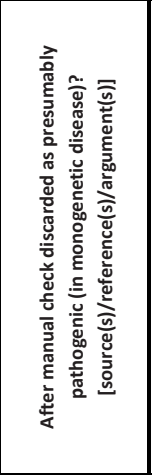 & 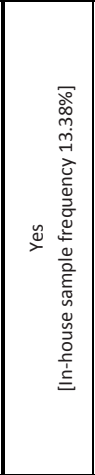 & 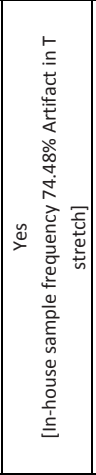 & 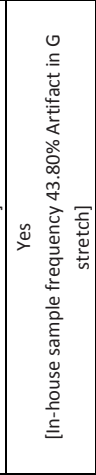 & 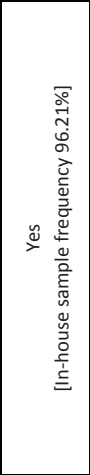 & 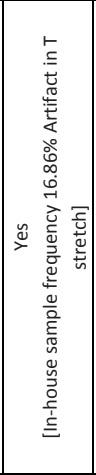 & 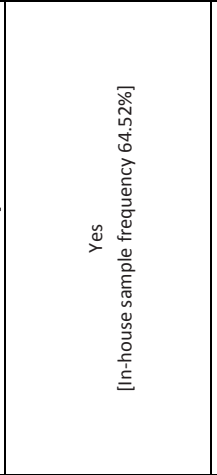 & 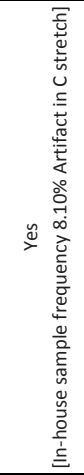 & 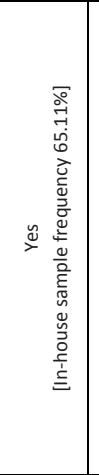 & 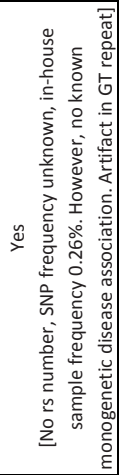 \\
\hline 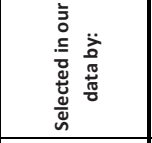 & 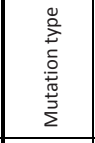 & 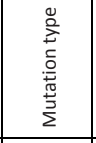 & 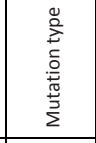 & 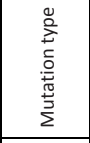 & 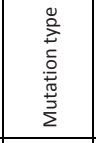 & 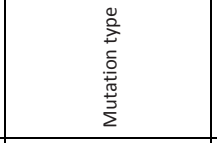 & 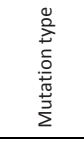 & 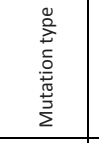 & 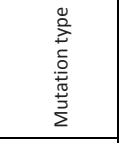 \\
\hline 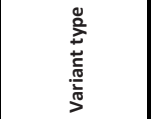 & 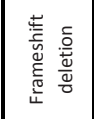 & 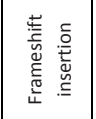 & 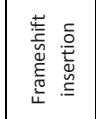 & 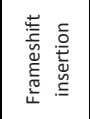 & 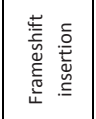 & 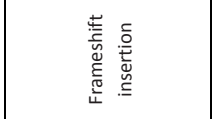 & 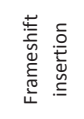 & 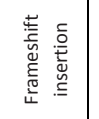 & 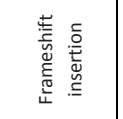 \\
\hline 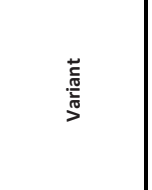 & 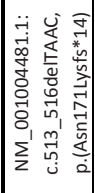 & 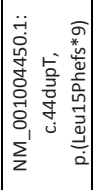 & 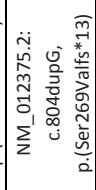 & 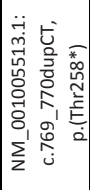 & 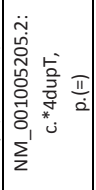 & 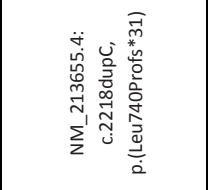 & 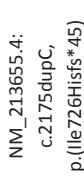 & 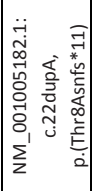 & 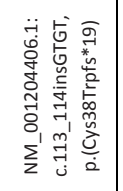 \\
\hline 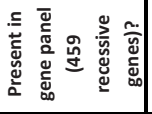 & $\stackrel{0}{z}$ & z & z & $\stackrel{0}{z}$ & z & $\stackrel{0}{z}$ & $\stackrel{0}{z}$ & $\stackrel{0}{2}$ & z \\
\hline 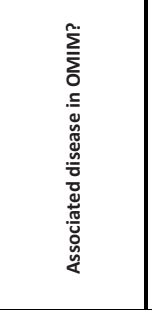 & 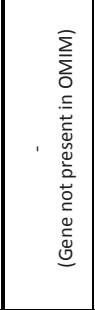 & 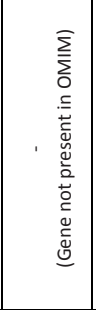 & 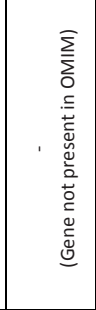 & 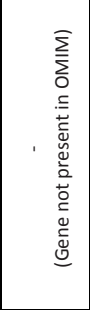 & 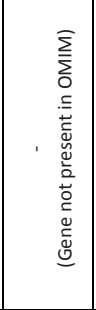 & 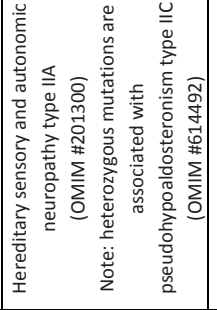 & & 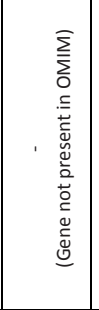 & 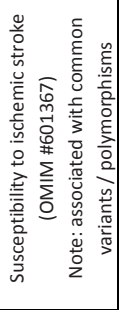 \\
\hline 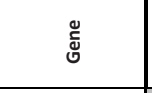 & $\begin{array}{l}\text { Oु } \\
\tilde{y} \\
\tilde{c} \\
\end{array}$ & $\begin{array}{l}\overrightarrow{\vec{d}} \\
\text { वे }\end{array}$ & 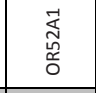 & $\begin{array}{l}\text { 势 } \\
\text { वे } \\
\text { o } \\
\end{array}$ & 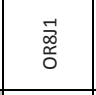 & $\frac{\vec{y}}{3}$ & & $\begin{array}{l}\text { 己े } \\
\text { ơ } \\
\end{array}$ & 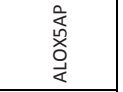 \\
\hline 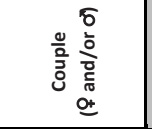 & 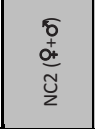 & 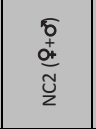 & 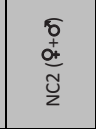 & 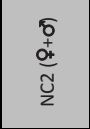 & 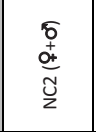 & $\frac{\bar{\partial}}{\frac{\partial}{z}}$ & $\frac{\frac{\pi}{2}}{\frac{\pi}{5}}$ & $\begin{array}{l}5 \\
\vdots \\
\frac{1}{0+} \\
\tilde{N} \\
z\end{array}$ & $\begin{array}{l}\frac{\partial+}{\tilde{O}} \\
\frac{\partial}{z}\end{array}$ \\
\hline
\end{tabular}


A comprehensive strategy for exome-based preconception carrier screening

\begin{tabular}{|c|c|c|c|c|c|c|c|c|c|c|c|}
\hline 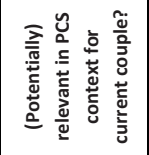 & $\stackrel{\circ}{z}$ & $\stackrel{0}{2}$ & $\stackrel{0}{z}$ & $\stackrel{\circ}{2}$ & $\stackrel{\circ}{2}$ & $\stackrel{\circ}{z}$ & $\stackrel{0}{2}$ & $\stackrel{\circ}{z}$ & $\stackrel{0}{z}$ & $\stackrel{\circ}{z}$ & $\stackrel{0}{2}$ \\
\hline 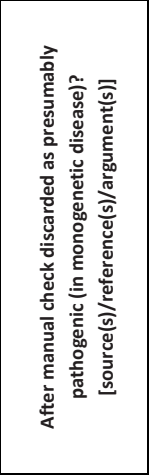 & 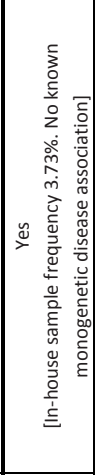 & 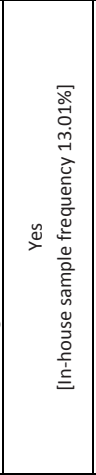 & 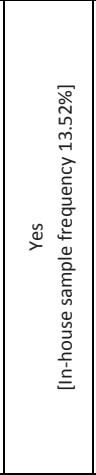 & 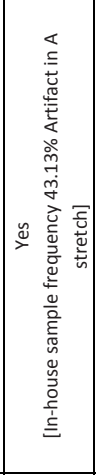 & 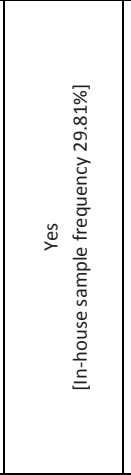 & 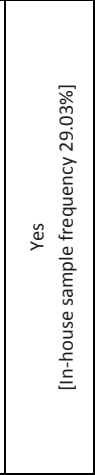 & 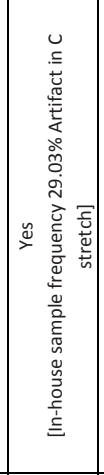 & 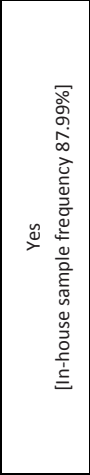 & 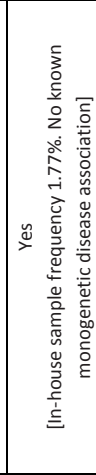 & 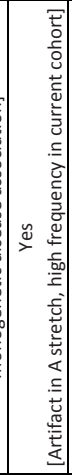 & 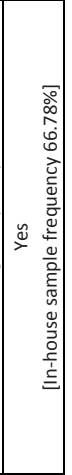 \\
\hline 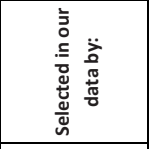 & 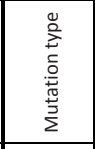 & 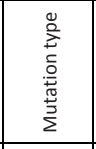 & 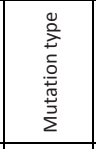 & 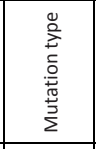 & 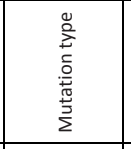 & 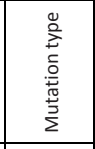 & 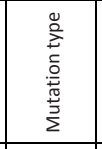 & 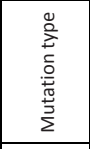 & 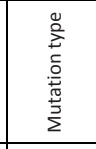 & 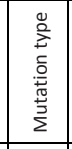 & 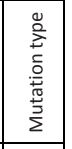 \\
\hline 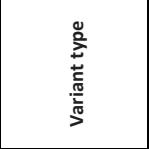 & 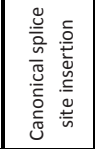 & 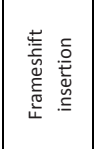 & 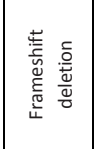 & 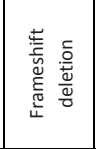 & 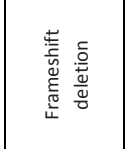 & 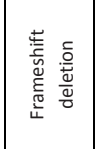 & 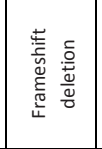 & 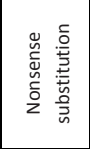 & 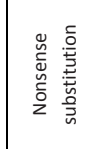 & 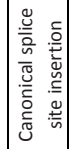 & 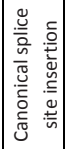 \\
\hline 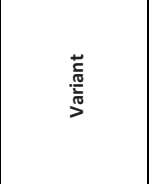 & 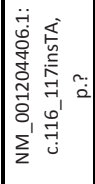 & 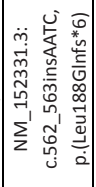 & 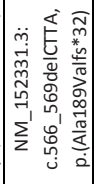 & 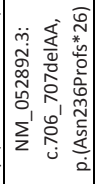 & 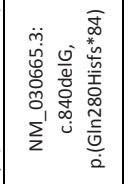 & 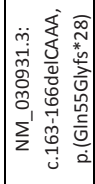 & 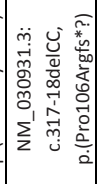 & 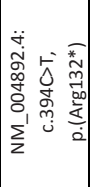 & 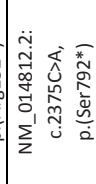 & 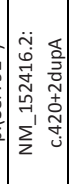 & 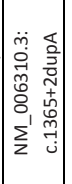 \\
\hline 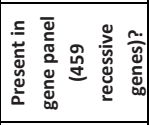 & & 운 & & 울 & 울 & zo & & 울 & $\frac{0}{2}$ & 2 & 20 \\
\hline 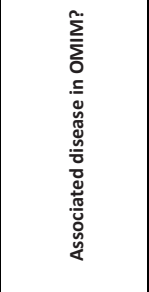 & & 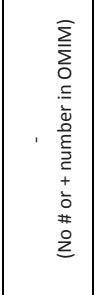 & & 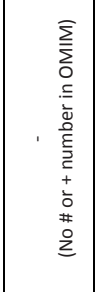 & 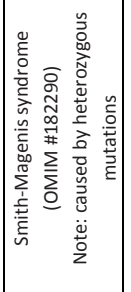 & 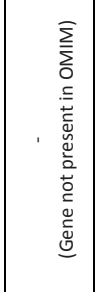 & & 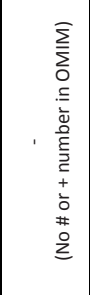 & 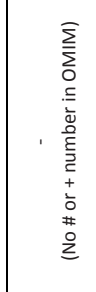 & 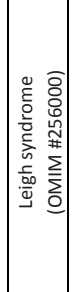 & 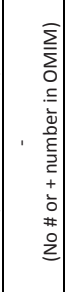 \\
\hline 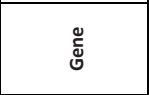 & & 莺 & & $\begin{array}{l}\overrightarrow{\overrightarrow{1}} \\
\frac{\partial}{2}\end{array}$ & $\frac{\vec{\pi}}{\propto}$ & 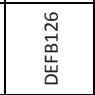 & & 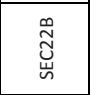 & 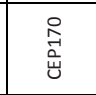 & 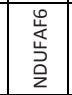 & $\begin{array}{l}\text { ñ } \\
\frac{\hat{\omega}}{2} \\
\frac{\hat{n}}{2}\end{array}$ \\
\hline 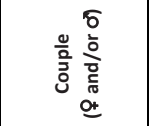 & $\begin{array}{l}\bar{\sigma} \\
\text { o+ } \\
\tilde{\sigma} \\
z\end{array}$ & $\begin{array}{l}\bar{\sigma} \\
\stackrel{+}{+} \\
\frac{1}{\tilde{T}} \\
z\end{array}$ & 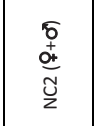 & 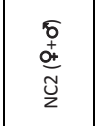 & 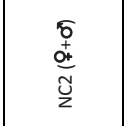 & $\begin{array}{l}\frac{\sigma+}{\sigma} \\
\frac{\sigma}{z}\end{array}$ & 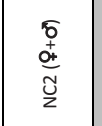 & 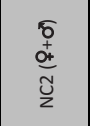 & 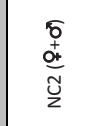 & $\begin{array}{l}\bar{\delta} \\
\text { ot } \\
\tilde{N} \\
z\end{array}$ & 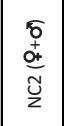 \\
\hline
\end{tabular}




\begin{tabular}{|c|c|c|c|c|c|c|}
\hline 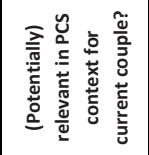 & $\stackrel{0}{z}$ & $\stackrel{\check{~}}{\rightleftharpoons}$ & $\stackrel{0}{2}$ & $\stackrel{0}{2}$ & $\stackrel{0}{2}$ & $\stackrel{0}{z}$ \\
\hline 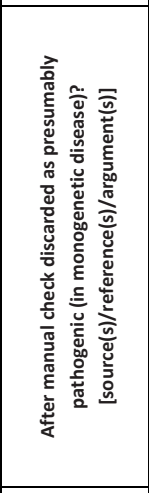 & 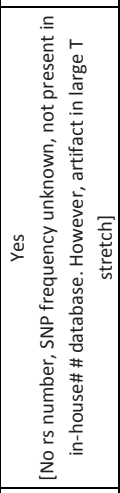 & 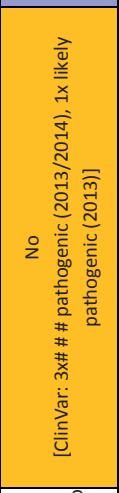 & 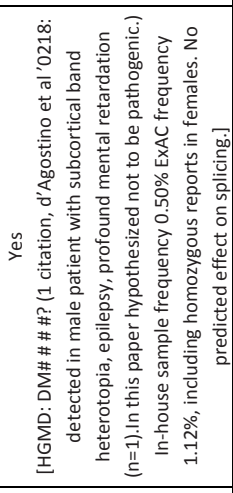 & 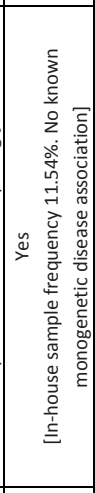 & 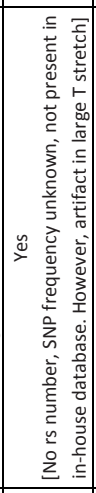 & 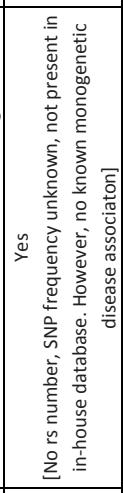 \\
\hline 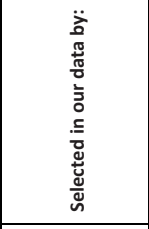 & 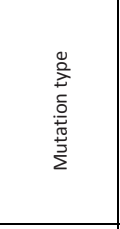 & 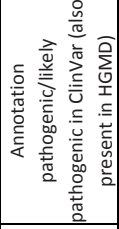 & 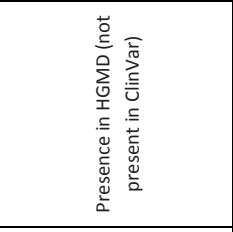 & 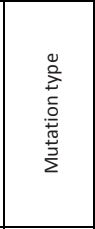 & 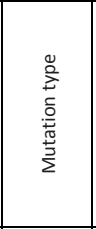 & 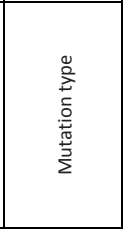 \\
\hline 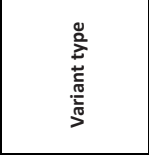 & 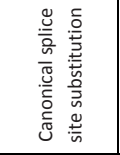 & 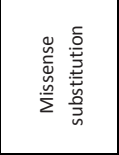 & 旁 & 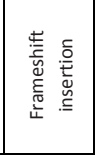 & 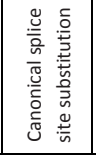 & 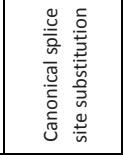 \\
\hline 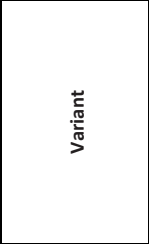 & 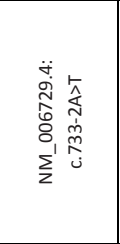 & 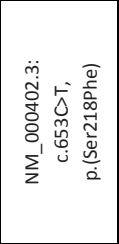 & 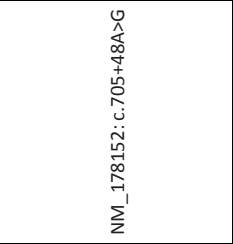 & 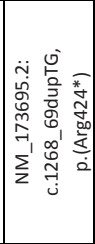 & 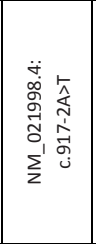 & 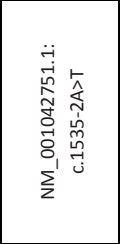 \\
\hline 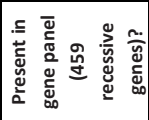 & zo & $\stackrel{\check{\varpi}}{\rightleftharpoons}$ & $\stackrel{\check{\nu}}{\rightleftharpoons}$ & 울 & 울 & zo \\
\hline 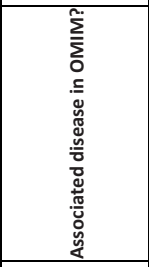 & 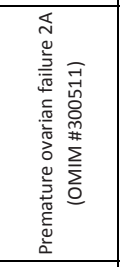 & 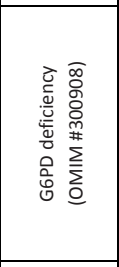 & 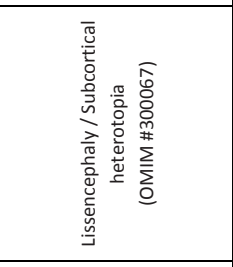 & 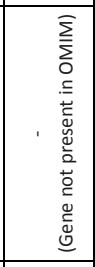 & 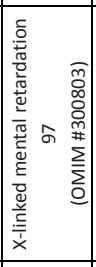 & 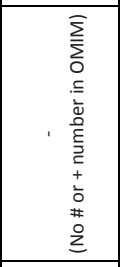 \\
\hline 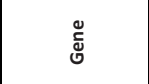 & $\begin{array}{l}\frac{1}{2} \\
\frac{1}{4} \\
\frac{1}{\alpha}\end{array}$ & $\begin{array}{l}\text { ㅇ⿺ㅇ } \\
\text { on }\end{array}$ & ఫ્ઠ & $\begin{array}{l}\text { Õ } \\
\text { 종 }\end{array}$ & 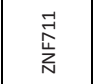 & $\begin{array}{l}\frac{\tilde{Q}}{5} \\
\stackrel{5}{n}\end{array}$ \\
\hline 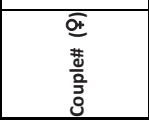 & $\widetilde{J}$ & $\widetilde{\sigma}$ & $\widetilde{\sigma}$ & $\tilde{\sigma}$ & $\widetilde{\sigma}$ & $\widetilde{\sigma}$ \\
\hline
\end{tabular}




\begin{tabular}{|c|c|c|c|c|}
\hline 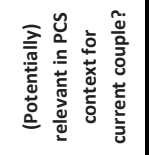 & z & z & $\stackrel{\check{\nu}}{\check{\nu}}$ & $\frac{0}{2}$ \\
\hline 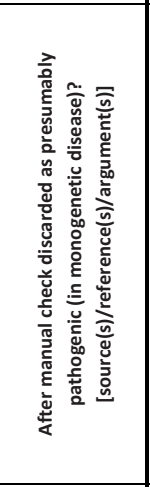 & 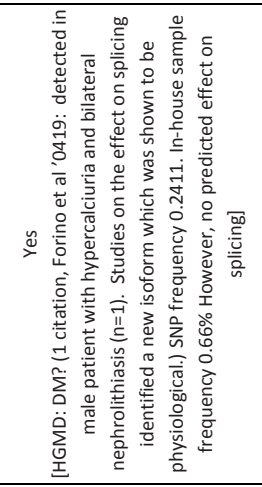 & 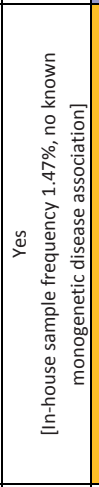 & 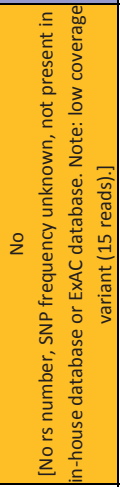 & 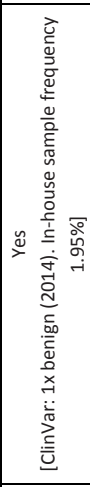 \\
\hline 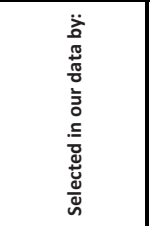 & 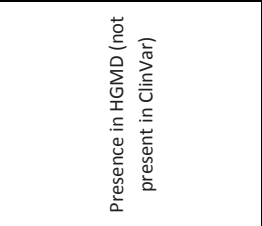 & 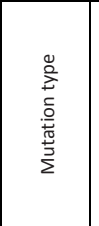 & 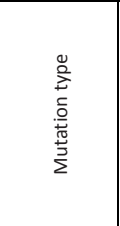 & 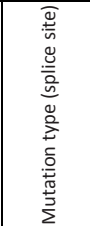 \\
\hline 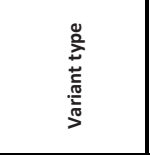 & 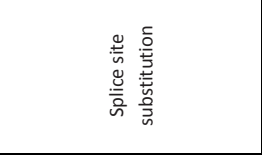 & 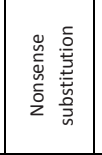 & 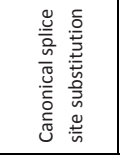 & 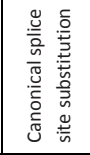 \\
\hline 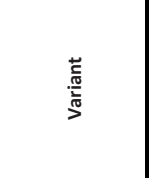 & 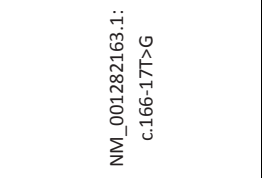 & 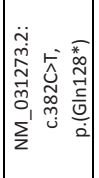 & 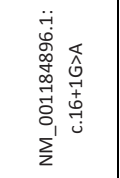 & 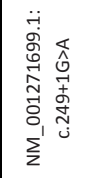 \\
\hline 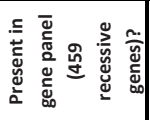 & $\stackrel{\circ}{2}$ & $\stackrel{0}{2}$ & $\stackrel{0}{z}$ & 울 \\
\hline 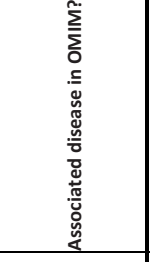 & 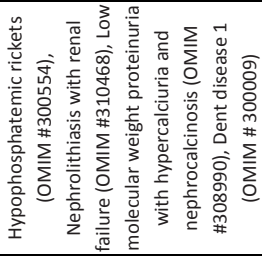 & 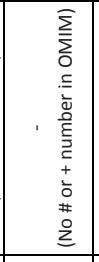 & 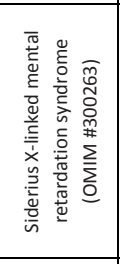 & 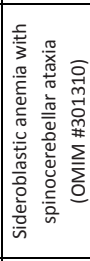 \\
\hline 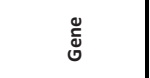 & 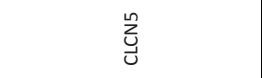 & 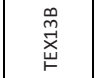 & 喜 & $\begin{array}{l}\hat{0} \\
\text { Q }\end{array}$ \\
\hline 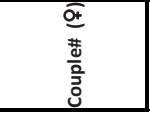 & $\tilde{z}$ & $\tilde{Z}$ & 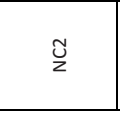 & $\tilde{O}$ \\
\hline
\end{tabular}




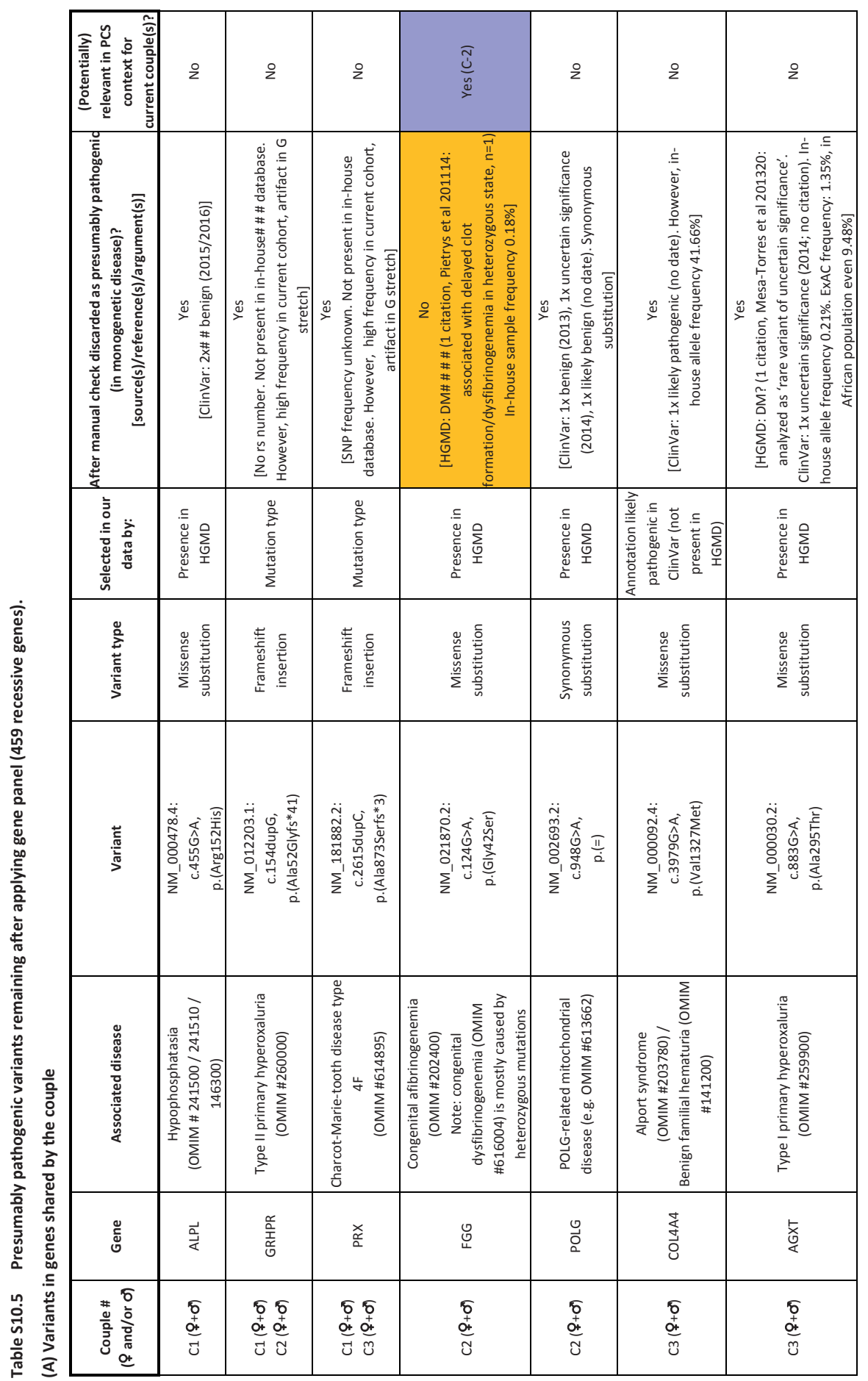




\begin{tabular}{|c|c|c|c|c|c|c|}
\hline 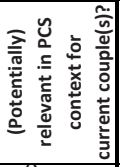 & $\stackrel{0}{z}$ & $\stackrel{0}{z}$ & $\begin{array}{l}\frac{\bar{\varphi}}{\dot{u}} \\
\stackrel{\tilde{y}}{x}\end{array}$ & $\stackrel{2}{2}$ & 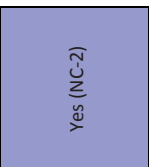 & 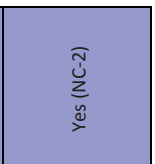 \\
\hline 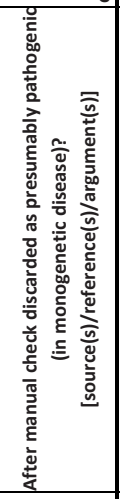 & 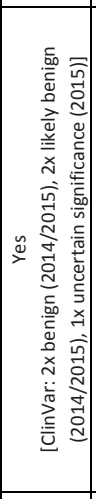 & 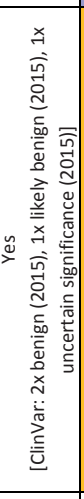 & 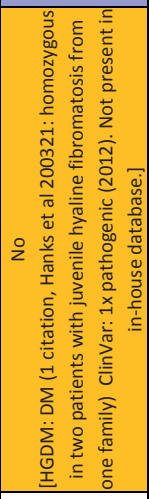 & 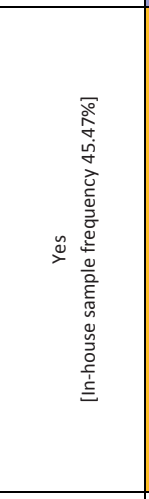 & 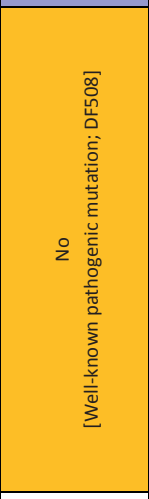 & 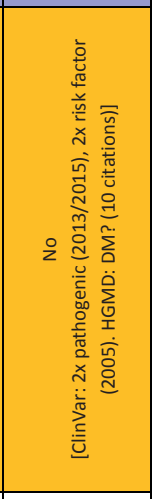 \\
\hline 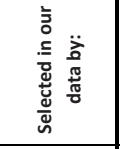 & 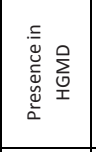 & 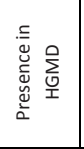 & 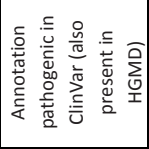 & 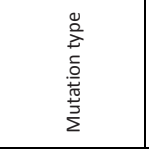 & 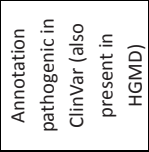 & 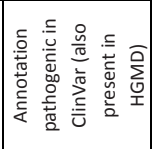 \\
\hline 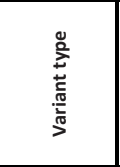 & 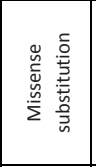 & 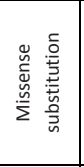 & 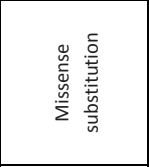 & 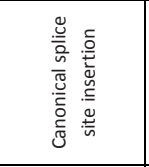 & 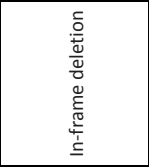 & 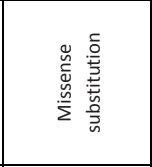 \\
\hline 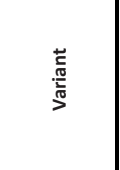 & 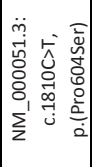 & 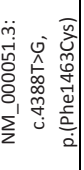 & 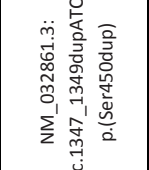 & 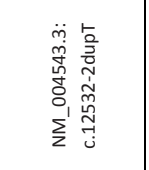 & 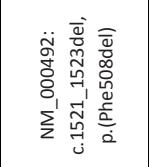 & 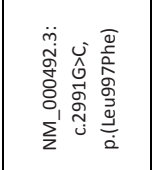 \\
\hline 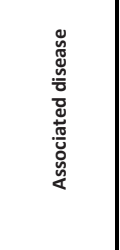 & 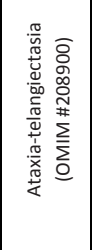 & & 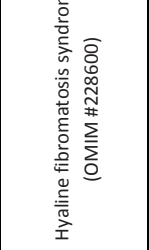 & 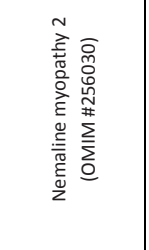 & 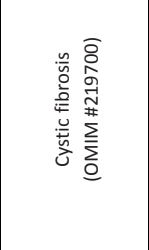 & \\
\hline $\begin{array}{l}\text { ๊๊̃ } \\
\text { }\end{array}$ & $\sum_{\mathbf{k}}$ & & $\underset{\tilde{x}}{\tilde{x}}$ & $\underline{\tilde{z}}$ & 誉 & \\
\hline 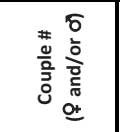 & $\frac{\overline{\alpha f}}{\tilde{O}}$ & $\frac{\bar{\sigma}}{0}$ & $\begin{array}{l}5 \\
+ \\
+1 \\
\text { o+ } \\
8\end{array}$ & 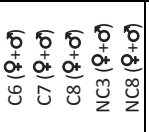 & $\frac{\overline{\partial f}}{\tilde{N}}$ & $\frac{\delta}{\tilde{N}}$ \\
\hline
\end{tabular}




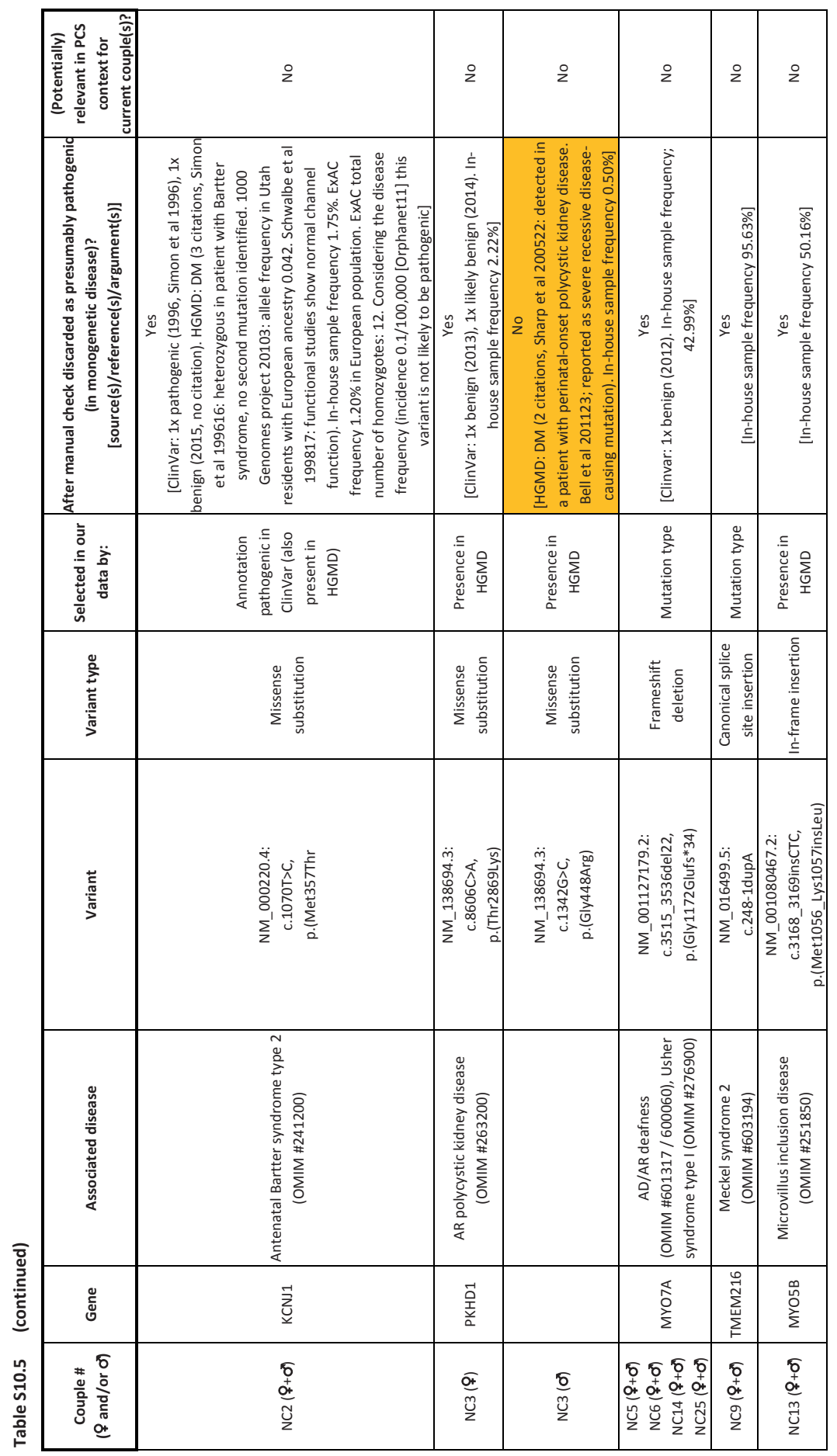




\begin{tabular}{|c|c|c|}
\hline 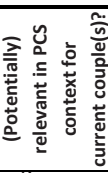 & $\stackrel{0}{z}$ & $\stackrel{2}{2}$ \\
\hline 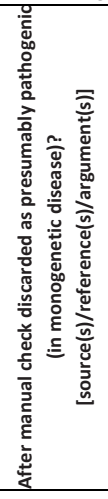 & 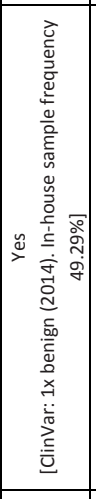 & 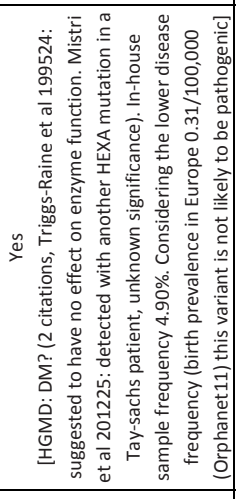 \\
\hline 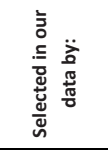 & 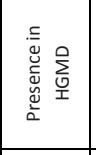 & 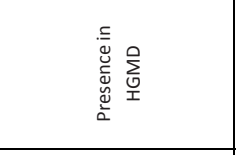 \\
\hline 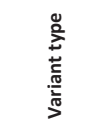 & 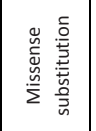 & 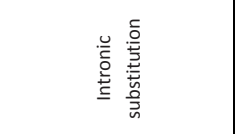 \\
\hline 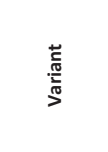 & 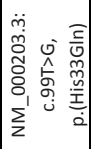 & 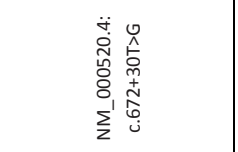 \\
\hline 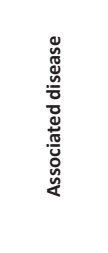 & 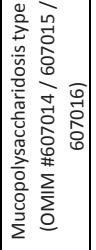 & 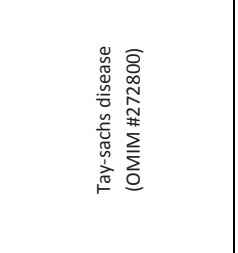 \\
\hline 芯 & $\stackrel{\mathbb{J}}{\underline{a}}$ & $\begin{array}{l}\underset{\mathbb{x}}{\underline{x}} \\
\end{array}$ \\
\hline 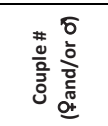 & 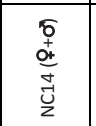 & $\begin{array}{l}5 \\
0 \\
+ \\
o+ \\
o \\
己 \\
z\end{array}$ \\
\hline
\end{tabular}




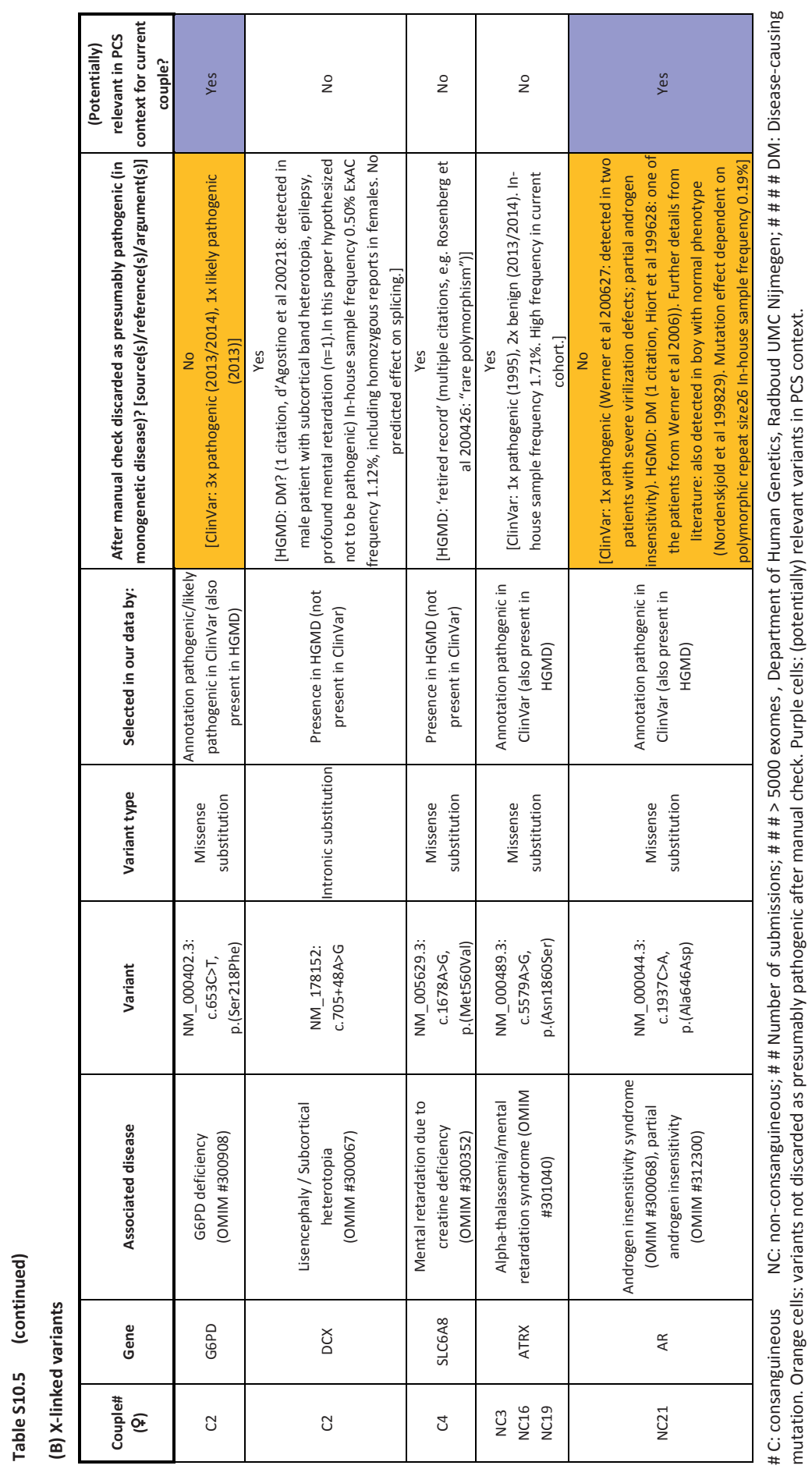




\section{Supplementary references}

1. Allikmets $\mathrm{R}$, Singh $\mathrm{N}$, Sun $\mathrm{H}$, et al. A photoreceptor cell-specific ATP-binding transporter gene (ABCR) is mutated in recessive Stargardt macular dystrophy. Nat Genet. 1997;15(3):236-246.

2. Jacobson S.G. CAV, Bascom R.A., McInnes R.R., Sheffield V.C., Stone E.M. Variable expression of retinitis pigmentosa in patients with digenic inheritance of peripherin/RDS and ROM-1 gene mutations. Invest.Ophthalmol.Vis.Sci. 1995;36(S913).

3. Abecasis GR, Altshuler D, Auton A, et al. A map of human genome variation from population-scale sequencing. Nature. 2010;467(7319):1061-1073.

4. Coco RM, Telleria JJ, Sanabria MR, Rodriguez-Rua E, Garcia MT. PRPH2 (Peripherin/RDS) mutations associated with different macular dystrophies in a Spanish population: a new mutation. Eur J Ophthalmol. 2010;20(4):724-732.

5. Kruer MC, Jepperson T, Dutta S, et al. Mutations in gamma adducin are associated with inherited cerebral palsy. Ann Neurol. 2013;74(6):805-814.

6. Jimenez-Cervantes C, Germer S, Gonzalez P, Sanchez J, Sanchez CO, Garcia-Borron JC. Thr40 and Met122 are new partial loss-of-function natural mutations of the human melanocortin 1 receptor. FEBS Letters. 2001;508(1):44-48.

7. Ibarrola-Villava M, Pena-Chilet M, Llorca-Cardenosa MJ, et al. Modeling MC1R rare variants: a structural evaluation of variants detected in a Mediterranean case-control study. J Invest Dermatol. 2014;134(4):1146-1149.

8. Puig-Butille JA, Carrera C, Kumar R, et al. Distribution of MC1R variants among melanoma subtypes: p.R163Q is associated with lentigo maligna melanoma in a Mediterranean population. Br J Dermatol. 2013;169(4):804-811.

9. Mahjabeen I, Baig RM, Masood N, et al. Genetic variations in XRCC1 gene in sporadic head and neck cancer (HNC) patients. Pathol Oncol Res. 2013;19(2):183-188.

10. Calvo SE, Tucker EJ, Compton AG, et al. High-throughput, pooled sequencing identifies mutations in NUBPL and FOXRED1 in human complex I deficiency. Nat Genet. 2010;42(10):851-858.

11. Orphanet. Orphanet Report Series - Prevalence of rare diseases: Bibliographic data.

http://www.orpha.net/orphacom/cahiers/docs/GB/Prevalence_of_rare_diseases_by_alphabetical_list. pdf and

http://www.orpha.net/orphacom/cahiers/docs/GB/Prevalence_of_rare_diseases_by_decreasing_prev alence_or_cases.pdf. 2016.

12. Lin $\mathrm{H}$, Sinner MF, Brody JA, et al. Targeted sequencing in candidate genes for atrial fibrillation: the Cohorts for Heart and Aging Research in Genomic Epidemiology (CHARGE) Targeted Sequencing Study. Heart Rhythm. 2014;11(3):452-457.

13. Bernard O, Tojcic J, Journault K, Perusse L, Guillemette C. Influence of nonsynonymous polymorphisms of UGT1A8 and UGT2B7 metabolizing enzymes on the formation of phenolic and acyl glucuronides of mycophenolic acid. Drug Metab Dispos. 2006;34(9):1539-1545.

14. Pietrys D, Balwierz W, Iwaniec T, Vorjohann S, Neerman-Arbez M, Undas A. Two different fibrinogen gene mutations associated with bleeding in the same family (A alphaGly13Glu and gammaGly16Ser) and their impact on fibrin clot properties: fibrinogen Krakow II and Krakow III. Thromb Haemos. 2011;106(3):558-560.

15. Guipponi M, Antonarakis SE, Scott HS. TMPRSS3, a type II transmembrane serine protease mutated in non-syndromic autosomal recessive deafness. Front Biosci. 2008;13:1557-1567.

16. Simon DB, Karet FE, Rodriguez-Soriano J, et al. Genetic heterogeneity of Bartter's syndrome revealed by mutations in the K+ channel, ROMK. Nat Genet. 1996;14(2):152-156.

17. Schwalbe RA, Bianchi L, Accili EA, Brown AM. Functional consequences of ROMK mutants linked to antenatal Bartter's syndrome and implications for treatment. Hum Mol Genet. 1998;7(6):975-980.

18. D'Agostino MD, Bernasconi A, Das S, et al. Subcortical band heterotopia (SBH) in males: clinical, imaging and genetic findings in comparison with females. Brain. 2002;125(Pt 11):2507-2522.

19. Forino M, Graziotto R, Tosetto E, Gambaro G, D'Angelo A, Anglani F. Identification of a novel splice site mutation of CLCN5 gene and characterization of a new alternative 5' UTR end of CIC-5 mRNA in human renal tissue and leukocytes. J Hum Genet. 2004;49(1):53-60. 
20. Mesa-Torres N, Fabelo-Rosa I, Riverol D, et al. The role of protein denaturation energetics and molecular chaperones in the aggregation and mistargeting of mutants causing primary hyperoxaluria type I. PloS One. 2013;8(8):e71963.

21. Hanks S, Adams S, Douglas J, et al. Mutations in the gene encoding capillary morphogenesis protein 2 cause juvenile hyaline fibromatosis and infantile systemic hyalinosis. Am J Hum Genet. 2003;73(4): 791-800.

22. Sharp AM, Messiaen LM, Page G, et al. Comprehensive genomic analysis of PKHD1 mutations in ARPKD cohorts. J Med Genet. 2005;42(4):336-349.

23. Bell CJ, Dinwiddie DL, Miller NA, et al. Carrier testing for severe childhood recessive diseases by nextgeneration sequencing. Sci Transl Med. 2011;3(65):65ra64.

24. Triggs-Raine B, Richard M, Wasel N, Prence EM, Natowicz MR. Mutational analyses of Tay-Sachs disease: studies on Tay-Sachs carriers of French Canadian background living in New England. Am J Hum Genet. 1995;56(4):870-879.

25. Mistri M, Tamhankar PM, Sheth F, et al. Identification of novel mutations in HEXA gene in children affected with Tay Sachs disease from India. PloS One. 2012;7(6):e39122.

26. Rosenberg EH, Almeida LS, Kleefstra T, et al. High prevalence of SLC6A8 deficiency in X-linked mental retardation. Am J Hum Genet. 2004;75(1):97-105.

27. Werner R, Holterhus PM, Binder G, et al. The A645D mutation in the hinge region of the human androgen receptor (AR) gene modulates $A R$ activity, depending on the context of the polymorphic glutamine and glycine repeats. J Clin Endocrinol Metab. 2006;91(9):3515-3520.

28. Hiort O, Sinnecker GH, Holterhus PM, Nitsche EM, Kruse K. The clinical and molecular spectrum of androgen insensitivity syndromes. Am J Med Genet. 1996;63(1):218-222.

29. Nordenskjold A, Soderhall S. An androgen receptor gene mutation (A645D) in a boy with a normal phenotype. Hum Mutat. 1998;11(4):339. 
Discussion and summary 



\section{CHAPTER 11}

General discussion and future perspectives 

The work presented in this thesis provides new opportunities to prevent the transmission of mitochondrial diseases based on novel insights in mitochondrial genetics and the occurrence and transmission of mitochondrial diseases, as well as guidance for their reproductive genetic counseling. Reproductive choices have been introduced in the general overview (Chapter 2 ) and (for mtDNA mutations) summarized in the flowchart in Chapter 7. Here this topic will be further discussed in a broader context, incorporating our findings as well as international developments in the field. Genetic testing initiated by affected individuals or their family (part 1 and part 2) is distinguished from genetic testing as screening in individuals without a known genetic defect in the family (part 3).

For a patient and his or her parents, the very start is identifying the genetic defect that causes the mitochondrial disease in the patient, hereby not forgetting to remain critical whether the mutation is indeed explanatory. This may seem a 'sine qua non', however in clinical practice this is not always that obvious as illustrated by case 5 , a patient carrying a low percentage of the m.3243A $>\mathrm{G}$ mutation in combination with being compound heterozygous for POLG1 mutations, in Chapter 7 and presumably by some more reported cases. ${ }^{\text {e.g., } 1}$ Recurrence risk and preferred reproductive option(s) depend on multiple factors, the most important one being the primary genetic defect (mitochondrial DNA versus nuclear DNA).

\section{Part 1: Preventing the transmission of mutations in the mitochondrial DNA (mtDNA)}

Figure 11.1 summarizes reproductive counseling, i.e. reproductive options related to recurrence risks, for mtDNA diseases and serves as a framework for this part of the general discussion.

Here, we provide guidelines for reproductive genetic counseling of mtDNA diseases, but, these are no black-and-white 'prescriptions'. For each couple, determining the most appropriate reproductive option, both from a medical and a personal point of view, will involve a process, including several decision points. The role of the counselor, not only in informing but also in structuring and sorting out what is most important for the couple, is crucial. Reproductive options that will be particularly addressed below are prenatal diagnosis (PND), preimplantation diagnosis (PGD) and mitochondrial replacement techniques (MRT). Genomic editing will be shortly mentioned as future addition. Obviously, accepting the risk for offspring without any testing is also an option for at risk couples. Finally, oocyte donation and adoption are effective ways to prevent mtDNA disease transmission, but will not result in biologically-own children. These options will not be further discussed here, but it is relevant to mention that oocyte donation is not permitted in every country. In the Netherlands, oocyte donation is allowed only non-anonymously, in contrast to some other countries where only 
anonymous oocyte donation is permitted (http://www.freya.nl). The unavailability of enough donors to meet the demands, resulting in waiting lists, is another issue (which will also apply to MRT).

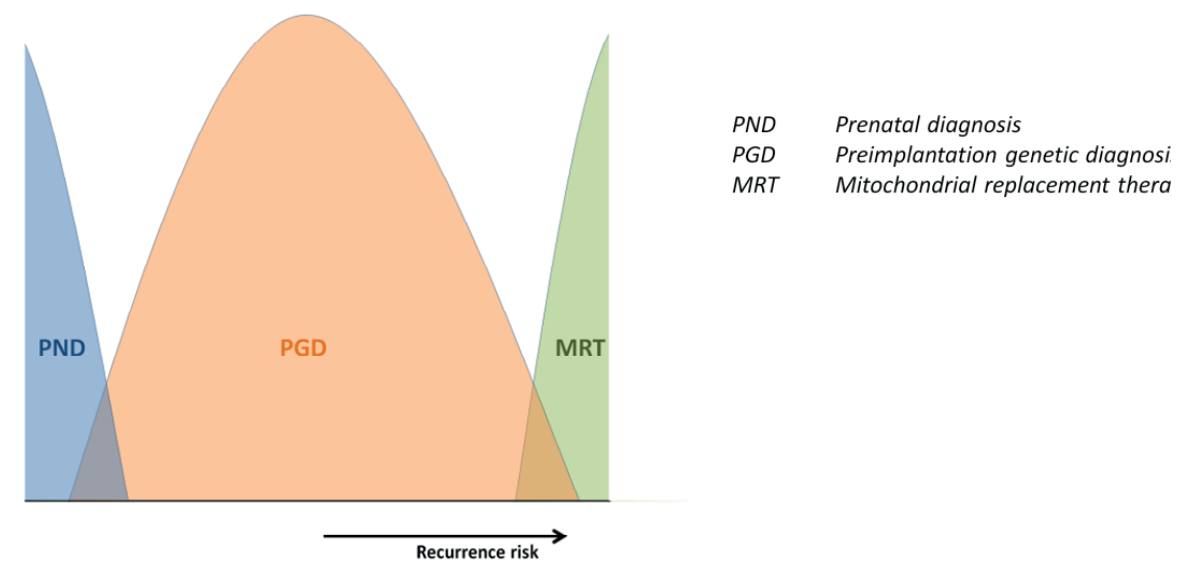

Figure 11.1 Reproductive options for mtDNA mutations, related to recurrence risk (X-axis) and accuracy/burden/risks of the treatment. The recurrence risk depends on de novo versus familial occurrence, nature of the mutation and maternal mutation load (further discussed in text).

\section{$>$ De novo mtDNA point mutations}

For de novo mtDNA point mutations, the recurrence risk is low and probably close to $0 \%$. However, as discussed in Chapter 7, it is hardly possible to establish de novo occurrence with complete certainty, and, mainly due to potential gonadal mosaicism in the mother, a recurrence risk of $0 \%$ cannot be provided. When the presence of the mutation in the mother has been excluded in several tissues, including a postmitotic tissue such as muscle or for some mutations urine (m.3243A>G), and with adequately sensitive techniques, no recurrence has been described. Based on recurrence cases from the literature where no postmitotic tissue in the mother was analyzed, and thus a mutation in the mother may have been missed, the recurrence risk is $~ 4 \%$ (Chapter 7 ). This probably implies a lower actual recurrence risk in cases where the lack of detectable mutation levels is demonstrated in a postmitotic maternal tissue. Regarding reproductive counseling, this stresses the importance of including such tissues in evaluating de novo occurrence. For skewing mutations in particular, one should be aware that (near-)homoplasmic levels in an affected child may well result from very low mutation load in the mother. Thus, one should be careful in concluding a mutation arose de novo.

Because of the low recurrence risk of (presumably) de novo mtDNA point mutations, prenatal diagnosis (PND) seems to be the preferred choice if a couple wishes 
reproductive testing in a subsequent pregnancy. As discussed in Chapters 2 and 3, PND for mtDNA mutations has certain limitations, the most important one being the potential difficulties in interpreting the prenatal test result with respect to the phenotype of the child. PND for de novo cases merely serves as reassurance, given the high likelihood that the mutation will be absent in prenatal samples in a subsequent pregnancy of the mother. An alternative reproductive option is preimplantation genetic diagnosis (PGD), discussed in Chapters 2, 3, 4 and 5. The invasive, lengthy and costly nature of this treatment, with generally smaller pregnancy rates than conceiving spontaneously, renders PGD a less obvious choice when recurrence risk is low. If the couple undergoes an assisted reproductive treatment (ART) for other reasons (e.g. fertility problems, nuclear gene defect) considerations could obviously be different.

\section{$>$ Single large-scale mtDNA deletions}

Other situations with low recurrence risk relate to single large-scale mtDNA deletions, which arise mostly de novo and consequently involve a low recurrence risk for mothers of affected children. ${ }^{2-4}$ Also, for affected women harboring a single large-scale mtDNA deletion themselves, the recurrence risk is low. The latter has been established at 1:24 (4.11\%, $\mathrm{Cl}$ 0.86-11.54\%) based on 40 women with an mtDNA disorder due to an mtDNA deletion and their 73 offspring, in which recurrence was reported in three. ${ }^{3}$ Therefore, also for single large-scale deletions PND is the most feasible reproductive option.

\section{$>$ Inherited mtDNA point mutations}

\section{Low recurrence risk due to a low-level mtDNA point mutation in the female}

Females carrying low heteroplasmy levels of mtDNA point mutations in post-mitotic tissue generally have a low recurrence risk, but are a potentially challenging category regarding reproductive counseling, since the choice between PND and PGD may be difficult. Low recurrence risk and low mutation load are obviously linked to each other in this context, but not by definition. Mutation load is preferably established in multiple tissues, and again these ideally include muscle. 'Low' mutation load could be arbitrarily defined as $<10 \%$, although this is not an evidence-based margin and may differ between mutations. As for the question 'how to define low' for the maternal mutation load, determining a concrete recurrence risk for low-level carriers is difficult, as different mtDNA mutations have different transmission patterns, illustrated by Chapter 6 and Chapter 8.

\section{Prenatal diagnosis (PND)}

The skewed segregation of the $\mathrm{m} .8993 \mathrm{~T}>\mathrm{G}$, resulting from positive selection (Chapter 6), means that the risk of affected offspring is higher for the m.8993T>G 
mutation than for other mtDNA mutations, not positively selected for upon transmission, with comparable maternal mutation loads. Nevertheless, carriers of the m.8993T>G mutation, particularly with low mutation load, are considered suitable PND candidates because the correlation between mutation load and phenotype is rather $\operatorname{good}^{5}$ and mutation loads in oocytes/embryos show a distribution to the extremes; in the majority either 0 or $\sim 100 \%$ (Chapter 2 and Chapter 3 ). Due to this combination, prenatal samples are less likely to pose interpretation issues. Furthermore, recurrence risk can be quite adequately predicted based on maternal mutation load (Chapter $\mathbf{3}$ and reference $5^{5}$ ). Another prerequisite for reliable PND is the representativeness of the mutation load in the prenatal sample for the mutation load in postnatal tissues of the child. This includes stability of the mutation throughout embryofetal development and a relatively uniform distribution between fetal tissues, which has been shown for $\mathrm{m} .8993 \mathrm{~T}>\mathrm{G}$ (and one $\mathrm{m} .8993 \mathrm{~T}>\mathrm{C}$ case) and $\mathrm{m} .9176 \mathrm{~T}>\mathrm{C}$ (one case) but, somewhat unexpectedly considering its postnatal behaviour, also for m.3243A $>\mathrm{G}$ (reviewed for example in Chapter 2). Although data are limited, this points to differences in segregation prenatally compared to postnatally, which might be related to the shorter prenatal time window, or to different demands to the metabolic system. Finally, the prenatal sample itself has to correspond to the fetal tissues, to be predictive. In line with this, should a couple opt for PND, the question comes up whether chorionic villus sampling (CVS) or amniocentesis is the preferred choice. CVS has the advantage of being performed earlier in the pregnancy, at around 11-12 weeks, enabling earlier termination in case of an unfavorable result. However, for mutations other than those at nucleotide 8993, data on representativeness of chorionic villi for fetal mutant load have been conflicting or insufficient. For the m.3243A>G mutation, heteroplasmy differences of $>10 \%$ have been shown within fetal placenta ${ }^{6}$ and between placenta and embryonic tissues. ${ }^{7}$ This might point to potential differences in mutation segregation between fetal tissues at the one hand and placenta on the other. Importantly however, intra-placental variation particularly seems a potential issue when the placental sample is very small ( $\leq 10 \mathrm{mg}$ or single cells), ${ }^{6-8}$ which is unsurprising considering the variability in single cell mutation load in postnatal tissues, collectively resulting in a representative average mutant load upon testing. Intra-placental mtDNA mutation load variations up to $55 \%$ have been reported without further details on the specific mutations and samples. ${ }^{9}$ Other cases indicate analogy between CVS, amniocytes and fetal tissues for the m.3243A>G mutation. 7,10 Taken together, a sufficiently large sample should be obtained upon CVS, and an amniocentesis in addition to CVS would be optimal for mtDNA mutations in order to collect the most available data for a diagnosis as reliable as possible. Similarly, one may consider to perform DNA-testing both on cultured and uncultured chorionic villi as long as data are lacking on potential heteroplasmy differences between both. For m.8993T>G/C such double testing (cultured/uncultured, CVS/amniocentesis) is probably not necessary given the relatively stable average mutant load between tissues and over time. Obviously, carrying out both CVS and 
amniocentesis has the drawback of necessitating two invasive procedures, each accompanied by a small but relevant risk of inducing a miscarriage ( $1-2 \%$ for CVS, $\sim 0.5-$ $1 \%$ for amniocentesis, respectively). The need of performing an additional amniocentesis cannot entirely be predicted beforehand and therefore represents one of the decision points in the process. It depends for example on the mutation load identified in the CVS, and its proximity to the expression threshold. This threshold may be mutation-specific, if sufficient data are available, although, as mentioned, is often difficult to define. Nevertheless, a systematic review provides some guidance, showing that individuals with muscle mutant loads of below $\sim 18 \%$ are considered to be unaffected in $>95 \%$ of cases. ${ }^{11}$ This 'generic threshold' was calculated after pooling various mtDNA mutations. For declining mutation loads, the likelihood that an individual will develop symptoms obviously further decreases. ${ }^{11}$

If mutation load identified with CVS is either very high or very low, additional amniocentesis may not be needed since even with a significant error rate, expressed as increase or decrease relative to the identified heteroplasmy level, the mutation load could remain below or above the established threshold. If a couple does not wish to risk the advice of obtaining a second prenatal sample, they may prefer amniocentesis only, which seems a priori more appropriate than CVS as single test. Still, even though by amniocentesis fetal cells from multiple organs are analyzed (epithelial cells from the urinary, respiratory, and gastrointestinal tracts, and from the epidermis), it is only one, and dividing, cell type. Therefore, combined with the scantiness of the available data, differential segregation cannot be excluded. An alternative approach is to hold on to a larger safety margin below the threshold, to take potential segregation differences between tissues and over time into account. Obviously, the lower the threshold, the safer it is, but at the same time the chance increases of terminating a pregnancy of a child that would not have been affected. The chance that the female produces offspring below the threshold should also be taken into account. When such an 'extra safe' threshold is chosen, CVS may be regarded sufficiently reliable when the result is below this threshold. If slightly above, subsequent amniocentesis can still be considered.

\section{Preimplantation genetic diagnosis (PGD)}

An advantage of PGD for female carriers with a low-level mtDNA mutation, irrespective of the mutation, is that large numbers of mutation-free (or low-level) and thus transferrable embryos are to be expected. Nevertheless, the earlier-mentioned disadvantages of PGD compared to spontaneous conception are also applicable. In all, making a choice between PND and PGD can be difficult for carriers with relatively low mutation load, depicted by an overlap in Figure 11.1. Patient preferences play an important role as well; considerations regarding for example impact of terminating a pregnancy in case of a (likely) affected fetus and burden of a PGD treatment are very personal. These are also likely to be influenced by a couple's personal history, for example whether they have already had an affected child and if so, how severely 
affected he/she was. The same can be true for a carrier's own disease and/or family history. Furthermore, female's age and disease manifestations can both be a factor in eligibility for an ART (assisted reproductive technology)/PGD treatment.

Moderate-to-high recurrence risk due to a higher-level mtDNA point mutation in the female

With increasing mtDNA mutation loads, recurrence risks become higher and more difficult to predict.

\section{PGD: blastomere biopsy in the cleavage-stage embryo}

For carriers with moderate to high recurrence risks, which are the majority in clinical practice, we consider PGD as the best reproductive option available at present. It should be emphasized that PGD for mtDNA mutations is a strong risk reduction for clinically affected offspring, albeit in case no embryos without detectable mutation load are available and low-level embryos are transferred not a complete risk exclusion. Notably, the policy and professional opinions regarding transfer differs between countries. $^{12,13}$ In most centers the genetic analysis is performed in blastomeres derived from the cleavage stage embryo at day 3 postfertilization. At this point in embryogenesis, the fertilized oocyte, containing between 50,000 and 550,000 copies of the mtDNA, ${ }^{14}$ has divided into 6-8 blastomeres. Upon cleavage, the mtDNA is presumed to be distributed randomly over the daughter cells. Although some replication may occur during early preimplantation development in human embryos, it is yet unknown whether this takes place during the first three days. ${ }^{14,15}$ Furthermore, available data suggest this to be limited to embryos with a mitochondrial deficit at this stage, ${ }^{14,15}$ which seems corroborated by findings in pig embryos. ${ }^{16}$ Should this prove to be true, it means that mtDNA replication in the cleavage stage embryo is presumably not relevant in a PGD context where embryos with absent or low-level mutations, and thus no mitochondrial failure, are selected. In bovine ${ }^{17}$ and mouse ${ }^{18}$ embryos a (sharp) decrease in total mtDNA content between the 2 cell- and 4/8 cell-stage was shown but this has not been reported in humans (reviewed in reference $14^{14}$ ). Thus, for viable human embryos the total mtDNA amount of the embryo appears to remain largely constant. As a result, the mtDNA copy number per cell decreases upon cleavage. If a pathogenic mtDNA mutation is present, the mutation load per cell is expected to remain grossly the same in the early embryo due to the high copy number, although small differences may occur by chance. The heteroplasmy variation between cells increases upon progressive cleavage, as mtDNA amount per cell further decreases. This is illustrated in a mouse study, showing a strong mutation load correlation among individual blastomeres of 2-, 4- and 8-cell stage embryos but with increasing 95\% prediction intervals with increasing cleavage stage. ${ }^{19}$ Our initial results in human embryos showed that mutation loads are generally very consistent between blastomeres in most 
embryos, although (single) blastomeres can diverge in some (Chapter 3). Based on these findings, the biopsy and analysis of two blastomeres was advised and as such incorporated in our initial clinical protocol. The main drawback of analyzing two blastomeres instead of one is the decrease in live birth deliveries, ${ }^{20}$ illustrating a dilemma between diagnostic certainty and pregnancy chances. Now we have performed more PGD treatments, a false negative error rate (i.e. classifying an embryo erroneously as below the threshold) of $0.34 \%$ (Chapter 4 ) could be confidentially calculated for the m.3243A $>\mathrm{G}$ mutation, based on a single blastomere analysis. For the other mtDNA mutations the numbers are (and will be) insufficient. However, the passive division of mitochondria among daughter cells makes our m.3243A>G data also applicable to other mtDNA mutations, as the mutation itself does not seem to play a role, and we changed the biopsy policy to one blastomere instead of two for every mtDNA mutation, with favorable consequences for embryo viability and implantation rate. An additional advantage is the potential to select for two treats, each on one cell. Although in that case the positive effect of 1-blastomere biopsy on live birth rate is abrogated, couples may wish so anyway after careful counseling. This is exemplified by requests we encountered in clinical practice for additional testing of a nuclear gene defect (POLG, BRCA2). Another concerned the simultaneous selection for male gender, in order to prevent the transmission of even low-level mutation to a female fetus who could be facing comparable dilemmas regarding reproductive options. This question is understandable; after all, selecting male offspring is an effective way to prevent transmission of mtDNA mutations to future generations, and as such is deemed morally acceptable, ${ }^{21}$ but currently not legally allowed in the Netherlands. A potential drawback of the simultaneous selection for gender is the reduced number of remaining embryos eligible for transfer, thereby diminishing the chance of a successful PGD treatment for the couple. The consequences for future generations in case of transmission by female offspring have been modeled, showing that for a clinical threshold of $60 \%$, the reduction of heteroplasmy levels to $<5 \%$ dramatically reduces the chance of disease recurrence in subsequent generations, whereas transmitting $>5 \%$ mutant mtDNA was associated with a significant risk of disease recurrence. ${ }^{22}$ Notably, female offspring born after PGD and harboring low mutation levels will likely produce mutation-free embryos which can be selected to clear the mtDNA mutation from the next generation. This is also true for preferentially-transmitted mutations that show skewing upon transmission.

\section{PGD: trophectoderm biopsy in the blastocyst}

A PGD technique that enables biopsy of a larger number of cells for genetic analysis is trophectoderm (TE) biopsy in the blastocyst stage at day 5 or 6 postfertilization, which is increasingly replacing blastomere biopsy in clinical practice. In the blastocyst, comprising of a trophectoderm, a blastocoele and an inner cell mass, respectively, the first cellular differentiation has occurred. The trophectoderm will develop into most of 
the fetal part of the placenta, the blastocoele provides growth factors and other prerequisites for blastocyst development, and the inner cell mass will later give rise to the embryo proper and the fetus. At this point in embryogenesis, more cell divisions have occurred (the human blastocyst consists of $70-100$ cells) and consequently the mtDNA amount per cell is expected to have further decreased as long as mtDNA replication remains silenced. Although human data at this stage are lacking, animal studies indeed show a continued suppression of mtDNA replication in the inner cell mass cells ${ }^{23}$ (Figure 11.2), which are estimated to contain 1000 mtDNA copies per cell. $^{24}$ In the trophectoderm mtDNA replication resumes, evidenced by non-human ${ }^{23}$ and human ${ }^{25}$ data (Figure 11.2). mtDNA replication in inner cell mass cells is not believed to initiate before gastrulation ( day 16 postfertilization) has taken place.

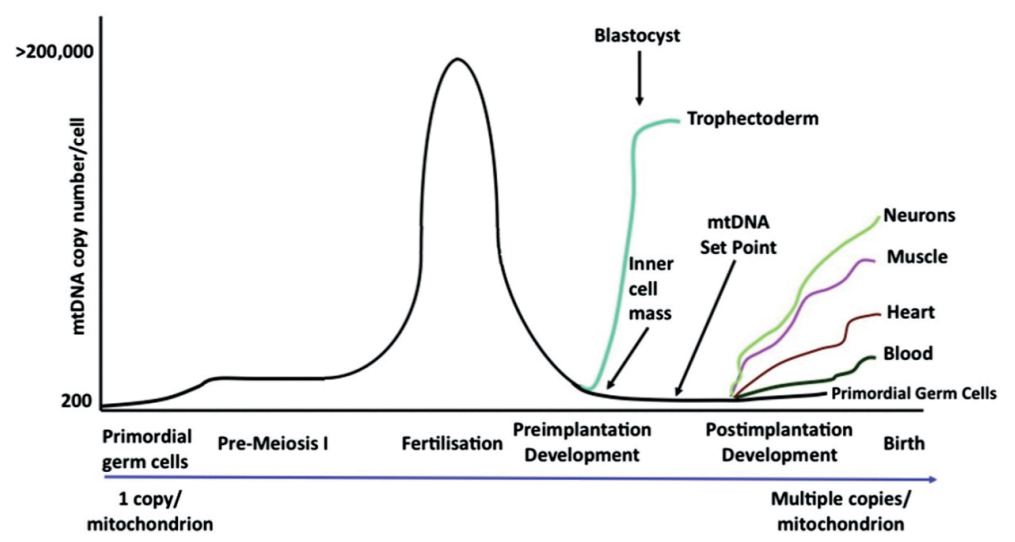

Figure 11.2 mtDNA copy number throughout embryonic development stages. During oogenesis, mtDNA copy number increases up to the metaphase II stage. In larger mammals, it then undergoes significant reduction during preimplantation development. At the blastocyst stage, replication is initiated at the blastocyst stage but this is confined to the trophectoderm. MtDNA copy number undergoes continual reduction in the inner cell mass cells to establish the 'mtDNA set point'. This ensures that specialized cell types, once they have differentiated, acquire the appropriate mtDNA copy number to meet their needs for ATP production in a cell type specific manner (reprinted with permission ${ }^{23}$ )

Blastocyst-stage TE biopsy has several advantages, including less detrimental effects on embryo viability and implantation potential. ${ }^{26,27}$ Also, the necessity for the embryos to develop to the blastocyst phase results in a further selection of the most viable embryos. Disadvantages include the necessity to vitrify all embryos after biopsy. ${ }^{27}$ For mtDNA mutations, a major advantage of TE biopsy would be a lower risk of missing potential differences in heteroplasmy levels among cells, as multiple cells can be biopsied. However, paradoxically, heteroplasmy mosaicism is at the same time a potential drawback due to the mtDNA replication in the trophectoderm. So far very 
limited human data exist on the representativeness of TE biopsy for the entire embryo and for the child with regard to mtDNA heteroplasmy levels. TE biopsy followed by PGD has been reported in two m.3243A>G mutation carriers ${ }^{28,29}$ (Table 11.1). In one of these, conflicting postpartum results have been reported in the resulting child. ${ }^{28,30}$ Until now these discrepancies have not been explained by the author groups. Anyhow, considering the timing of TE biopsy being closer to the lowest point of the bottleneck, blastomere biopsy in the cleavage-stage embryo seems preferable for PGD for mtDNA mutations as this reflects the entire embryo better and somatic mosaicism occurring during development is less an issue.

Table 11.1 Published and unpublished children born after PGD for a pathogenic mtDNA mutation (as of May 2016).

\begin{tabular}{|c|c|c|c|c|}
\hline $\begin{array}{l}\text { Blastomere/ } \\
\text { trophectoderm } \\
\text { biopsy (embryo } \\
\text { stage) }\end{array}$ & Mutation & Embryonic mutation load & Postpartum mutation load & Reference \\
\hline $\begin{array}{l}\text { Blastomere } \\
\text { (cleavage-stage) }\end{array}$ & $\mathrm{m} .8993 \mathrm{~T}>\mathrm{G}$ & $\begin{array}{l}0 \% \text { (single blastomere) / } \\
0 \% \text { (single blastomere) }\end{array}$ & $0 \%$ (cord blood) & $\begin{array}{l}\text { Sallevelt et al } \\
2013^{31} \\
\text { (Chapter } 3 \text { ) }\end{array}$ \\
\hline $\begin{array}{l}\text { Blastomere } \\
\text { (cleavage-stage) }\end{array}$ & $\mathrm{m} .8993 \mathrm{~T}>\mathrm{G}$ & $\begin{array}{l}0 \% \text { (single blastomere) / } \\
0 \% \text { (single blastomere) }\end{array}$ & $0 \%$ (cord blood) & $\begin{array}{l}\text { Unpublished } \\
\text { data }\end{array}$ \\
\hline $\begin{array}{l}\text { Blastomere } \\
\text { (cleavage-stage) }\end{array}$ & $\mathrm{m} .8993 \mathrm{~T}>\mathrm{G}$ & $\begin{array}{l}0 \% \text { (single blastomere) / } \\
0 \% \text { (single blastomere) }\end{array}$ & 0\% (cord blood) & $\begin{array}{l}\text { Steffann et al } \\
2006^{32}\end{array}$ \\
\hline $\begin{array}{l}\text { Blastomere } \\
\text { (cleavage-stage) }\end{array}$ & $\mathrm{m} .8993 \mathrm{~T}>\mathrm{G}$ & $\begin{array}{l}2.5 \% \text { (no. of analyzed } \\
\text { blastomeres not reported) }\end{array}$ & $4 \%$ (cord blood) & $\begin{array}{l}\text { Thorburn et al } \\
2009^{33}\end{array}$ \\
\hline $\begin{array}{l}\text { Blastomere } \\
\text { (cleavage-stage) }\end{array}$ & $\mathrm{m} .14487 \mathrm{~T}>\mathrm{C}$ & $\begin{array}{l}5.4 \% \text { (single blastomere) / } \\
6.1 \% \text { (single blastomere) }\end{array}$ & $5 \%$ (cord blood) & $\begin{array}{l}\text { Unpublished } \\
\text { data } \\
\text { (Chapter 5) }\end{array}$ \\
\hline $\begin{array}{l}\text { Blastomere } \\
\text { (cleavage-stage) }\end{array}$ & $\mathrm{m} .3243 \mathrm{~A}>\mathrm{G}$ & $\begin{array}{l}12.6 \% \text { (single blastomere) } \\
\text { / } 13.4 \% \text { (single } \\
\text { blastomere) }\end{array}$ & $16 \%$ (cord blood) & $\begin{array}{l}\text { Unpublished } \\
\text { data }\end{array}$ \\
\hline $\begin{array}{l}\text { Blastomere } \\
\text { (cleavage-stage) }\end{array}$ & $\mathrm{m} .3243 \mathrm{~A}>\mathrm{G}$ & $\begin{array}{l}5 \% \text { (single blastomere) / } \\
5 \% \text { (single blastomere) }\end{array}$ & $5 \%$ (cord blood) & $\begin{array}{l}\text { Monnot et al } \\
2011^{7}\end{array}$ \\
\hline $\begin{array}{l}\text { Trophectoderm } \\
\text { (blastocyst) }\end{array}$ & m.3243A>G & $\begin{array}{l}12 \% \text { (no. of analyzed TE } \\
\text { cells not reported) }\end{array}$ & 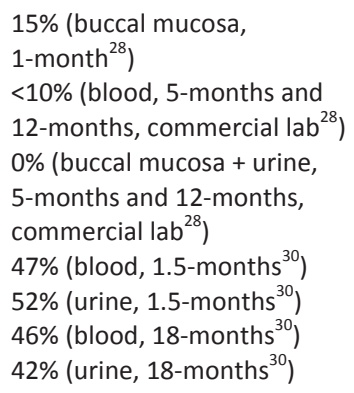 & $\begin{array}{l}\text { Treff et al } \\
2012^{28} \\
\text { Mitalipov et al } \\
2014^{30}\end{array}$ \\
\hline $\begin{array}{l}\text { Trophectoderm } \\
\text { (blastocyst) }\end{array}$ & $\mathrm{m} .3243 \mathrm{~A}>\mathrm{G}$ & $\begin{array}{l}0 \% \text { ( } 4 \text { TE cells) / 0\% ( } 2 \text { TE } \\
\text { cells) }\end{array}$ & $\begin{array}{l}0 \% \text { (blood + saliva + urine, } \\
\text { postpartum) }\end{array}$ & $\begin{array}{l}\text { Heindryckx et al } \\
2014^{29}\end{array}$ \\
\hline
\end{tabular}




\section{PGD: implications of selection events and random drift?}

An interesting aspect regarding PGD in light of this thesis is the question whether selection events in the female germline upon transmission of mtDNA mutations (Chapter 6) might have implications for the reliability of PGD. The genetic analysis in the context of a PGD treatment is performed after the germline genetic bottleneck from mother to the embryo has taken place, since this has happened during oogenesis in mother's own embryonic phase. Therefore, the PGD-results represent the mutation loads resulting from potential germline selection and are not expected to be altered by it anymore. However, a 'second bottleneck' could be expected in the embryo's somatic cells, since not only the cells destined to become germ cells undergo cell divisions postfertilization. Indeed, evidence for such a bottleneck comes from mouse, ${ }^{23}$ monkey $^{34}$ and zebrafish ${ }^{35}$ studies. Postimplantation, the mtDNA amount increases, whereby specialized cell types, once differentiated from pluripotent embryonic stem cells, acquire the appropriate mtDNA copy number to meet their needs for ATP production in a cell type specific manner. ${ }^{23}$ Also in human fetal and postnatal tissues, varying and increasing mtDNA copy numbers have been demonstrated in different tissues. ${ }^{9}$ This process may be regulated by epigenetic processes as suggested in mice. ${ }^{36}$ In zebrafish, a vertebrate model convenient to investigate bottleneck events, the initiation of mtDNA replication seems to coincide with somitogenesis, when early organ development starts and cells shift from glycolysis to the use of oxidative phosphorylation (OXPHOS). ${ }^{35}$ Interestingly, there is a partial parallel with the germ cells, as the metabolic shift observed in the human germline is coinciding with mtDNA replication: primordial germ cells are highly dependent on glycolysis, whereas developing oocytes, in which mtDNA content increases, rely on the OXPHOS for their ATP production (Chapter 6). Should the selection events, predicted to occur in the female germline (Chapter 6), also take place during embryogenesis, PGD outcome might be influenced when positive selection for the mutation occurs, as is the case for the m.8993T>G mutation, due to increased mutation in the somatic tissues of the embryo/child. A selective advantage of the m.8993T>G mutation has been shown in somatic cell lines, ${ }^{37}$ which implies this is not a germline-specific process. Important to note is that the initial m.8993T>G heteroplasmy levels in this study were high (85-95\%). This may corroborate another study, showing an increase of $0.75 \%$ per year in blood of an individual with high (79\%) m.8993T>G mutation load whereas in an individual with low $(7 \%)$ mutation load this percentage remained stable over 23 years. ${ }^{38}$ Although generally no significant differential segregation throughout embryofetal development is reported at varying heteroplasmy levels, ${ }^{39-42}$ at a closer look, three cases with somewhat larger differences between PND samples and fetal tissues are in the higher mutant load range. ${ }^{39,42}$ Whether these differences indeed result from preferential segregation or merely from random drift is unclear. The only embryo transferred after PGD which contained the m.8993T>G mutation harbored $2.5 \%$ mutation load in the blastomere(s) and $4 \%$ in postpartum cord blood ${ }^{33}$ (Table 11.1). Taken together, as in 
the germline (Chapter 6), in somatic tissues the positive selection seems to be a relevant factor at high but not at low mutation loads. Conveniently, for this mutation often mutation-free embryos are available due to the skewed segregation. Not only selection processes, also random genetic drift has to be taken into account and for this too the mutation load of the transferred embryo is of importance. An increase in average mutation load may by chance occur upon random genetic drift. Our work has revealed that selection and drift should be taken into account when establishing a threshold for transfer, instead of merely relying on clinical expression in relation to mutation load. The generic threshold of $18 \%$ seems safe, but caution is warranted if higher threshold is considered for a given mtDNA mutation. This is illustrated by the one m.3243A $>G$ embryo that showed a relative increase of $\sim 20 \%$ when comparing the PGD and postnatal results ( $13 \%$ and $16 \%$ heteroplasmy, respectively, Table 11.1). Mutation loads above the threshold are at increased risk of segregating to clinically significant levels. The lower the initial mutation load is, the higher the chances are that it remains stable upon random drift or, more likely, disappears pre- or postnatally. Although data are yet limited, stable segregation is observed in the other cleavagestage m.3243A $>G$ and the m.14487T $>C$ PGD results (Table 11.1). With the current progressed insight, the transfer threshold of $60 \%$ which we considered appropriate for the m.9176T $>$ C mutation, based on a high ( $>90 \%)$ phenotypic threshold, should be critically re-evaluated. Given the possible skewed pattern of this mutation, ${ }^{43-45}$ it is advisable to investigate whether in addition to genetic drift positive selection is involved. In fact, each mtDNA mutation should ideally be evaluated this way.

\section{PGD: further prerequisites for successful outcome}

The success of PGD furthermore depends on the availability of embryos with either $0 \%$ or below-threshold mutation loads. As of May 2016, we have performed a total of 26 PGD treatment cycles in 13 mtDNA mutation carriers (1-3 treatments per carrier). Nearly all (12/13) carriers produced one or more embryo(s) with either absent or below-threshold mutation levels in at least one of the cycles. The chance of mutationfree or low-level embryos is in general inversely correlated with the mutation load of the carrier. So, at some point, mutation load in the female carrier may be too high to produce sufficient embryos eligible for transfer in a PGD setting. The number of embryos permitted to develop during a PGD treatment has a maximum, related to the risk of overstimulation in the female. The maternal mutation load at which not enough suitable embryos for transfer are produced anymore is not a black-and-white threshold. It is a chance event which also depends on the segregation pattern of the mutation concerned. When the mutated mtDNAs are preferentially transmitted, similarly to the m.8993T>G mutation (Chapter 6), a female with high heteroplasmy levels is less likely to have transferable embryos. Interestingly, for the m.9176T>G mutation an increased mitochondrial membrane potential (MMP), which is the suggested mechanism underlying the positive selection of the m.8993T>G mutation (Chapter 6), has been 
demonstrated as well. ${ }^{46}$ Although only two families with this mutation have been reported to date, it is striking that in both a healthy carrier mother with $\sim 50 \%$ mutation load in blood conceived two children affected by Leigh syndrome resulting from (near)homoplasmic levels, ${ }^{46,47}$ which may indeed be compatible with preferential transmission of mutated mtDNAs. Given the moderate mutation load of this particular mother, she is expected to have oocytes with low mutation load as well, 'escaping' the (potential) positive selection, and thus she would probably benefit from PGD.

A final determinant in the outcome of PGD is the pregnancy rate. Unfortunately, not every embryo transfer results in a viable pregnancy. Chances are on average $\sim 22 \%$ per cycle, ${ }^{48}$ depending on age and other known and unknown factors.

With respect to legislation, one should be aware that PGD may not, or not for the same conditions, be allowed in every country.

High recurrence risk due to a (very-)high-level to homoplasmic mtDNA point mutation in the female

The one carrier in our series who did not produce embryos below the transfer level so far is an m.9176T>C carrier harboring very high mutation loads herself $(\geq 90 \%)$. She underwent two treatment cycles which yielded only (near-)homoplasmic embryos except for one, harboring $\sim 66 \%$ mutant load. Besides the high mutation level in the carrier, an attributing factor seems the apparently skewed transmission pattern. Homoplasmic mtDNA mutation carriers constitute the category for whom PGD is by definition not able to decrease or exclude mutation levels transmitted to the offspring, since all embryos will harbor $100 \%$ mutant load. The most prevalent and well-known homoplasmic mtDNA mutations are associated with Leber hereditary optic neuropathy (LHON), resulting in severe vision loss. ${ }^{49}$ There is a reduced penetrance which is genderdependent, with female carriers having on average $~ 10 \%$ risk of developing LHON and male carriers $\sim 40 \%{ }^{49}$ Accordingly, each pregnancy of a homoplasmic LHON mutation carrier has a risk of $\sim 20 \%$ to result in a son who will experience vision loss and $\sim 5 \%$ risk to result in a daughter with vision loss. In our center, a handful of couples has requested sex-selective PGD with transfer of girl embryos in order to reduce the risk of an affected child. Although this may pose some ethical questions, PGD for LHON is generally deemed morally acceptable, ${ }^{13,50}$ as it is the only risk-reducing strategy towards offspring currently available for homoplasmic LHON carriers. When there is another reason necessitating an IVF treatment, the 'proportionality', in this context referring to the (additional) treatment risks and burden in relation to the achieved risk reduction, is more balanced compared to couples without IVF indication. Still, the view on this of the couples themselves can be very personal and variable, whereby for example differences in the perceived severity of the disease, heaviness of the treatment and/or worthiness of the risk reduction play a role. More importantly, despite general ethical consensus, legislation may not allow PGD for this indication. 


\section{Mitochondrial replacement technology}

For mtDNA mutation carriers not able to benefit from the existing reproductive options for a biologically-own child, promising developments are ongoing in the field of mitochondrial replacement. The mitochondria are located in the cytoplasm of the oocyte (ooplasm). Theoretically, two ways exist to replace mutated mitochondria by healthy ones: either healthy mitochondria are transferred between oocytes from the donor to the mutation carrier, or the nuclear DNA is transferred the other way around. The former has originally been developed for fertility purposes and still commercially applied in this field, although safety concerns exist due to the resulting mixture of donor ooplasm with still a significant proportion of the recipient's mtDNA. ${ }^{51-55}$ Another disputed, experimentally applied fertility-increasing approach uses the female's own mitochondria to augment the mtDNA copy number in the oocyte, thereby circumventing the issue of mtDNA mixing. ${ }^{56}$ Although in theory, healthy mitochondria could be used accordingly to dilute the mutated mtDNA population, it seems unattainable to achieve generally a switch to levels under the threshold of expression. Thus, this ooplasm donation is unsuitable for preventing the transmission of mitochondrial diseases. The focus has therefore shifted to transferring the nuclear DNA instead of the ooplasm. Currently four variants exist on germ cells: germinal vesicle (GV) transfer, $^{57-59}$ meiosis II (MII) spindle transfer, ${ }^{60-65}$ pronuclear (PN) transfer ${ }^{25,66-68}$ and, most recently described, polar body transfer. ${ }^{69,70}$ Each has its specific challenges and advantages, reviewed for example in references 55 and 70-73, ${ }^{55,71-74}$ although GV transfer is generally not considered a fruitful option in humans. Remarkably, the results of PN transfer in abnormally fertilized human oocytes recently appeared not to be applicable to normally fertilized zygotes: the techniques used in the proof-of-concept studies (abnormally fertilized zygotes) were not well tolerated in the first preclinical studies (normally fertilized zygotes) and an alternative approach had to be developed. ${ }^{25,68}$ This underscores the potential limitations of studies in non-viable human zygotes and the need for studies in viable ones.

Essentially the four mitochondrial transfer techniques have the same principle, with the (mainly ethical) difference that in GV transfer, spindle transfer and first polar body transfer unfertilized oocytes are the starting point, whereas in pronuclear transfer and second polar body transfer these are the fertilized oocytes (zygotes). The chromosomes are obtained from the mtDNA mutation carrier's oocyte/zygote and fused to the donor oocyte/zygote, of which the chromosome-containing spindle or nucleus has been removed. Consequently, the resulting oocyte/zygote has the nuclear DNA from the female carrier, and the (healthy) mtDNA from the female donor. This is why media often refer to "three-parent IVF", although it is more correct to speak of mitochondrial donation, or, as has been playfully introduced, "2.00005-parent IVF" considering the small contribution of genetic material, which is often suggested not to influence characteristics normally associated with individual identity (http://nuffieldbioethics.org/ project/mitochondrial-dna-disorders/). On the other hand, disease is considered part of 
one's identity, which will as such be altered by MRT, but this is regarded an acceptable change as it improves the child's health and options in life. ${ }^{75}$ Thus, MRT has to be considered as a form of gene therapy without modifying the genes, whereby the advantages outweigh the drawbacks. Nevertheless, some concerns exist about the mixing up of the donor's mtDNA with the carrier's nuclear DNA, potentially resulting in mito-nuclear mismatches affecting health outcome ${ }^{76}$ although these are not evenly shared by most other scientist in the field (e.g. http://www.hfea.gov.uk/docs/ Third_Mitochondrial_replacement_scientific_review.pdf), supported by recent research. ${ }^{65}$ A more broadly acknowledged potential risk underlies the small amount of the mutation carrier's mitochondrial DNA that is co-transferred with her chromosomes. The (expected) amount is dependent on the type of transfer, since mitochondrial localization varies throughout the oocyte stages. ${ }^{55}$ Human data have established these carry-over levels at $\leq 1 \%$ for spindle transfer ${ }^{63,64}$ and, in normally fertilized human oocytes, mostly $<2 \%$ (always $<5 \%$ ) for pronuclear transfer after optimization in experienced hands. ${ }^{25}$ These data reflect the expected difference between spindle transfer (when the mitochondria are scattered in the cytoplasm with a tendency to concentrate in the inner cytoplasm) and pronuclear transfer (when mitochondria are concentrated in a peri-nuclear position). For polar body transfer no human data are available, but in mice the carry-over was less than for spindle and pronuclear transfer, which also makes sense considering the small size and cellular content of the polar bodies compared to the oocyte. ${ }^{69}$ The mtDNA carry-over in all three techniques is well below the expected phenotypic threshold, ${ }^{11}$ implying reassurance with respect to the risk of affected offspring despite MRT. However, recent studies showed incidental upward drift originating from low mtDNA carryover after nuclear transfer, resulting in wide heteroplasmy variation. ${ }^{25,65}$ This may be the result of random genetic drift, as has been concluded from a bovine study on ooplasmic transfer, ${ }^{77}$ or of a replication advantage of one mtDNA population over the other. The latter has been demonstrated in a recent study in human oocytes, and was attributed to a certain D-loop polymorphism ${ }^{78}$ A specific characteristic of MRT, and important difference with PGD, is the mixing of 2 mtDNA populations. Several 'artificial' animal models with mixed mtDNA populations exist. In mice zygotes constructed with a procedure similar to pronuclear transfer, variant mtDNA segregated less uniformly between blastomeres than in unmanipulated controls, $^{79}$ corroborating observations in monkeys $^{34}$ and in human embryos resulting from PNT of abnormally fertilized human oocytes. ${ }^{68}$ Since mtDNA replication presumably does not occur in the cleavage-stage, interblastomere heteroplasmy differences may rather result from a non-random localization of the carried-over mtDNA after injection. In the mice there was furthermore a significant increase in the proportion of the carried-over mtDNA molecules from the zygote to the blastocyst stage. This has been hypothesized to result from preferential and/or earlier replication of mtDNAs in the vicinity of the nucleus during early embryonic stages, due to better access to nuclear-derived transcription and replication factors. ${ }^{79}$ At this point 
it is not clear what the meaning of this mouse study is for the human situation, but it implies that preferential replication may not only occur due to the nature of certain variants, but also due to the location of carried-over mitochondria within the cell.

Besides replication-differences, other selection processes can be relevant in the context of mtDNA carry-over. Preferential carry-over due to positive selection (Chapter 6) would obviously have potential implications for the child itself, and in a girl also for future offspring. Different distribution patterns of low- and high-inner membrane potential mitochondria have been reported in oocytes/embryos. ${ }^{80}$ In normal human metaphase II oocytes for example (the stage where spindle transfer is carried out), high-polarized mitochondria have been localized in a narrow circumferential band just beneath the plasma membrane, whereas mitochondria throughout the interior of the cytoplasm appear to possess a comparatively lower state of polarity. ${ }^{80}$ This distribution seems to continue into the blastomere stage. ${ }^{80}$ These data imply that upon transfer of the MII spindle, which is located closely to the plasma membrane, the risk of cotransferring mitochondria harboring MMP-increasing pathogenic mtDNA mutations might be higher compared to transfer of the pronuclei which are centered in the middle of the zygote. At which mutation loads this would have an increased risk of being affected, is not clear yet, but it demonstrates that MRT has to be viewed as riskreducing strategy rather than completely preventing the transmission of mtDNA disease $^{25,65}$ and that mtDNA carry-over should be reduced to the lowest possible levels. One way to accomplish this may be centrifugation, which causes mitochondria to concentrate in one of the zygote's poles away from the nucleus. ${ }^{77,81}$ Alternatively, genomic editing technologies ${ }^{82,83}$ may in time be of particular interest to reduce/exclude mtDNA carry-over. As a single reproductive option these could be an option for heteroplasmic mutations, although 'cutting away' of the mutated molecules is not yet efficient enough and, for carriers with high mutation loads, the resulting mtDNA copy number may become too low to enable implantation. But, as a complement to MRT they may prove very helpful. Besides, genomic editing evolves from 'cutting' techniques to 'correction' techniques (CRISPR/Cas9 ${ }^{82,84}$ ), whereby the above-mentioned issues are no longer applicable and which therefore may be applicable as separate reproductive option in the future for both heteroplasmic and homoplasmic mtDNA mutations.

The possibility of transferring only male embryos in order to prevent potential transmission of mtDNA mutations to future generations, already addressed in the previous paragraph, is again, and perhaps even more, relevant in the context of MRT, especially in initial studies, permitting safety evaluation without transmitting potentially heritable risks related to manipulated mtDNA genomes. This has indeed been proposed (American Institute of Medicine (IOM) report, see below). 
From a practical point of view, the latest studies indicate that vitrification of patient rather than donor oocytes results in more efficient development to the blastocyst stage, and is likely to minimize mtDNA carryover. ${ }^{25,65}$ Thus, patient oocytes can be frozen, circumventing potential difficulties in cycle synchronizing between the donor and the recipient. An additional advantage is the possibility to stockpile patient oocytes before they become susceptible to age-related meiotic aneuploidy. ${ }^{25}$

In order to be able to apply MRT in vivo in humans in due course, among others legislation and regulation issues had and have to be settled. The UK law had to be altered, which in 2015 indeed happened, the UK Parliament approving legislation to allow mitochondrial replacement to be used in the clinic under tight regulations. This means that as of 29 October 2015, when the law came into force, IVF clinics can apply to the Human Fertilisation and Embryology Authority (HFEA) for licenses to carry out one or both of the two techniques of maternal spindle transfer (MST) and pronuclear transfer (PNT). An expert panel convened by the HFEA, which has considered the safety and efficacy of MST and PNT in three reports (http://www.hfea.gov.uk/8807.html), had recommended a number of tests which it believed should be completed before the treatment is offered. The recent results ${ }^{25}$ are being considered by an expert panel (call for evidence) to evaluate whether the HFEA can initiate the processing of license applications. Only after satisfaction by the panel and acceptance of its recommendations by the Authority the license process will be initiated. On November 30,2016 , the panel has stated in an advisory report that the therapy is ready for clinical testing, which should be limited to mtDNA mutation carriers who do not have an alternative such as PGD. Whether to indeed allow clinical trials, the HFEA will decide at a meeting on 15 December. Once a clinic is licensed to perform mitochondrial donation, it can submit patient applications (called 'license variations'), which the HFEA will consider on a case-by-case basis. In the United States, the FDA started a process in 2014 to evaluate whether to approve preclinical or clinical trials using MRT (http://www.fda.gov/advisorycommittees/

committeesmeetingmaterials/bloodvaccinesandotherbiologics/cellulartissueandgeneth erapiesadvisorycommittee/ucm406412.htm). As part of this, the Institute of Medicine (IOM) of the National Academies of Sciences, Engineering, and Medicine was asked to assemble an expert committee to consider the ethical, social and policy issues raised by the techniques. Recently this committee concluded that it is ethically permissible to conduct clinical investigations of $\mathrm{MRT}(\mathrm{s})$, subject to certain conditions and principles (http://www.nationalacademies.org/hmd/Reports/2016/Mitochondrial-ReplacementTechniques.aspx). However, due to federal legislation passed in December 2015, the FDA will not be able to grant permission for any MRT efforts for fiscal year 2016 (http://www.sciencemag.org/news/2016/02/boys-only-panel-endorses-mitochondrialtherapy-says-start-male-embryos). So, in the US, restrictions on funding rather than federal laws on human embryo research hamper further developments. In the 
Netherlands, the current Dutch Embryo Act forbids the creation of embryos for research (http://wetten.overheid.nl/BWBR0013797/2013-09-27). However, recently (May 27, 2016) the Minister for Health has announced the adjustment of the Embryo Act, permitting this in some specific situations (https://www.rijksoverheid.nl/ documenten/kamerstukken/2016/05/27/kamerbrief-met-kabinetsreactie-op-rapportover-wetenschappelijk-onderzoek-embryo-s), including MRT research. This means preclinical safety studies will be legally allowed. However, given the probably limited patient group opting for MRT and the potential risks it seems most logical to concentrate the expertise. Of note, should MRT prove to be effective and safe, clinical application of MRT would in fact be allowed following Dutch legislation: although the 1997 Oviedo Convention (Declaration on Human Rights and Biomedicine) of the Council of Europe was signed, which includes a general prohibition of germline gene modification, the Netherlands has made a provision allowing her upon ratification to exclude the mitochondrial DNA from this ban. ${ }^{85}$ The usefulness of adopting MRT from our colleagues abroad also depends on the number of couples that are expected to undergo this treatment. Such a theoretical calculation has been made for the UK, indicating that around 150 births per year would potentially benefit from MRT. ${ }^{86}$ Accordingly, for the United States a number of $\sim 780$ was calculated. ${ }^{86}$ For the Netherlands, the similarly obtained estimation is 25 yearly births (http://www.medischcontact.nl/Nieuws/Laatste-nieuws/Nieuwsbericht/149054/

Gentherapie-voorkomt-niet-elke-mitochondriale-ziekte.htm). It should be noted that only the mtDNA mutation carriers that have come to the authors' attention have been implicated, ${ }^{86}$ meaning the actual total number of childbearing-aged carriers is probably higher. However, in the calculations all identified mtDNA mutation carriers of childbearing age are considered as potential MRT candidates, whereas a substantial subset of these is expected to be able to benefit from PGD (Fig. 11.1). All the more because $\sim 2 / 3$ of the female carriers used in this calculation are unaffected, implying that carriers with relatively low mutation loads are expected to be included. Furthermore, $43 \%$ of the females carried a primary LHON mutation. Although as discussed such carriers are preeminent MRT candidates given the homoplasmic nature of the mutation, the non-lethal phenotype might pose ethical questions concerning the applicability of MRT. The latter is especially true as long as the (long-term) effects of the treatment are still unknown. The potential risks are more likely to outweigh the treatment effects for severe multisystem/lethal diseases. For the Netherlands, we estimate that $<10$ women per year would opt for MRT. Either way, MRT has the potential to become a valuable addition to the portfolio of reproductive options for mtDNA mutation carriers. Time will tell whether it is in fact going to replace PGD, which may only happen if MRT proves to be at least as effective and 'easily' applicable, and the in theory lower residual risk of affected offspring proves to be true. But for this moment, we do not consider MRT as replacement for PGD yet, and disagree with others who seem to have discarded PGD already. ${ }^{87,88}$ 
Very recently (abstract published on October 19, 2016) MRT (spindle nuclear transfer) has been applied in Mexico, where there are no laws preventing it, and resulted in the birth of the first child following this treatment. ${ }^{89}$ The mother carried the m.8993T>G mutation with a mutation load of $24.5 \%$. Based on this information, in our opinion she would have been a good PGD candidate as well.]

In conclusion, recurrence risks and preferred reproductive option(s) for mtDNA diseases depend on de novo or familial occurrence, on the nature of the specific mutation, on the maternal mutation load and on national legislation. Obviously, also patient preferences are part of the decision-making, a well-informed choice being important. The severity of the disease associated with the mtDNA mutation and particularly the couple's personal experience with it in the family are likely to influence the risks a couple is willing to take versus the treatment burden they deem acceptable. At present reproductive options to pursue likely unaffected, biologically-own offspring exist for the majority of mtDNA mutation cases in the form of PND or PGD. For the couples who do not currently have such options and do not wish, or are not allowed, to opt for alternatives such as oocyte donation or adoption, MRT may offer a solution in the semi-near future.

\section{Part 2: Preventing the transmission of mutations in the nuclear DNA}

Although the mitochondrial DNA is entirely dedicated to the synthesis of the 13 essential proteins, or subunits, of the respiratory chain, it does not by far provide all components necessary for mitochondrial function. Rather, mitochondria also greatly rely on an estimated number of $>1500$ proteins, encoded by nuclear genes, ${ }^{90,91}$ which provide the majority of the OXPHOS components and play important roles in mtDNA replication and maintenance as well as in mitochondrial network dynamics. Obviously, there is a close and complex mito-nuclear interaction (Figure 11.3).

Unlike the complex, maternal transmission of mitochondrial DNA mutations, nuclear DNA (nDNA) mutations implicated in mitochondrial disease follow Mendelian transmission patterns and are mostly autosomal recessively (AR) inherited or occur de novo (either X-linked or autosomal). Therefore, both prenatal diagnosis and preimplantation genetic diagnosis can be applied in a rather straightforward way. However, such reproductive options are only possible when the causative genetic defect of the disorder concerned is known. Exactly that is the major challenge in mitochondrial diseases. Considering the huge number of potentially involved nuclear genes, of which $\sim 250$ genes have already been implicated in mitochondrial diseases to 
date, $^{92}$ and a large and mostly not gene-specific variability in clinical and biochemical phenotypes, the difficulty in establishing a genetic diagnosis is not surprising.

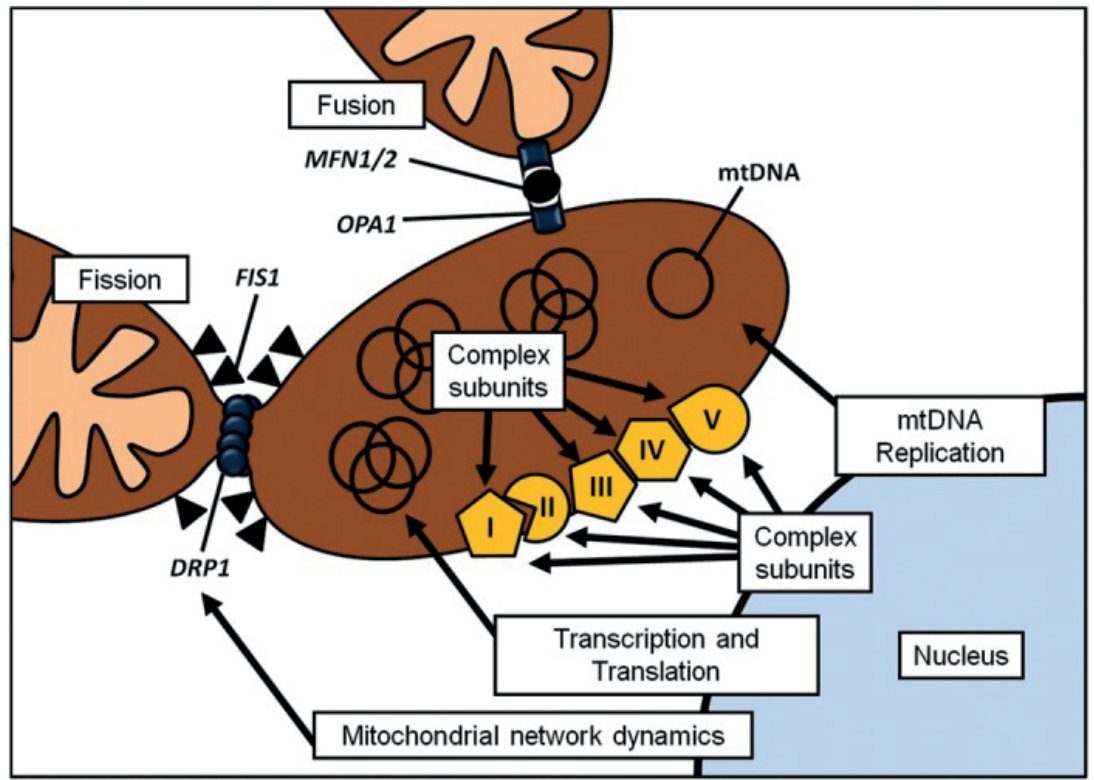

Figure 11.3 Interaction between nDNA and mtDNA Cartoon demonstrating the complex interaction between genes encoded by nDNA and the processes they control in the mitochondrion (reprinted with permission ${ }^{4}$ ).

With conventional DNA sequencing methods the genetic cause could be identified in $\sim 25 \%$ of the patients (personal experience). However, with the introduction of Next Generation Sequencing (NGS) technologies a large subset of, or even all, genes can be analyzed simultaneously. It was expected that by Whole Exome Sequencing (WES), when all genes are being analyzed simultaneously, the causative mutation(s) could be identified in the majority of mitochondrial disease patients. This is indeed our experience; so far, after exclusion of the mtDNA, the genetic cause could be firmly established in $\sim 50 \%$ of families, and in a further $\sim 20 \%$ one or more candidate variants which necessitate further follow-up were found (Hellebrekers, unpublished data). In time, WES will evolve to Whole Genome Sequencing (WGS), whereby not only the coding regions but also the non-coding regions are sequenced, further increasing the diagnostic yield. ${ }^{93}$ 


\section{$>$ Whole exome sequencing (WES) in the context of reproductive testing options}

Identifying the genetic cause enables parents to opt for reproductive testing options before (PGD), or in (PND), a subsequent pregnancy. Although defects in nuclearencoded genes are not as complex as mtDNA mutations with regard to transmission, other challenges exist.

\section{Monogenic versus multigenic phenotypes}

When a couple with a child affected by a disease of unknown etiology, like a mitochondrial(-like) disorder, asks for recurrence risk and potential options to prevent the birth of another affected child, the primary approach is to identify the cause of their child's disease. In the targeted approach, genetic testing of individual genes fitting the phenotype could either result in 1 ) the establishment of a clear cause (pathogenic variant), or 2 ) in the identification of a variant of unknown significance (VUS), or 3 ) in the inability to find genetic abnormalities at all. Only in the first situation reproductive testing options can be 'simply' offered and merely the nature of the disorder in relation to the desirability to perform reproductive testing for, can be a point of discussion. For a VUS, reproductive testing is technically possible but obviously not useful as long as its role in causing the disease is uncertain. At least a subclassification of pathogenicity is necessary to decide whether or not reproductive testing may be approved for suspicious variants in certain cases. Functional studies or the presence of mutations in the same gene in comparable patients may provide convincing evidence for the pathogenicity, but these are often lengthy or difficult to obtain in rare disease. For couples with a child wish this time factor is difficult; the context is different for parents who would like to know the cause of their child's disease but do not have the desire for further children. As mentioned above, for mitochondrial diseases the category, for which reproductive testing is not possible due to the lack of a genetic defect, has long been the majority. By performing WES, a significant subset fortunately shifts to the first category. Notably, the more unbiased the genetic analysis is, the more relevant and challenging it becomes for clinical laboratory specialists and clinicians to critically evaluate whether an identified (likely) pathogenic mutation fits the phenotype. Besides solving more cases, which obviously is the primary goal, also more VUS are being identified, not only in genes fitting the specific phenotype, but also in genes of which the association with the phenotype may be uncertain. This, as well as the for WES still more time-consuming diagnostic process, should be discussed with the couple so they can decide whether or not they wish to proceed or rather take their chances with a subsequent pregnancy. The severity of the disease in their child as well as the suspected mode of inheritance will likely influence their choice.

Not just for monogenic disease the diagnostic yield is increased by WES. Also complex phenotypes consisting of multiple genetic defects, each responsible for a part of the 
phenotype, can be unraveled by this approach, as illustrated by the two families described in Chapter 9. Multigenic phenotypes concern diseases, caused by multiple genetic defects which are individually categorized as 'monogenic'. Thus, patients have more than one pathogenic variant, which are unrelated to each other and would cause a phenotype when solely present as well, but together result in a mixed/complex phenotype. Although disease penetrance and expression may vary, with monogenic disease it is certain that an individual will not develop symptoms if the pathogenic variant is absent. In other words, the pathogenic variant is an obligatory factor to develop disease. This contrasts with multifactorial disease where a single genetic defect will not result in a phenotype unless other, genetic or environmental, factors are present, and, conversely, the absence of the genetic variant may still result in the disease. Multifactorial diseases are left out of account here.

In multigenic disease it is essential to determine which clinical features can be ascribed to which genetic defects and what the expected mode of inheritance is. The couple may have to decide for which inherited trait(s) they wish reproductive testing, considering both the severity of the diseases and the eventual chance of having an unaffected child, which reduces with the number of conditions tested for. Again, the question may also rise as to which disorder warrants or permits reproductive testing, which obviously also depends on the couple's perspective. When testing for multiple disorders in a PND setting, one could end up in a situation where the couple not just have to decide on whether or not to terminate the pregnancy for one disorder, but rather they have to make up their mind for different scenarios / combinations. Guidance of couples, dealing with such difficult processes, in a specialized setting is paramount. In such cases PGD has an advantage to PND as the a priori frequency of an unaffected embryo can be low, and that embryo can be selected for by PGD. Currently, PGD on cleavage-stage embryos is possible for a maximum of two separate gene defects but selection for three or more diseases is possible after TE biopsy, or with new single cell analysis methods. One should be aware that an increase in the number of conditions tested reduces the number of embryos suitable for transfer.

\section{WES: informed consent and pre-test counseling on incidental findings}

A more unbiased genetic test not only results in identifying more variants of uncertain significance with respect to the phenotype. Also, variants causally involved with other phenotypes may be encountered. The extent to which this may happen depends on the approach of the data analysis. After performing WES, only genes implicated in a certain disease group (e.g., OXPHOS disorders, muscle disorders, movement disorders etc.) may be analyzed and the findings are supposed to be at least related to the patient's phenotype. Identifying a causative mutation which not only explains the phenotype present in the patient, but is also associated with an increased risk to still develop other features, is possible. However, this is not unexpected since the genes included in the 
panel are known before testing. This possibility is discussed with patients during pretest counseling, and the information that can be provided will be increasingly aspecific when higher numbers of genes are tested. Such additional clinical manifestations associated with an established gene defect may influence a couple's considerations regarding reproductive testing of a subsequent pregnancy. If the WES analysis includes 'all known disease genes' ('Mendeliome'), pathogenic variants not at all related to the phenotype concerned, but to another disease may be identified. Different terminology is being used to refer to such findings that are unrelated to the reason for the test (e.g., unsolicited, unexpected, or secondary findings) but incidental findings seems to be the preferred term. ${ }^{94} \mathrm{~A}$ complete exome analysis may determine presumably pathogenic variants in genes with unknown function or disease association. Not only for the patient, also in the context of recurrence risk and reproductive choices incidental findings may have consequences.

It is controversial whether patients/parents should have a choice regarding which findings they wish to receive and which they do not.

Several studies on the topic of incidental findings have been published. Townsend et al. for example conducted group interviews from different perspectives ${ }^{95}$ and concluded that from a 'lay' point of view the patient's autonomy and his/her right to decide which findings he/she wants to know is the most important focus point. Patients accept potential anxiety or insecurity that could be the consequence. They also state that the terms 'relevance' or 'severity' can have a different meaning for each individual and that this cannot be defined in advance by the healthcare professional. Another study is cited, ${ }^{96}$ in which the public opinion with respect to incidental findings in a hypothetical pharmacogenetic test was investigated. $69 \%$ of the questioned individuals wanted to receive information involving insecure risks and $96 \%$ wanted information about serious and treatable diseases. Notably, is it known from other study areas (such as PCS) that a positive intention does not necessarily results in a high test-uptake. ${ }^{\text {e.g.97 }}$ Other papers, reporting the public's perspective by means of focus groups and interviews $(n=63){ }^{98}$ and the perspective of patients undergoing clinical WES and their family members by means of 55 in-depth interviews, ${ }^{99}$ respectively, also showed that participants wish to have a choice in receiving incidental findings. This follows from a large discrete-choice experiment $(n=1200)$ among the Canadian population ${ }^{100}$ as well, illustrating that most but not all participants wanted to receive incidental findings, and that personal utility depended on the type of finding. This corroborates our own experience that individuals clearly have different opinions and desires with respect to incidental findings, advocating the inclusion of their own choice in the process. Choices may also depend on ethnicity or culture. Although the numbers are limited, we observed a tendency towards the desire for more extensive receiving of findings among non-Dutch parents, whereas the Dutch were more often conservative in their choice. 
Other papers focus more on the perspective of the counselor or researcher. ${ }^{101-105}$ Wellknown dilemmas from the counselor's point of view are for example "how informed is informed?", i.e. is the patient able to really understand the implications of the test and of (the refusal of receiving) incidental findings, are there incidental findings that should always be reported irrespective of the patient's preference, and should the patient have a choice at all, or is it unethical to place our own conscience above the patient's autonomy? It has even been stated that caregivers have a duty to actively search for actionable genetic diseases when WES is performed, and report these findings to the patient. ${ }^{106}$

Tiered consent has been proposed as strategy to deal with incidental findings. ${ }^{107-110}$ Tiered consent is differentiated consent, subdividing the potential findings in categories of which patients may, at least partly, decide whether or not they desire to receive them. Such categories may be based on 1) clinical consequences of the associated diseases, including for example the availability of preventive measures and/or treatment options (clinical utility), or the possibility of reproductive options (personal utility) 2) the risk to develop the associated diseases (clinical validity) and 3) the extent to which the clinical implications of variants are clear (pathogenicity). The exact nature and criteria of such categories are not easy to define. Regarding the pathogenicity, it is obvious that VUS are undesirable and should be redefined as either pathogenic or benign as soon as possible, necessitating adequate and up-to-date variant databases.

\section{Consanguineous versus non-consanguineous families}

The diagnostic yield in our WES cohort of mitochondrial(-like) disease patients so far is $\sim 65 \%$ for the non-consanguineous couples and $\sim 35 \%$ for the consanguineous (unpublished data). The majority of solved cases being non-consanguineous may seem surprising at first, but the consanguinity hampers the analysis because the homozygous regions are often large and include many potentially pathogenic variants. Moreover, the causative gene or variant is more likely to be very rare (also illustrated by Chapter 10) and therefore yet unknown to be associated with disease. Indeed, more VUS are identified in patients with consanguineous parents. Thus, it is more difficult to (firmly) establish the genetic cause in a subset of cases, which has consequences for the applicability of reproductive testing options.

Identifying causative mutations in consanguineous families not only provides recurrence risks and reproductive options for the family itself, but, for specific ethnic groups, it can also be of relevance for their countries of origin. In isolated subpopulations, ${ }^{\text {e.g.,111 }}$ or subpopulations where consanguinity is custom, carrier frequency of rare variants can be high. There are global differences in the availability of (extensive) genetic testing, for example due to varying financial resources. When founder mutations are identified, ${ }^{\text {e.g.,112 }}$ it is useful to register these in population- 
specific databases ${ }^{\text {e.g.,113 }}$ as this enables targeted testing, which is more widely available than WES, in the populations concerned. This way, a far greater number of couples can be informed about the specific risks for their subsequent offspring and may opt for reproductive testing. These benefits in our opinion outweigh the potential delicate issues of stigmas based on ethnicity.

\section{Part 3: Population-wide preconception carrier screening}

The rapidly declining costs of WES enable its increasing use, not only for individual families or subpopulations, but on a population-wide scale. Analysis in search of carriership in parents not per se having an increased risk of affected offspring is defined as preconception carrier screening (PCS), the topic of Chapter 10, and brings the application of WES to yet another level. Although the concept of PCS goes back several years, until recently this was mainly performed in a targeted manner, in specific subpopulations where the frequency of the mutations concerned is known to be increased. Currently PCS is becoming available for each couple who wishes so. The most important rationale behind this is that couples who are confronted with the birth of a child affected by an autosomal or X-linked disease, could often not have anticipated this beforehand due to a negative family history. Also for consanguineous couples, having a known increased risk of affected offspring irrespective of ethnicity, genetic testing to qualify this risk was not possible, particularly when family history was negative. WES enables PCS in an unbiased way and can therefore be applied for all couples, whether or not they have an increased risk of being carriers. Not only for couples without a priori increased risks but also for known carrier couples PCS may still be advantageous. Perhaps even more so in certain situations. Couples who already have an affected child will often be more aware and motivated to minimize the risk of another affected child, not only regarding the same disorder but also regarding another. The same applies to couples going through an intensive PGD treatment. They may be eager to carry out PCS and this has indeed been requested repeatedly in our experience.

A major difference of PCS compared to identifying the genetic cause of a patient's disease is the lack of an affected index patient to relate the findings to. Therefore, the clinical consequences of a certain defect have to be predicted based on the at that point available data. Obviously, this is rather straightforward for well-known gene defects/diseases but the rarer the finding, the more difficult this will be. If possible, it is relevant to inform the couple about the clinical spectrum of a given disease. In case the clinical consequences of one or more identified gene defects are not entirely clear, this brings along dilemmas with regard to reproductive testing. We advocate to embed WES-based PCS in a specialized setting, whereby the findings, and whether or not they 
should be reported, are discussed in a multidisciplinary PCS team. Close collaboration between clinical and laboratory geneticists in an academic setting is therefore increasingly important. Which diseases should be tested/reported in a PCS context is somewhat controversial, not for the highly severe and untreatable diseases with onset in infancy or childhood, but for example for diseases with variable expression, adult onset, milder phenotype, or availability of a treatment. In our opinion, 'filters' on what to report and what not, should be applied after the analysis, because, as shown in Chapter 10, this way the chances of missing highly relevant pathogenic mutations or genes is smallest. Defining the exact criteria of the findings to be reported is a difficult/arbitrary discussion upfront and can be more easily done on the small number of remaining variants, in case-by-case decisions. Even if certain criteria are agreed upon, creating a gene panel which is, given these criteria, complete, is hardly possible. Not only because (rare) disease genes may be overlooked even by careful consideration, but also due to the discovery of new disease genes almost on a daily basis. Thus, in our opinion, defining gene panels beforehand not only filters out 'undesired' findings (which is the main rationale of gene panels), but most likely also mutations/genes that are in fact the reason for carrying out PCS. Chances of the latter are highest in consanguineous couples (Chapter 10) but are also relevant for nonconsanguineous couples. Another discussion concerns in- or excluding analysis for X-linked carriership, mainly because its identification may have implications for the female's own health (note: although to a lesser extent, this may also apply to some autosomal diseases). If X-linked analysis is included, this consequence should be discussed in pretest counseling, and couples may opt-in or opt-out. Still, the associated phenotypes in affected boys are generally much more severe, which obviously is the main argument to test for them. Moreover, parents often worry more about the health of their (future) children than about their own.

Although opinions on PCS obviously vary and some concerns and barriers exist, in general, positive attitudes are observed both among professionals and the public. ${ }^{114}$ Data on the public's perception of WES/WGS-based PCS in particular are yet scarce but indicated positive attitudes as well (10/16 individuals desiring all possible screening information) and illustrate, similar to the studies on incidental findings, that opinions are very personal. ${ }^{115}$ With the introduction of PCS at a broader scale, one may fear a 'slippery slope', whereby PND/PGD will be offered for increasingly milder diseases. To prevent this from evolving to unethical / immoral dimensions it is paramount that reproductive testing indications are carefully set in the afore-mentioned specialized and professional academic settings with multidisciplinary evaluation and, if necessary, independent review by a national committee.

In all, despite the dilemmas and differences in opinions that more extensive genetic testing may bring along, the advantages are evident. Reproductive autonomy is enhanced, including the possibility of choosing for reproductive testing options to 
prevent the transmission of disease. ${ }^{116}$ Importantly, genetic testing is available for individuals who desire it, but can be omitted by those who do not. We feel that people, when they receive adequate information, are well capable of making this choice.

\section{$>$ Preconception carrier screening for mtDNA mutations?}

As WES is a genome-wide approach, one would expect to be able to obtain the whole mitochondrial DNA sequence as well. Indeed, this has been achieved at least for heteroplasmy levels of $\sim 40 \%$ and above. ${ }^{117}$ The potential to simultaneously analyze the nDNA and mtDNA with a single sequencing method brings up the question whether, in time, the mtDNA should be included in a female's PCS test. Crucial differences of PCS for mtDNA mutations compared to nuclear gene mutations are the heteroplasmy and inter-tissue variation aspects.

Based on the current performance ${ }^{117}$ WES would be a rough filter for mtDNA mutations. The disadvantage obviously is that mutations at lower, but still relevant, heteroplasmy levels would be missed, but at the same time this has the advantage that pathogenic variants (Mitomap; http://www.mitomap.org/MITOMAP) identified at these mutation loads are by definition clinically relevant, at least in the context of transmission risk. Therefore they should be followed up in all cases (e.g., testing of postmitotic tissue, as the initial screening is most likely performed on blood). Such mutations may also have consequences for the female's own health, an issue mentioned above for (particularly X-linked) nuclear variants as well, but the frequency of finding these, at $>40 \%$ mutation load, in healthy women is expected to be very low. ${ }^{118,119}$

For PCS a more sensitive screening is desired, making a separate method necessary, with greater sequencing depths. Adding this to the screening would undermine the advantage of applying a single test and therefore, most importantly, brings up a cost aspect. The approach could be analogous to our current diagnostic protocol, using an mtDNA-specific Next Generation Sequencing technique with a detection level of $>1-2 \%$ (personal experience), as a first screening in blood. Variants should be evaluated for pathogenicity, as described for the nuclear genes, and only confirmed pathogenic mtDNA variants (Mitomap) should be followed up, by testing postmitotic tissues in order to estimate the recurrence risk properly. For the 10 most common pathogenic mtDNA mutations a carrier frequency of 1:200 (15/3168) was shown, ${ }^{118}$ but by applying a detection level of $>1-2 \%$ not all of these would be detected. This does not seem a major disadvantage as very low heteroplasmy levels, even in blood, will often have limited clinical relevance, both for the female and for her (future) offspring. Detecting these in a screening setting may result in unnecessary anxiety. 


\section{Further perspectives for treatment of patients}

Preventing disease transmission will not prevent the birth of patients affected by mitochondrial disorders, for example due to de novo mutations. Therefore, investing in the development of cures remains relevant. Although the advantages of genetic testing in that context are currently limited, there are some promising starting points. The work presented in this thesis in fact also contributes to this. Identifying the genetic cause of a disease is the first step to gain insight in the pathologic processes responsible for the patient's phenotype and may result in clues on potential treatment options. ${ }^{\text {e.g.,112,120-124 }}$ With regard to mtDNA mutations, gaining an understanding of their heteroplasmic nature and transmission patterns, provides clues for an entirely different way of treating these diseases. Looking at observed selection processes both in the germline and in somatic dividing tissues, it all comes down to selecting healthy, and discarding mutated mitochondria. Following these biological principles, myogenic stem cell therapy is currently being developed for mtDNA mutations. Hereby, the patient's own muscle stem cells that do not contain the mtDNA mutation are used, aiming to safely induce muscle regeneration and to increase mitochondrial functioning. As such, this approach is similar to PGD for mtDNA mutations whereby (preferably) mutationfree cells (embryos) are selected as well. Thus, our work not only evolved new strategies, optimized existing strategies and provided useful insights for developing/future strategies to prevent the transmission of mitochondrial diseases, it also imparts clues for the development of their treatment. 


\section{References}

1. Wong LJ. Pathogenic mitochondrial DNA mutations in protein-coding genes. Muscle Nerve. 2007;36(3): 279-293.

2. Zeviani M, Antozzi C. Mitochondrial disorders. Mol Hum Reprod. 1997;3(2):133-148.

3. Chinnery PF, DiMauro S, Shanske S, et al. Risk of developing a mitochondrial DNA deletion disorder. Lancet (London, England). 2004;364(9434):592-596.

4. Chinnery PF, Hudson G. Mitochondrial genetics. Br Med Bull. 2013;106:135-159.

5. White SL, Collins VR, Wolfe R, et al. Genetic counseling and prenatal diagnosis for the mitochondrial DNA mutations at nucleotide 8993. Am J Hum Genet. 1999;65(2):474-482.

6. Marchington D, Malik S, Banerjee A, et al. Information for genetic management of mtDNA disease: sampling pathogenic mtDNA mutants in the human germline and in placenta. $J$ Med Genet. 2010;47(4):257-261.

7. Monnot S, Gigarel N, Samuels DC, et al. Segregation of mtDNA throughout human embryofetal development: m.3243A>G as a model system. Hum Mutat. 2011;32(1):116-125.

8. Marchington DR, Scott-Brown M, Barlow DH, Poulton J. Mosaicism for mitochondrial DNA polymorphic variants in placenta has implications for the feasibility of prenatal diagnosis in mtDNA diseases. Eur $J$ Hum Genet. 2006;14(7):816-823.

9. Steffann J, Monnot S, Bonnefont JP. mtDNA mutations variously impact mtDNA maintenance throughout the human embryofetal development. Clin Genet. 2015;88(5):416-424.

10. Bouchet C, Steffann J, Corcos J, et al. Prenatal diagnosis of myopathy, encephalopathy, lactic acidosis, and stroke-like syndrome: contribution to understanding mitochondrial DNA segregation during human embryofetal development. J Med Genet. 2006;43(10):788-792.

11. Hellebrekers DM, Wolfe R, Hendrickx AT, et al. PGD and heteroplasmic mitochondrial DNA point mutations: a systematic review estimating the chance of healthy offspring. Hum Reprod Update. 2012;18(4):341-349.

12. Bredenoord AL, Krumeich A, De Vries MC, Dondorp W, De Wert G. Reproductive decision-making in the context of mitochondrial DNA disorders: views and experiences of professionals. Clin Genet. 2010;77(1):10-17.

13. Poulton J, Bredenoord AL. 174th ENMC international workshop: Applying pre-implantation genetic diagnosis to mtDNA diseases: implications of scientific advances 19-21 March 2010, Naarden, The Netherlands. Neuromuscul Disord. 2010;20(8):559-563.

14. Fragouli E, Spath K, Alfarawati S, et al. Altered levels of mitochondrial DNA are associated with female age, aneuploidy, and provide an independent measure of embryonic implantation potential. PLoS Genet. 2015;11(6):e1005241.

15. Monnot S, Samuels DC, Hesters L, et al. Mutation dependance of the mitochondrial DNA copy number in the first stages of human embryogenesis. Hum Mol Genet. 2013;22(9):1867-1872.

16. Cagnone GL, Tsai TS, Makanji Y, et al. Restoration of normal embryogenesis by mitochondrial supplementation in pig oocytes exhibiting mitochondrial DNA deficiency. Sci Rep. 2016;6:23229.

17. May-Panloup P, Vignon X, Chretien MF, et al. Increase of mitochondrial DNA content and transcripts in early bovine embryogenesis associated with upregulation of mTTFA and NRF1 transcription factors. Reprod 'Biol Endocrinol. 2005;3:65.

18. Diot A, Dombi $E$, Lodge $T$, et al. Modulating mitochondrial quality in disease transmission: towards enabling mitochondrial DNA disease carriers to have healthy children. Biochem Soc Trans. In press.

19. Neupane J, Vandewoestyne M, Heindryckx B, et al. A systematic analysis of the suitability of preimplantation genetic diagnosis for mitochondrial diseases in a heteroplasmic mitochondrial mouse model. Hum Reprod. 2014;29(4):852-859.

20. De Vos A, Staessen C, De Rycke M, et al. Impact of cleavage-stage embryo biopsy in view of PGD on human blastocyst implantation: a prospective cohort of single embryo transfers. Hum Reprod. 2009;24(12):2988-2996.

21. Bredenoord AL, Dondorp W, Pennings G, De Wert G. Avoiding transgenerational risks of mitochondrial DNA disorders: a morally acceptable reason for sex selection? Hum Reprod. 2010;25(6):1354-1360. 
22. Samuels DC, Wonnapinij P, Chinnery PF. Preventing the transmission of pathogenic mitochondrial DNA mutations: Can we achieve long-term benefits from germ-line gene transfer? Hum Reprod. 2013;28(3):554-559.

23. St John J. The control of mtDNA replication during differentiation and development. Biochim Biophys Acta. 2014;1840(4):1345-1354.

24. Shamsi MB, Govindaraj P, Chawla L, et al. Mitochondrial DNA variations in ova and blastocyst: implications in assisted reproduction. Mitochondrion. 2013;13(2):96-105.

25. Hyslop LA, Blakeley P, Craven L, et al. Towards clinical application of pronuclear transfer to prevent mitochondrial DNA disease. Nature. 2016;534(7607):383-386.

26. Scott RT, Jr., Upham KM, Forman EJ, Zhao T, Treff NR. Cleavage-stage biopsy significantly impairs human embryonic implantation potential while blastocyst biopsy does not: a randomized and paired clinical trial. Fertil Steril. 2013;100(3):624-630.

27. Cimadomo D, Capalbo A, Ubaldi FM, et al. The Impact of Biopsy on Human Embryo Developmental Potential during Preimplantation Genetic Diagnosis. BioMed Res Int. 2016;2016:7193075.

28. Treff NR, Campos J, Tao X, Levy B, Ferry KM, Scott RT, Jr. Blastocyst preimplantation genetic diagnosis (PGD) of a mitochondrial DNA disorder. Fertil Steril. 2012;98(5):1236-1240.

29. Heindryckx B, Neupane J, Vandewoestyne M, et al. Mutation-free baby born from a mitochondrial encephalopathy, lactic acidosis and stroke-like syndrome carrier after blastocyst trophectoderm preimplantation genetic diagnosis. Mitochondrion. 2014;18:12-17.

30. Mitalipov S, Amato P, Parry S, Falk MJ. Limitations of preimplantation genetic diagnosis for mitochondrial DNA diseases. Cell Rep. 2014;7(4):935-937.

31. Sallevelt SC, Dreesen JC, Drusedau M, et al. Preimplantation genetic diagnosis in mitochondrial DNA disorders: challenge and success. J Med Genet. 2013;50(2):125-132.

32. Steffann J, Frydman N, Gigarel N, et al. Analysis of mtDNA variant segregation during early human embryonic development: a tool for successful NARP preimplantation diagnosis. J Med Genet. 2006;43(3):244-247.

33. Thorburn DR, Wilton L, Stock-Myer S. Healthy baby girl born following pre-implantation genetic diagnosis for mitochondrial DNA m.8993T>G mutation. Mol Genet Metab. 2009;98:5-6.

34. Lee HS, Ma H, Juanes RC, et al. Rapid mitochondrial DNA segregation in primate preimplantation embryos precedes somatic and germline bottleneck. Cell Rep. 2012;1(5):506-515.

35. Otten $A B$, Theunissen TE, Derhaag JG, et al. Differences in Strength and Timing of the mtDNA Bottleneck between Zebrafish Germline and Non-germline Cells. Cell Rep. 2016;16(3):622-630.

36. Kelly RD, Mahmud A, McKenzie M, Trounce IA, St John JC. Mitochondrial DNA copy number is regulated in a tissue specific manner by DNA methylation of the nuclear-encoded DNA polymerase gamma $A$. Nucleic Acids Res. 2012;40(20):10124-10138.

37. Vergani L, Rossi R, Brierley $\mathrm{CH}$, Hanna M, Holt IJ. Introduction of heteroplasmic mitochondrial DNA (mtDNA) from a patient with NARP into two human rho degrees cell lines is associated either with selection and maintenance of NARP mutant mtDNA or failure to maintain mtDNA. Hum Mol Genet. 1999;8(9):1751-1755.

38. White SL, Shanske S, McGill JJ, et al. Mitochondrial DNA mutations at nucleotide 8993 show a lack of tissue- or age-related variation. J Inherit Metab Dis. 1999;22(8):899-914.

39. Harding $A E$, Holt IJ, Sweeney MG, Brockington M, Davis MB. Prenatal diagnosis of mitochondrial DNA8993 T----G disease. Am J Hum Genet. 1992;50(3):629-633.

40. Bartley J, Senadheera D, Park P, Brar H, Abad D, Wong L. Prenatal diagnosis of T8993G mitochondrial DNA point mutation in amniocytes by heteroplasmy detection. Am J Hum Genet. 1996;59:A316.

41. Ferlin T, Landrieu P, Rambaud C, et al. Segregation of the G8993 mutant mitochondrial DNA through generations and embryonic tissues in a family at risk of Leigh syndrome. J Pediatr. 1997;131(3):447-449.

42. Steffann J, Gigarel N, Corcos J, et al. Stability of the m.8993T->G mtDNA mutation load during human embryofetal development has implications for the feasibility of prenatal diagnosis in NARP syndrome. $J$ Med Genet. 2007;44(10):664-669.

43. Jacobs LJ, de Coo IF, Nijland JG, et al. Transmission and prenatal diagnosis of the T9176C mitochondrial DNA mutation. Mol Hum Reprod. 2005;11(3):223-228. 
44. Jacobs LJAM, Drusedau M, De Coo IFM, Lindsey PJ, Smeets HJM. Skewed heteroplasmy levels in single fibroblasts and lymphocytes of carriers of the Leigh syndrome m.9176T>C mutation [Dissertation]. Someren, Maastricht University; 2007.

45. Hung PC, Wang HS. A previously undescribed leukodystrophy in Leigh syndrome associated with T9176C mutation of the mitochondrial ATPase 6 gene. Dev Med Child Neurol. 2007;49(1):65-67.

46. Carrozzo R, Tessa A, Vazquez-Memije ME, et al. The T9176G mtDNA mutation severely affects ATP production and results in Leigh syndrome. Neurology. 2001;56(5):687-690.

47. Akagi $\mathrm{M}$, Inui $\mathrm{K}$, Tsukamoto $\mathrm{H}$, et al. A point mutation of mitochondrial ATPase 6 gene in Leigh syndrome. Neuromuscul Disord. 2002;12(1):53-55.

48. De Rycke M, Belva F, Goossens V, et al. ESHRE PGD Consortium data collection XIII: cycles from January to December 2010 with pregnancy follow-up to October 2011. Hum Reprod. 2015;30(8):1763-1789.

49. Yu-Wai-Man P, Chinnery PF. Leber Hereditary Optic Neuropathy. In: Pagon RA, Adam MP, Ardinger HH, et al., eds. GeneReviews(R). Seattle (WA): University of Washington, Seattle University of Washington, Seattle. All rights reserved.; 1993.

50. Bredenoord AL, Dondorp W, Pennings G, De Die-Smulders CE, De Wert G. PGD to reduce reproductive risk: the case of mitochondrial DNA disorders. Hum Reprod. 2008;23(11):2392-2401.

51. Muggleton-Harris A, Whittingham DG, Wilson L. Cytoplasmic control of preimplantation development in vitro in the mouse. Nature. 1982;299(5882):460-462.

52. Brenner CA, Barritt JA, Willadsen S, Cohen J. Mitochondrial DNA heteroplasmy after human ooplasmic transplantation. Fertil Steril. 2000;74(3):573-578.

53. Barritt JA, Brenner CA, Malter HE, Cohen J. Mitochondria in human offspring derived from ooplasmic transplantation. Hum Reprod. 2001;16(3):513-516.

54. Brenner CA, Kubisch HM, Pierce KE. Role of the mitochondrial genome in assisted reproductive technologies and embryonic stem cell-based therapeutic cloning. Reprod Fertil Dev. 2004;16(7):743751.

55. Cree L, Loi P. Mitochondrial replacement: from basic research to assisted reproductive technology portfolio tool-technicalities and possible risks. Mol Hum Reprod. 2015;21(1):3-10.

56. Fakih MH, El Shmoury M, Szeptycki J, et al. The AUGMENT ${ }^{\text {sm }}$ Treatment: Physician Reported Outcomes of the Initial Global Patient Experience. JFIV Reprod Med Genet. 2015;3(3).

57. Zhang J, Wang CW, Krey L, et al. In vitro maturation of human preovulatory oocytes reconstructed by germinal vesicle transfer. Fertil Steril. 1999;71(4):726-731.

58. Takeuchi T, Gong J, Veeck LL, Rosenwaks Z, Palermo GD. Preliminary findings in germinal vesicle transplantation of immature human oocytes. Hum Reprod. 2001;16(4):730-736.

59. Takeuchi T, Rosenwaks Z, Palermo GD. A successful model to assess embryo development after transplantation of prophase nuclei. Hum Reprod. 2004;19(4):975-981.

60. Tanaka A, Nagayoshi M, Awata S, et al. Metaphase II karyoplast transfer from human in-vitro matured oocytes to enucleated mature oocytes. Reprod Biomed Online. 2009;19(4):514-520.

61. Tanaka A, Nagayoshi M, Awata S, Tanaka I, Kusunoki H, Watanabe S. A novel trial of nuclear transfer for repairing an aged oocyte. Fertil Steril. 2009;92(3):S58.

62. Tachibana $M$, Sparman $M$, Sritanaudomchai $H$, et al. Mitochondrial gene replacement in primate offspring and embryonic stem cells. Nature. 2009;461(7262):367-372.

63. Tachibana $M$, Amato $P$, Sparman $M$, et al. Towards germline gene therapy of inherited mitochondrial diseases. Nature. 2013;493(7434):627-631.

64. Paull D, Emmanuele V, Weiss KA, et al. Nuclear genome transfer in human oocytes eliminates mitochondrial DNA variants. Nature. 2013;493(7434):632-637.

65. Yamada M, Emmanuele V, Sanchez-Quintero MJ, et al. Genetic Drift Can Compromise Mitochondrial Replacement by Nuclear Transfer in Human Oocytes. Cell Stem Cell. 2016;18(6):749-754.

66. McGrath J, Solter D. Nuclear transplantation in the mouse embryo by microsurgery and cell fusion. Science (New York, N.Y.). 1983;220(4603):1300-1302.

67. Sato A, Kono T, Nakada K, et al. Gene therapy for progeny of mito-mice carrying pathogenic mtDNA by nuclear transplantation. Proc Natl Acad Sci U S A. 2005;102(46):16765-16770.

68. Craven L, Tuppen HA, Greggains GD, et al. Pronuclear transfer in human embryos to prevent transmission of mitochondrial DNA disease. Nature. 2010;465(7294):82-85. 
69. Wang $\mathrm{T}$, Sha $\mathrm{H}$, Ji D, et al. Polar body genome transfer for preventing the transmission of inherited mitochondrial diseases. Cell. 2014;157(7):1591-1604.

70. Ma H, O'Neil RC, Marti Gutierrez N, et al. Functional Human Oocytes Generated by Transfer of Polar Body Genomes. Cell Stem cell. 2016.

71. Craven L, Elson JL, Irving L, et al. Mitochondrial DNA disease: new options for prevention. Hum Mol Genet. 2011;20(R2):R168-174.

72. Smeets HJ. Preventing the transmission of mitochondrial DNA disorders: selecting the good guys or kicking out the bad guys. Reprod Biomed Online. 2013;27(6):599-610.

73. Amato $P$, Tachibana $M$, Sparman M, Mitalipov S. Three-parent in vitro fertilization: gene replacement for the prevention of inherited mitochondrial diseases. Fertil Steril. 2014;101(1):31-35.

74. Richardson J, Irving L, Hyslop LA, et al. Concise reviews: Assisted reproductive technologies to prevent transmission of mitochondrial DNA disease. Stem Cells (Dayton, Ohio). 2015;33(3):639-645.

75. Bredenoord AL, Dondorp W, Pennings G, De Wert G. Nuclear transfer to prevent mitochondrial DNA disorders: revisiting the debate on reproductive cloning. Reprod Biomed Online. 2011;22(2):200-207.

76. Morrow EH, Reinhardt K, Wolff JN, Dowling DK. Risks inherent to mitochondrial replacement. $E M B O$ Rep. 2015;16(5):541-544.

77. Ferreira CR, Burgstaller JP, Perecin F, et al. Pronounced segregation of donor mitochondria introduced by bovine ooplasmic transfer to the female germ-line. Biol Reprod. 2010;82(3):563-571.

78. Kang E, Wu J, Gutierrez NM, et al. Mitochondrial replacement in human oocytes carrying pathogenic mitochondrial DNA mutations. Nature. 2016;540(7632):270-275.

79. Meirelles FV, Smith LC. Mitochondrial genotype segregation during preimplantation development in mouse heteroplasmic embryos. Genetics. 1998;148(2):877-883.

80. Van Blerkom J. Mitochondria as regulatory forces in oocytes, preimplantation embryos and stem cells. Reprod Biomed Online. 2008;16(4):553-569.

81. Chiaratti MR, Bressan FF, Ferreira CR, et al. Embryo mitochondrial DNA depletion is reversed during early embryogenesis in cattle. Biol Reprod. 2010;82(1):76-85.

82. Cong L, Ran FA, Cox D, et al. Multiplex genome engineering using CRISPR/Cas systems. Science (New York, N.Y.). 2013;339(6121):819-823.

83. Reddy P, Ocampo A, Suzuki K, et al. Selective elimination of mitochondrial mutations in the germline by genome editing. Cell. 2015;161(3):459-469.

84. Ceasar SA, Rajan V, Prykhozhij SV, Berman JN, Ignacimuthu S. Insert, remove or replace: A highly advanced genome editing system using CRISPR/Cas9. Biochim Biophys Acta. 2016;1863(9):2333-2344.

85. Hens K, Dondorp W, de Wert G. A leap of faith? An interview study with professionals on the use of mitochondrial replacement to avoid transfer of mitochondrial diseases. Hum Reprod. 2015;30(5):12561262.

86. Gorman GS, Grady JP, Ng Y, et al. Mitochondrial donation--how many women could benefit? N Engl J Med. 2015;372(9):885-887.

87. Falk MJ, Decherney A, Kahn JP. Mitochondrial Replacement Techniques--Implications for the Clinical Community. N Engl J Med. 2016;374(12):1103-1106.

88. Engelstad K, Sklerov M, Kriger J, et al. Attitudes toward prevention of mtDNA-related diseases through oocyte mitochondrial replacement therapy. Hum Reprod. 2016;31(5):1058-1065.

89. Zhang J, Liu H, Luo S, et al. First live birth using human oocytes reconstituted by spindle nuclear transfer for mitochondrial DNA mutation causing Leigh syndrome. Fertil Steril. 2016;106(3):e375-e376.

90. Calvo S, Jain M, Xie X, et al. Systematic identification of human mitochondrial disease genes through integrative genomics. Nat Genet. 2006;38(5):576-582.

91. Pagliarini DJ, Calvo SE, Chang B, et al. A mitochondrial protein compendium elucidates complex I disease biology. Cell. 2008;134(1):112-123.

92. Kohda M, Tokuzawa Y, Kishita Y, et al. A Comprehensive Genomic Analysis Reveals the Genetic Landscape of Mitochondrial Respiratory Chain Complex Deficiencies. PLoS Genet. 2016;12(1):e1005679.

93. Gilissen C, Hehir-Kwa JY, Thung DT, et al. Genome sequencing identifies major causes of severe intellectual disability. Nature. 2014;511(7509):344-347.

94. Shkedi-Rafid S, Dheensa S, Crawford G, Fenwick A, Lucassen A. Defining and managing incidental findings in genetic and genomic practice. J Med Genet. 2014;51(11):715-723. 
95. Townsend A, Adam S, Birch PH, Lohn Z, Rousseau F, Friedman JM. "I want to know what's in Pandora's Box": comparing stakeholder perspectives on incidental findings in clinical whole genomic sequencing. Am J Med Genet. Part A. 2012;158a(10):2519-2525.

96. Haga SB, Tindall G, O'Daniel JM. Public perspectives about pharmacogenetic testing and managing ancillary findings. Genet Test Mol Biomarkers. 2012;16(3):193-197.

97. Lakeman P, Plass AM, Henneman L, Bezemer PD, Cornel MC, ten Kate LP. Preconceptional ancestrybased carrier couple screening for cystic fibrosis and haemoglobinopathies: what determines the intention to participate or not and actual participation? Eur J Hum Genet. 2009;17(8):999-1009.

98. Daack-Hirsch S, Driessnack M, Hanish A, et al. 'Information is information': a public perspective on incidental findings in clinical and research genome-based testing. Clin Genet. 2013;84(1):11-18.

99. Clift KE, Halverson CM, Fiksdal AS, Kumbamu A, Sharp RR, McCormick JB. Patients' views on incidental findings from clinical exome sequencing. Appl Trans/ Genom. 2015;4:38-43.

100. Regier DA, Peacock SJ, Pataky R, et al. Societal preferences for the return of incidental findings from clinical genomic sequencing: a discrete-choice experiment. CMAJ. 2015;187(6):E190-197.

101. Meacham MC, Starks H, Burke W, Edwards K. Researcher perspectives on disclosure of incidental findings in genetic research. J Empir Res Hum Res Ethics. 2010;5(3):31-41.

102. Haga SB, Tindall G, O'Daniel JM. Professional perspectives about pharmacogenetic testing and managing ancillary findings. Genet Test Mol Biomarkers. 2012;16(1):21-24.

103. Downing NR, Williams JK, Daack-Hirsch S, Driessnack M, Simon CM. Genetics specialists' perspectives on disclosure of genomic incidental findings in the clinical setting. Patient Educ Couns. 2013;90(1):133138.

104. Lemke AA, Bick D, Dimmock D, Simpson P, Veith R. Perspectives of clinical genetics professionals toward genome sequencing and incidental findings: a survey study. Clin Genet. 2013;84(3):230-236.

105. Christenhusz GM, Devriendt K, Dierickx K. To tell or not to tell? A systematic review of ethical reflections on incidental findings arising in genetics contexts. Eur J Hum Genet. 2013;21(3):248-255.

106. Green RC, Berg JS, Grody WW, et al. ACMG recommendations for reporting of incidental findings in clinical exome and genome sequencing. Genet Med. 2013;15(7):565-574.

107. Bredenoord AL, Onland-Moret NC, Van Delden JJ. Feedback of individual genetic results to research participants: in favor of a qualified disclosure policy. Hum Mutat. 2011;32(8):861-867.

108. Berg JS, Khoury MJ, Evans JP. Deploying whole genome sequencing in clinical practice and public health: meeting the challenge one bin at a time. Genet Med. 2011;13(6):499-504.

109. Lolkema MP, Gadellaa-van Hooijdonk CG, Bredenoord AL, et al. Ethical, legal, and counseling challenges surrounding the return of genetic results in oncology. J Clin Oncol. 2013;31(15):1842-1848.

110. Bijlsma RM, Bredenoord AL, Gadellaa-Hooijdonk CG, et al. Unsolicited findings of next-generation sequencing for tumor analysis within a Dutch consortium: clinical daily practice reconsidered. Eur J Hum Genet. 2016;24(10):1496-500.

111. Mathijssen IB, Henneman L, van Eeten-Nijman JM, et al. Targeted carrier screening for four recessive disorders: high detection rate within a founder population. Eur J Med Genet. 2015;58(3):123-128.

112. Gerards M, Kamps R, van Oevelen J, et al. Exome sequencing reveals a novel Moroccan founder mutation in SLC19A3 as a new cause of early-childhood fatal Leigh syndrome. Brain. 2013;136(Pt 3):882-890.

113. Charoute H, Nahili H, Abidi O, et al. The Moroccan Genetic Disease Database (MGDD): a database for DNA variations related to inherited disorders and disease susceptibility. Eur J Hum Genet. 2014; 22(3):322-326.

114. Henneman L, Borry P, Chokoshvili D, et al. Responsible implementation of expanded carrier screening. Eur J Hum Genet. 2016;24(6):e1-e12.

115. Schneider JL, Goddard KA, Davis J, et al. "Is It Worth Knowing?" Focus Group Participants' Perceived Utility of Genomic Preconception Carrier Screening. J Genet Couns. 2016;25(1):135-145.

116. De Wert GM, Dondorp WJ, Knoppers BM. Preconception care and genetic risk: ethical issues. J Community Genet. 2012;3(3):221-228.

117. Griffin HR, Pyle A, Blakely EL, et al. Accurate mitochondrial DNA sequencing using off-target reads provides a single test to identify pathogenic point mutations. Genet Med. 2014;16(12):962-971.

118. Elliott HR, Samuels DC, Eden JA, Relton CL, Chinnery PF. Pathogenic mitochondrial DNA mutations are common in the general population. Am J Hum Genet. 2008;83(2):254-260. 
119. Li M, Rothwell R, Vermaat M, et al. Transmission of human mtDNA heteroplasmy in the Genome of the Netherlands families: support for a variable-size bottleneck. Genome Res. 2016;26(4):417-426.

120. Gerards $M$, van den Bosch $B$, Calis $C$, et al. Nonsense mutations in CABC1/ADCK3 cause progressive cerebellar ataxia and atrophy. Mitochondrion. 2010;10(5):510-515.

121. Gerards M, van den Bosch BJ, Danhauser K, et al. Riboflavin-responsive oxidative phosphorylation complex I deficiency caused by defective ACAD9: new function for an old gene. Brain. 2011;134(Pt 1):210-219.

122. Haack TB, Klee D, Strom TM, et al. Infantile Leigh-like syndrome caused by SLC19A3 mutations is a treatable disease. Brain. 2014;137(Pt 9):e295.

123. Gerards M, de Coo R, Smeets H. Reply: Infantile Leigh-like syndrome caused by SLC19A3 mutations is a treatable disease. Brain. 2014;137(Pt 9):e296.

124. Schiff M, Veauville-Merllie A, Su CH, et al. SLC25A32 Mutations and Riboflavin-Responsive Exercise Intolerance. N Engl J Med. 2016;374(8):795-797. 

Summary 

Mitochondrial diseases represent the most common inborn errors of metabolism and are associated with serious morbidity and mortality. This, combined with the current lack of treatment, results in requests for reproductive counseling and options to prevent the birth of an affected child. The challenge lies in the complex genetics of these disorders, as the primary genetic cause can be mutations in the mitochondrial DNA (mtDNA) or in the nuclear DNA (nDNA). Recurrence risks for mitochondrial diseases are often difficult to establish due to either the biology - unpredictable segregation patterns of mtDNA mutations- or the lack of a known genetic cause of the disease -particularly an issue for nuclear gene defects-. The same issues influence the applicability of prenatal diagnosis (PND) and preimplantation genetic diagnosis (PGD) as reproductive testing options for these disorders.

The central aim of this thesis was to develop new and optimize existing strategies to prevent the transmission of mitochondrial diseases, either caused by mtDNA mutations or nuclear gene defects, and to incorporate these choices in reproductive counseling for mitochondrial disorders. This central aim was broken down in a number of concrete objectives:

- Determine the reliability and success of PND and PGD for inherited or de novo mtDNA mutations and establish an optimal diagnostic protocol

- Characterize the different mechanisms affecting the germline transmission of mtDNA mutations and the effect this might have for recurrence risks

- Determine the frequency and distribution of de novo mtDNA mutations among mtDNA patients

- Determine the value of whole exome sequencing (WES) for establishing a genetic diagnosis in mitochondrial disease patients

- Develop a protocol for preconception carrier screening (PCS) based on whole exome sequencing

Chapter 1 describes the aims and outline of the thesis, and introduces some terminology related to mtDNA mutations, such as heteroplasmy -the mixture of mutated and wild-type mtDNA- and the genetic bottleneck -an event occurring upon transmission of mtDNA from a female to her offspring-.

Chapter 2 provides a general introduction to the reproductive options available to prevent the transmission of mitochondrial diseases. The options are considered in relation to the primary genetic causes of the disease, whereby challenges, successes and limitations are reviewed.

Chapter 3 presents nine PGD cycles in four female carriers of mitochondrial diseases, three MELAS (m.3243A>G) and one Leigh (m.8993T>G) case, and shows the applicability of PGD for mtDNA mutations. All carriers produced embryos with a 
mutation load below the threshold of disease expression, which were genetically eligible for transfer to the uterus. The mutation load of a single blastomere turned out to be representative for the mutation load of the entire embryo in the majority of cases. Because of incidental heteroplasmy deviations in single blastomeres and having limited data available, the biopsy of two blastomeres was advised for the most reliable diagnosis.

As the biopsy of two blastomeres from the embryo instead of one diminishes the chance of pregnancy, in Chapter 4 the 2-blastomere diagnostic protocol was reevaluated in our meanwhile expanded dataset. 294 single blastomeres analyzed in 73 embryos of nine female m.3243A>G mutation carriers were used to establish the error rate of a single-blastomere biopsy protocol. The risk of a false-negative diagnosis was $0.34 \%(1 / 294)$. This is defined as the risk that an embryo is classified as eligible for transfer based on a single blastomere below the threshold, whereas the other blastomeres of the embryo in fact harbor mutation loads above the threshold. Falsepositive diagnoses were not observed. The low diagnostic error rate supports a singleblastomere biopsy PGD protocol for the m.3243A>G mutation. As in the early preimplantation embryo no mtDNA replication seems to occur and the mtDNA is divided randomly among the daughter cells, we conclude this result to be independent of the specific mutation and therefore applicable to all mtDNA mutations.

Chapter 5 describes the first and successful PGD treatment for the m.14487T>C mtDNA mutation, in a 41-year old carrier, illustrating the increasing use of PGD for mtDNA mutations.

In Chapter 6, PGD data (mutation loads in oocytes, zygotes and embryos) of three mtDNA mutations ( $m .3243 A>G, m .8993 T>G, m .14487 T>C$ ) were used to further unravel the mechanisms involved in the transmission of mtDNA mutations. It was shown that both random drift and selection events (negative and positive) occur and explain the observed differences in transmission patterns between the different mutations. High m.3243A>G mutation loads are selected against in the germline, because the resulting complete oxidative phosphorylation (OXPHOS) deficiency prohibits oocyte growth and maturation. In contrast, the OXPHOS deficiency of the m.8993T>G mutation is, even at high mutation loads, less severe. In fact, this mutation is positively selected for during oogenesis, because of an increased mitochondrial membrane potential (MMP), normally a hallmark of healthy mitochondria. The m.14487T $>C$ mutation seems transmitted by random genetic drift only.

In contrast to the situation where a female carries an mtDNA mutation and the risk of affected offspring is often unpredictable and potentially high, de novo mtDNA mutations in an affected child are expected to have a low recurrence risk for a 
subsequent child. However, this is no common knowledge among health care professionals, resulting in incorrect reproductive genetic counseling. As exact data on recurrence risks for de novo point mutations were lacking, we investigated (Chapter 7) frequency and recurrence risk of de novo mtDNA mutations were investigated in data from our own laboratory (105 patients, of which 17 with a de novo mutation) and from the literature (137 de novo cases), including five illustrative cases. It was shown that de novo mtDNA point mutations are a common cause ( $25 \%)$ of mtDNA disease, and indeed have a low recurrence risk of less than $4 \%$. Prenatal diagnosis can be offered for reassurance. When determining whether an mtDNA mutation occurred de novo, it is important that multiple tissues of the mother, including postmitotic (muscle), are tested.

Chapter $\mathbf{8}$ describes the results of a study on the distribution of mutations in different mtDNA gene types (tRNA genes and protein coding genes) among mitochondrial disease patients (datasets from Chapter 7). This distribution was compared between patients with de novo and inherited mtDNA mutations and related to the total number of possible pathogenic tRNA and protein coding mutations in the mtDNA. It is known that of all mtDNA patients, most have a mutation in a tRNA gene, even though the tRNA genes together make up only $\sim 10 \%$ of the mtDNA. Remarkably, we found an entirely different ratio in de novo patients, of which the majority ( $70 \%)$ harbored a mutation in a protein-coding gene. Still, the proportion of tRNA mutations in de novo patients ( $30 \%)$ was already larger than would be expected based on their proportion in all potential mtDNA mutations ( $\sim \%)$. In the subgroup with inherited mtDNA mutations the proportion of tRNA mutations was largest ( $\sim 6 \%$ ). Besides frequency differences between tRNA and protein coding mutations as groups, also individual mutations showed varying frequencies in patients. We hypothesize that the observed patterns are explained by random occurrence of mtDNA mutations, followed by selection events, which differ for mtDNA mutations in tRNA and protein-coding genes. These selection events either occur in the germline or result from reproductive fitness related to the postnatal phenotype. Also, the heteroplasmy range where a mutation gives rise to a clinical phenotype plays a role.

Chapter 9 illustrates the value of WES in identifying nuclear gene defects as cause of mitochondrial(-like) diseases by describing two families where complex phenotypes were resolved by WES. In both families the affected patients harbored multiple genetic defects.

In Chapter 10 the application of WES on a population-wide scale was explored by developing a strategy for WES-based PCS. The proposed strategy, selecting pathogenic variants and discarding variants of unknown significance and benign variants, was applied to 8 consanguineous and 25 non-consanguineous (fictive) couples. Our results 
show for the first time that PCS based on WES is very well possible, detecting the majority of pathogenic mutations, whereas the number of variants remaining for posthoc manual evaluation remains manageable in a clinical setting. This also means that it is not necessary to apply gene panels as additional filters. In fact, a gene panel reduced the sensitivity of PCS as relevant pathogenic mutations were missed. Our study furthermore stressed the importance of recording pathogenic variants in databases, because this will in time further increase the sensitivity of PCS.

In Chapter 11, our work was incorporated into clinical practice by providing guidelines for reproductive testing strategies of mitochondrial diseases. Furthermore, overseas developments in the field of preventing the transmission of mitochondrial diseases, particularly mitochondrial replacement therapies (MRT), and the potential implications of our findings for these, were discussed in relation to the current options available. WES and PCS were more broadly discussed, in the context of counseling and reproductive options, including WES for multigenic disease, incidental findings, the potential benefits of the identified recessive mutations for carrier-screening in the population of origin, and PCS for mtDNA mutations. 
PART V

Addendum 

Nederlandse samenvatting 

Mitochondriële ziekten vormen de meest voorkomende erfelijke stofwisselingsziekten en leiden tot ernstige morbiditeit en mortaliteit. Dit, in combinatie met het vooralsnog ontbreken van behandelingsmogelijkheden, creëert een grote behoefte aan reproductieve counseling en opties om de geboorte van een aangedaan kind te voorkomen. Dit is niet eenvoudig, aangezien de primaire genetische oorzaak zowel een mutatie in het mitochondriële DNA (mtDNA) als in het nucleaire DNA (nDNA) kan zijn. Herhalingsrisico's voor mitochondriële ziekten zijn vaak moeilijk vast te stellen omdat de erfelijke oorzaak niet altijd bekend is en omdat in geval van mtDNA mutaties de overerving vaak onvoorspelbaar is. Dit beperkt ook de toepasbaarheid van prenatale diagnostiek (PND) en preimplantatie genetische diagnostiek (PGD) als reproductieve opties voor deze aandoeningen.

Het primaire doel van dit proefschrift was het ontwikkelen van nieuwe en het optimaliseren van bestaande strategieën om de transmissie van mitochondriële ziekten te voorkomen. Dit omvat ziekten zowel veroorzaakt door mtDNA mutaties als door nucleaire gendefecten. De verschillende opties zijn geïncorporeerd in de reproductieve counseling voor mitochondriële ziekten. Concreet is erop ingezet om

- de betrouwbaarheid en succes van PND en PGD voor geërfde of de novo mtDNA mutaties te bepalen en een optimaal diagnostisch protocol vast te stellen

- de verschillende mechanismen die de kiembaantransmissie van mtDNA mutaties beïnvloeden te karakteriseren alsmede het effect dat dit kan hebben op herhalingsrisico's

- de frequentie en distributie van de novo mtDNA mutaties onder mtDNA patiënten te bepalen

- de betekenis van whole exome sequencing (WES) te bepalen voor het identificeren van het nucleaire gendefect bij patiënten met een mitochondriële ziekte

- een protocol voor preconceptie dragerschapsscreening (PCS) te ontwikkelen gebaseerd op whole exome sequencing

Hoofdstuk 1 beschrijft de doelen en opbouw van het proefschrift, en introduceert een aantal kernthema's van het proefschrift, zoals mtDNA mutaties, heteroplasmie (de mix van gemuteerd en wildtype mtDNA) en de genetische mtDNA bottleneck (een fenomeen dat optreedt tijdens de transmissie van mtDNA van een vrouw naar haar nageslacht).

Hoofdstuk 2 geeft een overzicht van de reproductieve opties die beschikbaar zijn om de transmissie van mitochondriële ziekten te voorkomen. De opties worden besproken in relatie tot de primaire genetische oorzaken van de ziekte, waarbij mogelijkheden en beperkingen naast elkaar zijn gezet. 
In Hoofdstuk 3 worden de resultaten gepresenteerd van negen PGD cycli bij vier vrouwelijke draagsters van een mitochondriële ziekte; drie dragen de m.3243A>Gmutatie, die kan leiden tot het MELAS syndroom en een de m.8993T>G mutatie, die kan leiden tot Leigh syndroom. Alle draagsters produceerden op basis van het onderzoek van 2 blastomeren uit het 8-cellig stadium, embryo's met een mutatiepercentage, dat dermate laag was dat het onwaarschijnlijk was dat dit tot een aangedaan kind kon leiden. Deze zijn op basis van dit criterium geschikt om in de baarmoeder geplaatst te worden. Uit onderzoek van restembryo's bleek dat het mutatiepercentage van individuele blastomeren in de grote meerderheid van de gevallen representatief was voor het mutatiepercentage van het gehele embryo en het blastomeer-onderzoek dus betrouwbaar is als voorspellend onderzoek. Omdat er incidenteel een enkele blastomeer een afwijkend percentage liet zien en omdat de omvang van de beschikbare data nog beperkt is, werd de biopsie van twee blastomeren geadviseerd om de kans op een misdiagnose te minimaliseren.

Echter, de biopsie van twee blastomeren in plaats van één verkleint de kans op zwangerschap en daarom is in Hoofdstuk 4 het diagnostische 2-blastomeren protocol geherevalueerd op basis van een inmiddels uitgebreidere dataset. 294 individuele blastomeren, geanalyseerd uit 73 embryo's van negen vrouwelijke m.3243A>G mutatiedraagsters, zijn gebruikt. Bij het single-blastomeer biopsie protocol was het risico op een fout-negatieve diagnose, waarbij de geteste blastomeer onder de drempelwaarde van ziekte-expressie zit en de rest van het embryo erboven, $0.34 \%$ (1/294). Fout-positieve diagnoses werden niet waargenomen. Deze zeer lage kans op een foute diagnose ondersteunt een PGD-protocol met single-blastomeer biopsie voor de m.3243A>G mutatie. Aangezien in het vroege preïmplantatie embryo geen mtDNA replicatie lijkt plaats te vinden en het mtDNA willekeurig verdeeld wordt over de dochtercellen, concludeerden we dat deze resultaten onafhankelijk zijn van de specifieke mutatie en derhalve toepasbaar zijn op alle mtDNA mutaties.

Hoofdstuk 5 beschrijft de eerste en succesvolle PGD behandeling voor de m.14487T>C mtDNA mutatie, in een 41-jarige draagster, en illustreert de steeds bredere toepassing van PGD voor mtDNA mutaties.

In Hoofdstuk 6 zijn PGD data (mutatiepercentages in oocyten, zygoten en embryo's) van drie mtDNA mutaties (m.3243A>G, m.8993T>G, m.14487T>C) gebruikt om mechanismen, die een rol spelen bij de transmissie van mtDNA mutaties, verder te ontrafelen. De verschillen in transmissiepatronen tussen verschillende mutaties wijzen op een per mutatie verschillende rol van zowel willekeurige genetische drift als (negatieve en positieve) selectie. Hoge mutatiepercentages van de m.3243A>G overleven de kiembaan niet, doordat de resterende oxidatieve fosforylering (OXPHOS) capaciteit onvoldoende is voor groei en rijping van oocyten. De OXPHOS deficiëntie van 
de m.8993T $>C$ mutatie daarentegen is, zelfs bij hogere mutatiepercentages, minder ernstig, waardoor groei en rijping van oocyten normaal plaats kan vinden. Het is zelfs zo dat er positieve selectie op deze mutatie plaatsvindt tijdens de oogenese, als gevolg van een door deze mutatie hogere mitochondriale membraanpotentiaal (MMP) hetgeen normaliter een kenmerk van gezonde mitochondria is. De m.14487T >C mutatie lijkt uitsluitend door genetische drift te worden doorgegeven.

In tegenstelling tot de situatie waarbij een vrouw een mtDNA mutatie draagt en het risico op aangedaan nageslacht onvoorspelbaar en potentieel hoog is, verwachtten wij dat de novo mtDNA mutaties die zijn ontstaan in een aangedaan kind een laag herhalingsrisico hebben. Echter, in deze situatie worden deze draagsters vaak gecounseld met hoge herhalingsrisico's gebaseerd op het hoge mutatiepercentage in het kind en niet op het de novo karakter. Aangezien exacte gegevens over deze herhalingsrisico's van de novo puntmutaties ontbraken, onderzochten we (Hoofdstuk 7) de frequentie en de herhalingsrisico's van de novo mtDNA mutaties in data uit ons eigen laboratorium (105 patiënten, waarvan 17 met een de novo mutatie) en uit de literatuur (137 de novo cases). Aangetoond werd dat de novo mtDNA puntmutaties een veelvoorkomende oorzaak ( 25\%) van mtDNA-ziekten zijn, en dat deze inderdaad een laag herhalingsrisico hebben, van minder dan 4\%. Prenatale diagnostiek kan aangeboden worden ter geruststelling. Bij het vaststellen of een mtDNA mutatie de novo is opgetreden, is het belangrijk om meerdere weefsels van de moeder, inclusief postmitotisch (spier), te onderzoeken.

Hoofdstuk 8 beschrijft de resultaten van een studie naar de distributie van mutaties in het mtDNA bij patiënten met mitochondriële ziekten (datasets van Hoofdstuk 7). Deze distributie is vergeleken tussen patiënten met de novo mtDNA mutaties en met mtDNA mutaties, die van de moeder geërfd zijn. Het was bekend dat van alle mtDNA patiënten, de meeste een mutatie in een tRNA gen hebben, ondanks dat de tRNA genen samen slechts $\sim 10 \%$ van het mtDNA uitmaken. Opvallend was dat we een volledig andere ratio vonden in de novo patiënten, van wie de meerderheid ( $70 \%)$ een mutatie in een eiwitcoderend gen had. Toch was ook daar al de proportie tRNA mutaties ( $30 \%$ ) hoger dan verwacht zou worden op basis van het aandeel in alle potentiële mtDNA mutaties ( 5\%). Bij de geërfde mtDNA mutaties was het aandeel tRNA mutaties het grootst ( $86 \%)$. Afgezien van verschillen in frequentie tussen tRNA- en eiwitcoderende mutaties als groepen, waren er ook verschillen tussen individuele mutaties in patiënten. De distributiepatronen kunnen verklaard worden uit een proces, waarbij mtDNA mutaties willekeurig ontstaan, gevolgd door selectieprocessen die verschillen voor mutaties in de tRNA- en eiwitcoderende genen. Deze selectieprocessen treden ofwel in de kiembaan op, ofwel volgen uit de reproductieve fitness welke is gerelateerd aan de ernst van de ziekte. Ook de heteroplasmie-range waarbinnen een mutatie een klinisch fenotype geeft, speelt hierbij een rol. 
Hoofdstuk 9 illustreert de betekenis van WES om nucleaire gendefecten te identificeren als oorzaak van mitochondriële, en hierop lijkende, ziekten. Twee families worden beschreven waarbij complexe klinische beelden zijn opgelost met WES. In beide families hadden de aangedane patiënten meerdere gendefecten.

In Hoofdstuk 10 is een strategie ontwikkeld om op basis van WES preconceptiescreening aan te bieden op populatieniveau. In deze strategie worden zoveel mogelijk zekere pathogene varianten geselecteerd en worden varianten van onbekende significantie en benigne varianten weggefilterd. Deze benadering is uitgetest bij 8 consanguine en 25 niet-consanguine (fictieve) paren. Onze resultaten lieten voor het eerst zien dat PCS gebaseerd op WES de meerderheid van pathogene mutaties detecteert terwijl het aantal varianten dat overblijft voor post-hoc manuele evaluatie hanteerbaar is voor de klinische setting. Dit betekent ook dat het niet nodig is om genpanels toe te voegen als additionele filters. Sterker nog, een genpanel verminderde de sensitiviteit van PCS aangezien relevante pathogene varianten werden gemist. Onze studie benadrukte verder het belang om pathogene varianten in databases te registreren, omdat dit op termijn de sensitiviteit van PCS verder zal doen toenemen.

In Hoofdstuk 11 zijn onze bevindingen geïncorporeerd in de richtlijnen voor reproductief testen van mitochondriële ziekten in de klinische praktijk. Verder zijn nieuwe technieken om de transmissie van van mtDNA ziekten te voorkomen, zoals mitochondriële vervanging (MRT), besproken in relatie tot de huidige beschikbare opties. WES en PCS zijn verder bediscussieerd, in de context van counseling en reproductieve opties, inclusief WES voor multigene aandoeningen, het probleem van bijbevindingen, de bredere betekenis van geïdentificeerde recessieve mutaties voor carrier-screening in de oorspronkelijke populatie, en PCS voor mtDNA mutaties. 
Valorisation 



\section{Relevance of this thesis in patient care}

The work presented here is not only relevant from a scientific point of view, but is of importance in clinical genetic practice as well, and for patients and patient organizations.

\section{Reproductive options (prenatal diagnosis, PND / preimplantation genetic diagnosis,} PGD)

Mitochondrial diseases are often severe hereditary disorders for which couples regularly seek genetic counseling to prevent transmission to a (subsequent) child. In this thesis, guidelines for reproductive testing strategies are provided (General Discussion), which can be applied in reproductive genetic counseling. As such, it serves both the clinician and the patient. For the clinician it is an evidence-based, yet practical guideline in an area of genetics which is often considered complex. The patient can thus be informed in an optimal way regarding available reproductive options and the pros and cons in the couples specific situation, enabling an informed choice. Especially for mitochondrial diseases caused by mutations in the mitochondrial DNA (mtDNA) this adds value to the current situation, as even many health care professionals are unaware of the available options, resulting in incorrect counseling of couples (as illustrated for example in Chapter 7). To further increase knowledge of reproductive options and awareness of the possibility of PGD for mtDNA mutation carriers, we plan to collect the PGD data for mtDNA mutations from our center in an online and continuously curated database, which will be accessible for other centers as well. We expect that the (few) other centers worldwide performing PGD for mtDNA mutations will join and add their data as well, to be able to provide patients and centers offering PGD for mtDNA mutation carriers with the most up-to-date information.

Looking at health care costs, our study showing that a single-blastomere biopsy protocol is sufficient for a reliable PGD diagnosis for mtDNA mutations (Chapter 4) has a direct effect as analysing one blastomere instead of two is cheaper, whereas the success rate of the treatment (chance of a viable/ongoing pregnancy) will increase. Furthermore, our work shows that many mtDNA mutation carriers will benefit from PGD, a cheaper alternative compared to mitochondrial replacement techniques (MRT), which is currently being developed. MRT will only be necessary in a limited and selected carrier subgroup. For those carriers, not having other options for unaffected offspring, the higher costs are justifiable. The successful PGD treatment in a 41-year-old mtDNA mutation carrier (Chapter 5) who produced a quite remarkable number of oocytes considering her age, showed the benefit of establishing ovarian reserve parameters for IVF eligibility. Although not the primary objective of the paper, this illustrates that the use of such fertility markers adds to a more cost-effective 
deployment of IVF treatments by selecting the females with a realistic chance of success.

\section{Using whole exome sequencing (WES) for diagnostics}

Both in our own experience and that of others, ${ }^{1}$ the application of WES as diagnostic tool for mitochondrial diseases, where the mtDNA has been excluded as the cause, enables the identification of the causative gene defect(s) in the majority of patients, instead of the $\sim 16^{2}-25 \%$ that could be solved with conventional diagnostic DNA sequencing methods. As discussed in Chapter 11 (General discussion) this potentially has favourable consequences for the patient (altered patient medical management including treatment options in specific cases) but certainly also for the parents, opting for reproductive testing options to prevent the transmission of the disease to a subsequent child. Not only for individual patients / parent couples the identification of causative mutations is evidently advantageous, also in a broader context this can, and has been shown to, be relevant for subpopulations around the world (see General discussion).

The combination of WES being a generic diagnostic method and having a higher diagnostic yield makes it an efficient and undoubtedly cost-effective approach for genetically diagnosing mitochondrial patients. Costs will be saved due to preventing the conduction of unnecessary investigations. The cost-effectiveness of WES for mitochondrial disorders may further be confirmed in a formal cost-effectiveness (MTA) study, although the clinical impact and cost-effectiveness of WES have already been shown in for example a broader pediatric setting, ${ }^{3}$ in the context of four genetically heterogeneous conditions including mitochondrial disease, ${ }^{4}$ or in another clinically and genetically heterogeneous pediatric subgroup, ${ }^{5}$ respectively.

\section{Preconception carrier screening (PCS)}

PCS enables couples at risk of having (severely) affected offspring, for example by a mitochondrial disorder, to be identified prior to their first pregnancy. If a shared carriership is revealed in the parents, an affected child does not need to be born first. The concept of PCS in itself is not new, but PCS based on WES, for which we developed a strategy suitable for the clinical setting, has not been described before. Therefore, WES-based PCS can be viewed as a new 'product' for clinical practice, resulting from this thesis. The major advantage compared to existing PCS approaches is the increased sensitivity, because not just a selection of genes, but all genes are included. Our strategy combines the potential of identifying the mutations of interest in the broadest possible way at one hand, and minimizing findings with unknown significance on the other. 
Several groups studied the cost-effectiveness of screening for single disorders, sometimes in specific populations, showing cost-effectiveness in certain contexts. ${ }^{6-8}$ Furthermore, for a set of 14 genes, PCS by next-generation sequencing (NGS) was modeled to be cost-effective compared with no screening or targeted mutation analysis of these genes. ${ }^{9}$ Cost-effectiveness of WES-based PCS has not been investigated so far. As already mentioned above, WES for PCS can be efficiently implemented in the existing diagnostic flow. Moreover, costs for WES are rapidly declining. Considering the tremendous costs of raising a child with a (severe) genetic disorder, PCS is presumed to be cost-effective, although cost-effectiveness is obviously not the main reason to perform PCS. Nevertheless, it is a recurring question when the application of PCS on a broader scale is considered. The most important cost parameters involved are the expenses of the test, the costs (treatment of a patient) that are saved, and the recurrence of the disease(s) in the population. Therefore, a gross indication of costeffectiveness for WES-based PCS in the Netherlands can already be obtained by comparing carrier frequency and the average patient lifetime costs for the most prevalent disorders. In a hypothetical number of 20,000 couples, 20 CF carrier couples will be present (carrier frequency 1 in 30, ${ }^{10}$ carrier couple frequency 1 in 900). By identifying them, the birth of 20 unexpected CF patients can potentially be prevented. As lifetime costs for a CF patient are $€ 895.291,{ }^{11} \sim € 17,905,820$ can be saved when only considering this single disorder. For spinal muscular atrophy (SMA) with 10 carrier couples in this hypothetical population (carrier frequency of $\sim 1$ in $45^{12}$ ) and average lifetime costs per patient of $€ 336,726^{13}$ (calculated from $2009 \$$ ), a saving of $\sim € 3,367,260$ can be calculated. Accordingly, for Pompe's disease, with a carrier frequency of 1 in $100^{14}$ and average costs of $€ 7,032,899$ for the classic form when treated with enzyme replacement therapy, ${ }^{15}$ the number is $€ 14,065,798$. Smith Lemli Opitz (SLO) and Congenital Disorder of Glycosylation type 1A (CDG1A) are two other relatively frequent autosomal recessive disorders in our population, both with carrier frequencies of $\sim 1$ in $70,{ }^{16,17}$ but unfortunately no data on lifetime costs are available. However, when considering the lifetime costs per patient $\sim € 500,000$ for each disorder (which is probably an underestimation), for these diseases together PCS would save $\sim 4,000,000$. So, in total, only for these five disorders the costs saved would be $\sim 40,000,000$. This means that a PCS test for these disorders combined should not exceed $€ 40,000,000 / 20,000 \approx € 2000$ per couple, thus $\approx € 1000$ per individual. PCS costs include the (whole exome) sequencing and analysis costs, pretest counseling, specific posttest counseling of carrier couples, and the costs for reproductive options (PND or PGD) if a carrier couple opt for these. Exome sequencing can currently be performed for well below $€ 750$ per individual and costs are rapidly declining. Only including these 5 most frequent diseases already point to cost-effectiveness of WES-based PCS. Considering that it is estimated that about 1-2 in 100 couples are at risk of having a child affected by any recessive genetic condition, ${ }^{18}$ in addition to the 37 couples from the calculation above, between 163 and 363 other carrier couples are expected to be 
present in the total of 20,000 hypothetical couples. Evidently, the cost-effectiveness question is hereby in fact answered already.

The most important question in this context probably is whether WES-based PCS is more cost-effective compared to other PCS approaches. A limited panel may be less expensive but is also less universally applicable in our nowadays mostly multicultural communities. Panels need customization for specific populations, which necessitates investment of time and money as well. Despite the higher costs of WES, the obvious advantage is that carriership for more diseases can be detected, and in a universal way. Does the extra yield outweigh the extra costs? For this question to answer, one must quantify the extra yield. For one of the PCS panels currently available in the Netherlands (Groningen), consisting of $\sim 70$ genes causing $\sim 50$ serious autosomal recessive disorders, it was reported that the cumulative carrier couple frequency of the diseases included is 1 in 150 for the Dutch population ${ }^{19}$. When applying this number, in the 20,000 hypothetical couples the panel would identify 133 carrier couples, and WES would potentially be able to identify 200-400 carrier couples. This difference seems to justify the use of WES. The WES-PCS strategy we developed could serve as a starting point to address this issue in a (cost-)effectiveness study. For this goal it will be necessary to perform WES-based PCS in a larger patient group, and compare the results to (hypothethical) results of targeted PCS based on limited gene panels in the same group. Consanguineous couples and couples opting for PGD could be the first candidates. Depending on these results, expanding PCS to a broader patient group or eventually the general population can be considered.

In the above-mentioned gross calculations, obviously several relevant factors have been left out. For example, whether or not a couple chooses to prevent the birth of an affected child, and the fact that not every carrier couple necessarily gets an affected child. The risk of the latter also depends on the total number of children a couple will have. We furthermore assumed that both the panel and WES would identify all carrier couples (in case of the panel only of the included genes of course), which in fact will not be the case. Also, for certain diseases a separate test will have to be added to the (whole exome) sequencing because otherwise they cannot be detected, and thus the PCS-test will likely be in fact a set of tests. Another factor is the variability of life-time costs of a disease, dependend on the severity in a given patient. However, such variables are in our opinion not expected to undermine the cost-effectiveness of (WES-based) PCS.

Apart from costs, and the willingness of for example health insurance companies to pay these, evidently other important factors determine whether or not PCS will eventually be applied in a population-wide manner. Examples are political opinions, societal acceptance, and awareness among the couples with a child wish. Thus, support needs 
to be secured and knowledge spread among different layers of society. This is a task for the Dutch working group PCS, which was founded in 2014 as part of the "Vereniging Klinische Genetica Nederland (VKGN)" and the "Vereniging Klinisch Genetische Laboratoriumdiagnostiek (VKGL)" and contains representatives from all 8 Academic centers in the Netherlands, including me from Maastricht.

\section{Concrete activities carried out to inform patients}

Besides informing clinicians about our work it is important to reach patients, since they are the actual 'target group'. The 'modern patient' is active and will approach health care professionals with requests if they are aware of certain opportunities. Therefore, our results and activities have not only been presented at scientific meetings, but were also reported in magazines from patient societies (e.g., magazine "Wisselstof" from "Volwassenen, Kinderen en Stofwisselingsziekten", magazine "Oog" from "Oogvereniging Nederland"), and as poster and oral presentations at patient meetings (e.g., LHON meeting for couples with a child wish, organized by "Patiëntengroep LOA/LHON", annual meeting for muscular disorders, organized by "Vereniging Spierziekten Nederland"). Also, there is close contact with for example "Stichting Metakids" and "Prinses Beatrix Spierfonds" which funded parts of our research. They receive reports of our findings and they are informed when the results are published in a scientific journal. Often they prepare, in collaboration with us, a notification / summary to publish on their websites. Furthermore, a press release is intended regarding the PCS paper. This thesis will be propagated as well and we will prepare a press release upon its publication. 


\section{References}

1. Wortmann SB, Koolen DA, Smeitink JA, van den Heuvel L, Rodenburg RJ. Whole exome sequencing of suspected mitochondrial patients in clinical practice. J Inherit Metab Dis. 2015;38(3):437-443.

2. Carroll CJ, Brilhante V, Suomalainen A. Next-generation sequencing for mitochondrial disorders. $\mathrm{Br} J$ Pharmacol. 2014;171(8):1837-1853.

3. Valencia CA, Husami A, Holle J, et al. Clinical Impact and Cost-Effectiveness of Whole Exome Sequencing as a Diagnostic Tool: A Pediatric Center's Experience. Front Pediatr. 2015;3:67.

4. Neveling K, Feenstra I, Gilissen C, et al. A post-hoc comparison of the utility of sanger sequencing and exome sequencing for the diagnosis of heterogeneous diseases. Hum Mutat. 2013;34(12):1721-1726.

5. Monroe GR, Frederix GW, Savelberg SM, et al. Effectiveness of whole-exome sequencing and costs of the traditional diagnostic trajectory in children with intellectual disability. Genet Med. 2016;18(9): 949-956.

6. Musci TJ, Caughey AB. Cost-effectiveness analysis of prenatal population-based fragile $\mathrm{X}$ carrier screening. Am J Obstet Gynecol. 2005;192(6):1905-1912; discussion 1912-1905.

7. Wei S, Quigg MH, Monaghan KG. Is cystic fibrosis carrier screening cost effective? Community Genet. 2007;10(2):103-109.

8. Tsai MC, Cheng CN, Wang RJ, Chen KT, Kuo MC, Lin SJ. Cost-effectiveness analysis of carrier and prenatal genetic testing for X-linked hemophilia. J Formos Med Assoc. 2015;114(8):722-728.

9. Azimi M, Schmaus K, Greger V, Neitzel D, Rochelle R, Dinh T. Carrier screening by next-generation sequencing: health benefits and cost effectiveness. Mol Genet Genom Med. 2016;4(3):292-302.

10. Ten Kate LP. Cystic fibrosis in the Netherlands. Int J Epidemiol. 1977;6(1):23-34.

11. van der Ploeg CP, van den Akker-van Marle ME, Vernooij-van Langen AM, et al. Cost-effectiveness of newborn screening for cystic fibrosis determined with real-life data. J Cyst Fibros. 2015;14(2):194-202.

12. Wilson RB, Ogino S. Carrier frequency of spinal muscular atrophy. Lancet (London, England). 2008;372(9649):1542; author reply 1542.

13. Little SE, Janakiraman V, Kaimal A, Musci T, Ecker J, Caughey AB. The cost-effectiveness of prenatal screening for spinal muscular atrophy. Am J Obstet Gynecol. 2010;202(3):253.e251-257.

14. Leslie N, Tinkle BT. Glycogen Storage Disease Type II (Pompe Disease). In: Pagon RA, Adam MP, Ardinger $\mathrm{HH}$, et al., eds. GeneReviews(R). Seattle (WA): University of Washington, Seattle University of Washington, Seattle. All rights reserved.; 1993.

15. Kanters TA, Hoogenboom-Plug I, Rutten-Van Molken MP, Redekop WK, van der Ploeg AT, Hakkaart L. Cost-effectiveness of enzyme replacement therapy with alglucosidase alfa in classic-infantile patients with Pompe disease. Orphan J Rare Dis. 2014;9:75.

16. Porter FD. Smith-Lemli-Opitz syndrome: pathogenesis, diagnosis and management. Eur J Hum Genet. 2008;16(5):535-541.

17. Schollen E, Kjaergaard S, Legius E, Schwartz M, Matthijs G. Lack of Hardy-Weinberg equilibrium for the most prevalent PMM2 mutation in CDG-la (congenital disorders of glycosylation type la). Eur J Hum Genet. 2000;8(5):367-371.

18. Henneman L, Borry P, Chokoshvili D, et al. Responsible implementation of expanded carrier screening. Eur J Hum Genet. 2016;24(6):e1-e12.

19. Plantinga $M$, Birnie $E$, Abbott $K M$, et al. Population-based preconception carrier screening: how potential users from the general population view a test for 50 serious diseases. Eur J Hum Genet. 2016;24(10):1417-1423. 
Dankwoord 



\section{Dankwoord}

De afronding van dit promotietraject, een mooie en leerzame periode van wetenschappelijk onderzoek doen, nadert. Aan het einde van dit proefschrift rest mij het dankwoord. En dat vind ik misschien wel een van de moeilijkste onderdelen. Gaat het lukken om zo op schrift goed over te brengen hoe je mensen waardeert? Ga ik niemand vergeten? Toch is het dankwoord natuurlijk niet voor niets belangrijk, want een proefschrift schrijf je nooit alleen. Ik denk, of hoop, dat iedereen die heeft bijgedragen, en dat kan op zoveel verschillende manieren zijn, dit van zichzelf wel weet. En ook weet dat ik hem/haar daar dankbaar voor ben. Graag begin ik ermee om dat hier ook expliciet te zeggen, dus hierbij iedereen die betrokken is geweest: heel veel dank!

Ik heb overwogen om het hierbij te laten ;-) maar hier volgt dan toch een dankwoord zoals het hoort.

\section{Patiënten:}

Allereerst: de patienten en families die bereid waren hun medewerking te verlenen aan wetenschappelijk onderzoek en/of toestemming te geven hun medische gegevens te publiceren. Zoals in de inleiding ook al vermeld: $u$ bent de belangrijkste reden om dit werk te doen. En het omgekeerde is niet minder waar: zonder u was dit proefschrift er niet geweest.

\section{Mijn promotieteam:}

Prof. dr. H.J.M. Smeets, beste Bert, mijn eerste promotor. Wat een fijne en onmisbare begeleiding heb ik van jou mogen krijgen de afgelopen jaren. Woorden die bij me opkomen zijn onder andere stimulerend en inspirerend. Ik ging steevast met een positief gevoel weg na een overleg. En dat kwam niet alleen door een grapje waar ook altijd ruimte voor was, maar zeker ook door je vermogen om dingen toch nog net weer beter te maken, met een andere invalshoek of een ontbrekend element. En dat op een opbouwende manier. Ik heb me altijd door jou gesteund gevoeld, of het nu om mijn werk ging of om aanmoediging langs de kant van een Charity Run in Lissabon (ESHRE 2015) :-). Maar zonder gekheid, dat illustreert je betrokkenheid waarbij de persoonlijke kant niet uit het oog verloren wordt. Wat betreft het schrijven zijn me vooral 'de lijn in het verhaal houden' en je kunst om teksten in te korten zonder informatieverlies bijgebleven, beide goede leerpunten voor mij. Je motto "ik heb altijd overal tijd voor", hetgeen je ook waarmaakte als ik er een (krappe) planning doorheen wilde krijgen, zie ik als voorbeeld. Tot slot: de week in Vancouver was een gezellige afsluiting van deze periode!

Prof. dr. C.E.M. de Die-Smulders, beste Christine, mijn tweede promotor. Bedankt voor je waardevolle bijdrage vanuit jouw ervaren klinisch perspectief, waarin je voor mij een 
voorbeeld bent. Ook stel ik je betrokkenheid op prijs, en dan heb ik het uiteraard niet alleen over het uitwisselen van shoptips of het feit dat je het altijd opmerkt als ik iets nieuws aan heb. Jeroen noemt jou nog wel eens gekscherend mijn 'surrogaatmoeder', misschien nog niet eens zo'n hele vreemde vinding; je bent in elk geval wel een beetje mijn 'geneticamoeder' als tevens opleider geweest zijnde. Ik ben blij om zo nauw met je samen te werken binnen het reproductieve team op onze afdeling. Wat betreft congressen in het buitenland: daar was het aan jou wel toevertrouwd om met hulp van je tripadvisor-app een leuk kleinschalig restaurantje te vinden.

Dr. I.F.M. de Coo, beste René, mijn co-promotor. We hebben vooral veel met elkaar te maken gehad rondom jouw patienten die geïncludeerd werden in het 'Metakids project' met als doel de genetische oorzaak van hun mitochondriele aandoening aan te tonen. Een deel van deze families is later ook nog meegenomen in de ontwikkeling van de preconceptiescreening-methode. Je hebt dan ook een essentieel aandeel in de 'patiëntenlevering' voor dit proefschrift. Ik heb je leren kennen als enthousiast en hartelijk, met veel kennis over mitochondriële aandoeningen en hart voor je patiënten. Gastvrij ook, ik mocht zelfs eens logeren bij jou en je vrouw Mechteld toen ik twee dagen achter elkaar patiënten kwam zien in Rotterdam. Leuk dat je me uitnodigde voor de MCRN meeting in Kopenhagen waar ik gezellige en hilarische herinneringen aan heb overgehouden.

Leden van de beoordelingscommissie en corona:

Prof. dr. C.G. Faber (voorzitter), prof. dr. J.L.H. Evers, dr. P. Lakeman en prof. dr. J.A.M. Smeitink, u allen wil ik als leden van de beoordelingscommissie hartelijk danken voor de aandacht die $\mathrm{u}$ heeft besteed aan de beoordeling van dit proefschrift. Prof. dr. M. Herbert, prof. dr. M. Bonduelle en prof. dr. G. de Wert wil ik danken voor het aannemen van de uitnodiging om aan de oppositie deel te nemen tijdens de verdediging van mijn proefschrift.

\section{Collega's:}

Naast mijn promotieteam zijn er uiteraard nog diverse andere collega's die hebben bijgedragen aan mijn proefschrift. Jos, jou wil ik hier apart noemen; de PGD-data inclusief heranalyses die jij allemaal zo keurig bijhoudt zijn van onschatbare waarde geweest. Daarnaast dank aan alle anderen die een rol hebben gehad in het genereren van data en/of bij wie ik altijd terecht kon met vragen variërend van mito, stand van zaken in research-families, filteren van exoom data tot ICT-perikelen: o.a. Debby, Alexandra, Bianca, Tom, Bart, Rick, Dimitri, Aimée, Ralph, Iris, Patrick, Radek, Jo Beerens en Jo Vanoevelen. Alle (overige) co-auteurs van de papers bedankt voor jullie bijdragen en samenwerking. Auke, mijn 'zebravis-counterpart', we hebben in ons beider promotie-traject deels samen opgetrokken op het bottleneck-onderwerp, en dat schiep een band, die tevens geresulteerd heeft in een vriendschap. Binnenkort maar 
weer eens wat gaan eten en hopelijk proosten op de publicatie van ons gezamenlijke artikel :-).

Verder mijn collega's van de afdeling klinische genetica. Altijd geïnteresseerd in hoe het ging met mijn onderzoek, en in de drie maanden 'schrijftijd' die ik kreeg heb ik bergen kunnen verzetten voor de eindspurt. Naast hard werken op deze bevlogen afdeling is er gelukkig ook af en toe tijd voor een lunch, koffiemomentje, borrel of etentje, waardevol om elkaar ook eens op een andere manier te spreken. Maaike, gezellig dat we toch nog wat langer kamergenoten blijven.

Hulp bij het boekje:

Tiny bedankt voor je hulp bij het opmaken van dit proefschrift. Jennie, leuk dat jij als mijn nicht geholpen hebt met het ontwerp van de omslag, je hebt wat ik aan ideeën in mijn hoofd had mooi weten weer te geven, dankjewel!

\section{Buiten het werk:}

Ook niet-werk-gerelateerd zijn er zovelen die hun bijdrage hebben geleverd, in de vorm van welkome afleiding, gezelligheid en belangstelling.

Smurfen; Anniek, Mieke, Ninne, Nina, Anne, Irma, Kristel en Kirsten, ook al ligt de middelbare schooltijd al ver achter ons, we delen nog altijd lief, leed en lol samen en dat is me enorm dierbaar en waardevol! Lang leve de groepsapp (waarbij jullie me er nooit op aankijken dat ik de 'weekend-apper' ben), maar gelukkig ook nog regelmatig in real-life want daar gaat toch niets boven. Feestjes, theekransjes, BBQ's, festivals, Sinterkersternieuw, op stap, of weekendjes weg, alles is even leuk met jullie; ik verheug me alweer op de volgende smurfendate!

Marie-Claire; samen gestart met de studie geneeskunde in Maastricht en vanaf het eerste moment (introductiekamp) dikke vriendinnen. En ook al worden we ouder, onze vriendschap blijft hetzelfde; we kunnen uren kletsen over serieuze (waarbij de wederzijdse herkenbaarheid op diverse terreinen altijd zo fijn is) en niet-serieuze dingen, shoppen, lachen, en ook nog steeds gekke dingen doen. Ik kijk ernaar uit dat we nu als het goed is allebei weer wat meer tijd hebben om samen leuke dingen te doen. Om dat te vieren wordt het om te beginnen toch echt weer eens tijd voor een ouderwets Zsa-zsa \& Loopy avondje vind je niet?

Soetinah; de introductiecursus voor nieuwe werknemers van het azM bracht ons bij elkaar, en sindsdien hebben we menig gezellig avondje samen gegeten, een theetje en/of wijntje gedronken, gesport, of een bezoekje aan de (ladies night) bioscoop gebracht. Ik ben blij om jou tijdens mijn promotie aan mijn zijde te hebben als paranimf. Jammergenoeg ga je Maastricht verlaten, ik ga je missen! Maar ik kom je zeker een weekend opzoeken in Londen en ook daarna, als je in Utrecht gaat wonen, weet ik zeker dat we elkaar blijven zien.

Ingrid; mijn andere paranimf, waarvan ik net zo blij ben dat je aan mijn zijde staat. Onze vriendschap ontstond op het moment dat we collega's werden bij de klinische genetica. 
Je bent iemand die er is als je het nodig hebt en met je no-nonsense instelling kun je verhelderende adviezen geven. Verder koester ik de gezellige avonden met onze partners (Jeroen en Bart) erbij, eten, drinken en vaak een spelletje, en moeten we gauw weer een weekendje weg gaan plannen!

Dolce Far Niente; Irene, Laura, Miranda, Elsa, Marjanneke, Debbie en Marie, ook al zien we elkaar nog maar sporadisch, de gezelligheid op die momenten wordt er niet minder op. En mijn agenda wordt vanaf nu leger is de bedoeling, dus dat biedt perspectief. Misschien leuk om te vermelden dat jullie gegraveerde vulpen ("Dolce Far Niente", vrij vertaald "Het zoete nietsdoen") nog altijd trouw dienst doet en ook aan dit proefschrift zijn woordje heeft bijgedragen.

Verder alle anderen met wie ik (of wij) geregeld of minder geregeld een koffietje, drankje of iets anders leuks doe(n): Margje, Bianca, Kim, Céline, Deborah, Rélana, AnneMarthe, Malou (in het voorjaar ga ik weer eens mee tennissen!), Jenda \& Marius, Mariëlle \& Ralph.

Ook onze buurtjes, Maurice \& Danycia en Rob \& Kelly, niet te vergeten; een warm welkom toen we ruim twee jaar geleden in de Ravensbosch kwamen wonen, de gezellige avondjes houden we erin!

En natuurlijk de maatjes (Mathieu, Jos, Heidi, Annemijn, Valerie, Esther, Roos, Juella en anderen) en paarden van manege 't Vroendael, dank voor het wekelijkse avondje ontspanning, zowel in de les als daarna aan de bar! Daarnaast de buitenritten (Jos en Heidi) en op pad met de motor (Mathieu, Paul en Annemijn), zeker voor herhaling vatbaar, en de wijnproeverij wordt ongetwijfeld ook geslaagd.

Mijn schoonfamilie; Ineke en Fred, Niels en Tanja, ook altijd belangstellend hoe het vorderde met de promotie. Jammergenoeg is Groningen (Niels en Tanja) niet echt naast de deur maar gelukkig weerhoudt ons dat er niet van elkaar toch af en toe te zien.

En dan natuurlijk mijn lieve familie. Pap en mam: een cliché maar daarom niet minder waar: zonder jullie stond ik hier niet! Jullie hebben mij alle kansen gegeven om mijn mogelijkheden te benutten. De grote betrokkenheid die er altijd is waardeer ik zeer. Joost en Bas, dat geldt ook voor jullie. Ik ben blij met de goede band die ik met jullie als broertjes heb. Een groot deel van dit proefschrift is tot stand gekomen onder toeziend oog van jullie wijze uil op mijn bureau thuis. Joost, dankjewel ook dat je altijd beschikbaar bent als vraagbaak voor computervraagjes. Lisanne en Jay, inmiddels ook al een tijd part of the family, dus jullie mogen hier niet ontbreken. Tot slot ook alle andere familieleden dank voor jullie interesse, waarbij ik jou, Lenie, als mijn peettante hier graag apart nog even noem.

En het is geloof ik een beetje een terugkerend thema in dit dankwoord, maar ook met jullie, familie, hoop ik met een minder volle agenda nog wat vaker leuke dingen te kunnen plannen (frequentere moeder-dochterdagjes, paardrijden met pap, broers-zusweekendje deel 3 en eens met $z$ 'n zessen, Polman/Burgers-damesdagje etc.). 
Lieve Jeroen: bedankt voor jouw liefde en steun, ik hou van je! Je humor en af en toe de noodzakelijke rem die ik zelf niet altijd kan vinden, maar ook je bereidheid om iets door te lezen of mee te denken als ik weer eens een keuzedilemma had, zijn slechts voorbeelden van jouw hulp. De uiltjes voor aan de deur tijdens mijn 'uitzending in eigen huis' blijven zo'n leuk idee dat ik ze hier toch ook even vermeld. Een promotie wordt wel eens een bruiloft voor één persoon genoemd; het volgende feestje wordt voor twee!

Suzanne 

Curriculum vitae 



\section{Curriculum vitae}

Suzanne Sallevelt werd op 23 maart 1985 geboren in Boxmeer. Haar gymnasiumopleiding volgde ze aldaar op het Elzendaalcollege, waar zij in 2003 cum laude haar diploma behaalde. In datzelfde jaar verhuisde ze naar Maastricht om geneeskunde te gaan studeren. In 2009 behaalde ze cum laude haar artsexamen. Tijdens haar geneeskundestudie koos zij voor een keuzeblok "Erfelijke stofwisselingsziekten bij kinderen". Buitenlandervaring deed zij op in Suriname, waar zij zowel een keuze-coschap kindergeneeskunde (onderwerp van de bijbehorende scriptie: Pathofysiologie van multi-orgaanfalen bij perinatale asfyxie) als een wetenschapsstage farmacologie/ fysiologie (Evaluation of plant extracts with presumed antihypertensive properties for their capacity to relax isolated guinea pig aorta rings precontracted with phenylephrine) volgde. Ook is zij tijdens de eerste Suriname-periode op medische zending in de binnenlanden geweest. Haar laatste klinische keuzestage voor het afstuderen vond plaats op de afdeling Klinische Genetica in Maastricht. Hier startte zij aansluitend in 2009 met de opleiding tot klinisch geneticus (opleider: Prof. dr. C.E.M. de DieSmulders). Al snel werd duidelijk dat haar hart bij de reproductieve genetica ligt. Haar interesse voor de erfelijkheid en transmissie van mitochondriële aandoeningen werd aangewakkerd door een project over MELAS syndroom in het kader van haar DNAstage. Eind 2011 begon zij tijdens haar opleiding aan het in dit proefschrift beschreven promotieonderzoek, met Prof. dr. H.J.M. Smeets en Prof. dr. C.E.M. de Die-Smulders als promotoren, waarin de beide interesses werden gecombineerd. Als afronding van de opleiding koos zij voor een differentiatiestage Preimplantatie Genetische Diagnostiek (PGD). Sinds eind 2013 werkt ze als klinisch geneticus in Maastricht en is daarnaast aan haar promotieonderzoek blijven werken. Het proefschrift gaat van bench naar bedside en combineert inzichten uit het basale onderzoek met klinische observaties en vice versa. Fundamentele inzichten in de overerving van de mitochondriële ziektes en de preventie van nieuwe ziektegevallen zijn omgezet in concrete praktische handvatten voor de klinisch genetische praktijk, die zowel op wetenschappelijke congressen als ook op nascholingscursussen en patiëntenbijeenkomsten zijn gepresenteerd. Haar aandachtsgebieden binnen de klinische genetica zijn de reproductieve en mitochondriële genetica. Ze is voornemens ook na haar promotie kliniek en research te blijven combineren. Als onderdeel hiervan zal zij zich om te beginnen binnen de specialty Erfelijkheid, Voortplanting en Aanleg (EVA) van het MUMC+ inzetten om de implementatie van preconceptie dragerschapscreening op basis van whole exome sequencing, zoals ontwikkeld in dit proefschrift, te realiseren.

Zij heeft een relatie met Jeroen van Rheenen, met wie zij samenwoont in Maastricht. 

List of publications 



\section{List of publications}

\section{Scientific}

\section{Wi-1}

Sallevelt SCEH, Dreesen JCFM, Drusedau M, Hellebrekers DMEI, Paulussen ADC, Coonen E, van Golde RJT, Geraedts JPM, Gianaroli L, Magli MC, Zeviani M, Smeets HJM, de Die-Smulders CEM. Preimplantation genetic diagnosis for the m.14487T>C mitochondrial DNA mutation resulted in the birth of a healthy boy. Hum Reprod. (Accepted for publication) IF 4.621

Hellebrekers DMEI, Sallevelt SCEH, Theunissen TEJ, Hendrickx ATM, Gottschalk RW, Hoeijmakers JGJ, Habets DD, Bierau J, Schoonderwoerd KG, Smeets HJM. Novel SLC25A32 mutation in a patient with a severe neuromuscular phenotype. Eur J Hum Genet. (Accepted for publication) IF 4.580

Theunissen TEJ, ${ }^{\#}$ Sallevelt SCEH, ${ }^{\#}$ Hellebrekers DMEI, de Koning B, Hendrickx ATM, van den Bosch BJC, Kamps R, Schoonderwoerd K, Szklarczyk R, Mulder-den Hartog ENM, de Coo IFM, Smeets HJM. Resolving blended or composite multigenic disease by whole exome sequencing. J Pediatr. (Accepted for publication) IF $3.890^{\#}$ these authors share first authorship

Sallevelt SCEH, de Koning B, Szklarczyk R, Paulussen ADC, de Die-Smulders CEM, Smeets HJM. A comprehensive strategy for exome-based preconception carrier screening. Genet Med. 2016 Oct 27. doi: 10.1038/gim.2016.153. (Epub ahead of print) IF 7.710

Sallevelt SCEH, de Die-Smulders CEM, Hendrickx ATM, Hellebrekers DMEI, de Coo IFM, Alston CL, Knowles C, Taylor RW, McFarland R, Smeets HJM. De novo mtDNA point mutations are common and have a low recurrence risk. J Med Genet. 2016 Jul 22. pii: jmedgenet-2016-103876. doi: 10.1136/jmedgenet-2016-103876. (Epub ahead of print) IF 5.650

Gerards M, Sallevelt SCEH, Smeets HJM. Leigh syndrome: Resolving the clinical and genetic heterogeneity paves the way for treatment options. Mol Genet Metab. 2016;117(3):300-12. IF 3.093 
Wilson IJ, Carling PJ, Alston CL, Floros VI, Pyle A, Hudson G, Sallevelt SCEH, Lamperti C, Carelli V, Bindoff LA, Samuels DC, Wonnapinij P, Zeviani M, Taylor RW, Smeets HJM, Horvath R, Chinnery PF. Mitochondrial DNA sequence characteristics modulate the size of the genetic bottleneck. Hum Mol Genet. 2016;25(5):1031-41. IF 5.985

Meuwissen MEC, Halley DJJ, Smit LS, Lequin MH, Cobben JM, de Coo IFM, van Harssel J, Sallevelt SCEH, Woldringh G, van der Knaap MS, de Vries LS, Mancini GMS. The expanding phenotype of COL4A1 and COL4A2 mutations: clinical data on 13 newly identified families and a review of the literature. Genet Med. 2015;17(11):843-53. IF 7.710

Smeets HJM, Sallevelt SCEH, Dreesen JCFM, de Die-Smulders CEM, de Coo IFM. Preventing the transmission of mitochondrial DNA disorders using prenatal or preimplantation genetic diagnosis. Ann N Y Acad Sci. 2015;1350:29-36. IF 4.518

Vreeburg M, Sallevelt SCEH, Stegmann APA, van Geel M, Detisch YJHA, SchranderStumpel CTRM, van Steensel MAM, Marcus-Soekarman D. Cutaneous clues for diagnosing X-chromosomal disorders. Clin Genet. 2014;85(4):328-35. IF 3.931

Sallevelt SCEH, Dreesen JCFM, Drüsedau M, Spierts S, Coonen E, van Tienen FHJ, van Golde RJT, de Coo IFM, Geraedts JPM, de Die-Smulders CEM*, Smeets HJM*. Preimplantation genetic diagnosis in mitochondrial DNA disorders: challenge and success. J Med Genet. 2013;50:125-132. IF 5.703* these authors share senior authorship

\section{Book contribution}

Sallevelt SCEH, Dreesen JCFM, de Coo IFM, de Die-Smulders CEM, Smeets HJM. Selecting the right embryo in mitochondrial disorders. (C) Springer International Publishing Switzerland. Screening the Single Euploid Embryo - Molecular Genetics in Reproductive Medicine 2015;Chapter 17:231-246.

\section{Dissertation contribution}

Van Tienen FHJ, Lindsey PJ, Sallevelt SCEH, Frints SG, de Coo IFM, Tiranti V, Smeets HJM. Gene expression profiling reveals differences in pathological processes in skeletal muscle of affected carriers of the m.3243A>G (MELAS) mutation. Thesis F.H.J. van Tienen, Deciphering mitochondrial and molecular pathology in Type 2 Diabetes Mellitus, Chapter 3, 2011. 


\section{Wi-2}

Mans DRA, Sallevelt SCEH, Soekhoe R, Bipat R, Toelsie JR. Evaluation of plants with presumed antihypertensive properties for their potential to decrease peripheral resistance using isolated guinea pig aortic rings precontracted with phenylephrine. Acad J Sur. 2010;1:15-19.

\section{Submitted papers}

Sallevelt SCEH, Dreesen JCFM, Coonen E, Paulussen ADC, Hellebrekers DMEI, de DieSmulders CEM, Smeets HJM, Lindsey P. Preimplantation genetic diagnosis for mitochondrial DNA mutations: analysis of one blastomere suffices.

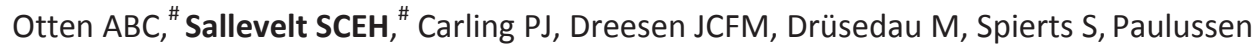
ADC, de Die-Smulders CEM, Herbert M, Chinnery PF, Samuels DC, Smeets HJM. Mitochondrial DNA inheritance is determined by genetic drift and negative and positive selection. " these authors share first authorship

Haer-Wigman L, van Zelst-Stams WAG, Pfundt R, van den Born LI, Klaver CCW, Verheij JBGM, Hoyng CB, Breuning MH, Boon CJF, Kievit AJ, Verhoeven VJM, Pott JWR, Sallevelt SCEH, van Hagen JM, Plomp AS, Kroes HY, Lelieveld SH, Hehir Kwa JY, Castelein S, Nelen M, Scheffer H, Lugtenberg D, Cremers FPM, Hoefsloot L, Yntema HG. Diagnostic exome sequencing in 266 Dutch patients with visual impairment.

Sallevelt SCEH, de Die-Smulders CEM, HJM. The distribution of mtDNA mutations in human patients is defined by random de novo occurrence followed by pre- and postnatal selection.

Kamps R, Szklarczyk R, Theunissen TEJ, Hellebrekers DMEI, Sallevelt SCEH, Boesten I, de Koning B, van den Bosch B, Schoonderwoerd K, de Coo IFM, Vanoevelen J*, Smeets HJM*. Aminoacyl-tRNA synthetase gene defects cause pediatric, mitochondrial cardiomyopathy with early-onset brain disease. * these authors share senior authorship

\section{Conference contributions}

Sallevelt SCEH, de Koning B, Szklarczyk R, Paulussen ADC, de Die-Smulders CEM, Smeets HJM. A comprehensive strategy for exome-based preconception carrier screening. Annual American Society of Human Genetics (ASHG) meeting, October 18-22, 2016, Vancouver, Canada (poster presentation) 
De Die-Smulders CEM, Smeets HJM, Hendrickx ATM, Hellebrekers DMEI, de Coo IFM, Alston CL, Knowles C, Taylor RW, McFarland R, Sallevelt SCEH. De novo mtDNA point mutations are common and have a low recurrence risk. Annual American Society of Human Genetics (ASHG) meeting, October 18-22, 2016, Vancouver, Canada (poster presentation)

Sallevelt SCEH, de Koning B, Szklarczyk R, Paulussen ADC, de Die-Smulders CEM, Smeets HJM. A comprehensive strategy for exome-based preconception carrier screening. NVHG autumn symposium, October $6^{\text {th }}, 2016$, Amsterdam, The Netherlands (oral presentation)

Sallevelt SCEH, Hellebrekers DMEI, Dreesen JCFM, Drüsedau M, Spierts S, Hendrickx ATM, Calis CMH, de Coo IFM, Geraedts JPM, de Die-Smulders CEM, Smeets HJM. Reproductive options for mtDNA diseases. 4th Joint Meeting of the UK /Dutch Clinical Genetics Societies \& Cancer Genetics groups, March 17-18, 2014, Leiden, The Netherlands (oral presentation)

Sallevelt SCEH, Hellebrekers DMEI, Dreesen JCFM, Drüsedau M, Spierts S, Hendrickx ATM, Calis CMH, de Coo IFM, Geraedts JPM, de Die-Smulders CEM, Smeets HJM. Reproductive options for mtDNA disease. Euromit Conference, June 15-19, 2014, Tampere, Finland (poster presentation)

Sallevelt SCEH, Hellebrekers DMEI, Dreesen JCFM, Drüsedau M, Spierts S, Hendrickx ATM, Calis CMH, de Coo IFM, Geraedts JPM, de Die-Smulders CEM, Smeets HJM. Reproductive options exist for most women with affected offspring due to a maternal or de novo mtDNA mutation. ESHG European Human Genetics Conference, June 8-11, 2013, Paris, France (poster presentation)

Sallevelt SCEH, Theunissen TEJ, Eijkenboom I, de Coo IFM, Smeets HJM. AARS2 mutations: case solved by exome sequencing and literature overview. MCRN meeting, May $6^{\text {th }}, 2013$, Copenhagen, Denmark (oral presentation)

Sallevelt SCEH, Hellebrekers DMEI, van den Bosch BJC, Kamps R, Boesten I, Jongen ELC, de Koning B, Gottschalk WHR, Calis CMH, de Die-Smulders CEM, Schoonderwoerd K, de Coo IFM, Smeets HJM. Next-generation sequencing in mitochondrial disease. NVHG autumn symposium, September 20-21, 2012, Arnhem, The Netherlands (oral presentation) 
Sallevelt SCEH, Dreesen JCFM, Drüsedau M, Spierts S, Coonen E, van Golde RJT, Geraedts JPM, de Die-Smulders CEM, Smeets HJM. PGD in mtDNA disorders: challenge and success. 28th Annual Meeting of ESHRE, July 1-4, 2012, Istanbul, Turkey (oral presentation)

Sallevelt SCEH, Dreesen JCFM, Drüsedau M, Spierts S, Coonen E, van Golde RJT, de Coo IFM, Geraedts JPM, de Die-Smulders CEM, Smeets HJM. PGD in mtDNA disorders: the Maastricht experience. 22nd Genetics Retreat, March 15-16, 2012, Kerkrade, The Netherlands (oral presentation)

Sallevelt SCEH, Dreesen JCFM, Drüsedau $M$, Spierts $S$, Coonen E, van Golde RJT, Geraedts JPM, de Die-Smulders CEM, Smeets HJM. PGD in mtDNA disorders: the Maastricht experience. LOG VKGN/VKGL/NVGC, December $15^{\text {th }}, 2011$, Rotterdam, The Netherlands (oral presentation)

Sallevelt SCEH, Gómez García EB. Looking at Gorlin syndrome from a new perspective: the cilia of hedgehog. ESHG European Human Genetics Conference, May 28-31, 2011, Amsterdam, The Netherlands (poster presentation)

Sallevelt SCEH, Gómez García EB. Erfelijk medulloblastoom: cilia afwijkingen en therapeutische implicaties. KNOP symposium Erfelijkheid en kinderhersentumoren, May 16th, 2011, Rotterdam, The Netherlands (oral presentation)

Sallevelt SCEH, Alders M, Stegmann APA, Delhaas T, Maas SM, Schrander JJP, de DieSmulders CEM, Frints SGM. Long QT syndrome in a girl with Beckwith Wiedemann syndrome and clinical features of Simpson Golabi Behmel syndrome: just coincidence? 21st European meeting on Dysmorphology, September 2-3, 2010, Strasbourg, France (oral presentation)

Sallevelt SCEH, Stegmann APA, van Oort M, Helderman-van den Enden ATJM, MarcusSoekarman D. Co-incidental finding of deletion of a tumor suppressor gene: consequences for counseling? 1st Joint Meeting of the UK /Dutch Clinical Genetics Societies \& Cancer Genetics groups, March 10-12, 2010, Amsterdam, The Netherlands (poster presentation) 


\section{Nominations and awards}

Sallevelt SCEH, de Koning B, Szklarczyk R, Paulussen ADC, de Die-Smulders CEM, Smeets HJM. A comprehensive strategy for exome-based preconception carrier screening. NVHG autumn symposium, October $6^{\text {th }}, 2016$, Amsterdam, The Netherlands (oral presentation): annual young investigators award for best presentation

Sallevelt SCEH, Dreesen JCFM, Drüsedau M, Spierts S, Coonen E,van Golde R, Geraedts JPM, de Die-Smulders CEM, Smeets HJM. PGD in mtDNA disorders: the Maastricht experience. LOG VKGN/VKGL/NVGC, December $15^{\text {th }}, 2011$, Rotterdam, The Netherlands (oral presentation): annual award for best presentation

Sallevelt SCEH, Gómez García EB. Looking at Gorlin syndrome from a new perspective: the cilia of hedgehog. European Human Genetics Conference, May 28-31, 2011, Amsterdam, The Netherlands (poster presentation): among the best scored posters of that year's submissions, candidate for ESHG Poster Award for Young Investigators

\section{Public/patients}

\section{$\mathrm{Wn} / \mathrm{Vp}$}

Krapels IPC, Helderman-van den Enden ATJM, Sallevelt SCEH, Hoos MB, de Vos-Houben JMJ, Hazebroek MR, van den Wijngaard A, Heymans SRB. Familiaire gedilateerde cardiomyopathie. Modern Medicine 2012;7/8.

Martens JHA, Sallevelt SCEH, Vrinte S, van de Ven P.H.M. Peer tutoring op het Elzendaalcollege; een palet aan teksten. Moer Tijdschrift voor het onderwijs in het Nederlands 2003;35(4):110-127.

\section{Patient magazine}

Sallevelt SCEH, de Coo IFM, Smeets HJM. De zoektocht naar de erfelijke oorzaak van mitochondriële ziekten. Wisselstof, uitgave van de Vereniging voor Volwassenen en Kinderen met Stofwisselingsziekten (VKS) maart 2013;19e jaargang. 


\section{Patient meetings}

Sallevelt SCEH. Genetische diagnostiek van congenitale / metabole spierziekten. Spierziektecongres van Spierziekten Nederland en het Prinses Beatrix Spierfonds, 10 september 2016, Veldhoven, Nederland (mondelinge presentatie)

Sallevelt SCEH, Hellebrekers DMEI, Dreesen JCFM, Wolfe R, Drüsedau M, Hendrickx ATM, Spierts S, van Golde RJT, Coonen E, de Coo IFM, Geraedts JPM, Chinnery PF, de Die-Smulders CEM, Smeets HJM. Preïmplantatie genetische diagnostiek (PGD) biedt draagsters van mtDNA-mutaties een reële kans op gezonde nakomelingen. Spierziektencongres van Spierziekten Nederland en het Prinses Beatrix Spierfonds, 15 september 2012, Veldhoven, Nederland (poster presentatie)

Van den Bosch BJC, Sallevelt SCEH, Hellebrekers DMEI, Gerards M, Kamps R, Boesten I, Jongen ELC, de Koning B, Schoonderwoerd K, Sefiani A, Ratbi I, Coppieters W, Karim L, de Coo IFM, Smeets HJM. Ontrafeling van genetische oorzaken van mitochondriële ziekten. Spierziektencongres van Spierziekten Nederland en het Prinses Beatrix Spierfonds, 15 september 2012, Veldhoven, Nederland (poster presentatie) 

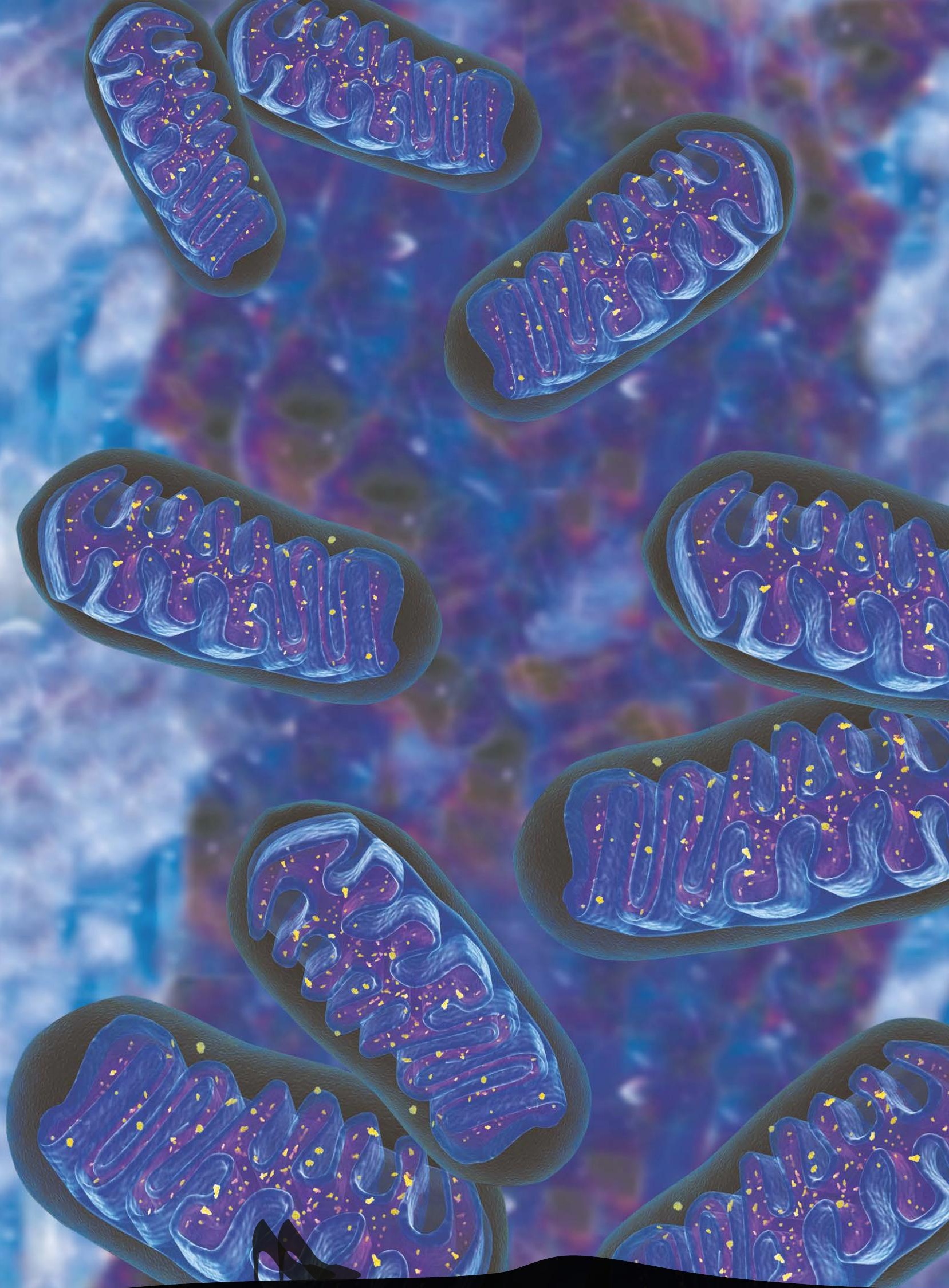

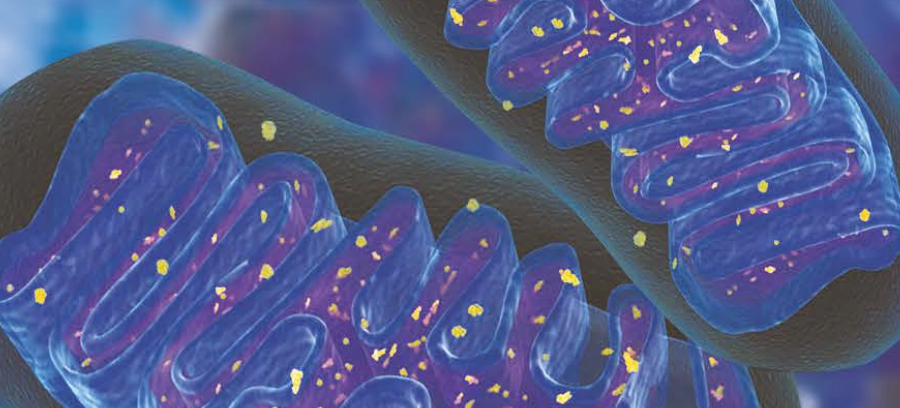

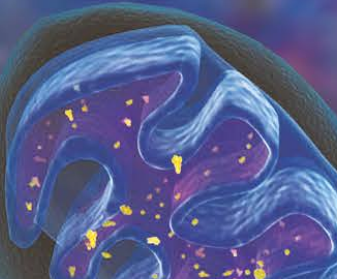
(1) $3=0$ 